


\section{Estudio de sistemas combinados de combustión catalítica de VOCs}

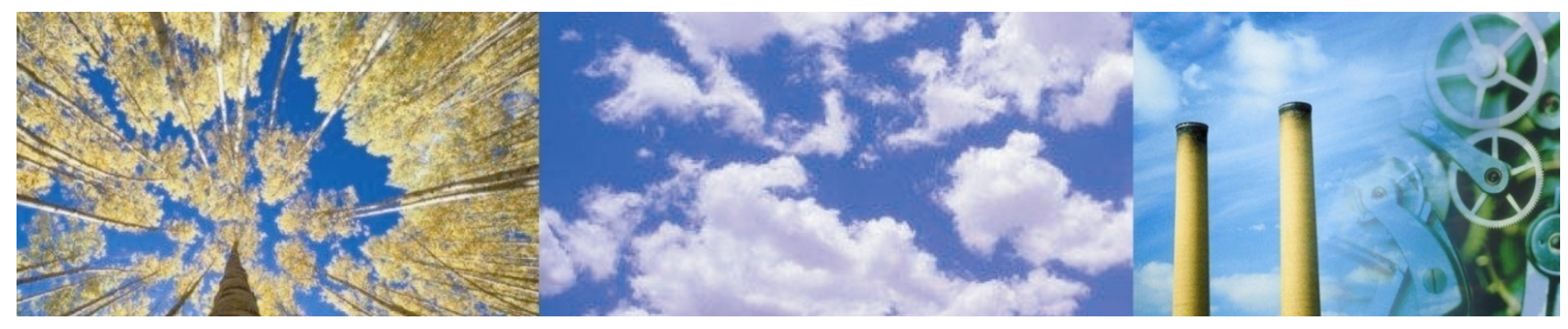

María Agustina Campesi 

A mi mamá y mi papá, Corina y Miguel. 

ESTUDIO DE SISTEMAS

COMBINADOS DE

COMBUSTION

V catalitica de VOCs

agRADECIMIENTOS 

Agradecimientos

A mi Director Osvaldo M. Martínez, Co-director Guillermo F. Barreto por su prolífica enseñanza, dedicación exclusiva y contención.

Mis agradecimientos al Centro de Investigación y Desarrollo en Procesos Catalíticos y a la Facultad de Ingeniería de la Universidad Nacional de La Plata.

Al Consejo Nacional de Investigaciones Científicas y Técnicas por su apoyo económico. A mis compañeros de trabajo por la colaboración brindada en diferentes aspectos del desarrollo de mi Tesis.

A Clarisa por haberme motivado y apoyado en la concreción de esta etapa de mi vida.

A mis Amigas por haberme acompañado en todo momento.

A mi Familia por su apoyo incondicional y contención permanente. 

ESTUDIO DE SISTEMAS

COMBINADOS DE

COMBUSTION

$\checkmark$ CATALITICA DE VOCs

INDICE GENERAL 

CAPÍTULO 1: Introducción y planteo de la problemática

pág.19

1.1 Técnicas de destrucción de VOCs

pág. 20

1.1.1 Incineración o combustión

pág. 20

1.1.1.1 Combustión térmica

pág. 20

1.1.1.2 Combustión catalítica

pág. 24

1.1.2 Biofiltración

pág. 26

1.1.3 Fotocatálisis

pág. 28

1.2 Técnicas de recuperación de VOCs

pág.29

1.2.1 Adsorción

pág.29

1.2.2 Absorción

pág.31

1.2.3 Condensación

pág.32

1.2.4 Membrana

pág.32

pág.33

1.3 Criterios de selección de las técnicas de tratamiento de VOCs

pág.35

1.4 Objetivos para el desarrollo de la Tesis

pág.36

CAPÍTULO 2: Sistema combinado de eliminación de VOCs

pág.41

2.1 Elección de la técnica de eliminación de VOCs

pág.41

2.2 Conclusiones

pág.47

CAPÍTULO 3: Adsorción

pág.51

3.1 Técnicas de adsorción/desorción

3.1.1 Temperature Swing Adsorption (TSA)

pág.52

3.1.2 Pressure Swing Adsorption (PSA)

pág.53

pág.53

3.1.2.1 Layered Bed Pressure Swing Adsorption (LBSA)

pág.54

3.1.2.2 Vacuum Swing Adsorption (VSA)

pág.54

3.1.3 Thermally Coupled Pressure Swing (TCPS)

pág. 55

pág.56

pág.57

3.2.1 Carbón activado

pág.57

3.2.2 Zeolitas

pág. 58

3.2.3 Adsorbentes poliméricos

pág. 58

3.3.1 Tamaño de la molécula de adsorbato vs tamaño de poro del adsorbente

pág.59 
3.3.2 Microporosidad

pág.59

3.3.3 Afinidad del adsorbente por algunas sustancias

pág. 59

3.3.4 Costos y frecuencia de reemplazo

pág.59

3.3.5 Temperatura de desorción

pág.59

pág.60

pág.64

3.5 Disposición del material adsorbente

pág.66

CAPÍTULO 4: Simulación del sistema de adsorción/desorción sin considerar las resistencias a la transferencia de calor y materia

pág.71

4.1 Sistema isotérmico de un componente

pág.71

4.2 Sistema adiabático multicomponente

pág. 80

4.2.1 Construcción de la solución continua para la aplicación de un escalón

pág. 83

4.2.2 Construcción de las soluciones discontinuas para la aplicación de un escalón

4.2.2.1 Discontinuidad de contacto

pág. 88

4.2.2.2 Ondas de choque

pág. 88

pág.90

4.2.2.3 Soluciones combinadas para la aplicación de un escalón

pág.95

pág.98

4.3 Adsorción simultánea de acetato de etilo y etanol

pág.100

4.3.1 Etapa de adsorción

pág.108

4.3.3 Conclusiones obtenidas a partir de modificar las condiciones operativas del sistema

pág.116

4.4 Conclusiones

pág.117

Apéndice 4.1 / Componentes de la matriz $\mathcal{F}\left(\mathrm{q}_{\mathrm{i}, \mathrm{j}}\right.$ : Derivadas primeras de $\left.\mathrm{q}_{\mathrm{i}}(\underline{\mathrm{C}})\right)$ pág. 120

Apéndice 4.2 / Derivadas primeras de las componentes de la matriz $\mathcal{F}$

pág. 121

Apéndice 4.3 / Determinación de las derivadas de los autovalores $\sigma^{\mathrm{k}}$ de la matriz $\mathcal{F}$ respecto de $\mathrm{C}_{1}, \mathrm{C}_{2}$ y $\mathrm{T}$

pág. 123

CAPÍTULO 5: Simulación del sistema de adsorción/desorción considerando

las resistencias a la transferencia de calor y materia

pág. 127

5.1 Balances de materia dentro y fuera del sólido adsorbente para un único componente en un sistema isotérmico

pág. 127

5.2 Método de Colocaciones Ortogonales

pág. 129

5.2.1 Un punto de colocación ortogonal

pág.133

5.2.2 $\mathrm{N}$ puntos de colocación ortogonal

pág.134 
5.3 Resolución del balance en la corriente de fluido

pág.136

5.3.1 Tanques agitados en serie

pág.136

5.3.2 Método Cuasi-Analítico

pág.137

5.3.2.1 N puntos de colocación ortogonal

pág.137

5.3.3 Comparación entre la aproximación del sistema de tanques agitados en serie y el Método Cuasi-Analítico

pág.139

5.4 Adsorción/desorción simultánea de acetato de etilo y etanol considerando las resistencias a la transferencia de calor y materia

pág.140

5.4.1 Propiedades del material adsorbente y selección de las características geométricas del adsorbedor

pág.143

5.4.1.1Características del material adsorbente: carbón activado

pág.143

5.4.1.2 Características geométricas del monolito

pág.146

5.4.1.3 Características geométricas del lecho relleno

pág.149

5.4.2 Monolitos vs. Lecho Relleno: efecto de las resistencias internas y externas

pág.150

5.4.3 Monolito vs. Lecho Relleno: efecto del número de puntos de colocación

pág.155

5.5 Efecto de las resistencias externas e internas a la transferencia de calor y materia

5.6 Influencia mutua de los componentes en el proceso de adsorción/desorción

pág.159

5.7 Conclusiones

pág.163

pág.170

APÉNDICE 5.1 / Determinación de los puntos de colocación para el caso de un lecho relleno con partículas esféricas

pág.173

APÉNDICE 5.2 / Evaluación de propiedades termodinámicas y de transporte

pág. 175

CAPÍTULO 6: Análisis de los ciclos de adsorción/desorción y caudal mínimo de desorción

pág.183

6.1 Sistema de dos lechos que operan alternativamente vs. concentrador rotativo

6.1.1 Características geométricas del concentrador rotativo

pág.185

6.2 Determinación del período que define el ciclo de adsorción/desorción

pág. 186

6.3 Determinación del caudal mínimo ( $\mathbf{W}_{\mathrm{des}}^{\mathrm{mín}}$ )

pág.189

6.4 Influencia de la relación $\mathrm{D} / \mathrm{L}$ en el valor de $\mathbf{W}_{\mathrm{des}}^{\mathrm{mí}}$

pág.190

6.5 Efecto de la relación entre áreas de adsorción y desorción

pág.191

6.6 Influencia de la cantidad de adsorbente sobre el caudal mínimo

de desorción: $\mathbf{W}_{\text {des }}^{\text {mín }}$

pág.195 
6.7 Criterio de diseño: Zona de Transferencia de Masa (ZTM) pág.198

6.8 Método aproximado de cálculo_ pág.199

6.9 Conclusiones pág.204

CAPÍTULO 7: Combustión catalítica: determinación de parámetros cinéticos pág.209

7.1 Estudio cinético de la combustión de acetato de etilo y etanol sobre un catalizador de $\mathrm{MnCu}$

pág. 210

7.1.1 Descripción del sistema experimental

pág. 210

7.1.2 Análisis cualitativo de los resultados experimentales

pág. 213

7.1.3 Interacción en la combustión conjunta de etanol y acetato de etilo

pág. 217

7.1.4 Modelo del sistema experimental

pág.222

7.1.5 Procedimiento de regresión

pág. 224

7.1.6 Resultados

pág. 225

7.2 Conclusiones

pág.234

APÉNDICE 7.1 / Presentación de las características del catalizador y evaluación de la difusividad efectiva

pág. 236

CAPÍTULO 8: Simulación del Reactor

pág.241

8.1 Modelo del reactor catalítico

pág.242

8.1.1 Expresiones cinéticas y evaluación de las velocidades efectivas de consumo

pág.244

8.1.1.1 Procedimiento aproximado para evaluar las velocidades efectivas de consumo $\left(\mathrm{r}_{\mathrm{j}}^{\mathrm{ef}}\right)$

pág.247

8.2. Resultados y discusión

pág.252

8.2.1 Comparación de la aproximación con el procedimiento numérico

pág. 252

8.2.2 Análisis del comportamiento del reactor

pág. 254

8.3 Conclusiones

pág. 258

CAPÍTULO 9: Sistema de recuperación térmica

pág.263

9.1 Selección del sistema de intercambio térmico

pág.264

9.2 Características geométricas y modelo de cálculo de un intercambiador de flujo cruzado tipo "plate-fin" (con ambos fluidos "unmixed") pág.266

9.3 Definición de las variables geométricas

pág.271

9.4 Conclusiones

pág. 273 
APÉNDICE 9.1 / Intercambiadores de flujo cruzado con ambos fluidos unmixed

CAPÍTULO 10: Análisis del impacto que causa la incorporación de un concentrador sobre el reactor catalítico e intercambiadores de calor

pág.281

10.1 Factor de concentración, Fc

pág.281

10.2 Impacto del Fc sobre el reactor catalítico

pág. 282

10.3 Presentación de tres sistemas de eliminación de VOCs

pág. 285

10.3.1 $\mathrm{Fc}=1$

pág. 286

10.3.2 $\mathrm{Fc}=3.67$

pág.287

10.3.3 Fc $=5.88$

pág. 290

10.4 Evaluación de resultados

pág.292

10.5 Selección de la unidad impulsora

pág. 294

10.6 Conclusiones

pág. 297

Conclusiones y recomendaciones

pág.301

Nomenclatura

pág. 313

Bibliografía

pág.321 

ESTUDIO DE SISTEMAS

COMBINADOS DE

COMBUSTION

CATALITICA DE VOCs

\section{1/ INTRODUCCION Y PLANTEO DE LA PROBLEMATICA}

Técnicas de destrucción de VOCs

Técnicas de recuperación de VOCs

Criterios de selección de las técnicas de tratamiento de VOCs

Objetivos para el desarrollo de la Tesis

Descripción del contenido de la Tesis 



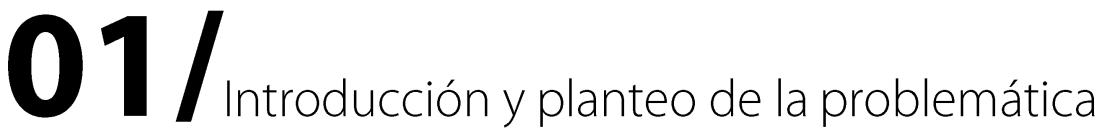

En las últimas décadas, el desmedido aumento en la contaminación del aire ha convertido al control de la polución en uno de los objetivos prioritarios a analizar desde la investigación, el desarrollo de tecnologías y la puesta en marcha de las regulaciones ambientales. Entre los contaminantes del aire más comunes, se encuentran los Compuestos Orgánicos Volátiles (denominados VOCs por sus siglas en inglés). Los VOCs emitidos a la atmósfera pueden ser de origen natural (cloroplastos vegetales) o antropogénicos (emisiones fugitivas de escape, procedentes de fuentes móviles o estacionarias). Las plantas industriales representan gran parte de las fuentes de escape estacionarias.

Las industrias elaboradoras de envases ocasionan una importante contaminación del aire, con solventes que utilizan en sus procesos de impresión. Entre los solventes típicamente empleados se encuentran el acetato de etilo y el etanol. Esta situación, que requiere la purificación de una corriente de aire que contiene estas sustancias, constituye el caso base sobre el cual se realizará este estudio.

Las técnicas de tratamiento de efluentes gaseosos pueden clasificarse en dos grupos:

a- Las que realizan alguna modificación en el proceso y en los equipos (técnicas de prevención).

b- Las que implican algún método de tratamiento de las emisiones de VOCs.

Las medidas preventivas (a) incluyen mejoras en el proceso, desde la inspección y mantenimiento para evitar fugas, hasta el rediseño completo de instalaciones para minimizar los productos orgánicos residuales. Cuando no son posibles o suficientes dichas actuaciones sobre productos o los propios procesos, deben aplicarse sistemas de control de final de tubería (b). Las distintas alternativas disponibles para el tratamiento de VOCs presentes en efluentes gaseosos, principalmente aire, se clasifican en tecnologías de eliminación y tecnologías de recuperación. El objetivo específico de la aplicación y/o un análisis económico del proceso son los factores que permiten determinar la utilización de una u otra opción (Khan y Ghoshal, 2000; Everaert y Baeyens, 2002). 
Si bien existe una abundante bibliografía sobre diferentes aspectos del tratamiento de eliminación de VOCs, la mayor parte de los trabajos abordan aspectos específicos de cada una de las técnicas disponibles. Teniendo en cuenta que aquí solo se pretende realizar una revisión general de las mismas, destacando sus características distintivas, se utilizarán las descripciones realizadas en un conjunto de trabajos de revisión (de la Fuente García y Gutiérrez Martín, 1998; Khan y Goshal, 2000; Everaert y col., 2002; Moretti, 2002).

\subsection{Técnicas de destrucción de VOCs}

Como su nombre lo indica el objetivo de estas técnicas es eliminar los VOCs presentes en la corriente a tratar. Los VOCs pueden ser eliminados mediante una combustión, térmica o catalítica, mediante un proceso de biofiltración o por un procedimiento fotocatalítico. Tales tecnologías se describen a continuación:

\subsubsection{Incineración o combustión}

La incineración o combustión es, definitivamente, el método destructivo más importante en la actualidad, ya sea por vía térmica o catalítica. Los sistemas de incineración constan de tres subsistemas básicos, el quemador (para iniciar y mantener la reacción), la cámara de oxidación (en general un recipiente de tipo cilíndrico construido de un material resistente a la temperatura) y el economizador de calor de los gases depurados (para precalentar la corriente de entrada u otros propósitos como generar vapor).

\subsubsection{Combustión térmica}

La combustión térmica consiste en la oxidación de VOCs en presencia de oxígeno a una elevada temperatura, durante el tiempo necesario, para lograr una combustión completa a $\mathrm{CO}_{2}$ y $\mathrm{H}_{2} \mathrm{O}$ como únicos productos. El tiempo de contacto, la temperatura y la relación $\mathrm{O}_{2} / \mathrm{VOCs}$ afectan la velocidad y la eficiencia del proceso de combustión.

Los productos de la combustión completa, $\mathrm{CO}_{2}$ y $\mathrm{H}_{2} \mathrm{O}$, son relativamente innocuos, haciendo a la incineración un método atractivo de disposición de residuos. Cuando están presentes en la mezcla compuestos clorados o que contienen azufre, los productos de la combustión completa incluyen los 
componentes ácidos $\mathrm{HCl}$ y $\mathrm{SO}_{2}$ respectivamente, además de $\mathrm{CO}_{2}$ y $\mathrm{H}_{2} \mathrm{O}$. En general, estas corrientes requerirán la remoción de los componentes ácidos por medio de una unidad absorbedora.

El incinerador térmico (Figura 1) consiste principalmente en un equipo de combustión en el cual la llama se mantiene por la combinación de un combustible auxiliar, los componentes presentes en la corriente de gas a tratar y aire suplementario. Cuando el gas a tratar pasa a través de la llama es calentado desde la temperatura obtenida en el precalentamiento a la entrada, hasta la temperatura de ignición. Ésta última varía para diferentes compuestos y se la suele determinar empíricamente; ésta es la temperatura a la cual la energía aportada por la reacción de combustión, excede las pérdidas de calor, elevando por esta razón la temperatura de los gases a algún valor más alto. Así, cualquier mezcla de compuestos orgánicos/aire se encenderá si sus temperaturas son elevadas a un nivel suficientemente alto.

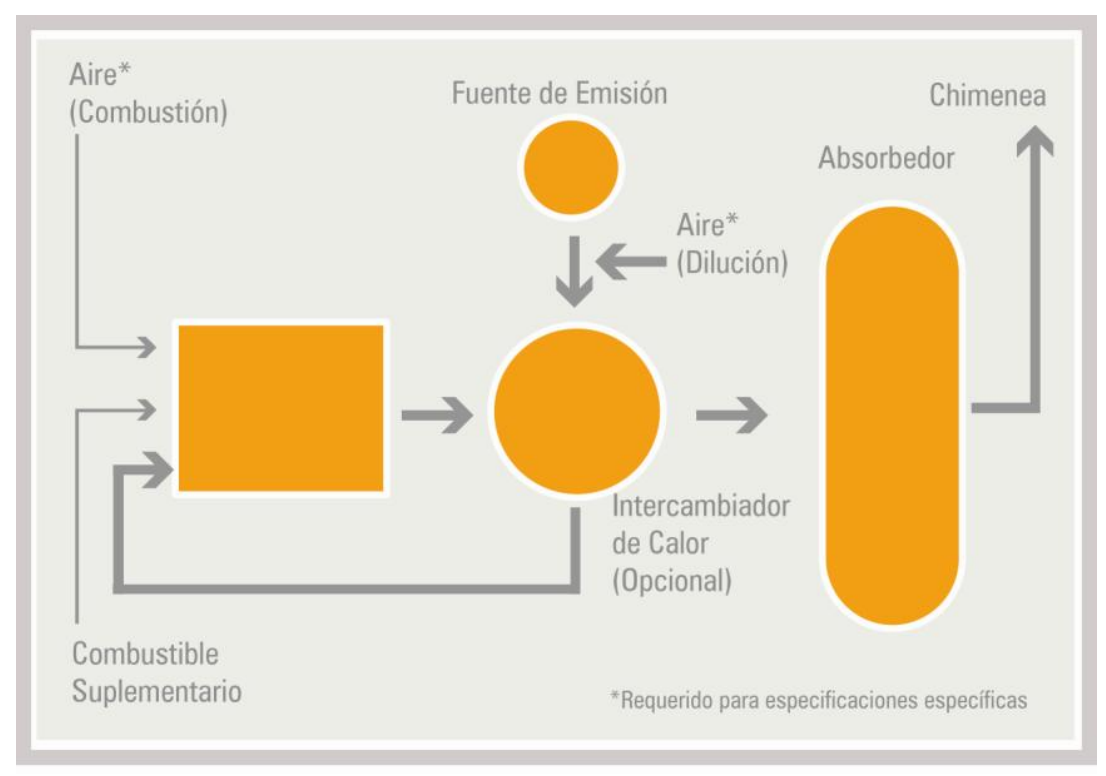

Fig. 1 - Incinerador térmico

Por consideraciones de seguridad, la máxima concentración de VOCs en el gas residual a tratar debe ser sustancialmente menor que el límite inferior de explosividad (en inglés - LEL - lower explosive limit) de los componentes específicos que están siendo controlados.

El LEL de un compuesto orgánico dado, es la concentración mínima de dicho compuesto en aire capaz de mantener la combustión (Lewandowski, 2000). 
Como regla, se utiliza un factor de seguridad de 4 en menos (25\% del LEL); a pesar de que algunos equipos de oxidación son capaces de operar en forma segura sobre este nivel. En caso de ser necesario, el gas residual debe ser diluido con aire del ambiente para, de esta manera, poder disminuir su concentración. Si bien este tipo de consideración debe ser tenida en cuenta por cuestiones de seguridad, normalmente no es una cuestión significativa cuando se tratan corrientes con VOCs mediante métodos destructivos, ya que las mismas suelen encontrarse muy diluidas. Cuando los niveles de concentración de VOCs son altos, generalmente se imponen los sistemas de recuperación de los mismos, como se discutirá posteriormente.

El nivel de control requerido de $\mathrm{VOC}$ en el gas residual que debe alcanzarse durante el tiempo en el que el gas se encuentra en la cámara de combustión térmica, determina la temperatura del reactor. Cuanto menor sea el tiempo de residencia del gas dentro de la cámara, mayor debe ser la temperatura del reactor. Muchos incineradores térmicos están diseñados para proveer no más de un segundo de tiempo de residencia del gas residual con temperaturas típicas de 650 a $1000{ }^{\circ} \mathrm{C}$. Una vez que la unidad fue diseñada y construida no es fácil modificar el tiempo de residencia, de esta manera, la temperatura de reacción requerida, resultará una función de las especies gaseosas particulares y del nivel de control deseado (Moretti, 2002).

Para asegurar un $98 \%$ de destrucción de compuestos orgánicos no halogenados, los incineradores comerciales generalmente deben ser operados por encima de $870^{\circ} \mathrm{C}$ con un tiempo de residencia nominal de 0.75 segundos. La destrucción de compuestos orgánicos halogenados puede requerir una temperatura de oxidación cercana a $1100{ }^{\circ} \mathrm{C}$ y resulta aconsejable implementar una post-oxidación (Moretti, 2002).

Las altas temperaturas requeridas en los procesos de combustión térmica pueden conducir a la formación de NOx, lo cual representa una desventaja en este tipo de tratamiento de VOCs. Aunque existen alternativas para el control de la formación de NOx, de producirse los mismos, debería realizarse un tratamiento ulterior de la corriente de salida de la cámara de combustión, por su carácter contaminante (Lewandowski, 2000). 
Existen tres tipos de sistemas de oxidación térmica, que se pueden diferenciar de acuerdo a la inclusión, o no, de recuperación de calor y a la forma en que se lleva a cabo la misma. Estas alternativas son:

- de llama directa

- recuperativo

- regenerativo

El incinerador térmico de llama directa, también conocido como "afterburner", consiste en una cámara de combustión y no incluye recuperación de calor (Moretti, 2002).

El incinerador recuperativo incluye un precalentamiento de la corriente a tratar haciendo uso de la energía térmica obtenida en la reacción de combustión. Con esta finalidad se dispone de un intercambiador de calor. Un esquema de la configuración descripta se puede observar con mayor detalle en la Figura 2.

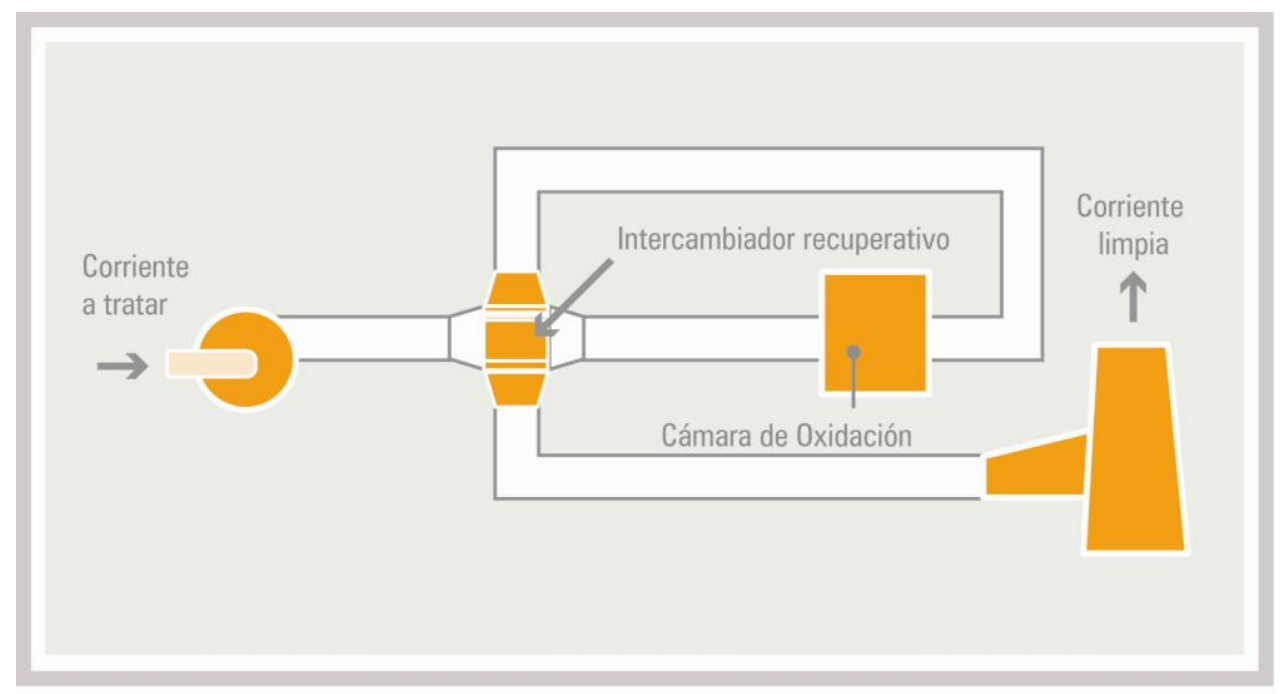

Fig. 2 - Incineración con sistema recuperativo

Los sistemas regenerativos pueden operar con dos lechos paralelos de material de alta capacidad y resistencia térmica, de acuerdo al esquema mostrado en la Figura 3. Los lechos "almacenan" el calor de los gases calientes provenientes de la cámara de combustión y lo ceden a la corriente fría de entrada en forma alternativa. Esta operación se logra mediante válvulas especiales que invierten el flujo (15-60 ciclos/h) (de la Fuente García y Gutierrez Martin, 1998). Otra alternativa dentro de los sistemas regenerativos son los intercambiadores rotatorios, consistentes en un lecho cilíndrico, con una matriz de relleno, y con 
una partición, de tal manera que se mantiene una posición espacial fija para las corrientes frías y calientes (se eliminan válvulas) y gira el propio intercambiador, permitiendo de esa manera el contacto alternado de las corrientes fría y caliente con la matriz de relleno (Shah y Sekulic, 2003).

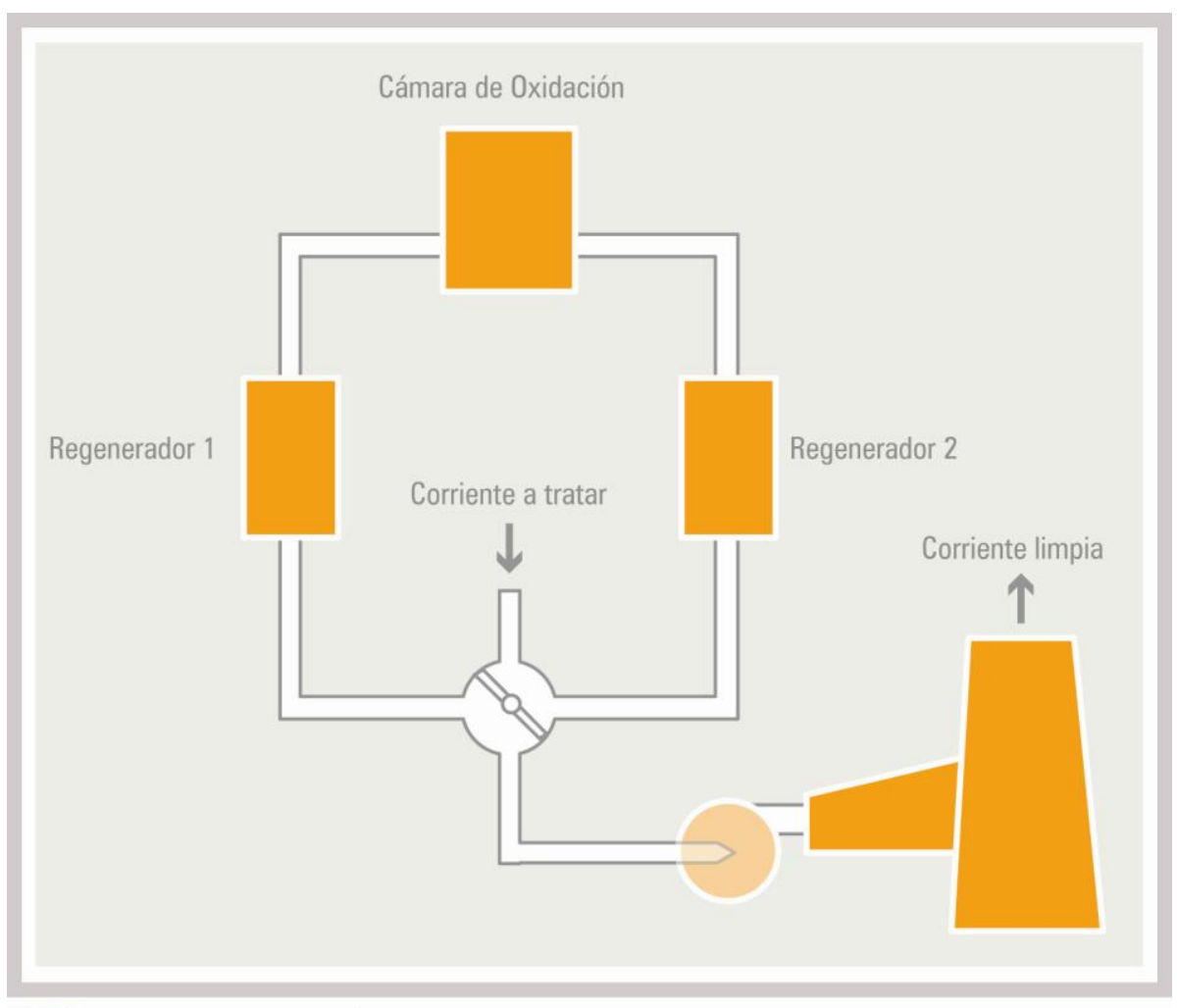

Fig. 3 - Incineración con sistema regenerativo

Estos sistemas, para grandes volúmenes de aire y bajas concentraciones de VOCs, alcanzan eficiencias térmicas superiores al 90\%, lo que permite trabajar con bajo, e incluso nulo, consumo de combustible (de la Fuente García y Gutierrez Martin, 1998).

La ventaja que el sistema recuperativo presenta respecto al sistema regenerativo, es que el primero requiere de tiempos más cortos para que el intercambiador de calor alcance las condiciones de operación y una mayor sencillez operativa.

\subsubsection{Combustión catalítica}

Los reactores de combustión catalítica consisten en lechos fijos o estructurados. En la Figura 4 puede observarse un sistema de combustión catalítica. 


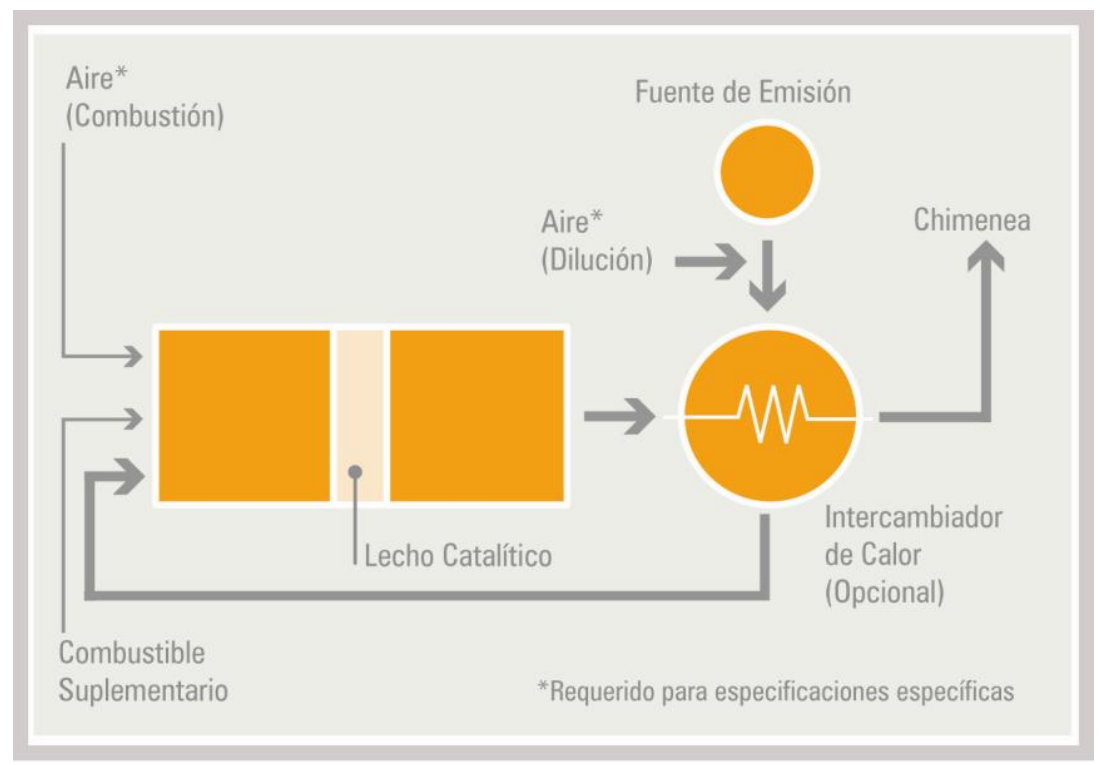

Fig. 4 - Incineración con combustión catalítica

En la oxidación catalítica, la corriente de gas es introducida al reactor catalítico donde se produce la combustión. Una vez que la corriente de gas caliente ingresa al reactor catalítico, el oxígeno y los VOCs difunden, desde la corriente de gas hacia los sitios catalíticos, donde se produce la oxidación. Los productos resultantes de la reacción son luego desorbidos de los sitios activos y difunden de regreso a la corriente gaseosa. El gas a tratar debe ser precalentado para ingresar al reactor a la temperatura adecuada (depende del catalizador empleado), con esta finalidad se suele hacer pasar el gas a través de un intercambiador de calor, en el cual se eleva la temperatura de la corriente empleando el calor generado en la combustión.

El sistema catalítico debe optimizarse en función de los requisitos de aplicación diseñando correctamente sus componentes: soporte y material activo (actividad, superficie específica, estabilidad, difusión de reactivos y productos).

El catalizador provee mecanismos alternativos para que se produzca la reacción de oxidación, permitiendo que la misma ocurra a menores temperaturas que las requeridas para la incineración térmica. Los catalizadores usados para la eliminación de VOCs incluyen como materiales activos a los óxidos metálicos o metales nobles (Moretti, 2002).

El empleo de la eliminación catalítica presenta ventajas significativas respecto a la incineración térmica: repercute en un ahorro de energía, permite trabajar con 
una menor cantidad de oxígeno y genera bajos niveles de óxidos de nitrógeno $\left(\mathrm{NO}_{\mathrm{x}}\right)$, un contaminante muy agresivo para el medio ambiente.

Tiene además la ventaja de poder emplearse para flujos variables; sus principales inconvenientes respecto a la oxidación térmica son el posible envenenamiento del catalizador y su costo. Adicionalmente, aunque siempre se trata de oxidaciones, existen combinaciones más adecuadas del tipo de VOC y el material catalítico a utilizar, lo que requiere estudios específicos.

\subsubsection{Biofiltración}

Este proceso (Figura 5), desarrollado originalmente con el objetivo de disminuir el olor de gases residuales, ha probado ser un método de remoción de VOCs efectivo y económicamente accesible. La biofiltración es una técnica basada en la habilidad de microorganismos (generalmente bacterias) para convertir, bajo condiciones aeróbicas (en presencia de oxígeno), contaminantes orgánicos en $\mathrm{H}_{2} \mathrm{O}, \mathrm{CO}_{2}$ y biomasa.

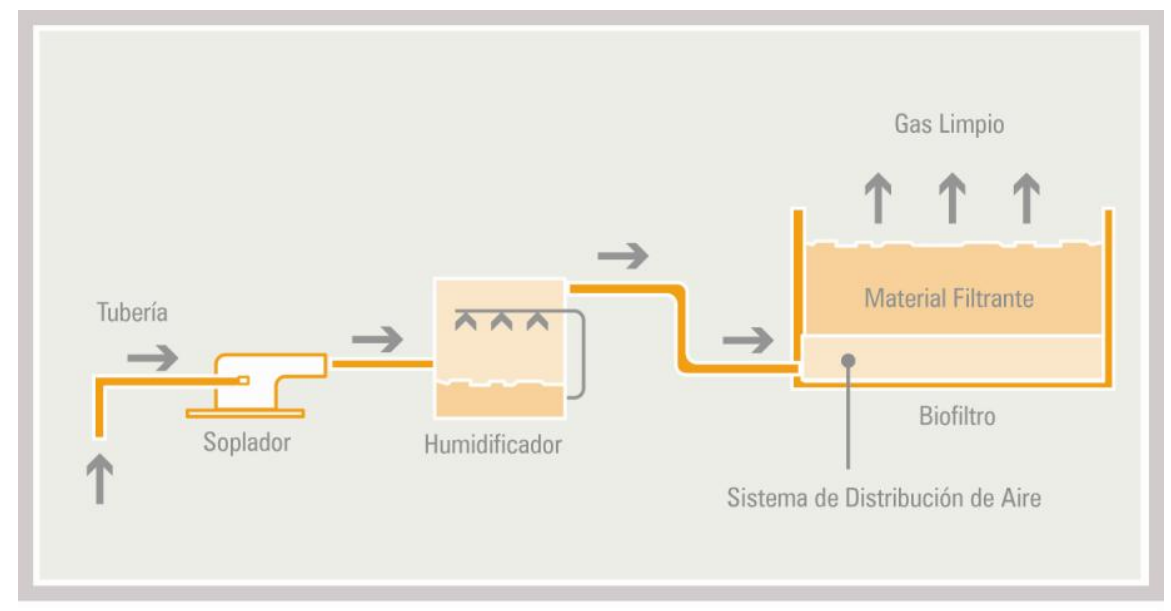

Fig. 5 - Sistema de biofiltración

El componente clave de la biofiltración es el biofiltro, el cual consiste en un lecho relleno de materiales naturales como compost, tierra, etc., rodeados de una microflora inmovilizada. El biofiltro debe mantenerse a una humedad elevada, y para esto se lo trata de conservar mojado mediante el uso de dispersores los cuales proveen agua continuamente. El nivel de humedad es importante para el funcionamiento adecuado y la eficiencia del biofiltro, debido a que el proceso de degradación es exotérmico y tiende a secar los lechos filtrantes. 
Los contaminantes orgánicos en la corriente de aire a tratar se disuelven en agua y luego son convertidos por los microorganismos en una biopelícula. El tipo de microorganismo depende del tipo de VOC a eliminar. Muchos microorganismos requieren condiciones de $\mathrm{pH}$ neutras.

Los materiales naturales usados como sustrato filtrante brindan los nutrientes necesarios para soportar el crecimiento y la supervivencia de los microorganismos. La descomposición de las células también lleva a la pérdida de nutrientes. El tamaño de la partícula de sustrato es seleccionado para proveer una mayor superficie de absorción y una mínima resistencia al flujo (Moretti, 2002).

El grado de absorción es una función de las propiedades físico-químicas de los contaminantes como: solubilidad en el agua, constante de Henry, peso molecular. Una vez absorbidos los contaminantes, los microorganismos los convierten en $\mathrm{CO}_{2}$, $\mathrm{H}_{2} \mathrm{O}$, productos inorgánicos y biomasa.

El tipo más adecuado de biofiltro para emisiones de plantas químicas es un lecho fijo, donde la temperatura y la humedad pueden ser controladas de una manera efectiva y el monitoreo de los efluentes es sencillo. Algunos diseños incluyen una pre-humidificación de la corriente de gas.

Componentes de bajo peso molecular que son solubles y contienen átomos de oxígeno son buenos candidatos para la biofiltración. Aldehídos, cetonas, alcoholes, éteres, ésteres y ácidos orgánicos se degradan rápidamente en biofiltros, pero hidrocarburos halogenados e hidrocarburos poliaromáticos no.

Se han alcanzado eficiencias de remoción superiores al 90\% para compuestos fácilmente degradables (Moretti, 2002).

La degradación de compuestos con azufre, nitrógeno y halógenos llevan a la formación de ácidos que bajan el pH de las capas en biofiltros. Compuestos alcalinos se suman al material filtrante para el control de $\mathrm{pH}$; sin embargo, después de un período de tiempo de operación se forma una acumulación de sales que obstruye la circulación en el lecho. Los lechos tienen una vida útil de entre 2 y 5 años, que con lavados periódicos puede extenderse.

Los biofiltros no presentan los problemas energéticos de las oxidaciones térmica (en mayor medida) o catalítica (en menor medida), y pueden ser competitivos para bajas concentraciones de compuestos que sean fácilmente biodegradables, pero pierden ventajas para altas cargas de contaminantes $\mathrm{y}$, particularmente, si son compuestos clorados (Leson y Winer, 1991). 
Debido a la incertidumbre en el funcionamiento del proceso biológico, se hacen indispensables las pruebas en escala piloto para la mayoría de las aplicaciones. Las pruebas piloto proveen un orden de magnitud para los parámetros que permite estimar la eficiencia y el volumen de lecho filtrante necesario para lograr el nivel deseado de destrucción de VOCs.

\subsubsection{Fotocatálisis}

Este proceso está basado en el empleo de luz ultravioleta (UV), la que, al iluminar un tipo específico de semiconductor (nanopartículas de $\mathrm{TiO}_{2}$ ) posibilita que se lleven a cabo reacciones superficiales que permiten oxidar el o los VOCs a tratar. Este proceso ha recibido considerable atención en los últimos años, lo que puede justificar que no siempre se menciona en algunos trabajos de revisión (Khan y Ghoshal, 2000; Everaert y col., 2002) como una de las alternativas de tratamientos de VOCs para sistemas industriales.

Por las características del sistema una gran cantidad de trabajos están orientados a la aplicación de purificación del aire en ambientes interiores (indoor air quality), ya que tienen la gran ventaja de trabajar a temperatura ambiente, con baja pérdida de carga y llegando a límites muy bajos de concentración de VOCs, lo que los hace atractivos para su empleo en nuevos, o existentes, sistemas de ventilación, calefacción y acondicionamiento de aire (Jacoby y col., 1996). Se ha estudiado su aplicación en la eliminación de diversos tipos de VOCs, tanto de un único compuesto (Jacoby y col., 1996), como mezclas de los mismos (Hodgson y col., 2007), encontrándose que una fracción significativa de los estudios se ha direccionado a la eliminación de formaldehído (Passalia y col., 2012), un compuesto que puede ser difícil de eliminar con otros métodos.

El empleo de este proceso en escalas industriales, donde deben tratarse grandes caudales de aire, requiere estudios ulteriores, a efectos de evitar algunos de los inconvenientes que pueden presentar el cambio de escala, como ha señalado Ray (2000). 


\subsection{Técnicas de recuperación de VOCs}

El objetivo de este tipo de técnicas es remover los VOCs presentes en la corriente de aire a tratar y recuperarlos para su posterior utilización. De esta forma aparecería un doble objetivo, que es cumplir con las normas ambientales que limitan la emisión de VOCs, pero también alcanzar una recuperación y reutilización de los mismos. Existen distintas tecnologías que permiten la recuperación de los VOCs, las mismas se basan en procesos de adsorción, absorción, condensación o mediante el empleo de membranas. A continuación se describen las distintas técnicas.

\subsubsection{Adsorción}

En el control de la contaminación del aire, la adsorción se emplea para remover VOCs de corrientes de gas con baja o media concentración, cuando deba alcanzarse alguna concentración restringida de salida y/o se desea la recuperación del VOC.

El diseño de un sistema de adsorción depende de las características del VOC que vaya a ser recuperado, de las condiciones operativas de la corriente de gas a la entrada de la columna (temperatura, presión, caudal volumétrico) y las propiedades físicas del adsorbente.

La corriente de proceso circula a través del adsorbedor donde los VOCs resultan ser selectivamente retenidos por el material adsorbente hasta que la presencia de los mismos se evidencia en el efluente. Alcanzada una cierta concentración máxima permitida a la salida del adsorbedor, se debe discontinuar la operación, para que el mismo pueda ser regenerado. La regeneración del lecho es típicamente realizada por calentamiento o uso de un aspirador que desorbe los gases adsorbidos; posteriormente el lecho es enfriado y secado, lo que usualmente se hace alimentando aire mediante un ventilador. El lecho así regenerado ya queda disponible para actuar nuevamente como adsorbedor. El proceso de regeneración puede ser repetido varias veces; sin embargo, el adsorbente debe ser periódicamente reemplazado debido a un descenso gradual de su capacidad de adsorción. Los vapores obtenidos son llevados a un sistema de recuperación, como un condensador, decantador o torre de destilación.

En la Figura 6 se puede observar un sistema de adsorción típico, formado por dos lechos, que operan en forma alternada. Siempre uno de los lechos está trabajando como adsorbedor, mientras que el otro está en espera o en proceso de regeneración. 
Una variante al sistema de dos lechos es un concentrador rotativo, alternativa que será analizada con mayor detalle en el Capítulo 3.

Para este tipo de sistemas se alcanzan eficiencias de remoción superiores a un 95\% (Moretti, 2002). La adsorción es aplicable a corrientes continuas e intermitentes y tiene la capacidad de manejar un rango extendido de concentraciones. Si bien los sistemas de adsorción resultan aptos para la recuperación de una gran variedad de VOCs, en ciertas circunstancias su empleo no es recomendado:

* Para la adsorción de altas concentraciones de VOCs puede producirse un excesivo aumento de temperatura en el lecho debido a la gran cantidad de calor liberada en la adsorción. No obstante, debe aclararse que en presencia de vapores inflamables los requerimientos de seguridad limitan las concentraciones de los VOCs a la entrada a un LEL menor del $25 \%$ (Moretti, 2002).

* Los compuestos de pesos moleculares muy elevados (>130) que son caracterizados por baja volatilidad $\left(\mathrm{P}_{\mathrm{ebn}}>204^{\circ} \mathrm{C}\right)$ son fuertemente adsorbidos, haciendo difícil su desorción durante la regeneración. Por otro lado, los compuestos de bajo peso molecular no presentan una adecuada adsorción en adsorbentes típicos como el carbón activado.

* Los sistemas de adsorción operados de manera apropiada pueden ser muy efectivos cuando se manejan corrientes que contienen una mezcla de componentes de bajo y alto peso molecular. Sin embargo, los componentes orgánicos más livianos tienden a ser desplazados de la superficie adsorbente por los más pesados (mayor $\left.\mathrm{P}_{\mathrm{ebn}}\right)$, reduciendo considerablemente la eficiencia del sistema.

* Finalmente, los niveles de humedad de las corrientes de gas que superen el $50 \%$ pueden afectar la capacidad del adsorbente a concentraciones menores de 1000 ppm de adsorbato. La humedad relativa puede reducirse agregando aire seco a la corriente o calentando el gas en un intercambiador de calor.

Los sistemas de adsorción típicos empleados en la industria hacen uso de carbón activado, zeolita o adsorbentes poliméricos. 


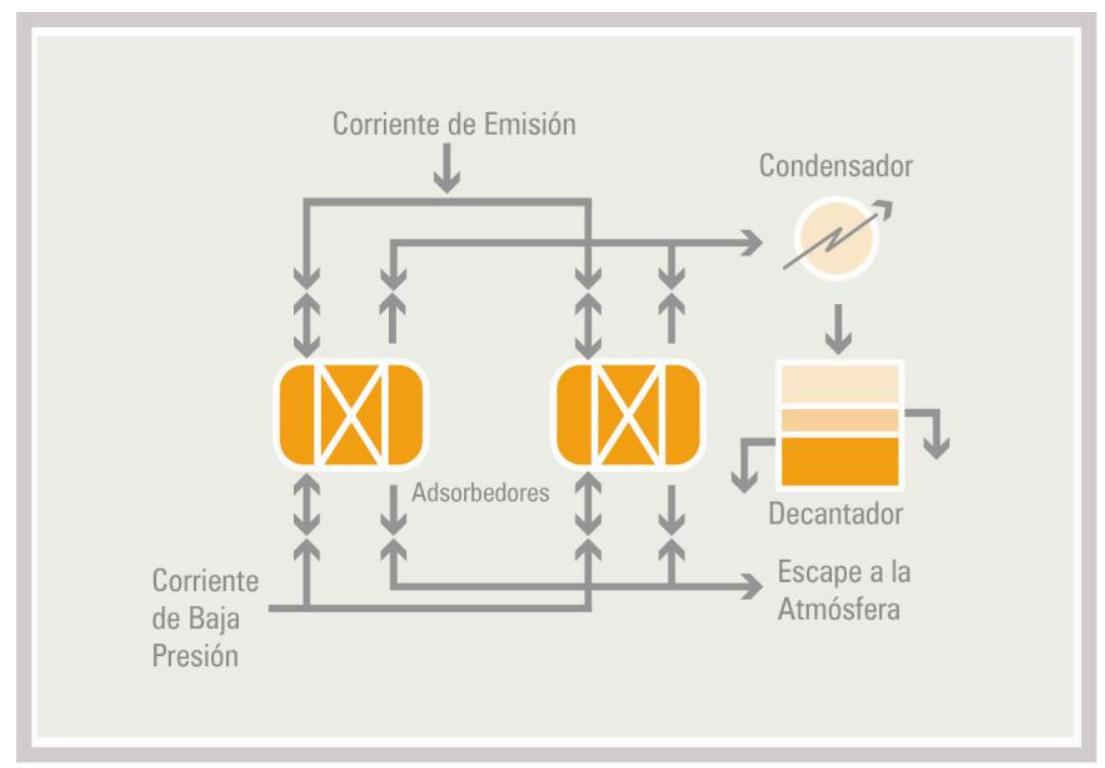

Fig. 6 - Sistema de adsorción

\subsubsection{Absorción}

Esta alternativa consiste en remover los VOCs contenidos en una corriente gaseosa poniéndola en contacto con un solvente líquido. Todos los VOCs solubles resultan transferidos a la fase líquida y de esta manera se logra limpiar la corriente gaseosa.

Este tipo de separación se lleva a cabo por medio de una torre de absorción diseñada para proveer el área necesaria de contacto líquido-vapor y así facilitar la transferencia de masa.

La absorción es generalmente usada para limpiar corrientes gaseosas con altas concentraciones de componentes orgánicos, especialmente aquellos solubles en agua, como metanol, etanol, isopropanol, butanol, acetona y formaldehído.

Existen algunos factores que limitan su uso:

* Disponibilidad de un solvente adecuado. Los VOCs deben ser solubles en el líquido absorbente (agua, aceites minerales).

* Disponibilidad de información del equilibrio líquido-vapor para sistemas específicos orgánico-solventes en cuestión. Esta información es necesaria para diseñar el sistema absorbedor, sin embargo, en muchas ocasiones no está disponible para compuestos orgánicos no comunes.

Otro aspecto a tener en cuenta para este tipo de sistemas es el tratamiento del solvente luego del proceso de extracción del VOC. En muchos casos el líquido que 
absorbió los VOCs es recuperado por desorción del VOC, típicamente por elevación de temperaturas o por vacío. El VOC es luego recuperado como líquido en un condensador.

\subsubsection{Condensación}

La separación por condensación puede ser llevada a cabo incrementando la presión del sistema a una dada temperatura (condensación-compresión) o disminuyendo la temperatura a una presión constante (condensación-refrigeración).

En un sistema de dos componentes donde uno de ellos es no condensable (aire), la condensación ocurre cuando la presión parcial del componente volátil iguala a la presión de vapor (punto de saturación). Para los compuestos más volátiles, una gran cantidad de los mismos se mantiene como vapor a una dada temperatura. Por lo tanto, para remover o recuperar el compuesto, se requiere una temperatura más baja para producir la saturación y condensación. En estos casos, se utiliza la refrigeración para obtener las temperaturas bajas necesarias para lograr las eficiencias de remoción requeridas.

El equipamiento básico de un sistema de condensación refrigerado incluye esencialmente un condensador y una unidad de refrigeración. Para la obtención de temperaturas muy bajas puede utilizarse nitrógeno líquido. Los condensadores pueden ser usados para remover VOCs no halogenados y halogenados sin necesidad de equipamiento auxiliar costoso. Si la corriente de venteo contiene vapor de agua o si el VOC tiene un alto punto de congelación, pueden formarse hielo o hidrocarburos congelados en los tubos o placas del condensador. Esto puede reducir la eficiencia en la transferencia de calor, y aumentar la caída de presión en el condensador. En estos casos puede ser usado un pre-enfriador para remover la humedad antes de que la corriente ingrese al condensador.

Las eficiencias de los condensadores usualmente varían desde un 50 a un $95 \%$ (Everaert y col., 2002).

\subsubsection{Membrana}

En un separador típico de membrana, la corriente de gas a tratar es alimentada a un arreglo de módulos de membranas, donde éstas son preferentemente permeables a los solventes orgánicos. Los VOCs en la corriente permeable son luego condensados 
y removidos como un líquido para su recuperación. El gas purificado es removido como un residuo.

El paso a través de la membrana es inducido por una diferencia de presión a ambos lados de la misma. El compuesto atraviesa la membrana a una determinada velocidad dependiendo de la permeabilidad del material y de la fuerza impulsora.

Los sistemas de separación por membranas pueden tener uno o múltiples pasos tantos como sean necesarios para lograr las eficiencias de recuperación deseadas.

Las separaciones por membranas deben ser consideradas para bajos caudales y altas concentraciones, casos en los cuales otros sistemas, como la adsorción, no son económicamente viables o capaces de lograr el nivel de eficiencia de remoción requerida. La vida útil de las membranas es, en promedio, de tres años.

Existen diversos factores que limitan la aplicabilidad de los sistemas de membrana para la recuperación de VOCs (Khan y Ghoshal, 2000):

* Adecuación al tratamiento de un conjunto de orgánicos

* Costo de la membrana

* Mantenimiento de la membrana

* Velocidad del proceso

* Reutilización de la membrana

* Humedad de la corriente

\subsection{Criterios de selección de las técnicas de tratamiento de VOCs}

Descriptas las principales técnicas para el tratamiento de los VOCs, resta formular criterios que permiten la selección entre las mismas. En términos generales el criterio a aplicar sería seleccionar la técnica que permita alcanzar el objetivo (nivel de VOCs requerido) con el menor costo posible. Aunque para cada caso específico se pueden definir criterios que permiten retener ciertas metodologías y desechar otras, una primera selección entre las diferentes metodologías puede apoyarse en las siguientes características del sistema a tratar:

* concentración de VOCs

* caudal de la corriente gaseosa

* nivel de control requerido

El caudal de aire y la concentración de VOCs en la corriente son los dos aspectos más importantes para la selección del método de tratamiento. Muchas de las alternativas 
de tratamiento mencionadas no presentan impedimentos de tipo técnico para ser llevadas a cabo fuera de ciertos rangos, pero las mismas dejan de ser económicamente convenientes.

Se han elaborado diagramas, normalmente con entradas de caudal y concentración de VOCs, que permiten seleccionar, en una primera instancia, las metodologías más adecuadas para el tratamiento de una corriente dada. Los mismos se presentarán en el Capítulo 2, donde se definen las características del sistema a tratar.

Aunque todos los procesos mencionados se utilizan en la práctica, y se pueden considerar como alternativas convenientes de acuerdo a las características de las corrientes a tratar, una posibilidad de gran interés tecnológico es combinar algunos de estos procesos a efectos de remediar alguna desventaja que pueden presentar individualmente. Estas alternativas de procesos combinados conducen inexorablemente a sistemas más complejos, pero pueden permitir alcanzar una disminución de costos en la operación del proceso, en las de los propios equipos tomados individualmente y además, extender el rango de aplicación de los procesos individuales. Si bien no todas las combinaciones de los procesos individuales resultan razonables, existen diversos estudios en los cuales se han desarrollado alternativas de procesos combinados. Algunos ejemplos de diferentes combinaciones se mencionarán a continuación.

Buzek y col. (1999) realizan un análisis comparativo de los costos para dos sistemas alternativos de tratamiento de VOCs: separación con membrana seguida de condensación o separación con membrana seguida de combustión no catalítica. El proceso más adecuado depende de las condiciones de operación (concentración de VOCs y caudal de la corriente a tratar).

La combinación de un sistema de adsorción/desorción, empleando un cilindro rotativo, y un reactor fotocatalítico fue estudiada por Shiraishi y col. (2003) para la eliminación de formaldehído. Los autores señalan la notable performance del sistema propuesto que se alcanza por el "trabajo cooperativo" entre el sistema de adsorción con carbón activado y el reactor fotocatalítico. En este caso debe emplearse un calentador para lograr el aire caliente que facilite el proceso de desorción. Posteriormente, el mismo grupo, Shiraishi e Ishimatsu (2009), empleó un sistema similar para estudiar la eliminación de tolueno.

Los sistemas de tratamientos biológicos, como la biofiltración, también pueden ser combinados con otros procesos, globalmente consignados como "físicoquímicos", para diferenciarlos del tratamiento "biológico". Dependiendo del tipo de VOC y su 
concentración, y especialmente teniendo en cuenta la posibilidad de fluctuaciones significativas en la carga de VOCs, los biofiltros pueden ser combinados, a través de diferentes alternativas, con procesos de adsorción, absorción o fotoreactores, para mejorar la performance del proceso. Una detallada revisión de estas alternativas ha sido presentada recientemente por Rene y col. (2012).

Los procesos de oxidación catalítica también han sido empleados en forma combinada. Si bien la oxidación catalítica se distingue dentro de las tecnologías de eliminación debido a que el uso de un catalizador permite alcanzar elevadas velocidades de oxidación posibilitando operar a menores temperaturas que en los sistemas térmicos, aún así, se requieren temperaturas bien por encima de la ambiente que, aunque dependiente del tipo de VOC, normalmente no será inferior a los $200^{\circ} \mathrm{C}$. Puesto que los caudales de aire a tratar en una vasta cantidad de casos prácticos son muy elevados $\left(10 \mathrm{Nm}^{3} / \mathrm{s}\right.$, como orden de magnitud típico) y se encuentran a temperaturas cercanas a la ambiente, el tratamiento catalítico requiere de lechos voluminosos y el precalentamiento del efluente con los gases de salida del reactor obliga a disponer de intercambiadores con una extensa superficie de intercambio de calor, debido a los elevados caudales y a la pequeña fuerza impulsora determinada por el bajo salto térmico adiabático. El empleo de una etapa previa de concentración de los VOCs con lechos adsorbentes permite aliviar en gran medida los requerimientos de catalizador y superficie de intercambio. Algunos de estos sistemas combinados (Adsorción - Oxidación catalítica) han sido reportados en la bibliografía (Cho y col., 1999; Sramek y col., 2004). Alternativas similares han sido propuestas en patentes (Greene, 1995) o por empresas que comercializan este tipo de sistemas (Anguil Environmental Systems Inc., Chemisch Thermische Prozesstechnik GmbH, Formia Emissions Control Oy, Fusion Environmental, Munters Corporation, Zeol Division, Met-Pro Environmental Air Solutions)

\subsection{Objetivos para el desarrollo de la Tesis}

El objetivo general de este trabajo es estudiar sistemas de eliminación de compuestos orgánicos volátiles (VOCs) por medio de la oxidación catalítica de los mismos. A partir de un estudio particular, se pretende alcanzar conclusiones y desarrollos metodológicos que puedan extenderse a otros sistemas, a efectos de definir criterios de selección de diversas alternativas tecnológicas en la eliminación catalítica 
de VOCs.

Se estudia en forma específica la destrucción catalítica de acetato de etilo y etanol producidos en corrientes efluentes de plantas de impresión de envases para alimentos.

Los estudios se orientan hacia la propuesta de distintas alternativas utilizando reactores catalíticos, recuperación térmica en un intercambiador de calor y la inclusión o no de un adsorbedor, que actúe como concentrador de la corriente de VOCs, constituyendo un sistema combinado en caso de incluirse el proceso de adsorción/desorción.

Para la obtención y análisis de resultados se realiza la simulación de cada uno de los equipos en particular y de la planta en conjunto.

Para las simulaciones se estudian distintas metodologías de cálculo de acuerdo al grado de precisión requerido.

Dentro de estos objetivos generales, se presentan objetivos específicos como el conocimiento necesario de la interacción de los VOCs, acetato de etilo y etanol, con los materiales adsorbente y catalítico, y los parámetros, de adsorción y cinéticos, que gobiernan estos procesos. Dada la falta de información cinética confiable, surge como un objetivo específico la obtención de la misma a través de la regresión de datos experimentales obtenidos en el INTEQUI sobre un catalizador desarrollado en dicho Instituto.

\subsection{Descripción del contenido de la Tesis}

En el Capítulo 2 se presentan las propiedades operativas de la corriente a tratar, una corriente de aire contaminada con acetato de etilo y etanol, y se sugiere un posible sistema de eliminación de VOCs apto para el caso particular de estudio. El mismo consta de un reactor catalítico como proceso final para la destrucción del VOC, pero incorpora, en forma previa, un sistema de concentración de VOCs en la corriente a tratar, mediante un procedimiento equivalente a la purificación a través de la adsorción/ desorción y uno o más intercambiadores para la recuperación de calor

En el Capítulo 3 se describen las distintas alternativas empleadas para llevar a cabo los procesos de adsorción/desorción, seleccionándose una técnica apropiada para el caso particular de estudio presentado en el Capítulo 2. También se mencionan las características de los posibles materiales adsorbentes junto con los criterios a tener en cuenta para la selección de los mismos. El empleo de estos criterios para el caso 
específico en estudio permite seleccionar el material adsorbente que se utilizará en el diseño del sistema de adsorción/desorción. Finalmente se presentan dos alternativas de diseño para sistemas de adsorción/ desorción, y se describe en que forma es factible la disposición del material adsorbente.

En los Capítulos 4 y 5 se presentan los métodos numéricos empleados para la simulación del sistema de adsorción/desorción. Para los mismos se tuvieron en cuenta dos enfoques. La diferencia entre los mismos se basó en incluir, o no, las resistencias a la transferencia de calor y materia dentro y fuera del material adsorbente.

Además, en el Capítulo 5 se analiza la influencia de las resistencias a la transferencia de materia y energía según el tipo de lecho, i.e. relleno o monolito. A partir de los resultados obtenidos se selecciona el tipo de lecho a emplear en el sistema de eliminación propuesto en el Capítulo 2.

En el Capítulo 6 se introduce el concepto de ciclo de adsorción/desorción y se presenta una modalidad de cálculo para la determinación del período que define dicho ciclo. A partir de haber definido el tipo de lecho en el Capítulo 5, se sugiere un tipo de configuración para el mismo. Se estudia la influencia de algunas variables operativas sobre el proceso. Finalmente se presenta una aproximación que, sin tener en cuenta las resistencias a la transferencia de calor y materia, permite determinar el tiempo correspondiente a la etapa de adsorción y la cantidad de material adsorbente requerida para el diseño del sistema de adsorción/desorción.

En el Capítulo 7 se presenta un estudio cinético de la combustión del acetato de etilo $\mathrm{y}$ el etanol sobre un catalizador de $\mathrm{MnCu}$. El objetivo es determinar un esquema cinético de reacción, proponer las expresiones para las velocidades de reacción de cada componente y obtener los valores de los parámetros cinéticos a partir de la regresión de los datos experimentales obtenidos en el INTEQUI a partir de un catalizador desarrollado en dicho Instituto (Pramparo, 2010).

En el Capítulo 8 se propone el modelo de cálculo para un reactor de lecho fijo con catalizador granular de forma esférica. Se adopta un sistema de flujo radial, con el propósito de reducir sustancialmente la pérdida de carga (Rase, 1990). También se presenta una aproximación explícita para el cálculo de las velocidades efectivas de reacción.

En el Capítulo 9 se propone, en base a las características de las corrientes a tratar, un sistema de recuperación de calor y se presenta el modelo de cálculo para llevar a cabo la simulación del mismo. 
En el Capítulo 10 se evalúa el impacto que causa la incorporación de un sistema de adsorción/desorción al sistema de eliminación catalítica de VOCs sobre los requerimientos de catalizador y superficie de intercambio térmico.

Finalmente se presentan las conclusiones del trabajo y se proponen recomendaciones para trabajos futuros. 


ESTUDIO DE SISTEMAS

COMBINADOS DE

$\bigcirc$ COMBUSTION

CATALITICA DE VOCs

\section{2/ SISTEMA COMBINADO DE ELIMINACION DE VOCs}

Elección de la técnica de eliminación de VOCs

Conclusiones 



\section{$02 /$

Tanto el acetato de etilo como el etanol son compuestos orgánicos volátiles (VOCs) típicamente utilizados en la industria gráfica como solventes; gran parte de los mismos es liberada a la atmósfera en forma de vapores. Ambos compuestos son altamente inflamables y la exposición a los mismos puede traer severas consecuencias a corto y largo plazo para la salud humana y el medio ambiente. Por esta razón es necesario disminuir su concentración a niveles que puedan considerarse inocuos. Con esta finalidad, se tomaron como referencia los estándares internacionales. Siguiendo los niveles de concentración recomendados por la Comisión Europea (http://ec.europa.eu/environment/air/pollutants/stationary/solvents/legislation.htm), para los cálculos se asumió una tolerancia de $20 \mathrm{mg} / \mathrm{kg}$ de VOCs (5 ppm) en la corriente descargada a la atmósfera. Este valor corresponde a un promedio temporal, dado que, de acuerdo al sistema empleado, es factible que se verifiquen fluctuaciones en la concentración de la corriente de emisión a la atmósfera.

En la siguiente tabla se detallan las características de una corriente de aire, contaminada por acetato de etilo y etanol, proveniente de una planta de impresión de envases. Estos valores serán tomados en cuenta para el análisis y diseño del sistema de eliminación.

Tabla 1: Características de la corriente a tratar

\begin{tabular}{|c|c|c|}
\hline \multicolumn{2}{|c|}{ Caudal $\left[\mathbf{m}^{3} / \mathbf{s}\right]$} & 11.7 \\
\hline $\begin{array}{c}\text { Composición } \\
{\left[\mathbf{m o l} / \mathbf{m}^{3}\right]}\end{array}$ & Acetato de Etilo & $2.3510^{-2}$ \\
\cline { 2 - 3 } Tipo de emisión & Etanol & $9.6310^{-3}$ \\
\hline \multicolumn{2}{|c|}{ Presión [atm] } & Permanente \\
\hline \multicolumn{2}{|c|}{ Temperatura $\left[{ }^{\circ} \mathbf{C}\right]$} & 50 \\
\hline
\end{tabular}

\subsection{Elección de la técnica de eliminación de VOCs}

De acuerdo al relevamiento bibliográfico presentado en el Capítulo 1 existen diversas técnicas mediante las cuales se pueden tratar los VOCs presentes en una corriente 
gaseosa. Teniendo en cuenta el caudal de la corriente a tratar, la concentración de VOCs, el nivel de control requerido y los costos, se pueden seleccionar a priori la o las técnicas que podrían resultar más apropiadas para el caso particular analizado.

Debido a que se plantea como objetivo específico del trabajo la eliminación de los vapores de acetato de etilo y etanol, y no la recuperación de los mismos, las técnicas de oxidación de VOCs a $\mathrm{CO}_{2}$ y $\mathrm{H}_{2} \mathrm{O}$ surgen como una opción adecuada. Se ha mencionado en el Capítulo 1 que las oxidaciones pueden llevarse a cabo con o sin la presencia de un catalizador sólido. La diferencia entre ambos esquemas radica en el nivel térmico en el reactor y, por consiguiente, en la energía necesaria para alcanzarlo. La idea es utilizar la menor cantidad de energía posible. La situación ideal sería implementar un esquema de tipo autotérmico, donde el calor generado en la reacción de combustión fuese suficiente para precalentar la alimentación (corriente de aire conteniendo los VOCs) hasta una temperatura estipulada para la entrada del reactor. La temperatura estipulada será aquella que permita alcanzar un diseño y operación eficientes a fin de obtener la concentración requerida a la salida del mismo. En este contexto, y teniendo en cuenta el continuo desarrollo de nuevos catalizadores, que presentan alta actividad a relativamente bajas temperaturas de operación, la oxidación catalítica surge como una alternativa de marcado interés.

Por otra parte, de acuerdo al nivel de concentración y caudal a tratar, es posible realizar un análisis de las distintas alternativas de tratamiento de efluentes desde un punto de vista económico. Como se mencionara en el capítulo anterior existen cuadros y gráficos construidos en función de la experiencia recogida de las unidades en funcionamiento. Las Figuras 1 (Shah y col., 2000) y 2 (Everaert y col., 2002) son dos gráficos típicos en los cuales se han indicado las condiciones del caso a tratar, Tabla 1. De los mismos se desprende que la oxidación catalítica resulta una de las opciones más convenientes en cuanto al costo. Sin embargo, a pesar de la ventaja que representa el uso de reactores catalíticos, el nivel térmico de operación sigue siendo relativamente alto. Esto implica un costo operativo elevado para llevar los reactivos desde la temperatura de salida de la planta hasta la de operación del reactor; un problema que se ve agravado por los altos caudales de la corriente a tratar. Como ya fue mencionado, la posibilidad de una recuperación energética es una alternativa que no se puede ignorar y que es considerada en todos los procesos, catalíticos o no, de eliminación de VOCs por oxidación. La necesidad de recuperar parte de la energía de la corriente de salida del reactor genera un conjunto muy amplio de alternativas tecnológicas. En primer lugar 
por el tipo de operación del sistema en conjunto (reactor e intercambiador) que puede concebirse como una operación típica de estado estacionario o como una operación periódica, con reorientación temporal del flujo de cada corriente (Van de Beld, 1994), y por otra parte, por la posibilidad de emplear combustible adicional o llevar a cabo una operación totalmente autotérmica, la cual es factible en virtud de que las reacciones de oxidación son exotérmicas.

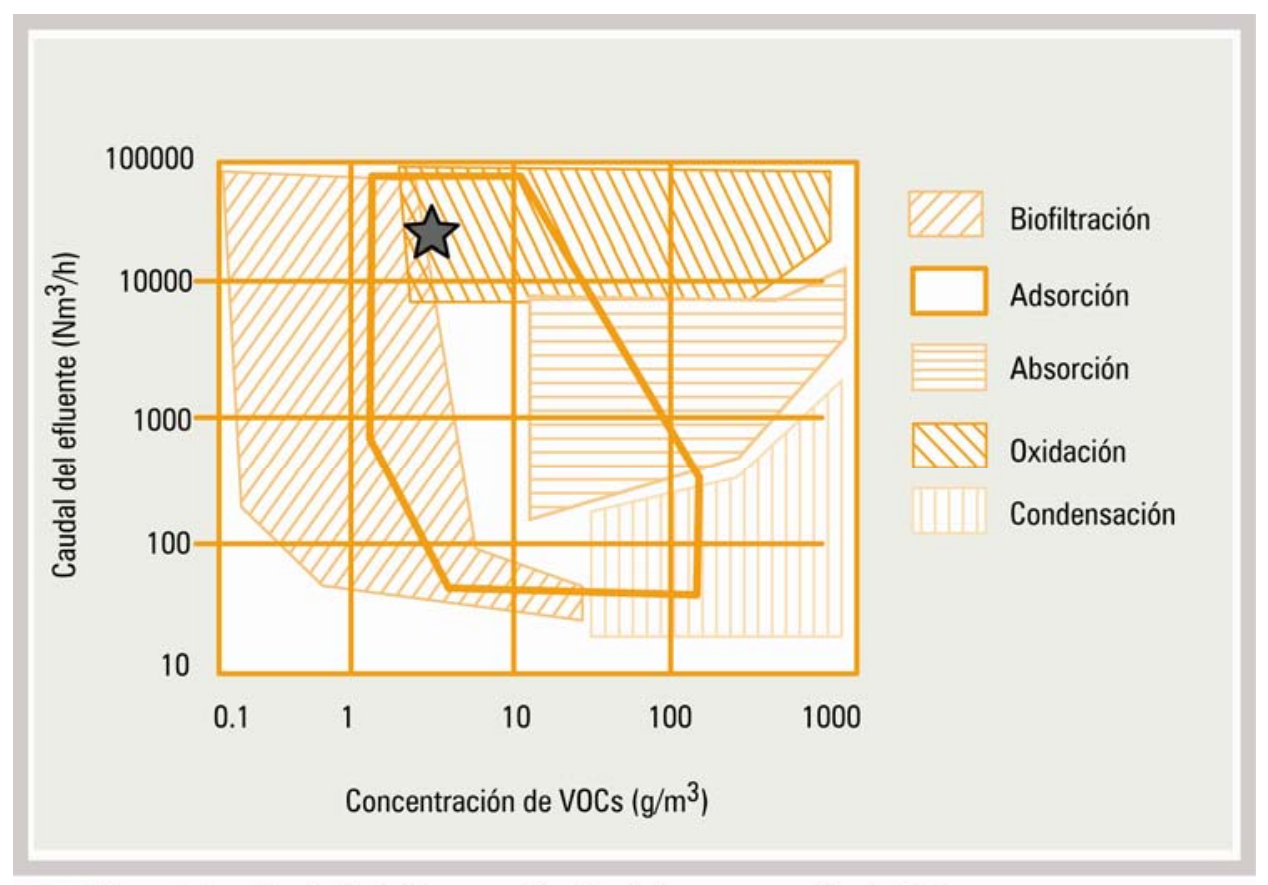

Fig. 1 - Caudal del efluente en función de la concentración de VOCs.

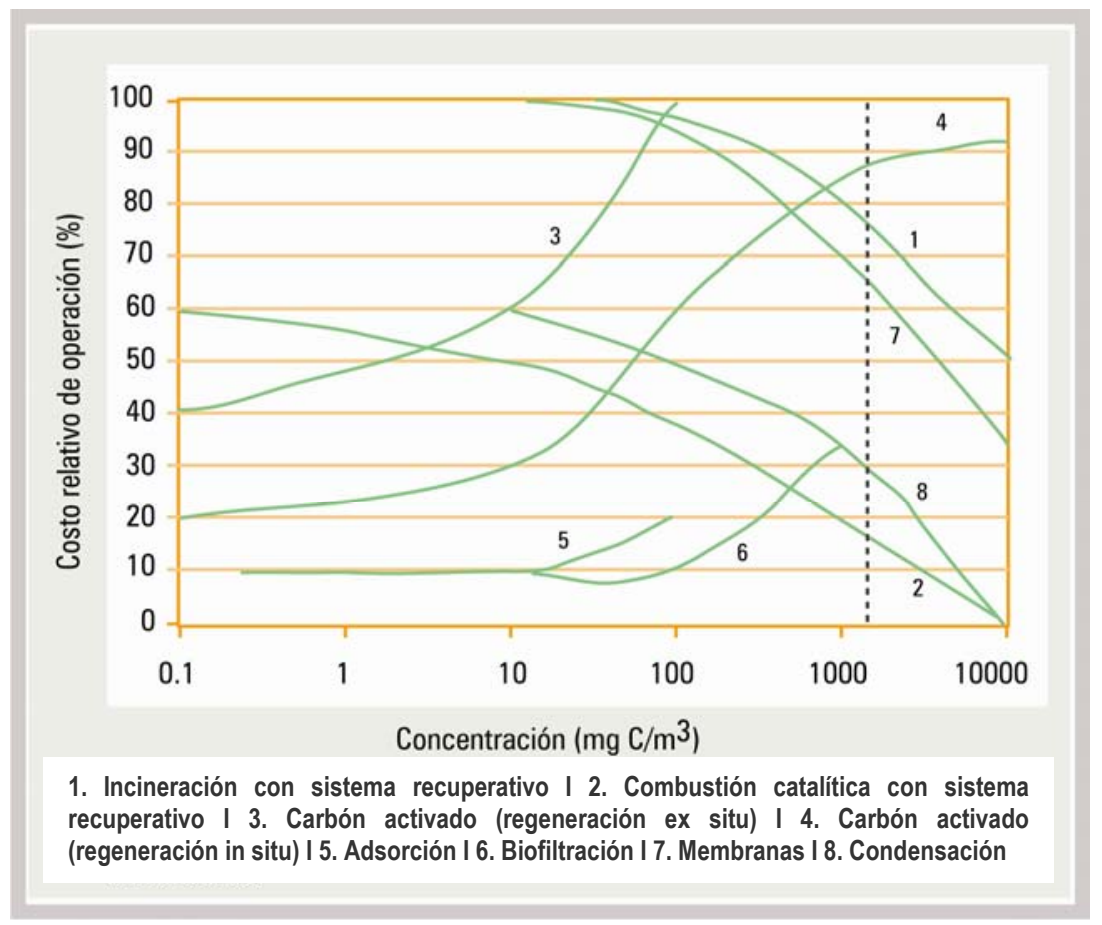

Fig. 2 - Costos relativos de operación en función a la concentración de VOCs. 
La utilización de combustible adicional aparece como una necesidad ineludible por dos razones: la puesta en marcha del proceso y ganar flexibilidad para el control de la planta ante una desactivación del catalizador o cambio en las condiciones de alimentación (Campesi y col., 2007). Sin embargo, la utilización de combustible adicional para la operación “en condiciones de diseño”, implica admitir que exista un costo operativo adicional que puede ser importante. Por otra parte, para un catalizador y un tipo de VOCs definidos, una corriente con mayor concentración de VOCs permitiría una mayor liberación de energía por unidad de volumen mediante su oxidación, mayor elevación de la temperatura en el reactor y, en definitiva, menor requerimiento de combustible adicional o que el mismo no sea necesario.

Una posibilidad de disminuir el costo de combustible en el proceso de eliminación, para un catalizador y corriente de VOCs definida, es mediante la utilización de un sistema combinado. Esta alternativa mantiene la utilización del reactor catalítico como proceso final para la destrucción del VOC, pero incorpora, en forma previa, un sistema de concentración de VOCs en la corriente a tratar, mediante un procedimiento equivalente a la purificación a través de la adsorción/desorción. Adicionalmente, el circuito se completa con sistemas de intercambio térmico. Este tipo de sistema combinado ha sido mencionado en la revisión presentada en el Capítulo 1. Como se señaló en el mismo, el empleo de una etapa previa de concentración de los VOCs con lechos adsorbentes puede provocar una disminución de los requerimientos de catalizador y superficie de intercambio.

En la Figura 3 puede observarse el esquema del sistema de eliminación de VOCs propuesto para este trabajo. El mismo incluye un reactor catalítico, un sistema de adsorción/desorción, 2 intercambiadores de calor y un impulsor (no se indica en la figura un posible quemador que se utilizaría en la puesta en marcha y control del proceso).

El efluente con VOCs ingresa al adsorbedor, que bien puede ser de tipo rotativo, como se encuentra representado en la Fig. 3, o un sistema de lechos, para su uso en ciclos de adsorción/desorción. Durante el proceso de adsorción los VOCs (acetato de etilo y etanol) son adsorbidos. Una fracción del aire limpio que abandona el adsorbedor es utilizada para la regeneración del lecho, el resto del aire es liberado a la atmósfera. Para alcanzar la temperatura de desorción necesaria, el aire es precalentado por la corriente que sale del reactor mediante un intercambiador gas-gas. La corriente que egresa, luego de la desorción, presenta una mayor concentración de VOCs y un menor 
caudal que la corriente de ingreso al sistema, y se envía al reactor adiabático para su incineración catalítica. Con el fin de obtener una temperatura estipulada a la entrada del reactor, que permita alcanzar la concentración requerida a la salida del mismo, la corriente es precalentada en un segundo intercambiador gas-gas. Para dicho precalentamiento también se emplea el calor liberado por la reacción de combustión.

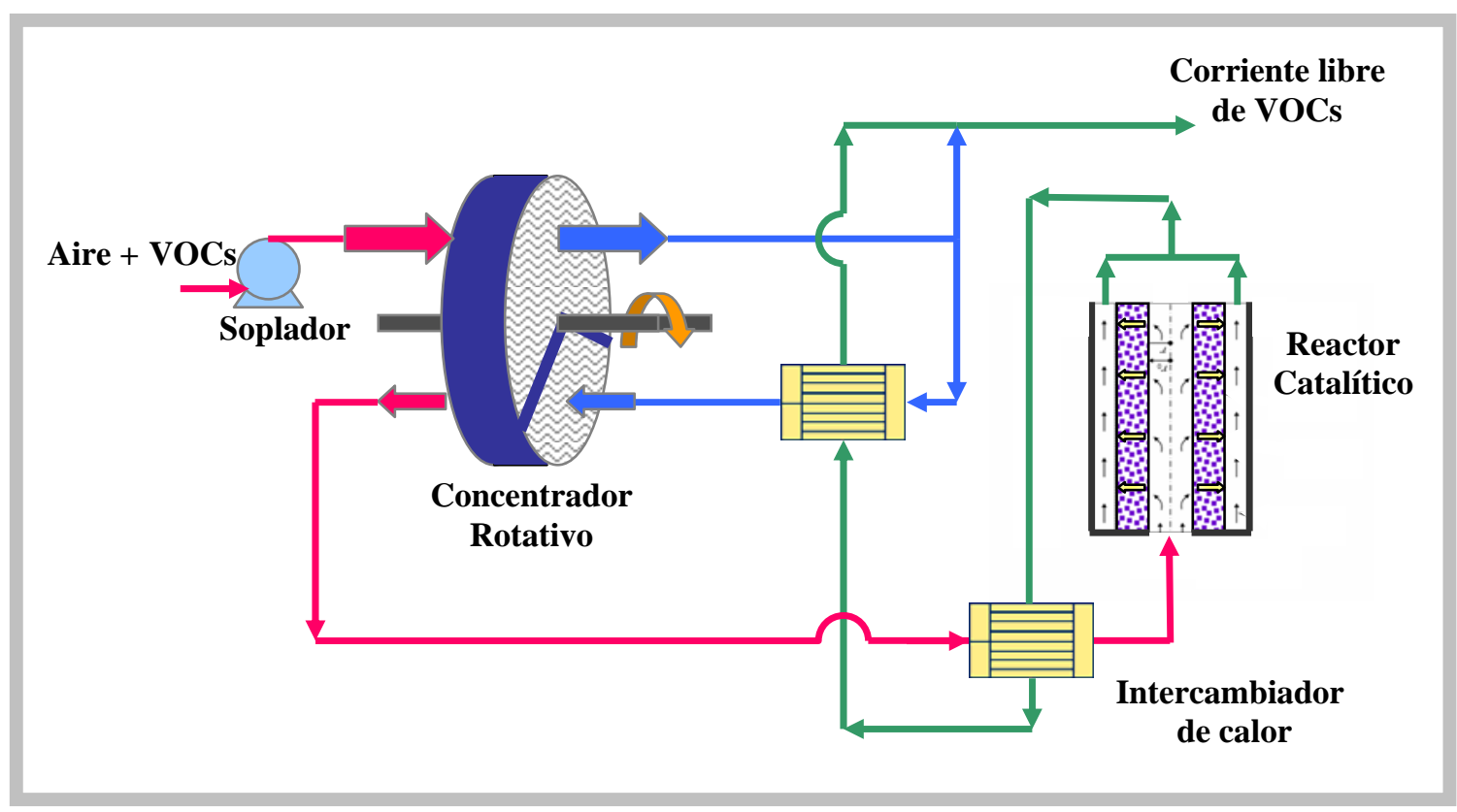

Fig. 3 - Sistema combinado: combustión catalítica de VOCs con concentración de los mismos en un sistema de adsorción/desorción.

Una vez que la corriente egresa del reactor, a la mayor temperatura del sistema, la misma se dirige hacia los intercambiadores de calor mencionados previamente. Por una cuestión de aprovechamiento energético, primero se dirige al intercambiador empleado para precalentar la corriente que alimenta al reactor y posteriormente se dirige al intercambiador en el cual se calienta la corriente utilizada en el proceso de desorción. Luego de pasar por este último intercambiador, la corriente en cuestión es liberada a la atmósfera junto con la fracción del efluente que abandona el adsorbedor y que no es empleada para la regeneración del lecho.

Para el caso particular en que la corriente que lleva a cabo el proceso de desorción estuviese a una temperatura equivalente a la estipulada para la entrada del reactor, se podría prescindir del intercambiador gas-gas.

Si bien esta alternativa de sistema combinado ha sido considerada en la bibliografía, y permite un ahorro en combustible adicional, conduce a plantas operativamente más complejas. Existen diversas variantes que, dentro de los sistemas combinados, pueden 
agruparse en diferentes niveles del diseño y de la operación del proceso. Un nivel es el esquema del proceso que presenta, entre otras alternativas, la inclusión o no del adsorbedor o el número de intercambiadores a utilizar. Un segundo nivel es el tipo de equipo, ya que para los tres equipos principales (reactor, adsorbedor, intercambiador) existen diversas variantes. Finalmente, el último nivel es de tipo operativo, ya que corresponde a las características de las diversas corrientes (caudales, temperatura), incluyendo el combustible adicional a emplear. En consecuencia resulta de interés realizar un estudio que permita discriminar, sobre una base racional, cual de las alternativas puede resultar más conveniente, conociendo las características de la fuente emisora de VOCs y las restricciones impuestas por la normativa legal vigente y eventualmente futura, atento a la cada vez más exigente legislación sobre la eliminación de compuestos contaminantes. Para alcanzar la simulación de todo el proceso, los componentes básicos son las simulaciones individuales de cada equipo principal, teniendo presentes los compuestos a tratar y sus condiciones de trabajo.

El proceso de simulación de cada equipo se concibe mediante los planteos, y su resolución, de los balances de materia, energía y cantidad de movimiento en cada uno de los mismos. Dentro de los equipos involucrados, se consideró adecuado enfatizar la simulación del reactor, intercambiador y adsorbedor, ya que un adecuado diseño de los mismos, y teniendo en cuenta las diversas alternativas que se pueden plantear para cada equipo, son los que determinan la eficiencia de la planta. Para la selección del tipo de impulsor se consideraron los requerimientos generales del proceso. Siendo que el quemador es concebido para la puesta en marcha del proceso, no se lo estudia de manera específica. El desarrollo de los simuladores de cada equipo individual permite su integración en diversos esquemas de la planta, lo que posibilita el análisis global de la misma.

Una etapa importante es la obtención de información específica sobre el sistema en estudio. Si bien en cada etapa de simulación se debe obtener información de parámetros termodinámicos y de transporte, coeficientes de transferencia de calor y materia, factores de fricción, etc., existe amplia literatura sobre los mismos. En cambio, la información cinética sobre la oxidación catalítica de acetato de etilo y etanol, así como sobre la adsorción/desorción de estas sustancias sobre diferentes tipos de adsorbentes es mucho más restringida, dado que es específica del par sólido (ya sea catalizador o adsorbente) y VOC a tratar, por lo que se requiere un esfuerzo particular de búsqueda e interpretación de la información disponible. 
Dentro de los datos obtenidos de bibliografía se ha alcanzado a seleccionar el material a emplear como adsorbente y los parámetros del mismo. En cambio, a pesar de una exhaustiva revisión bibliográfica, no se obtuvo información cinética confiable, por lo tanto se decidió llevar a cabo la regresión de datos experimentales disponibles para la obtención de los parámetros cinéticos. Los datos experimentales fueron obtenidos en el INTEQUI (CONICET/UNSL) y presentados por Pramparo (2010), para lo cual se empleó un catalizador de laboratorio en base a óxidos de Cu y Mn. La obtención de la información cinética se encuentra desarrollada en el Capítulo 7.

\subsection{Conclusiones}

El objetivo de este trabajo (Capítulo 1) está basado principalmente en el estudio de técnicas de eliminación de VOCs. Particularmente se estudia la eliminación de acetato de etilo y etanol producidos en corrientes efluentes de plantas de impresión de envases.

Tanto el acetato de etilo como el etanol son perjudiciales para la salud humana y el medio ambiente, por lo que se pretende disminuir su concentración a niveles inocuos recomendados por la Comisión Europea. En el capítulo anterior se presentaron una serie de técnicas apropiadas para el tratamiento de corrientes gaseosas.

Teniendo en cuenta el objetivo del trabajo, las características de la corriente a tratar (caudal, concentración), y los costos, la combustión catalítica surge “a priori” (Figs. 1 y 2) como una de las técnicas de control más adecuada para el caso particular analizado. Este tipo de sistemas permite emplear el calor generado en la reacción de combustión para precalentar la alimentación (corriente de aire conteniendo los VOCs) hasta la temperatura estipulada a la entrada del reactor que permita alcanzar la concentración requerida a la salida del mismo. Sin embargo, a pesar de la ventaja que representa el uso de reactores catalíticos, el nivel térmico de operación sigue siendo relativamente alto, lo que considerando el combustible a emplear, puede implicar un costo operativo adicional demasiado elevado. Con el propósito de disminuir los costos se propuso un sistema combinado de eliminación de VOCs (Fig. 3). Esta alternativa mantiene la utilización del reactor catalítico como proceso final para la destrucción del VOC, pero incorpora, en forma previa, un sistema de concentración de VOCs en la corriente a tratar, mediante un procedimiento equivalente a la purificación a través de la adsorción/desorción. Adicionalmente, el circuito se completa con sistemas de intercambio térmico.

El sistema combinado proporciona una corriente más concentrada y de menor caudal 
en la alimentación al reactor, lo que permite una mayor liberación de energía por unidad de volumen mediante su oxidación, mayor elevación de la temperatura en el reactor y, como consecuencia, menor, o eventualmente nulo, requerimiento de combustible adicional. Asimismo, al operar con un caudal bajo y concentrado en VOCs, el reactor catalítico y los intercambiadores de recuperación de calor resultarán más pequeños. 


ESTUDIO DE SISTEMAS

COMBINADOS DE

COMBUSTION

CATALITICA DE VOCs

\section{3/ ADSORCION}

Técnicas de adsorción/desorción

Materiales adsorbentes

Criterios de selección de material adsorbente

Diseño del sistema de adsorción/desorción

Disposición del material adsorbente

Conclusiones 

La adsorción es un fenómeno en el cual las moléculas de una fase fluida, i.e. gas o líquido, son retenidas sobre una superficie sólida. Las moléculas adsorbidas en el sólido son denominadas adsorbatos, mientras que la superficie sobre la cual se adsorben se denomina adsorbente. En los casos prácticos, el material adsorbente está constituido por un sólido altamente poroso, de manera tal de obtener una alta área superficial por unidad de volumen, en consecuencia, la adsorción ocurre esencialmente sobre las paredes de los poros. Los adsorbatos pueden ser retenidos sobre el sólido tanto por fuerzas de atracción débiles, “fisisorción”, como por verdaderas uniones químicas, “quimisorción”. Durante la fisisorción, se libera energía, identificada como “calor de adsorción”. Cuando se pretende llevar a cabo un proceso de recuperación del adsorbato resulta conveniente orientar la elección hacia una adsorción por fisisorción, a efectos de emplear una menor cantidad de energía para la regeneración del adsorbente. En general, la capacidad de adsorción sobre un determinado material adsorbente, depende específicamente de la naturaleza de los adsorbatos (concentración, peso molecular, polaridad, punto de ebullición, etc.).

En el control de la contaminación del aire, la adsorción se emplea para remover VOCs de corrientes de gas de baja o media concentración, cuando se desea la recuperación del VOC y/o se requiere obtener una corriente más concentrada. Esta última opción es la considerada en el sistema propuesto en el Capítulo 2 (Fig. 3). Desde el punto de vista operativo las técnicas de recuperación o concentración mediante adsorción conducen a una operación de tipo “periódica” ya que, inevitablemente, deben ocurrir ciclos de adsorción, para retener el contaminante en un lecho de adsorbente, y de desorción, para recuperar las condiciones iniciales del lecho a efectos de comenzar un nuevo proceso de adsorción. Este tipo de procedimiento ha sido empleado extensamente en la purificación de corrientes gaseosas, conduciendo al desarrollo de diferentes técnicas para llevar a cabo los procesos de adsorción y desorción. Entre las mismas pueden mencionarse: Temperature Swing Adsorption (TSA), Pressure Swing Adsorption (PSA), Vacuum Swing Adsorption (VSA) o combinaciones de las mismas, como Thermally Coupled Pressure Swing (TCPS). Estas técnicas han sido estudiadas para su aplicación en el tratamiento de corrientes de VOCs (Ghoshal y Manjare, 2002). 
Los sistemas de adsorción típicos empleados en la industria utilizan como material adsorbente carbones activados, zeolitas o adsorbentes poliméricos.

La adsorción es aplicable a corrientes continuas o intermitentes y tiene la capacidad de manejar un rango extendido de concentraciones. Se alcanzan eficiencias de remoción superiores a un 95\%, llegándose en determinados casos a que sea factible lograr una remoción de hasta un 99\% (Moretti, 2002). Es claro que estas técnicas pueden presentar una alta eficiencia de concentración del VOC, proceso que puede emplearse para una posterior recuperación o eliminación del mismo. Sin embargo, debe tenerse presente que la necesidad de la desorción incrementa la complejidad del proceso.

En este capítulo se describen las distintas técnicas empleadas para llevar a cabo los procesos de adsorción/desorción, seleccionándose una técnica apropiada para nuestro caso particular de estudio (Capítulo 2). También se mencionan las características de los posibles materiales adsorbentes a utilizar, junto con criterios a tener en cuenta para la selección de los mismos. A partir de estos criterios se propone el material adsorbente a utilizar para poder llevar a cabo el análisis y diseño del sistema.

Finalmente se presentan dos alternativas de diseño para sistemas de adsorción/ desorción, y se describe en que forma es factible la disposición del material adsorbente.

\subsection{Técnicas de adsorción/desorción}

Las técnicas de separación mediante el proceso de adsorción/desorción son frecuentemente empleadas en la purificación de corrientes con VOCs dado que, respecto a otros procesos de separación como la absorción y la destilación, pueden requerir de menor energía y por ende menores costos.

Como fue mencionado en la introducción, existen diferentes técnicas que permiten llevar a cabo el proceso de adsorción/desorción. A continuación se realiza una descripción general de las mismas. Al analizar las características de cada una, y sus ventajas y desventajas, se debe tener presente el fin para el cual se utilizan. Aunque el proceso de adsorción/desorción puede ser similar, el objetivo final es muy diferente si se pretende lograr una recuperación del VOC o sólo una concentración de los mismos en la corriente a tratar, para luego llevar a cabo su combustión. Esta diferencia se aprecia claramente cuando se trata de una mezcla, ya que en el caso de la recuperación puede buscarse obtener cada VOC por separado, mientras que en el caso de una posterior combustión, lograr la separación es irrelevante. 


\subsubsection{Temperature Swing Adsorption (TSA)}

Esta técnica consiste en un ciclo que opera en 2 etapas:

* Adsorción de la corriente que se desea tratar (aire + VOCs) a relativamente bajas temperaturas.

* Desorción con gas caliente generalmente en sentido inverso a la corriente de adsorción. Típicamente se han empleado para la desorción corrientes de vapor de agua, aire o $\mathrm{N}_{2}$ caliente. Otras alternativas, con calentamiento con diferentes sistemas eléctricos, como microondas, han sido analizadas, especialmente a nivel laboratorio o escala piloto (EPA, 1999; Bathen, 2003).

En general se incorpora una tercera etapa de enfriamiento del lecho con gas inerte a baja temperatura. Para el caso aquí estudiado, dado que la corriente a adsorber se encuentra a una temperatura suficientemente baja para llevar a cabo la adsorción y que, como posteriormente se describirá, el frente de temperatura avanza más rápido que el de saturación del lecho, es factible no incluir la etapa de enfriamiento.

El TSA presenta un modo de operación simple y más económico comparado con el resto de los métodos, pero no puede utilizarse para la recuperación de compuestos sensibles a la temperatura, ni para aquellos que polimerizan a altas temperaturas (Ghoshal y Manjare, 2002).

Es importante aclarar que para llevar a cabo la recuperación de distintos componentes adsorbidos por separado, se requiere de más de una unidad de TSA (Ghoshal y Manjare, 2002). Obviamente, este no sería un problema en nuestro caso ya que, aunque la corriente contiene dos VOCs, no se plantea una recuperación, sino que el objetivo final es concentrar la corriente.

\subsubsection{Pressure Swing Adsorption (PSA)}

Esta técnica se suele utilizar para la separación de gases o vapores del aire. El ciclo consta de 4 etapas:

Presurización de la corriente a tratar.

Adsorción con alimentación de la corriente a tratar (aire + VOCs) a alta presión.

Despresurización del gas no adsorbido.

Desorción del gas adsorbido a baja presión. 
Desde los años 80, esta técnica ha ganado una gran aceptación a nivel comercial (Ruthven, 1984; Yang, 1997) y su desarrollo y comercialización ha ido creciendo en las últimas décadas. La principal razón a la cual se debe el progreso y crecimiento de esta técnica es la de proveer una separación y purificación de gases de manera flexible y eficiente.

La selectividad del proceso está dada por la diferencia en el equilibrio y velocidad de adsorción entre los componentes a separar. Algunos gases se adsorben más rápido que otros y la frecuencia del ciclo es usada para separarlos. Esta técnica tiene la ventaja de que los lechos son rápidamente regenerados disminuyendo la presión total y purgando a bajas presiones. Diversos autores (Ritter y Yang, 1991; Friday y col., 1993) han empleado este tipo de técnica para la purificación del aire, por ejemplo buscando la recuperación de solventes, sin embargo Ghoshal y Manjare (2002) han presentado objeciones a la misma, señalando que resulta dificultosa la desorción cuando se requiere la remoción de bajas concentraciones de componentes con baja presión de vapor.

Otras técnicas de adsorción que se basan en las mismas condiciones de operación del PSA son:

\subsubsection{Layered Bed Pressure Swing Adsorption (LBSA)}

Dentro de una misma columna se tienen varias capas de distintos adsorbentes con el objetivo de aprovechar las propiedades de cada uno de ellos. Este procedimiento puede resultar económicamente más efectivo que utilizar un único adsorbente en la columna. Por ejemplo, es muy común tener “carbón activado” seguido de “zeolita” (Ghoshal y Manjare, 2002).

\subsubsection{Vacuum Swing Adsorption (VSA)}

Para la desorción de contaminantes fuertemente adsorbidos, y la posibilidad de obtener una completa regeneración del lecho, se utiliza una bomba de vacío, que permite alcanzar una presión de operación menor a la presión de vapor del VOC haciendo que este se desprenda del adsorbente a temperatura ambiente (EPA, 1999). 
Existen otras alternativas aplicadas a sistemas más específicos, que también se basan en las condiciones del PSA, entre ellas se encuentra la denominada Tapered Pressure Swing Adsorption (TPSA), donde, a diferencia del PSA convencional, las columnas de adsorción adoptan una forma cónica. Esta técnica fue estudiada por Ritter y Liu (1998).

\subsubsection{Thermally Coupled Pressure Swing (TCPS)}

Los pasos del ciclo son los mismos que se siguen en el PSA convencional, la diferencia es que para esta técnica de operación (TCPS) además de variarse la presión del sistema, también se varía la temperatura (Figura 1).

En la etapa de la presurización y adsorción (Etapas I y II en la Fig. 1) el lecho es enfriado con una corriente de agua que circula por una camisa de enfriamiento (Water, en la Fig. 1). Durante el resto del ciclo se opera con la camisa vacía y se calienta el lecho haciendo uso de un sistema eléctrico de calentamiento (Heater on, en la Fig. 1) (Gales y col., 2003).

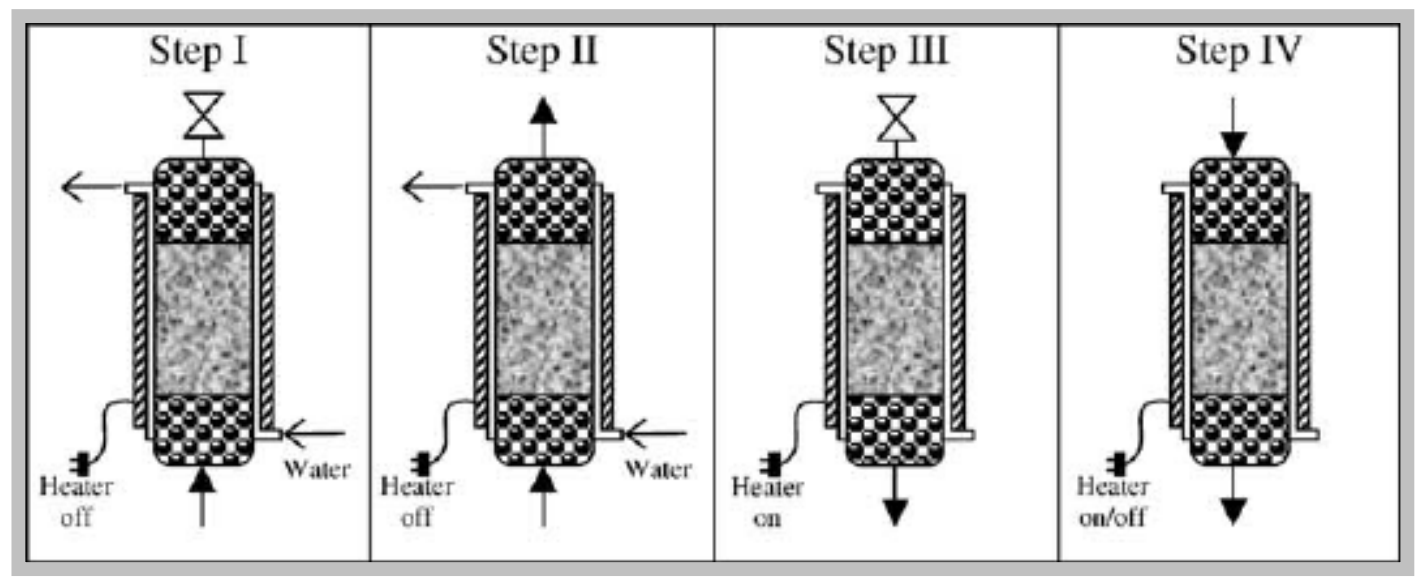

Fig. 1 - Diferentes etapas que abarca el proceso Thermally Coupled Pressure Swing (TCPS).

\section{Selección de la técnica de adsorción/desorción}

Entre las técnicas mencionadas previamente se destacan las Temperature Swing Adsorption (TSA) y Pressure Swing Adsorption (PSA). Las otras son derivaciones o combinaciones de las mismas por lo que, para nuestro caso particular de estudio presentado en el Capítulo 2, se decidió realizar una selección entre las técnicas más convencionales, TSA y PSA.

En principio, la selección se realizó en base al requerimiento de energía, ya sea para 
calentar o presurizar según sea el caso. Teniendo en cuenta el sistema combinado propuesto en el Capítulo 2 (Fig. 3), es factible el uso del calor liberado durante la reacción de oxidación para la obtención de la energía necesaria que permita el calentamiento de la corriente empleada para la desorción. Esto permitiría orientarse a un proceso autosustentable energéticamente cuando se lleva a cabo el proceso de adsorción/desorción mediante el empleo de la técnica TSA. En cambio, cuando la técnica a emplear es la PSA, necesariamente se debe recurrir a un equipo adicional (compresor) y el empleo de energía eléctrica para la presurización de la corriente a tratar.

Por otro lado, también resulta significativo el destino que tiene la corriente concentrada. En este caso, por enviarse a un proceso de oxidación catalítica, para lo cual se requiere una temperatura relativamente alta, surge la necesidad de calentar la corriente una vez que abandona el desorbedor. Esto implica que automáticamente la TSA y las oxidaciones catalíticas tengan un proceso común, que es tener que calentar la corriente empleada para desorber, que luego se envía al reactor. La PSA, en cambio, no requiere una corriente caliente, sino que produce una corriente de menor temperatura, en consecuencia, la incorporación de un intercambiador para elevar la temperatura no se puede evitar.

En síntesis, para emplear PSA se requiere energía para comprimir la corriente a adsorber y para elevar la temperatura de la corriente en forma previa a su entrada al reactor, mientras que en TSA solo se requiere energía para elevar la temperatura.

Debido a estas consideraciones resulta apropiado optar por un sistema adsorción/desorción TSA (Temperature Swing Adsorption).

\subsection{Materiales adsorbentes}

Como se mencionó previamente, los sistemas de adsorción empleados a nivel industrial utilizan carbón activado, zeolita o adsorbentes poliméricos como material adsorbente. La descripción de las características básicas de cada tipo de adsorbente se puede encontrar en diversas fuentes bibliografícas (Ruthven, 1984; Yang, 2003). Dado que un análisis detallado de las propiedades y funcionamiento de cada adsorbente está fuera del objetivo de esta Tesis, a continuación se realiza una breve descripción de los mismos, mencionando sus ventajas y limitaciones, e indicando algunos trabajos 
representativos de las diferentes posibilidades de utilización de los mismos en el tratamiento de VOCs.

\subsubsection{Carbón activado}

El carbón activado es el adsorbente más antiguo y más utilizado industrialmente. Tiene la característica de no ser completamente hidrofóbico ni hidrofílico, por lo que tiene afinidad tanto por las moléculas polares como las no polares. Por esta razón, la humedad de la corriente puede afectar el comportamiento del carbón activado como adsorbente, modificando las curvas de ruptura (breakthrough) (Huang y col., 2003).

Asimismo, debido a su gran superficie interna adsorbe mayor cantidad de moléculas no polares o levemente polares que el resto de los adsorbentes.

Una de sus ventajas destacable sobre el resto de los adsorbentes es que generalmente presenta menor calor de adsorción o fuerza de enlace intermolecular, esto implica un menor requerimiento de energía para la regeneración del adsorbente (Yang, 1997). Además son relativamente económicos comparado con las zeolitas o los adsorbentes poliméricos.

Sin embargo el carbón activado presenta algunas desventajas:

- $\quad$ Es inflamable.

- Resulta difícil de regenerar para sustancias con alto punto de ebullición.

- $\quad$ Empieza a oxidarse a temperaturas entre los $180-200{ }^{\circ} \mathrm{C}$, este valor debe ser considerado al seleccionar la temperatura de regeneración.

- $\quad$ Promueve la polimerización u oxidación de algunos solventes.

Existen innumerables trabajos publicados que reportan la utilización de carbón activado como material adsorbente para el tratamiento de emisiones de VOCs, tanto para sistemas de lechos rellenos con partículas (Shepherd, 2001; Ko y col., 2002), como monolitos (Yates y col., 2000; Moreno-Castilla y Pérez-Cadenas, 2010), ya sea en lechos fijos o en sistemas rotativos (Shiraishi y col., 2007).

\subsubsection{Zeolitas}

Las zeolitas son un adsorbente alternativo al carbón activado para la purificación de corrientes contaminadas con VOCs, que mejoran algunos aspectos deficientes de este último. Presentan ventajas de interés, como:

- $\quad$ Poseer estabilidad térmica. 
- $\quad$ No son inflamables.

- $\quad$ Pueden ser manufacturadas con un preciso tamaño de poros, permitiendo una adsorción selectiva de algunos componentes junto a la exclusión de otros.

Sin embargo, poseen la desventaja de ser más costosas que el carbón activado, en consecuencia se trata de materiales “competitivos”, cuya elección está fuertemente ligada al tipo de sustancia a tratar.

$\mathrm{Al}$ igual que el carbón activado, las zeolitas se han empleado profusamente para el tratamiento de corrientes con VOCs (Carsten y col., 2000; Bathia y col., 2009), tanto en lechos fijos de partículas (Brosillon y col., 2001) como en forma estructurada en sistemas rotativos (Chang y col., 2003).

\subsubsection{Adsorbentes poliméricos}

Los adsorbentes poliméricos, en comparación con el carbón activado, resultan menos susceptibles al fuego, a la fractura y al ensuciamiento. Por lo tanto, no necesitan ser reemplazados con tanta frecuencia. Se pueden destacar sus propiedades hidrofóbicas, que resultan de interés para corrientes con una humedad significativa, y su muy baja probabilidad de presentar propiedades catalíticas. Sin embargo, estos tipos de adsorbentes, como las zeolitas, poseen un costo inicial sustancialmente mayor que el carbón activado.

Si bien se han llevado a cabo estudios experimentales de isotermas de adsorción de VOCs sobre diferentes materiales poliméricos (Choung y col., 2001), así como simulaciones teóricas de la utilización de Thermal Swing Adsorption (Ambrozek, 2004), tomando como caso de estudio la adsorción de tolueno sobre Dowex Optipore V493 (un polímero comercializado como adsorbente por Dow Chemical Company), el volumen de información experimental sobre la capacidad de adsorción de materiales poliméricos es sustancialmente menor que sobre zeolitas y carbones activados, siendo claramente este último material el que más ha sido estudiado, tanto en trabajos experimentales como de simulación.

\subsection{Criterios de selección de material adsorbente}

Existen una serie de criterios a considerar $(E P A, 1999)$ para la selección del material adsorbente: 


\subsubsection{Tamaño de la molécula de adsorbato vs tamaño de poro del adsorbente}

Las zeolitas cuyo tamaño de poro resulta uniforme en un rango de 0.3 - $3 \mathrm{~nm}$, no pueden proveer un área de adsorción para moléculas de mayor tamaño que sus poros y menos aún para aquellas por las que no tienen afinidad. Para el carbón y los polímeros sucede lo mismo, a diferencia de que el rango de tamaño de poro es mucho más amplio, por lo que este criterio es mucho menos estricto que para las zeolitas.

\subsubsection{Microporosidad}

Debido al espacio reducido en el interior del poro se obtiene una velocidad de difusión más lenta de la molécula lo que dificulta la regeneración del material adsorbente. Este fenómeno suele darse en adsorbentes como carbón, zeolitas y algunos polímeros.

\subsubsection{Afinidad del adsorbente por algunas sustancias}

Cada adsorbente tiene una cierta afinidad por algunas sustancias y no por otras. La molécula de mayor afinidad será preferentemente adsorbida frente a moléculas de menor afinidad. En el momento de elegir el adsorbente se debe estudiar qué afinidad tiene este último con la sustancia que se desea adsorber.

\subsubsection{Costos y frecuencia de reemplazo}

Este criterio es de importancia al considerar los balances económicos. No sólo se debe conocer el costo inicial del material adsorbente sino también su frecuencia de reemplazo.

El carbón presenta un menor costo inicial que las zeolitas o polímeros, sin embargo su frecuencia de reemplazo es mayor. Esta última depende del tipo de carbón, la frecuencia de regeneración y la temperatura de operación.

\subsubsection{Temperatura de desorción}

La temperatura máxima de desorción debe ser superior a la temperatura de ebullición del VOC pero menor a la temperatura de fusión del adsorbente. La temperatura de fusión del polímero es relativamente baja, por lo que la temperatura 
máxima de desorción, cuando se opera con adsorbentes poliméricos, es menor que la permitida cuando se utiliza carbón activado o zeolita.

El límite real de temperatura para las zeolitas puede depender de la temperatura de pirólisis, la de catálisis del VOC adsorbido o del rango de temperaturas entre las cuales la estructura cristalina se mantiene estable.

Es obvio que cuanto mayor es la temperatura de regeneración más se favorece la desorción de los VOCs. Sin embrago, para temperaturas de desorción excesivamente altas, mayor es el esfuerzo para enfriar el lecho previamente a la etapa de adsorción. Esto puede implicar una reducción en la eficiencia global del proceso (Chang y col, 2003).

\section{Selección del material adsorbente}

Para la selección del material adsorbente se deben tener en cuenta los criterios mencionados previamente $\mathrm{y}$, obviamente, cuando se propone llevar a cabo una simulación, información específica y confiable sobre la capacidad del adsorbente elegido frente a las sustancias a tratar, en este caso acetato de etilo y etanol.

Para el sistema seleccionado para este estudio (eliminación de acetato de etilo y etanol) se han realizado estudios de purificación empleando adsorbentes de diferentes tipos, como diversos tipos de zeolitas (Manjare y Ghoshal, 2005) o carbones activados (Gales y col., 2003). Teniendo en cuenta el tipo y concentración de VOC, la afinidad entre el VOC y el adsorbente, y el costo del adsorbente, se optó por el uso de carbón activado.

\subsection{Diseño del sistema de adsorción/desorción}

El diseño de un sistema de adsorción depende de las características del VOC que vaya a ser retenido, de las condiciones operativas de la corriente de gas a la entrada de la columna (temperatura, presión, caudal volumétrico) y de las propiedades físicas del adsorbente (Ruthven, 1984).

En principio, se consideró para el estudio un sistema de adsorción/desorción constituido por dos lechos de igual tamaño. Ambos lechos operan alternativamente para adsorber o desorber. Un dado lecho permite adsorber los VOCs de la corriente a tratar hasta que la presencia de los mismos se evidencia en el efluente. Alcanzada una cierta concentración máxima permitida, se debe discontinuar la operación en ese lecho. La 
corriente a tratar es derivada al segundo lecho, mientras que el lecho usado debe regenerarse, desorbiendo los VOCs con una corriente caliente en sentido inverso a la corriente de adsorción. Una vez que el lecho se encuentra regenerado ya queda disponible para actuar nuevamente como adsorbedor. El ciclo de adsorción/desorción descrito demanda un cierto período, que dependerá de la masa de adsorbente empleada en los lechos (Capítulo 6).

Para la desorción, se utiliza una corriente de aire puro, cuyo caudal suele ser menor al utilizado en la etapa de adsorción, y se encuentra a una temperatura lo suficientemente alta como para permitir una desorción rápida sin desestabilizar el material adsorbente. Para una determinada temperatura de desorción, cuanto menor es el caudal empleado en esta etapa, se alcanza una mayor concentración en la corriente a la salida, la cual se envía al incinerador catalítico. De esta manera, al operar con un caudal bajo y concentrado en VOCs, el reactor catalítico y los intercambiadores de recuperación de calor resultarán convenientemente pequeños.

Para este tipo de lechos, se consideraron dos opciones para disponer el material adsorbente: un lecho fijo relleno con gránulos del adsorbente o un lecho estructurado con canales de sección uniforme en una matriz sólida (monolito).

Alternativamente al uso de dos lechos, surge el concentrador rotativo. La aparición en el mercado de este tipo de sistemas para la remoción de VOCs abrió, en la década del 80, un nuevo camino hacia la investigación y desarrollo de procesos de tratamiento de aire (Rezaei y Webley, 2010).

Los concentradores rotativos constan de una estructura fija que se encuentra dividida en dos compartimientos. En uno de ellos se impulsa la corriente a tratar, que luego circula a través de una fracción de un cilindro rotativo que contiene el material adsorbente (Process zone en la Fig. 2) y finalmente se conduce hasta la descarga. En otro compartimiento, generalmente más pequeño, se introduce la corriente de desorción, que, luego de pasar por una segunda fracción del cilindro (Cooling zone en la Fig. 2), sucesivamente se precalienta para luego retornar a la tercer zona del cilindro (Desorption zone en la Fig. 2), donde se regenera el material adsorbente, y finalmente se lo impulsa para su descarga (Fig.2).

Por razones de visualización, en la Fig. 2 no se ilustra la estructura fija que determina ambos compartimientos.

El material adsorbente normalmente se dispone en el cilindro en módulos del tipo monolito de canales regulares, con el material adsorbente depositado sobre las paredes o 
alternativamente constituyendo la propia estructura de los módulos (Rezaei y Webley, 2010; Yates y col., 2010). La rotación del cilindro permite que la porción de adsorbente cargada de VOCs ingrese sucesivamente a la zona de desorción y luego a la de enfriamiento para que, una vez regenerada, complete el ciclo ingresando nuevamente a la zona de adsorción.

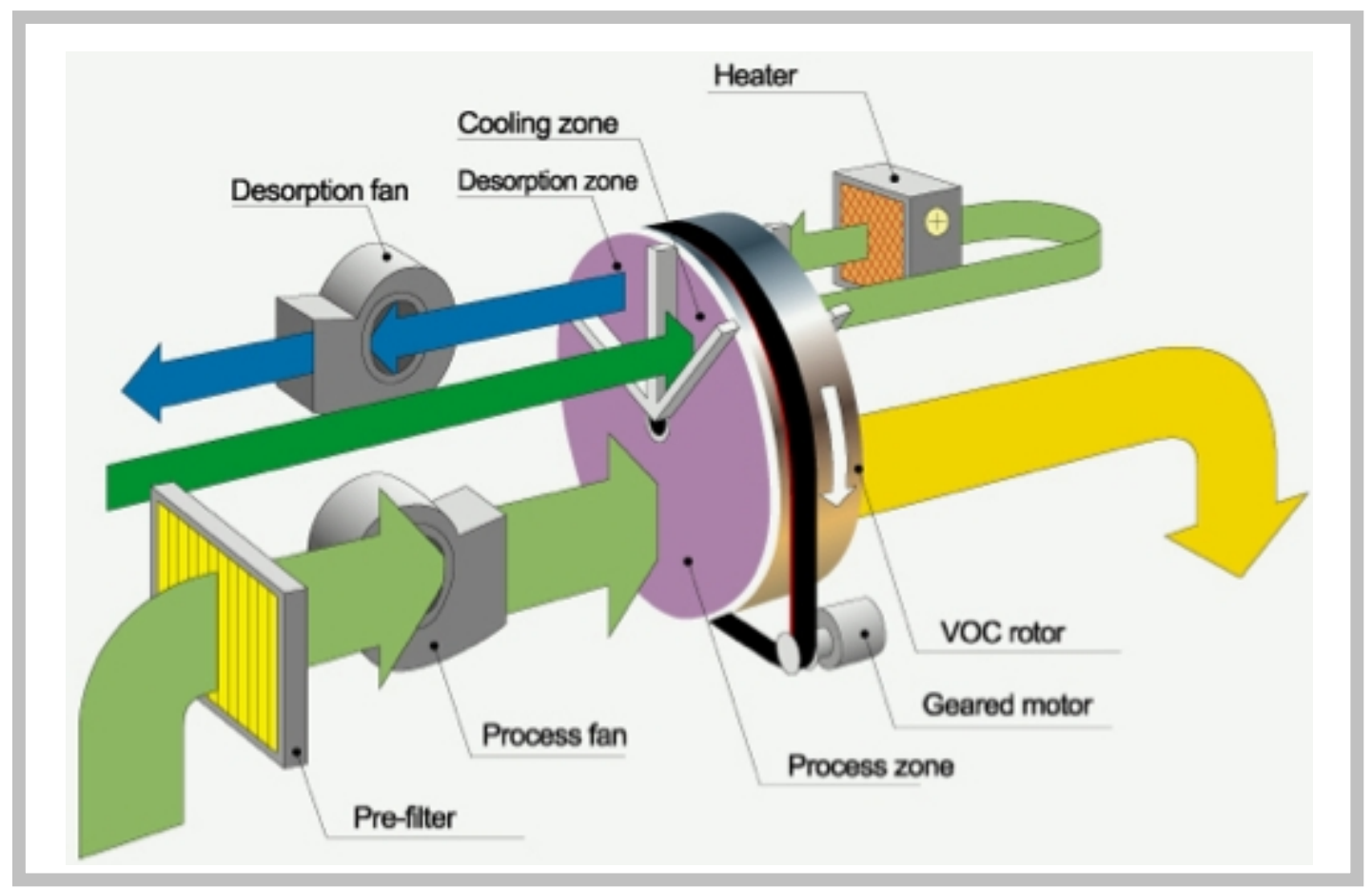

Fig. 2 - Esquema del proceso utilizando un concentrador rotativo.

En la Figura 3 se ha aislado el cilindro rotativo para mejor visualización de las zonas. Como fuera previamente discutido, en la aplicación específica de este trabajo no se incluirá la zona de enfriamiento. Fotografías de cilindros rotativos se incluyen en la Figura 4, donde además se ilustran los canales de la estructura monolítica.

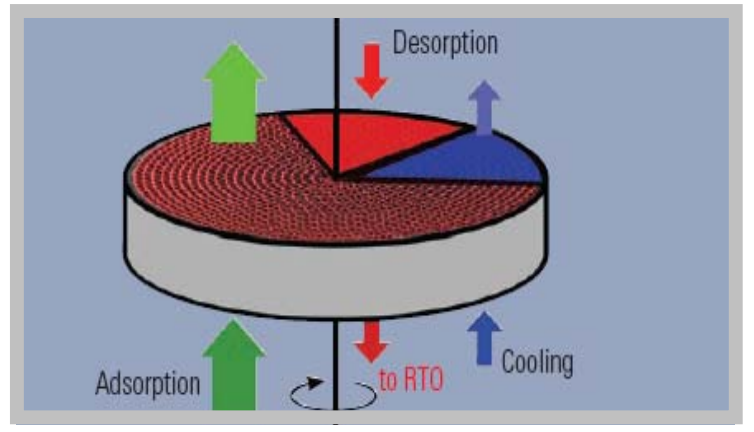

Fig. 3 - Concentrador rotativo de flujo axial y de instalación vertical. 


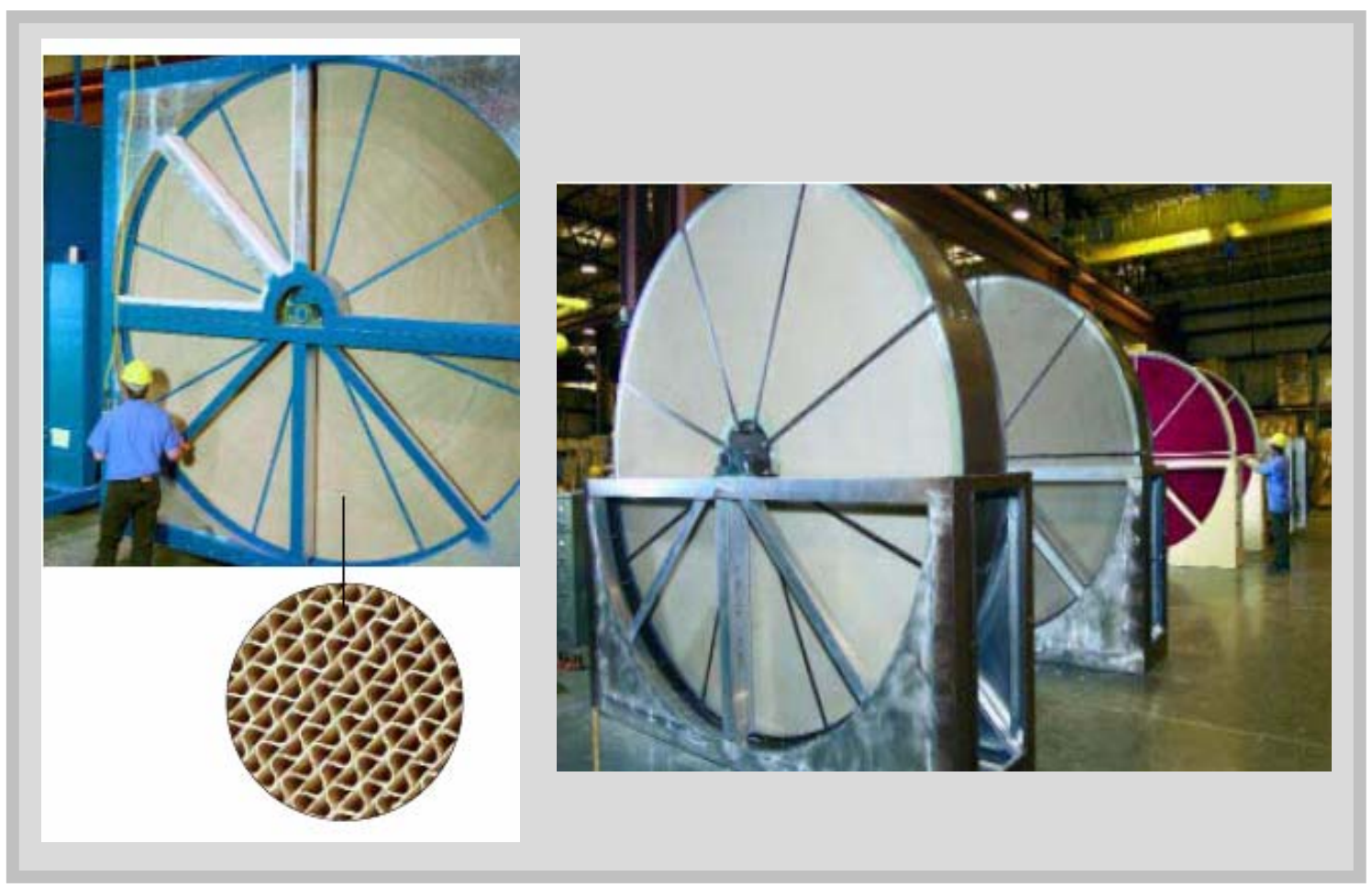

Fig. 4- Concentrador rotativo de flujo axial y de instalación horizontal.

El cilindro puede disponerse, para su operación, tanto de manera vertical (Figs. 2 y 4) como horizontal (Fig. 3). En ambos casos, donde el flujo es de tipo axial, el equipo posee una relación diámetro/longitud relativamente elevada (Fig. 4) adoptando una forma similar a un disco o rueda. Para elevados caudales de la corriente a tratar, el diámetro del disco puede resultar muy elevado y por lo tanto de difícil construcción e instalación. Surge como alternativa el uso de un cilindro rotativo con flujo radial, como se indica en la Figura 5, donde solamente está representada la zona de desorción. En este caso, el tamaño requerido de la sección de flujo para una pérdida de carga tolerable se alcanza usando un diámetro técnicamente razonable junto con una considerable longitud del cilindro. La cantidad de adsorbente en ambas modalidades, con flujo axial o radial, debe ser similar, a fin de mantener la capacidad de adsorción. El cilindro es identificado usualmente en la bibliografía como carrusel. Cualquiera sea el tipo de instalación del cilindro o el sentido del flujo, el modo operativo es el mismo. 


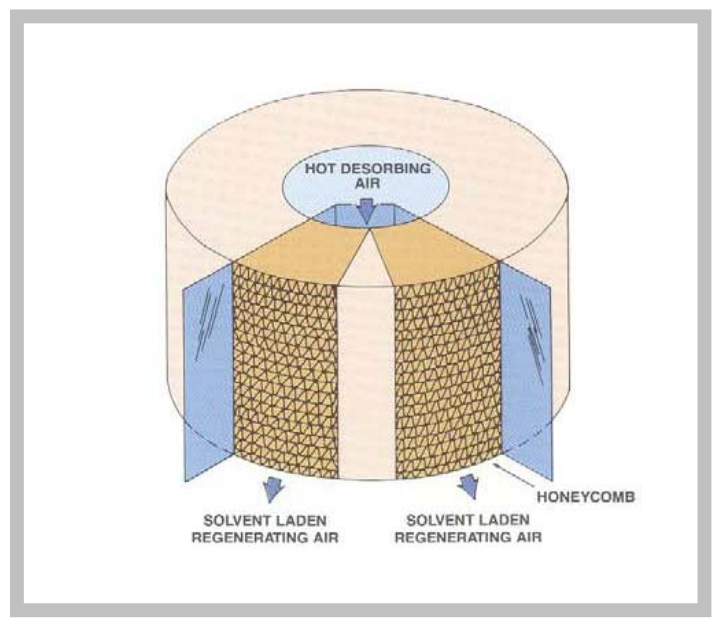

Fig. 5 - Concentrador rotativo de flujo radial y de instalación vertical.

Tanto el sistema de adsorción/desorción constituido por dos lechos como el concentrador rotativo, presentan muchas ventajas cuando, con el fin de concentrar, se opera en conjunto con un sistema de combustión térmica o catalítica como se propuso en el Capítulo 2 (Fig. 3). La principal ventaja es la posibilidad de usar el calor de la combustión del solvente para abastecer el precalentamiento del aire requerido para la desorción y el de la corriente de entrada al reactor. Este tipo de sistemas se emplea en aquellas industrias cuyas emisiones son muy diluidas y se encuentran a temperatura ambiente. Algunas aplicaciones típicas de este tipo de sistemas son:

- Pintura y revestimiento

- Producción de semiconductores

- Imprenta

Ambas alternativas de diseño pueden simularse empleando la misma metodología, que se describe en capítulos posteriores (Capítulos 4 y 5).

\subsection{Disposición del material adsorbente}

Como fue mencionado en la sección anterior, se consideraron dos opciones para disponer el material adsorbente: un lecho fijo relleno con gránulos del adsorbente o un lecho estructurado con canales de sección uniforme en una matriz sólida (monolito).

Para el caso del monolito, se analizaron dos alternativas en cuanto a su construcción: o bien depositar el adsorbente sobre las paredes de la matriz del monolito (Gadkaree, 1998) o conformar las paredes con el propio adsorbente (Yates y col., 2000; Rezaei y Webley, 2010). En el primer caso, se propuso una matriz cerámica (cordierita) revestida 
por una capa de carbón activado. Para la segunda alternativa directamente se consideró que la propia matriz está construida con carbón activado. Se retuvo esta última opción en virtud de la diferencia de capacidades caloríficas entre ambas estructuras. La cordierita tiene una capacidad calorífica relativamente alta, con lo que el valor promedio del sólido resultó ser muy elevada $\left(3.810^{6} \mathrm{~J} /{ }^{\circ} \mathrm{C} \mathrm{m}{ }^{3}\right.$ ), promoviendo un retardo en el enfriamiento del lecho durante la etapa de adsorción y por ende aumentando los tiempos del proceso. En cambio, el carbón activado presenta una capacidad calorífica con un orden más bajo que la anterior $\left(4.910^{5} \mathrm{~J} /{ }^{\circ} \mathrm{C} \mathrm{m}{ }^{3}\right)$.

Con respecto a las alternativas de tipo de lecho mencionadas, relleno o estructurado, es aceptado que ambos sistemas presentan ventajas y desventajas que los hacen competitivos. Los sistemas con rellenos han sido empleados desde hace un largo tiempo en adsorbedores $\mathrm{y}$, genéricamente, tienen un costo menor que los sistemas estructurados. Recientemente, Rezaei y Webley (2010) realizaron una revisión de sistemas estructurados y puntualizaron qué características deberían cumplir los mismos para ser competitivos con un lecho relleno. Estos autores indican que se debería tener altas velocidades, o sea bajas resistencias a la transferencia de materia, tanto en la fase gaseosa (coeficiente pelicular) como en el medio poroso. Se debe alcanzar un alto "volume working capacity", o sea la capacidad de adsorción por unidad de volumen, y una baja porosidad, a efectos de minimizar el tamaño del equipo. El flujo de fluidos debe ser tal que conduzca a una baja pérdida de carga, para minimizar el consumo energético. Asimismo, se debe lograr una adecuada eficiencia en los procesos térmicos, tanto en cuanto a los procesos de acumulación como de transferencia de calor. Obviamente, algunos de estos requerimientos derivan en situaciones de compromiso que requieren la optimización de las características geométricas del sistema estructurado. Crittenden y col. (2005) a través de estudios experimentales con monolitos de carbón activado y Patton y col. (2004), mediante un estudio teórico, demostraron la posibilidad de alcanzar, en la etapa de adsorción, performances similares en cuanto a la transferencia de materia en monolitos y en lechos rellenos. Simultáneamente, verificaron que la pérdida de carga era sustancialmente menor en los monolitos.

En resumen, no resulta obvia la elección en cuanto a la disposición del material en el adsorbedor, por lo que en el Capítulo 5 se analizan los resultados obtenidos a partir de comparar un lecho fijo relleno de pellets esféricos y un monolito, teniendo en cuenta las condiciones operativas de nuestro caso particular de estudio descripto en el Capítulo 2 (adsorción de acetato de etilo y etanol). 


\subsection{Conclusiones}

En este capítulo se analizó el proceso de adsorción y su vinculación con el tratamiento de efluentes gaseosos, particularmente corrientes de aire contaminadas con VOCs. La adsorción se emplea para remover VOCs de corrientes diluidas de gas de baja o media concentración, cuando se desea la recuperación del VOC y/o se requiere obtener una corriente más concentrada. El sistema de eliminación de VOCs propuesto en el Capítulo 2 (Fig. 3), incluye un adsorbedor con el fin de concentrar la corriente a tratar. De esta manera, al operar con un caudal bajo y concentrado en VOCs, el reactor catalítico y los intercambiadores de recuperación de calor resultarán convenientemente pequeños.

Se describieron las distintas técnicas empleadas para llevar a cabo los procesos de adsorción/desorción. Teniendo en cuenta los requerimientos energéticos, la TSA resulta ser la técnica más apropiada para nuestro caso particular de estudio. Cabe recordar que, para el sistema combinado del Capítulo 2 (Fig. 3), es factible el uso del calor liberado durante la reacción de oxidación para el calentamiento de la corriente empleada para la regeneración del adsorbedor.

También se presentaron los posibles materiales adsorbentes a utilizar junto con los criterios a tener en cuenta para la selección de los mismos. Teniendo en cuenta el tipo y concentración de VOC, la afinidad entre el VOC y el adsorbente, y el costo del adsorbente, se optó por el uso de carbón activado para poder obtener el diseño del sistema.

Se retuvieron dos alternativas de diseño para los sistemas de adsorción/desorción, consistentes en un sistema de dos lechos que operan alternativamente o un concentrador rotativo (Figs. 2-5). En el Capítulo 6 se realiza una selección entre dichas alternativas en base a las ventajas que presenta una sobre la otra.

Finalmente se describió en que forma es factible disponer el material adsorbente, ya sea un lecho fijo relleno con gránulos del adsorbente o un lecho estructurado con canales de sección uniforme en una matriz sólida (monolito). En el Capítulo 5 se realiza un análisis en base a la comparación entre un lecho fijo relleno de partículas esféricas y un monolito.

Para el caso del monolito, el adsorbente se supuso depositado sobre las paredes de la matriz monolítica, o bien, constituyendo el material de las mismas. En el primer caso, se propuso una matriz cerámica (cordierita) revestida por una capa de carbón activado, 
mientras que para la segunda alternativa directamente se consideró que la propia matriz se encuentra construida con carbón activado.

Dado que, al emplear cordierita el valor promedio de la capacidad calorífica del sólido resulta relativamente alta, se optó por considerar que la matriz del monolito está construida con carbón activado. 

N ESTUDIO DE SISTEMAS

COMBINADOS DE

COMBUSTION

CATALITICA DE VOCs

\section{4/ SIMULACION DEL SISTEMA DE ADSORCION/DESORCION SIN CONSIDERAR LAS RESISTENCIAS A LA TRANSFERENCIA DE CALOR Y MATERIA}

Sistema isotérmico de un componente Sistema adiabático multicomponente

Adsorción simultánea de acetato de etilo y etanol

Conclusiones 



\section{$04 /$

Tanto para el uso de 2 lechos como para la alternativa de un concentrador rotativo, es posible emplear la misma metodología de simulación.

Para la simulación del sistema de adsorción/desorción se tuvieron en cuenta dos enfoques. La diferencia entre los mismos se basó en incluir, o no, las resistencias a la transferencia de calor y materia, dentro y fuera del material adsorbente.

En una primera etapa de análisis del sistema de adsorción/desorción, no se tuvieron en cuenta las resistencias a la transferencia de calor y materia, tanto dentro como fuera del sólido. Para resolver el sistema sin resistencias se aplicó el Método de las Características (Rhee y col., 1970 a, b; Rhee y col., 1972). Este método permite reemplazar el sistema de ecuaciones diferenciales de derivadas parciales de primer orden con dos variables independientes (tiempo y espacio) por un sistema de ecuaciones diferenciales ordinarias con una única variable independiente.

A lo largo de este capítulo se describe el Método de las Características para un sistema isotérmico con un único componente y para un sistema adiabático de multicomponentes. Finalmente, se aplica dicho método a nuestro caso particular de estudio, donde se deben adsorber acetato de etilo y etanol presentes en una corriente de aire (Capítulo 2).

\subsection{Sistema isotérmico de un componente}

A continuación se describe el Método de las Características para el caso particular de una columna de adsorción isotérmica, donde un único soluto es transportado por la fase móvil y se adsorbe en la fase estacionaria. Ignorando los efectos difusivos en ambas fases, surge el siguiente balance de materia diferencial:

$$
u \frac{\partial \mathrm{C}}{\partial \mathrm{Z}}+\left[\frac{\partial \mathrm{C}}{\partial \mathrm{t}}+\mathrm{v} \frac{\partial \mathrm{q}(\mathrm{C})}{\partial \mathrm{t}}\right]=0
$$

donde $\mathrm{C}$ es la concentración de la especie a adsorber, $\mathrm{Z}$ es la variable espacial medida desde la entrada del adsorbedor $(Z=0)$ hasta la salida $(Z=L)$, t es el tiempo, $u$ la velocidad intersticial, $v$ la relación volumétrica entre las fases sólida y fluida en 
función de la porosidad: $\left(1-\varepsilon_{\mathrm{L}}\right) / \varepsilon_{\mathrm{L}}$, y q representa los moles adsorbidos por unidad de volumen de adsorbente. En rigor, dado que en la práctica los adsorbentes son porosos, resulta apropiado incluir en el balance (Ec. [1]) la acumulación de adsorbato en el volumen de poros de la partícula. Sin embargo, puesto que nuestro caso particular de estudio, cuyas características se mencionan en el Capítulo 2, consta de una corriente gaseosa diluida a presión atmosférica, el hecho de considerar o no la cantidad de adsorbato dentro de los poros del adsorbente resulta irrelevante. En consecuencia, en esta presentación, el término correspondiente a la acumulación de VOCs dentro de los poros no fue incluido en los balances de materia. Dicha simplificación pudo ser verificada empleando condiciones específicas de operación y las isotermas de adsorción.

Entre C y q debe existir una relación de equilibrio que dependerá de la isoterma de adsorción establecida.

$$
\mathrm{q}=f(C)
$$

Definiendo $\mathrm{h}(\mathrm{C})$ como la cantidad de moles totales por unidad de volumen de fase fluida se obtiene:

$$
\mathrm{h}(\mathrm{C})=\mathrm{C}+v \mathrm{q}(\mathrm{C})
$$

reemplazando en [1] resulta:

$$
u \frac{\partial \mathrm{C}}{\partial \mathrm{Z}}+\left[\frac{\partial \mathrm{h}(\mathrm{C})}{\partial \mathrm{t}}\right]=0
$$

Equivalentemente, de [4] surge:

$$
u \frac{\partial \mathrm{C}}{\partial \mathrm{Z}}+\mathrm{h}^{\prime}(\mathrm{C}) \frac{\partial \mathrm{C}}{\partial \mathrm{t}}=0
$$

donde $h^{\prime}(C)=\frac{d h}{d C}$

Analizamos la posibilidad de encontrar una dirección $\Psi$ en el plano $(\mathrm{Z}, \mathrm{t})$ a lo largo de la cual la ecuación diferencial [1] pueda transformarse en una ecuación diferencial ordinaria. A tal fin recordamos que:

$$
\mathrm{dC}=\frac{\partial \mathrm{C}}{\partial \mathrm{Z}} \mathrm{dZ}+\frac{\partial \mathrm{C}}{\partial \mathrm{t}} \mathrm{dt}
$$

La curva $\Psi$ puede expresarse en forma paramétrica, 


$$
\mathrm{Z}=\mathrm{Z}(\omega) ; \quad \mathrm{t}=\mathrm{t}(\omega)
$$

Siendo $\omega$ un parámetro que varía a lo largo de $\Psi$. Dividiendo [7a] por d $\omega$ se obtiene:

$$
\frac{\mathrm{dC}}{\mathrm{d} \omega}=\frac{\partial \mathrm{C}}{\partial \mathrm{Z}} \frac{\mathrm{dZ}}{\mathrm{d} \omega}+\frac{\partial \mathrm{C}}{\partial \mathrm{t}} \frac{\mathrm{dt}}{\mathrm{d} \omega}
$$

La curva $\Psi$, hasta ahora indefinida, la determinaremos de forma tal que $\mathrm{dC} / \mathrm{d} \omega$, sujeta a la ecuación diferencial, no dependa de las derivadas parciales $\partial \mathrm{C} / \partial \mathrm{Z}$ y $\partial \mathrm{C} / \partial \mathrm{t}$. Con dicho fin, igualamos los factores que multiplican a las derivadas parciales en la Ec. [5], con los correspondientes a la Ec. [8], resultando:

$$
\frac{\mathrm{dZ}}{\mathrm{d} \omega}=u \quad[9 \mathrm{a}] \quad \frac{\mathrm{dt}}{\mathrm{d} \omega}=\mathrm{h}^{\prime}(\mathrm{C})
$$

Si se satisfacen las Ecs. [9a] y [9b], resultará:

$$
\frac{\mathrm{dC}}{\mathrm{d} \omega}=0
$$

La ecuación de derivadas parciales [1] se ha descompuesto en tres ecuaciones diferenciales ordinarias $[9 a, b, c]$, que tienen a $\omega$ como variable independiente.

Las proyecciones sobre el plano $(\mathrm{Z}, \mathrm{t})$ de variables independientes, corresponden a la curva $\Psi$ y dependen del valor de la variable C. Estas proyecciones se denominan curvas características y a las Ecs. [9a, b, c] se las denomina ecuaciones características.

La Ec. [9c] establece que $\mathrm{C}$ es constante a lo largo de una curva característica.

Supongamos inicialmente una columna con concentración uniforme, es decir

$$
\mathrm{C}(\mathrm{Z}, 0)=\mathrm{C}^{0} \quad 0<\mathrm{Z}<\mathrm{L}
$$

$y$, además, que se establece un escalón de concentración en la alimentación $(Z=0)$, al tiempo $\mathrm{t}=0$,

$$
\mathrm{C}(0, \mathrm{t})=\mathrm{C}^{\mathrm{F}} \quad \mathrm{t} \geq 0
$$

$\operatorname{con} \mathrm{C}^{\mathrm{F}}>\mathrm{C}^{0}$.

Habrá un grupo de características que arrancan desde el eje Z, identificadas con $\Psi_{0}$, cuya pendiente $\left(1 / u_{\omega, 0}\right)$, evaluada a partir de las Ecs. [9a] y [9b] con $C=C^{0}$, resulta, 


$$
\frac{\mathrm{dt}}{\mathrm{dZ}}=\frac{1}{u_{\omega, 0}}=\frac{\mathrm{h}^{\prime}\left(\mathrm{C}^{0}\right)}{u}
$$

Un segundo grupo de características con concentración $C^{F}$, identificadas con $\Psi_{F}$, que arrancan en el eje t estarán definidas por la pendiente $1 / u_{\omega, \mathrm{F}}:$

$$
\frac{\mathrm{dt}}{\mathrm{dZ}}=\frac{1}{u_{\omega, \mathrm{F}}}=\frac{\mathrm{h}^{\prime}\left(\mathrm{C}^{\mathrm{F}}\right)}{u},
$$

La inversa de las pendientes, e.i. $u_{\omega, 0}$ y $u_{\omega, \mathrm{F}}$, indica la velocidad con la que se propaga la curva característica sobre el plano $(\mathrm{Z}, \mathrm{t})$. La velocidad de propagación es inversamente proporcional a $\mathrm{h}^{\prime}(\mathrm{C})$, por lo que la funcionalidad de la velocidad con $\mathrm{C}$ dependerá estrictamente de la isoterma que haya sido empleada (Ec. [3]). Dependiendo de la relación que exista entre las velocidades $u_{\omega, 0} \mathrm{y} u_{\omega, \mathrm{F}}$, se presentan dos situaciones diferentes.

En primer lugar suponemos que $u_{\omega, 0}>u_{\omega, \mathrm{F}}$. Teniendo en cuenta la funcionalidad de la velocidad con $h^{\prime}(C)$, para que tal relación se verifique, $h(C)$, o lo que es lo mismo la isoterma de adsorción $\mathrm{q}(\mathrm{C})$ (ver Ec. [3]), tendrá que tener pendiente creciente con $\mathrm{C}$, para la situación considerada con $\mathrm{C}^{\mathrm{F}}>\mathrm{C}^{0}$. Se ilustra en la Figura 1 una isoterma de este tipo, que corresponde a las isotermas de adsorción de tipo III (según la clasificación adoptada por IUPAC).

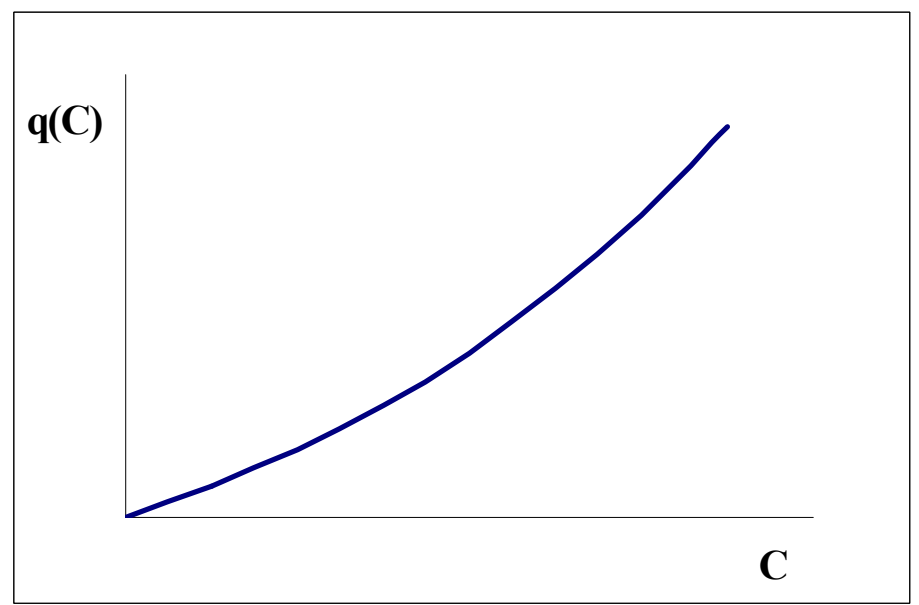

Fig. 1 - Isoterma de adsorción tipo III.

La situación es representada sobre el plano $(\mathrm{Z}, \mathrm{t})$ en la Figura 2, donde se puede observar que del origen, $(\mathrm{Z}, \mathrm{t})=(0,0)$, parten dos líneas características, las rectas a y $\mathbf{b}$, 
correspondientes a cada uno de los grupos de características $\left(\Psi_{0}, \Psi_{\mathrm{F}}\right)$. Entre dichas líneas a y $b$ surge un abanico de rectas cuya pendiente $\left(1 / u_{\omega}\right)$ va creciendo de $\left(1 / u_{\omega, 0}\right)$ a $\left(1 / u_{\omega, \mathrm{F}}\right)$. Cada una de estas curvas lleva asociada un valor diferente de $\mathrm{C}$, la cual va variando de manera continua desde $\mathrm{C}^{0}$ hasta $\mathrm{C}^{\mathrm{F}}$.

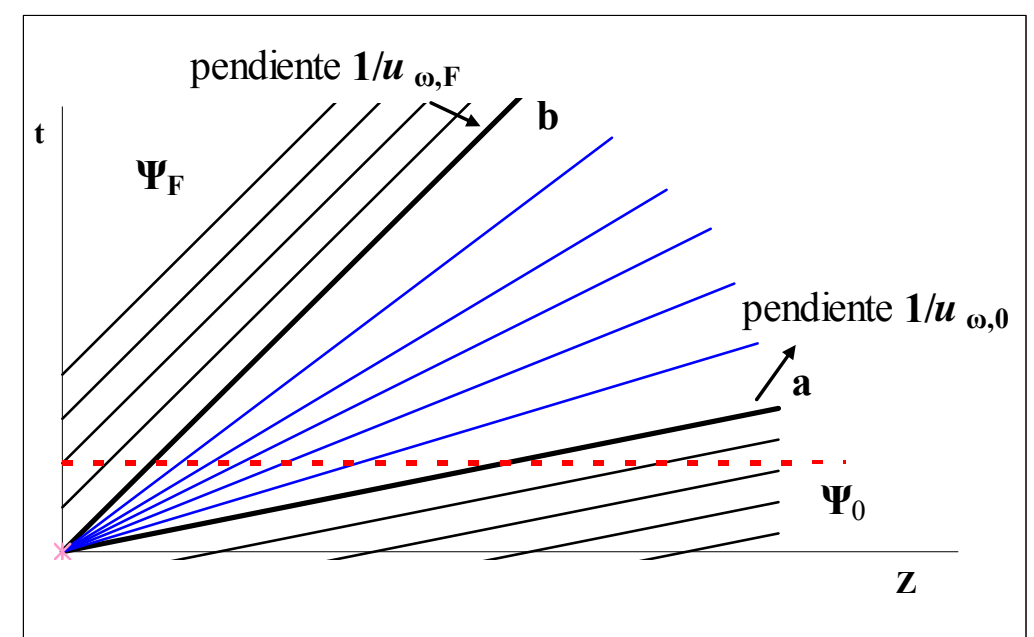

Fig. 2 - Proyecciones de las características para un escalón de concentración $C^{\mathrm{F}} \operatorname{con} u_{\omega, 0}>u_{\omega, \mathrm{F}}$.

Para un tiempo dado (indicado con línea punteada en la Fig. 2) se obtiene un perfil continuo de concentración, C, en el espacio Z. Dicho perfil puede observarse en la Figura 3. Siendo que $u_{\omega, 0}>u_{\omega, \mathrm{F}}$, a medida que el tiempo aumenta la región intermedia se ensancha $\mathrm{y}$, para un dado $\mathrm{Z}$, la concentración en dicha zona va aumentado; este comportamiento está vinculado al conjunto de rectas comprendidas entre las rectas a y b en la Fig. 2.

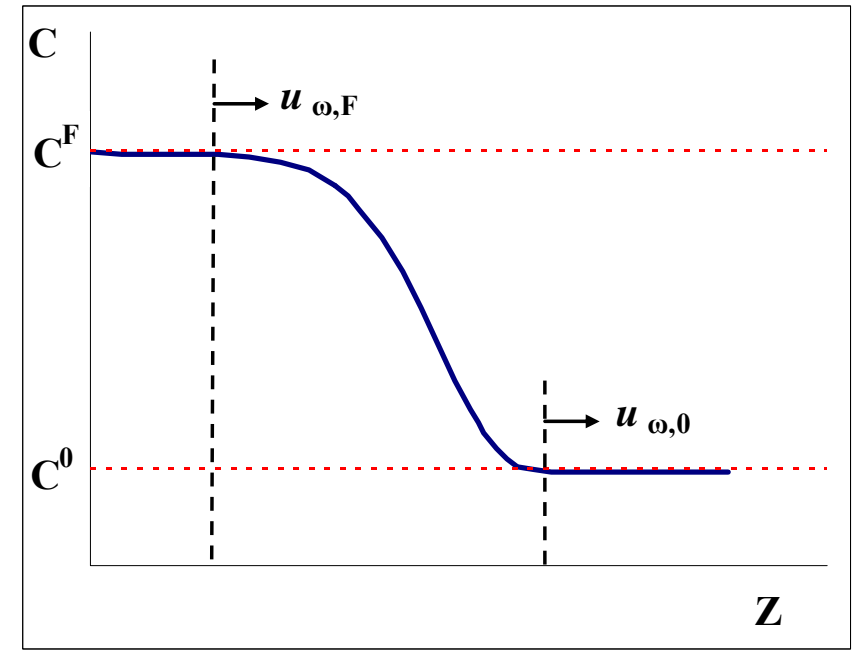

Fig. 3 - Perfil de concentración en la columna de adsorción a un tiempo t. 
En la segunda situación, o sea si $u_{\omega, 0}<u_{\omega, \mathrm{F}}$, sucede la situación inversa a la desarrollada previamente. Esto se da para una isoterma de adsorción tipo I (según la clasificación adoptada por IUPAC) como se puede observar en la Figura 4. Este es el caso de la isoterma de adsorción de Langmuir: $\mathrm{q}=\frac{\alpha \mathrm{C}}{1+\beta \mathrm{C}}$.

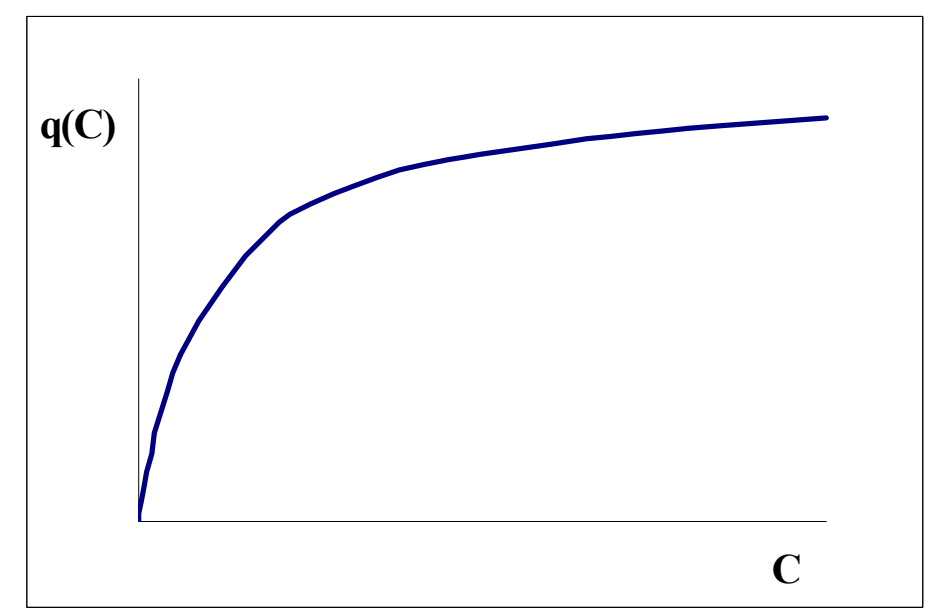

Fig. 4 - Isoterma de adsorción tipo I.

En la Figura 5, se encuentra representada esta situación sobre el plano $(\mathrm{Z}, \mathrm{t})$, donde del origen, $(\mathrm{Z}, \mathrm{t})=(0,0)$, parten dos líneas características, las rectas a y $\mathbf{b}$, correspondientes a cada uno de los grupos de características $\left(\Psi_{0}, \Psi_{\mathrm{F}}\right)$. Estas líneas confinan una zona en donde existe entrecruzamiento de proyecciones de características. Dado que cada característica lleva asociada un valor diferente de $\mathrm{C}$, esta variable resulta indefinida en dicha zona. A fin de asignarle un valor físicamente aceptable a la concentración en la zona en cuestión, partimos del hecho de que en el origen se presenta un caso de discontinuidad y analizamos como se propaga dicha discontinuidad en el tiempo y espacio a través de la línea $\Lambda$ en la Fig. 5. Este fenómeno se conoce como onda de choque.

Para determinar la trayectoria de la recta $\Lambda$, y contemplar si su existencia resulta ser físicamente aceptable, consideramos un pequeño elemento rectangular de lados $\delta \mathrm{Z}$ y $\delta \mathrm{t}$ (ver Figura 6), tal que la relación $1 / u_{s}=\delta \mathrm{t} / \delta \mathrm{Z}$ defina la pendiente de $\Lambda$. $u_{s}$ definirá la velocidad con la que se propaga la onda de choque. Para determinar el valor de dicha pendiente emplearemos la forma integrada del balance de materia en el pequeño elemento (Fig. 6), donde uno de sus vértices, el punto A (de coordenadas $\left(Z_{A}\right.$, $\left.\mathrm{t}_{\mathrm{A}}\right)$ ), representa un punto de discontinuidad por encontrarse sobre la línea $\Lambda$. 


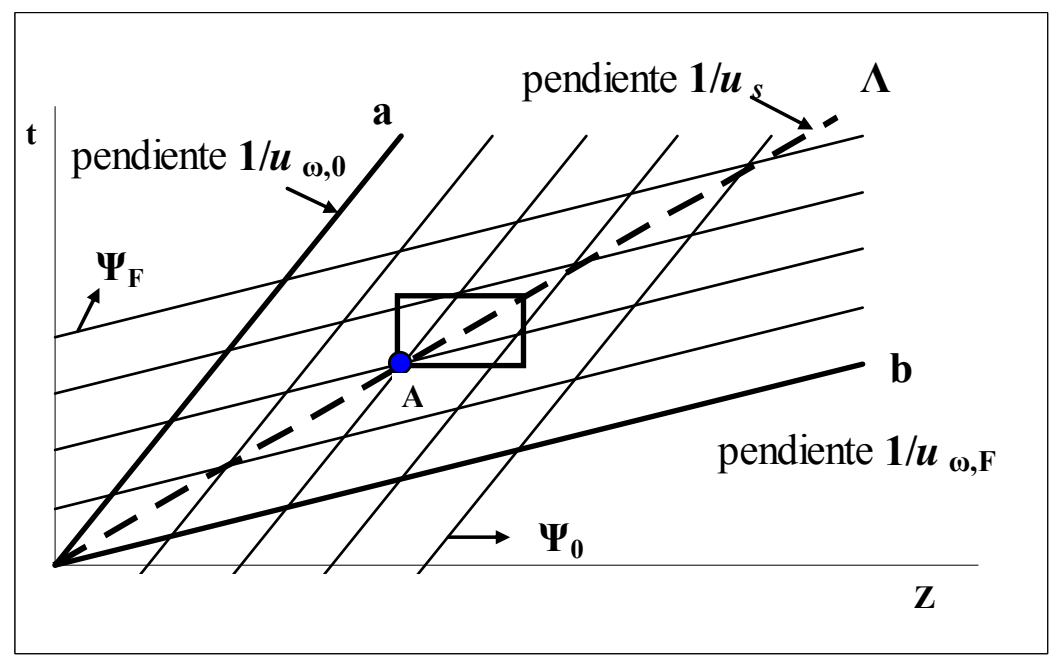

Fig. 5 - Proyecciones de las características para un escalón de concentración $\mathrm{C}^{\mathrm{F}}$.

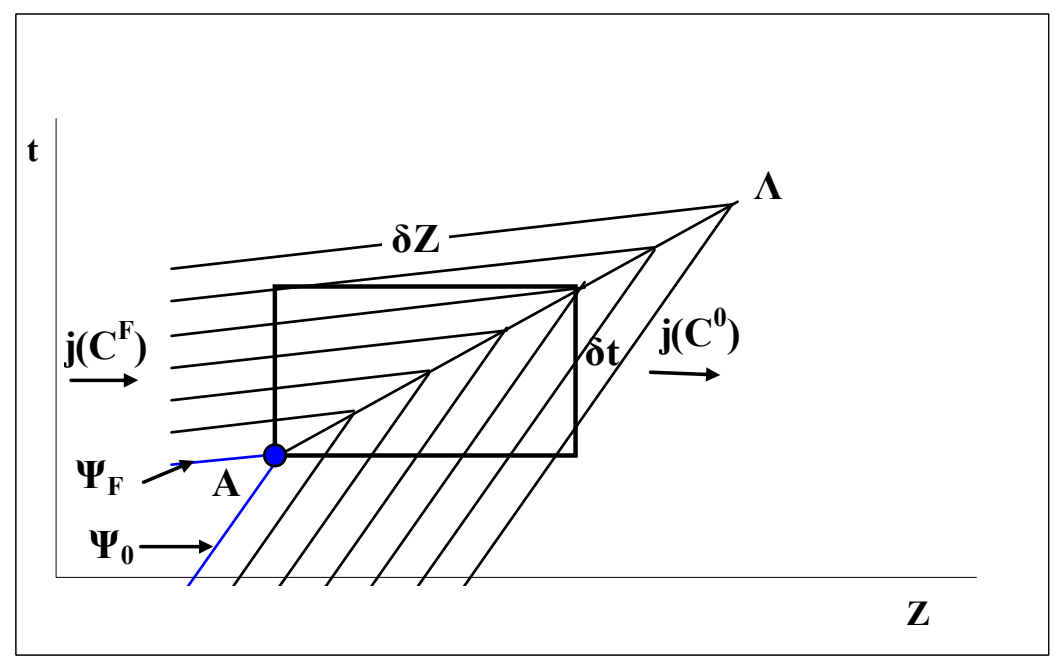

Fig. 6 - Generación de la onda de choque $\Lambda$.

Previo a la resolución de los balances, identificamos como $\mathrm{j}$ al flujo de un componente en la dirección $\mathrm{Z}$, por lo que para el caso particular de adsorción que venimos tratando resulta $\mathrm{j}=u \mathrm{C}$, con $u$ constante. De esta manera el balance de materia diferencial estará dado por la siguiente expresión:

$$
\frac{\partial \mathrm{j}(\mathrm{C})}{\partial \mathrm{Z}}+\frac{\partial \mathrm{h}(\mathrm{C})}{\partial \mathrm{t}}=0
$$

Integrando la Ec.[11] entre $\mathrm{t}_{\mathrm{A}}<\mathrm{t}<\mathrm{t}_{\mathrm{A}}+\delta \mathrm{t}$ y $\mathrm{Z}_{\mathrm{A}}<\mathrm{Z}<\mathrm{Z}_{\mathrm{A}}+\delta \mathrm{Z}$, siendo $\left(\mathrm{Z}_{\mathrm{A}}, \mathrm{t}_{\mathrm{A}}\right)$ las coordenadas del punto A, resulta,

$$
\left.\int_{t_{A}}^{t_{A}+\delta t} j\right|_{Z_{A}+\delta Z} d t-\left.\int_{t_{A}}^{t_{A}+\delta t} j\right|_{Z_{A}} d t+\left.\int_{Z_{A}}^{Z_{A}+\delta Z} h\right|_{t_{A}+\delta t} d Z-\left.\int_{Z_{A}}^{Z_{A}} h\right|_{t_{A}} d Z=0
$$


El flujo de la primera integral $\mathrm{j}_{\mathrm{z}_{\mathrm{A}}+\delta \mathrm{z}}$ en [12a] estará evaluado sobre todo el intervalo $\mathrm{t}_{\mathrm{A}}<\mathrm{t}<\mathrm{t}_{\mathrm{A}}+\delta \mathrm{t}$ a una concentración $\mathrm{C}^{0}$, por lo que se tiene:

$$
\left.\int_{\mathrm{t}_{\mathrm{A}}}^{\mathrm{t}_{\mathrm{A}}+\delta \mathrm{t}} \mathrm{j}\right|_{\mathrm{Z}_{\mathrm{A}}+\delta \mathrm{Z}} \mathrm{dt}=\mathrm{j}\left(\mathrm{C}^{0}\right) \delta \mathrm{t}
$$

Análogas consideraciones pueden hacerse para las restantes tres integrales del primer miembro de [12a]. Por lo tanto podremos escribir:

$$
\left[\mathrm{j}\left(\mathrm{C}^{0}\right)-\mathrm{j}\left(\mathrm{C}^{\mathrm{F}}\right)\right] \delta \mathrm{t}-\left[\mathrm{h}\left(\mathrm{C}^{0}\right)-\mathrm{h}\left(\mathrm{C}^{\mathrm{F}}\right)\right] \delta \mathrm{Z}=0
$$

donde la pendiente de la curva $\Lambda$ es

$$
\frac{\delta \mathrm{t}}{\delta \mathrm{Z}}=\frac{1}{u_{s}}=\frac{\left[\mathrm{h}\left(\mathrm{C}^{0}\right)-\mathrm{h}\left(\mathrm{C}^{\mathrm{F}}\right)\right]}{\left[\mathrm{j}\left(\mathrm{C}^{0}\right)-\mathrm{j}\left(\mathrm{C}^{\mathrm{F}}\right)\right]}
$$

Hemos determinado así la pendiente del camino de la onda de choque a través de un balance de materia, por lo que su existencia es físicamente aceptable y proporciona la frontera que delimita el cruce de las características a partir de un dado punto A donde se identifica una discontinuidad.

Mediante la aplicación del concepto de ondas de choque se elige una solución entre las soluciones múltiples que en realidad admite la ecuación diferencial y sus condiciones de contorno. Estas soluciones, que corresponden a la aplicación de balances de conservación en forma integrada, del tipo de la Ec. [12a], se denominan soluciones débiles.

Notemos que para la Ec. [11] las trayectorias de las características vienen definidas por,

$$
\frac{\mathrm{dZ}}{\mathrm{d} \omega}=\frac{\partial \mathrm{j}}{\partial \mathrm{C}} \quad \frac{\mathrm{dt}}{\mathrm{d} \omega}=\frac{\partial \mathrm{h}}{\partial \mathrm{C}}
$$

De donde la pendiente de las mismas en el plano $(\mathrm{Z}, \mathrm{t})$ es:

$$
\frac{1}{u_{\omega}}=\frac{\partial \mathrm{h} / \partial \mathrm{C}}{\partial \mathrm{j} / \partial \mathrm{C}}
$$

Para que el balance de materia, integrado en la forma de la Ec. [12b], sea válido, y la trayectoria $\Lambda$ con pendiente definida por [13] permita confinar a cada lado de la misma cada grupo de características, es necesario que el valor de la velocidad $u_{s}$ sea 
intermedio entre aquellos correspondientes a las velocidades de propagación de las curvas características $\Psi_{0}$ y $\Psi_{\mathrm{F}}$, es decir,

$$
u_{\omega, 0} \leq u_{s} \leq u_{\omega, \mathrm{F}}
$$

Para que la relación [15] se satisfaga, es fácil demostrar que la condición suficiente es que $u_{\omega}$, Ec. [14], sea una función monótonamente creciente de C para valores comprendidos entre $\mathrm{C}^{0}$ y $\mathrm{C}^{\mathrm{F}}$.

La línea $\Lambda$ de pendiente $1 / u_{s}$ que pasa por el origen de coordenadas es la trayectoria de la onda de choque (Fig. 5). Por debajo de la misma, las características físicamente aceptables son aquellas con concentración $\mathrm{C}=\mathrm{C}^{0}$. Los perfiles de concentración transitorios serán escalones del tipo de la Figura 7, con el frente de choque viajando con una velocidad $u_{s}$. El transitorio demorará un tiempo dado por $\mathrm{t}_{\mathrm{f}}=\mathrm{L} / \mathrm{u}_{s}$, siendo $\mathrm{L}$ la longitud de la columna.

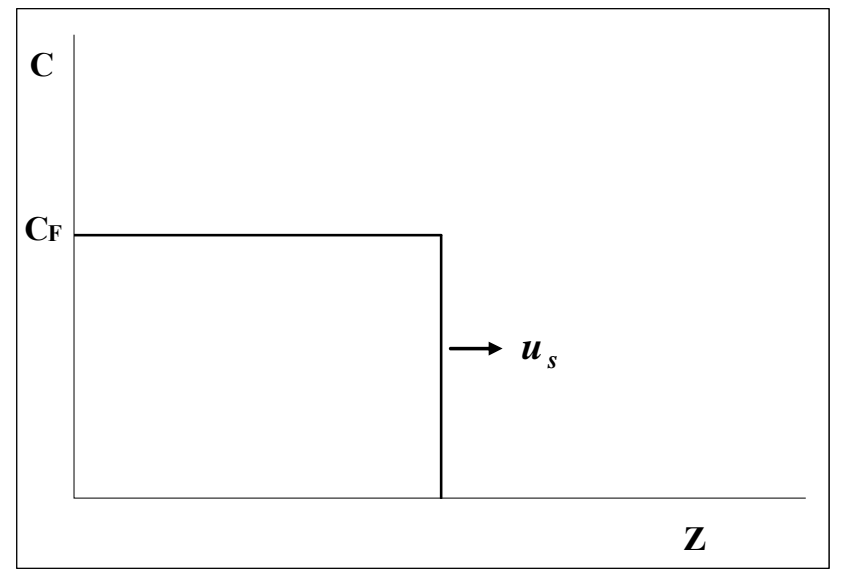

Fig. 7 - Perfiles de concentración en una columna de adsorción.

El Método de las Características puede extenderse a sistemas de multicomponentes. Para el caso particular de que el sistema sea isotérmico, y que se verifique la isoterma de Lagmuir generalizada para los distintos componentes, existe un procedimiento que permite aplicar el Método de las Características de manera práctica y sencilla. El desarrollo del mismo se puede consultar en el libro de Yang, (1987).

Para nuestro caso particular de estudio, donde se pretende concentrar una corriente de aire con acetato de etilo y etanol (Capítulo 2) empleando un sistema de adsorción/desorción mediante la técnica Temperature Swing Adsorption (Capítulo 3), no es posible emplear el procedimiento descrito por Yang (1987), debido a la existencia 
de distintos niveles de temperatura. Para un caso de este tipo, y para cualquier otro caso en que no se verifiquen las condiciones impuestas previamente, surge una metodología de cálculo alternativa que se describirá en la siguiente sección.

\subsection{Sistema adiabático multicomponente}

A continuación se describe el Método de las Características para el caso general de una columna de adsorción adiabática donde m componentes son transportados por la fase móvil y se adsorben en la fase estacionaria. Para el desarrollo presentado a lo largo de esta sección, nos basamos en los trabajos de Rhee y col. (1970 a, b; 1972).

Cuando ambas fases se encuentran en equilibrio los balances de materia por componente y entalpía, considerando concentraciones y temperatura uniformes en la sección transversal del lecho, son:

$$
\begin{aligned}
& u \frac{\partial \mathrm{C}_{\mathrm{i}}}{\partial \mathrm{Z}}+\left[\frac{\partial \mathrm{C}_{\mathrm{i}}}{\partial \mathrm{t}}+\mathrm{v} \frac{\partial \mathrm{q}_{\mathrm{i}}}{\partial \mathrm{t}}\right]=0 \quad \mathrm{i}=1, \ldots, \mathrm{m} \\
& \mathrm{c}_{\mathrm{p}, \mathrm{g}} u \frac{\partial \mathrm{T}}{\partial \mathrm{Z}}+\left[\left(\mathrm{c}_{\mathrm{p}, \mathrm{g}}+v \mathrm{c}_{\mathrm{p}, \mathrm{s}}\right) \frac{\partial \mathrm{T}}{\partial \mathrm{t}}-v \sum_{\mathrm{i}=1}^{\mathrm{m}}\left(-\Delta \mathrm{H}_{\mathrm{i}}\right) \frac{\partial \mathrm{q}_{\mathrm{i}}}{\partial \mathrm{t}}\right]=0
\end{aligned}
$$

Por regla de la cadena se tiene:

$$
\frac{\partial \mathrm{q}_{\mathrm{i}}}{\partial \mathrm{t}}=\mathrm{q}_{\mathrm{i}, \mathrm{T}} \frac{\partial \mathrm{T}}{\partial \mathrm{t}}+\sum_{\mathrm{k}=1}^{\mathrm{m}} \mathrm{q}_{\mathrm{i}, \mathrm{k}} \frac{\partial \mathrm{C}_{\mathrm{k}}}{\partial \mathrm{t}} \quad \mathrm{i}=1, \ldots, \mathrm{m}
$$

donde: $\quad \mathrm{q}_{\mathrm{i}, \mathrm{T}}=\partial \mathrm{q}_{\mathrm{i}} / \partial \mathrm{T} ; \quad \mathrm{q}_{\mathrm{i}, \mathrm{k}}=\partial \mathrm{q}_{\mathrm{i}} / \partial \mathrm{C}_{\mathrm{k}} \quad \mathrm{i}, \mathrm{k}=1, \ldots, \mathrm{m}$

Reemplazando [18] en las Ecs. [17] se obtiene:

$$
\begin{aligned}
& u \frac{\partial \mathrm{C}_{\mathrm{i}}}{\partial \mathrm{Z}}+\left[\frac{\partial \mathrm{C}_{\mathrm{i}}}{\partial \mathrm{t}}+v \mathrm{q}_{\mathrm{i}, \mathrm{T}} \frac{\partial \mathrm{T}}{\partial \mathrm{t}}+v \sum_{\mathrm{k}=1}^{\mathrm{m}} \mathrm{q}_{\mathrm{i}, \mathrm{k}} \frac{\partial \mathrm{C}_{\mathrm{k}}}{\partial \mathrm{t}}\right]=0 \quad \mathrm{i}=1, \ldots, \mathrm{m} \\
& \mathrm{c}_{\mathrm{p}, \mathrm{g}} u \frac{\partial \mathrm{T}}{\partial \mathrm{Z}}+\left(\mathrm{c}_{\mathrm{p}, \mathrm{g}}+v \mathrm{c}_{\mathrm{p}, \mathrm{s}}-v \sum_{\mathrm{i}=1}^{\mathrm{m}}\left(-\Delta \mathrm{H}_{\mathrm{i}}\right) \mathrm{q}_{\mathrm{i}, \mathrm{T}}\right) \frac{\partial \mathrm{T}}{\partial \mathrm{t}}-v \sum_{\mathrm{k}=1}^{\mathrm{m}}\left(\sum_{\mathrm{i}=1}^{\mathrm{m}}\left(-\Delta \mathrm{H}_{\mathrm{i}}\right) \mathrm{q}_{\mathrm{i}, \mathrm{k}}\right) \frac{\partial \mathrm{C}_{\mathrm{k}}}{\partial \mathrm{t}}=0
\end{aligned}
$$

Para compactar la formulación definimos:

$\mathrm{C}_{\mathrm{m}+1} \equiv \mathrm{T}$,

$\mathrm{q}_{\mathrm{m}+1} \equiv \mathrm{q}_{\mathrm{T}} \equiv \gamma \mathrm{T}-\sum_{\mathrm{i}=1}^{\mathrm{m}} \mathrm{J}_{\mathrm{i}} \mathrm{q}_{\mathrm{i}}$ 
donde $\quad \gamma=\mathrm{c}_{\mathrm{p}, \mathrm{s}} / \mathrm{c}_{\mathrm{p}, \mathrm{g}} ; \quad \mathrm{J}_{\mathrm{i}}=-\Delta \mathrm{H}_{\mathrm{i}} / \mathrm{c}_{\mathrm{p}, \mathrm{g}} \quad \mathrm{i}=1, \ldots, \mathrm{m}$

Suponiendo que $\gamma$ y $\mathrm{J}_{\mathrm{i}}$ son constantes, podemos sustituir las nuevas variables en las Ecs. [19] resultando:

$$
u \frac{\partial \underline{\mathrm{C}}}{\partial \mathrm{Z}}+(\mathrm{I}+v \mathcal{F}) \frac{\partial \underline{\mathrm{C}}}{\partial \mathrm{t}}=\underline{0}
$$

donde $\mathcal{F}$ es una matriz de $(\mathrm{m}+1) \times(\mathrm{m}+1)$ componentes definidos por $\mathcal{F}_{\mathrm{ij}}=\partial \mathrm{q}_{\mathrm{i}} / \partial \mathrm{C}_{\mathrm{j}}$, I es la matriz identidad de $(\mathrm{m}+1) \times(\mathrm{m}+1)$ componentes y $\underline{\mathrm{C}}$ es el vector definido por $\underline{\mathrm{C}}=\left(\mathrm{C}_{1}, \mathrm{C}_{2}, \ldots, \mathrm{C}_{\mathrm{m}}, \mathrm{C}_{\mathrm{m}+1}\right)^{\mathrm{T}}$. De ahora en más los vectores serán considerados como vectores columna para las operaciones que los involucran.

Supongamos que perturbamos una columna, que inicialmente se encuentra en equilibrio y con composición y temperatura uniformes, mediante la aplicación de una corriente de alimentación. Esta situación se la conoce como un problema de Riemann (Yang, 1987), para el cual se verifica que $\mathrm{C}_{\mathrm{i}}=\mathrm{C}_{\mathrm{i}}(\mathrm{Z} / \mathrm{t}), \mathrm{i}=1,2, \ldots, \mathrm{m}, \mathrm{m}+1$. Esto implica que la solución depende de un único parámetro, $\mathrm{Z} / \mathrm{t}$, y por lo tanto representa una curva en el espacio de las variables $C_{i}$, el cual es denominado hodógrafo. Un ejemplo se presenta en la Figura 8 donde por una cuestión de visualización se consideraron solo dos variables $\left(\mathrm{C}_{1} \mathrm{y} \mathrm{T}\right)$. Tal curva se puede reparametrizar con un parámetro $\omega$, escribiendo $C_{i}=C_{i}(\omega)$. Deberá verificarse que $\omega=\omega(Z / t)$. Luego,

$$
\frac{\partial \mathrm{C}_{\mathrm{i}}}{\partial \mathrm{t}}=\frac{\mathrm{dC}_{\mathrm{i}}}{\mathrm{d} \omega} \frac{\partial \omega}{\partial \mathrm{t}} ; \quad \frac{\partial \mathrm{C}_{\mathrm{i}}}{\partial \mathrm{Z}}=\frac{\mathrm{dC}_{\mathrm{i}}}{\mathrm{d} \omega} \frac{\partial \omega}{\partial \mathrm{Z}} \quad(\mathrm{i}=1, \ldots, \mathrm{m}+1)
$$

Reemplazando en la Ec. [20],

$$
\left[\left(1-u / u_{\omega}\right) I+v \mathcal{F}\right] \frac{\mathrm{d} \underline{\mathrm{C}}}{\mathrm{d} \omega}=\underline{0}
$$

donde se ha definido:

$$
u_{\omega}=-\frac{\partial \omega / \partial \mathrm{t}}{\partial \omega / \partial Z}
$$

Puesto que a partir de la relación $\omega=\omega(Z / t)$ resulta $d \omega=\frac{\partial \omega}{\partial Z} d Z+\frac{\partial \omega}{\partial t} d t$, vemos que si $d \omega=0$ (es decir, si $\omega$ permanece constante), y teniendo en cuenta [22a]:

$$
\left(\frac{\mathrm{dZ}}{\mathrm{dt}}\right)_{\omega}=u_{\omega}=-\frac{\partial \omega / \partial \mathrm{t}}{\partial \omega / \partial \mathrm{Z}}
$$


La [22b] establece que $u_{\omega}$ es la velocidad con la que se traslada una onda con $\omega \equiv$ constante. Puesto que $\omega \equiv$ constante implica que $\underline{\mathrm{C}} \equiv$ constante, $u_{\omega}$ es la velocidad de propagación de una onda con $\underline{\mathrm{C}} \equiv$ constante.

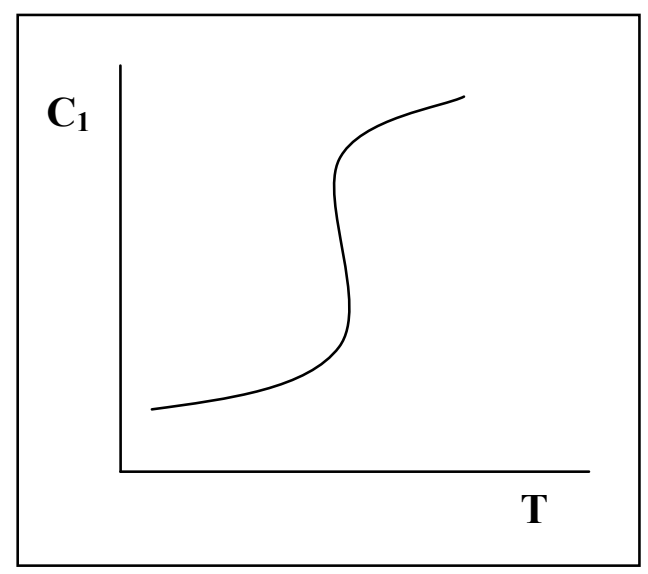

Fig. 8 - Hodógrafo.

Para que [21] admita una solución no trivial (es decir, para que $\mathrm{d} \underline{\mathrm{C}} / \mathrm{d} \omega \neq 0$ ), es necesario que la matriz $\left[\left(1-u / u_{\omega}\right) I+v \mathcal{F}\right]$ sea singular, por lo que el determinante de la matriz debe ser nulo, $\left|\left(1-u / u_{\omega}\right) I+v \mathcal{F}\right|=0$. Podemos re-escribir esta condición como:

$$
|\mathcal{F}-\sigma \mathrm{I}|=0
$$

donde

$$
\sigma=-\left(1-\frac{u}{u_{\omega}}\right) \frac{1}{v}
$$

con lo que:

$$
u_{\omega}=\frac{u}{(1+\sigma v)}
$$

De acuerdo a [23a], $\sigma$ es un autovalor de la matriz $\mathcal{F}$ y por lo tanto pueden existir hasta $\mathrm{m}+1$ valores diferentes y reales, $\sigma^{\mathrm{k}}(\mathrm{k}=1, \ldots, \mathrm{m}+1)$. Si efectivamente es así, el problema se denomina problema totalmente hiperbólico (PTH). Aquí solamente consideraremos este tipo de problema, $\mathrm{PTH}$. 
Recordando el significado físico de $u_{\omega}$, si para un PTH se establece el vector de variables de estado $\underline{\mathrm{C}}$, existirán $\mathrm{m}+1$ velocidades diferentes, $u_{\omega}^{\mathrm{k}}(\mathrm{k}=1, \ldots, \mathrm{m}+1)($ cada una calculada de la Ec. [23b] con cada $\sigma^{\mathrm{k}}$ ), con las que pueden viajar ondas con la composición y temperatura $(\underline{\mathrm{C}})$ establecidas. Las velocidades $u_{\omega}^{\mathrm{k}}$ serán ordenadas de mayor a menor, es decir

$$
u>u_{\omega}^{1}>u_{\omega}^{2}>\ldots>u_{\omega}^{m+1}>0
$$

Puede demostrarse que la primera y la última condición se verifican cuando se cumple que $\mathrm{q}_{\mathrm{i}, \mathrm{i}}>0$ y $\mathrm{q}_{\mathrm{i}, \mathrm{j}}<0$ si $\mathrm{i} \neq \mathrm{j}(\mathrm{i}, \mathrm{j}=1, \ldots, \mathrm{m}+1)$, lo que resulta afirmativo para las isotermas de adsorción usadas normalmente.

A continuación se presentará la construcción de la solución para la aplicación de un escalón para tres situaciones diferentes, que conducen a tres tipos de soluciones denominadas continua, discontinua y combinada.

\subsubsection{Construcción de la solución continua para la aplicación de un escalón}

El problema de Riemann se verifica cuando se aplica un escalón de composición y temperatura $\underline{C}^{\mathrm{F}}$ en la alimentación a un lecho inicialmente en equilibrio con una composición y temperatura uniformes $\underline{\mathrm{C}}^{0}$. Veremos aquí como puede resultar una solución continua (para $\mathrm{t}>0$ ) en este caso.

Considerando que $\underline{\mathrm{C}}^{\mathrm{F}}$ y $\underline{\mathrm{C}}^{0}$ han sido establecidos, la solución quedará definida por las Ecs. [21] para obtener la variación de $\underline{\mathrm{C}}$, las [23] para evaluar $\sigma^{\mathrm{k}}$ y $u_{\omega}^{\mathrm{k}}$ y la [22b] para relacionar $\mathrm{Z}$ con t. Con cada autovalor $\sigma^{\mathrm{k}}$, podemos re-escribir las Ecs. [21]:

$$
\left(-\sigma^{\mathrm{k}} \mathrm{I}+\mathcal{F}\right)\left(\frac{\mathrm{d} \underline{\mathrm{C}}}{\mathrm{d} \omega}\right)^{\mathrm{k}}=\underline{0}
$$

y en forma escalar,

$$
\sigma^{\mathrm{k}}\left(\frac{\mathrm{d} C_{\mathrm{i}}}{\mathrm{d} \omega}\right)^{\mathrm{k}}-\underline{\mathcal{F}}_{\mathrm{i}}^{\mathrm{T}}\left(\frac{\mathrm{d} \underline{\mathrm{C}}}{\mathrm{d} \omega}\right)^{\mathrm{k}}=0 \quad \mathrm{i}=1, \ldots, \mathrm{m}+1
$$

donde: $\quad \mathcal{F}_{\mathrm{i}}^{\mathrm{T}}=\left(\frac{\partial \mathrm{q}_{\mathrm{i}}}{\partial \mathrm{C}_{1}}, \frac{\partial \mathrm{q}_{\mathrm{i}}}{\partial \mathrm{C}_{2}}, \ldots, \frac{\partial \mathrm{q}_{\mathrm{i}}}{\partial \mathrm{C}_{\mathrm{m}+1}}\right) \quad \mathrm{i}=1, \ldots, \mathrm{m}+1$ 
Antes de proseguir formulando la solución del problema, notemos que para cualquier $\mathrm{d} \underline{\mathrm{C}}$, la correspondiente variación diferencial $\mathrm{dq}_{\mathrm{i}}$ estará dada por $\mathrm{dq}_{\mathrm{i}}=\underline{\mathcal{F}}_{\mathrm{i}}^{\mathrm{T}} \mathrm{d} \underline{\mathrm{C}}$. Por lo tanto, de [25] vemos que para una variación diferencial d $\omega$ en la dirección definida por $\sigma^{\mathrm{k}}$ deberá cumplirse que

$$
\sigma^{\mathrm{k}}=\left(\frac{\mathrm{dq}_{\mathrm{i}}}{\mathrm{dC}_{\mathrm{i}}}\right)^{\mathrm{k}}, \quad \mathrm{i}=1, \ldots, \mathrm{m}+1
$$

Las Ecs. [26] suelen ser denominadas las condiciones de compatibilidad y $\left(\mathrm{dq}_{\mathrm{i}} / \mathrm{dC}_{\mathrm{i}}\right)^{\mathrm{k}}$ son las derivadas direccionales.

Para la integración de las Ecs. [25], es conveniente eliminar el parámetro $\omega \mathrm{y}$ emplear directamente como parámetro a la variable $\mathrm{T}$ (recordemos que $\mathrm{C}_{\mathrm{m}+1} \equiv \mathrm{T}$ ). Las Ecs. [25] pueden reescribirse:

$$
\begin{aligned}
& \mathrm{q}_{\mathrm{i}, 1} \frac{\mathrm{dC}_{1}}{\mathrm{dT}}+\ldots+\mathrm{q}_{\mathrm{i}, \mathrm{i}-1} \frac{\mathrm{dC}_{\mathrm{i}-1}}{\mathrm{dT}}+\left(\mathrm{q}_{\mathrm{i}, \mathrm{i}}-\sigma^{\mathrm{k}}\right) \frac{\mathrm{dC}_{\mathrm{i}}}{\mathrm{dT}}+\mathrm{q}_{\mathrm{i}, \mathrm{i}+1} \frac{\mathrm{dC}_{\mathrm{i}+1}}{\mathrm{dT}}+\ldots+\mathrm{q}_{\mathrm{i}, \mathrm{m}} \frac{\mathrm{dC}_{\mathrm{m}}}{\mathrm{dT}}=-\mathrm{q}_{\mathrm{i}, \mathrm{T}} \\
& \mathrm{i}=1, \ldots, \mathrm{m}
\end{aligned}
$$

Para las derivadas $\mathrm{dC}_{\mathrm{i}} / \mathrm{dT}$ se ha eliminado el índice (k), a fin de simplificar la notación, considerando que la dirección de integración queda determinada con el valor específico $\sigma^{\mathrm{k}}$. Para determinar una posible solución a partir de una cierta condición inicial $\underline{\mathrm{C}}^{0}$, se deberá elegir un valor de $\mathrm{k}$ y se integrarán las Ecs. [27]. Tal solución será, como ya fuera dicho, una curva en el espacio de las variables $\mathrm{C}_{\mathrm{i}}$ $(\mathrm{i}=1, \ldots, \mathrm{m}+1)$ que se indicará con $\Gamma^{\mathrm{k}}$. A tal fin, es importante tener en cuenta que sobre cada punto de la curva los autovalores (en particular $\sigma^{\mathrm{k}}$, para el k elegido) se van modificando, por lo que es necesario recalcularlos usando la Ec. [23a].

La solución obtenida debiera pasar por el punto $\underline{\mathrm{C}}^{\mathrm{F}}$, pero como la condición de alimentación es independiente de la inicial, solo podrá ocurrir fortuitamente. En rigor, teniendo en cuenta todos los valores posibles $\sigma^{\mathrm{k}}(\mathrm{k}=1, \ldots, \mathrm{m}+1)$, podremos generar $\mathrm{m}+1$ curvas $\Gamma^{\mathrm{k}}$, pero en general ninguna de ellas contendrá al punto $\underline{\mathrm{C}}^{\mathrm{F}}$. Por lo tanto, para unir $\underline{\mathrm{C}}^{0}$ con $\underline{\mathrm{C}}^{\mathrm{F}}$ de manera continua es necesario cambiar de dirección $\mathrm{k}$ en algunos puntos del recorrido, a estos puntos se los denomina puntos de quiebre. Veremos el caso para 1 componente $(\mathrm{m}=1)$, para visualizar dicho cambio.

En este caso existe una única ecuación del tipo de las Ecs.[27]: 


$$
\left(\mathrm{q}_{1,1}-\sigma^{\mathrm{k}}\right) \frac{\mathrm{dC}}{\mathrm{dT}}=-\mathrm{q}_{1, \mathrm{~T}}
$$

Si integramos la [28] a partir del punto $\mathrm{P}^{0}$ (ver Figura 9) sobre la dirección $\mathrm{k}=1$ obtenemos la curva $\Gamma^{1}$. Si también integramos la Ec. [28] a partir del punto $\mathrm{P}^{\mathrm{F}}$ sobre $\mathrm{k}=2$, obtenemos la curva $\Gamma^{2}$. El punto de quiebre $\mathrm{P}^{1}$ queda definido por la intersección de ambas curvas.

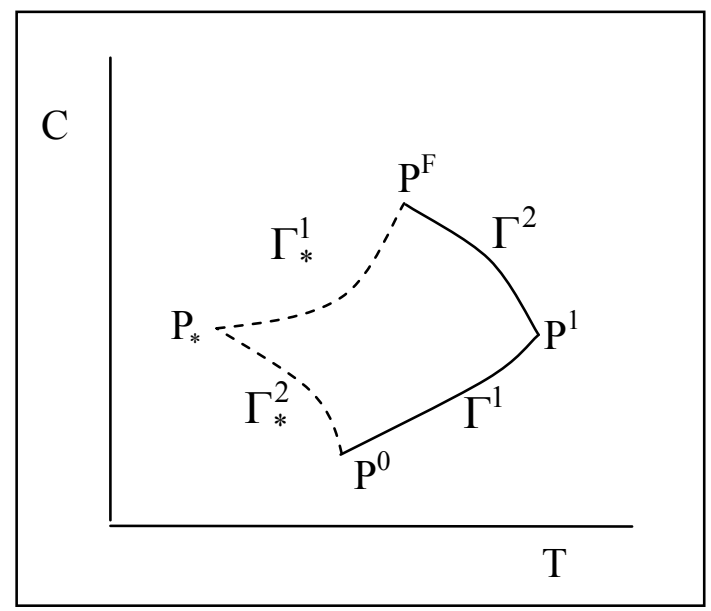

Fig. 9 - Hodógrafo. Solución para un componente.

Sobre la trayectoria $\mathrm{P}^{0}-\mathrm{P}^{1}-\mathrm{P}^{\mathrm{F}}$ la composición varía continuamente desde $\underline{\mathrm{C}}^{0}$ hasta $\underline{\mathrm{C}}^{\mathrm{F}}, \mathrm{y}$ constituye una solución potencialmente factible para nuestro problema, pero en el punto $\mathrm{P}^{1}$ se ha tenido que apelar a un cambio de dirección, lo que implica que el vector de variables de estado $\underline{\mathrm{C}}^{1}$ del punto $\mathrm{P}^{1}$ tendrá físicamente dos velocidades de propagación, $u_{\omega, 1}^{1}$ y $u_{\omega, 1}^{2}$; en su conexión con el tramo $\Gamma^{1}$ su velocidad deberá ser $u_{\omega, 1}^{1}$ y con respecto al tramo $\Gamma^{2}$ su velocidad deberá ser $u_{\omega, 1}^{2}$. Puesto que el tramo $\Gamma^{1}$ se encuentra espacialmente adelante del $\Gamma^{2}$ y dado que $u_{\omega, 1}^{1}>u_{\omega, 1}^{2}$ (convención adoptada en [23c]), existirá a lo largo de la columna (para un dado t>0) una zona de composición y temperatura uniformes $\underline{\mathrm{C}}^{1}$, con el extremo superior (frente) avanzando con velocidad $u_{\omega, 1}^{1}$ y con el extremo inferior (fondo) a la velocidad $u_{\omega, 1}^{2}$. Por lo tanto, la longitud de la zona crece con el tiempo, tendencia que permite concebir su existencia. 
El tramo $\Gamma^{1}$ se presentará en el lecho como una zona de composición y $\underline{\text { temperatura variables }}$ (desde $\underline{\mathrm{C}}^{0}$ hasta $\underline{\mathrm{C}}^{1}$ ). Para que efectivamente pueda existir, es necesario que $u_{\omega}^{1}$ disminuya desde el punto $\mathrm{P}^{0}$ hasta $\mathrm{P}^{1}$. De esta forma, la longitud de la zona correspondiente al tramo $\Gamma^{1}$ también aumentará con t. Finalmente, para que la solución descripta sea definitivamente admisible, también es necesario respecto al tramo $\Gamma^{2}$, que $u_{\omega}^{2}$ disminuya desde el punto $\mathrm{P}^{1}$ hasta el punto $\mathrm{P}^{\mathrm{F}}$, para que exista en el lecho una segunda zona de composición variable.

De cumplirse las anteriores condiciones de velocidad respecto a los tramos $\Gamma^{1} \mathrm{y}$ $\Gamma^{2}$, la solución descripta es la única posible. Para convencernos, podemos considerar la alternativa resultante al invertir las direcciones de integración a partir de los puntos $\mathrm{P}^{0}$ y $\mathrm{P}^{\mathrm{F}}$, generando un punto de quiebre $\mathrm{P}_{*}$ para el cambio de dirección (ver Fig. 9). El extremo superior de la zona correspondiente a $\mathrm{P}_{*}$ debería avanzar con velocidad $u_{\omega, 2}^{2}$ y su extremo inferior con $u_{\omega, 2}^{1}$, pero dado que $u_{\omega, 2}^{1}>u_{\omega, 2}^{2}$, la existencia de una zona uniforme con composición $\mathrm{P}_{*}$ es imposible, y la solución alternativa no es factible.

Tampoco es posible ninguna solución con más de un punto de cambio de dirección, dado que uno o más de tales puntos requerirían ser del tipo $\mathrm{P}_{*}$.

En la Figura 10 se encuentra representado el perfil de concentración en la variable $\mathrm{Z}$ para un cierto valor del tiempo, t. Para la temperatura se obtienen perfiles equivalentes.

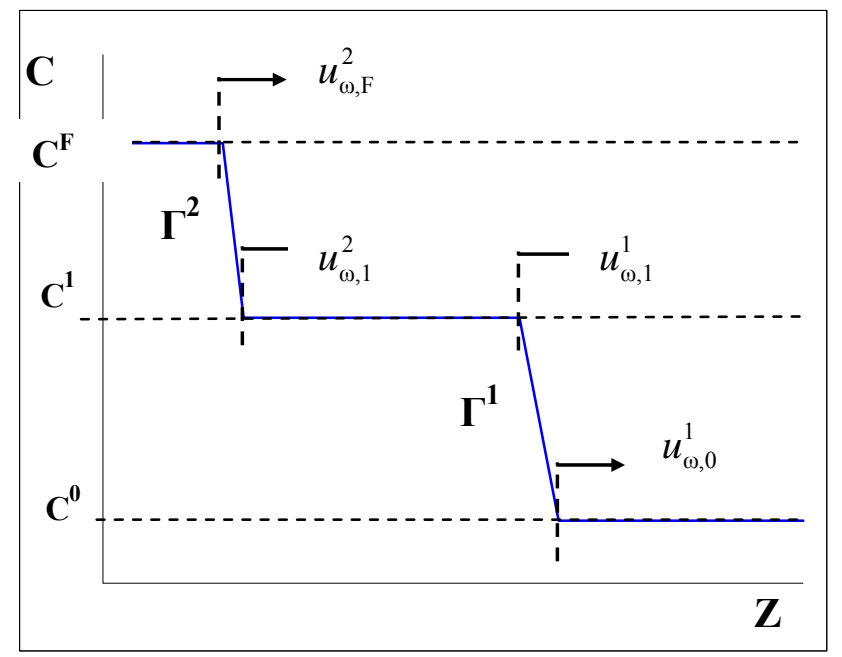

Fig. 10 - Perfil de concentración en la variable $\mathrm{Z}$ a un cierto t. 
Para valores de $m>1$, es usualmente necesario re-escribir las Ecs. [27] explicitando cada $\mathrm{dC}_{\mathrm{i}} / \mathrm{dT}$. A tal fin se debe considerar que, una vez fijado $\underline{\mathrm{C}}$, los coeficientes que multiplican las derivadas $\mathrm{dC}_{\mathrm{i}} / \mathrm{dT}$ en las Ecs. [27] serán valores numéricos definidos y el conjunto de ecuaciones resulta lineal a fin de calcular las derivadas $\mathrm{dC}_{\mathrm{i}} / \mathrm{dT}$. Supongamos que la solución de tal sistema permite escribir:

$$
\mathrm{dC}_{\mathrm{i}} / \mathrm{dT}=\mathrm{f}_{\mathrm{i}}^{\mathrm{k}}(\underline{\mathrm{C}}), \mathrm{i}=1, \ldots, \mathrm{m}
$$

donde el supraíndice $\mathrm{k}$ de $\mathrm{f}_{\mathrm{i}}^{\mathrm{k}}$ alude al correspondiente valor $\sigma^{\mathrm{k}}$ de [27].

En general, se necesitan llevar a cabo $\mathrm{m}+1$ tramos de integración $\Gamma^{\mathrm{k}}$, empezando a integrar las Ecs. [29] sobre la dirección $\mathrm{k}=1$, desde el punto inicial $\mathrm{P}^{0}$ hasta un punto $\mathrm{P}^{1}$ con temperatura $\mathrm{T}^{1}$ (tramo $\Gamma^{1}$ ), que definirá el primer punto de quiebre. En $\mathrm{P}^{1}$ se cambia de dirección, $\mathrm{k}=2$, $\mathrm{y}$ se integra hasta un segundo punto $\mathrm{P}^{2}$ con temperatura $\mathrm{T}^{2}$, definiendo el tramo $\Gamma^{2}$. Si continuamos de esta manera, llegaremos al m-ésimo tramo (desde $\mathrm{P}^{\mathrm{m}-1}$ hasta $\mathrm{P}^{\mathrm{m}}$ ) sobre la dirección $\mathrm{k}=\mathrm{m}$, cuyos extremos de temperatura son $\mathrm{T}^{\mathrm{m}-1} \mathrm{y} \mathrm{T}^{\mathrm{m}}$. El último tramo (desde $\mathrm{P}^{\mathrm{m}}$ hasta $\mathrm{P}^{\mathrm{F}}$ ) lo llevamos a cabo sobre $\mathrm{k}=\mathrm{m}+1\left(\right.$ con $\mathrm{T}$ desde $\mathrm{T}^{\mathrm{m}}$ hasta $\left.\mathrm{T}^{\mathrm{F}}\right) . \mathrm{El}$ valor $\mathrm{T}=\mathrm{T}^{\mathrm{F}}$ se habrá alcanzado junto con las concentraciones $\mathrm{C}^{\prime}{ }_{1}, \ldots, \mathrm{C}_{\mathrm{m}}$, que deberían ser iguales a $\mathrm{C}_{1}^{\mathrm{F}}, \ldots, \mathrm{C}_{\mathrm{m}}^{\mathrm{F}}$ (los valores de alimentación). Para que se satisfagan tales igualdades se disponen como grados de libertad las temperaturas de los puntos de quiebre $\mathrm{T}^{1}, \ldots, \mathrm{T}^{\mathrm{m}}$. Por lo tanto, se puede concebir un proceso iterativo sobre las mismas hasta que se verifiquen todos $\operatorname{los} C^{\prime}{ }_{i}=C_{i}^{F}(i=1, . ., m)$ (si se decide llevar a cabo la integración a partir del punto $\mathrm{P}^{\mathrm{F}}$ sobre la dirección $\mathrm{m}+1$, como se describió para el caso particular $\mathrm{m}=1$, y se conservan los resultados, es posible reducir en una unidad el número de variables a iterar). Las razones para que los tramos tengan que seguir las direcciones $1,2, \ldots, \mathrm{m}+1$ son las mismas que las discutidas para el caso $\mathrm{m}=1$.

La composición y temperatura de los m puntos de quiebre resultantes, digamos $\underline{C}^{\mathrm{k}}$ $(\mathrm{k}=1, \ldots, \mathrm{m})$, se presentan a lo largo del lecho como $\mathrm{m}$ zonas uniformes (en total habrán $\mathrm{m}+2$ zonas uniformes, si adicionamos las de la composición y temperatura inicial $\underline{C}^{0}$ y de alimentación $\underline{C}^{\mathrm{F}}$ ). Los tramos $\Gamma^{\mathrm{k}}$ se deberán presentar en el lecho como $\mathrm{m}+1$ zonas de composición y temperatura variables, que se alternan con las uniformes. Para que la k-ésima zona variable pueda existir, resultará necesario que $u_{\omega}^{k}$ no aumente, parcial o monótonamente, desde $\mathrm{P}^{\mathrm{k}-1}$ hasta $\mathrm{P}^{\mathrm{k}}$. Si una o más de las 
zonas de composición y temperatura variables no pueden existir, la solución continua no podrá existir.

A continuación se describen las soluciones para los casos particulares en que se presenta una discontinuidad.

\subsubsection{Construcción de las soluciones discontinuas para la aplicación de un escalón}

\subsubsection{Discontinuidad de contacto}

El caso en que $u_{\omega}^{\mathrm{k}}$ permanezca constante en el tramo $\Gamma^{\mathrm{k}}$ resulta una situación particular. El caso más común de esta particularidad puede presentarse cuando el punto $\mathrm{P}^{0}$ o el $\mathrm{P}^{\mathrm{F}}$ no contiene ningún componente, $\mathrm{C}_{\mathrm{i}}=0, \mathrm{i}=1, \ldots, \mathrm{m}$. De esta manera se tendrá que $\mathrm{q}_{\mathrm{i}}=0, \mathrm{i}=1, \ldots, \mathrm{m} ; \mathrm{q}_{\mathrm{m}+1}=\gamma \mathrm{T}$. En estas condiciones, definimos las derivadas $\mathrm{q}_{\mathrm{ii}}=\Phi_{\mathrm{i}}(\mathrm{i}=1, \ldots, \mathrm{m})$, y para cualquier isoterma termodinámicamente consistente será $\Phi_{\mathrm{i}}>0$. Se verificará que $\mathrm{q}_{\mathrm{i}, \mathrm{j}}=0,(\mathrm{i} \neq \mathrm{j} ; \mathrm{i}, \mathrm{j}=1, \ldots, \mathrm{m}+1)$ y $\mathrm{q}_{\mathrm{m}+1, \mathrm{~m}+1}=\gamma$. La matriz $\mathcal{F}$ solo tiene componentes en la diagonal principal y la Ec. [23a] que define los autovalores $\sigma$ resulta:

$$
\left(\Phi_{1}-\sigma\right)\left(\Phi_{2}-\sigma\right) \ldots\left(\Phi_{\mathrm{m}}-\sigma\right)(\gamma-\sigma)=0
$$

Los autovalores son: $\sigma^{\mathrm{j}}=\Phi_{\mathrm{j}}, \mathrm{j}=1, \ldots, \mathrm{m}, \sigma^{\mathrm{m}+1}=\gamma$ (el superíndice de $\sigma \underline{\text { no }}$ involucra ordenamiento por magnitud).

Consideramos que al punto $\mathrm{P}^{0}$ le corresponde concentraciones nulas, $\mathrm{C}_{\mathrm{i}}=0$, $\mathrm{i}=1, \ldots, \mathrm{m}$; con $\mathrm{T}=\mathrm{T}^{0}$. Supongamos ahora que se verifica que $\gamma<\Phi_{\mathrm{i}}, \mathrm{i}=1, \ldots, \mathrm{m}$. El menor valor de $\sigma$ será $\sigma^{1}=\gamma$, y la mayor velocidad de propagación será (Ec. [23b]) $u_{\omega}^{1}=u /(1+v \gamma)$. Esta velocidad $u_{\omega}^{1}$ se denomina onda térmica pura. En este caso, para determinar el tramo $\Gamma^{1}$ las Ecs.[27] resultan en el punto 0:

$$
\left(\Phi_{\mathrm{i}}-\gamma\right) \mathrm{dC}_{\mathrm{i}} / \mathrm{dT}=0 ; \quad \mathrm{i}=1, \ldots, \mathrm{m}
$$

Estas ecuaciones indican que el tramo se inicia con pendiente nula para todas las concentraciones y mantendrán su valor nulo frente a un incremento diferencial de $\mathrm{T}$; condición que también permanecerá inalterada para cualquier incremento finito de T. En definitiva, el tramo $\Gamma^{1}$ será un segmento en el eje T. Además, todos lo puntos en ese tramo tendrán la misma velocidad de propagación $u_{\omega}^{1}=u /(1+v \gamma)$. 
El tramo $\Gamma^{1}$ se presentará espacialmente como una discontinuidad (respecto a $\mathrm{T}$, que varía entre $\mathrm{T}^{0} \mathrm{y} \mathrm{T}^{1}$ : temperatura en el punto de quiebre), pero en realidad consiste en una sucesión de estados (a diferente temperatura) que se mueven con la misma velocidad de propagación $u_{\omega}^{1}$ (notar la diferencia con la onda de choque, que se describe a continuación).

Vale la pena aclarar que para el caso en que no se verifique que $\gamma<\Phi_{\text {i }}$, $\mathrm{i}=1, \ldots, \mathrm{m}$, no se está en presencia de una onda térmica pura.

En la Figura 11 se presenta una situación de discontinuidad de contacto para un componente $(\mathrm{m}=1)$ con $\underline{\mathrm{C}}^{0}=\left(0, \mathrm{~T}^{0}\right)$ y con $\gamma<\Phi_{\mathrm{i}}$, por lo que el tramo $\Gamma^{1}$ resulta un segmento en el eje T. En la Figura 12 se encuentran los perfiles de concentración $\mathrm{y}$ temperatura a lo largo de la variable $\mathrm{Z}$ para un cierto valor del tiempo $\mathrm{t}$.

Un caso similar se produce para el tramo $\Gamma^{\mathrm{m}}$ cuando la alimentación no contiene ningún adsorbato y se verifica que $\gamma>\Phi_{i}, i=1, \ldots, m$.

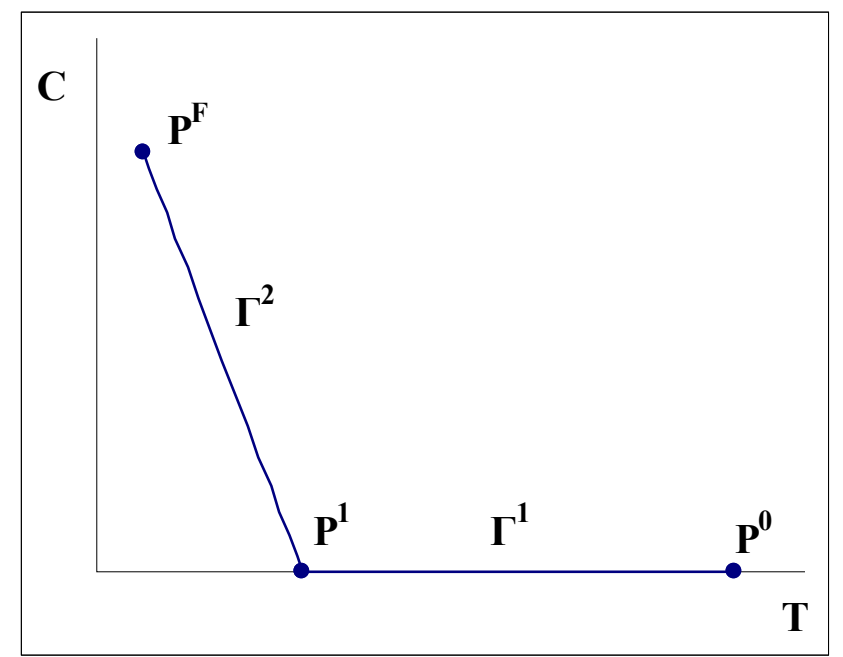

Fig. 11 - Hodógrafo. Solución para un componente. 


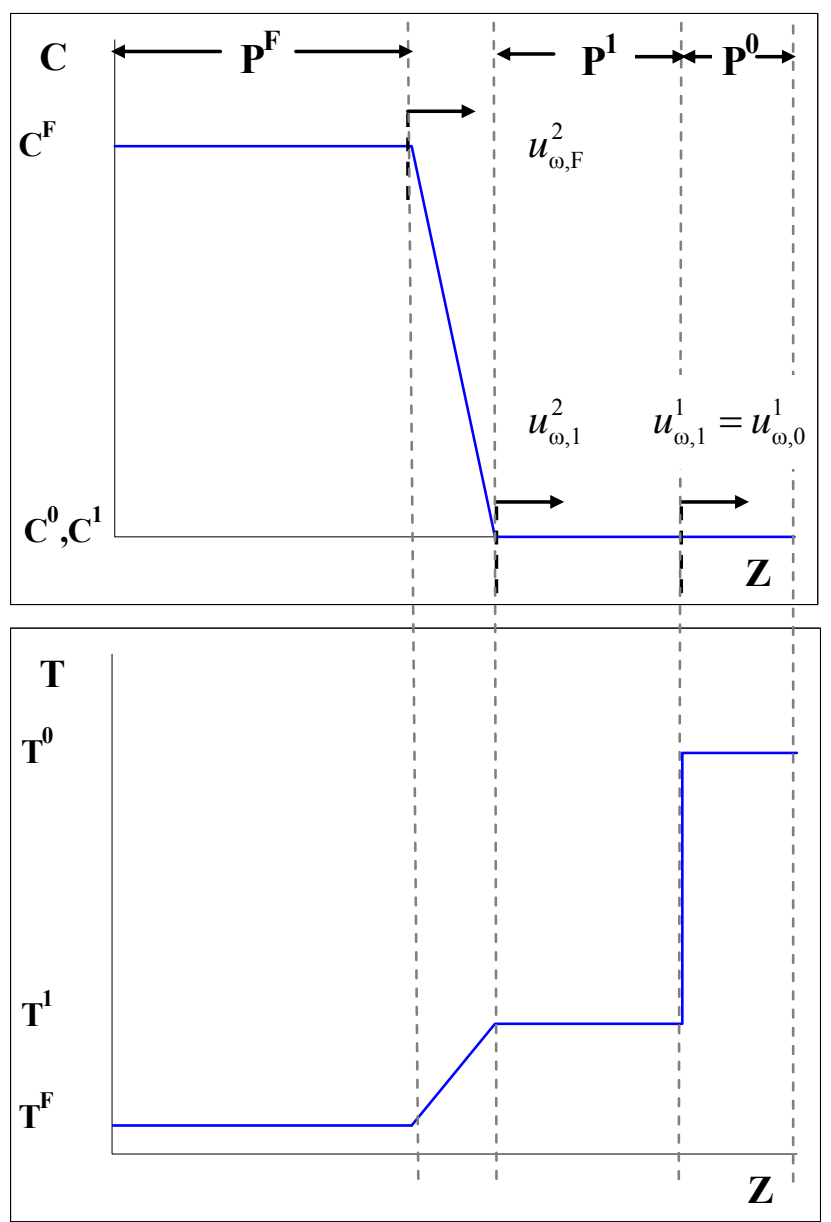

Fig. 12a,b - Perfiles de concentración y temperatura a lo largo de la variable $\mathrm{Z}$ a un cierto tiempo.

\subsubsection{Ondas de choque}

Cuando una o más de las zonas de composición y temperatura variables no pueda existir debido a que el "frente" es más lento que el "fondo" $\left(u_{\omega, \mathrm{k}-1}^{\mathrm{k}}<u_{\omega, \mathrm{k}}^{\mathrm{k}}\right)$, es necesario admitir que existirán ondas de choque, cada una reemplazando cada tramo $\Gamma^{\mathrm{k}}$ que no verifique la condición de existencia. Veremos someramente la formulación. Detalles adicionales se pueden consultar en las Partes I, II y III del trabajo de Rhee y col. (1970a,b; 1972). Supondremos, en principio, que en cada tramo $\Gamma^{\mathrm{k}}$ que deba reemplazarse por una onda de choque, $u_{\omega}^{\mathrm{k}}$ aumenta monótonamente desde $\mathrm{P}^{\mathrm{k}-1}$ hasta $\mathrm{P}^{\mathrm{k}}$.

Consideremos que la onda de choque debe suplantar un dado tramo $\Gamma^{\mathrm{B}}$, entre

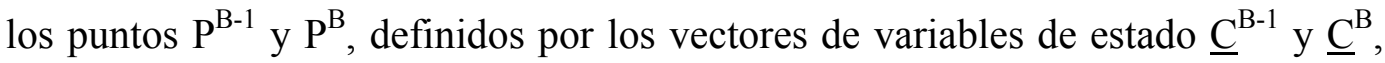
respectivamente. La velocidad de la onda de choque debe ser la misma para todos 
los componentes. Para determinar la trayectoria de la onda de choque tomamos como referencia la situación planteada en la Sección 4.1 para el caso isotérmico con un único componente. Al aplicar los balances de conservación (Ec. [17a]), para cada uno de los componentes, incluida la temperatura, sobre un elemento tal como el ubicado en la Fig. 6, surgen las siguientes expresiones:

$$
\begin{aligned}
& \frac{\mathrm{q}_{\mathrm{i}}^{\mathrm{B}}-\mathrm{q}_{\mathrm{i}}^{\mathrm{B}-1}}{\mathrm{C}_{\mathrm{i}}^{\mathrm{B}}-\mathrm{C}_{\mathrm{i}}^{\mathrm{B}-1}}=\frac{\mathrm{q}_{\mathrm{T}}^{\mathrm{B}}-\mathrm{q}_{\mathrm{T}}^{\mathrm{B}-1}}{\mathrm{~T}^{\mathrm{B}}-\mathrm{T}^{\mathrm{B}-1}}, \quad \mathrm{i}=1, \ldots, \mathrm{m} \\
& \frac{u / u_{\mathrm{s}}-1}{\mathrm{v}}=\frac{\mathrm{q}_{\mathrm{T}}^{\mathrm{B}}-\mathrm{q}_{\mathrm{T}}^{\mathrm{B}-1}}{\mathrm{~T}^{\mathrm{B}}-\mathrm{T}^{\mathrm{B}-1}}
\end{aligned}
$$

donde $u_{s}=\delta Z / \delta$ t es la velocidad de la onda de choque.

La Ec. [30b] permitirá calcular $u_{\mathrm{s}}$, mientras que las Ecs. [30a] son m condiciones a satisfacer que sustituyen a las ecuaciones diferenciales [29] del tramo $\Gamma^{\mathrm{B}}$. Consideremos por un momento que el vector $\underline{\mathrm{C}}^{\mathrm{B}-1}$ está definido, las Ecs. [30a] solo permiten un grado de libertad para las variables $\underline{C}^{B}$, por ejemplo podríamos fijar $\mathrm{T}^{\mathrm{B}}$, mientras que los $\mathrm{C}_{\mathrm{i}}^{\mathrm{B}}(\mathrm{i}=1, \ldots, \mathrm{m})$ se deberán evaluar de las [30a], procedimiento que normalmente será iterativo y sobre el que luego veremos algunos aspectos adicionales. Por lo tanto, salvo casos singulares, el vector de variables de estado $\underline{\mathrm{C}}^{\mathrm{B}}$ de la onda de choque será distinto al vector de variables de estado $\underline{C}^{\mathrm{B}}$ del tramo $\Gamma^{\mathrm{B}}$ que debe sustituirse. Teniendo en cuenta que la composición y temperatura en el punto $\mathrm{P}^{\mathrm{B}}\left(\underline{\mathrm{C}}^{\mathrm{B}}\right)$ corresponderá a un punto de quiebre, concluimos que las coordenadas del mismo resultarán modificadas.

Se desprende que todo el procedimiento iterativo para conectar la trayectoria desde $\mathrm{P}^{0}$ hasta $\mathrm{P}^{\mathrm{F}}$ debe modificarse, debido a que el cambio de composición, de al menos uno de los puntos de quiebre, afectará a todos los demás. No obstante, seguirán existiendo $\mathrm{m}$ grados de libertad para cerrar la trayectoria desde $\mathrm{P}^{0}$ hasta $\mathrm{P}^{\mathrm{F}}$, correspondientes a las temperaturas $\mathrm{T}^{\mathrm{k}}(\mathrm{k}=1, \ldots, \mathrm{m})$ de los puntos de quiebre. Espacialmente, cada punto de quiebre genera una zona de composición y temperatura uniformes, y su extremo superior podrá estar conectada hacia adelante mediante una onda de choque o mediante una zona de composición y temperatura continuamente variables (un tramo $\Gamma^{\mathrm{k}}$ ); las mismas alternativas surgen para la conexión del extremo inferior hacia atrás. 
Como fuera expuesto, cuando deba existir una onda de choque para conectar $\mathrm{P}^{\mathrm{B}-1}$ con $\mathrm{P}^{\mathrm{B}}$, las Ecs. [30a] reemplazan la integración de las Ecs. [29]. Sin embargo, supuesto fijo el vector $\underline{\mathrm{C}}^{\mathrm{B}-1}$, para un dado valor $\mathrm{T}^{\mathrm{B}}$ las Ecs. [30a] proporcionarán normalmente $(\mathrm{m}+1)$ soluciones distintas para el conjunto de concentraciones $C_{i}^{B}(i=1, \ldots, m)$.

Para visualizar la existencia de dicho número de soluciones y elegir entre ellas cual es la correcta, tengamos en cuenta que las Ecs. [30] pueden escribirse:

$$
\sigma_{s}=\frac{\mathrm{q}_{\mathrm{i}}^{\mathrm{B}}-\mathrm{q}_{\mathrm{i}}^{\mathrm{B}-1}}{\mathrm{C}_{\mathrm{i}}^{\mathrm{B}}-\mathrm{C}_{\mathrm{i}}^{\mathrm{B}-1}}, \quad \mathrm{i}=1, \ldots, \mathrm{m}+1
$$

donde el parámetro $\sigma_{s}$ se relaciona con $u_{s}(c f s$. Ec. [30b]) mediante

$$
u_{s}=\frac{u}{1+v \sigma_{s}}
$$

Si consideramos un valor de $\mathrm{T}^{\mathrm{B}}$ próximo a $\mathrm{T}^{\mathrm{B}-1}$, para cualquier solución arrojada por [31] resultarán pequeños incrementos $\left(\mathrm{C}_{i}^{B}-\mathrm{C}_{i}^{\mathrm{B}-1}\right)$ y $\left(\mathrm{q}_{\mathrm{i}}^{\mathrm{B}}-\mathrm{q}_{\mathrm{i}}^{\mathrm{B}-1}\right)$, por lo que los cocientes en [31] se aproximarán a derivadas en el punto $\mathrm{P}^{\mathrm{B}-1}$. Una comparación directa entre las Ecs. [31] y las Ecs. [26] (aplicadas al punto $\mathrm{P}^{\mathrm{B}-1}$ ) indica que el parámetro $\sigma_{s}$ se aproximará a $\sigma_{\mathrm{B}-1}^{\mathrm{k}}(\mathrm{el}$ subíndice indica evaluación en $\left.\mathrm{P}^{\mathrm{B}-1}\right)$ y por lo tanto podrá asumir hasta $(\mathrm{m}+1)$ valores diferentes, semejantes a $\operatorname{los} \mathrm{m}+1$ autovalores $\sigma_{\mathrm{B}-1}^{\mathrm{k}}$.

Podemos ahora definir $(\mathrm{m}+1)$ curvas en el hodógrafo usando las [31] con uno de los extremos fijo, para el cual supondremos que se elije el punto $\mathrm{P}^{\mathrm{B}-1}$, y el segundo variable, es decir:

$$
\begin{aligned}
& \frac{\mathrm{q}_{\mathrm{i}}-\mathrm{q}_{\mathrm{i}}^{\mathrm{B}-1}}{\mathrm{C}_{\mathrm{i}}-\mathrm{C}_{\mathrm{i}}^{\mathrm{B}-1}}=\frac{\mathrm{q}_{\mathrm{T}}-\mathrm{q}_{\mathrm{T}}^{\mathrm{B}-1}}{\mathrm{~T}-\mathrm{T}^{\mathrm{B}-1}}, \quad \mathrm{i}=1, \ldots, \mathrm{m} \\
& \sigma_{s}=\frac{u / u_{s}-1}{v}=\frac{\mathrm{q}_{\mathrm{T}}-\mathrm{q}_{\mathrm{T}}^{\mathrm{B}-1}}{\mathrm{~T}-\mathrm{T}^{\mathrm{B}-1}}
\end{aligned}
$$

En las $\left[30^{*}\right]$ se considera a $\mathrm{T}$ como variable continua y a $\sigma_{s}$ como parámetro, con cada curva diferenciándose de las demás por el valor en el origen: $\sigma_{s}=\sigma_{\mathrm{B}-1}^{\mathrm{k}}$. 
A tales curvas se las indica con $\Sigma^{\mathrm{k}}, \mathrm{k}=1, \ldots, \mathrm{m}+1$, y vemos que para un dado punto $\mathrm{P}^{\mathrm{B}-1}$ las curvas $\Sigma^{\mathrm{k}} \mathrm{y} \Gamma^{\mathrm{k}}$ tendrán la misma pendiente en $\mathrm{P}^{\mathrm{B}-1}$.

Si el punto $\mathrm{P}^{\mathrm{B}}$, que pretendemos determinar, no se encuentra demasiado lejos de $\mathrm{P}^{\mathrm{B}-1}$, las curvas $\Sigma^{\mathrm{k}} \mathrm{y} \Gamma^{\mathrm{k}}$ no diferirán demasiado una de otra. En rigor, ambas curvas se aproximan aún más de lo que sugiere la mencionada igualdad de pendientes en $\mathrm{P}^{\mathrm{B}-1} \mathrm{y}$ difieren solo en un tercer orden de magnitud, según Rhee y col. (1970a), quienes en tales condiciones mencionan que la solución al problema de la onda de choque existe y se debe elegir la curva $\Sigma^{\mathrm{B}}($ i.e., $\mathrm{k}=\mathrm{B})$ como opción válida para obtener la solución global. Para la misma se deberá verificar que:

$$
\begin{aligned}
& u_{\omega, \mathrm{B}-1}^{\mathrm{B}}<u_{\omega, \mathrm{B}}^{\mathrm{B}} \\
& u_{s} \in\left[u_{\omega, \mathrm{B}-1}^{\mathrm{B}}, u_{\omega, \mathrm{B}}^{\mathrm{B}}\right]
\end{aligned}
$$

donde $u_{\omega, \mathrm{B}-1}^{\mathrm{B}}$ es la B-ésima velocidad de propagación para el punto $\mathrm{P}^{\mathrm{B}-1} \mathrm{y} u_{\omega, \mathrm{B}}^{\mathrm{B}}$ es la B-ésima velocidad de propagación para la solución $\mathrm{P}^{\mathrm{B}}$ determinada por la resolución de las Ecs. [30a]. La Ec. [33a] establece que $u_{\omega, \mathrm{B}-1}^{\mathrm{B}}$ será estrictamente menor que $u_{\omega, \mathrm{B}}^{\mathrm{B}}$, y la Ec. [33b] que $u_{s}$ será normalmente un valor intermedio, aunque podría coincidir con alguno de los extremos, $u_{\omega, \mathrm{B}-1}^{\mathrm{B}}$ o $u_{\omega, \mathrm{B}}^{\mathrm{B}}$.

En base a lo expuesto, para determinar la solución adecuada correspondiente a un valor definido $\mathrm{T}^{\mathrm{B}}$ se puede, en primer lugar, "localizar" la curva $\Sigma^{\mathrm{B}}$. A tal efecto, se toma un valor de $\mathrm{T}$ cercano a $\mathrm{T}^{\mathrm{B}-1}$ y se resuelven las $\left[30 \mathrm{a}^{*}\right]$ iterando a partir de valores de prueba $C_{i}(i=1, \ldots, m)$ cercanos a los de la curva $\Gamma^{\mathrm{B}}(\mathrm{se}$ pueden obtener por linealización a partir de las derivadas en el punto $\mathrm{P}^{\mathrm{B}-1}$, que a su vez se calculan de [29] con el valor $\left.\sigma_{\mathrm{B}-1}^{\mathrm{B}}\right)$. Las Ecs. [30a*] pueden seguir resolviéndose para incrementos en $\mathrm{T}$, hasta alcanzar el valor deseado $\mathrm{T}^{\mathrm{B}}$. En cada incremento se deberá comprobar que los valores $\mathrm{C}_{\mathrm{i}}$ obtenidos se mantengan sobre la curva deseada $\Sigma^{\mathrm{B}}$, para lo cual los mismos deberán conservar continuidad (se puede monitorear con la sucesión de valores $\sigma_{s}$, Ec.[31]). Asimismo, las iteraciones de las Ec. [30a*] para cada incremento deberán iniciarse con los valores de $\mathrm{C}_{\mathrm{i}}$ de la etapa anterior o con alguna proyección basada en los 
incrementos previos. Al arribar al valor deseado $\mathrm{T}^{\mathrm{B}}$, el valor de $u_{s}$ deberá satisfacer la Ec.[33b].

En general, debido a la proximidad de las curvas $\Gamma^{\mathrm{k}} \mathrm{y} \Sigma^{\mathrm{k}}$, los puntos de quiebre $\mathrm{P}^{\mathrm{k}}$ obtenidos como solución global, suponiendo que todos los tramos son continuos, se aproximarán mucho a los puntos definitivos resultantes de sustituir aquellos tramos donde deban introducirse ondas de choque.

A continuación se presentan un hodógrafo (Figura 13) y los perfiles de concentración y temperatura a lo largo de la variable $\mathrm{Z}$ para un cierto valor del tiempo (Figura 14), para el caso particular de un componente $(\mathrm{m}=1)$.

En este caso el punto de quiebre $\mathrm{P}^{1}$, de composición y temperatura uniformes, presenta su extremo superior (ver Fig. 14) conectado hacia delante mediante una onda de choque $\left(\Sigma^{1}\right)$, mientras que su extremo inferior se encuentra conectado hacia atrás mediante una zona de composición y temperatura continuamente variables $\left(\Gamma^{2}\right)$. Existen casos, como se verá en la sección siguiente, donde ambos extremos del punto de quiebre pueden encontrarse conectados a ondas de choque.

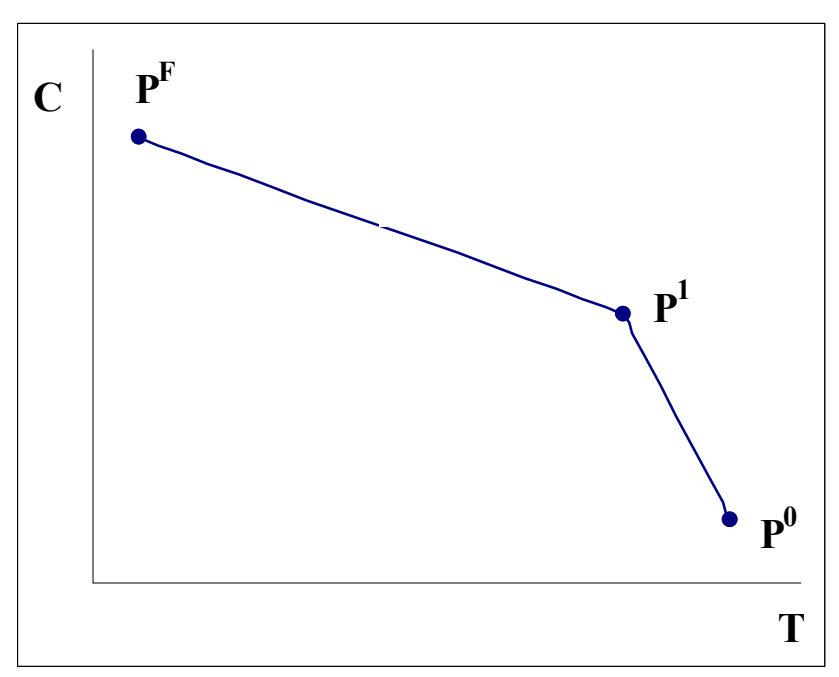

Fig. 13 - Hodógrafo. Solución discontinua para un componente. 


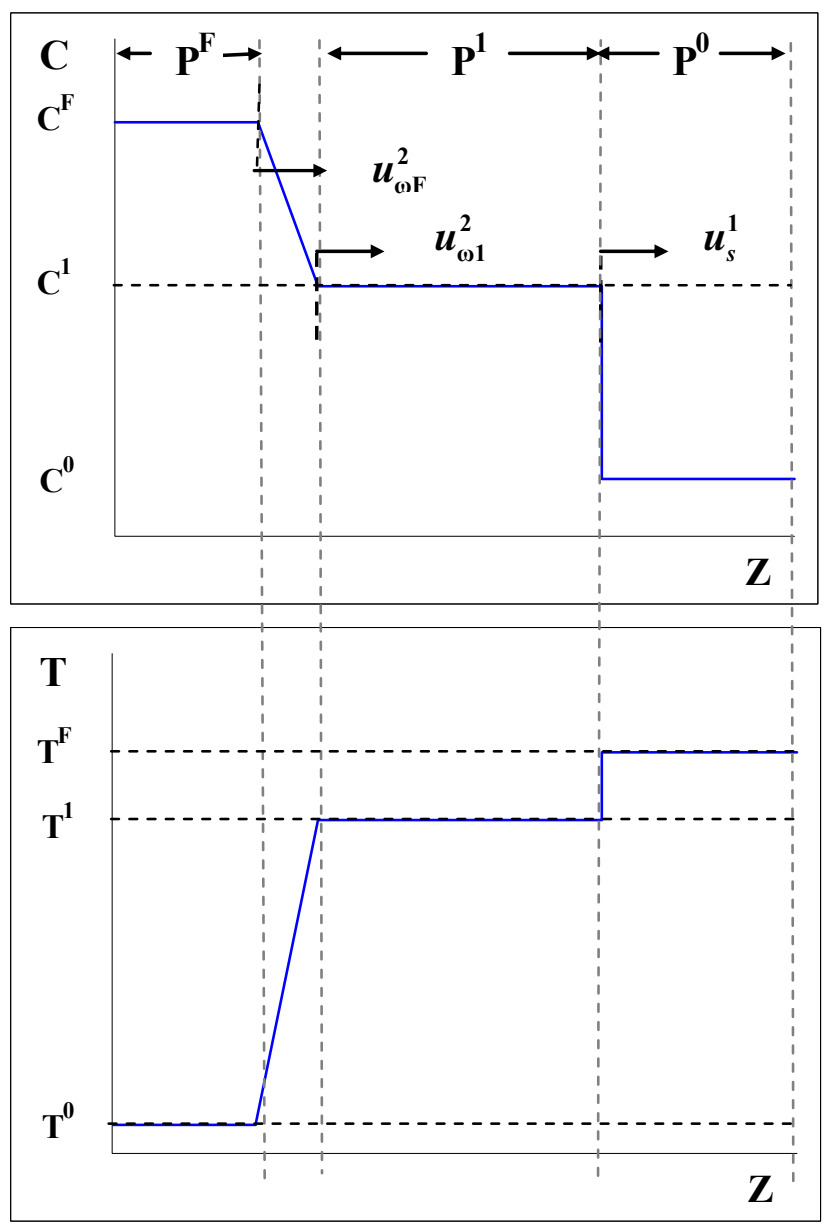

Fig. 14 - Perfiles de concentración y temperatura a lo largo de la variable $\mathrm{Z}$ a un cierto tiempo.

\subsubsection{Soluciones combinadas para la aplicación de un escalón}

Puede ocurrir que en un dado tramo continuo $\Gamma^{\mathrm{j}}$ la velocidad $u_{\omega}^{\mathrm{j}}$ no varíe monótonamente entre el punto $\mathrm{P}^{\mathrm{j}-1}$ y $\mathrm{P}^{\mathrm{j}}$. Para visualizar esta posibilidad y sus consecuencias, es conveniente analizar las curvas $q_{i}$ vs. $C_{i}$ que resultan sobre $\Gamma^{j}$. Es suficiente elegir un dado i, puesto que todas las curvas resultan equivalentes, según surge de la relación [26]. Elijamos $\mathrm{i}=\mathrm{m}+1$, es decir $\mathrm{q}_{\mathrm{m}+1}$ vs. $\mathrm{T}, \mathrm{y}$ analicemos el caso de la Figura 15a. De acuerdo a las Ecs. [23b] y [26] la derivada en cada punto de la curva permite evaluar $u_{\omega}^{\mathrm{j}}$.

$$
\frac{u / u_{\omega}^{\mathrm{j}}-1}{v}=\left(\frac{\mathrm{dq}_{\mathrm{m}+1}}{\mathrm{dT}}\right)^{\mathrm{j}}
$$

Los valores de $u_{\omega}^{\mathrm{j}}$ entre $\mathrm{P}^{\mathrm{j}-1}$ y $\mathrm{P}^{\mathrm{j}}$ se grafican en la Figura $15 \mathrm{~b}$. 


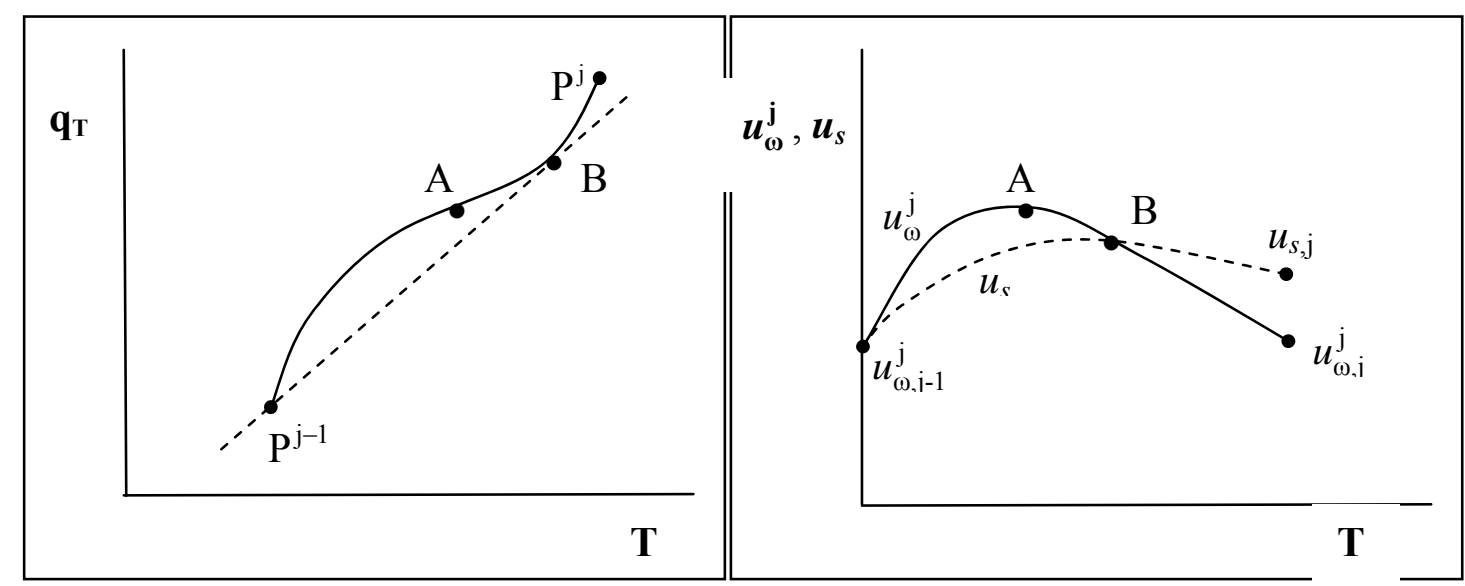

Fig.15a - Curva $\mathrm{q}_{\mathrm{T}}$ vs. $\mathrm{T}$

Fig.15b - Curva $u_{\omega}^{\mathrm{j}}$ vs. T

El punto de inflexión A en la Fig. 15a corresponde al máximo $u_{\omega}^{\mathrm{j}}$ que se observa en la Fig. $15 b$.

El tramo $\Gamma^{\mathrm{j}}$, discutido en las Figs.15, genera el perfil de $\mathrm{T}$ que se grafica en línea de trazos en la Figura 16 para un dado tiempo $\mathrm{t}>0$ (en vez de $\mathrm{T}$ podría haberse empleado cualquier $C_{i}$ para representar a cada estado del tramo $\Gamma^{\mathrm{j}}$ ). Los valores de $Z$ son calculados mediante $Z=t u_{\omega}^{j}$. Resulta claro que existe un intervalo de valores de $\mathrm{Z}$ para el cual existirían hasta tres niveles de temperatura, lo cual es inadmisible. La solución real deberá involucrar una onda de choque.

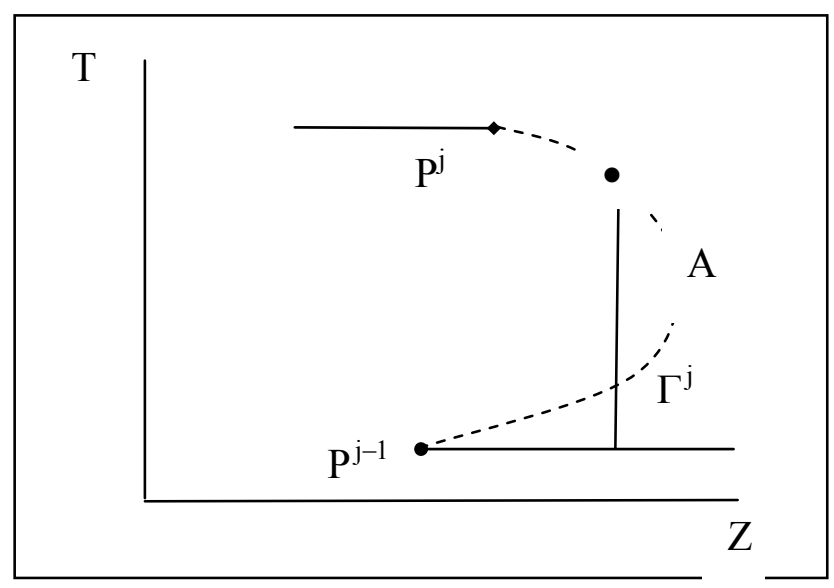

Fig. 16 - Perfil de temperatura a lo largo de la variable $\mathrm{Z}$ a un dado $\mathrm{t}>0$.

Para analizar la onda de choque, supondremos que la curva $\Sigma^{j}$, a los fines prácticos, coincide con la curva $\Gamma^{\mathrm{j}}$. Esta aproximación permite visualizar el tipo de soluciones resultante con mucha mayor sencillez que cuando se consideran las diferencias entre $\Sigma^{\mathrm{j}}$ y $\Gamma^{\mathrm{j}}$. Considerando a $\mathrm{P}^{\mathrm{j}-1}$ como punto fijo de análisis, esta 
suposición involucra que el punto $\mathrm{P}^{\mathrm{j}}$, que satisfizo la solución global asumiendo tramos continuos, se conservará tal cual al considerar la aparición de una onda de choque.

La velocidad $u_{s}$ de la onda de choque entre cualquier punto de la curva $\Sigma^{\mathrm{j}}\left(=\Gamma^{\mathrm{j}}\right)$ y el punto $\mathrm{P}^{\mathrm{j}-1}$ (es decir, considerando a $\mathrm{P}^{\mathrm{j}-1}$ como punto fijo de análisis) puede evaluarse a partir de la Ec. [30b],

$$
\frac{u / u_{s}-1}{v}=\frac{\mathrm{q}_{\mathrm{T}}-\mathrm{q}_{\mathrm{T}}^{\mathrm{j}-1}}{\mathrm{~T}-\mathrm{T}^{\mathrm{j}-1}}
$$

El segundo miembro de [34b] es la pendiente de la cuerda que une un punto genérico de la curva $\Sigma^{\mathrm{j}}$ con $\mathrm{P}^{\mathrm{j}-1}$. No resulta difícil visualizar que los valores de $u_{s}$ resultantes de [34b] corresponden, cualitativamente, a la curva de trazos de la Fig. 15b. Existe un valor máximo de $u_{s}$ correspondiente al punto donde la derivada coincide con la cuerda que pasa por $\mathrm{P}^{\mathrm{j}-1}$ (punto $\mathrm{B}$ ).

Si consideramos cualquier punto intermedio entre $\mathrm{P}^{\mathrm{j}-1}$ y $\mathrm{B}$, vemos de la Fig. $15 \mathrm{~b}$ que una eventual onda de choque entre tal punto y el $\mathrm{P}^{\mathrm{j}-1}$ satisface las [33], que al considerar $\mathrm{P}^{\mathrm{j}-1}$ y un punto genérico pueden re-escribirse como

$$
\begin{aligned}
& u_{\omega, \mathrm{j}-1}^{\mathrm{j}}<u_{\omega}^{\mathrm{j}} \\
& u_{s} \in\left[u_{\omega, \mathrm{j}-1}^{\mathrm{j}}, u_{\omega}^{\mathrm{j}}\right]
\end{aligned}
$$

Por lo tanto, si el punto $\mathrm{P}^{\mathrm{j}}$ hubiera correspondido al tramo entre $\mathrm{P}^{\mathrm{j}-1}$ y $\mathrm{B}$, la solución al problema planteado sería una onda de choque "simple" entre tal punto y $\mathrm{P}^{\mathrm{j}-1}$. Pero para el punto $\mathrm{P}^{\mathrm{j}}$ considerado en las Figs. 15, una onda de choque "simple" no resulta posible, porque no se verifican las [35] para cualquier punto a la derecha de B.

La imposibilidad de ocurrencia, tanto de una solución continua como de la existencia de una onda de choque "simple", hace que deba buscarse una solución combinada. Puesto que entre $\mathrm{P}^{\mathrm{j}-1}$ y B es posible una onda de choque y entre $\mathrm{A}$ y $\mathrm{P}^{\mathrm{j}}$ la solución continua es factible (Figs. 15), la solución combinada podrá componerse de una onda de choque entre $\mathrm{P}^{\mathrm{j}-1}$ y algún punto de transición comprendido entre A y B, y de un sub-tramo continuo entre el punto de transición y $\mathrm{P}^{\mathrm{j}}$. Como el punto de transición debe simultáneamente sustentar una onda de 
choque y pertenecer a un tramo continuo, deberá cumplirse para el mismo pueda existir que

$$
u_{\omega}^{\mathrm{j}}=u_{s}
$$

Resulta claro de las Figs. 15 que el punto B satisface la Ec. [36] y por lo tanto será el punto de transición.

En definitiva, la solución combinada en el presente caso se compone de un subtramo continuo entre $\mathrm{P}^{\mathrm{j}}$ y el punto $\mathrm{B}$ (ver perfil en la Fig. 16) y de una onda de choque entre el punto $\mathrm{B}$ y el punto $\mathrm{P}^{\mathrm{j}-1}$.

A raíz de la suposición $\Sigma^{\mathrm{j}}=\Gamma^{\mathrm{j}}$, el punto $\mathrm{B}$ queda geométricamente bien definido como el punto sobre la curva $\Gamma^{\mathrm{j}}$ en el que la tangente iguala a la pendiente de la cuerda que pasa por $\mathrm{P}^{\mathrm{j}-1}$. En el caso general $\Sigma^{\mathrm{j}} \neq \Gamma^{\mathrm{j}}$, la Ec. [36] aún será la condición para definir el punto de transición, pero la interpretación geométrica se distorsiona, porque $u_{s}$ se evaluá a partir de la curva $\Sigma^{\mathrm{j}}$ originada en $\mathrm{P}^{\mathrm{j}-1}$ mientras que $u_{\omega}^{\mathrm{j}}$ resulta a partir de la curva $\Gamma^{\mathrm{j}}$ que pasa localmente por cada punto de $\Sigma^{\mathrm{j}}$.

Existen otros ejemplos de soluciones combinadas, pero dado que en nuestro caso particular no se presentan situaciones de este tipo, no las abordaremos con mayor detalle.

\subsection{Adsorción simultánea de acetato de etilo y etanol}

En está sección se aplica el Método de las Características, previamente desarrollado, a nuestro caso particular de estudio donde se pretende purificar una corriente de aire contaminada con acetato de etilo y etanol (Capítulo 2). El sistema de eliminación de VOCs presentado en el Capítulo 2 incluye un adsorbedor con el fin de concentrar la corriente gaseosa para luego ingresar al reactor catalítico. Para aplicar el Método de las Características al sistema de adsorción/desorción se considera una columna adiabática, donde el acetato de etilo (“1”) y el etanol (“2”) son transportados por la corriente de aire y adsorbidos en la fase estacionaria de carbón activado.

Para el sistema sin resistencias los balances de materia por componente y entalpía, considerando concentraciones y temperatura uniformes en la sección transversal del lecho, son: 


$$
\begin{aligned}
& u \frac{\partial \mathrm{C}_{\mathrm{i}}}{\partial \mathrm{Z}}+\left[\frac{\partial \mathrm{C}_{\mathrm{i}}}{\partial \mathrm{t}}+\mathrm{v} \frac{\partial \mathrm{q}_{\mathrm{i}}}{\partial \mathrm{t}}\right]=0 \quad \mathrm{i}=1,2 \\
& \mathrm{c}_{\mathrm{p}, \mathrm{g}} u \frac{\partial \mathrm{T}}{\partial \mathrm{Z}}+\left[\left(\mathrm{c}_{\mathrm{p}, \mathrm{g}}+v \mathrm{c}_{\mathrm{p}, \mathrm{s}}\right) \frac{\partial \mathrm{T}}{\partial \mathrm{t}}-v \sum_{\mathrm{i}=1}^{2}\left(-\Delta \mathrm{H}_{\mathrm{i}}\right) \frac{\partial \mathrm{q}_{\mathrm{i}}}{\partial \mathrm{t}}\right]=0
\end{aligned}
$$

Los moles adsorbidos por unidad de volumen de adsorbente, $\mathrm{q}_{\mathrm{i}}(\mathrm{i}=1,2)$, son función de las concentraciones, $\mathrm{C}_{\mathrm{i}}(\mathrm{i}=1,2)$, y la temperatura, $\mathrm{T}$, a partir de una relación de equilibrio. Para el acetato de etilo y el etanol, Gales y col. (2003), sugieren el empleo de la isoterma de Langmuir generalizada para un sistema de dos componentes:

$$
\mathrm{q}_{\mathrm{i}}=\frac{Q_{\mathrm{i}} \mathrm{K}_{\mathrm{i}} \mathrm{C}_{\mathrm{i}}}{1+\sum_{\mathrm{j}=1}^{2} \mathrm{~K}_{\mathrm{j}} \mathrm{C}_{\mathrm{j}}} \quad \mathrm{i}=1,2
$$

donde $Q_{\mathrm{i}}$ es el valor de saturación de la especie i adsorbida por unidad de volumen de adsorbente, y $\mathrm{K}_{\mathrm{i}}$ la constante de equilibrio de adsorción de la especie $\mathrm{i}$.

$\mathrm{K}_{\mathrm{i}}$ es una función de la temperatura, representada por la siguiente expresión adoptada del trabajo de Gales y col. (2003):

$$
\mathrm{K}_{\mathrm{i}}=\mathrm{bo}_{\mathrm{i}} \mathrm{T}^{3 / 2} \exp \left(-\Delta \mathrm{H}_{\mathrm{i}} /(\mathrm{RT})\right) \quad \mathrm{i}=1,2
$$

Los valores de $Q_{\mathrm{i}}$, bo $_{\mathrm{i}}$ y $\Delta \mathrm{H}_{\mathrm{i}}$ se obtuvieron del trabajo de Gales y col. (2003). En la Tabla 1 se presentan dichos parámetros, empleando las unidades correspondientes a considerar concentraciones molares para los adsorbatos y considerando el volumen de adsorbente en lo que respecta a la cantidad adsorbida. Dado que en el trabajo original las unidades eran diferentes, para el cambio de unidades se requirió la densidad aparente del adsorbente $\left(750 \mathrm{~kg} / \mathrm{m}^{3}\right)$; este valor y toda la información referida a la caracterización del carbón activado utilizado como adsorbente se obtuvo del trabajo de Gales y col. (2000).

Tabla 1: Parámetros para la expresión de Langmuir generalizada para un sistema multicomponente

\begin{tabular}{lccc}
\hline & $\boldsymbol{Q}_{\mathbf{i}}\left[\mathbf{m o l} / \mathbf{m}^{3}\right]$ & $\mathbf{b o}_{\mathbf{i}}\left[\mathbf{m}^{\mathbf{3}} /\left(\mathbf{K}^{\mathbf{3} / \mathbf{2}} \mathbf{m o l}\right)\right]$ & $\left(-\Delta \mathbf{H}_{\mathbf{i}}\right)[\mathbf{J} / \mathbf{m o l}]$ \\
\hline 1: Acetato de Etilo & $3.5210^{3}$ & $2.4810^{-7}$ & 19600 \\
2: Etanol & $4.9410^{3}$ & $1.4710^{-10}$ & 42100
\end{tabular}


Definiendo $\mathrm{C}_{3} \equiv \mathrm{T} \quad$ y $\quad \mathrm{q}_{3} \equiv \mathrm{q}_{\mathrm{T}} \equiv \gamma \mathrm{T}-\sum_{\mathrm{i}=1}^{2} \mathrm{~J}_{\mathrm{i}} \mathrm{q}_{\mathrm{i}}, \mathrm{y}$ suponiendo que $\gamma=\mathrm{c}_{\mathrm{p}, \mathrm{s}} / \mathrm{c}_{\mathrm{p}, \mathrm{g}} \quad \mathrm{y}$ $\mathrm{J}_{\mathrm{i}}=-\Delta \mathrm{H}_{\mathrm{i}} / \mathrm{c}_{\mathrm{p}, \mathrm{g}}(\mathrm{i}=1,2)$ son constantes, compactamos la formulación [37] a la siguiente expresión:

$$
u \frac{\partial \underline{\mathrm{C}}}{\partial \mathrm{Z}}+(\mathrm{I}+v \mathcal{F}) \frac{\partial \underline{\mathrm{C}}}{\partial \mathrm{t}}=\underline{0}
$$

donde $\mathcal{F}$ es un matriz de $3 \times 3$ componentes definidos por $\mathcal{F}_{\mathrm{ij}}=\partial \mathrm{q}_{\mathrm{i}} / \partial \mathrm{C}_{\mathrm{j}}(\mathrm{i}, \mathrm{j}=1,2,3)$, I es la matriz identidad de $3 \times 3$ componentes y $\underline{\mathrm{C}}$ es el vector definido por $\underline{\mathrm{C}}=\left(\mathrm{C}_{1}, \mathrm{C}_{2}, \mathrm{~T}\right)^{\mathrm{T}}$. Las componentes de la matriz $\mathcal{F}$ se encuentran definidas en el Apéndice 4.1 para el caso particular de considerar una isoterma del tipo Langmuir, Ec. [38a].

Para la obtención de resultados se adoptó un sistema de adsorción/desorción constituido por dos lechos estructurados idénticos operando alternativamente. Se consideraron lechos de sección $\mathrm{S}=2.25 \mathrm{~m}^{2}$ y porosidad, $\varepsilon_{\mathrm{L}}=0.73$, cuyas paredes están constituidas por el propio material adsorbente, carbón activado (para el cual se adoptó $\left.\mathrm{c}_{\mathrm{p}, \mathrm{s}}=486850 \mathrm{~J} / \mathrm{K} \mathrm{m}^{3}\right)$.

\subsubsection{Etapa de adsorción}

Las propiedades de la corriente a adsorber (alimentación) a presión atmosférica son las siguientes (Tabla 1, Capítulo 2):

- Caudal volumétrico $\left(Q_{\text {ads }}\right): 11.7 \mathrm{~m}^{3} / \mathrm{s}$

- Temperatura $\left(\mathrm{T}^{\mathrm{F}}\right): 50^{\circ} \mathrm{C}$

- Concentración de acetato de etilo $\left(\mathrm{C}_{1}^{\mathrm{F}}\right): 2.3510^{-2} \mathrm{~mol} / \mathrm{m}^{3}$

- Concentración de etanol $\left(\mathrm{C}_{2}^{\mathrm{F}}\right): 9.6310^{-3} \mathrm{~mol} / \mathrm{m}^{3}$

- Capacidad calorífica $\left(\mathrm{c}_{\mathrm{p}, \mathrm{g}}\right): 1086.7 \mathrm{~J} / \mathrm{K} \mathrm{m}^{3}$

A $t=0$ s se definió la composición $\left(\mathrm{C}_{\mathrm{i}}, \mathrm{i}=1,2\right)$ y temperatura $(\mathrm{T})$ a lo largo del adsorbedor como:

$$
\mathrm{C}_{1}^{0}=0 \mathrm{~mol} / \mathrm{m}^{3} \quad \mathrm{C}_{2}^{0}=0 \mathrm{~mol} / \mathrm{m}^{3} \quad \mathrm{~T}^{0}=150^{\circ} \mathrm{C}
$$

El valor de $\mathrm{T}^{0}$ corresponde al valor de temperatura a la que se encuentra el lecho luego de ser regenerado, por lo que coincide con la temperatura de la corriente utilizada para la desorción. 
Teniendo en cuenta las condiciones operativas planteadas se identifica la presencia de un escalón de composición y temperatura $\underline{\mathrm{C}}^{\mathrm{F}}$ en la alimentación a un lecho inicialmente en equilibrio con una composición y temperatura uniformes $\underline{\mathrm{C}}^{0}$, verificándose así el problema de Riemann. A continuación se verá como se construye la solución para $\mathrm{t}>0$ según lo desarrollado previamente (Sección 4.2).

En principio se construirá un hodógrafo para una solución continua, es decir con puntos de quiebre unidos por tramos $\Gamma^{\mathrm{k}}$, del tipo de la Fig. 9. Recordemos que la condición inicial $\underline{C}^{0}$ (Ec. [40]), correspondiente al punto $\mathrm{P}^{0}, \mathrm{y}$ de alimentación $\underline{\mathrm{C}}^{\mathrm{F}}$, correspondiente al punto $\mathrm{P}^{\mathrm{F}}$, se encuentran definidas. Para este caso particular de dos componentes (acetato de etilo y etanol), surgen dos puntos de quiebre $\mathrm{P}^{1}$ y $\mathrm{P}^{2}$, por lo que se tienen tres tramos de composición y temperatura variables $\Gamma^{\mathrm{k}}(\mathrm{k}=1,2,3)$ que determinan la trayectoria $\mathrm{P}^{0}-\mathrm{P}^{1}-\mathrm{P}^{2}-\mathrm{P}^{\mathrm{F}}$.

Para la obtención de los tramos $\Gamma^{\mathrm{k}}(\mathrm{k}=1,2,3)$ se deben integrar las Ecs. [27] adaptadas a un sistema de dos componentes, como se muestra a continuación:

$$
\begin{aligned}
& \left(\mathrm{q}_{1,1}-\sigma^{\mathrm{k}}\right) \frac{\mathrm{dC}_{1}}{\mathrm{dT}}+\mathrm{q}_{1,2} \frac{\mathrm{dC}_{2}}{\mathrm{dT}}=-\mathrm{q}_{1, \mathrm{~T}} \\
& \mathrm{q}_{2,1} \frac{\mathrm{dC}_{1}}{\mathrm{dT}}+\left(\mathrm{q}_{2,2}-\sigma^{\mathrm{k}}\right) \frac{\mathrm{dC}_{2}}{\mathrm{dT}}=-\mathrm{q}_{2, \mathrm{~T}}
\end{aligned}
$$

donde:

$$
\mathrm{q}_{\mathrm{i}, \mathrm{j}}=\frac{\partial \mathrm{q}_{\mathrm{i}}}{\partial \mathrm{C}_{\mathrm{j}}} \quad \mathrm{y} \quad \mathrm{q}_{\mathrm{i}, \mathrm{T}}=\frac{\partial \mathrm{q}_{\mathrm{i}}}{\partial \mathrm{T}} \quad \mathrm{i}, \mathrm{j}=1,2
$$

Comenzamos con la construcción del tramo $\Gamma^{1}$ para lo cual se integran las Ecs. [41] en la dirección $\mathrm{k}=1$ partiendo del punto $\mathrm{P}^{0}$, cuyas condiciones operativas se encuentran definidas en [40]. En este punto se obtiene que:

$$
\mathrm{q}_{1}=0 \mathrm{~mol} / \mathrm{m}^{3} \quad \mathrm{q}_{2}=0 \mathrm{~mol} / \mathrm{m}^{3} \quad \mathrm{q}_{\mathrm{T}}=\gamma \mathrm{T}=1.9010^{5}{ }^{\circ} \mathrm{C}
$$

Para el caso particular de tener concentraciones nulas $\left(C_{i}=0, i=1,2\right)$ los autovalores de la matriz $\mathcal{F}, \sigma^{\mathrm{k}}(\mathrm{k}=1,2,3)$, resultan ser las componentes de su diagonal principal: $\mathrm{q}_{1,1}, \mathrm{q}_{2,2}$ y $\mathrm{q}_{\mathrm{T}, \mathrm{T}}$; siendo $\mathrm{q}_{\mathrm{T}, \mathrm{T}}=\gamma$, con $\gamma$ constante. Como se vio en la Sección 4.2, existe la posibilidad de que se presente un caso de discontinuidad de contacto, para lo cual resta verificar si $\sigma^{1}=\gamma$.

Para las condiciones establecidas en [40] se tiene: 


$$
\mathrm{q}_{1,1}=2003.1 \mathrm{~mol} / \mathrm{m}^{3} \quad \mathrm{q}_{2,2}=1000 \mathrm{~mol} / \mathrm{m}^{3} \quad \gamma=448.02
$$

resultando $\gamma<\mathrm{q}_{2,2}<\mathrm{q}_{1,1}$. De esta manera, considerando la convención adoptada por las Ec. [23c] en la Sección 4.2, queda definido:

$$
\sigma^{1}=\gamma \quad \sigma^{2}=q_{2,2} \quad \sigma^{3}=q_{1,1}
$$

Puesto que se verifica que el autovalor correspondiente a la dirección indicada $(k=1)$ es $\sigma^{1}=\gamma$, nos encontramos con un caso de discontinuidad de contacto donde la velocidad con la que se propaga la denominada onda térmica pura será $u_{\omega}^{1}=\frac{u}{1+v \gamma}$. El tramo $\Gamma^{1}$ resulta un segmento en el eje $\mathrm{T}$ que va desde $\mathrm{T}^{0}$ hasta un cierto valor $\mathrm{T}^{1}$ aún no determinado. Todos los puntos de este tramo tienen la misma velocidad $u_{\omega}^{1}$.

Ahora bien, para obtener la curva $\Gamma^{2}$ se deben integrar las ecuaciones [41a] y [41b] en la dirección $\mathrm{k}=2$ partiendo del punto de quiebre $\mathrm{P}^{1}$, donde $\mathrm{C}_{\mathrm{i}}=0, \mathrm{i}=1,2$; $\mathrm{T}=\mathrm{T}^{1}$. Dado que las concentraciones de los componentes son nulas se verifica que los autovalores correspondientes al punto $\mathrm{P}^{1}$ son las componentes de la diagonal principal de la matriz $\mathcal{F}$ evaluada en dicho punto (Sección 4.2).

Recordando que $\mathrm{T}^{1}<\mathrm{T}^{0}$, las constantes de adsorción $\mathrm{K}_{\mathrm{i}}$ (Ec. [38b]) aumentan, implicando un aumento de $\mathrm{q}_{1,1} \mathrm{y}_{2,2}$. Dado que $\gamma$ es constante, va a seguir siendo un valor menor a $\mathrm{q}_{1,1}$ y $\mathrm{q}_{2,2}$, por lo que a $\sigma^{2}$ se le asignará el menor valor que surja entre $\mathrm{q}_{1,1}$ y $\mathrm{q}_{2,2}$. Para nuestro caso planteado, y como se podrá confirmar más adelante al exponer los resultados finales, se tiene que $\sigma^{2}=\mathrm{q}_{1,1}$ por ser $\gamma<\mathrm{q}_{1,1}<\mathrm{q}_{2,2}$.

Considerando que $\sigma^{2}=\mathrm{q}_{1,1}$ y teniendo en cuenta que $\mathrm{q}_{1,2}=\mathrm{q}_{1, \mathrm{~T}}=0$, la Ec. [41a] resulta idénticamente nula para cualquier valor de $\frac{\mathrm{dC}_{1}}{\mathrm{dT}}$ y $\frac{\mathrm{dC}_{2}}{\mathrm{dT}}$. De la Ec. [41b] resulta que $\frac{\mathrm{dC}_{2}}{\mathrm{dT}}=0$ por ser $\mathrm{q}_{2,1}=\mathrm{q}_{2, \mathrm{~T}}=0$. Por lo tanto, de las Ecs. [41] se tiene que $\frac{\mathrm{dC}_{1}}{\mathrm{dT}}$ se encuentra indeterminada en el punto $\mathrm{P}^{1}$, impidiendo iniciar la integración en este punto. Cuando se presenta una indeterminación como la indicada, diremos que se tiene un "punto singular". 
Para poder determinar el valor de $\frac{\mathrm{dC}_{1}}{\mathrm{dT}} \mathrm{y}$ así poder iniciar la integración, se debe encontrar una expresión sustituta a la Ec. [41a], la cual se obtiene al derivar a esta última respecto de la temperatura, T, (Ec. [42]).

$$
\left(\frac{\mathrm{dq}_{1,1}}{\mathrm{dT}}-\frac{\mathrm{d} \sigma^{2}}{\mathrm{dT}}\right) \frac{\mathrm{dC}_{1}}{\mathrm{dT}}+\left(\mathrm{q}_{1,1}-\sigma^{2}\right) \frac{\mathrm{d}^{2} \mathrm{C}_{1}}{\mathrm{dT}^{2}}+\frac{\mathrm{dq}_{1,2}}{\mathrm{dT}} \frac{\mathrm{dC}_{2}}{\mathrm{dT}}+\mathrm{q}_{1,2} \frac{\mathrm{d}^{2} \mathrm{C}_{2}}{\mathrm{dT}^{2}}=-\frac{\mathrm{dq}_{1, \mathrm{~T}}}{\mathrm{dT}}
$$

Considerando que $\frac{\mathrm{dC}_{2}}{\mathrm{dT}}=0$ y que $\sigma^{2}=\mathrm{q}_{1,1}$, y que se tiene que $\mathrm{q}_{1,2}=0$, de [42] resulta:

$$
\left(\frac{\mathrm{dq}_{1,1}}{\mathrm{dT}}-\frac{\mathrm{d} \sigma^{2}}{\mathrm{dT}}\right) \frac{\mathrm{dC}_{1}}{\mathrm{dT}}=-\frac{\mathrm{dq}_{1, \mathrm{~T}}}{\mathrm{dT}}
$$

Por regla de la cadena se tiene:

$$
\begin{aligned}
& \frac{\mathrm{dq}_{1, \mathrm{j}}}{\mathrm{dT}}=\mathrm{q}_{1, \mathrm{j}, 1} \frac{\mathrm{dC}_{1}}{\mathrm{dT}}+\mathrm{q}_{1, \mathrm{j}, 2} \frac{\mathrm{dC}_{2}}{\mathrm{dT}}+\mathrm{q}_{1, \mathrm{j}, \mathrm{T}} \quad \text { para } \mathrm{j}=1, \mathrm{~T} \\
& \text { con: } \mathrm{q}_{1, \mathrm{j}, 1}=\frac{\partial \mathrm{q}_{1, \mathrm{j}}}{\partial \mathrm{C}_{1}} ; \quad \mathrm{q}_{1, \mathrm{j}, 2}=\frac{\partial \mathrm{q}_{1, \mathrm{j}}}{\partial \mathrm{C}_{2}} ; \quad \mathrm{q}_{1, \mathrm{j}, \mathrm{T}}=\frac{\partial \mathrm{q}_{1, \mathrm{j}}}{\partial \mathrm{T}} \quad \text { para } \mathrm{j}=1, \mathrm{~T}
\end{aligned}
$$

En el Apéndice 4.2 se encuentran las expresiones correspondientes a las derivadas $\mathrm{q}_{1, \mathrm{j}, 1}, \mathrm{q}_{1, \mathrm{j}, 2}$ y $\mathrm{q}_{1, \mathrm{j}, \mathrm{T}}$ para una isoterma tipo Langmuir.

$$
\text { Además } \frac{\mathrm{d} \sigma^{2}}{\mathrm{dT}}=\frac{\partial \sigma^{2}}{\partial \mathrm{C}_{1}} \frac{\mathrm{dC}_{1}}{\mathrm{dT}}+\frac{\partial \sigma^{2}}{\partial \mathrm{C}_{2}} \frac{\mathrm{dC}_{2}}{\mathrm{dT}}+\frac{\partial \sigma^{2}}{\partial \mathrm{T}}
$$

Las expresiones de $\frac{\partial \sigma^{2}}{\partial \mathrm{C}_{1}}, \frac{\partial \sigma^{2}}{\partial \mathrm{C}_{2}}$ y $\frac{\partial \sigma^{2}}{\partial \mathrm{T}}$ se pueden ver en el Apéndice 4.3.

Reemplazando en [43] y recordando que $\frac{\mathrm{dC}_{2}}{\mathrm{dT}}=0 \quad$ y que se tiene que $\mathrm{q}_{1, \mathrm{~T}, \mathrm{~T}}=0$ resulta:

$$
\frac{\mathrm{dC}_{1}}{\mathrm{dT}}=\frac{-\mathrm{q}_{1, \mathrm{~T}, 1}-\mathrm{q}_{1,1, \mathrm{~T}}+\frac{\partial \sigma^{2}}{\partial \mathrm{T}}}{\mathrm{q}_{1,1,1}-\frac{\partial \sigma^{2}}{\partial \mathrm{C}_{1}}}
$$

Una vez que se inicia la integración de la Ec. [47] a partir de $\mathrm{P}^{1}, \mathrm{C}_{1}$ adopta un valor distinto de cero por lo que desaparece la indeterminación inicial y nos permite 
seguir integrando normalmente (empleando las Ecs. [41]) hasta un cierto valor de $\mathrm{T}^{2}$ aun no definido.

A continuación se presenta un hodógrafo (Figura 17) construido a partir de la obtención de los resultados finales. El mismo resulta práctico para entender el análisis descripto sobre la construcción de los tramos $\Gamma^{\mathrm{k}}$.

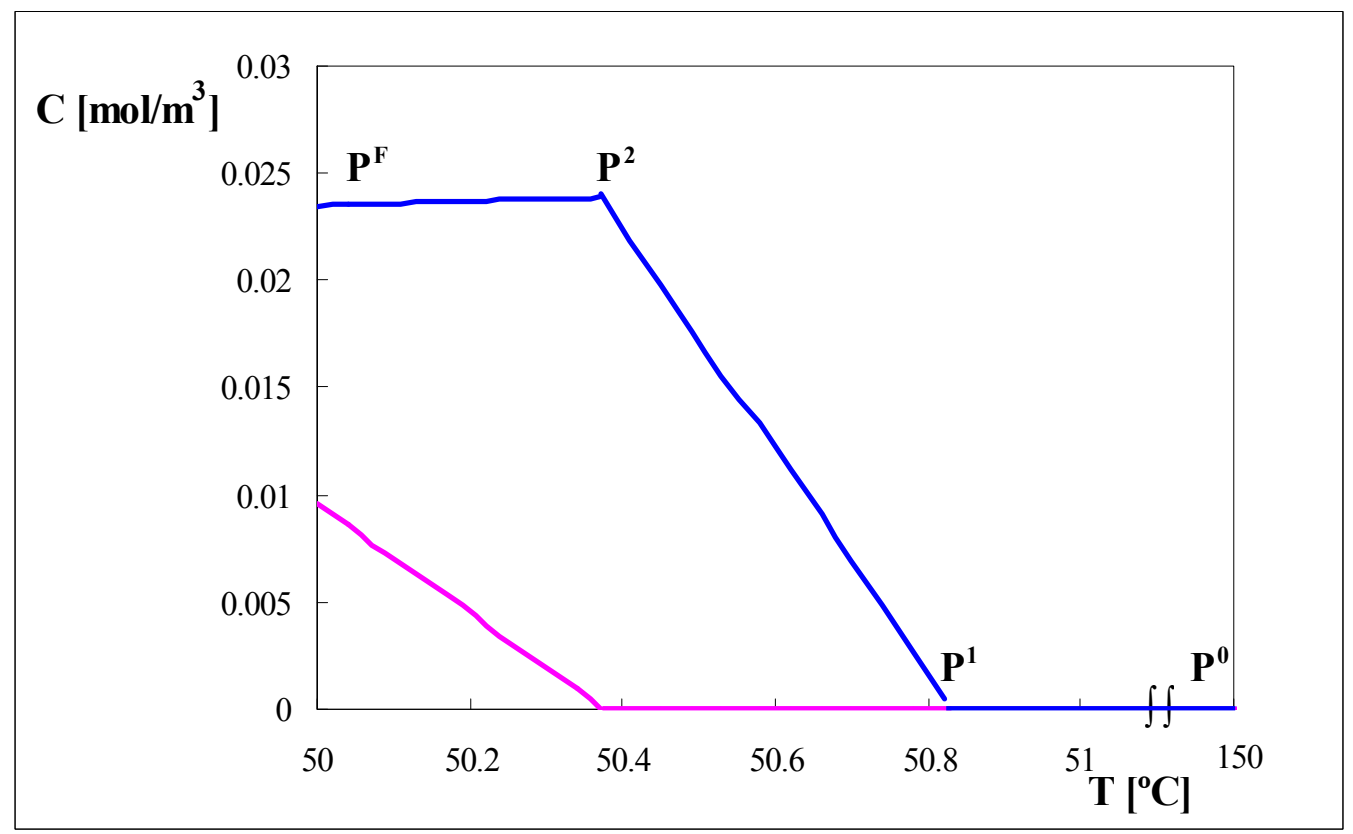

Fig. 17 - Hodógrafo. Solución para dos componentes ( —acetato de etilo; —etanol).

Para obtener el tramo $\Gamma^{3}$ debemos integrar las Ecs. [41] en dirección $\mathrm{k}=3$, partiendo del punto de quiebre $\mathrm{P}^{2}$ (Fig. 17). Al ser $\mathrm{C}_{2}^{2}=0, \mathrm{q}_{2,2}$ coincide con uno de los autovalores. Para nuestro caso particular, y como se verificará más adelante, $\mathrm{q}_{2,2}$ resulta ser el autovalor de mayor valor, por lo que $\sigma^{3}=\mathrm{q}_{2,2}$.

Reemplazando este valor de $\sigma^{3}$ en la Ec. [41b], y considerando que $\mathrm{q}_{2,1}=\mathrm{q}_{2, \mathrm{~T}}=0$, se observa que dicha expresión resulta idénticamente nula para cualquier valor de $\frac{\mathrm{dC}_{1}}{\mathrm{dT}}$ y $\frac{\mathrm{dC}_{2}}{\mathrm{dT}}$. Nuevamente nos encontramos con un punto singular, por lo que debemos encontrar una situación alternativa a la Ec [41b], la cual se obtiene derivando esta expresión respecto de $\mathrm{T}$.

Teniendo en cuenta que $\mathrm{q}_{2,1,1}=\mathrm{q}_{2,1, \mathrm{~T}}=\mathrm{q}_{2, \mathrm{~T}, 1}=\mathrm{q}_{2, \mathrm{~T}, \mathrm{~T}}=0$, resulta:

$$
\left(\mathrm{q}_{2,1,2}+\mathrm{q}_{2,2,1}-\frac{\partial \sigma^{3}}{\partial \mathrm{C}_{1}}\right) \frac{\mathrm{dC}_{1}}{\mathrm{dT}}+\left(\mathrm{q}_{2,2,2}-\frac{\partial \sigma^{3}}{\partial \mathrm{C}_{2}}\right) \frac{\mathrm{dC}_{2}}{\mathrm{dT}}=-\mathrm{q}_{2, \mathrm{~T}, 2}-\mathrm{q}_{2,2, \mathrm{~T}}+\frac{\partial \sigma^{3}}{\partial \mathrm{T}}
$$


De manera equivalente a la situación descripta para el punto $\mathrm{P}^{1}$, una vez que se inicia la integración de las Ecs. [48] y [41a] a partir de $\mathrm{P}^{2}, \mathrm{C}_{2}^{2}$ adopta un valor distinto de cero por lo que desaparece la indeterminación inicial y nos permite seguir integrando normalmente (Ecs. [41]) hasta $\mathrm{T}=\mathrm{T}^{\mathrm{F}}$ en la dirección $\mathrm{k}=3$. Si en este punto las composiciones alcanzadas no coinciden con las condiciones de alimentación definidas previamente $\left(\mathrm{C}_{1}^{\mathrm{F}}, \mathrm{C}_{2}^{\mathrm{F}}\right)$, es necesario variar iterativamente los valores de $\mathrm{T}^{1}$ y $\mathrm{T}^{2}$ supuestos hasta obtener la composición fijada en el punto $\mathrm{P}^{\mathrm{F}}$.

Este sería el procedimiento para poder obtener la trayectoria $\mathrm{P}^{0}-\mathrm{P}^{1}-\mathrm{P}^{2}-\mathrm{P}^{\mathrm{F}}$ en el plano de variables $C_{i}(i=1,2,3)$ suponiendo una solución continua.

En la práctica, la obtención de la solución requiere de un esfuerzo bastante más modesto. Puesto que ya se conoce que en $\mathrm{P}^{2}$, deberá ser $\mathrm{C}_{2}^{2}=0$, se puede comenzar integrando las Ecs. [41] desde el punto $\mathrm{P}^{\mathrm{F}}$, definido previamente, hasta un valor de $\mathrm{T}$ tal que $\mathrm{C}_{2}^{2}$ resulte cercano a cero. De esta manera evitamos la situación particular de caer en un punto singular. Luego se calculan $\mathrm{C}_{1}^{2}$ y $\mathrm{T}^{2}$ determinando así el vector de variables de estado $\underline{\mathrm{C}}^{2}$ en el punto $\mathrm{P}^{2}$. Con las condiciones operativas del punto $\mathrm{P}^{2}$ ya establecidas, queda comprobado que $\sigma^{3}=\mathrm{q}_{2,2}$. Finalmente se integran las Ecs. [41] a partir de $\mathrm{P}^{2}$ en dirección $\mathrm{k}=2$ hasta un valor de $\mathrm{T}$ tal que $\mathrm{C}_{1}^{1}$ resulte un valor cercano de cero. Con $\mathrm{P}^{1}$ definido se verifica que $\mathrm{q}_{1,1}<\mathrm{q}_{2,2}$ en dicho punto.

Para esta alternativa de cálculo se necesita del análisis previamente desarrollado que nos permitió definir que $C_{1}^{1}=C_{2}^{1}=C_{2}^{2}=0$.

Para las condiciones operativas iniciales (Ec. [40]) y las de alimentación, se obtuvieron los siguientes valores de concentración, temperatura y velocidades de propagación en cada uno de los puntos (Tabla 2):

Tabla 2: Composición y temperatura en cada uno de los puntos que definen la trayectoria $\mathrm{P}^{0}-\mathrm{P}^{1}-\mathrm{P}^{2}-\mathrm{P}^{\mathrm{F}}$

\begin{tabular}{lcccc}
\hline \multicolumn{1}{c}{$\mathbf{P}^{\mathbf{j}}$} & $\mathbf{P}^{\mathbf{0}}$ & $\mathbf{P}^{\mathbf{1}}$ & $\mathbf{P}^{\mathbf{2}}$ & $\mathbf{P}^{\mathbf{F}}$ \\
\hline $\mathbf{C}_{\mathbf{1}}\left[\mathbf{m o l} / \mathbf{m}^{\mathbf{3}}\right]$ & 0 & 0 & $2.3910^{-2}$ & $2.3510^{-2}$ \\
$\mathbf{C}_{\mathbf{2}}\left[\mathbf{m o l} / \mathbf{m}^{3}\right]$ & 0 & 0 & 0 & $9.6310^{-3}$ \\
$\mathbf{T}\left[{ }^{\mathbf{0}} \mathbf{C}\right]$ & 150 & 50.83 & 50.37 & 50 \\
$\mathbf{u}_{\mathbf{\omega}, \mathbf{j}}^{\mathbf{j}}[\mathbf{m} / \mathbf{s}]$ & & 0.04344 & 0.00287 & 0.00082 \\
$\mathbf{u}_{\mathbf{\omega}, \mathbf{j}}^{\mathbf{j}+\mathbf{1}}[\mathbf{m} / \mathbf{s}]$ & 0.04344 & 0.00265 & 0.00077 &
\end{tabular}


Para que efectivamente puedan existir los tramos $\Gamma^{2}$ y $\Gamma^{3}$, es necesario que $u_{\omega}^{2} \mathrm{y}$ $u_{\omega}^{3}$ disminuyan desde $\mathrm{P}^{1}$ hasta $\mathrm{P}^{2} \mathrm{y}$ desde $\mathrm{P}^{2}$ hasta $\mathrm{P}^{\mathrm{F}}$ respectivamente. Sin embargo, según los resultados obtenidos (Tabla 2), esto no sucede para ambos tramos. Por lo tanto es necesario admitir que existirán sendas ondas de choque, cada una reemplazando los tramos $\Gamma^{2}$ y $\Gamma^{3}$.

Como se discutiera en la Sección 4.2, salvo casos singulares, la composición de los extremos de $\Gamma^{\mathrm{k}}(\mathrm{k}=2,3)$ no podrán conectarse a través de una onda de choque, sino que al menos uno de ellos resultará con una composición diferente. Recordando que al menos uno de los extremos de $\Gamma^{\mathrm{k}}(\mathrm{k}=2,3)$ será un punto de quiebre, concluimos que las coordenadas del mismo resultarán modificadas y el nuevo tramo pasará a llamarse $\Sigma^{\mathrm{k}}(\mathrm{k}=2,3)$. Se debe tener en cuenta que el cambio de composición de al menos uno de los puntos de quiebre afectará a todos los demás.

Para la obtención de los tramos $\Sigma^{2}$ y $\Sigma^{3}$ se deben resolver las Ecs. [30] vistas en la Sección 4.2 para $\mathrm{m}=2$.

Para nuestro caso en particular las curvas $\Gamma^{\mathrm{k}} \mathrm{y} \Sigma^{\mathrm{k}}$ dieron muy próximas entre sí, lo cual se debe a que las derivadas de $\Gamma^{\mathrm{k}}$ son prácticamente constantes. Por lo tanto, los puntos de quiebre $\mathrm{P}^{1}$ y $\mathrm{P}^{2}$ obtenidos se aproximan mucho a los puntos definitivos resultantes de sustituir ambos tramos por ondas de choque.

La velocidad de la onda de choque debe ser la misma para todos los componentes, en el tramo $\Sigma^{2}$ la velocidad a la cual se propaga la onda es $u_{s}^{2}=0.0028 \mathrm{~m} / \mathrm{s}$ y en el tramo $\Sigma^{3}$ la velocidad es $u_{s}^{3}=0.0008 \mathrm{~m} / \mathrm{s}$. Estos valores fueron obtenidos a partir de las Ecs. [30] considerando la composición y temperatura de los puntos $\mathrm{P}^{1}, \mathrm{P}^{2}$ y $\mathrm{P}^{\mathrm{F}}$, resultando:

$$
\begin{aligned}
& u_{s}^{2}=u\left(\frac{\mathrm{q}_{\mathrm{T}}^{2}-\mathrm{q}_{\mathrm{T}}^{1}}{\mathrm{~T}^{2}-\mathrm{T}^{1}} \mathrm{v}+1\right)^{-1} \\
& u_{s}^{3}=u\left(\frac{\mathrm{q}_{\mathrm{T}}^{3}-\mathrm{q}_{\mathrm{T}}^{2}}{\mathrm{~T}^{\mathrm{F}}-\mathrm{T}^{2}} \mathrm{v}+1\right)^{-1}
\end{aligned}
$$


En la Figura 18 se pueden observar los perfiles de concentración $\left(\mathrm{C}_{1}, \mathrm{C}_{2}\right)$ y temperatura $(\mathrm{T})$ a lo largo de la variable axial $(\mathrm{Z})$ a los $700 \mathrm{~s}$, para lo cual se consideró un lecho de $3 \mathrm{~m}$ de largo (L).

En el instante en que alguno de los componentes se hace presente a la salida del lecho, se detiene la etapa de adsorción para pasar a la etapa de desorción donde es regenerado. Para las condiciones geométricas y operativas estipuladas previamente, el tiempo requerido para completar la etapa de adsorción con $\mathrm{L}=3 \mathrm{~m}$ es de $1087.47 \mathrm{~s}$ (18.13 min), y ocurre cuando la onda de choque con mayor velocidad, $u_{s}^{2}$, alcanza el fin del lecho.

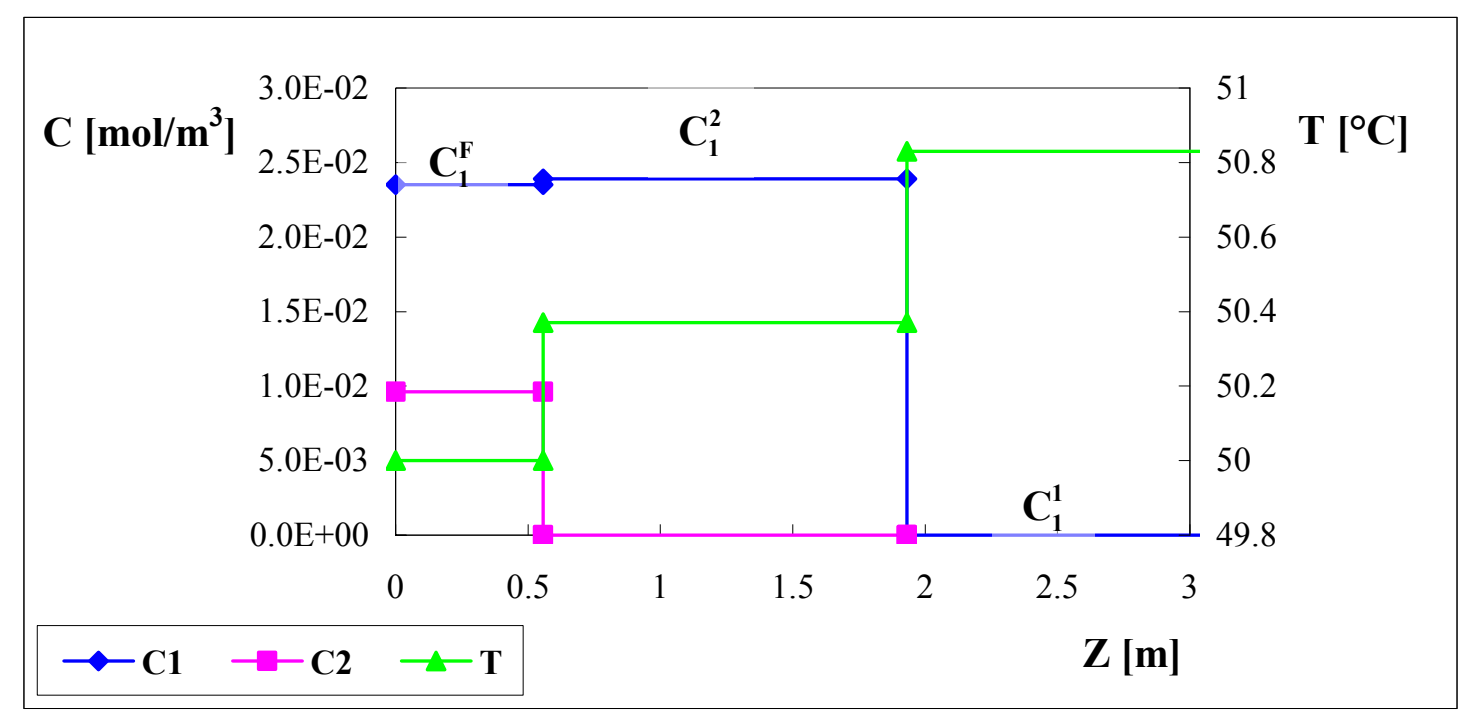

Fig. 18 - Variación de la concentración de cada uno de los componentes $\left(\mathrm{C}_{1}\right.$ y $\left.\mathrm{C}_{2}\right)$ y de la temperatura $(\mathrm{T})$ a lo largo del lecho a $t_{\text {ads }}=700 \mathrm{~s}$. Se indican los valores de $\mathrm{C}_{1}$ para los distintos puntos de quiebre. (1: acetato de etilo, 2: etanol)

En virtud de la resolución numérica alcanzada se pudo inferir que el comportamiento físico del sistema está caracterizado por un enfriamiento rápido, desde 150 hasta un valor cercano a $50{ }^{\circ} \mathrm{C}$, determinado por la velocidad del avance del frente de temperatura. Esta situación es la que posibilita la posterior adsorción de los VOCs, la cual se produce avanzando como ondas de choque. Una vez adsorbidos el acetato de etilo y el etanol, la temperatura del lecho alcanza el valor de la temperatura de la corriente de alimentación $\left(50^{\circ} \mathrm{C}\right)$. La finalización del proceso está asociada con la detección en la corriente de salida de una concentración superior a la admisible, en este caso correspondiente exclusivamente al acetato de etilo, que tiene un frente de avance más rápido que el etanol. 
A continuación se presentan los resultados obtenidos a partir de la aplicación del Método de las Características a la etapa de desorción.

\subsubsection{Etapa de desorción}

Para llevar a cabo la desorción del lecho se simuló la circulación de una corriente de aire puro a presión atmosférica y a una temperatura de $150{ }^{\circ} \mathrm{C}$ en contracorriente respecto a la corriente que circulara durante la adsorción.

En la Figura 19, puede apreciarse el estado del lecho una vez finalizada la etapa de adsorción. Dado que la desorción se llevará a cabo en contracorriente con el sentido de flujo empleado durante la adsorción, en la Fig. 19 se ha invertido el eje correspondiente a $\mathrm{Z}$ respecto al utilizado en la Fig. 18.

En la Fig. 19 se observan dos escalones, uno en el origen al que llamaremos Escalón I y otro a los 2.14 m, identificado como Escalón II, correspondiente a la posición que alcanzó la onda de choque más lenta en la etapa de adsorción con velocidad $u_{s}^{3}(0.0008 \mathrm{~m} / \mathrm{s})$.

El Escalón I (Fig. 19) separa dos estados de composición y temperatura uniformes definidos como $\mathrm{P}_{\text {(I) }}^{\mathrm{F}}$ y $\mathrm{P}_{(\mathrm{I})}^{0}$ (el subíndice I hace referencia al escalón en cuestión). La composición y temperatura en $\mathrm{P}_{(\mathrm{I})}^{\mathrm{F}}$ corresponden a las de la corriente de alimentación en el proceso de desorción $\left(\mathrm{P}_{(\mathrm{DES})}^{\mathrm{F}}\right)$, siendo $\mathrm{C}_{1}^{\mathrm{F}}=\mathrm{C}_{2}^{\mathrm{F}}=0$ y $\mathrm{T}^{\mathrm{F}}=150^{\circ} \mathrm{C}$. Mientras que la composición y temperatura en $\mathrm{P}_{(\mathrm{I})}^{0}$ son las establecidas para el segundo punto de quiebre en la etapa de adsorción, $\mathrm{P}_{(\mathrm{ADS})}^{2}$ (ver Tabla 2). 


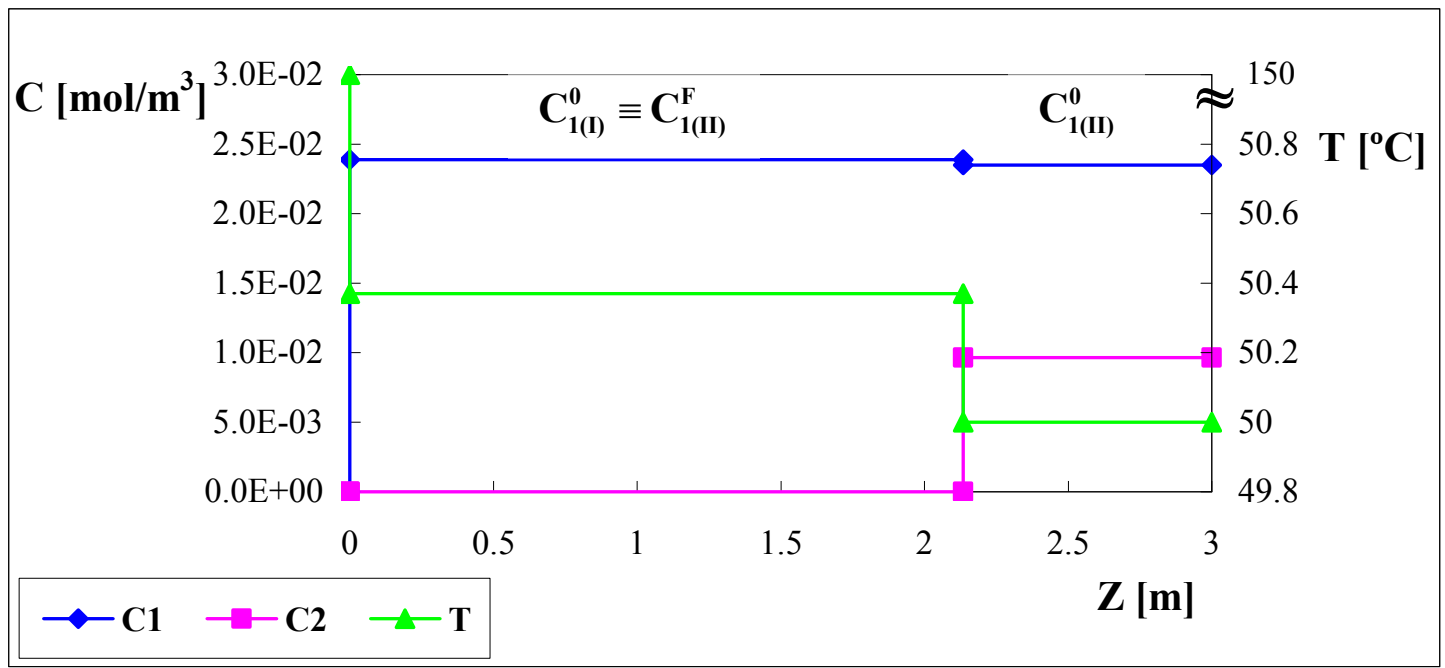

Fig. 19 - Variación de la concentración de cada uno de los componentes $\left(\mathrm{C}_{1}\right.$ y $\left.\mathrm{C}_{2}\right)$ y de la temperatura (T) a lo largo del lecho a $t_{\text {des }}=0 \mathrm{~s}$. Se indican los valores de $\mathrm{C}_{1}$ para los distintos puntos de quiebre. (1: acetato de etilo, 2: etanol)

Por otro lado el Escalón II también separa dos estados de composición y temperatura uniformes a los que llamaremos $\mathrm{P}_{\text {(II) }}^{\mathrm{F}}$ y $\mathrm{P}_{(\text {II) }}^{0}$. La composición y temperatura en $\mathrm{P}_{(\mathrm{II})}^{\mathrm{F}}$ es la misma que la establecida para $\mathrm{P}_{(\mathrm{I})}^{0}\left(\equiv \mathrm{P}_{(\mathrm{ADS})}^{2}\right)$, mientras que las variables de estado en $\mathrm{P}_{(\text {II) }}^{0}$ corresponden a las definidas para la alimentación en la etapa de adsorción, $\mathrm{P}_{(\mathrm{ADS})}^{\mathrm{F}}$ (ver Tabla 2).

Como ya fue mencionado, el principal objetivo de la incorporación de un sistema de adsorción/desorción al sistema combinado de eliminación de VOCs sugerido en el Capítulo 2, es concentrar la corriente de aire a tratar, lo que permitirá disminuir el volumen del reactor e intercambiadores presentes en el sistema. Cuanto más concentrada la corriente, más pequeños resultarán los equipos previamente mencionados. La etapa de desorción está determinada esencialmente por la temperatura y el caudal de la corriente utilizada para desorber. Para una temperatura definida (su impacto se analizará posteriormente) de dicha corriente, el grado de concentración de la corriente depende del valor del caudal de aire con el que se lleve a cabo la desorción. El valor mínimo de dicho caudal se definirá cuando el período correspondiente a la etapa de desorción coincida con el de la etapa de adsorción. Retomando nuestro ejemplo, para que esto suceda, y como se verificará a posteriori, la corriente utilizada durante la desorción debe circular a una velocidad intersticial de $2 \mathrm{~m} / \mathrm{s}$, menos de un tercio del valor de la velocidad a la cual circula la corriente durante la adsorción $(7.08 \mathrm{~m} / \mathrm{s})$. 
Se debe destacar que, al no encontrarse el lecho con composición y temperatura uniformes al iniciar la etapa de desorción, no se verifica el problema de Riemann. Sin embargo, mientras que los Escalones I y II no interactúen entre sí, podemos abarcar el problema a partir de dividir al lecho en 2 zonas independientes, cada una con composición y temperatura uniformes $\left(\mathrm{P}_{(\mathrm{I})}^{0} \mathrm{y} \mathrm{P}_{\text {(II) }}^{0}\right)$ a las cuales se les aplica un escalón de alimentación diferente $\left(\mathrm{P}_{(\mathrm{I})}^{\mathrm{F}} \mathrm{y} \mathrm{P}_{(\mathrm{II})}^{\mathrm{F}}\right)$. De esta manera es factible aplicar el problema de Riemann a cada una de estas zonas de forma individual.

A continuación analizaremos el Escalón $I(e n Z=0 \mathrm{~m}, \mathrm{t}=0 \mathrm{~s})$, para lo cual recordamos la composición y temperatura correspondientes a $\mathrm{P}_{(\mathrm{I})}^{0} \quad \mathrm{y} \quad \mathrm{P}_{(\mathrm{I})}^{\mathrm{F}}$ en la siguiente tabla.

Tabla 3: Composición y temperatura en $\mathrm{P}_{(\mathrm{I})}^{0}$ y $\mathrm{P}_{(\mathrm{I})}^{\mathrm{F}}$

\begin{tabular}{lcc}
\hline \multicolumn{1}{c}{$\mathbf{P}_{(\mathbf{I})}^{\mathbf{j}}$} & $\mathbf{P}_{(\mathrm{I})}^{0}$ & $\mathbf{P}_{(\mathrm{I})}^{\mathbf{F}}$ \\
\hline $\mathbf{C}_{\mathbf{1}}\left[\mathbf{m o l} / \mathbf{m}^{3}\right]$ & $2.3910^{-2}$ & 0 \\
$\mathbf{C}_{2}\left[\mathbf{m o l} / \mathbf{m}^{3}\right]$ & 0 & 0 \\
$\mathbf{T}\left[{ }^{\mathbf{0}} \mathbf{C}\right]$ & 50.37 & 150
\end{tabular}

En este caso particular, siendo que la concentración del etanol es nula en $\mathrm{P}_{\text {(I) }}^{0} \mathrm{y}$ $\mathrm{P}_{(\mathrm{I})}^{\mathrm{F}}$, podemos no considerarla para el análisis, e incluir solo la concentración del acetato de etilo y la temperatura de la corriente (dos autovalores). De esta manera, al considerar un único componente (acetato de etilo) se obtendrá un único punto de quiebre $\mathrm{P}_{(\mathrm{I})}^{1}$ y las Ecs. [41] resultan en una única expresión que es la siguiente:

$$
\left(\mathrm{q}_{1,1}-\sigma^{k}\right) \frac{\mathrm{dC}_{1}}{\mathrm{dT}}=-\mathrm{q}_{1, \mathrm{~T}}
$$

Como fuera realizado para la etapa de adsorción, para la obtención de la curva $\Gamma_{(\mathrm{I})}^{1}$ se debe integrar la Ec. [49] a partir de $\mathrm{P}_{(\mathrm{I})}^{0}$ en dirección $\mathrm{k}=1$ hasta un cierto valor de la temperatura.

Para determinar la curva $\Gamma_{(\mathrm{I})}^{2}$ que une los puntos $\mathrm{P}_{(\mathrm{I})}^{1}$ y $\mathrm{P}_{(\mathrm{I})}^{\mathrm{F}}$ en el hodógrafo (Figura 20), resulta práctico integrar la Ec. [49] en dirección $\mathrm{k}=2$ partiendo de las condiciones de alimentación anteriormente establecidas $\left(\underline{\mathrm{C}}_{(\mathrm{I})}^{\mathrm{F}}\right)$ hasta un cierto valor 
de $\mathrm{T}$ sobre la curva $\Gamma_{(\mathrm{I})}^{1}$. La intersección de ambas curvas resulta en el punto $\mathrm{P}_{(\mathrm{I})}^{1}$ (Fig. 20) cuya composición se presenta en la Tabla 4.

Se debe tener en cuenta que en el punto $P_{(\mathrm{I})}^{F}$ al ser $C_{1,(\mathrm{I})}^{\mathrm{F}}$ nula se tiene que $\mathrm{q}_{1, \mathrm{~T}}=0$, y además siendo que $\mathrm{q}_{1,1}>\gamma$, surge que $\sigma^{2}=\mathrm{q}_{1,1}$, por lo tanto la Ec. [49] resulta nula para cualquier valor de $\frac{\mathrm{dC}_{1}}{\mathrm{dT}}$. Nuevamente nos encontramos con un punto singular, por lo que de manera equivalente a como se procedió en la etapa de adsorción, debemos encontrar una situación alternativa a la Ec [49], la cual se obtiene derivando esta expresión respecto de T. Una vez iniciada la integración, $\mathrm{C}_{1,(\mathrm{I})}^{\mathrm{F}}$ toma un valor distinto de cero y desaparece la indeterminación inicial, lo que nos permite seguir integrando normalmente empleando las Ec. [49].

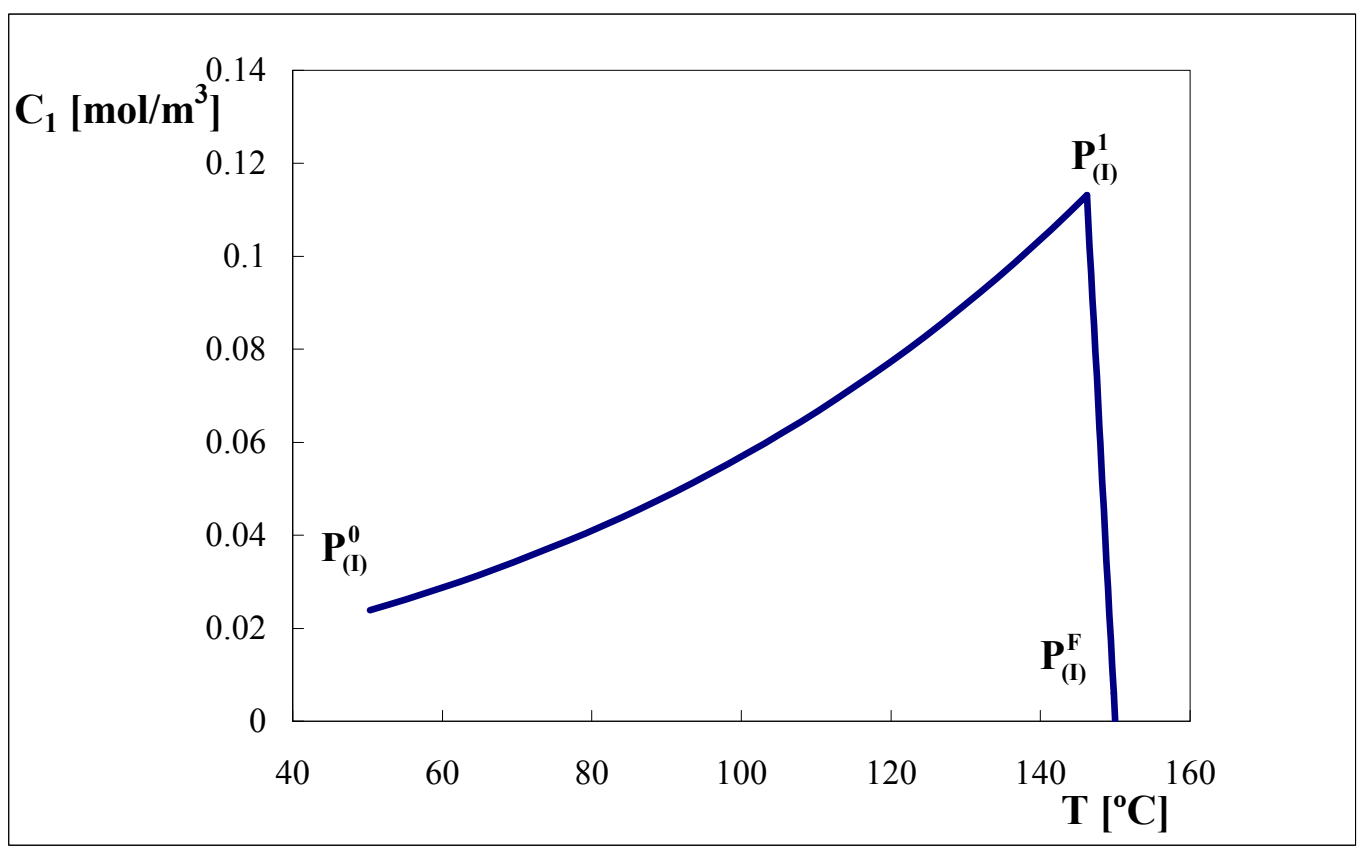

Fig. 20 - Hodógrafo. Solución para un componente (acetato de etilo).

Tabla 4: Composición y temperatura en el punto de quiebre $\mathrm{P}_{(\mathrm{I})}^{1}$

\begin{tabular}{|c|c|}
\hline$P_{I}^{j}$ & $\mathbf{P}_{(\mathrm{I})}^{1}$ \\
\hline $\mathrm{C}_{1}\left[\mathrm{~mol} / \mathrm{m}^{3}\right]$ & $1.1310^{-1}$ \\
\hline $\mathrm{C}_{2}\left[\mathrm{~mol} / \mathrm{m}^{3}\right]$ & 0 \\
\hline $\mathrm{T}\left[{ }^{\circ} \mathrm{C}\right]$ & 146.2 \\
\hline
\end{tabular}

Las velocidades de propagación en cada uno de los puntos resultan (Tabla 5): 
Tabla 5: Velocidades de propagación de los puntos $\mathrm{P}_{(\mathrm{I})}^{0}, \mathrm{P}_{(\mathrm{I})}^{1}, \mathrm{P}_{(\mathrm{I})}^{\mathrm{F}}$

\begin{tabular}{cccc}
\hline $\mathbf{P}^{\mathbf{j}}$ & $\mathbf{P}_{(\mathrm{I})}^{\mathbf{0}}$ & $\mathbf{P}_{(\mathrm{I})}^{\mathbf{1}}$ & $\mathbf{P}_{(\mathrm{I})}^{\mathbf{F}}$ \\
\hline $\boldsymbol{u}_{\boldsymbol{\omega}, \mathbf{j}(\mathbf{I})}^{\mathbf{j}}[\mathbf{m} / \mathbf{s}]$ & & 0.00978 & 0.00276 \\
$\boldsymbol{u}_{\boldsymbol{\omega}, \mathbf{j}(\mathbf{I})}^{\mathbf{j}+\mathbf{1}}[\mathbf{m} / \mathbf{s}]$ & 0.00953 & 0.00292 &
\end{tabular}

A partir de los resultados presentados en la Tabla 5 se observa que $u_{\omega, 1(\mathrm{I})}^{1}>u_{\omega, 0(\mathrm{I})}^{1}$, por lo que el tramo $\Gamma_{(\mathrm{I})}^{1}$ debe sustituirse por una onda de choque $\sum_{(\mathrm{I})}^{1}$. Para determinar la velocidad de la onda de choque a partir de las Ecs. [30], se asumió a la composición y temperatura del nuevo punto $\mathrm{P}_{(\mathrm{I})}^{1}$ equivalentes a las obtenidas para dicho punto de quiebre al considerar el tramo continuo $\Gamma_{(\mathrm{I})}^{1}$. A fin de estimar el error cometido por dicha aproximación, se compararon los valores de la velocidad de la onda de choque obtenidos a partir de las siguientes expresiones:

$$
\begin{aligned}
& u_{s,(\mathrm{I})}^{1}=u\left(\frac{\mathrm{q}_{\mathrm{T}, \mathrm{I})}^{1}-\mathrm{q}_{\mathrm{T}, \mathrm{I}}^{0}}{\mathrm{~T}_{(\mathrm{I})}^{1}-\mathrm{T}_{(\mathrm{I})}^{0}} \mathrm{v}+1\right)^{-1}=0.00963 \mathrm{~m} / \mathrm{s} \\
& u_{s,(\mathrm{I})}^{1}=u\left(\frac{\mathrm{q}_{1,(\mathrm{I})}^{1}-\mathrm{q}_{1,(\mathrm{I})}^{0}}{\mathrm{C}_{1,(\mathrm{I})}^{1}-\mathrm{C}_{1,(\mathrm{I})}^{0}} \mathrm{v}+1\right)^{-1}=0.00964 \mathrm{~m} / \mathrm{s}
\end{aligned}
$$

Se obtuvo un error de $0.1 \%$, por lo que se consideró razonable el hecho de adoptar dicha aproximación.

Por otro lado, el tramo $\Gamma_{(\mathrm{I})}^{2}$ se presenta en el lecho como una zona de composición variable, siendo que $u_{\omega,(\mathrm{I})}^{2}$ disminuye desde $\mathrm{P}_{(\mathrm{I})}^{1}$ hasta $\mathrm{P}_{(\mathrm{I})}^{\mathrm{F}}$, haciendo que la longitud del tramo aumente con $\mathrm{t}$.

Para analizar el Escalón II $(Z=2.14 \mathrm{~m}, \mathrm{t}=0 \mathrm{~s})$ recordemos que en la etapa de adsorción resultó factible considerar a la curva $\Sigma_{(\mathrm{ADS})}^{3}$ equivalente a la curva $\Gamma_{(\mathrm{ADS})}^{3}$. Al invertir la dirección de la alimentación con respecto a la de la etapa de adsorción los puntos $\mathrm{P}_{(\mathrm{II})}^{0}\left(\equiv \mathrm{P}_{(\mathrm{ADS})}^{\mathrm{F}}\right)$ y $\mathrm{P}_{(\mathrm{II})}^{\mathrm{F}}\left(\equiv \mathrm{P}_{(\mathrm{ADS})}^{2}\right)$ están efectivamente unidos por una curva $\Gamma^{3}$ y no por una onda de choque, por ser $u_{\omega, 0(\mathrm{II})}^{3}>u_{\omega, \mathrm{F}(\mathrm{II})}^{3}$ (ver Tabla 6). Para este caso en particular, resulta suficiente con integrar las Ecs. [41] en dirección k $=3$ para la 
obtención de la curva $\Gamma_{\text {(II) }}^{3}$ que une los puntos $\mathrm{P}_{\text {(II) }}^{0}$ y $\mathrm{P}_{\text {(II) }}^{\mathrm{F}}$, sin la necesidad de cambiar de dirección.

Por otro lado, por ser $u_{\omega, 0(\mathrm{II})}^{3} \mathrm{y} u_{\omega, \mathrm{F}(\mathrm{II})}^{3}$ valores muy próximos entre sí (Tabla 6), se consideró que todos los puntos de la curva $\Gamma_{\text {(II) }}^{3}$, incluyendo los extremos $\mathrm{P}_{(\text {II) }}^{0}$ y $\mathrm{P}_{\text {(II) }}^{\mathrm{F}}$, viajan a la misma velocidad con un valor aproximado de $u_{\omega, \text { (II) }}^{3}=2.18 \times 10^{-4} \mathrm{~m} / \mathrm{s}$.

En la Tabla 6 se muestran la composición, temperatura y velocidades de propagación correspondientes a $\mathrm{P}_{(\mathrm{II})}^{0} \mathrm{y} \mathrm{P}_{(\mathrm{II})}^{\mathrm{F}}$.

Tabla 6: Composición y temperatura en los puntos $\mathrm{P}_{(\mathrm{II})}^{0}$ y $\mathrm{P}_{(\mathrm{II})}^{\mathrm{F}}$

\begin{tabular}{lcc}
\hline \multicolumn{1}{c}{$\mathbf{P}_{(\mathbf{I})}^{\mathbf{j}}$} & $\mathbf{P}_{(\mathrm{II})}^{\mathbf{0}}$ & $\mathbf{P}_{(\mathrm{II})}^{\mathbf{F}}$ \\
\hline $\mathbf{C}_{\mathbf{1}}\left[\mathbf{m o l} / \mathbf{m}^{\mathbf{3}}\right]$ & $2.3510^{-2}$ & $2.3910^{-2}$ \\
$\mathbf{C}_{\mathbf{2}}\left[\mathbf{m o l} / \mathbf{m}^{\mathbf{3}}\right]$ & $9.6310^{-3}$ & 0 \\
$\mathbf{T}\left[{ }^{\mathbf{0}} \mathbf{C}\right]$ & 50 & 50.37 \\
$\boldsymbol{u}_{\omega, \mathbf{j}(\mathrm{II})}^{\mathbf{3}}$ & $2.3110^{-4}$ & $2.1810^{-4}$
\end{tabular}

En la Figura 21 se pueden observar los perfiles de concentración $\left(\mathrm{C}_{1}, \mathrm{C}_{2}\right)$ y temperatura $(\mathrm{T})$ a lo largo de la variable axial $(\mathrm{Z})$ a los 150 segundos de haber comenzado la etapa de desorción.

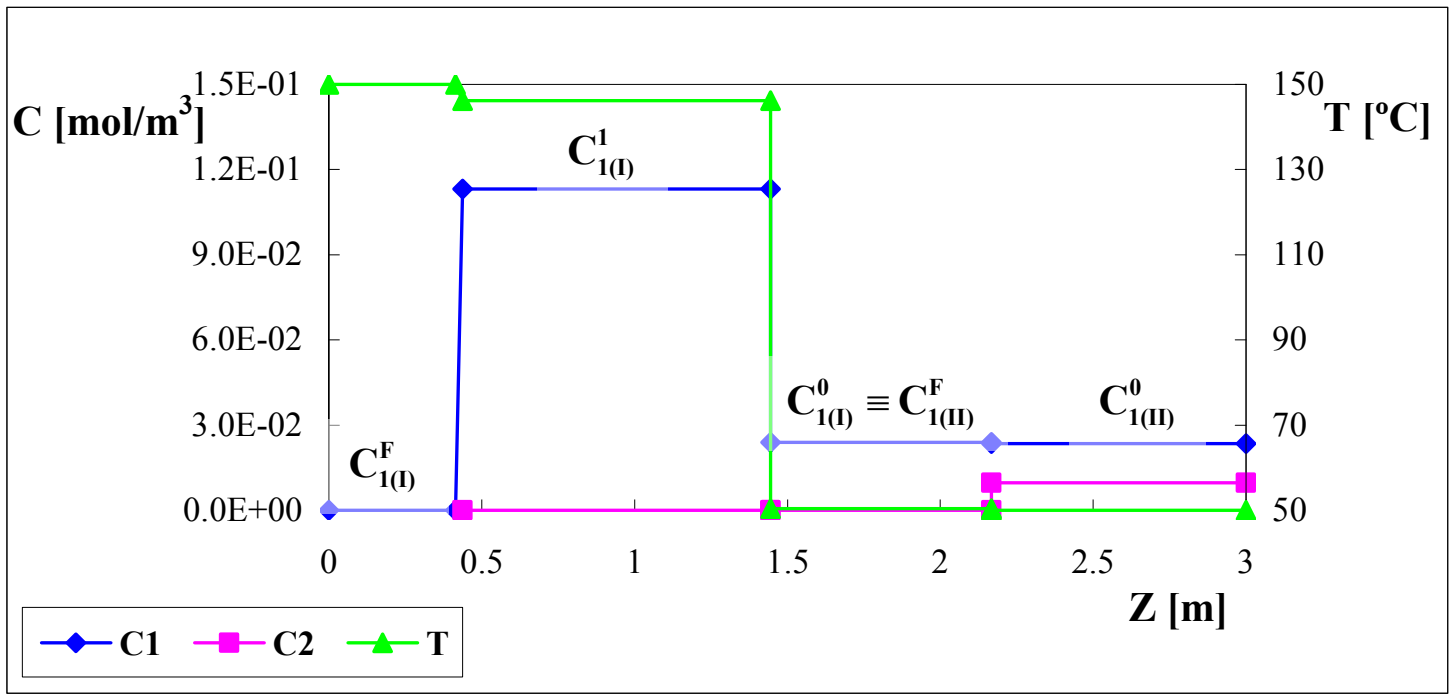

Fig. 21 - Variación de la concentración de cada uno de los componentes $\left(\mathrm{C}_{1}, \mathrm{C}_{2}\right)$ y de la temperatura $(\mathrm{T})$ a lo largo del lecho a $t_{\text {des }}=150 \mathrm{~s}$. Se indican los valores de $\mathrm{C}_{1}$ para los distintos puntos de quiebre. (1: acetato de etilo, 2: etanol) 
Siendo que $u_{s,(\mathrm{I})}^{1} \gg>u_{\omega,(I I)}^{3}$, el Escalón I alcanza al Escalón II previamente a que este abandone el lecho, creándose un nuevo escalón denominado Escalón III. Esto sucede a los a los 226.86 segundos para $Z=2,185 \mathrm{~m}$, e implica la desaparición de la zona de composición uniforme $\mathrm{P}_{\text {(I) }}^{0} \equiv \mathrm{P}_{\text {(II) }}^{\mathrm{F}}$. Podemos observar la situación mencionada en la Figura 22.

Para el Escalón III, la solución fue obtenida con la misma metodología de cálculo aplicada para el Escalón I, con la diferencia de que ahora deben ser considerados los dos componentes para el análisis, por lo que en este caso se tienen dos puntos de quiebre.

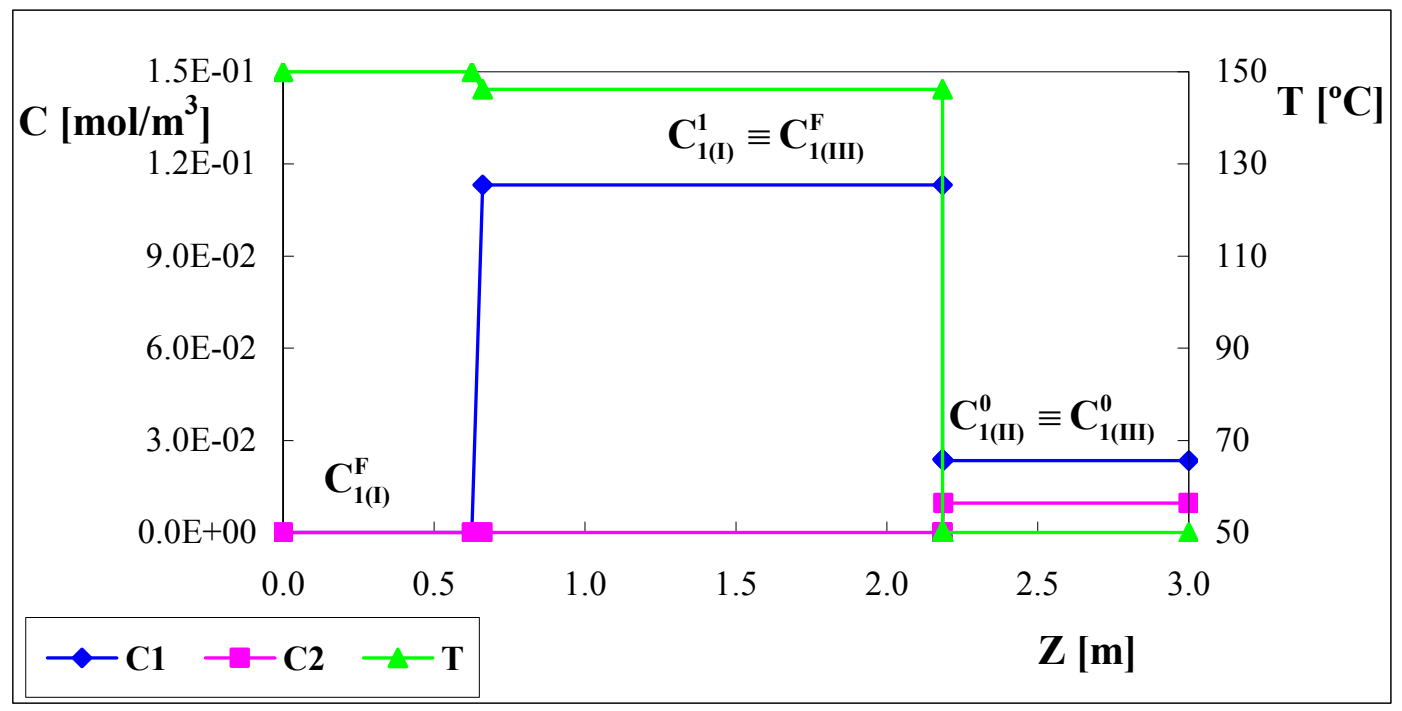

Fig. 22- Variación de la concentración de cada uno de los componentes $\left(\mathrm{C}_{1}, \mathrm{C}_{2}\right)$ y de la temperatura $(\mathrm{T})$ a lo largo del lecho a $\mathrm{t}_{\text {des }}=226.86 \mathrm{~s}$. Se indican los valores de $\mathrm{C}_{1}$ para los distintos puntos de quiebre. (1: acetato de etilo, 2: etanol)

De los resultados obtenidos (Tabla 7) se tiene que la solución corresponde a dos ondas de choque, $\Sigma_{\text {(III) }}^{1}\left(\mathrm{P}_{\text {(III) }}^{0}-\mathrm{P}_{\text {(III) }}^{1}\right)$ y $\Sigma_{\text {(III) }}^{2}\left(\mathrm{P}_{\text {(III) }}^{0}-\mathrm{P}_{(\text {III) }}^{1}\right)$, y un tramo continuo $\Gamma_{\text {(III) }}^{3}$ uniendo los puntos $\mathrm{P}_{(\mathrm{III})}^{2} \mathrm{y} \mathrm{P}_{\text {(III) }}^{\mathrm{F}}$. De la misma manera que se realizó anteriormente se consideró aceptable la aproximación de las curvas $\Sigma_{(\mathrm{III})}^{1}$ y $\Sigma_{(\mathrm{III})}^{2}$ con las obtenidas para la solución continua, $\Gamma_{\text {(III) }}^{1}$ y $\Gamma_{\text {(III) }}^{2}$ respectivamente, obteniéndose un error de aproximadamente $4 \%$ para el tramo $\Sigma_{\text {(III) }}^{1}$ y del $2.5 \%$ para el tramo $\Sigma_{(\text {III) }}^{2}$. 
Tabla 7: Composición y temperatura en cada uno de los puntos que definen la trayectoria $\mathrm{P}_{(\mathrm{III})}^{0}-\mathrm{P}_{(\mathrm{III})}^{1}-\mathrm{P}_{\text {(III) }}^{2}-\mathrm{P}_{\text {(III) }}^{\mathrm{F}}$

\begin{tabular}{|c|c|c|c|c|}
\hline $\mathbf{P}^{\mathbf{j}}$ & $\mathbf{P}^{0}$ & $\mathbf{P}^{1}$ & $\mathbf{P}^{2}$ & $\mathbf{P}^{\mathbf{F}}$ \\
\hline $\mathrm{C}_{1}\left[\mathrm{~mol} / \mathrm{m}^{3}\right]$ & $2.3510^{-2}$ & $8.7810^{-2}$ & $1.4910^{-1}$ & $1.1310^{-1}$ \\
\hline $\mathrm{C}_{2}\left[\mathrm{~mol} / \mathrm{m}^{3}\right]$ & $9.6310^{-3}$ & $2.2010^{-1}$ & 0 & 0 \\
\hline $\mathrm{T}\left[{ }^{\circ} \mathrm{C}\right]$ & 50 & 128.7 & 145 & 146.2 \\
\hline$u_{\omega, j}^{\mathrm{j}}[\mathbf{m} / \mathbf{s}]$ & & 0.01377 & 0.00531 & 0.00292 \\
\hline$u_{\omega, j}^{j+1}[m / s]$ & 0.00976 & 0.00277 & 0.00297 & \\
\hline $\boldsymbol{u}_{\boldsymbol{s}(\mathrm{III})}^{\mathbf{1}}=0$ & $0.0112 \mathrm{~m} / \mathrm{s}$ & & (III) $=0.00$ & \\
\hline
\end{tabular}

La situación del lecho a los 290 segundos sería la siguiente:

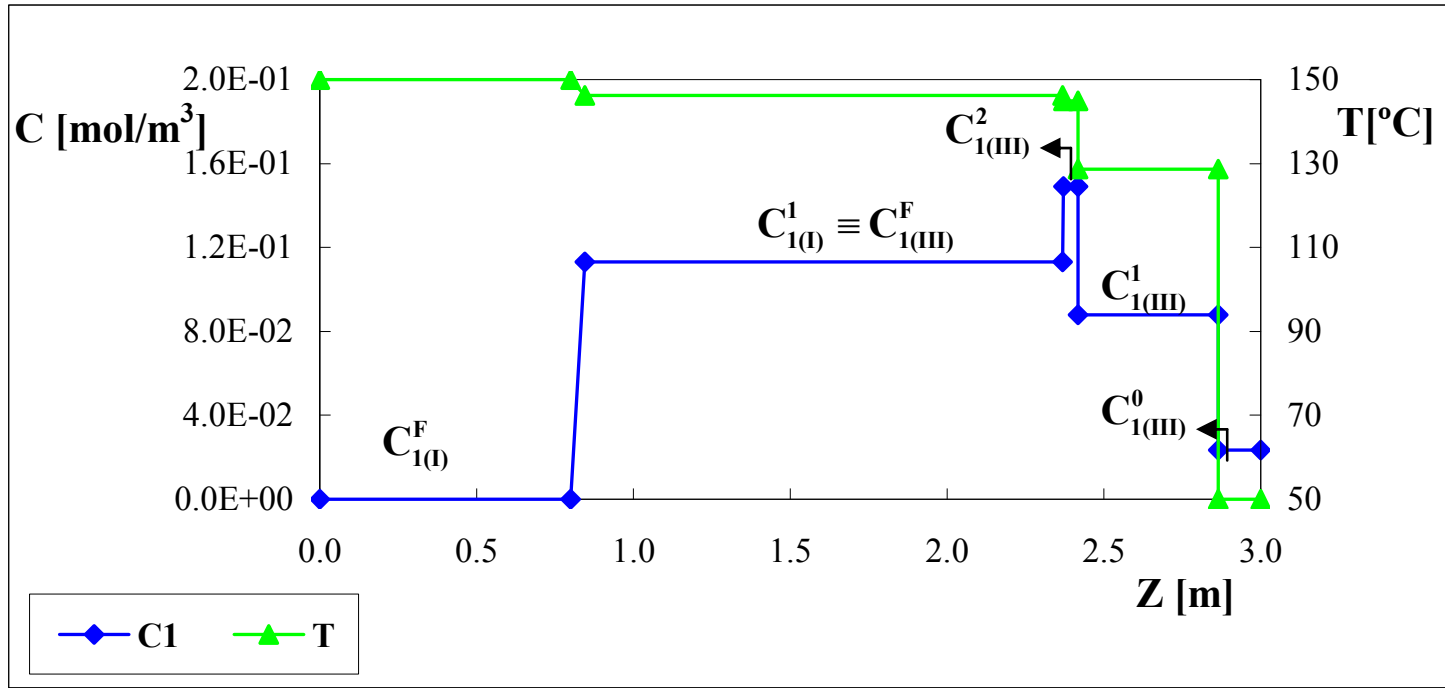

Fig. 23- Variación de la concentración del acetato de etilo $\left(\mathrm{C}_{1}\right)$ y de la temperatura $(\mathrm{T})$ a lo largo del lecho a $t_{\text {des }}=290 \mathrm{~s}$. Se indican los valores de $C_{1}$ para los distintos puntos de quiebre. (1: acetato de etilo, 2: etanol)

En la Figura 23 se pueden distinguir claramente las zonas de composición del acetato de etilo y temperatura uniformes creadas a partir de aplicar el problema de Riemann al Escalón III. Además se observa que el tiempo correspondiente a la etapa de desorción $\left(t_{\text {des }}\right)$ depende únicamente de la velocidad con la que se propaga el punto $\mathrm{P}_{(\mathrm{DES})}^{\mathrm{F}}\left(\equiv \mathrm{P}_{1(\mathrm{I})}^{\mathrm{F}}\right)$, i.e. $u_{\omega, \mathrm{F}(\mathrm{I})}^{2}$. Esto implica que más allá de lo que suceda con los Escalones II y III no se va a modificar el periodo correspondiente a la etapa de desorción. Como se dijo anteriormente, este periodo debe coincidir con el de la etapa de adsorción ( $\left.\mathrm{t}_{\mathrm{ads}}=1087.47 \mathrm{~s}\right)$. Para que esto suceda la velocidad de propagación $u_{\omega, \mathrm{F}(\mathrm{I})}^{2}$ debe coincidir con la velocidad de propagación que determina el tiempo de la 
etapa de adsorción, $u_{s(\mathrm{ADS})}^{2}(\mathrm{t}=u \mathrm{Z})$. El hecho de que se pueda garantizar dicha igualdad una vez definidas la composición y temperatura en la alimentación $\left(\sigma_{(\mathrm{I})}^{2}\right)$, va a depender de la velocidad intersticial de la corriente empleada para la regeneración, la cual se obtiene a partir de la siguiente relación:

$$
u=u_{s(\mathrm{ADS})}^{2}\left(1+v \sigma_{(\mathrm{I})}^{2}\right)
$$

Como fuera anticipado al comienzo de esta subsección resulta $u=2 \mathrm{~m} / \mathrm{s}$.

\subsubsection{Conclusiones obtenidas a partir de modificar las condiciones operativas del} sistema

A continuación se exponen las conclusiones alcanzadas al realizar modificaciones operativas $\left(T_{(D E S)}^{F}\right.$, concentración de VOCs) con respecto al caso original planteado. Dado que los procedimientos de cálculo son análogos al realizado previamente, no se reiterarán en esta instancia, exponiéndose solo los resultados más significativos.

- Variación de la temperatura de alimentación $\left(T_{(D E S)}^{F}\right)$ en la etapa de desorción

El hecho de modificar el valor de la temperatura a la que se encuentra la corriente de aire puro empleada para la desorción, implica que la temperatura a la que se encuentra el lecho al iniciar la etapa de adsorción también se modifique.

Supongamos que ahora la temperatura $\mathrm{T}_{(\mathrm{DES})}^{\mathrm{F}}$ equivale a $100^{\circ} \mathrm{C}$, por lo que $\mathrm{T}_{(\mathrm{ADS})}^{0}$ tomará el mismo valor. Del análisis de la etapa de adsorción surge que debido a la existencia de una discontinuidad de contacto (onda térmica pura) lo único que varía respecto al caso original es el $\Delta \mathrm{T}$ correspondiente al tramo $\Gamma^{1}$. Al no verse afectado el valor de la velocidad de la onda de choque $u_{s(\mathrm{ADS})}^{2}$ que determina el periodo correspondiente a la etapa de adsorción, el valor de dicho periodo permanece igual al obtenido para el caso originalmente planteado $\left(\mathrm{t}_{\mathrm{ads}}=1087.47 \mathrm{~s}\right)$.

Sin embargo en lo que respecta a la etapa de desorción, es necesario variar la velocidad de la corriente empleada para la regeneración del lecho.

Al disminuir la temperatura $\left(\mathrm{T}_{(\mathrm{DES})}^{\mathrm{F}}=100^{\circ} \mathrm{C}\right)$, la corriente tiene menos tendencia a recibir la impureza desorbida $\left(u_{\omega, \mathrm{F}(\mathrm{I})}^{2}\right.$ disminuye), por lo tanto de manera tal de poder mantener la relación: $t_{a d s}=t_{\text {des }}$, es necesario compensar con un aumento en el caudal. 
Para la situación planteada de disminuir la temperatura hasta los $100^{\circ} \mathrm{C}$, la velocidad intersticial de la corriente utilizada para la regeneración debe aumentar a $3.5 \mathrm{~m} / \mathrm{s}$, lo que claramente verifica que es mayor al valor requerido para una $\mathrm{T}_{(\mathrm{DES})}^{\mathrm{F}}=150{ }^{\circ} \mathrm{C}$ $(2 \mathrm{~m} / \mathrm{s})$.

- Aumento de la concentración de VOCs (acetato de etilo y etanol) de la corriente a tratar

Corrientes más concentradas provocan una saturación más rápida del lecho, por lo que la velocidad de propagación de la onda de choque que determina el tiempo correspondiente a la etapa de adsorción $\left(u_{s(\mathrm{ADS})}^{2}\right)$ aumenta.

Para el caso particular de aumentar un orden la concentración de los VOCs (acetato de etilo y etanol) presentes en la corriente a tratar, se produce una disminución del tiempo de adsorción de un 35\% $\left(\mathrm{t}_{\mathrm{tads}}=708.57 \mathrm{~s}\right)$ respecto al valor obtenido para el caso original $\left(t_{\mathrm{ads}}=1087.47 \mathrm{~s}\right)$. Si se pretende proporcionar un tiempo de desorción equivalente al de la etapa de adsorción se debe aumentar la velocidad intersticial de la corriente utilizada para la regeneración, llevándola, para el caso particular planteado, a $3.07 \mathrm{~m} / \mathrm{s}$, o sea un $50 \%$ mayor que el valor del caso original $(2 \mathrm{~m} / \mathrm{s})$.

Además, un aumento en la concentración de VOCs produce un aumento del calor liberado en la etapa de adsorción implicando un aumento de temperatura en los puntos de quiebre generados en dicha etapa. Este aumento resulta más significativo en el primer punto de quiebre donde $\mathrm{T}^{1}$ toma un valor de $58.61^{\circ} \mathrm{C}$, aproximadamente $8{ }^{\circ} \mathrm{C}$ más que lo que se obtuvo para el caso original $\left(50.83{ }^{\circ} \mathrm{C}\right)$.

\subsection{Conclusiones}

Se presentó el Método de las Características para un caso isotérmico con un único componente, como un ejemplo introductorio, y para el caso más general de un sistema adiabático de multicomponentes. Para esta última situación el desarrollo se encuentra restringido al caso de un lecho inicialmente en equilibrio con composición y temperatura uniformes $\underline{\mathrm{C}}^{0}$ al cual se le aplica una corriente de alimentación con composición y temperatura constantes $\underline{\mathrm{C}}^{\mathrm{F}}$ (problema de Riemann). Finalmente se aplicó el Método de las Características a nuestro caso particular de estudio donde se deben 
adsorber acetato de etilo y etanol presentes en una corriente de aire (Capítulo 2). Para la aplicación del Método se consideró una isoterma tipo Langmuir generalizada (Ec. [38a]) para representar la relación de equilibrio entre los moles adsorbidos y los moles en la corriente (Gales y col. 2003).

El empleo del Método de las Características requiere de un desarrollo de relativa complejidad, que involucra un trabajo de análisis en su presentación (para definir el comportamiento del sistema). Sin embargo, en la práctica el esfuerzo de resolución resultó ser muy modesto.

En la etapa de desorción el lecho no se encuentra inicialmente con composición y temperatura uniformes, por lo que no se verifica el problema de Riemann. Para sortear este problema surge como aproximación de la solución, dividir al lecho en dos zonas, cada una con composición y temperatura uniformes, a las cuales se les aplica una alimentación de composición y temperatura diferentes. En estas condiciones resulta factible obtener la solución para cada una de las zonas de forma independiente, puesto que ambas verifican el problema de Riemann.

Una variable crucial en la definición de cómo llevar a cabo el proceso es el tiempo correspondiente a la etapa de desorción. El mismo está determinado por el tiempo en que transcurre la etapa de adsorción, dado que se pretende que $t_{\text {des }}=t_{a d s}$, y está sujeto a las condiciones de alimentación de la corriente empleada para la regeneración del lecho.

Para las condiciones operativas establecidas en el caso particular de estudio, resultó que la velocidad intersticial de la corriente de regeneración es de $2 \mathrm{~m} / \mathrm{s}$, lo que equivale a menos de un tercio del valor de la velocidad a la cual circula la corriente durante la adsorción $(7.08 \mathrm{~m} / \mathrm{s})$.

Al variar alguna de las condiciones operativas en la corriente de alimentación, ya sea en el proceso de adsorción como de desorción, se obtuvieron las siguientes conclusiones:

- Una modificación en la temperatura de la corriente empleada para la regeneración del lecho no produce alteraciones en el periodo de adsorción, sin embargo en lo que respecta a la etapa de desorción, es necesario variar la velocidad de la corriente de desorción si se pretende mantener la relación: $t_{a d s}=t_{\text {des }}$.

- Al aumentar la concentración de VOCs en la corriente a tratar disminuye el periodo correspondiente a la etapa de adsorción. Esta disminución implica un aumento de la velocidad de la corriente empleada para la desorción, de manera tal de mantener la relación: $t_{\text {ads }}=t_{\text {des }}$. 
El Método de las Características resulta ser una muy buena alternativa de cálculo si se pretende analizar el comportamiento de un sistema de manera práctica. Posteriormente se verá que la solución obtenida a partir de aplicar el Método de las Características a nuestro caso particular de estudio se aproxima razonablemente a la solución obtenida considerando las resistencias a la transferencia de calor y materia dentro y fuera del material adsorbente. 


\section{1/ $/_{\text {seratice }}$}

\section{Componentes de la matriz $\mathcal{F}\left(\mathrm{q}_{\mathrm{i}, \mathrm{j}}\right.$ : Derivadas primeras de $\left.\mathrm{q}_{\mathrm{i}}(\underline{\mathrm{C}})\right)$}

A continuación se expresan las componentes de la matriz $\mathcal{F}$, habiendo considerado una isoterma de tipo Langmuir para 2 componentes (acetato de etilo (1) y etanol (2)).

$$
\mathrm{q}_{\mathrm{i}}=\frac{\mathrm{Q}_{\mathrm{i}} \mathrm{K}_{\mathrm{i}} \mathrm{C}_{\mathrm{i}}}{1+\sum_{\mathrm{j}=1}^{2} \mathrm{~K}_{\mathrm{j}} \mathrm{C}_{\mathrm{j}}} \quad \mathrm{i}=1,2 ; \quad \mathrm{q}_{\mathrm{T}}=\gamma \mathrm{T}-\sum_{\mathrm{i}=1}^{2} \mathrm{~J}_{\mathrm{i}} \mathrm{q}_{\mathrm{i}}
$$

Recordemos que:

$$
\begin{aligned}
& \mathrm{q}_{\mathrm{i}, \mathrm{j}}=\frac{\partial \mathrm{q}_{\mathrm{i}}}{\partial \mathrm{C}_{\mathrm{j}}} \text { y } \mathrm{q}_{\mathrm{i}, \mathrm{T}}=\frac{\partial \mathrm{q}_{\mathrm{i}}}{\partial \mathrm{T}} \quad \text { para } \mathrm{i}=1,2, \mathrm{~T}, \mathrm{j}=1,2 \\
& \mathrm{q}_{1,1}=Q_{1} \mathrm{~K}_{1}\left(1+\mathrm{K}_{2} \mathrm{C}_{2}\right) / \mathrm{D}^{2} \\
& \mathrm{q}_{2,2}=Q_{2} \mathrm{~K}_{2}\left(1+\mathrm{K}_{1} \mathrm{C}_{1}\right) / \mathrm{D}^{2} \\
& \mathrm{q}_{1,2}=-Q_{1} \mathrm{~K}_{1} \mathrm{~K}_{2} \mathrm{C}_{1} / \mathrm{D}^{2} \\
& \mathrm{q}_{2,1}=-Q_{2} \mathrm{~K}_{2} \mathrm{~K}_{1} \mathrm{C}_{2} / \mathrm{D}^{2} \\
& \mathrm{q}_{1, \mathrm{~T}}=Q_{1} \mathrm{C}_{1}\left(\mathrm{~b}_{1} \mathrm{D}-\mathrm{K}_{1} \mathrm{dD}\right) / \mathrm{D}^{2} \\
& \mathrm{q}_{2, \mathrm{~T}}=Q_{2} \mathrm{C}_{2}\left(\mathrm{~b}_{2} \mathrm{D}-\mathrm{K}_{2} \mathrm{dD}\right) / \mathrm{D}^{2} \\
& \mathrm{q}_{\mathrm{T}, \mathrm{i}}=-\mathrm{J}_{1} \mathrm{q}_{1, \mathrm{i}}-\mathrm{J}_{2} \mathrm{q}_{2, \mathrm{i}}, \mathrm{i}=1,2 \\
& \mathrm{q}_{\mathrm{T}, \mathrm{T}}=\gamma-\mathrm{J}_{1} \mathrm{q}_{1, \mathrm{~T}}-\mathrm{J}_{2} \mathrm{q}_{2, \mathrm{~T}}
\end{aligned}
$$

donde:

$$
\begin{aligned}
& \mathrm{K}_{\mathrm{i}}=\mathrm{bo}_{\mathrm{i}} \mathrm{T}^{3 / 2} \exp \left(-\Delta \mathrm{H}_{\mathrm{i}} /(\mathrm{RT})\right) \\
& \mathrm{D}=1+\sum_{\mathrm{i}=1}^{2} \mathrm{~K}_{\mathrm{i}} \mathrm{C}_{\mathrm{i}} \\
& \mathrm{b}_{\mathrm{i}}=\frac{\partial \mathrm{K}_{\mathrm{i}}}{\partial \mathrm{T}}=-\mathrm{bo}_{\mathrm{i}} \sqrt{\mathrm{T}} \exp \left(-\Delta \mathrm{H}_{\mathrm{i}} / \mathrm{RT}\right)\left(-\Delta \mathrm{H}_{\mathrm{i}} / \mathrm{RT}-3 / 2\right) \mathrm{i}=1,2 \\
& (\mathrm{dD})=\frac{\partial \mathrm{D}}{\partial \mathrm{T}}=\mathrm{b}_{1} \mathrm{C}_{1}+\mathrm{b}_{2} \mathrm{C}_{2}
\end{aligned}
$$




\section{2/ $/_{\text {ixtate }}$}

\section{Derivadas primeras de las componentes de la matriz $\mathcal{F}$}

A continuación se expresan las derivadas de las componentes de la matriz $\mathcal{F}$ respecto de $\mathrm{C}_{1}, \mathrm{C}_{2} \mathrm{y} \mathrm{T}$, para lo cual se define:

$$
\begin{aligned}
& q_{i, j, k}=\frac{\partial q_{i, j}}{\partial C_{k}} \quad y \quad q_{i, j, T}=\frac{\partial q_{i, j}}{\partial T} \text {, para } i=1,2, T ; j=1,2, T ; k=1,2 \\
& \mathrm{q}_{\mathrm{i}, \mathrm{i}, \mathrm{i}}=-2 Q_{\mathrm{i}} \mathrm{K}_{\mathrm{i}}^{2}\left(1+\mathrm{K}_{\mathrm{j}} \mathrm{C}_{\mathrm{j}}\right) / \mathrm{D}^{3} \quad \mathrm{i}, \mathrm{j}=1,2 ; \mathrm{i} \neq \mathrm{j} \\
& \mathrm{q}_{\mathrm{i}, \mathrm{i}, \mathrm{j}}=Q_{\mathrm{i}} \mathrm{K}_{\mathrm{i}} \mathrm{K}_{\mathrm{j}}\left[\mathrm{D}-2\left(1+\mathrm{K}_{\mathrm{j}} \mathrm{C}_{\mathrm{j}}\right)\right] / \mathrm{D}^{3} \quad \mathrm{i}, \mathrm{j}=1,2 ; \mathrm{i} \neq \mathrm{j} \\
& \mathrm{q}_{\mathrm{i}, \mathrm{j}, \mathrm{i}}=Q_{\mathrm{i}} \mathrm{K}_{\mathrm{i}} \mathrm{K}_{\mathrm{j}}\left(-\mathrm{D}+2 \mathrm{~K}_{\mathrm{i}} \mathrm{C}_{\mathrm{i}}\right) / \mathrm{D}^{3} \quad \mathrm{i}, \mathrm{j}=1,2 ; \mathrm{i} \neq \mathrm{j} \\
& \mathrm{q}_{\mathrm{i}, \mathrm{j}, \mathrm{j}}=2 Q_{\mathrm{i}} \mathrm{K}_{\mathrm{j}}{ }^{2} \mathrm{~K}_{\mathrm{i}} \mathrm{C}_{\mathrm{i}} / \mathrm{D}^{3} \quad \mathrm{i}, \mathrm{j}=1,2 ; \mathrm{i} \neq \mathrm{j} \\
& \mathrm{q}_{\mathrm{i}, \mathrm{i}, \mathrm{T}}=\left\{\left[Q_{\mathrm{i}} \mathrm{b}_{\mathrm{i}}\left(1+\mathrm{K}_{\mathrm{j}} \mathrm{C}_{\mathrm{j}}\right)+Q_{\mathrm{i}} \mathrm{K}_{\mathrm{i}} \mathrm{b}_{\mathrm{j}} \mathrm{C}_{\mathrm{j}}\right] \mathrm{D}-2 Q_{\mathrm{i}} \mathrm{K}_{\mathrm{i}}\left(1+\mathrm{K}_{\mathrm{j}} \mathrm{C}_{\mathrm{j}}\right) \mathrm{dD}\right\} / \mathrm{D}^{3} \quad \mathrm{i}, \mathrm{j}=1,2 ; \mathrm{i} \neq \mathrm{j} \\
& \mathrm{q}_{\mathrm{i}, \mathrm{j}, \mathrm{T}}=Q_{\mathrm{i}} \mathrm{C}_{\mathrm{i}}\left[-\mathrm{D}\left(\mathrm{b}_{\mathrm{i}} \mathrm{K}_{\mathrm{j}}+\mathrm{b}_{\mathrm{j}} \mathrm{K}_{\mathrm{i}}\right)+2 \mathrm{~K}_{\mathrm{i}} \mathrm{K}_{\mathrm{j}} \mathrm{dD}\right] / \mathrm{D}^{3} \quad \mathrm{i}, \mathrm{j}=1,2 ; \mathrm{i} \neq \mathrm{j} \\
& \mathrm{q}_{\mathrm{i}, \mathrm{T}, \mathrm{i}}=Q_{\mathrm{i}}\left(\mathrm{b}_{\mathrm{i}} \mathrm{D}-\mathrm{K}_{\mathrm{i}} \mathrm{dD}\right)\left(\mathrm{D}-2 \mathrm{~K}_{\mathrm{i}} \mathrm{C}_{\mathrm{i}}\right) / \mathrm{D}^{3} \quad \mathrm{i}=1,2 ; \\
& \mathrm{q}_{\mathrm{i}, \mathrm{T}, \mathrm{j}}=Q_{\mathrm{i}} \mathrm{C}_{\mathrm{i}}\left[2 \mathrm{~K}_{\mathrm{i}} \mathrm{K}_{\mathrm{j}} \mathrm{dD}-\mathrm{D}\left(\mathrm{b}_{\mathrm{i}} \mathrm{K}_{\mathrm{j}}+\mathrm{b}_{\mathrm{j}} \mathrm{K}_{\mathrm{i}}\right)\right] / \mathrm{D}^{3} \quad \mathrm{i}, \mathrm{j}=1,2 ; \mathrm{i} \neq \mathrm{j} \\
& \mathrm{q}_{\mathrm{i}, \mathrm{T}, \mathrm{T}}=Q_{\mathrm{i}} \mathrm{C}_{\mathrm{i}}\left\{\mathrm{D}\left[\mathrm{db}_{\mathrm{i}} \mathrm{D}+\mathrm{K}_{\mathrm{i}}\left(\mathrm{db}_{\mathrm{i}} \mathrm{C}_{\mathrm{i}}+\mathrm{db}_{\mathrm{j}} \mathrm{C}_{\mathrm{j}}\right)\right]-2 \mathrm{dD}\left(\mathrm{b}_{\mathrm{i}} \mathrm{D}-\mathrm{K}_{\mathrm{i}} \mathrm{dD}\right)\right\} / \mathrm{D}^{3} \quad \mathrm{i}, \mathrm{j}=1,2 ; \mathrm{i} \neq \mathrm{j} \\
& \mathrm{q}_{\mathrm{T}, \mathrm{i}, \mathrm{j}}=-\mathrm{J}_{1} \mathrm{q}_{1, \mathrm{ij}}-\mathrm{J}_{2} \mathrm{q}_{2, \mathrm{ij}} \quad \mathrm{i}, \mathrm{j}=1,2, \mathrm{~T}
\end{aligned}
$$

donde:

$$
\begin{aligned}
& \mathrm{K}_{\mathrm{i}}=\mathrm{bo}_{\mathrm{i}} \mathrm{T}^{3 / 2} \exp \left[-\Delta \mathrm{H}_{\mathrm{i}} /(\mathrm{RT})\right] \\
& \mathrm{D}=1+\sum_{\mathrm{i}=1}^{2} \mathrm{~K}_{\mathrm{i}} \mathrm{C}_{\mathrm{i}} \\
& \mathrm{b}_{\mathrm{i}}=\frac{\partial \mathrm{K}_{\mathrm{i}}}{\partial \mathrm{T}}=-\mathrm{bo}_{\mathrm{i}} \sqrt{\mathrm{T}} \exp \left[-\Delta \mathrm{H}_{\mathrm{i}} /(\mathrm{RT})\right]\left[-\Delta \mathrm{H}_{\mathrm{i}} /(\mathrm{RT})-3 / 2\right] \mathrm{i}=1,2
\end{aligned}
$$




$$
\begin{aligned}
& \left(\mathrm{db}_{\mathrm{i}}\right)=\frac{\partial \mathrm{b}_{\mathrm{i}}}{\partial \mathrm{T}}=\mathrm{bo}_{\mathrm{i}} \mathrm{T}^{(-1 / 2)} \exp \left[-\Delta \mathrm{H}_{\mathrm{i}} /(\mathrm{RT})\right]\left\{3 / 4-\left[-\Delta \mathrm{H}_{\mathrm{i}} /(\mathrm{RT})\right]+\left[-\Delta \mathrm{H}_{\mathrm{i}} /(\mathrm{RT})\right]^{2}\right\} \quad \mathrm{i}, \mathrm{j}=1,2 \\
& (\mathrm{dD})=\frac{\partial \mathrm{D}}{\partial \mathrm{T}}=\mathrm{b}_{1} \mathrm{C}_{1}+\mathrm{b}_{2} \mathrm{C}_{2}
\end{aligned}
$$




\section{3/ $/_{\text {kenatide }}$}

Determinación de las derivadas de los autovalores $\sigma^{\mathrm{k}}$ de la matriz $\mathcal{F}$ respecto de $\mathrm{C}_{1}, \mathrm{C}_{2} \mathrm{y} \mathrm{T}$

A continuación se evalúan las derivadas parciales de los autovalores de la matriz $\mathcal{F}$, $\sigma^{\mathrm{k}}$, respecto de $\mathrm{C}_{1}, \mathrm{C}_{2} \mathrm{y}$ T. (i.e., $\frac{\partial \sigma^{\mathrm{k}}}{\partial \mathrm{T}}, \frac{\partial \sigma^{\mathrm{k}}}{\partial \mathrm{C}_{1}} \mathbf{y} \frac{\partial \sigma^{\mathrm{k}}}{\partial \mathrm{C}_{2}}$ )

Siendo que $\sigma^{\mathrm{k}}$ son los autovalores de la matriz $\mathcal{F}$, se puede expresar:

$$
\mathcal{F} \underline{x}^{k}=\sigma^{k} \underline{x}^{k}
$$

donde $\underline{x}^{\mathrm{k}}$ es el autovector derecho de la matriz $\mathcal{F}$.

Además se tiene:

$$
\left(\underline{y}^{\mathrm{k}}\right)^{\mathrm{T}} \mathcal{F}=\sigma^{\mathrm{k}}\left(\underline{y}^{\mathrm{k}}\right)^{\mathrm{T}}
$$

donde $\underline{y}^{\mathrm{k}}$ es el autovector izquierdo de la matriz $\mathcal{F}$.

Definimos a " $x$ " como una matriz cuyas columnas son los vectores $\underline{x}^{\mathrm{k}} \mathrm{y}$ a " $y$ " como una matriz cuyas columnas son los vectores $\underline{y}^{\mathrm{k}}$.

La relación que existe entre las matrices $x$ e $y$ es:

$$
(y)^{\mathrm{T}}=(x)^{-1}
$$

Derivamos la expresión [A 4.4.1] respecto a algún parámetro de $\mathcal{F}$, por ejemplo la temperatura $\mathrm{T}$.

$$
\frac{\partial \mathcal{F}}{\partial \mathrm{T}} x^{\mathrm{k}}+\mathcal{F} \frac{\partial x^{\mathrm{k}}}{\partial \mathrm{T}}=\frac{\partial \sigma^{\mathrm{k}}}{\partial \mathrm{T}} x^{\mathrm{k}}+\sigma^{\mathrm{k}} \frac{\partial x^{\mathrm{k}}}{\partial \mathrm{T}}
$$

Reordenando se tiene:

$$
\frac{\partial \sigma^{\mathrm{k}}}{\partial \mathrm{T}} x^{\mathrm{k}}=\frac{\partial \mathcal{F}}{\partial \mathrm{T}} x^{\mathrm{k}}+\left(\mathcal{F}-\sigma^{\mathrm{k}}\right) \frac{\partial x^{\mathrm{k}}}{\partial \mathrm{T}}
$$

Premultiplico [A 4.4.4] por $y^{\mathrm{k}}$ : 


$$
\frac{\partial \sigma^{\mathrm{k}}}{\partial \mathrm{T}} y^{\mathrm{k}} x^{\mathrm{k}}=y^{\mathrm{k}} \frac{\partial \mathcal{F}}{\partial \mathrm{T}} x^{\mathrm{k}}+y^{\mathrm{k}}\left(\mathcal{F}-\sigma^{\mathrm{k}}\right) \frac{\partial x^{\mathrm{k}}}{\partial \mathrm{T}}
$$

Reexpresando la Ec. [A 4.4.2] en la forma:

$$
y^{\mathrm{k}}\left(\mathcal{F}-\sigma^{\mathrm{k}}\right)=0
$$

Surge que:

$$
\frac{\partial \sigma^{\mathrm{k}}}{\partial \mathrm{T}}=\frac{y^{\mathrm{k}}(\partial \mathcal{F} / \partial \mathrm{T}) x^{\mathrm{k}}}{y^{\mathrm{k}} x^{\mathrm{k}}}
$$

De forma análoga se tiene:

$$
\begin{aligned}
& \frac{\partial \sigma^{\mathrm{k}}}{\partial \mathrm{C}_{1}}=\frac{y^{\mathrm{k}}\left(\partial \mathcal{F} / \partial \mathrm{C}_{1}\right) x^{\mathrm{k}}}{y^{\mathrm{k}} x^{\mathrm{k}}} \\
& \frac{\partial \sigma^{\mathrm{k}}}{\partial \mathrm{C}_{2}}=\frac{y^{\mathrm{k}}\left(\partial \mathcal{F} / \partial \mathrm{C}_{2}\right) x^{\mathrm{k}}}{y^{\mathrm{k}} x^{\mathrm{k}}}
\end{aligned}
$$

Para la determinación de $\frac{\partial \mathcal{F}}{\partial \mathrm{C}_{1}}, \frac{\partial \mathcal{F}}{\partial \mathrm{C}_{1}} y \frac{\partial \mathcal{F}}{\partial \mathrm{T}}$ se puede consultar el Apéndice 4.2. 




\section{5/ SIMULACION DEL SISTEMA DE ADSORCION/DESORCION CONSIDERANDO LAS RESISTENCIAS A LA TRANSFERENCIA DE CALOR Y MATERIA}

Balances de materia dentro y fuera del sólido adsorbente para un único componente en un sistema isotérmico

Método de Colocaciones Ortogonales

Resolución del balance en la corriente de fluido

Adsorción/desorción simultánea de acetato de etilo y etanol considerando las resistencias a la transferencia de calor y materia

Efecto de las resistencias externas e internas a la transferencia de calor y materia Influencia mutua de los componentes en el proceso de adsorción/desorción

Conclusiones 



\section{$05 /$

\section{las resistencias a la transferencia de calor y materia}

En el Capítulo 4 se describió la metodología de simulación para un sistema de adsorción/desorción sin considerar las resistencias a la transferencia de calor y materia, dentro y fuera del material adsorbente. Para la resolución del sistema de ecuaciones correspondiente a dicha situación se empleó el Método de las Características.

Al incluirse las resistencias a la transferencia de calor y materia, dentro y fuera del sólido, el Método de las Características ya no es válido, puesto que debe incorporarse al cálculo la resolución de los balances de calor y materia dentro del adsorbente.

En este capítulo se propone una metodología de cálculo que nos permite representar el sistema de adsorción/desorción al considerar las resistencias. La formulación se presentará en base al balance de materia para un único componente en un sistema isotérmico, y luego se lo extenderá al caso en estudio de dos componentes (Capítulo 2) en un sistema adiabático, tanto para un lecho relleno como para un monolito, analizándose el efecto de las resistencias sobre cada tipo de lecho.

Finalmente se realiza una comparación entre los resultados obtenidos a partir de considerar, o no, las resistencias externas e internas a la transferencia de calor y materia, con el objetivo de analizar la significación de las mismas.

En una última sección se estudia la influencia mutua entre los componentes (acetato de etilo y etanol) en el proceso de adsorción.

\subsection{Balances de materia dentro y fuera del sólido adsorbente para un único componente en un sistema isotérmico}

Si bien la metodología de simulación que se desarrollará a continuación es apta tanto para un sistema de lechos rellenos como estructurados, a fin de ser específicos nos referiremos a un sistema de lechos rellenos.

Para la corriente de fluido (gas) se consideró el siguiente balance de conservación, suponiendo uniformidad de las variables en la sección transversal del lecho:

$$
\frac{\partial \mathrm{C}_{\mathrm{g}}}{\partial \mathrm{t}}=-u \frac{\partial \mathrm{C}_{\mathrm{g}}}{\partial \mathrm{Z}}-v \mathrm{a}_{\mathrm{v}} \mathrm{k}_{\mathrm{m}}\left(\mathrm{C}_{\mathrm{g}}-\mathrm{C}_{\mathrm{s}}\right)
$$


donde $\mathrm{C}_{\mathrm{s}}$ y $\mathrm{C}_{\mathrm{g}}$ son la concentración en la superficie del material adsorbente y en la fase fluida respectivamente, $Z$ es la variable espacial medida desde la entrada del adsorbedor $(Z=0)$ hasta la salida $(Z=L)$, t es el tiempo, $u$ la velocidad intersticial, $v$ la relación volumétrica entre las fases sólida y fluido en función de la porosidad: $\left(1-\varepsilon_{\mathrm{L}}\right) / \varepsilon_{\mathrm{L}}$, $\mathrm{a}_{\mathrm{v}}$ el área específica (área externa de pastilla de adsorbente por unidad de volumen de la misma) y $\mathrm{k}_{\mathrm{m}}$ el coeficiente de transferencia de materia entre el seno del fluido y la superficie externa del adsorbente.

El balance de materia correspondiente a la adsorción/desorción de una sustancia sobre una pastilla porosa de adsorbente, cuya geometría es esférica, resulta:

$$
\frac{D_{e}}{z^{2}} \frac{\partial}{\partial z}\left(z^{2} \frac{\partial C}{\partial z}\right)-r_{p}^{2} \frac{\partial q(C)}{\partial t}=0
$$

donde $\mathrm{C}$ es la concentración de adsorbato dentro de los poros del adsorbente, $\mathrm{q}(\mathrm{C})$ es la concentración de la especie adsorbida por unidad de volumen de adsorbente que se encuentra en equilibrio local con la concentración de la fase gaseosa en el interior del poro, $\mathrm{D}_{\mathrm{e}}$ es el coeficiente de difusividad efectiva, que se considera constante dentro de la pastilla, $\mathrm{r}_{\mathrm{p}}$ el radio de la pastilla, $\mathrm{t}$ el tiempo y $\mathrm{z}$ corresponde al radio adimensional, que toma el valor cero en el centro de la pastilla y 1 en la superficie.

La Ec. [2] esta escrita de forma tal que incluye dos términos, el primero representa la difusión de los adsorbatos en el interior del sólido, y depende del coeficiente de difusión efectiva, $D_{e}$, mientras que el segundo término hace referencia a la cantidad de adsorbato acumulada en el adsorbente.

De manera equivalente a como se procedió en el Capítulo 4, el término correspondiente a la acumulación de VOCs dentro de los poros no fue incluido en el balance de materia [2].

Las condiciones de borde son las siguientes:

$$
\begin{aligned}
& \frac{\partial C}{\partial z}=0, \text { en } z=0 \\
& \frac{D_{e}}{r_{p}} \frac{\partial C}{\partial z}=k_{m}\left(C_{g}-C_{s}\right), \text { en } z=1
\end{aligned}
$$

Si consideramos la sustitución $u=z^{2}$ en las Ecs. [2] y [3] resulta:

$$
\mathrm{u} \frac{\partial^{2} \mathrm{C}}{\partial \mathrm{u}^{2}}+\frac{3}{2} \frac{\partial \mathrm{C}}{\partial \mathrm{u}}-\frac{\mathrm{r}_{\mathrm{p}}^{2}}{4 \mathrm{D}_{\mathrm{e}}} \frac{\partial \mathrm{q}(\mathrm{C})}{\partial \mathrm{t}}=0
$$




$$
2 \frac{D_{e}}{r_{p}} \frac{\partial C}{\partial u}=k_{m}\left(C_{g}-C_{s}\right), \text { en } u=1
$$

El cambio de variable garantiza automáticamente la condición impuesta en [3a] por lo que solo es necesario emplear la expresión [5] como condición de contorno.

Vale la pena mencionar que integrando el balance de materia [4] sobre el volumen de la pastilla y teniendo en cuenta la condición de borde [5], se satisface la siguiente condición:

$$
\mathrm{k}_{\mathrm{m}}\left(\mathrm{C}_{\mathrm{g}}-\mathrm{C}_{\mathrm{s}}\right)=\left.2 \frac{\mathrm{D}_{\mathrm{e}}}{\mathrm{r}_{\mathrm{p}}} \frac{\partial \mathrm{C}}{\partial \mathrm{u}}\right|_{\mathrm{u}=1}=\frac{\mathrm{r}_{\mathrm{p}}}{2} \int_{0}^{1} \frac{\partial \mathrm{q}(\mathrm{C})}{\partial \mathrm{t}} \mathrm{u}^{1 / 2} \mathrm{du}
$$

La expresión [6] establece que, en la superficie externa del adsorbente, el flujo de la sustancia a adsorber equivale a la velocidad de acumulación de la misma dentro de la pastilla.

Para la resolución de la ecuación diferencial de segundo orden, Ec. [4], se hizo uso del Método de Colocaciones Ortogonales (Villadsen y Michelsen, 1978). El método propuesto se expone en la sección siguiente. Con el propósito de simplificar la presentación del mismo se asume conocido al valor de la variable $\mathrm{C}_{\mathrm{g}}$, cuyo modo de obtención se presenta en la Sección 5.3.

\subsection{Método de Colocaciones Ortogonales}

Con el fin de resolver de manera aproximada la expresión [4] se propone usar un polinomio. Supondremos que dicho polinomio se expresa de la forma de un polinomio interpolatorio basado en ciertos puntos nodales en el interior del dominio más, usualmente, los extremos del intervalo. En nuestro caso particular, donde solo resulta necesario satisfacer la condición de contorno en $\mathrm{u}=1$, consideraremos únicamente dicho extremo, por lo que el polinomio queda expresado de la siguiente manera:

$$
\mathrm{C}=\sum_{\mathrm{j}=1}^{\mathrm{N}+1} \mathrm{~L}_{\mathrm{j}}(\mathrm{u}) \mathrm{C}_{\mathrm{j}}
$$

donde $\mathrm{L}_{\mathrm{j}}(\mathrm{u})$ son polinomios interpolatorios de Lagrange para el conjunto de nodos $\left\{\mathrm{u}_{\mathrm{j}}\right\}$ $(\mathrm{j}=1, \mathrm{~N})$ y $\mathrm{u}_{\mathrm{N}+1}=1$. Los valores $\left\{\mathrm{C}_{\mathrm{j}}\right\}$ constituyen los parámetros de la función aproximación y se corresponden a los valores nodales, $\mathrm{C}_{\mathrm{j}}=\mathrm{C}\left(\mathrm{u}_{\mathrm{j}}\right)$.

La determinación de los $\mathrm{N}+1$ parámetros $\left\{\mathrm{C}_{\mathrm{j}}\right\}$ se realizará mediante el ajuste de la condición de contorno más $\mathrm{N}$ condiciones impuestas por algún Método de Residuos 
Ponderados (MRP) (Villadsen y Michelsen, 1978). Dentro de los MRP se encuentra el Método de Colocaciones, el mismo se basa en anular el residuo $R(\mathrm{u})$ que resulta de aplicar la ecuación diferencial a la aproximación estipulada en un conjunto de puntos $\left\{\mathrm{u}_{\mathrm{i}}\right\}, \mathrm{i}=1, \ldots, \mathrm{N}$, denominados puntos de colocación (Ec. [8]). Estos puntos se hacen coincidir con los puntos de interpolación de la Ec. [7].

$$
R\left(\mathrm{u}_{\mathrm{i}}\right)=0 \quad \mathrm{i}=1, \ldots, \mathrm{N}
$$

Dependiendo de la elección de dichos puntos se define el tipo de método de colocación que resulta. Una opción es elegir puntos equidistantes dentro del intervalo de integración. Otra opción, más precisa, es la que se desarrollará a continuación y se conoce con el nombre de Método de Colocaciones Ortogonales.

Retomando nuestro caso particular, basado en la adsorción/desorción de una sustancia sobre una pastilla esférica, resulta relevante determinar la cantidad de adsorbato en los poros de la misma.

$$
\overline{\mathrm{C}}=\frac{\int_{\mathrm{V}_{\mathrm{P}}} \mathrm{CdV}}{\mathrm{V}_{\mathrm{P}}}
$$

donde $\overline{\mathrm{C}}$ es la concentración media en la pastilla y $\mathrm{V}_{\mathrm{p}}$ su volumen.

De la Ec. [9] surge que se debe calcular la siguiente integral:

$$
\mathrm{I}=\int_{\mathrm{V}_{\mathrm{P}}} \mathrm{C} \mathrm{dV}
$$

A partir de [10] se obtiene:

$$
\mathrm{I}=\overline{\mathrm{C}} \mathrm{V}_{\mathrm{P}}
$$

Para el caso en que la pastilla es esférica, el diferencial de volumen en términos de $u$ resulta,

$$
\mathrm{dV}=\frac{3}{2} \mathrm{~V}_{\mathrm{p}} \mathrm{u}^{1 / 2} \mathrm{du}, \quad \mathrm{V}_{\mathrm{p}}=\frac{4}{3} \pi \mathrm{r}_{\mathrm{p}}^{3}
$$

Por lo tanto resulta:

$$
I=\frac{3}{2} V_{p} \int_{0}^{1} u^{1 / 2} C(u) d u
$$

La integral [11] no puede ser evaluada de manera analítica, por lo que su resolución debe plantearse numéricamente, para lo cual son particularmente eficientes los Métodos 
de Cuadratura. Estos métodos se basan en la evaluación de una función a integrar en puntos de cuadratura. Para ello se plantea una fórmula del siguiente tipo:

$$
\frac{3}{2} \int_{0}^{1} \mathrm{u}^{1 / 2} \mathrm{C}(\mathrm{u}) \mathrm{du}=\sum_{\mathrm{i}=1-\mathrm{M}_{0}}^{\mathrm{N}+\mathrm{M}_{1}} \mathrm{C}\left(\mathrm{u}_{\mathrm{i}}\right) \omega_{\mathrm{i}}
$$

siendo $\left\{\omega_{\mathrm{i}}\right\}$ los factores de peso y $\left\{\mathrm{u}_{\mathrm{i}}\right\}$ el conjunto de valores denominados puntos de cuadratura.

$\mathrm{M}_{0}$ y $\mathrm{M}_{1}$ adoptan el valor de 0 ó 1 según se incluyan o no los puntos extremos del intervalo en la fórmula de cuadratura [12]. Si inducimos $\mathrm{u}_{0}=0$ como punto de cuadratura, $\mathrm{M}_{0}$ toma el valor de 1 , caso contrario $\mathrm{M}_{0}=0$. Lo mismo ocurre con $\mathrm{M}_{1}$ que resulta ser 1 en caso de considerar $\mathrm{u}_{\mathrm{N}+1}=1$ y $\mathrm{M}_{1}=0$ en caso contrario.

Definidos $\mathrm{N}, \mathrm{M}_{0}$ y $\mathrm{M}_{1}$, tanto los factores de peso como los puntos de cuadratura deben ser independientes de la función que se desee integrar. La precisión de la fórmula de cuadratura depende de la elección de los puntos de cuadratura elegidos. El criterio que se adopta para los puntos de cuadratura $\left\{\mathrm{u}_{\mathrm{i}}\right\}$ y los pesos $\left\{\omega_{\mathrm{i}}\right\}$ es maximizar el grado del polinomio para el cual la Ec. [12] resulta exacta. Surge que los valores $\left\{\mathrm{u}_{\mathrm{i}}\right\}$ deben coincidir con las raíces del siguiente polinomio de Jacobi $\mathrm{P}_{\mathrm{N}}(\mathrm{u})$, definido mediante las siguientes expresiones:

$$
\int_{0}^{1} u^{\mathrm{M}_{0}}(1-u)^{\mathrm{M}_{1}} \mathrm{u}^{\gamma^{*}} \mathrm{u}^{\mathrm{i}-1} \mathrm{P}_{\mathrm{N}}(\mathrm{u}) \mathrm{du}=0 \quad \mathrm{i}=1, \ldots, \mathrm{N}
$$

donde $\gamma^{*}=1 / 2$ para la geometría esférica de pastilla adoptada. Vale la pena aclarar que para el caso en que el material adsorbente se encuentre depositado sobre una placa plana, $\gamma^{*}=-1 / 2$ y para un adsorbente que adopte la forma de un cilindro infinitamente $\operatorname{largo}, \gamma^{*}=0$.

Una vez encontradas las raíces del polinomio de Jacobi a partir de las N Ecs. [13], es posible determinar los factores de peso $\omega_{\mathrm{i}}$ retomando la fórmula de cuadratura [12]. En el Apéndice 5.1 se presenta un ejemplo de cálculo de los factores de peso $\omega_{i}$ para el caso particular de $\mathrm{N}=2$.

Con la elección de los puntos $\left\{\mathrm{u}_{\mathrm{i}}\right\}$ como las raíces del polinomio $\mathrm{P}_{\mathrm{N}}(\mathrm{u})$, la Ec. [12] permite integrar exactamente hasta un polinomio de grado $2 \mathrm{~N}+\mathrm{M}_{0}+\mathrm{M}_{1}-1$. 
La familia de polinomios $\left\{\mathrm{P}_{\mathrm{N}}(\mathrm{u})\right\}$ (con grado $\mathrm{N}$ variable), definidos por [13], son ortogonales entre sí en el intervalo de integración $[0,1]$ respecto a la función $\left[\mathrm{u}^{\left(\mathrm{M}_{0}+\gamma^{*}\right)}(1-\mathrm{u})^{\mathrm{M}_{1}}\right]$, es decir:

$$
\int_{0}^{1} u^{\left(M_{0}+\gamma^{*}\right)}(1-u)^{M_{1}} P_{N}(u) P_{M}(u) d u=0, \text { con } N \neq M
$$

Dado que los puntos de cuadratura adecuados $\left\{\mathrm{u}_{\mathrm{i}}\right\}$ para evaluar la concentración media de acuerdo a la expresión [12] son las raíces del polinomio de Jacobi $\mathrm{P}_{\mathrm{N}}(\mathrm{u})$, el Método de Colocaciones Ortogonales propone elegir a tales puntos para hacer nulos los residuos de la ecuación diferencial, conforme a la Ec. [8]. Se presume que los valores $\left\{\mathrm{C}_{\mathrm{j}}\right\}$ (Ec. [7]) calculados de tal manera tendrán suficiente precisión para emplear la fórmula de cuadratura [12]. En el libro de Villadsen y Michelsen (1978) se demuestra que efectivamente la concentración media resulta evaluada con elevado grado de precisión. En dicho libro también se analiza la conveniencia o no de incluir los extremos $\mathrm{u}=0$ y $\mathrm{u}=1$ como puntos de cuadratura, resultando que deben incluirse en el caso en que los valores $\mathrm{C}(0)$ y $\mathrm{C}(1)$ se conozcan de antemano como condiciones de contorno. En nuestro caso no se conoce ninguno de estos valores de C, por lo que para definir los polinomios de Jacobi adecuados resulta, a partir de [13] con $\mathrm{M}_{0}=\mathrm{M}_{1}=0, \gamma^{*}=1 / 2$ :

$$
\int_{0}^{1} u^{1 / 2} u^{i-1} P_{N}(u) d u=0 \quad i=1, \ldots, N
$$

A partir de [12] la expresión de cuadratura para el cálculo de la concentración media resulta:

$$
\frac{3}{2} \int_{0}^{1} u^{1 / 2} C(u) d u=\sum_{i=1}^{N} C\left(u_{i}\right) \omega_{i}
$$

Dado que los puntos de colocación $\left\{\mathrm{u}_{\mathrm{i}}\right\}$ para las Ecs. [8] son las raíces de polinomios ortogonales, el procedimiento de resolución de la ecuación diferencial toma el nombre de Colocaciones Ortogonales.

A continuación se aplicará el Método de Colocaciones Ortogonales con el propósito de evaluar el valor de la concentración en función del tiempo en los puntos de colocación considerados. 


\subsubsection{Un punto de colocación ortogonal}

Para comenzar se considerará un único punto de colocación ortogonal en el interior de la pastilla. De acuerdo la expresión [7] la función aproximación para este caso resulta:

$$
\mathrm{C}=\mathrm{L}_{1}(\mathrm{u}) \mathrm{C}_{1}+\mathrm{L}_{2}(\mathrm{u}) \mathrm{C}_{\mathrm{s}}
$$

donde $\mathrm{C}_{1} \mathrm{y}_{\mathrm{s}} \mathrm{C}_{\mathrm{s}}$ son la concentración en el punto de colocación y en la superficie respectivamente.

Siendo el punto de colocación $\mathrm{u}_{1}=3 / 5$ (ver Apéndice 5.1), los polinomios de Lagrange $\mathrm{L}_{\mathrm{j}}(\mathrm{u})$ quedan definidos como:

$$
\begin{aligned}
& \mathrm{L}_{1}(\mathrm{u})=\frac{\mathrm{u}-1}{3 / 5-1} \\
& \mathrm{~L}_{2}(\mathrm{u})=\frac{\mathrm{u}-3 / 5}{1-3 / 5}
\end{aligned}
$$

Si reemplazamos en la Ec. [16] se obtiene la siguiente expresión:

$$
\mathrm{C}=\frac{5}{2}(1-\mathrm{u}) \mathrm{C}_{1}+\frac{5}{2}\left(\mathrm{u}-\frac{3}{5}\right) \mathrm{C}_{\mathrm{s}}
$$

Sea $R(\mathrm{u})$ el residuo que resulta de aplicar el balance de materia [4] a la aproximación [17]:

$$
R(\mathrm{u})=\frac{3}{2}\left(\frac{5}{2}\left(\mathrm{C}_{\mathrm{s}}-\mathrm{C}_{1}\right)\right)-\frac{\mathrm{r}_{\mathrm{p}}{ }^{2}}{4 \mathrm{D}_{\mathrm{e}}} \frac{\partial \mathrm{q}(\mathrm{C})}{\partial \mathrm{t}}
$$

Aplicando el Método de Colocaciones Ortogonales $\left(\mathrm{u}_{1}=3 / 5\right)$ se tiene que:

$$
R(3 / 5)=0
$$

Reemplazando en la Ec. [18] y reordenando resulta:

$$
\frac{\partial \mathrm{q}\left(\mathrm{C}_{1}\right)}{\partial \mathrm{t}}=15 \frac{\mathrm{D}_{\mathrm{e}}}{\mathrm{r}_{\mathrm{p}}{ }^{2}}\left(\mathrm{C}_{\mathrm{s}}-\mathrm{C}_{1}\right)
$$

Se verá a continuación que la concentración en el punto de colocación $\mathrm{u}=3 / 5$, equivale a la concentración media de la pastilla, de acuerdo a la expresión [15].

En este caso particular con un único punto de colocación en $\mathrm{u}_{1}=3 / 5$ resulta un factor de peso $\omega_{1}=1$. Reemplazando en la Ec. [15] se obtiene efectivamente 
$\overline{\mathrm{C}}=\mathrm{C}(3 / 5)$, por lo que se verifica la propiedad anunciada, $\overline{\mathrm{C}} \equiv \mathrm{C}_{1}$. De ahora en más utilizaremos $\overline{\mathrm{C}}$ en el lugar de $\mathrm{C}_{1}$.

Del balance de materia en la película resulta:

$$
15 \frac{\mathrm{D}_{\mathrm{e}}}{\mathrm{r}_{\mathrm{p}}^{2}}\left(\mathrm{C}_{\mathrm{s}}-\overline{\mathrm{C}}\right)=\mathrm{a}_{\mathrm{v}} \mathrm{k}_{\mathrm{m}}\left(\mathrm{C}_{\mathrm{g}}-\mathrm{C}_{\mathrm{s}}\right)
$$

Reemplazando en la Ec. [19] se obtiene la siguiente expresión:

$$
\frac{\partial \mathrm{q}(\overline{\mathrm{C}})}{\partial \mathrm{t}}=\mathrm{K}^{*}\left(\mathrm{C}_{\mathrm{g}}-\overline{\mathrm{C}}\right)
$$

donde:

$$
\mathrm{K}^{*}=\frac{1}{\frac{1}{\mathrm{a}_{\mathrm{v}} \mathrm{k}_{\mathrm{m}}}+\frac{\mathrm{r}_{\mathrm{p}}{ }^{2}}{15 \mathrm{D}_{\mathrm{e}}}}
$$

Finalmente se tiene que, asumiendo conocido el valor $\mathrm{C}_{\mathrm{g}}(\mathrm{t})$, se puede determinar la variación de la concentración media de dicha sustancia en el interior de la pastilla en el tiempo a partir de integrar la expresión [21].

\subsubsection{N puntos de colocación ortogonal}

Se consideran $\mathrm{N}$ puntos de colocación ortogonal en el interior de la pastilla. La función de aproximación [7] resulta:

$$
\mathrm{C}=\sum_{\mathrm{i}=1}^{\mathrm{N}} \mathrm{L}_{\mathrm{i}}(\mathrm{u}) \mathrm{C}_{\mathrm{i}}+\mathrm{L}_{\mathrm{s}}(\mathrm{u}) \mathrm{C}_{\mathrm{s}}
$$

donde $C_{i}$ son la concentraciones en los puntos de colocación $(i=1, \ldots, N)$ y $C_{s}$ representa la concentración en el punto de colocación $\mathrm{N}+1$, el cual se ubica en la superficie de la pastilla. $\mathrm{L}_{\mathrm{s}}$ corresponde al polinomio de Lagrange $\mathrm{L}_{\mathrm{N}+1}$.

Se debe cumplir que:

$$
\sum_{\mathrm{i}=1}^{\mathrm{N}} \mathrm{L}_{\mathrm{i}}(\mathrm{u})+\mathrm{L}_{\mathrm{s}}(\mathrm{u})=1
$$

Reemplazando en la Ec. [23] se tiene:

$$
\mathrm{C}=\mathrm{C}_{\mathrm{s}}+\sum_{\mathrm{i}=1}^{\mathrm{N}} \mathrm{L}_{\mathrm{i}}(\mathrm{u})\left(\mathrm{C}_{\mathrm{i}}-\mathrm{C}_{\mathrm{s}}\right)
$$


Para poder determinar el valor de la concentración en los $\mathrm{N}$ puntos de colocación se debe resolver el sistema de ecuaciones resultante de hacer nulo el residuo del balance de materia [4] en los puntos de colocación.

$$
\frac{\partial q\left(C_{j}\right)}{\partial t}=4 \frac{D_{e}}{r_{p}^{2}} \sum_{i=1}^{N}\left(\frac{3}{2} L_{i}^{\prime}\left(u_{j}\right)+u_{i} L_{i}^{\prime \prime}\left(u_{j}\right)\right)\left(C_{i}-C_{s}\right) \quad j=1, \ldots, N
$$

donde

$$
\mathrm{L}_{\mathrm{i}}^{\prime}=\frac{\partial \mathrm{L}_{\mathrm{i}}}{\partial \mathrm{u}} \quad \mathrm{y} \quad \mathrm{L}_{\mathrm{i}}^{\prime \prime}=\frac{\partial^{2} \mathrm{~L}_{\mathrm{i}}}{\partial \mathrm{u}^{2}}
$$

De la Ec. [25] se tiene que

$$
\frac{\partial \mathrm{C}}{\partial \mathrm{u}}=\sum_{\mathrm{i}=1}^{\mathrm{N}} \mathrm{L}_{\mathrm{i}}^{\prime}(\mathrm{u})\left(\mathrm{C}_{\mathrm{i}}-\mathrm{C}_{\mathrm{s}}\right)
$$

Reemplazando en la condición de contorno $(u=1)[5]$ resulta:

$$
\frac{2 D_{e}}{r_{p}} \sum_{i=1}^{N} L_{i}^{\prime}(1)\left(C_{i}-C_{s}\right)=k_{m}\left(C_{g}-C_{s}\right)
$$

Despejando $\mathrm{C}_{\mathrm{s}}$ de la Ec. [27] resulta:

$$
\mathrm{C}_{\mathrm{s}}=\frac{\mathrm{B}_{\mathrm{i}} \mathrm{C}_{\mathrm{g}}-\sum_{\mathrm{i}=1}^{\mathrm{N}} \mathrm{L}_{\mathrm{i}}^{\prime}(1) \mathrm{C}_{\mathrm{i}}}{\mathrm{B}_{\mathrm{i}}+\Omega}
$$

donde: $\Omega=-\sum_{\mathrm{i}=1}^{\mathrm{N}} \mathrm{L}_{\mathrm{i}}^{\prime}(1) \quad \mathrm{y} \quad \mathrm{B}_{\mathrm{i}}=\frac{\mathrm{r}_{\mathrm{p}} \mathrm{k}_{\mathrm{m}}}{2 \mathrm{D}_{\mathrm{e}}}$

A partir de la Ec. [27] se puede independizar el flujo en la interfase de la concentración $\mathrm{C}_{\mathrm{s}}$ :

$\mathrm{k}_{\mathrm{m}}\left(\mathrm{C}_{\mathrm{g}}-\mathrm{C}_{\mathrm{s}}\right)=\mathrm{k}_{\mathrm{m}}\left(\mathrm{C}_{\mathrm{g}}-\tilde{\mathrm{C}}\right) \frac{\Omega}{\mathrm{B}_{\mathrm{i}}+\Omega}$

donde $\tilde{\mathrm{C}}=-\frac{\sum_{\mathrm{i}=1}^{\mathrm{N}} \mathrm{L}_{\mathrm{i}}^{\prime}(1) \mathrm{C}_{\mathrm{i}}}{\Omega}$

Para $\mathrm{N}=1$ se cumple $\tilde{\mathrm{C}}=\overline{\mathrm{C}}$. 
Finalmente se tiene que, asumiendo conocido el valor $\mathrm{C}_{\mathrm{g}}(\mathrm{t})$, se puede determinar la variación de la concentración en cada uno de los puntos de colocación en el tiempo a partir de integrar la expresión [26].

\subsection{Resolución del balance en la corriente de fluido}

En esta sección se presentarán dos alternativas para resolver el balance de materia en la corriente gaseosa (Ec. [1]): mediante la aproximación de un sistema de tanques agitados en serie o por el Método Cuasi-Analítico de Matros y col. (1999).

\subsubsection{Tanques agitados en serie}

Esta alternativa de cálculo supone que el lecho puede dividirse en una serie de $\mathrm{N}_{\mathrm{t}}$ tanques agitados de igual tamaño, $\Delta \mathrm{V}\left(\Delta \mathrm{V}=\mathrm{S} \Delta \mathrm{Z}, \Delta \mathrm{Z}=\mathrm{L} / \mathrm{N}_{\mathrm{t}}\right)$, con composición uniforme en cada tanque. El balance de materia para un tanque resulta:

$$
\frac{\partial \mathrm{C}_{\mathrm{g}}^{\mathrm{i}}}{\partial \mathrm{t}}=u \frac{\mathrm{C}_{\mathrm{g}}^{\mathrm{i}-1}-\mathrm{C}_{\mathrm{g}}^{\mathrm{i}}}{\Delta \mathrm{Z}}-v \mathrm{a}_{\mathrm{v}} \mathrm{k}_{\mathrm{m}}\left(\mathrm{C}_{\mathrm{g}}^{\mathrm{i}}-\mathrm{C}_{\mathrm{s}}^{\mathrm{i}}\right) \quad \mathrm{i}=1, \ldots, \mathrm{N}_{\mathrm{t}}
$$

Es posible apreciar que si $\Delta \mathrm{Z} \rightarrow 0, \frac{\mathrm{C}_{\mathrm{g}}^{\mathrm{i}-1}-\mathrm{C}_{\mathrm{g}}^{\mathrm{i}}}{\Delta \mathrm{Z}} \rightarrow \frac{\partial \mathrm{C}}{\partial \mathrm{Z}}$ y la Ec. [32] se transforma en la Ec. [1].

Considerando la Ec. [30] es factible independizar la Ec. [32] de la concentración en la superficie de la pastilla, $\mathrm{C}_{\mathrm{s}}$. Resultando:

$$
\frac{\partial \mathrm{C}_{\mathrm{g}}^{\mathrm{i}}}{\partial \mathrm{t}}=u \frac{\mathrm{C}_{\mathrm{g}}^{\mathrm{i}-1}-\mathrm{C}_{\mathrm{g}}^{\mathrm{i}}}{\Delta \mathrm{Z}}-v \mathrm{a}_{\mathrm{v}} \mathrm{k}_{\mathrm{m}}\left(\mathrm{C}_{\mathrm{g}}-\tilde{\mathrm{C}}\right) \frac{\Omega}{B_{i}+\Omega}
$$

Para el primer tanque $(i=1), C_{g}^{0}$ (concentración en fase fluida para $Z=0$ ) se encuentra definida a partir de conocer las condiciones operativas de la corriente de alimentación. Mediante la integración temporal de la Ec. [33] conjuntamente con la integración de las Ecs. [26], se obtiene la composición en la fase fluida, $\mathrm{C}_{\mathrm{g}}^{1}$, y en los $\mathrm{N}$ puntos de colocación, $\mathrm{C}_{\mathrm{j}}^{1}$ con $\mathrm{j}=1, \ldots, \mathrm{N}$. La integración se lleva a cabo desde $\mathrm{t}=0$ hasta un cierto tiempo $t_{\mathrm{f}}$. Con esta información disponible se está en condiciones de resolver el segundo tanque $(i=2)$ de manera tal de obtener la composición en la fase fluida y los $N$ puntos de colocación a la salida del mismo $\left(C_{g}^{2}, C_{j}^{2}\right.$ con $\left.j=1, \ldots, N\right), y$ 
así sucesivamente hasta $\mathrm{i}=\mathrm{L} / \Delta \mathrm{Z}=\mathrm{N}_{\mathrm{t}}$. De esta manera es posible obtener el perfil de concentración a lo largo del lecho para cualquier valor del tiempo.

Para la resolución del sistema de ecuaciones diferenciales en la variable tiempo, esto es la Ec. [33] simultáneamente con las Ec. [26], se utilizó el software de programación MATLAB empleando la subrutina ODE 15.

\subsubsection{Método Cuasi-Analítico}

Esta alternativa fue empleada por Matros y col. (1999).

Previamente a la presentación del procedimiento de cálculo, se recuerda que el balance de materia en el lecho es el siguiente (Ec. [1]):

$$
\frac{\partial \mathrm{C}_{\mathrm{g}}}{\partial \mathrm{t}}=-u \frac{\partial \mathrm{C}_{\mathrm{g}}}{\partial Z}-v \mathrm{a}_{\mathrm{v}} \mathrm{k}_{\mathrm{m}}\left(\mathrm{C}_{\mathrm{g}}-\mathrm{C}_{\mathrm{s}}\right)
$$

Se verificó que el aporte del término de acumulación en la fase gaseosa en la Ec. [1] no resulta significativo frente al resto de los términos, por lo que se supone despreciable. De esta manera resulta:

$$
\frac{\partial \mathrm{C}_{\mathrm{g}}}{\partial Z}=-\frac{v \mathrm{a}_{\mathrm{v}} \mathrm{k}_{\mathrm{m}}}{u}\left(\mathrm{C}_{\mathrm{g}}-\mathrm{C}_{\mathrm{s}}\right)
$$

Teniendo en cuenta la verificación realizada, el Método sugerido por Matros y col. (1999) se aplicará a la Ec. [34], pero debe destacarse que dicho método también sería aplicable si dicha simplificación no fuese posible.

A continuación se buscará obtener una expresión para $\mathrm{C}_{\mathrm{g}}$ para el caso general de considerar N puntos de colocación (Sección 5.2.2).

\subsubsection{N puntos de colocación ortogonal}

En la Sección 5.2.2 se demostró que para el flujo interfacial se cumple (Ec. [30]):

$$
\mathrm{k}_{\mathrm{m}}\left(\mathrm{C}_{\mathrm{g}}-\mathrm{C}_{\mathrm{s}}\right)=\mathrm{k}_{\mathrm{m}}\left(\mathrm{C}_{\mathrm{g}}-\tilde{\mathrm{C}}\right) \frac{\Omega}{\mathrm{B}_{\mathrm{i}}+\Omega}
$$

Reemplazando en la Ec. [34] resulta:

$$
\frac{\partial \mathrm{C}_{\mathrm{g}}}{\partial Z}=-\alpha\left(\mathrm{C}_{\mathrm{g}}-\tilde{\mathrm{C}}\right)
$$


$\operatorname{con} \alpha=\frac{v \mathrm{a}_{\mathrm{v}} \mathrm{k}_{\mathrm{m}}}{u} \frac{\Omega}{\mathrm{B}_{\mathrm{i}}+\Omega}$

Se pretende resolver la Ec. [35] entre $Z_{0}$ y $Z_{0}+\Delta Z$. Suponemos una variación lineal de $\tilde{\mathrm{C}}$ con $\mathrm{Z}$, de manera tal que:

$$
\tilde{\mathrm{C}}(\mathrm{Z})=\tilde{\mathrm{C}}_{\mathrm{Z}_{0}}+\frac{\tilde{\mathrm{C}}_{\Delta \mathrm{Z}}-\tilde{\mathrm{C}}_{\mathrm{Z}_{0}}}{\Delta \mathrm{Z}}\left(\mathrm{Z}-\mathrm{Z}_{0}\right) \quad \mathrm{Z}_{0}<\mathrm{Z}<\mathrm{Z}_{0}+\Delta \mathrm{Z}
$$

con $\tilde{\mathrm{C}}_{\mathrm{Z}_{0}}=\tilde{\mathrm{C}}\left(\mathrm{Z}_{0}\right)$ y $\tilde{\mathrm{C}}_{\Delta \mathrm{Z}}=\tilde{\mathrm{C}}\left(\mathrm{Z}_{0}+\Delta \mathrm{Z}\right)$.

Luego, reemplazando en la Ec. [35] se tiene:

$$
\frac{\partial \mathrm{C}_{\mathrm{g}}}{\partial \mathrm{Z}}+\alpha \mathrm{C}_{\mathrm{g}}=\alpha\left(\tilde{\mathrm{C}}_{\mathrm{Z}_{0}}+\frac{\tilde{\mathrm{C}}_{\Delta \mathrm{Z}}-\tilde{\mathrm{C}}_{\mathrm{Z}_{0}}}{\Delta \mathrm{Z}}\left(\mathrm{Z}-\mathrm{Z}_{0}\right)\right)
$$

La [37] es una ecuación diferencial lineal en $\mathrm{C}_{\mathrm{g}}$ no homogénea y se resuelve por un procedimiento elemental que permite escribir $C_{\mathrm{g}, \Delta \mathrm{Z}}=\mathrm{C}_{\mathrm{g}}\left(\mathrm{Z}_{0}+\Delta \mathrm{Z}\right)$ en la forma:

$$
\mathrm{C}_{\mathrm{g}, \Delta \mathrm{Z}}=\tilde{\mathrm{C}}_{\Delta \mathrm{Z}}+\left(\mathrm{C}_{\mathrm{g}, 0}-\tilde{\mathrm{C}}_{0}\right) \mathrm{e}^{-\alpha \Delta \mathrm{Z}}-\frac{\tilde{\mathrm{C}}_{\Delta \mathrm{Z}}-\tilde{\mathrm{C}}_{0}}{\Delta \mathrm{Z}} \frac{1}{\alpha}\left(1-\mathrm{e}^{-\alpha \Delta \mathrm{Z}}\right)
$$

Se recuerda que:

$$
\tilde{\mathrm{C}}_{\Delta \mathrm{Z}}=-\frac{\sum_{\mathrm{i}=1}^{\mathrm{N}} \mathrm{L}_{\mathrm{i}}^{\prime}(1) \mathrm{C}_{\mathrm{i}}\left(\mathrm{Z}_{0}+\Delta \mathrm{Z}\right)}{\Omega}
$$

La Ec. [38] debe resolverse conjuntamente con las Ecs.[26]. Este sistema de ecuaciones permite obtener la variación temporal de la concentración en la corriente de fluido y en el sólido adsorbente generada por la perturbación de la concentración en la corriente de alimentación. El procedimiento de cálculo es análogo al empleado para la aproximación del sistema de tanques. También se utilizó MATLAB como software de programación, empleándose la subrutina ODE 15 para la resolución del sistema diferencial (Ecs. [26]). 
5.3.3 Comparación entre la aproximación del sistema de tanques agitados en serie y el Método Cuasi-Analítico

Se evaluó la eficiencia de las alternativas de cálculo a partir de analizar el porcentaje de error obtenido al variar el número de tanques o intervalos según el método adoptado. Dada la analogía del procedimiento de cálculo de ambos métodos, el concepto de un tanque resulta equivalente al de un intervalo del Método CuasiAnalítico, por lo tanto, para la comparación, el término intervalo también corresponderá a un tanque. En general, se observó que para un mismo número de intervalos el error obtenido al emplear la aproximación de tanques agitados en serie resultó ser mayor que el obtenido para el Método Cuasi-Análitico.

Para el caso particular de considerar una corriente de aire con acetato de etilo que circula por un lecho relleno de carbón activado operando isotérmicamente durante 1000 segundos, se obtuvo, al dividir al lecho en 50 intervalos, un error máximo de $0.27 \%$ para el Método Cuasi-Analítico y $3.82 \%$ al aproximar mediante tanques agitados en serie. Para la estimación del error relativo se tomó como referencia el perfil de concentración del acetato de etilo $\left(\mathrm{C}_{\mathrm{g}}\right)$ a los 1000 segundos, obtenido mediante el empleo del Método Cuasi-Análitico para 1000 intervalos $\left(\Delta \mathrm{Z}=610^{-4} \mathrm{~m}\right)$. Las características de la corriente y del lecho se presentan en la Tabla 1.

Tabla 1: Características de la corriente y del lecho relleno

\begin{tabular}{c|c}
\hline \multicolumn{2}{c}{ Corriente de alimentación } \\
\hline Caudal $\left[\mathrm{m}^{3} / \mathrm{s}\right]$ & 11.7 \\
Concentración $\left[\mathrm{mol} / \mathrm{m}^{3}\right]$ & $2.3510^{-2}$ \\
Temperatura $\left[{ }^{\circ} \mathrm{C}\right]$ & 50 \\
Presión $[\mathrm{atm}]$ & 1 \\
\hline \multicolumn{2}{c}{ Lecho Relleno } \\
\hline \multicolumn{2}{c}{$\mathrm{S}\left[\mathrm{m}^{2}\right]$} \\
$\mathrm{L}[\mathrm{m}]$ \\
$\varepsilon_{\mathrm{L}}$ \\
$\mathrm{d}_{\mathrm{p}}[\mathrm{m}]$
\end{tabular}


Dado a que el Método Cuasi-Analítico presentó una mayor eficiencia que el sistema de tanques agitados, se decidió optar por dicho método para la obtención de los resultados.

\subsection{Adsorción/desorción simultánea de acetato de etilo y etanol considerando las resistencias a la transferencia de calor y materia}

Retomando nuestro caso particular de estudio, presentado en el Capítulo 2, y considerando las resistencias a la transferencia de calor y materia dentro y fuera del sólido adsorbente, se emplea, para la resolución de dicho sistema, el Método de Colocaciones Ortogonales (Sección 5.2) acoplado con el Método Cuasi-Analítico (Sección 5.3.2). Empleando estos métodos se analizan los resultados obtenidos según el tipo de lecho (relleno o monolito) considerado.

Al igual que para el caso particular de no tener en cuenta las resistencias (Capítulo 4), se considera una columna adiabática donde el acetato de etilo (1) y el etanol (2) son transportados por la corriente de aire y adsorbidos/desorbidos en la fase estacionaria de carbón activado.

Los balances locales de materia (a un dado Z) para las especies que difunden y se adsorben en el interior del sólido adsorbente, junto con el balance de energía, para el caso específico de un adsorbente granular de geometría esférica resultan (para el planteo se emplea directamente como coordenada $\mathrm{u}=\mathrm{z}^{2}$ ):

$$
\begin{aligned}
& \mathrm{u} \frac{\partial^{2} \mathrm{C}_{\mathrm{k}}}{\partial \mathrm{u}^{2}}+\frac{3}{2} \frac{\partial \mathrm{C}_{\mathrm{k}}}{\partial \mathrm{u}}-\frac{\mathrm{r}_{\mathrm{p}}^{2}}{4 \mathrm{D}_{\mathrm{e}, \mathrm{k}}} \frac{\partial \mathrm{q}_{\mathrm{k}}\left(\mathrm{C}_{1}, \mathrm{C}_{2}, \mathrm{~T}\right)}{\partial \mathrm{t}}=0 \quad \mathrm{k}=1,2 \\
& \mathrm{u} \frac{\partial^{2} \mathrm{~T}}{\partial \mathrm{u}^{2}}+\frac{3}{2} \frac{\partial \mathrm{T}}{\partial \mathrm{u}}-\frac{\mathrm{r}_{\mathrm{p}}^{2} \mathrm{c}_{\mathrm{p}, \mathrm{g}}}{4 \lambda_{\mathrm{p}}} \frac{\partial \mathrm{q}_{\mathrm{T}}\left(\mathrm{C}_{1}, \mathrm{C}_{2}, \mathrm{~T}\right)}{\partial \mathrm{t}}=0
\end{aligned}
$$

donde $\lambda_{\mathrm{p}}$ es la conductividad térmica efectiva del sólido adsorbente. Se recuerda que $\mathrm{q}_{\mathrm{k}}=\frac{Q_{\mathrm{k}} \mathrm{K}_{\mathrm{k}} \mathrm{C}_{\mathrm{k}}}{1+\sum_{\mathrm{j}=1}^{2} \mathrm{~K}_{\mathrm{j}} \mathrm{C}_{\mathrm{j}}}, \mathrm{k}=1,2$ (Isoterma de Langmuir generalizada, Ec. [38a] Capítulo 4), $\mathrm{y}$ $\mathrm{q}_{\mathrm{T}} \equiv \gamma \mathrm{T}-\sum_{\mathrm{k}=1}^{2} \mathrm{~J}_{\mathrm{k}} \mathrm{q}_{\mathrm{k}}($ Capítulo 4$)$

Las expresiones [39a] son similares al balance de materia [4] planteado para un único componente en la Sección 5.1 . 
A partir de hacer cero el residuo de las ecuaciones diferenciales [39] en $\mathrm{N}$ puntos de colocación, resultan:

$$
\begin{aligned}
& \frac{\partial \mathrm{q}_{\mathrm{k}}\left(\mathrm{C}_{\mathrm{j}, 1}, \mathrm{C}_{\mathrm{j}, 2}, \mathrm{~T}\right)}{\partial \mathrm{t}}=4 \frac{\mathrm{D}_{\mathrm{e}}}{\mathrm{r}_{\mathrm{p}}^{2}} \sum_{\mathrm{i}=1}^{\mathrm{N}}\left(\frac{3}{2} \mathrm{~L}_{\mathrm{i}}^{\prime}\left(\mathrm{u}_{\mathrm{j}}\right)+\mathrm{u}_{\mathrm{i}} \mathrm{L}_{\mathrm{i}}^{\prime \prime}\left(\mathrm{u}_{\mathrm{j}}\right)\right)\left(\mathrm{C}_{\mathrm{j}, \mathrm{k}}-\mathrm{C}_{\mathrm{s}, \mathrm{k}}\right), \mathrm{j}=1, \ldots, \mathrm{N}, \quad \mathrm{k}=1,2 \quad \text { [40a] } \\
& \frac{\partial \mathrm{q}_{\mathrm{T}}\left(\mathrm{C}_{\mathrm{j}, 1}, \mathrm{C}_{\mathrm{j}, 2}, \mathrm{~T}\right)}{\partial \mathrm{t}}=4 \frac{\lambda_{\mathrm{p}}}{\mathrm{r}_{\mathrm{p}}^{2} \mathrm{c}_{\mathrm{p}, \mathrm{g}}} \sum_{\mathrm{i}=1}^{\mathrm{N}}\left(\frac{3}{2} \mathrm{~L}_{\mathrm{i}}^{\prime}\left(\mathrm{u}_{\mathrm{j}}\right)+\mathrm{u}_{\mathrm{i}} \mathrm{L}_{\mathrm{i}}^{\prime \prime}\left(\mathrm{u}_{\mathrm{j}}\right)\right)\left(\mathrm{T}_{\mathrm{j}}-\mathrm{T}_{\mathrm{s}}\right), \mathrm{j}=1, \ldots, \mathrm{N} \quad \quad \text { [40b] }
\end{aligned}
$$

Las condiciones de borde en la superficie externa del adsorbente $(u=1)$ son:

$$
\begin{aligned}
& \frac{2 \mathrm{D}_{\mathrm{e}, \mathrm{k}}}{\mathrm{r}_{\mathrm{p}}} \frac{\partial \mathrm{C}_{\mathrm{k}}}{\partial \mathrm{u}}=\mathrm{k}_{\mathrm{m}, \mathrm{k}}\left(\mathrm{C}_{\mathrm{g}, \mathrm{k}}-\mathrm{C}_{\mathrm{s}, \mathrm{k}}\right), \quad \mathrm{k}=1,2 \\
& \frac{2 \lambda_{\mathrm{p}}}{\mathrm{r}_{\mathrm{p}}} \frac{\partial \mathrm{T}}{\partial \mathrm{u}}=\mathrm{h}_{\mathrm{t}}\left(\mathrm{T}_{\mathrm{g}}-\mathrm{T}_{\mathrm{s}}\right)
\end{aligned}
$$

donde $\mathrm{T}_{\mathrm{g}}$ y $\mathrm{T}_{\mathrm{s}}$ son la temperatura en la corriente de fluido y en la superficie del sólido adsorbente respectivamente, $\mathrm{y} \mathrm{h}_{\mathrm{t}}$ es el coeficiente de transferencia de calor entre el fluido y la superficie externa del adsorbente.

Teniendo en cuenta la Ec. [25], para un sistema de dos componentes se tiene:

$$
\frac{\partial \mathrm{C}_{\mathrm{k}}}{\partial \mathrm{u}}=\sum_{\mathrm{i}=1}^{\mathrm{N}} \mathrm{L}_{\mathrm{i}}^{\prime}(\mathrm{u})\left(\mathrm{C}_{\mathrm{i}, \mathrm{k}}-\mathrm{C}_{\mathrm{s}, \mathrm{k}}\right), \mathrm{k}=1,2
$$

Por analogía, para la temperatura resulta:

$$
\frac{\partial \mathrm{T}}{\partial \mathrm{u}}=\sum_{\mathrm{i}=1}^{\mathrm{N}} \mathrm{L}_{\mathrm{i}}^{\prime}(\mathrm{u})\left(\mathrm{T}_{\mathrm{i}}-\mathrm{T}_{\mathrm{s}}\right)
$$

Reemplazando en las condiciones de contorno [41] surge:

$$
\begin{gathered}
\mathrm{C}_{\mathrm{s}, \mathrm{k}}=\frac{\mathrm{B}_{\mathrm{i}, \mathrm{k}} \mathrm{C}_{\mathrm{g}, \mathrm{k}}-\sum_{\mathrm{i}=1}^{\mathrm{N}} \mathrm{L}_{\mathrm{i}}^{\prime}(1) \mathrm{C}_{\mathrm{i}, \mathrm{k}}}{\mathrm{B}_{\mathrm{i}, \mathrm{k}}+\Omega}, \mathrm{k}=1,2 \\
\mathrm{~T}_{\mathrm{s}}=\frac{\mathrm{B}_{\mathrm{i}, \mathrm{c}} \mathrm{T}_{\mathrm{g}}-\sum_{\mathrm{i}=1}^{\mathrm{N}} \mathrm{L}_{\mathrm{i}}^{\prime}(1) \mathrm{T}_{\mathrm{i}}}{\mathrm{B}_{\mathrm{i}, \mathrm{c}}+\Omega}
\end{gathered}
$$

donde $\Omega=-\sum_{i=1}^{N} L_{i}^{\prime}(1), B_{i, k}=\frac{r_{p} k_{m, k}}{2 D_{e, k}}, k=1,2, y B_{i, c}=\frac{r_{p} h_{t}}{2 \lambda_{p}}$. 
Considerando el balance de materia [1] planteado para un único componente en la Sección 5.1 para la corriente de fluido, surgen los siguientes balances de conservación, suponiendo uniformidad de las variables en la sección transversal del lecho:

$$
\begin{array}{ll}
\frac{\partial \mathrm{C}_{\mathrm{g}, \mathrm{k}}}{\partial \mathrm{t}}=-u \frac{\partial \mathrm{C}_{\mathrm{g}, \mathrm{k}}}{\partial \mathrm{Z}}-v \mathrm{a}_{\mathrm{v}} \mathrm{k}_{\mathrm{m}, \mathrm{k}}\left(\mathrm{C}_{\mathrm{g}, \mathrm{k}}-\mathrm{C}_{\mathrm{s}, \mathrm{k}}\right) \quad \mathrm{k}=1,2 \\
\frac{\partial \mathrm{T}_{\mathrm{g}}}{\partial \mathrm{t}}=-u \frac{\partial \mathrm{T}}{\partial \mathrm{Z}}-\frac{1}{\mathrm{c}_{\mathrm{p}, \mathrm{g}}} v \mathrm{a}_{\mathrm{v}} \mathrm{h}_{\mathrm{t}}\left(\mathrm{T}_{\mathrm{g}}-\mathrm{T}_{\mathrm{s}}\right)
\end{array}
$$

donde $\mathrm{c}_{\mathrm{p}, \mathrm{g}}$ es la capacidad calorífica de la fase fluida.

Las Ecs. [43] son válidas tanto para lechos granulares como estructurados. Considerando las Ecs. [42] y el Método Cuasi-Analítico, de manera discretizada, las Ecs. [43] resultan:

$$
\begin{aligned}
& \mathrm{C}_{\mathrm{g}, \mathrm{k}, \Delta \mathrm{Z}}=\tilde{\mathrm{C}}_{\mathrm{k}, \Delta \mathrm{Z}}+\left(\tilde{\mathrm{C}}_{\mathrm{g}, \mathrm{k}, 0}-\tilde{\mathrm{C}}_{\mathrm{k}, 0}\right) \mathrm{e}^{-\alpha_{\mathrm{k}} \Delta \mathrm{Z}}-\frac{\tilde{\mathrm{C}}_{\mathrm{k}, \Delta \mathrm{Z}}-\tilde{\mathrm{C}}_{\mathrm{k}, 0}}{\Delta \mathrm{Z}} \frac{1}{\alpha_{k}}\left(1-\mathrm{e}^{-\alpha_{\mathrm{k}} \Delta \mathrm{Z}}\right), \mathrm{k}=1,2 \\
& \mathrm{~T}_{\mathrm{g}, \Delta \mathrm{Z}}=\tilde{\mathrm{T}}_{\Delta \mathrm{Z}}+\left(\mathrm{T}_{\mathrm{g}, 0}-\tilde{\mathrm{T}}_{0}\right) \mathrm{e}^{-\alpha_{\mathrm{T}} \Delta \mathrm{Z}}-\frac{\tilde{\mathrm{T}}_{\Delta \mathrm{Z}}-\tilde{\mathrm{T}}_{0}}{\Delta \mathrm{Z}} \frac{1}{\alpha_{\mathrm{T}}}\left(1-\mathrm{e}^{-\alpha_{\mathrm{T}} \Delta \mathrm{Z}}\right)
\end{aligned}
$$

donde $\alpha_{\mathrm{k}}=\frac{v \mathrm{a}_{\mathrm{v}} \mathrm{k}_{\mathrm{m}, \mathrm{k}}}{u} \frac{\Omega}{\mathrm{B}_{\mathrm{i}, \mathrm{k}}+\Omega}, \mathrm{k}=1,2, \mathrm{y} \alpha_{\mathrm{T}}=\frac{v \mathrm{a}_{\mathrm{v}} \mathrm{h}_{\mathrm{t}}}{\mathrm{c}_{\mathrm{p}, \mathrm{g}} u} \frac{\Omega}{\mathrm{B}_{\mathrm{i}, \mathrm{c}}+\Omega}$.

Se recuerda que:

$$
\tilde{\mathrm{C}}_{\mathrm{k}, \Delta Z}=-\frac{\sum_{\mathrm{i}=1}^{\mathrm{N}} \mathrm{L}_{\mathrm{i}}^{\prime}(1) \mathrm{C}_{\mathrm{i}, \mathrm{k}}\left(\mathrm{Z}_{0}+\Delta \mathrm{Z}\right)}{\Omega}, \mathrm{k}=1,2,
$$

y, por analogía se tiene:

$\tilde{\mathrm{T}}_{\Delta \mathrm{Z}}=-\frac{\sum_{\mathrm{i}=1}^{\mathrm{N}} \mathrm{L}_{\mathrm{i}}^{\prime}(1) \mathrm{T}_{\mathrm{i}}\left(\mathrm{Z}_{0}+\Delta \mathrm{Z}\right)}{\Omega}$

De manera similar a la presentada para un en un proceso isotérmico con único componente, el sistema de ecuaciones diferenciales [40] se resuelve de manera conjunta con las Ecs. [44] empleando el software de programación MATLAB mediante la subrutina ODE 15. 
5.4.1 Propiedades del material adsorbente y selección de las características geométricas del adsorbedor

A partir de las condiciones operativas de la corriente a tratar presentadas en el Capítulo 2 y reescritas en la Tabla 2, se realizó la simulación tanto para un lecho relleno (LR) como para un monolito $(\mathrm{M})$. A continuación se presentarán las propiedades del material adsorbente empleado y los criterios de selección adoptados para definir las características geométricas de los lechos.

Tabla 2: Características de la corriente a tratar

\begin{tabular}{|c|c|c|}
\hline \multicolumn{2}{|c|}{ Caudal $\left[\mathbf{m}^{3} / \mathbf{s}\right]$} & 11.7 \\
\hline $\begin{array}{c}\text { Composición } \\
{\left[\mathbf{m o l} / \mathbf{m}^{3}\right]}\end{array}$ & Acetato de Etilo & $2.3510^{-2}$ \\
\cline { 2 - 3 } Tipo de emisión & Etanol & $9.6310^{-3}$ \\
\hline \multicolumn{2}{|c|}{ Presión [atm] } & Permanente \\
\hline \multicolumn{2}{|c|}{${\text { Temperatura }\left[{ }^{\circ} \mathbf{C}\right]}^{2}$} \\
\hline
\end{tabular}

\subsubsection{Características del material adsorbente: carbón activado}

Según los criterios de selección de material adsorbente presentados en el Capítulo 3 (Sección 3.3) y teniendo en cuenta las características de la corriente a tratar (Tabla 2), se optó por el carbón activado como sólido adsorbente. Como se ha mencionado en el Capítulo 3, en la bibliografía, se encuentran numerosos trabajos que emplean carbón activado para la adsorción de VOCs. En particular, Gales y col. $(2000,2003)$ estudiaron la adsorción de acetato de etilo y etanol sobre carbón activado. Los valores de la porosidad $\left(\varepsilon_{\mathrm{p}}\right)$, macroporosidad $\left(\varepsilon_{\mathrm{p}(\operatorname{macro})}\right)$, superficie específica correspondiente a los macroporos $\left(\mathrm{S}_{\mathrm{g}(\operatorname{macro})}\right)$ y densidad aparente $\left(\rho_{\text {pap }}\right)$ del sólido adsorbente fueron adoptados del trabajo de Gales y col. (2000). Los mismos se presentan en la Tabla 3. A partir de estas propiedades se determina el radio de poro medio $\left(\mathrm{r}_{\text {poro }}\right.$ ) mediante el modelo de poros en paralelo (Apéndice 5.2, Ec. [A5.2.7]). Este valor junto con la tortuosidad, conductividad térmica y capacidad calorífica también son presentados en la Tabla 3.

Es importante mencionar que, a pesar de que la geometría y disposición del material adsorbente difieren para ambos tipos de lecho aquí estudiados (monolito o lecho relleno), se consideraron idénticas propiedades intrínsecas del mismo. 
Además teniendo en cuenta lo analizado en la Sección 3.6 del Capítulo 3, en el caso del monolito se supuso al adsorbente constituyendo el material de las paredes.

Tabla 3: Características del material adsorbente

\begin{tabular}{c|c}
\hline \multicolumn{2}{c}{ Material Adsorbente : Carbón Activado } \\
\hline$\varepsilon_{\mathrm{p}}$ & 0.7 \\
$\varepsilon_{\mathrm{p}(\text { macro })}$ & 0.31 \\
$\tau$ & 1.5 \\
$\mathrm{~S}_{\mathrm{g}(\text { macro })}\left[\mathrm{m}^{2} / \mathrm{kg}\right]$ & $53.110^{3}$ \\
$\rho_{\mathrm{pap}}\left[\mathrm{kg} / \mathrm{m}^{3}\right]$ & 750 \\
$\mathrm{r}_{\mathrm{poro}}[\mathrm{nm}]=2 \varepsilon_{\mathrm{p}(\text { macro })} /\left(\mathrm{S}_{\mathrm{g}(\text { macro })} \rho_{\mathrm{pap}}\right)$ & 16 \\
$\lambda_{\mathrm{p}}\left[\mathrm{W} / \mathrm{m} \mathrm{K}^{3}\right]$ & 0.5 \\
$\mathrm{c}_{\mathrm{p}, \mathrm{p}}\left[\mathrm{kJ} / \mathrm{m}^{3} \mathrm{~K}\right]$ & 486.85 \\
\hline
\end{tabular}

El carbón activado suele presentar una porosidad distribuida entre macro y microporos como consideraron Gales y col. (2000, 2003) (Tabla 3). En general está aceptado que dentro de los microporos la difusión superficial de los adsorbatos es muy rápida permitiendo que el proceso de adsorción/desorción alcance el equilibrio rápidamente (Ruthven, 1984). De esta forma, es posible considerar que los moles adsorbidos se encuentran en equilibrio con la concentración en los macroporos. En nuestro caso esa relación se describe por la isoterma de Langmuir generalizada presentada en el Capítulo 4 (Ec. [38a]).

Por otro lado, se tiene que en los macroporos los procesos dominantes son la difusión molecular y la difusión Knudsen de las especies. Esto implica que para el cálculo del coeficiente de difusión efectiva, $\mathrm{D}_{\mathrm{e}}$, a partir de la expresión de Bosanquet presentada en el Apéndice 5.2 (Ec. [A5.2.4]), se considere únicamente la macroporosidad. La expresión de Bosanquet resulta apropiada debido a que los adsorbatos, acetato de etilo y etanol, se encuentran muy diluidos (Tabla 2). Teniendo en cuenta las propiedades del carbón presentadas en la Tabla 3 y las condiciones de la corriente a tratar expuestas en la Tabla 2, se determinaron los siguientes valores de difusividad efectiva, $\mathrm{D}_{\mathrm{e}, 1}$ y $\mathrm{D}_{\mathrm{e}, 2}$, para el acetato de etilo y etanol respectivamente: 


$$
\begin{aligned}
& \mathrm{D}_{\mathrm{e}, 1}=4.7510^{-7} \mathrm{~m}^{2} / \mathrm{s} \\
& \mathrm{D}_{\mathrm{e}, 2}=6.5710^{-7} \mathrm{~m}^{2} / \mathrm{s}
\end{aligned}
$$

Gales y col. (2003), presentan una formulación empírica para determinar los coeficientes de difusión del acetato de etilo y etanol. Los valores de difusividad obtenidos a partir de dicha expresión $\left(D_{e, 1}=3.110^{-7} \mathrm{~m}^{2} / \mathrm{s}, D_{e, 2}=4.410^{-7} \mathrm{~m}^{2} / \mathrm{s}\right.$ ) están dentro de los márgenes de precisión que se pueden esperar para la estimación de $\mathrm{D}_{\mathrm{e}}$.

A fin de adoptar una temperatura de regeneración del material adsorbente apropiada, considerando la estabilidad del mismo, se realizaron análisis sobre una muestra de carbón activado para poder estimar el consumo de carbón al encontrarse expuesto a $200{ }^{\circ} \mathrm{C}$. Se observó, mediante un ensayo termogravimétrico, que se consume aproximadamente un $22 \%$ del material por año. Sin embargo, se debe considerar que, en la práctica, el adsorbente no se encuentra expuesto a esa temperatura de manera continua, sino que hay un tiempo, generalmente mayor (como se verá al analizar los concentradores rotativos, Capítulo 6), que está destinado a la etapa de adsorción donde la temperatura es del orden de los $50{ }^{\circ} \mathrm{C}$. Por lo tanto el porcentaje de consumo de carbón anual resulta al menos la mitad que el valor mencionado. Por otro lado, Yates y col. (2000), que estudiaron una serie de de carbones activados, anunciaron que los mismos se mantuvieron térmicamente estables hasta temperaturas de $300^{\circ} \mathrm{C}$.

Para la obtención de resultados, y teniendo en cuenta el grado de consumo del carbón al exponerse a relativamente altas temperaturas, se adoptaron valores de 150 y $200^{\circ} \mathrm{C}$ para la regeneración del lecho, aunque en este capítulo los resultados que se informan corresponden exclusivamente al nivel de $150^{\circ} \mathrm{C}$.

En el Capítulo 6 se analiza la influencia de la temperatura de regeneración sobre el valor de caudal de la corriente de desorción. 


\subsubsection{Características geométricas del monolito}

Se partió de la selección de un conjunto de características que permiten definir la estructura del monolito. La caracterización geométrica básica de la matriz de un monolito está dada por la forma de los canales, el número de canales por unidad de superficie transversal total del monolito $\left(\mathrm{n}_{\mathrm{c}}\right)$ y por el espesor del tabique $(t)$. Para esta presentación se consideraron canales de sección cuadrada (Figura 1) y se optó por una densidad de canales, $\mathrm{n}_{\mathrm{c}}$, lo suficientemente alta para proporcionar un área de transferencia considerable y que, con un valor de espesor de tabique adecuado, $t$, permitan aportar una resistencia mecánica aceptable para la estructura del lecho (Boger y col. 2004). Se despreció la acumulación de material adsorbente en los vértices de los canales, tomándose un radio de curvatura nulo $\left(\mathrm{R}_{\mathrm{c}}=0\right)$.

El lado del canal, $L_{c}$, el área específica, $\mathrm{a}_{\mathrm{v}}$, la porosidad del monolito, $\varepsilon_{\mathrm{L}}$, el diámetro hidráulico, $\mathrm{d}_{\mathrm{h}}, \mathrm{y}$ la longitud de difusión efectiva en el sólido, $\ell_{e}$, también conocida como longitud característica, se determinan a partir de los valores de $\mathrm{n}_{\mathrm{c}}$, $t$, de la forma del canal y del radio de curvatura $\mathrm{R}_{\mathrm{c}}$. En la Tabla 4 se presentan los valores de las características geométricas seleccionadas para el monolito junto con las expresiones correspondientes a $L_{c}, \mathrm{a}_{\mathrm{v}}, \varepsilon_{\mathrm{L}}, \mathrm{d}_{\mathrm{h}} \mathrm{y} \ell_{e}$.

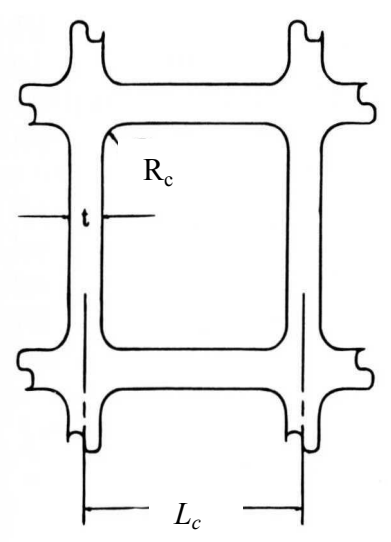

Fig. 1 - Esquema de un canal de sección cuadrada de un monolito

Una vez definidas las características geométricas del monolito, se debieron definir las dimensiones globales del lecho: sección, S, y longitud, L. Para la selección de dichas magnitudes se consideraron ciertos criterios. En primer lugar y al igual que como fuera adoptado en el Capítulo 4, se fijó un valor de $3 \mathrm{~m}$ para L, lo cual está ligado al proceso de desorción, como se justificará posteriormente 
en el Capítulo 6. Por otra parte, para completar la geometría del equipo resulta adecuado establecer una pérdida de carga admisible para el mismo. Esta debe ser moderada, dado que se deben tratar altos caudales, y una pérdida de carga elevada conduciría a un consumo energético elevado en el impulsor que se utilice para todo el sistema de eliminación propuesto (Capítulo 2). Con estas consideraciones, se adoptó como valor máximo, aproximadamente $0.5 \mathrm{~atm}$ para la pérdida de carga admisible $(\Delta \mathrm{P})$ en el adsorbedor. Esto último permite determinar la sección requerida (S). Estos valores también son reportados en la Tabla 4.

Tabla 4: Propiedades geométricas del monolito $\left(t^{*}=t / L_{c}\right)$

\begin{tabular}{c|c}
\hline \multicolumn{2}{c}{ Monolito } \\
\hline forma del canal & Cuadrada \\
$\mathrm{n}_{\mathrm{c}}\left[\right.$ canales $\left./ \mathrm{m}^{2}\right]$ & $2.4810^{6}$ \\
$t[\mathrm{~m}]$ & $45.410^{-6}$ \\
$\mathrm{Rc}[\mathrm{m}]$ & 0 \\
$L_{c}[\mathrm{~m}]=\left(1 / \mathrm{n}_{\mathrm{c}}\right)^{(1 / 2)}$ & $6.3510^{-4}$ \\
$\varepsilon_{\mathrm{L}}=\left(1-t^{*}\right)^{2}$ & 0.73 \\
$\mathrm{a}_{\mathrm{v}}\left[\mathrm{m}_{\text {ads }}^{2} / \mathrm{m}_{\text {ads }}^{3}=4\left(1-t^{*}\right) / L_{c} /\left(1-\varepsilon_{\mathrm{L}}\right)\right.$ & $20.3310^{3}$ \\
$\mathrm{~d}_{\mathrm{h}}[\mathrm{m}]=L_{c}-t$ & $5.4410^{-4}$ \\
$\ell_{e}[\mathrm{~m}]=1 / \mathrm{a}_{\mathrm{v}}$ & $4.9210^{-5}$ \\
$\mathrm{~S}\left[\mathrm{~m}^{2}\right]$ & 2.25 \\
$\mathrm{~L}[\mathrm{~m}]$ & 3 \\
\hline
\end{tabular}

La construcción del lecho puede concebirse como un arreglo modular de unidades monolíticas. Para materiales cerámicos se emplean módulos rectangulares cuyas dimensiones son del orden de los $20 \mathrm{~cm}$ de lado. Los mismos se acomodan de manera tal de alcanzar la sección total deseada del lecho (S) constituyendo un piso cuya profundidad equivale a la longitud de cada módulo del monolito $\left(\Delta Z_{\mathrm{M}}\right)$. Se deben ir agregando pisos en forma seriada hasta obtener la longitud total requerida para el lecho (L).

Con las dimensiones globales definidas, $\mathrm{S}$ y L, la porosidad del lecho $\left(\varepsilon_{\mathrm{L}}\right)$ y la densidad aparente del carbón $\left(\rho_{\text {pap }}\right)$ (Tabla 3 ), se puede determinar la masa de adsorbente. Una vez establecida la masa de adsorbente, a través de la resolución 
de las ecuaciones de balance se obtiene el período correspondiente a la etapa de adsorción. Definimos el período de adsorción como el tiempo requerido para el cual se obtiene a la salida del lecho una concentración de VOCs promedio de 5 ppm (European Comission).

Para la determinación del período de adsorción, se consideró al lecho inicialmente limpio $\left(\mathrm{C}_{1}=0, \mathrm{C}_{2}=0\right)$, a $150{ }^{\circ} \mathrm{C}$ y a presión atmosférica. Los valores correspondientes al período de adsorción, $\mathrm{t}_{\text {ads }}$, masa de adsorbente y pérdida de carga (evaluada con la expresión presentada en el Capítulo 6, Ec.[9]) se presentan en la Tabla 5, para las condiciones de la Tabla 2.

Tabla 5: Pérdida de carga $(\Delta \mathrm{P})$, período de adsorción $\left(\mathrm{t}_{\mathrm{ads}}\right)$ y masa de adsorbente $\left(\mathrm{m}_{\mathrm{ads}}\right)$ para el monolito

\begin{tabular}{l|c}
\hline$\Delta \mathbf{P}[\mathbf{a t m}]$ & 0.4 \\
$\mathbf{t}_{\text {ads }}[\mathbf{s}]$ & 839.9 \\
$\mathbf{m}_{\text {ads }}[\mathbf{k g}]$ & $1.3410^{3}$ \\
\hline
\end{tabular}

Así como se presentó para un lecho relleno al comienzo de la Sección 5.4, para la simulación del monolito debe resolverse un sistema de ecuaciones diferenciales similares a las Ecs. [40] para el interior del sólido conjuntamente con ecuaciones del tipo de las [44] para la corriente de fluido. Para plantear los balances en el interior del material adsorbente resulta factible aproximar la disposición del adsorbente como una placa plana de espesor efectivo $\ell_{e}$ (Tabla 4), resultando ( $c f r$. Ecs. [40]):

$$
\begin{aligned}
& \frac{\partial \mathrm{q}_{\mathrm{k}}\left(\mathrm{C}_{\mathrm{j}, 1}, \mathrm{C}_{\mathrm{j}, 2}, \mathrm{~T}\right)}{\partial \mathrm{t}}=4 \frac{\mathrm{D}_{\mathrm{e}}}{\ell_{e}^{2}} \sum_{\mathrm{i}=1}^{\mathrm{N}}\left(\frac{1}{2} \mathrm{~L}_{\mathrm{i}}^{\prime}\left(\mathrm{u}_{\mathrm{j}}\right)+\mathrm{u}_{\mathrm{i}} \mathrm{L}_{\mathrm{i}}^{\prime \prime}\left(\mathrm{u}_{\mathrm{j}}\right)\right)\left(\mathrm{C}_{\mathrm{j}, \mathrm{k}}-\mathrm{C}_{\mathrm{s}, \mathrm{k}}\right), \mathrm{j}=1, \ldots, \mathrm{N}, \mathrm{k}=1,2 \\
& \frac{\partial \mathrm{q}_{\mathrm{T}}\left(\mathrm{C}_{1}, \mathrm{C}_{2}, \mathrm{~T}\right)}{\partial \mathrm{t}}=4 \frac{\lambda_{\mathrm{p}}}{\ell_{e}^{2}} \sum_{\mathrm{i}=1}^{\mathrm{N}}\left(\frac{1}{2} \mathrm{~L}_{\mathrm{i}}^{\prime}\left(\mathrm{u}_{\mathrm{j}}\right)+\mathrm{u}_{\mathrm{i}} \mathrm{L}_{\mathrm{i}}^{\prime \prime}\left(\mathrm{u}_{\mathrm{j}}\right)\right)\left(\mathrm{T}_{\mathrm{j}}-\mathrm{T}_{\mathrm{s}}\right), \mathrm{j}=1, \ldots, \mathrm{N}
\end{aligned}
$$

donde $\mathrm{C}_{\mathrm{s}, \mathrm{k}}$ y $\mathrm{T}_{\mathrm{s}}$ se pueden expresar a partir de las condiciones de contorno como:

$$
\mathrm{C}_{\mathrm{s}, \mathrm{k}}=\frac{\mathrm{B}_{\mathrm{i}, \mathrm{k}} \mathrm{C}_{\mathrm{g}, \mathrm{k}}-\sum_{\mathrm{i}=1}^{\mathrm{N}} \mathrm{L}_{\mathrm{i}}^{\prime}(1) \mathrm{C}_{\mathrm{i}, \mathrm{k}}}{\mathrm{B}_{\mathrm{i}, \mathrm{k}}+\Omega}, \mathrm{k}=1,2
$$




$$
\mathrm{T}_{\mathrm{s}}=\frac{\mathrm{B}_{\mathrm{i}, \mathrm{c}} \mathrm{T}_{\mathrm{g}}-\sum_{\mathrm{i}=1}^{\mathrm{N}} \mathrm{L}_{\mathrm{i}}^{\prime}(1) \mathrm{T}_{\mathrm{i}}}{\mathrm{B}_{\mathrm{i}, \mathrm{c}}+\Omega}
$$

donde $\Omega=-\sum_{\mathrm{i}=1}^{\mathrm{N}} \mathrm{L}_{\mathrm{i}}^{\prime}(1), \mathrm{B}_{\mathrm{i}, \mathrm{k}}=\frac{\ell_{e} \mathrm{k}_{\mathrm{m}, \mathrm{k}}}{2 \mathrm{D}_{\mathrm{e}, \mathrm{k}}}, \mathrm{k}=1,2, \mathrm{y} \mathrm{B}_{\mathrm{i}, \mathrm{c}}=\frac{\ell_{e} \mathrm{~h}_{\mathrm{t}}}{2 \lambda_{\mathrm{p}}}$.

Se debe mencionar que, para el monolito, los puntos de colocación $\mathrm{u}_{\mathrm{j}}$ difieren de los del lecho relleno porque los polinomios de Jacobi $\mathrm{P}_{\mathrm{N}}$ para el caso de una placa plana son distintos que para una esfera $\left(\gamma^{*}=-1 / 2\right.$ en la Ec. [3]).

Teniendo en cuenta la aplicación del Método Cuasi-Analítico, los balances de conservación en la corriente de fluido para el monolito resultan idénticos a los presentados en las Ecs. [44], teniendo en cuenta, obviamente, las características geométricas y parámetros de transporte propios del monolito.

\subsubsection{Características geométricas del lecho relleno}

Con el fin de lograr una comparación consistente entre el monolito (M) y el lecho relleno (LR), las dimensiones del lecho relleno se seleccionaron de manera tal de que el valor de la pérdida de carga y el tiempo de adsorción fuesen del mismo orden en ambos sistemas. Además, se tomó una cota máxima de sección de $10 \mathrm{~m}^{2}$, de manera tal de evitar problemas de distribución de flujo y teniendo en cuenta que los costos de los cabezales para secciones mayores pueden resultar muy elevados. Considerando los criterios establecidos se pudieron definir la sección, longitud del lecho y el diámetro de pastilla $\left(\mathrm{d}_{\mathrm{p}}\right)$, para la cual se adoptó una geometría esférica. En la Tabla 6 se presentan las características geométricas que definen al lecho relleno. Los valores correspondientes al período de adsorción, $\mathrm{t}_{\mathrm{ads}}$, masa de adsorbente $\left(\mathrm{m}_{\mathrm{ads}}\right)$ y pérdida de carga $(\Delta \mathrm{P})$ (evaluada con la expresión propuesta por Ergun, 1952) se presentan en la Tabla 7.

Las simulaciones de ambos lechos se realizaron para dos puntos de colocación $(\mathrm{N}=2)$ en el interior del sólido. El hecho de haber considerado $\mathrm{N}=2$ se justificará posteriormente en la Sección 5.4.3. Dado que la variación de las propiedades del fluido con la temperatura no eran significativas, en todas las simulaciones se evaluaron las propiedades del fluido a su temperatura de alimentación. 
Tabla 6: Características geométricas del lecho relleno

\begin{tabular}{c|c}
\hline \multicolumn{2}{c}{ Lecho Relleno } \\
\hline$\varepsilon_{\mathrm{L}}$ & 0.4 \\
$\mathrm{~d}_{\mathrm{p}}[\mathrm{m}]$ & $110^{-3}$ \\
$\mathrm{a}_{\mathrm{v}}\left[\mathrm{m}^{2}{ }_{\text {ads }} / \mathrm{m}^{3}{ }_{\mathrm{ads}}\right]=6 / \mathrm{d}_{\mathrm{p}}$ & $610^{3}$ \\
$\mathrm{~d}_{\mathrm{h}}[\mathrm{m}]=4 \mathrm{~d}_{\mathrm{p}} \varepsilon_{\mathrm{L}} /\left[6\left(1-\varepsilon_{\mathrm{L}}\right)\right]$ & $4.4410^{-4}$ \\
$\ell_{e}[\mathrm{~m}]=1 / \mathrm{a}_{\mathrm{v}}$ & $1.6710^{-4}$ \\
$\mathrm{~S}\left[\mathrm{~m}^{2}\right]$ & 10 \\
$\mathrm{~L}[\mathrm{~m}]$ & 0.6 \\
\hline
\end{tabular}

Tabla 7: Pérdida de carga $(\Delta \mathrm{P})$, período de adsorción $\left(\mathrm{t}_{\mathrm{ads}}\right)$ y masa de adsorbente $\left(\mathrm{m}_{\mathrm{ads}}\right)$ para el lecho relleno

\begin{tabular}{l|c}
\hline$\Delta \mathbf{P}[\mathbf{a t m}]$ & 0.26 \\
$\mathbf{t}_{\text {ads }}[\mathbf{s}]$ & 808.11 \\
$\mathbf{m}_{\text {ads }}[\mathbf{k g}]$ & $2.7010^{3}$ \\
\hline
\end{tabular}

\subsubsection{Monolitos vs. Lecho Relleno: efecto de las resistencias internas y externas}

Analizando las Tablas 5 y 7, se tiene que, para tiempos de adsorción prácticamente similares, se requiere aproximadamente el doble de masa de carbón activado para un lecho relleno (LR) que para un monolito (M). Esto se debe principalmente a la disposición del material adsorbente, resultando para el monolito una longitud característica de la pared de adsorbente $\left(l_{e}\right)$ tres veces más pequeña que el valor correspondiente a la del lecho relleno, como se puede observar en las Tablas 4 y 6 . Esta diferencia en la longitud característica implica que la resistencia a la transferencia de materia en el sólido adsorbente sea mayor en el lecho relleno.

En la Sección 5.2.1, referida al caso particular de considerar un punto de colocación, se definió el coeficiente global de transferencia de materia $\mathrm{K}^{*}$ (Ec. [22]) correspondiente a la adsorción/desorción de un único componente en un lecho relleno isotérmico. Para un sistema multicomponente adiabático se debe considerar un $\mathrm{K}_{\mathrm{i}}^{*}$ para cada componente $\mathrm{i}$, y un coeficiente global de transferencia de energía $\mathrm{K}_{\mathrm{T}}^{*}$. Las expresiones correspondientes a $\mathrm{K}_{\mathrm{i}}^{*} \mathrm{y}_{\mathrm{T}}^{*}$ se adaptan según el tipo de lecho 
(LR, M), las mismas se encuentran en la Tabla 8. Los coeficientes de transferencia de materia, $\mathrm{k}_{\mathrm{m}, \mathrm{i}}, \mathrm{y}$ de transferencia de calor, $\mathrm{h}_{\mathrm{t}}$, en la corriente de fluido se determinan a partir de las expresiones presentadas en el Apéndice 5.2 según el tipo de lecho.

Tabla 8: Coeficientes globales de transferencia de materia y energía para $\mathrm{N}=1$ según el tipo de lecho

(M: $d=\ell_{e}$ y b $=3$, LR: $\left.d=\mathrm{r}_{\mathrm{p}} \mathrm{y} \mathrm{b}=15\right)\left(50^{\circ} \mathrm{C}, 1 \mathrm{~atm}\right)$

\begin{tabular}{l|cc}
\hline Tipo de Lecho & Monolito (M) & Lecho Relleno (LR) \\
$\mathbf{K}_{1}^{*}[\mathbf{1} / \mathbf{s}]$ & 390.59 & 27.97 \\
$\mathbf{K}_{2}^{*}[\mathbf{1} / \mathbf{s}]$ & 540.36 & 38.60 \\
$\mathbf{K}_{\mathrm{T}}^{*}\left[\mathbf{W} / \mathbf{K m}^{3}\right]$ & $3.0110^{6}$ & $2.7410^{6}$ \\
\hline $\mathrm{K}_{\mathrm{k}}^{*}=\frac{1}{\frac{1}{\mathrm{a}_{\mathrm{v}} \mathrm{k}_{\mathrm{m}, \mathrm{k}}}+\frac{d^{2}}{\mathrm{~b} \mathrm{D}}}, \mathrm{k}=1,2$ & $\mathrm{~K}_{\mathrm{T}, \mathrm{k}}^{*}=\frac{1}{\frac{1}{\mathrm{a}_{\mathrm{v}} \mathrm{h}_{\mathrm{T}}}+\frac{d^{2}}{\mathrm{~b} \lambda_{\mathrm{p}}}}$ \\
\hline
\end{tabular}

En la Tabla 8 se presentan los valores correspondientes a los coeficientes globales de transferencia de calor $\left(\mathrm{K}_{\mathrm{T}}^{*}\right)$ y materia $\left(\mathrm{K}_{\mathrm{i}}^{*}, \mathrm{i}=1,2\right)$ a la temperatura y presión de la corriente a tratar $\left(50{ }^{\circ} \mathrm{C}, 1 \mathrm{~atm}\right)$ para ambos tipos de lechos (LR y M). Los valores obtenidos demuestran que la resistencia a la transferencia de materia resulta un orden de magnitud mayor para el lecho relleno, tanto para el acetato de etilo como para el etanol. Vale la pena mencionar que para la resistencia a la transferencia de materia global $\left(1 / \mathrm{K}^{*}\right)$, el término dominante es el correspondiente a la resistencia dentro del sólido. Por otro lado, la resistencia a la transferencia de calor resulta del mismo orden para ambos lechos.

El efecto de las resistencias a la transferencia de materia, dentro y fuera del sólido adsorbente, puede apreciarse gráficamente en las Figuras 2 y 3 para el monolito y lecho relleno respectivamente. En las mismas se representan las concentraciones $C_{1, i}$ y $\mathrm{C}_{2, \mathrm{i}}$, para $\mathrm{i}=1,2$, del acetato de etilo (1) y el etanol (2) en los distintos puntos de colocación dentro del sólido adsorbente, siendo el punto 1 más interno que el punto 2, así como las concentraciones de cada componente en la superficie del sólido y el fluido, a lo largo del adsorbedor. Los perfiles representados en ambas figuras, corresponden a la finalización de la etapa de adsorción $\left(t=t_{a d s}\right)$. 


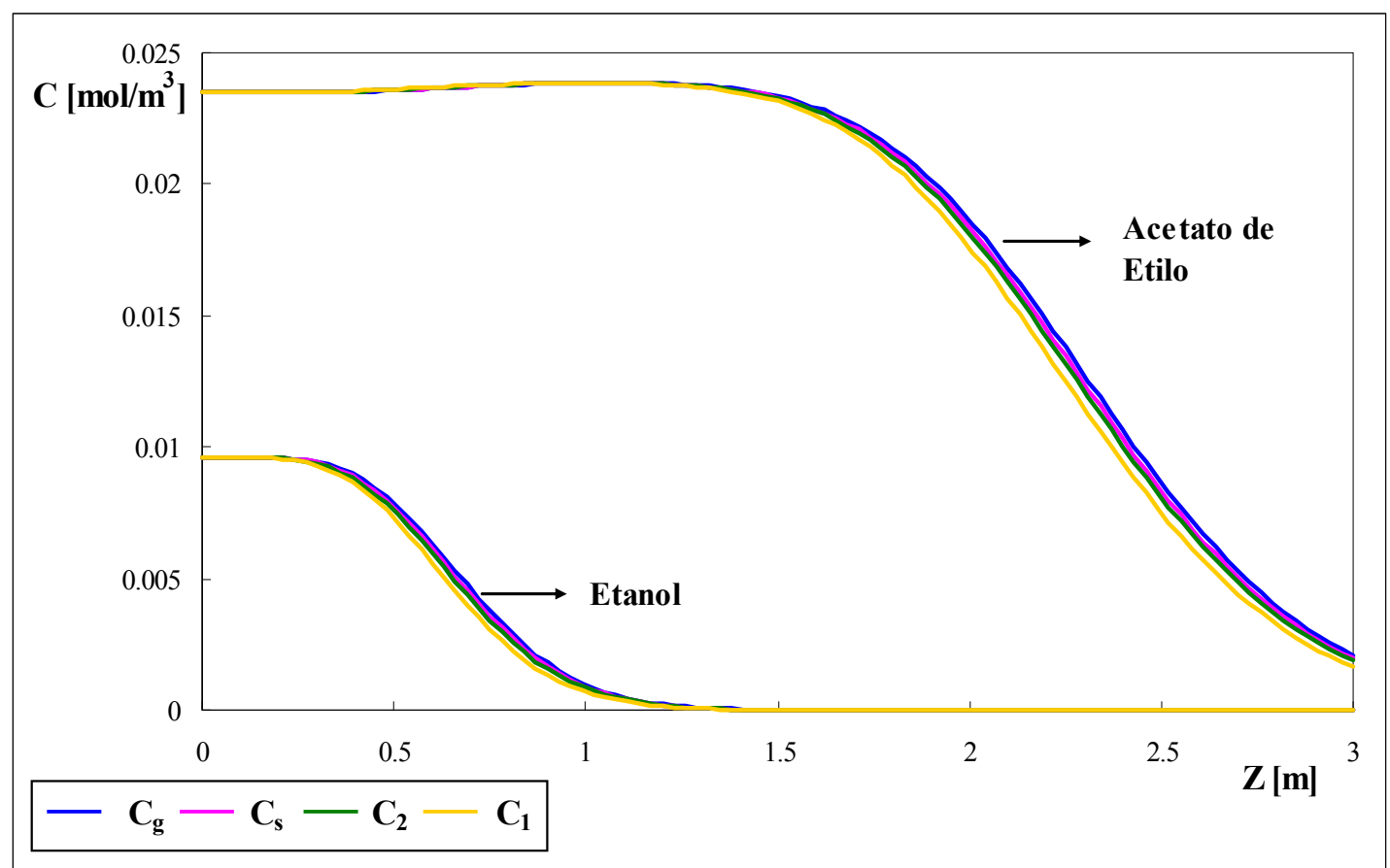

Fig. 2 - Perfiles de concentración en los canales, superficie del sólido adsorbente y puntos de colocación en el monolito al finalizar la etapa de adsorción.

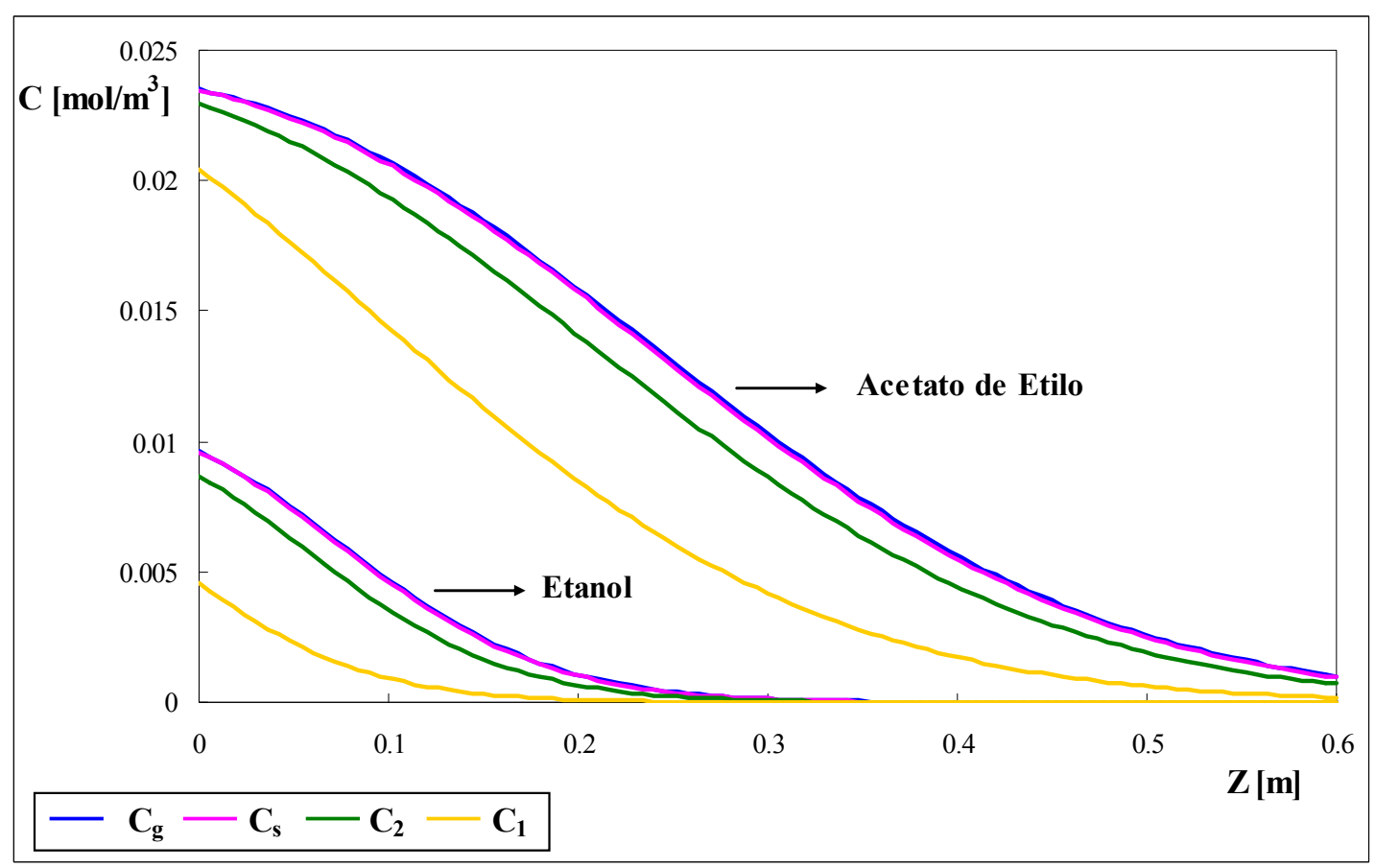

Fig. 3 - Perfiles de concentración en la corriente de fluido, superficie del sólido adsorbente y puntos de colocación en el lecho relleno al finalizar la etapa de adsorción.

Para el monolito (Fig. 2) podemos observar que las curvas correspondientes a cada uno de los componentes se encuentran muy próximas entre sí, lo que sugiere que las resistencias a la transferencia de materia dentro y fuera del sólido adsorbente no resultan significativas. No obstante, estas mínimas diferencias en los perfiles son 
suficientes para generar una dispersión del frente de avance de la concentración, afectando el período de adsorción.

Para el lecho relleno (Fig. 3), las curvas correspondientes a la concentración en el lecho $\left(\mathrm{C}_{\mathrm{g}}\right)$ y superficie $\left(\mathrm{C}_{\mathrm{s}}\right)$ prácticamente se superponen para ambos componentes, sin embargo, las correspondientes a la concentración en los puntos de colocación en el interior del sólido se diferencian claramente, por lo que el impacto de las resistencias resulta mayor que para el monolito, generando, por otra parte, una mayor dispersión del frente de avance de concentración.

Este efecto también puede apreciarse en la Figura 4 donde se adimensionalizó la variable espacial $\mathrm{Z}$ con el fin de obtener una comparación gráfica más efectiva de los perfiles de concentración para los dos casos (M y LR). A diferencia de las Fig. 2 y 3 se representa la concentración media $\mathrm{C}_{\mathrm{m}, \mathrm{i}}=\omega_{1} \mathrm{C}_{1, \mathrm{i}}+\omega_{2} \mathrm{C}_{2, \mathrm{i}}, \mathrm{i}=1,2$, donde $\omega_{1} \mathrm{y} \omega_{2}$ son los factores de peso (ver Apéndice 5.1 para la determinación de los mismos). Debido a la mayor dispersión del frente de avance de concentración obtenido en el lecho relleno, se tiene que ningún sector del mismo se encuentra saturado al finalizar la etapa de adsorción, mientras que una gran porción del monolito si lo está. Esto implica que el lecho relleno utiliza un porcentaje bastante menor de adsorbente en comparación al monolito, lo que lo hace menos eficiente.

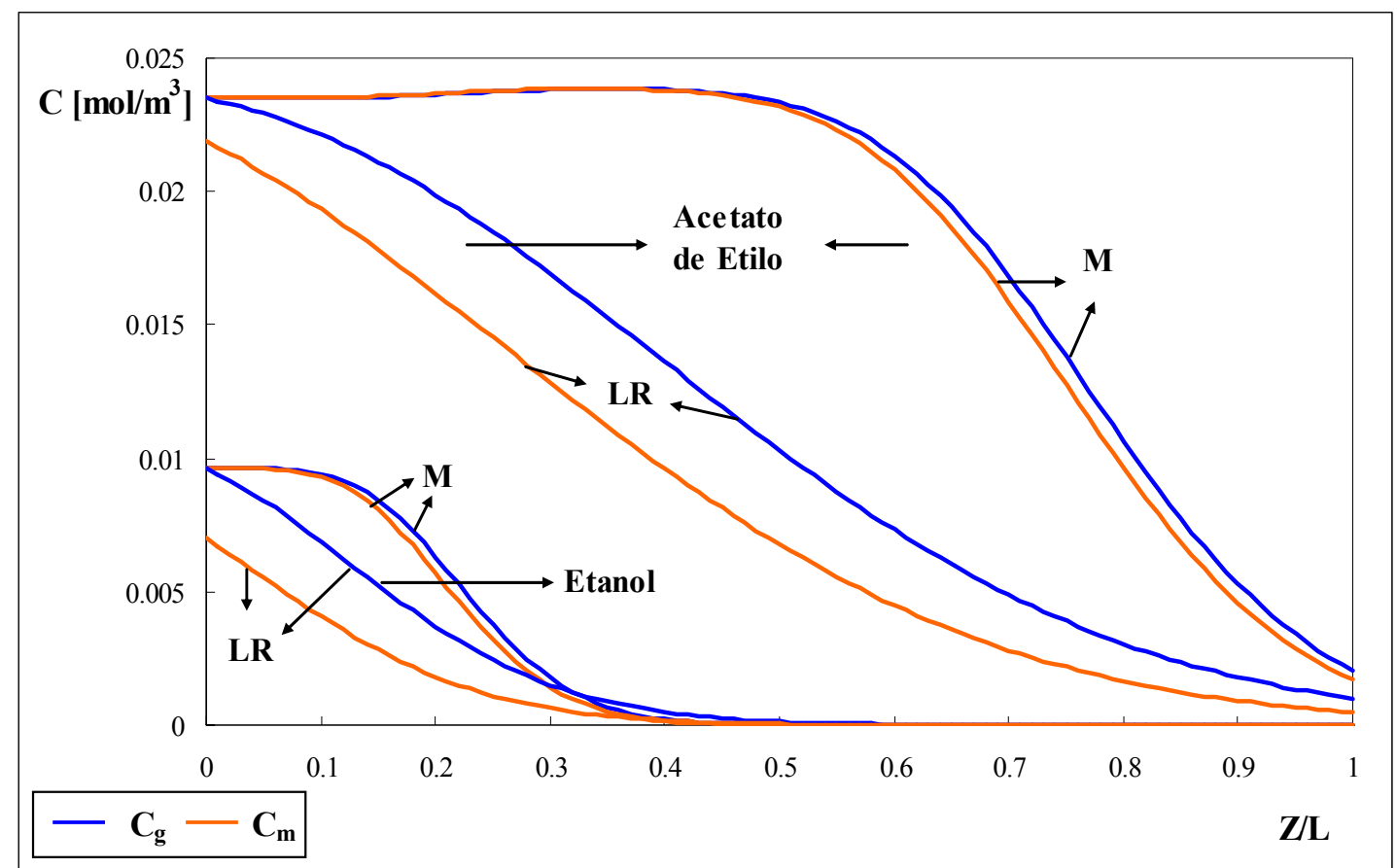

Fig. 4 - Perfiles de concentración en el lecho y concentración media dentro del sólido adsorbente $\left(\mathrm{C}_{\mathrm{m}, \mathrm{i}}=\omega_{1} \mathrm{C}_{1, \mathrm{i}}+\omega_{2} \mathrm{C}_{2, \mathrm{i}}\right)$ del acetato de etilo (1) y etanol (2) en el monolito (M) y lecho relleno (LR) al finalizar la etapa de adsorción. 
En la Figura 5 se presentan los perfiles de temperatura, tanto para el monolito como para el lecho relleno, a los 30 segundos de la etapa de adsorción. Si bien se ha buscado representar los perfiles correspondientes al seno del fluido, la superficie del sólido y los puntos de colocación dentro del mismo, todas las curvas prácticamente colapsan en una sola. Esto sugiere que las resistencias a la transferencia de calor dentro y fuera del sólido adsorbente no son significativas. Al comparar los dos sistemas, se aprecia que el perfil de temperatura avanza a mayor velocidad en el monolito que en el lecho relleno. Esto se debe a la velocidad intersticial con la que el fluido atraviesa el adsorbedor $\left(u_{\mathrm{M}}=7.1 \mathrm{~m} / \mathrm{s}-u_{\mathrm{LR}}=2.9 \mathrm{~m} / \mathrm{s}\right)$ y a la cantidad de masa de sólido adsorbente que se debe enfriar por unidad de longitud. Ambos efectos son adversos para el lecho relleno, donde, como ya se mencionó, la masa de sólido adsorbente es prácticamente el doble que para el monolito y la velocidad intersticial del fluido es menos de la mitad. Estas diferencias están fuertemente determinadas por la imposición establecida de alcanzar una pérdida de carga similar en ambos sistemas.

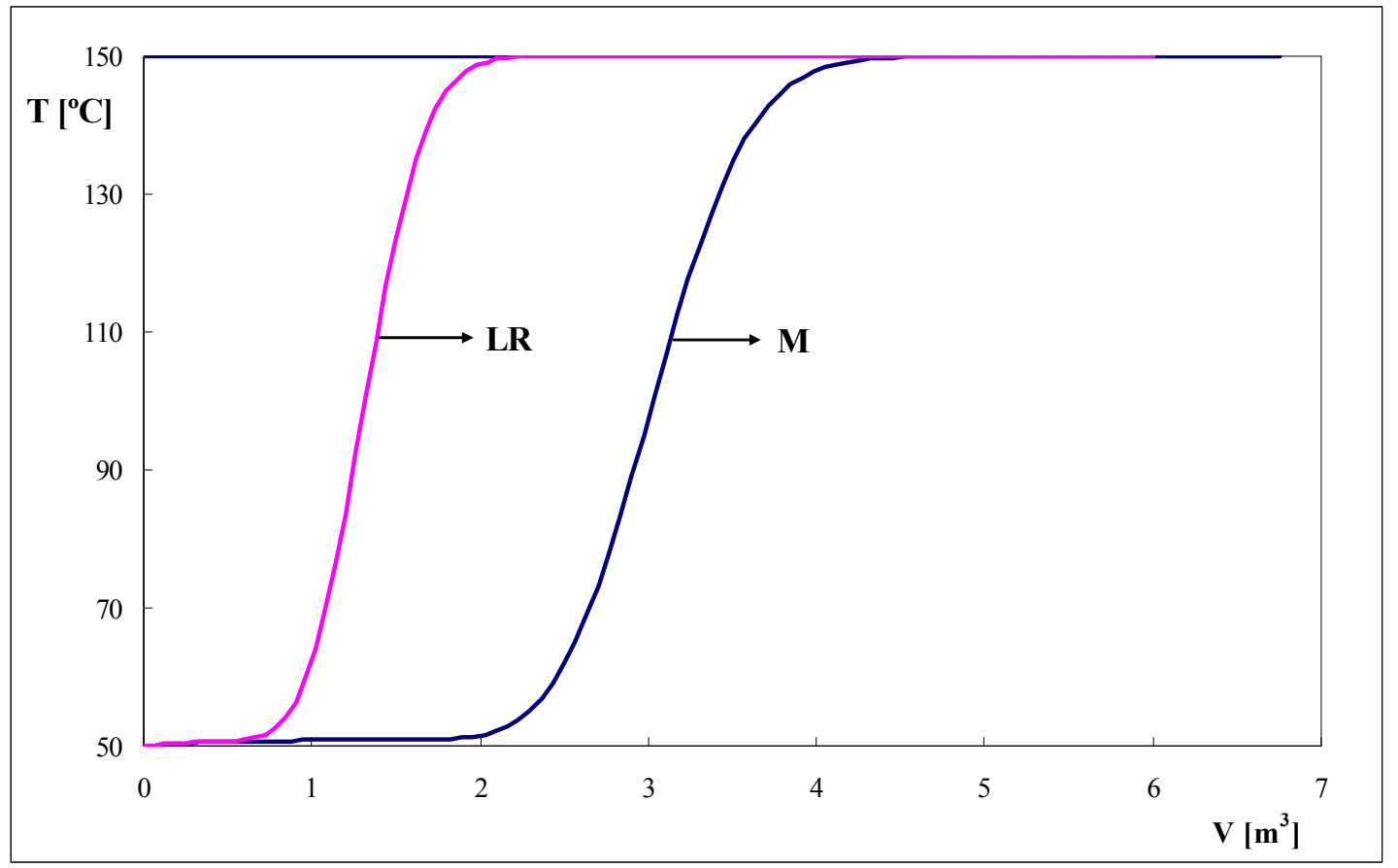

Fig. 5 - Perfiles de temperatura en el monolito (M) y lecho relleno (LR) a los $30 \mathrm{~s}$ de la etapa de adsorción.

Teniendo en cuenta los efectos que la disposición del material adsorbente genera sobre la cantidad de carbón requerida (Tabla 5 y 7), las resistencias a la transferencia de materia (Tabla 8), y el grado de saturación del lecho una vez finalizada la etapa de 
adsorción, resulta conveniente optar por un lecho monolítico para el sistema de eliminación propuesto en el Capítulo 2. Además, analizando las dimensiones globales de los lechos (Tabla 4 y 6), es esperable que el monolito permita una distribución de flujo más eficiente, al ser su sección $\left(2.25 \mathrm{~m}^{2}\right)$ prácticamente una cuarta parte de la correspondiente al lecho relleno $\left(10 \mathrm{~m}^{2}\right)$.

\subsubsection{Monolito vs. Lecho Relleno: efecto del número de puntos de colocación}

Además del efecto de las resistencias a la transferencia de calor y materia dentro y fuera del sólido adsorbente, se analizó la influencia de la elección del número de puntos de colocación según el tipo de lecho.

Se ensayó con 1 a 5 puntos de colocación para ambos tipos de lecho cuyas características fueron establecidas previamente en las Tablas 3, 4 y 6. Para el lecho relleno, a tiempos relativamente bajos (del orden de los minutos), se observaron diferencias al cambiar el número de puntos de colocación (Figura 6), sobre todo al pasar de 1 a 2 puntos. A medida que el número de puntos de colocación aumenta estas diferencias se vuelven prácticamente insignificantes $\mathrm{y}$, a tiempos suficientemente altos, las mismas desaparecen, haciendo indistinto el número de puntos de colocación considerados para la simulación. Esto se debe al abrupto perfil de concentración que surge por efecto de la condición inicial en el interior de la pastilla, al exponerla a la corriente de proceso con una concentración diferente. Para el monolito este efecto es prácticamente despreciable haciendo indistinto la elección del número de puntos de colocación para su simulación (Figura 7). 


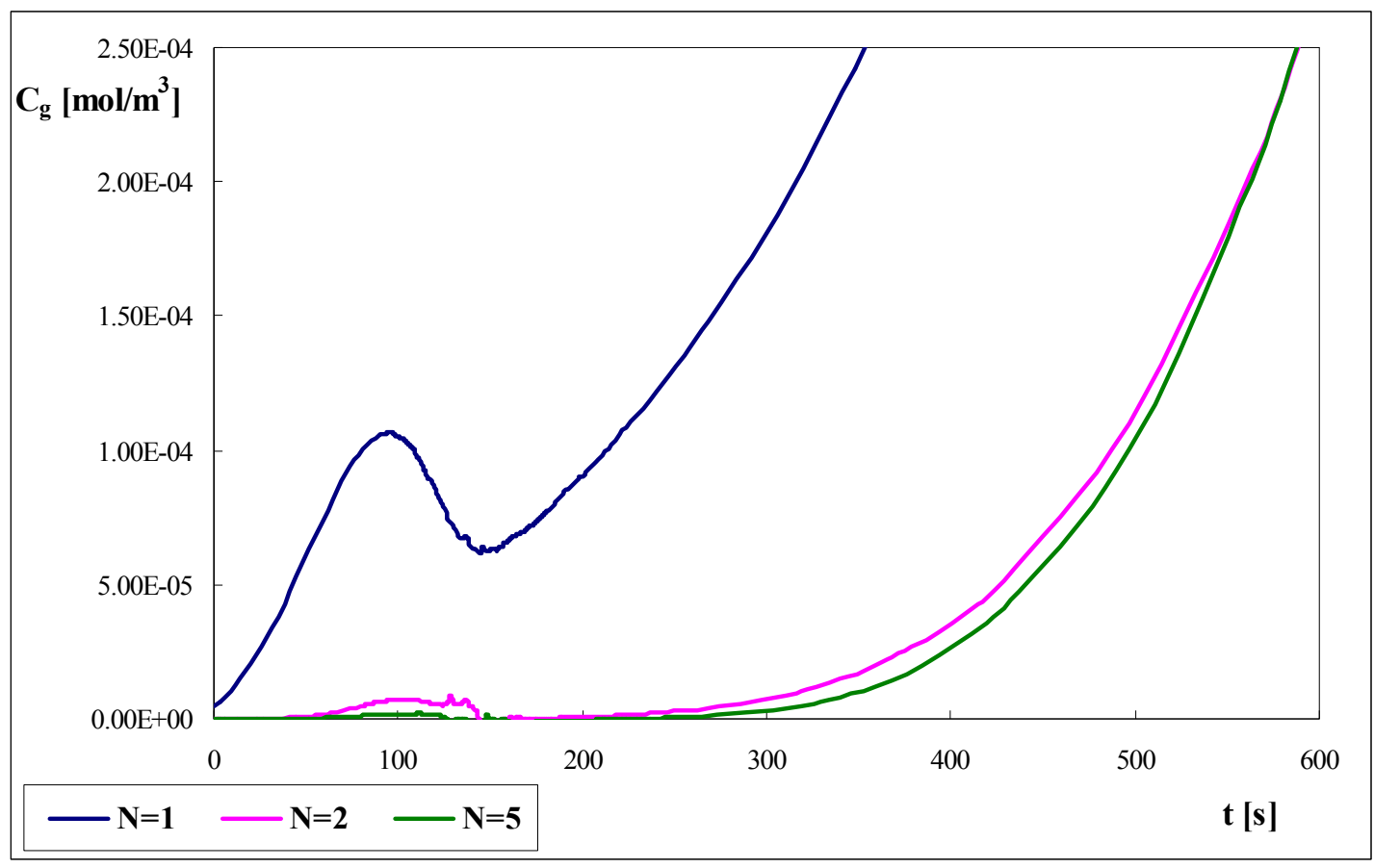

Fig. 6 - Concentración total de VOCs a la salida del lecho relleno (Tabla 6) durante la etapa de adsorción según el número de puntos de colocación seleccionado para la simulación.

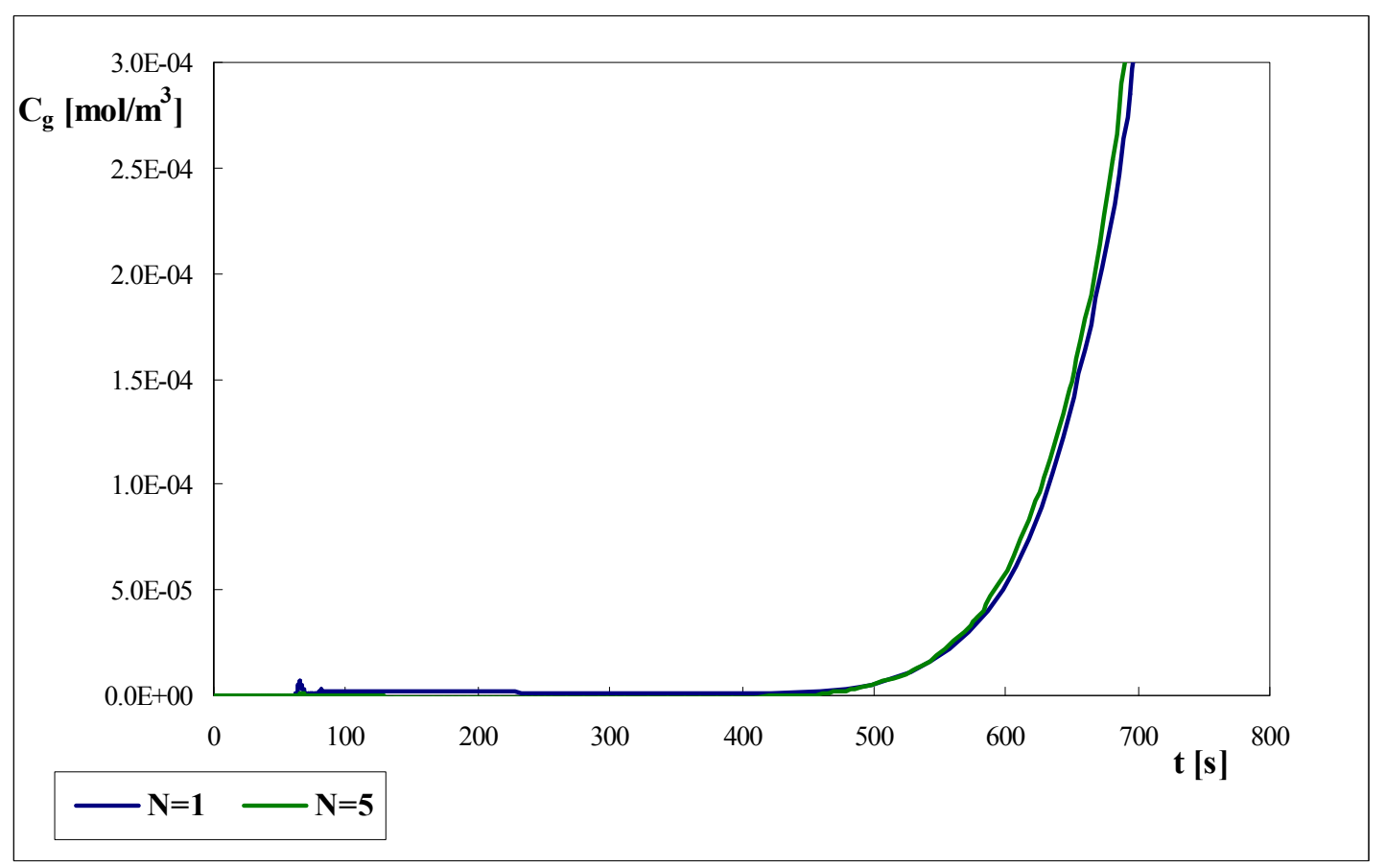

Fig. 7 - Concentración total de VOCs a la salida del monolito (Tabla 4) durante la etapa de adsorción según el número de puntos de colocación seleccionado para la simulación.

Para una sección de lecho determinada, la longitud total del mismo tiene incidencia en la elección del número de puntos de colocación. En las Figuras 8 y 9 , puede observarse dicho efecto sobre el período de adsorción, $t_{a d s}$, para el lecho relleno (condiciones en Tabla 6) y el monolito (condiciones en Tabla 4), 
respectivamente. Para el lecho relleno este efecto resulta ser más significativo que para el monolito.

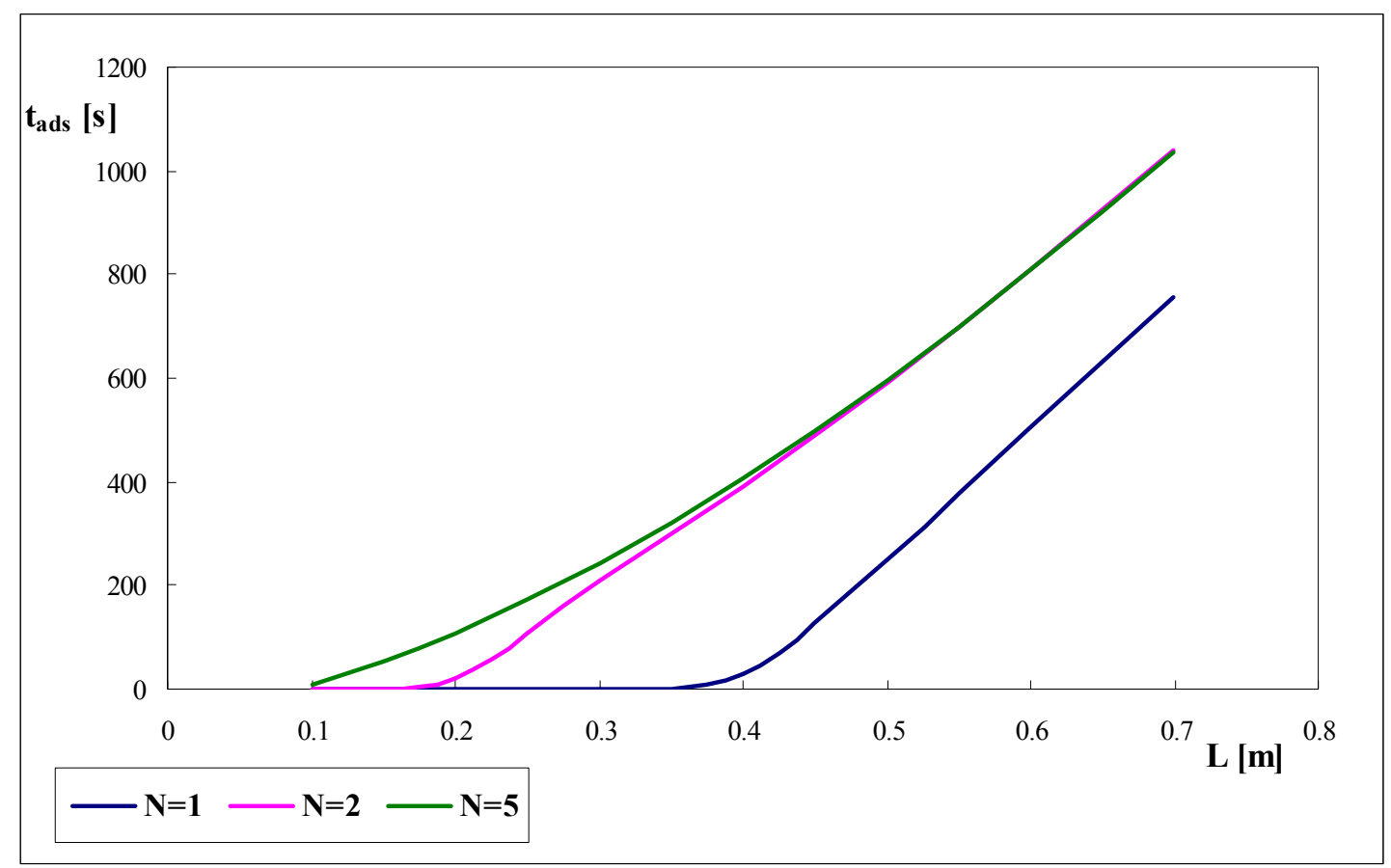

Fig. 8 - Influencia de la elección del número de puntos de colocación según el largo total del adsorbedor correspondiente al lecho relleno de características de la Tabla 6.

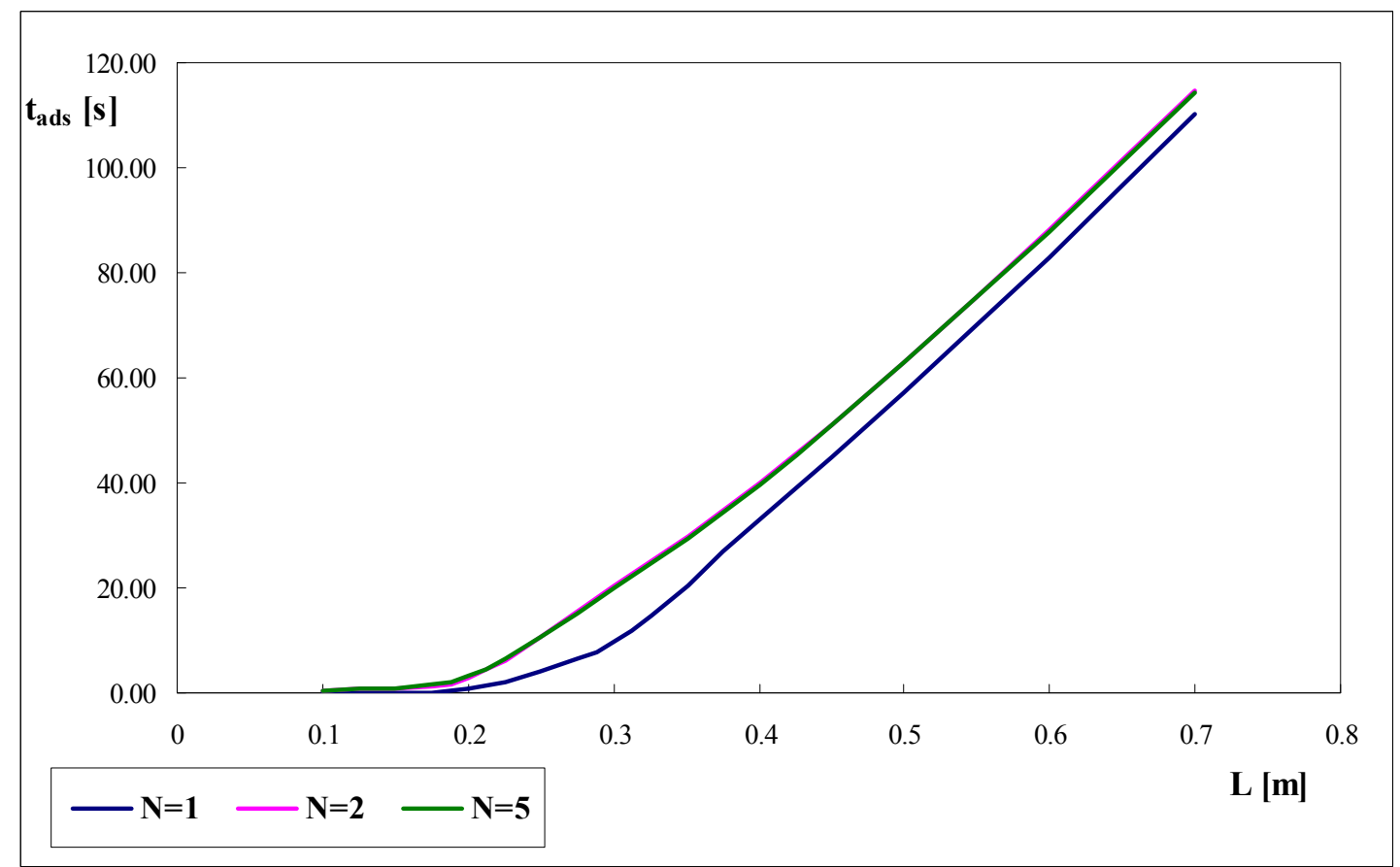

Fig. 9 - Influencia de la elección del número de puntos de colocación según el largo total del adsorbedor correspondiente a un monolito de características de la Tabla 4. 
Nuevamente la mayor diferencia se encuentra al pasar de 1 a 2 puntos de colocación, sin embargo a medida que la longitud del lecho aumenta esta diferencia se hace cada vez menor, resultando para el monolito propuesto $(\mathrm{L}=3 \mathrm{~m})$, indistinto el número de puntos de colocación considerados para su simulación. No sucede lo mismo para el lecho relleno cuya longitud es de $0.6 \mathrm{~m}$ donde todavía se aprecian diferencias entre la elección de 1 y más puntos de colocación. Por este motivo es que, para la comparación de resultados (Figs. 2 - 5) en la Sección 5.4.2, se realizaron las simulaciones correspondientes a cada lecho para 2 puntos de colocación.

Hasta ahora se analizó el efecto de la elección del número de puntos de colocación para la simulación de los lechos durante la etapa de adsorción, a continuación se analiza dicho efecto para la simulación de los mismos durante la etapa de desorción. Para este análisis se considera que inicialmente ambos lechos se encuentran completamente saturados $\left(\mathrm{C}_{1}=2.35 \times 10^{-2} \mathrm{~mol} / \mathrm{m}^{3}\right.$ y $\mathrm{C}_{2}=9.63 \times 10^{-3} \mathrm{~mol} / \mathrm{m}^{3}$ a $\mathrm{T}=50^{\circ} \mathrm{C}$ ) y se los desorbe mediante la circulación de una corriente de aire puro a $150{ }^{\circ} \mathrm{C}$ cuyo caudal volumétrico es igual al de la corriente a tratar durante la etapa de adsorción. En la Figura 10 se puede observar la concentración de VOCs total a la salida del lecho relleno en función del tiempo de desorción. Si bien se observan diferencias al pasar de 1 a 2 puntos de colocación a tiempos relativamente bajos, el efecto resulta ser menos importante que el que fue observado durante la etapa de adsorción. Por su parte, para el monolito no se observan diferencias al cambiar el número de puntos de colocación. Por lo tanto concluimos que sigue resultando apropiado realizar las simulaciones correspondientes a cada lecho para 2 puntos de colocación.

Según el análisis de los resultados obtenidos es factible concluir que el número de puntos de colocación seleccionados para la simulación de los lechos depende del tipo de lecho, relleno o monolito, y dimensiones del mismo. Sin embargo la elección de $\mathrm{N}=2$ permite una precisión razonable para todas las condiciones analizadas. 


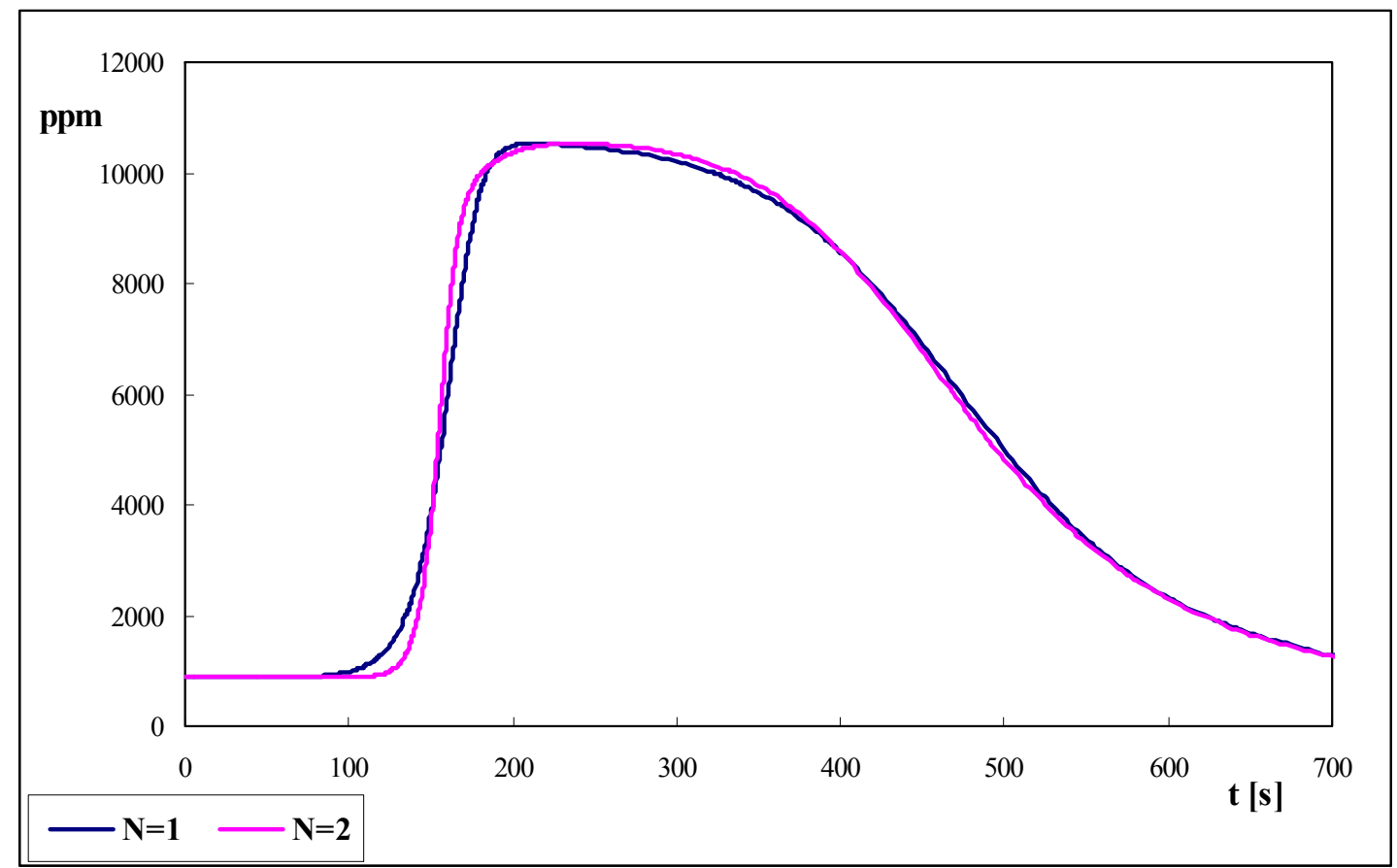

Fig. 10 - Concentración total de VOCs a la salida del lecho relleno (Tabla 6) durante la etapa de desorción según en número de puntos de colocación seleccionado para la simulación.

\subsection{Efecto de las resistencias externas e internas a la transferencia de calor y materia}

En la Sección 5.4.2 (Figura 2) se observó que para el monolito de características establecidas (Sección 5.4.1.2, Tabla 4), las resistencias a la transferencia de calor y materia dentro y fuera del sólido adsorbente generan una dispersión en el frente de avance de concentración. En esta sección se compararán dichos perfiles con los obtenidos al considerar al sistema sin resistencias, con el objetivo de analizar la significación de las mismas.

Para el caso sin resistencias, que identificaremos como caso ideal, se empleó el Método de las Características para un sistema adiabático de dos componentes, como el que se encuentra desarrollado en la Sección 4.3.

Para la comparación se consideró el mismo lecho monolítico definido en la Tabla 4 (Sección 5.4.1.2) y las condiciones operativas de la corriente de alimentación definidas en la Tabla 2 (Sección 5.4.1). Se consideró al lecho inicialmente limpio, a $150{ }^{\circ} \mathrm{C}$ y $1 \mathrm{~atm}$.

En las Figuras 11 y 12 se presentan los perfiles de concentración en la fase fluida $\left(\mathrm{C}_{\mathrm{g}}\right)$ del acetato de etilo y etanol a distintos tiempos de adsorción. En la Fig. 11 el tiempo de adsorción corresponde al tiempo en el que se evidencia la máxima 
concentración promedio de VOCs tolerable a la salida para el caso real $\left(\mathrm{t}_{\mathrm{ads}}=839.9 \mathrm{~s}\right)$, mientras que en la Fig. 12 el tiempo de adsorción se corresponde con el tiempo en que el perfil de concentración del acetato de etilo, para un caso ideal, alcanza la salida del lecho $\left(\mathrm{t}_{\mathrm{ads}}=1087.5 \mathrm{~s}\right)$. Esta diferencia de períodos (23\% apróx.) se debe a la influencia de la resistencia a la transferencia de materia dentro y fuera del sólido, que provoca la dispersión del frente de avance de la concentración $y$, en consecuencia, que la concentración tolerada a la salida se alcance más rápido.

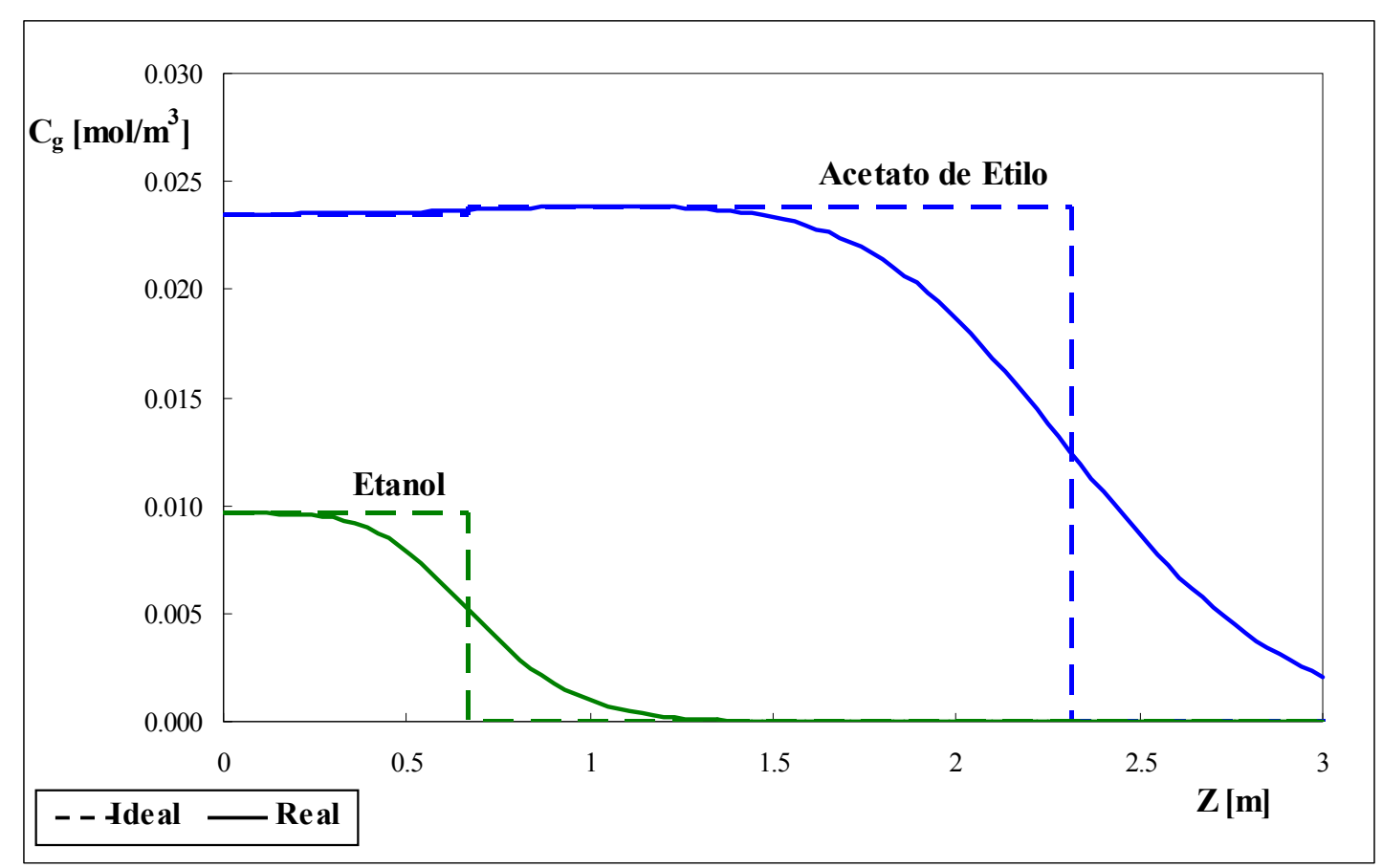

Fig. 11 - Perfiles de concentración de acetato de etilo y etanol a los 839.9 s de la etapa de adsorción del monolito. 


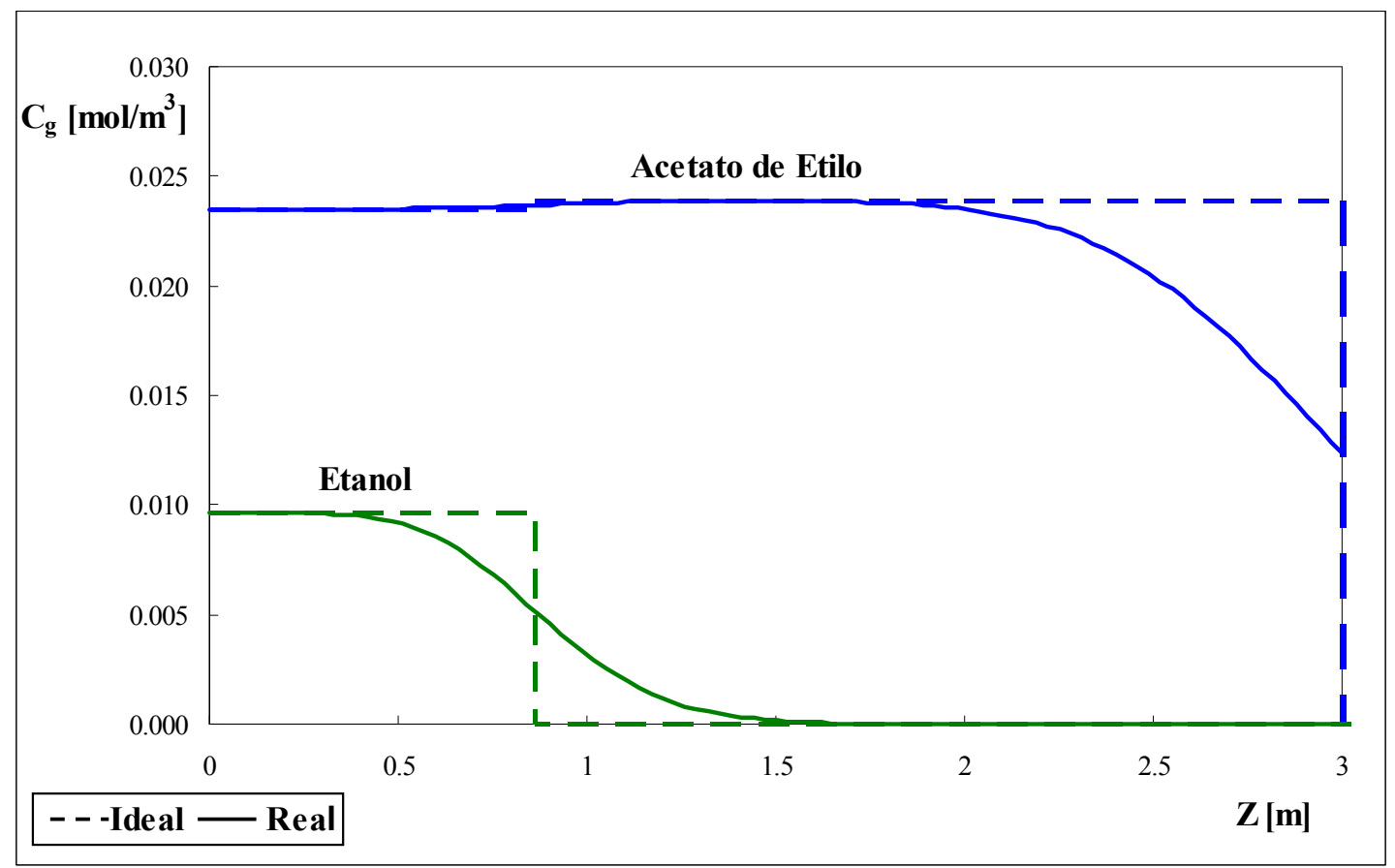

Fig. 12 - Perfiles de concentración de acetato de etilo y etanol a los $1087.5 \mathrm{~s}$ de la etapa de adsorción del monolito.

Los perfiles de temperatura correspondientes al caso real son prácticamente coincidentes con los del caso ideal; por esta razón no han sido representados. Asimismo, y dado el comportamiento observado en el Capítulo 4 para el caso ideal, para los tiempos indicados en las Figs. 11 y 12 el lecho ya ha alcanzado una temperatura uniforme, idéntica a la de la corriente de alimentación.

Adoptando como condición inicial el estado en el que resulta el lecho para el caso ideal luego de una etapa de adsorción de 1087.5 s, se lo desorbe con una corriente a $150{ }^{\circ} \mathrm{C}$ de aire puro con un caudal de $3.31 \mathrm{~m}^{3} / \mathrm{s}(u=2 \mathrm{~m} / \mathrm{s}$, ver Capítulo 4$)$. Los resultados de las simulaciones considerando, o no, las resistencias a la transferencia de calor y materia se presentan en la Figura 13. Se vuelve a comprobar que la diferencia en los tiempos en que se obtiene una desorción completa del lecho está relacionada con las resistencias a la transferencia materia.

Más allá de las diferencias en los tiempos requeridos, tanto para la etapa de adsorción como para la desorción completa del lecho, es importante destacar la similitud entre los perfiles de concentración (Figs. 11, 12 y 13) de ambos compuestos y en ambos procesos. Esta similitud también se aprecia en los perfiles de temperatura (ver Figura 14). Esto permite confirmar que para el tipo de lecho considerado, y tiempos relativamente altos de adsorción, cuando las resistencias a la transferencia de materia dentro y fuera del sólido tienen un peso poco significativo sobre los tiempos de 
adsorción/desorción (23\% aproximadamente), es posible adoptar el caso ideal como una buena aproximación para simular el comportamiento del sistema.

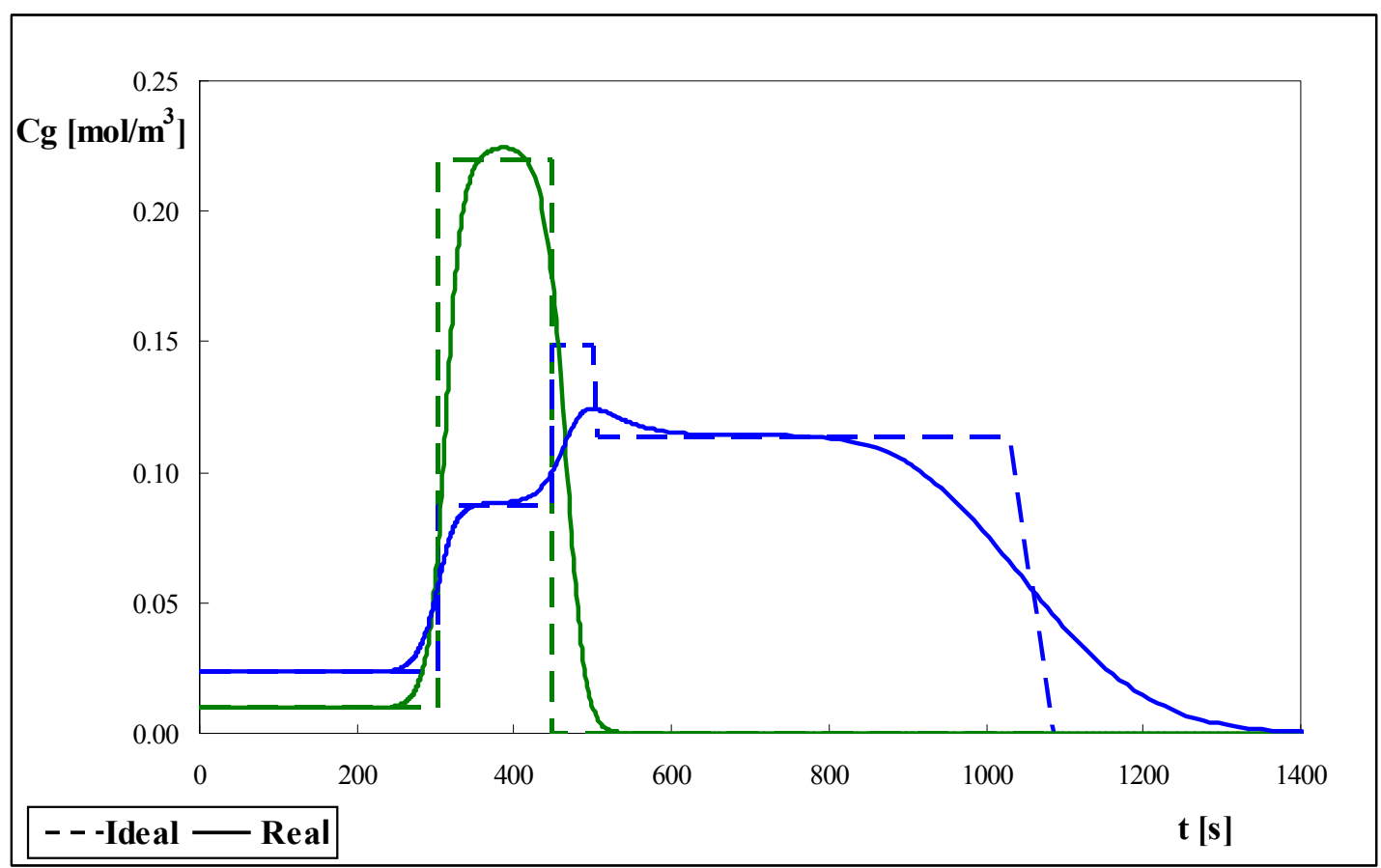

Fig. 13 - Concentración de acetato de etilo y etanol a la salida del monolito para un sistema ideal y real al llevarse a cabo el proceso de desorción.

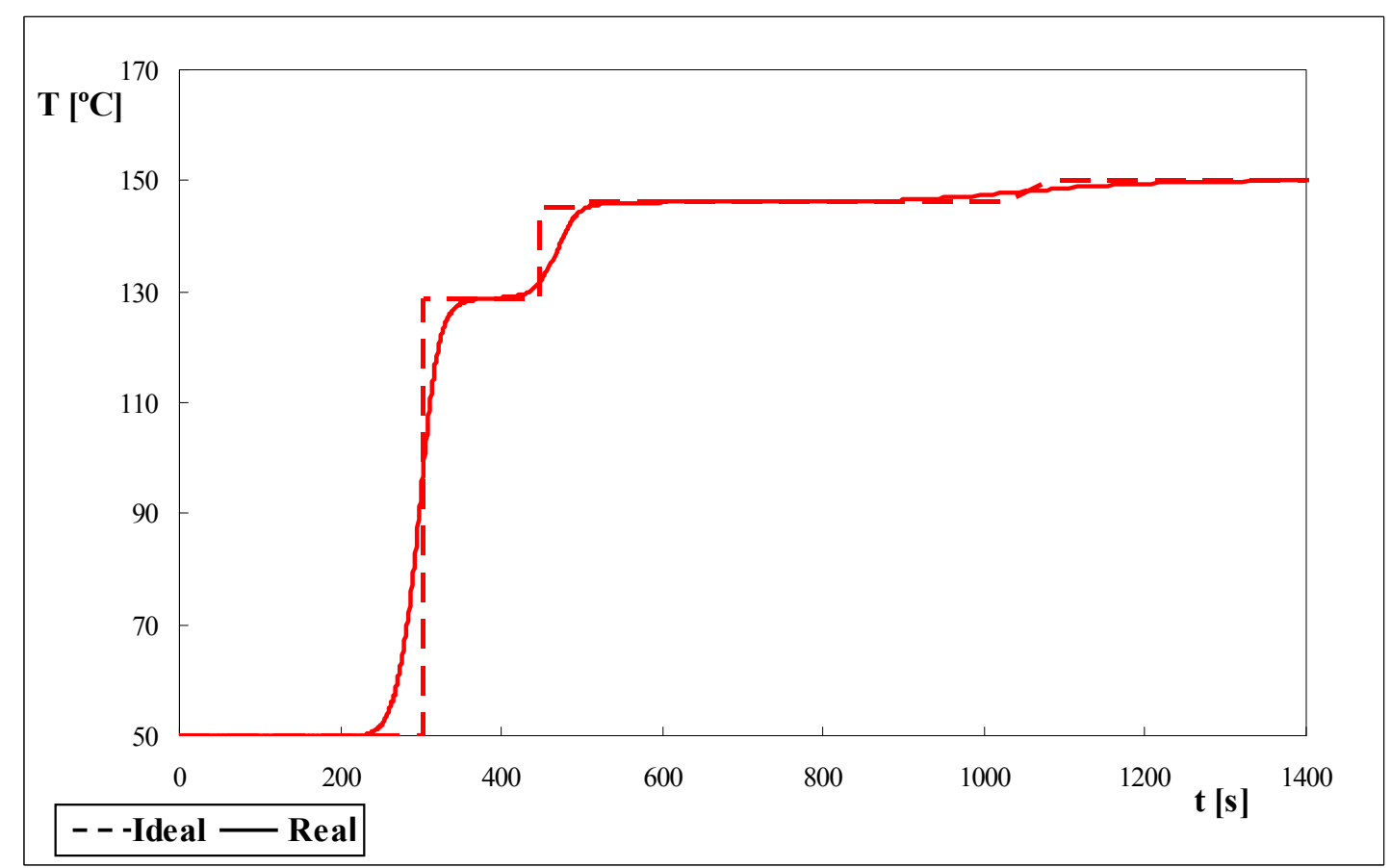

Fig. 14 - Temperatura a la salida del monolito para un sistema ideal y real al llevarse a cabo el proceso de desorción.

La simulación de este sistema a partir del Método de las Características requiere de un esfuerzo de programación y tiempo de ejecución significativamente menores que 
cuando se incluyen las resistencias a la transferencia de calor y materia. La economía en el tiempo de ejecución resulta muy ventajosa en los casos en que se pretenda realizar una optimización del sistema o cuando el sistema en sí sea más complejo, por ejemplo cuando se tiene un mayor número de componentes o isotermas de adsorción más complejas.

En nuestro caso particular de estudio el número de componentes a adsorber es dos, la isoterma de Langmuir adoptada es relativamente sencilla y además no se pretende una optimización del sistema, por lo que los tiempos de ejecución no han sido demasiado trascendentes para decidir si tomar o no en cuenta las resistencias. Considerando lo expuesto, y que la programación para el caso real ya fue realizada, de ahora en más se describirán resultados considerando las resistencias internas y externas a la transferencia de calor y materia, en virtud de que se obtienen resultados más precisos.

\subsection{Influencia mutua de los componentes en el proceso de adsorción/desorción}

Se analizó la influencia mutua de los VOCs durante las etapas de adsorción y desorción, como también el efecto que los mismos causan sobre la temperatura de la corriente. Para dicho análisis se simuló una etapa de adsorción seguida de una etapa de desorción variando las condiciones de la corriente a tratar: concentración de acetato de etilo $\left(\mathrm{C}_{1}^{0}\right)$ y etanol $\left(\mathrm{C}_{2}^{0}\right)$ en la corriente de alimentación al adsorbedor. Los valores adoptados para $\mathrm{C}_{1}^{0}$ y $\mathrm{C}_{2}^{0}$ se presentan en la Tabla 9.

Tabla 9: Concentraciones de los VOCs a la entrada del adsorbedor

\begin{tabular}{ccc}
\hline Situación & $\mathbf{C}_{\mathbf{1}}^{\mathbf{0}}\left[\mathbf{m o l} / \mathbf{m}^{3}\right]$ & $\mathbf{C}_{\mathbf{2}}^{\mathbf{0}}\left[\mathbf{m o l} / \mathbf{m}^{3}\right]$ \\
\hline $\mathbf{A}$ & $2.3510^{-2}$ & $2.3510^{-2}$ \\
$\mathbf{B}$ & $2.3510^{-2}$ & $9.6310^{-3}$ \\
$\mathbf{C}$ & $2.3510^{-2}$ & 0.00 \\
$\mathbf{D}$ & $9.6310^{-3}$ & $9.6310^{-3}$ \\
$\mathbf{E}$ & 0.00 & $9.6310^{-3}$ \\
$\mathbf{F}$ & 0.00 & 0.00 \\
\hline
\end{tabular}

La situación B corresponde al caso particular de estudio, mientras que el resto de las situaciones resultan de combinar 3 niveles de concentración: $2.35 \times 10^{-2}, 9.63 \times 10^{-3} \mathrm{y}$ 
$0 \mathrm{~mol} / \mathrm{m}^{3}$. La situación $\mathrm{F}$ se incluye a efectos de analizar posteriormente una comparación específica. En todos los casos la corriente a adsorber tiene una temperatura de $50{ }^{\circ} \mathrm{C}$ y se encuentra a 1 atm de presión, mientras que el lecho se encuentra inicialmente limpio a $150{ }^{\circ} \mathrm{C}(1 \mathrm{~atm})$. Se consideró que el caudal volumétrico a la entrada es de $11.7 \mathrm{~m}^{3} / \mathrm{s}$ y un tiempo de adsorción de $1087.5 \mathrm{~s}$.

Los procesos de adsorción y desorción se llevan a cabo en un lecho monolítico cuyas características geométricas se encuentran establecidas en la Tabla 4 de la Sección 5.4.1.2.

En la Figura 15 se presentan los perfiles correspondientes al acetato de etilo para las situaciones A, B y C, al finalizar la etapa de adsorción. En estas tres situaciones únicamente varía la concentración de etanol, lo que permite apreciar el efecto que la presencia del mismo causa sobre la adsorción del acetato de etilo. En la Figura 16 se presentan los perfiles correspondientes al etanol para las situaciones B, D y E (en las que varía la concentración de acetato de etilo), también al finalizar la etapa de adsorción.

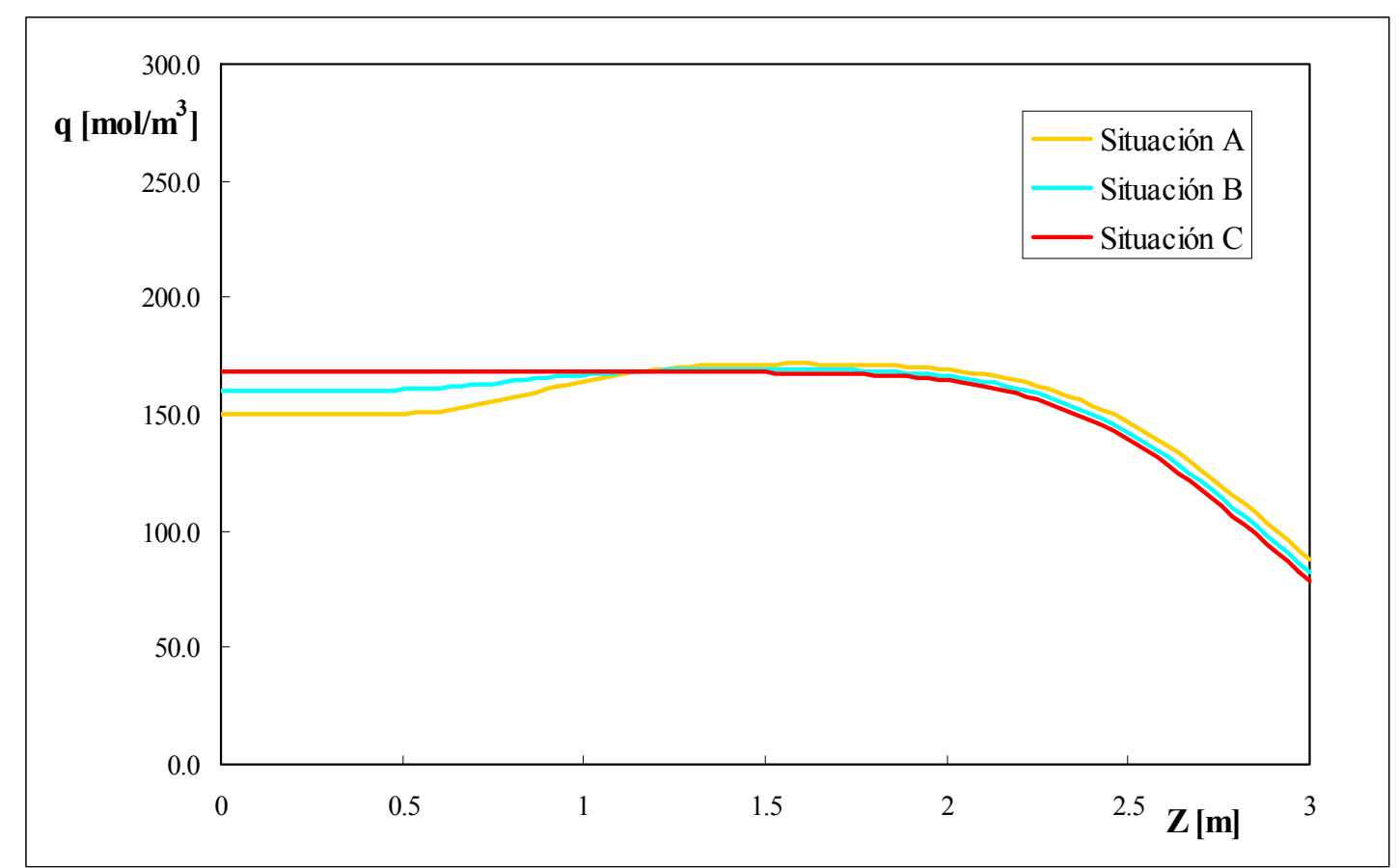

Fig. 15 - Perfil de concentración de acetato de etilo en el sólido a los 1087.5 s de la etapa de adsorción. 


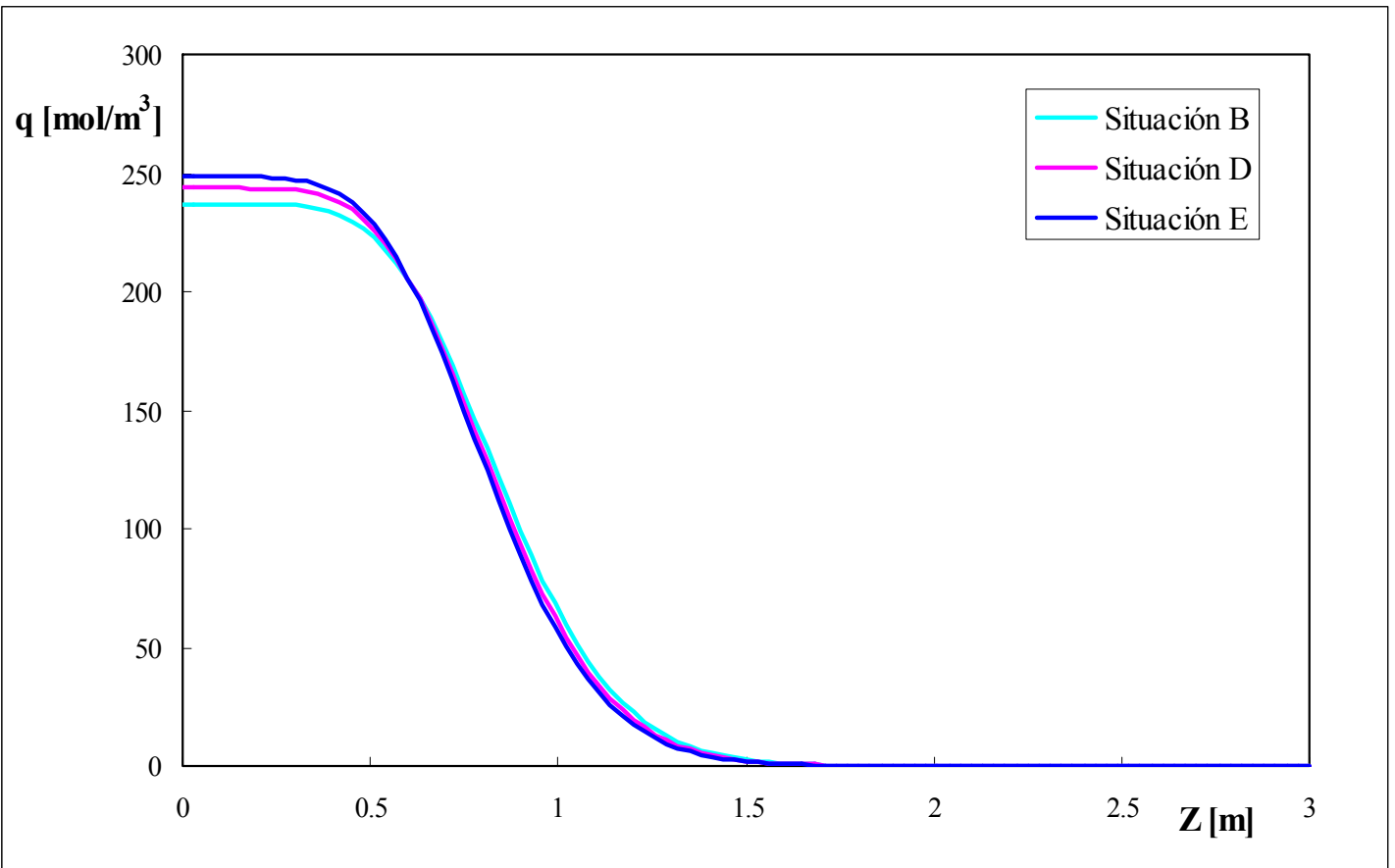

Fig. 16 - Perfil de concentración de etanol en el sólido a los $1087.5 \mathrm{~s}$ de la etapa de adsorción.

De la comparación de las curvas obtenidas en las Figs. 15 y 16, se observa que la presencia de etanol influye muy levemente sobre el acetato de etilo y viceversa, de manera tal que el proceso en sí resulta prácticamente inalterado obteniendo, a los 1087.5 segundos, aproximadamente la misma concentración de VOCs a la salida en todos los casos comparados.

Cuantitativamente dicho comportamiento se puede demostrar a partir de la expresión de la isoterma de adsorción, siendo considerada para este estudio la isoterma propuesta por Langmuir para un sistema multicomponente (Sección 4.3, Ec. [38a]).

$$
\mathrm{q}_{\mathrm{i}}=\frac{Q_{\mathrm{i}} \mathrm{K}_{\mathrm{i}} \mathrm{C}_{\mathrm{i}}}{1+\mathrm{K}_{1} \mathrm{C}_{1}+\mathrm{K}_{2} \mathrm{C}_{2}}, \quad \mathrm{i}=1,2
$$

Se recuerda que la constante de equilibrio de adsorción de la especie i $\left(\mathrm{K}_{\mathrm{i}}\right)$ es función de la temperatura según la siguiente expresión adoptada del trabajo de Gales y col. (2003) (Sección 4.3, Ec. [38b]):

$$
\mathrm{K}_{\mathrm{i}}=\mathrm{bo}_{\mathrm{i}} \mathrm{T}^{3 / 2} \exp \left(-\Delta \mathrm{H}_{\mathrm{i}} /(\mathrm{RT})\right)
$$

Los valores de $Q_{\mathrm{i}}$, bo ${ }_{\mathrm{i}}$ y $\Delta \mathrm{H}_{\mathrm{i}}$, obtenidos del trabajo de Gales y col. (2003), fueron presentados en la Tabla 1 de la Sección 4.3. Los mismos se reiteran (a efectos de facilitar la lectura) en la Tabla 10. 
Tabla 10: Parámetros para la expresión de Langmuir (Ec. [47a])

\begin{tabular}{lccc}
\hline & $\boldsymbol{Q}_{\mathbf{i}}\left[\mathbf{m o l} / \mathbf{m}^{3}\right]$ & $\mathbf{b o}_{\mathbf{i}}\left[\mathbf{m}^{\mathbf{3}} /\left(\mathbf{K}^{\mathbf{3} / \mathbf{2}} \mathbf{m o l}\right)\right]$ & $\left(-\Delta \mathbf{H}_{\mathbf{i}}\right)[\mathbf{J} / \mathbf{m o l}]$ \\
\hline 1: Acetato de Etilo & $3.5210^{3}$ & $2.4810^{-7}$ & 19600 \\
2: Etanol & $4.9410^{3}$ & $1.4710^{-10}$ & 42100 \\
\hline
\end{tabular}

Es posible verificar el grado de inhibición de cada uno de los VOCs sobre el otro analizando el peso de los términos $\mathrm{K}_{1} \mathrm{C}_{1} \mathrm{y}_{2} \mathrm{~K}_{2} \mathrm{C}_{2}$ en el denominador (Den) de la Ec. [47a]. En la Tabla 11 se presenta el valor y peso (\%) de cada término para cada una de las situaciones analizadas en las condiciones de alimentación, siendo ésta la posición para la cual los términos de inhibición tendrán mayor peso. Aún así, en todos los casos los términos $\mathrm{K}_{1} \mathrm{C}_{1}$ y $\mathrm{K}_{2} \mathrm{C}_{2}$ del denominador son suficientemente bajos como para concluir que no existe un efecto inhibitorio considerable, siendo el peor de los casos la situación A (ver Fig. 15), donde el término correspondiente al etanol representa un 11\% del denominador. De esta manera los resultados confirman las conclusiones establecidas previamente a partir de las curvas en las Fig. 15 y 16.

Tabla 11: Peso de cada uno de los términos en el denominador (Den) de la isoterma de Langmuir (Ec. [47a])

\begin{tabular}{cccc}
\hline Situación & Den & $\mathbf{K}_{\mathbf{1}} \mathbf{C}_{\mathbf{1}}^{\mathbf{0}}\left(\mathbf{5 0}{ }^{\circ} \mathbf{C}\right)$ & $\mathbf{K}_{\mathbf{2}} \mathbf{C}_{\mathbf{2}}^{\mathbf{0}}\left(\mathbf{5 0}{ }^{\circ} \mathbf{C}\right)$ \\
\hline $\mathbf{A}$ & 1.18 & $5.0110^{-2}(4.25 \%)$ & $12.9110^{-2}(10.95 \%)$ \\
$\mathbf{B}$ & 1.10 & $5.0110^{-2}(4.54 \%)$ & $5.2910^{-2}(4.80 \%)$ \\
$\mathbf{C}$ & 1.05 & $5.0110^{-2}(4.77 \%)$ & 0.00 \\
$\mathbf{D}$ & 1.05 & 0.00 & $5.2910^{-2}(5.02 \%)$ \\
$\mathbf{E}$ & 1.07 & $2.0510^{-2}(1.91 \%)$ & $5.2910^{-2}(4.93 \%)$ \\
$\mathbf{F}$ & 1.00 & 0.00 & 0.00 \\
\hline
\end{tabular}

También se observó que para cada una de las situaciones establecidas (A-F), el perfil de temperatura en el interior del lecho avanza a la misma velocidad, permitiendo un enfriamiento completo del lecho a $\operatorname{los} 80 \mathrm{~s}$ aproximadamente. Esto indica que la presencia de los VOCs, y su adsorción, no afecta al perfil de temperatura.

A continuación se llevará a cabo el análisis de la etapa de desorción en cada una de las situaciones establecidas (Tabla 9), para lo cual se consideró una corriente de aire puro a $150{ }^{\circ} \mathrm{C}$ y $1 \mathrm{~atm}$ con un caudal volumétrico de $3.31 \mathrm{~m}^{3} / \mathrm{s}(u=2 \mathrm{~m} / \mathrm{s})$. Durante esta 
etapa, la desorción del etanol influye sobre los perfiles de concentración de acetato de etilo a través de la variación que aquel provoca en la temperatura de la corriente. Este efecto se puede observar en la Figura 17, donde el perfil de concentración correspondiente al acetato de etilo a la salida del lecho varía su comportamiento según la cantidad de etanol presente en la corriente. De cualquier manera, el tiempo de elución total del acetato de etilo no resulta afectado.

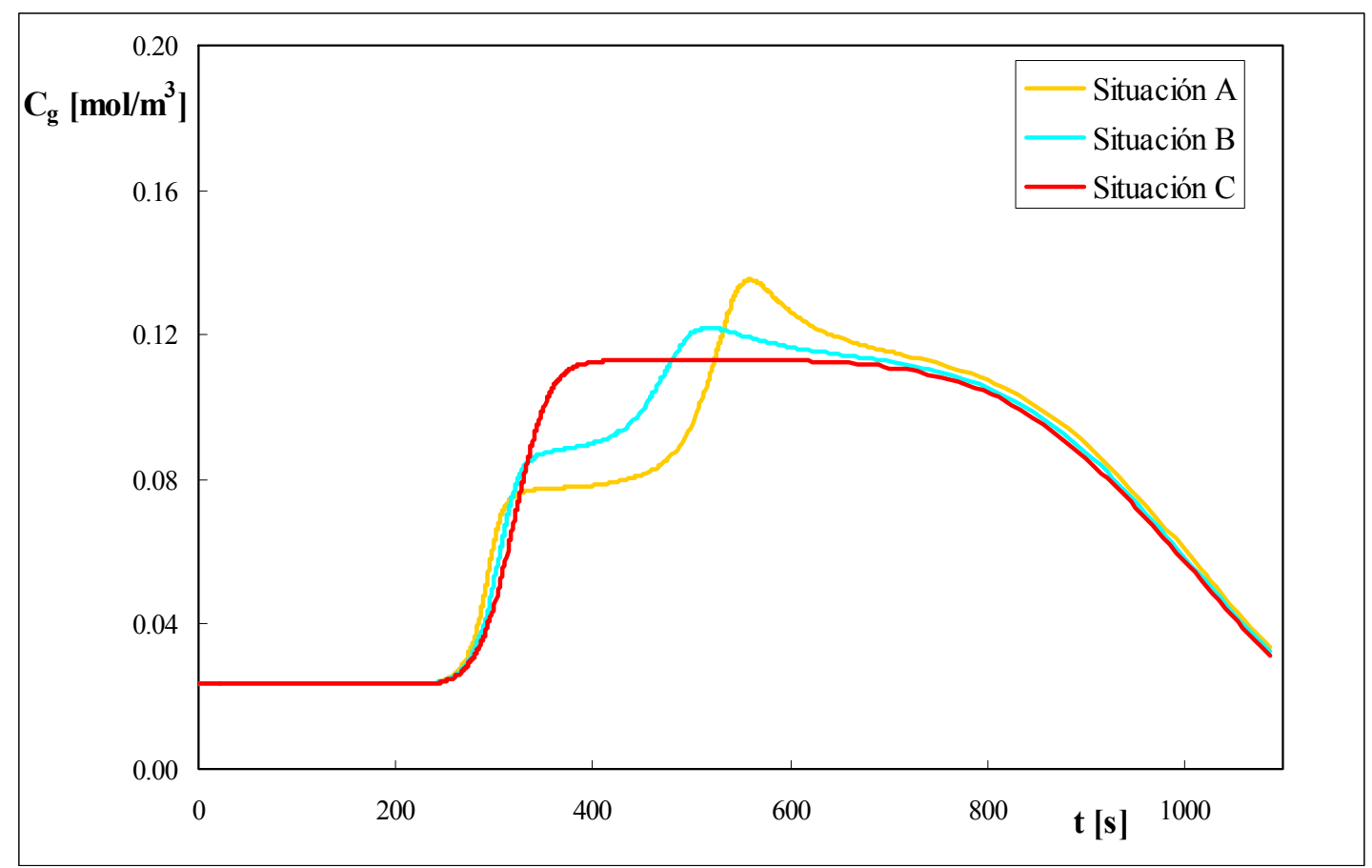

Fig. 17 - Concentración de acetato de acetato de etilo en función del tiempo a la salida del lecho.

Se puede observar en la Figura 18 para distintas concentraciones de acetato de etilo, que la curva correspondiente a la concentración de etanol en función del tiempo es prácticamente la misma. 


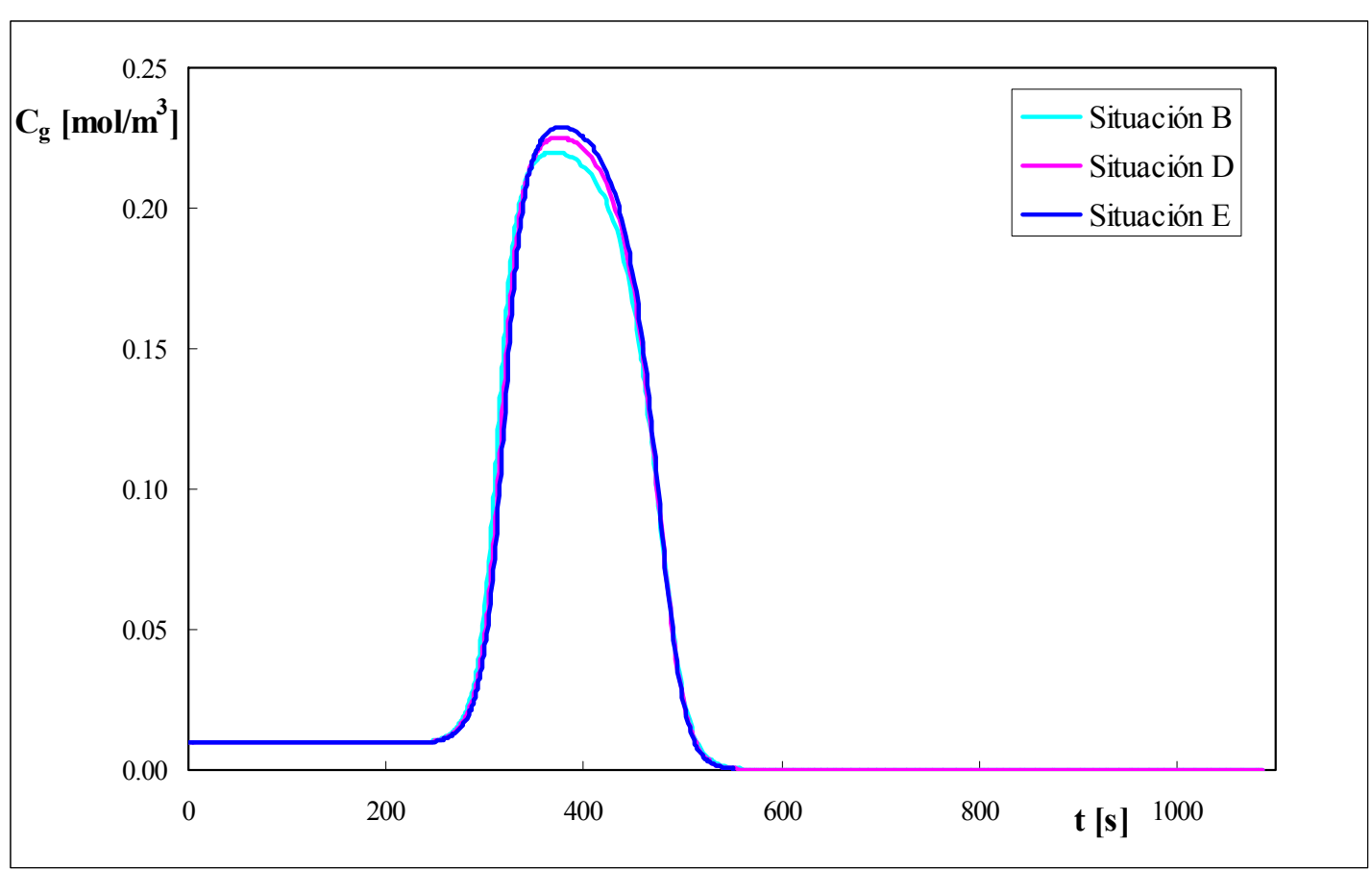

Fig.18 - Concentración de etanol en función del tiempo a la salida del lecho.

Estos comportamientos se pueden explicar considerando el efecto de los componentes sobre la temperatura de manera individual. Para esto se analizaron las situaciones $\mathrm{C}$ y $\mathrm{E}$, junto con los resultados del caso $\mathrm{F}$, donde únicamente se tiene calentamiento del lecho. En la Figura 19 se presentan los perfiles de concentración y temperatura a la salida del lecho para estas tres situaciones, C, E y F. Se observa que en todos los casos el frente de temperatura se hace presente a la salida a aproximadamente 200 s. La presencia del frente permite que ambos compuestos aceleren su desorción, incrementando la concentración de los mismos en la corriente. La desorción provoca una variación del frente térmico, aunque el efecto del etanol (caso E) resulta significativamente más pronunciado que el del acetato de etilo (caso $\mathrm{C}$ ).

Esta diferencia está relacionada con las variaciones de las constantes de adsorción $\mathrm{K}_{\mathrm{i}}$ al aumentar la temperatura (ver Ec. [47b]). En la Tabla 12 se presentan los valores de las constantes $\mathrm{K}_{\mathrm{i}}$ para ambos componentes a distintas temperaturas. A la temperatura de la etapa de adsorción $\left(\mathrm{T}=50{ }^{\circ} \mathrm{C}\right)$ la constante $\mathrm{K}_{2}$ del etanol es más del doble que la correspondiente al acetato de etilo $\left(\mathrm{K}_{1}\right)$, lo que hace que el etanol pueda adsorberse más fácilmente dentro del lecho (esto se aprecia en las Fig. 15 y 16, donde, para un mismo tiempo, el acetato de etilo requiere de más del doble de lecho para adsorberse). Al aumentar la temperatura la relación entre las constantes $\mathrm{K}_{\mathrm{i}}$ se invierte, debido a que el calor de adsorción del etanol es más del doble que el del acetato de etilo (Tabla 10), por lo que a $\mathrm{T}=150^{\circ} \mathrm{C}$ la constante del etanol resulta prácticamente tres veces menor que la 
del acetato de etilo. El menor valor de $\mathrm{K}_{2}$ favorece la velocidad de desorción del etanol y permite que alcance una mayor concentración a la salida. La rapidez de la desorción del etanol provoca que el frente de temperatura se desacelere notoriamente debido a la cantidad de calor involucrada (Fig. 19). Una vez que disminuye la cantidad de etanol a la salida, el crecimiento de la temperatura retoma su velocidad normal. Si bien la desorción del acetato de etilo también afecta el frente de temperatura (Fig. 19), el resultado es mucho más leve, debido a los mayores valores de $\mathrm{K}_{1}$ a altas temperaturas.

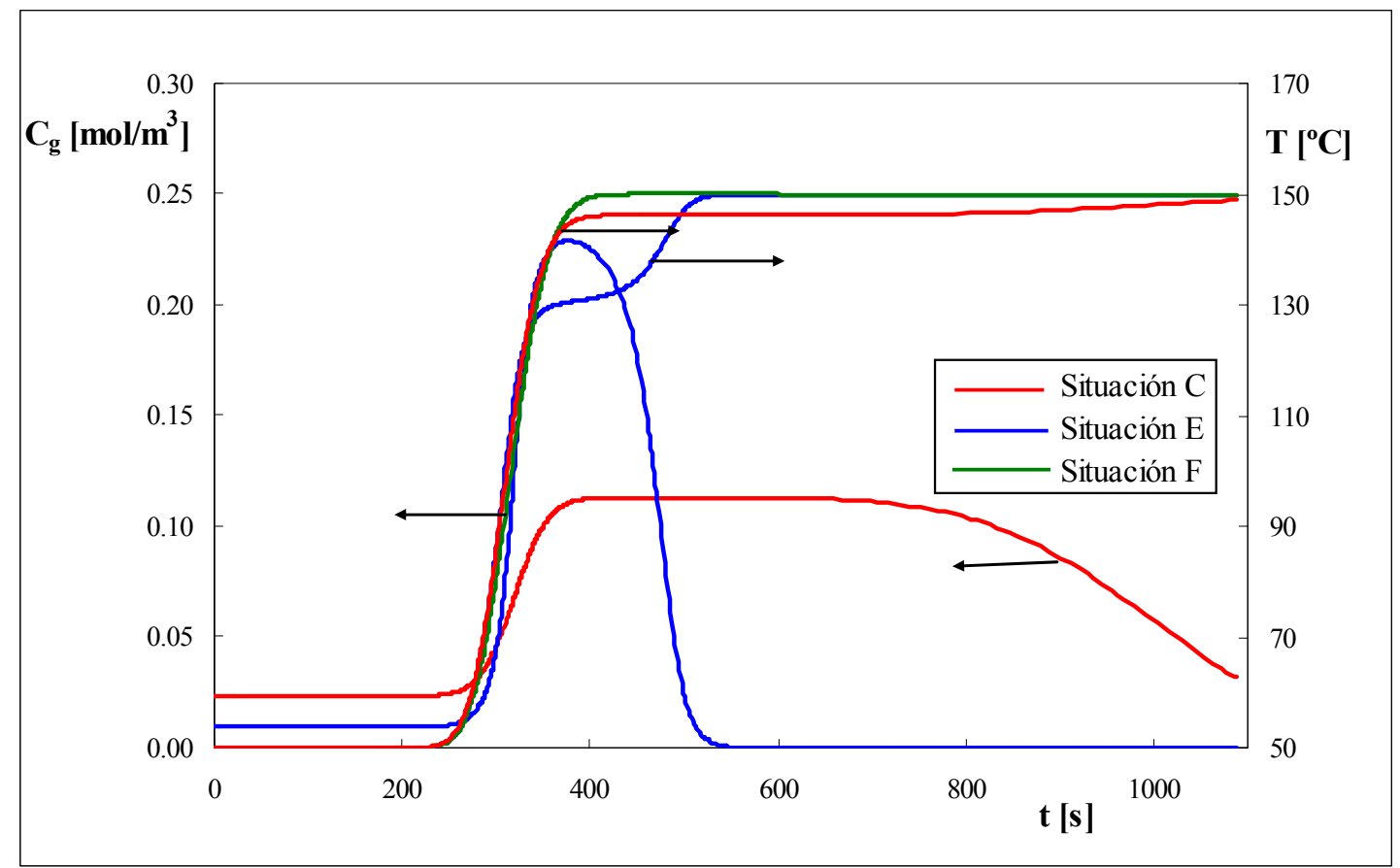

Fig.19 - Concentración de acetato de etilo, concentración de etanol y temperatura en función del tiempo a la salida del lecho.

Tabla 12: Variación de $\mathrm{K}_{\mathrm{i}}$ con la temperatura y calores de adsorción

\begin{tabular}{ccc}
\hline $\mathbf{T}\left[{ }^{\mathbf{0}} \mathbf{C}\right]$ & $\mathbf{K}_{\mathbf{1}}\left[\mathbf{m}^{\mathbf{3}} / \mathbf{m o l}\right]$ & $\mathbf{K}_{\mathbf{2}}\left[\mathbf{m}^{\mathbf{3}} / \mathbf{m o l}\right]$ \\
\hline 50 & 2.13 & 5.49 \\
130 & 0.70 & 0.34 \\
150 & 0.57 & 0.20 \\
\hline
\end{tabular}

Finalmente en la Figura 20, donde se representan las curvas del acetato de etilo, etanol y temperatura a la salida del lecho en función del tiempo, correspondiente a la situación B (Tabla 9), se observa claramente que la desaceleración del frente de temperatura afecta la velocidad de desorción del acetato de etilo, haciendo que la curva de salida del mismo resulte fuertemente correlacionada con la curva térmica. 


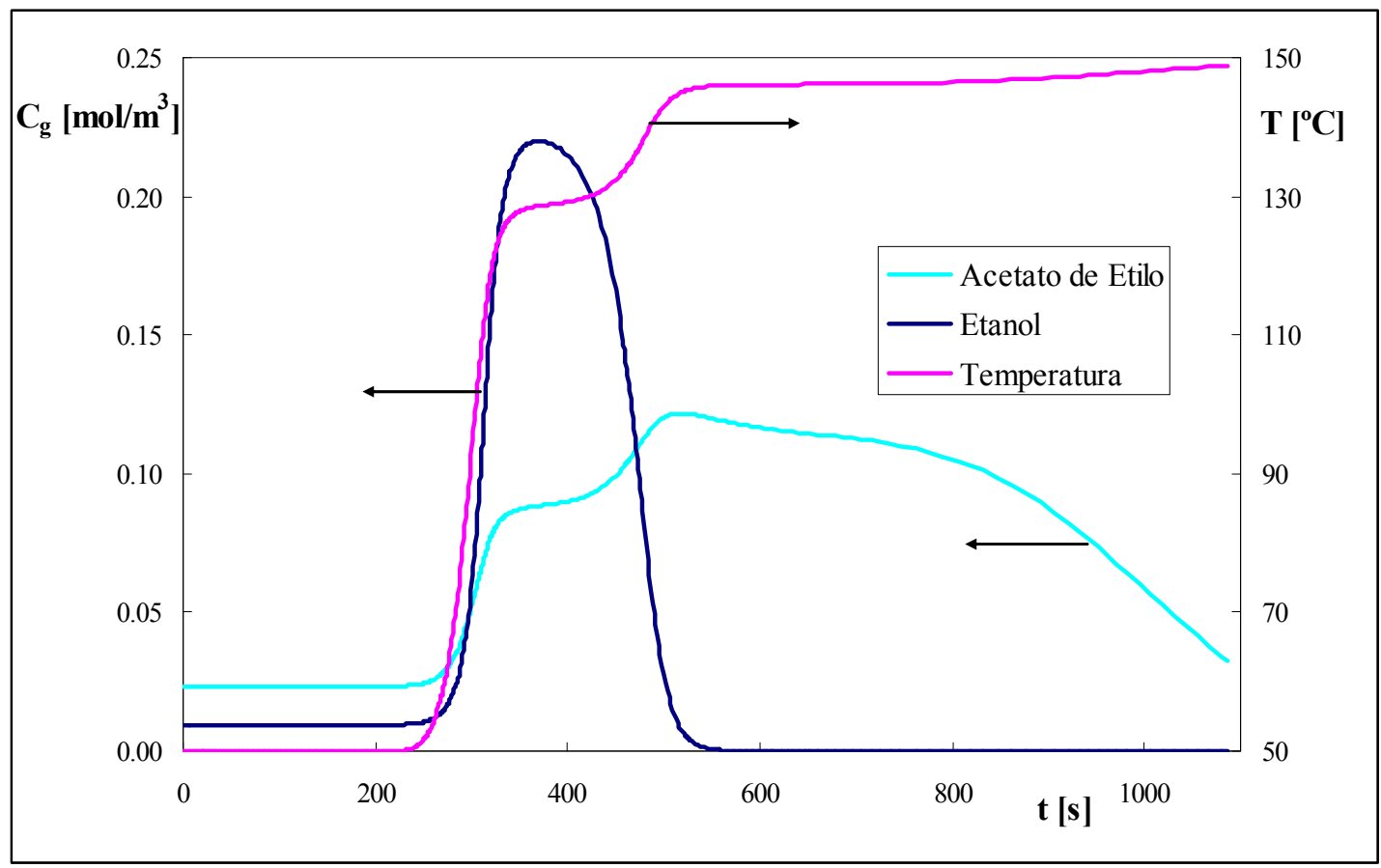

Fig. 20 - Concentración de acetato de etilo, concentración de etanol y temperatura en función del tiempo a la salida del lecho.

\subsection{Conclusiones}

Se propuso una metodología de cálculo para representar un sistema de adsorción/ desorción considerando las resistencias a la transferencia de calor y materia dentro y fuera del sólido adsorbente. Para resolver los balances de materia y energía en el interior del sólido, se propuso el Método de Colocaciones Ortogonales, mientras que para la resolución de los balances de materia y energía en la corriente de fluido se presentaron dos alternativas: la aproximación a un sistema de tanques agitados en serie y el Método Cuasi-Análitico propuesto por Matros y col. (1999).

En general, el Método Cuasi-Analítico resultó ser más eficiente, por lo que fue retenido para la obtención de resultados.

Para el caso particular de estudio, presentado en el Capítulo 2, se analizó el efecto de las resistencias a la transferencia de calor y materia según el tipo de lecho considerado, i.e., lecho relleno o monolito. Para un valor de pérdida de carga y tiempo de adsorción similares (Tablas 5 y 7), se observó que para el lecho relleno se requiere aproximadamente el doble de masa de material adsorbente. Esto se debe principalmente a la disposición del material adsorbente en cada uno de los lechos, lo que genera que en el lecho relleno exista una mayor resistencia a la transferencia de materia, especialmente en el medio poroso (Figs. 2, 3 y 4). Como consecuencia del efecto de la resistencia a la transferencia de materia surge una mayor dispersión del frente de concentración a lo 
largo del lecho. Para el lecho relleno (con las características presentadas en la Tabla 6), ningún sector del mismo se encuentra saturado al finalizar la etapa de adsorción, mientras que una gran porción del monolito (con las características presentadas en la Tabla 4) si lo está. Este comportamiento genera que el lecho relleno resulte menos eficiente frente al monolito, requiriendo una masa de adsorbente sustancialmente mayor para alcanzar el mismo objetivo. Asimismo, para los sistemas y condiciones analizados, se observó que en el monolito el frente de temperatura avanza a mayor velocidad que en el lecho relleno, debido a la mayor velocidad intersticial del fluido $\left(u_{\mathrm{M}}=7.1 \mathrm{~m} / \mathrm{s}-u_{\mathrm{LR}}=\right.$ $2.9 \mathrm{~m} / \mathrm{s}$ ) y a la menor masa por unidad de longitud de material adsorbente.

Considerando los efectos que proporciona la disposición del material adsorbente, el monolito resulta ser el tipo de lecho más apropiado para el sistema de eliminación propuesto en el Capítulo 2.

Para el empleo del Método de Colocaciones Ortogonales se debe definir el número de puntos de colocación adecuado, de manera tal de poder obtener, con el menor esfuerzo de cálculo, resultados lo suficientemente precisos. Teniendo en cuenta lo dicho, se analizó la influencia de la elección del número de puntos de colocación según el tipo de lecho, ensayándose con 1 a 5 puntos de colocación. Si bien para el monolito la influencia de la elección del número de puntos de colocación resultó menor que para el lecho relleno, se consideró apropiado un número de puntos $\mathrm{N}=2$ para realizar las simulaciones correspondientes a las etapas de adsorción y desorción.

Se compararon los perfiles de concentración obtenidos a partir de considerar, o no (caso ideal), las resistencias a la transferencia de calor y materia con el objetivo de analizar la significación de las mismas.

Para el caso particular de adsorción/desorción de acetato de etilo y etanol a lo largo de un monolito adiabático (Tabla 4), se observó una diferencia en los períodos de adsorción de aproximadamente el 23\%. Esta diferencia se debe a la influencia de las resistencias a la transferencia de materia dentro y fuera del sólido adsorbente. Las mismas provocan una dispersión del frente de avance de la concentración y en consecuencia la concentración tolerada a la salida es alcanzada más rápido. A pesar de esta diferencia en los períodos de adsorción, se observa una gran similitud entre los perfiles de concentración tanto para la etapa de adsorción como para la de desorción (Fig. 11, 12, 13). Por lo tanto es posible adoptar el caso ideal como una buena aproximación para simular el comportamiento del sistema. 
La simulación a partir, del Método de las Características, requiere de un esfuerzo de programación y tiempo de ejecución significativamente menores que cuando son incluidas las resistencias a la transferencia de calor y materia. En nuestro caso particular de estudio el número de componentes a adsorber es dos, la isoterma de Langmuir adoptada es relativamente sencilla y además no se pretende una optimización del sistema, por lo que los tiempos de ejecución no han sido demasiado trascendentes para decidir si tomar o no en cuenta las resistencias. Considerando lo expuesto, y que la programación para el caso real ya fuera realizada, se retuvo el modelo incluyendo las resistencias internas y externas a la transferencia de calor y materia, en virtud de que se obtienen resultados más precisos.

Finalmente se estudió la influencia mutua de los componentes en el proceso de adsorción/desorción y el efecto que los mismos causan sobre la temperatura de la corriente. Para el análisis se simuló una etapa de adsorción seguida de una etapa de desorción, variando la concentración de acetato de etilo y etanol presentes en la corriente a tratar (Tabla 9). Tanto para la etapa de adsorción como de desorción se consideró un lecho monolítico, cuyas características geométricas se encuentran en la Tabla 4 (Sección 5.4.1.2).

Durante la etapa de adsorción, se observó que la influencia mutua entre el etanol y el acetato de etilo es muy leve (Figs. 15 y 16). También se observó que para cada una de las situaciones establecidas en la Tabla 9, el perfil de temperatura en el interior del lecho avanza a la misma velocidad, obteniéndose un enfriamiento completo del lecho aproximadamente a los $80 \mathrm{~s}$, indicando que la presencia de VOCs no afecta al perfil de temperatura.

En cambio, se verificó que, al llevarse a cabo el proceso de desorción, la desorción del etanol influye sobre el perfil de concentración del acetato de etilo. Este comportamiento es debido a la variación provocada en la temperatura de la corriente por la desorción de etanol (Fig. 17). De todas maneras, el tiempo de elución total del acetato de etilo no resulta afectado. Por su parte la desorción del acetato de etilo prácticamente no tiene incidencia sobre las curvas de concentración del etanol a la salida del lecho a lo largo del tiempo. 


\section{$5.1 /_{\text {serented }}$}

Determinación de los puntos de colocación para el caso de un lecho relleno con partículas esféricas

A continuación se determinan los puntos de colocación para un lecho relleno de pastillas de adsorbente cuya geometría es esférica, para 1 y 2 puntos interiores.

Retomamos el caso particular de la adsorción de una especie considerando la condición de contorno impuesta [5]. De esta manera resulta $\mathrm{M}_{0}=0$ y $\mathrm{M}_{1}=0$, por lo que los polinomios $\mathrm{P}_{\mathrm{N}}(\mathrm{u})$ cuyas raíces generan los puntos de colocación corresponderán a los siguientes polinomios de Jacobi (Ec. [14]):

$$
\int_{0}^{1} \mathrm{u}^{\gamma^{*}} \mathrm{u}^{\mathrm{i}-1} \mathrm{P}_{\mathrm{N}}(\mathrm{u}) \mathrm{du}=0 \quad \mathrm{i}=1, \mathrm{~N}
$$

donde $\gamma^{*}=1 / 2$ por tratarse de una pastilla de geometría esférica y

$$
\mathrm{P}_{\mathrm{N}}(\mathrm{u})=\sum_{\mathrm{k}=0}^{\mathrm{N}} \mathrm{a}_{\mathrm{Nk}} \mathrm{u}^{\mathrm{k}}
$$

donde elegimos arbitrariamente $\mathrm{a}_{\mathrm{NN}}=1$.

Si consideramos $\underline{\mathrm{N}=1}$, el único punto de colocación se determina a partir de $\mathrm{P}_{1}(\mathrm{u})=\mathrm{a}_{10}+\mathrm{u}$. De la Ec. [A5.1.1] resulta:

$\int_{0}^{1} u^{1 / 2}\left(a_{10}+u\right) d u=0$

Resolviendo se obtiene:

$\mathrm{a}_{10}=-3 / 5$

La raíz de $\mathrm{P}_{1}(\mathrm{u})$ resulta:

$\mathrm{u}_{1}=3 / 5$

En este caso particular, donde se tiene un único punto de colocación, resulta un factor de peso $\omega_{1}=1$.

Consideremos ahora $\underline{\mathrm{N}=2}$, por lo que el polinomio de Jacobi $\mathrm{P}_{2}(\mathrm{u})$ puede escribirse como $a_{20}+a_{21} u+u^{2}$. Luego de [A.5.1.1] se tienen: 


$$
\begin{aligned}
& \int_{0}^{1} u^{1 / 2}\left(a_{20}+a_{21} u+u^{2}\right) d u=0 \\
& \int_{0}^{1} u^{1 / 2} u\left(a_{20}+a_{21} u+u^{2}\right) d u=0
\end{aligned}
$$

De la resolución conjunta de [A5.1.4] y [A5.1.5] surge:

$$
\begin{aligned}
& \mathrm{a}_{20}=0.238 \\
& \mathrm{a}_{21}=-1.111
\end{aligned}
$$

Por lo tanto las raíces de $\mathrm{P}_{2}(\mathrm{u})$ resultan:

$$
\begin{aligned}
& \mathrm{u}_{1}=0.29 \\
& \mathrm{u}_{2}=0.82
\end{aligned}
$$

Una vez encontradas las raíces del polinomio de Jacobi $\mathrm{P}_{2}$, es posible determinar los factores de peso $\omega_{1}$ y $\omega_{2}$ a partir de la siguiente fórmula de cuadratura:

$$
\frac{3}{2} \int_{0}^{1} u^{1 / 2} C(u) d u=\sum_{i=1}^{2} C\left(u_{i}\right) \omega_{i}
$$

Se recuerda que la elección de los puntos de colocación $\mathrm{u}_{1} \mathrm{y} \mathrm{u}_{2}$ como las raíces del polinomio $\mathrm{P}_{2}$ permite que la Ec. [A5.1.6] resulte exacta para integrar cualquier polinomio de hasta grado 3 .

Para determinar los factores de peso $\omega_{1}$ y $\omega_{2}$ a partir de la fórmula de cuadratura [A5.1.6] se deben elegir 2 polinomios de hasta grado 3. Resulta práctico definir:

$$
\begin{aligned}
& \mathrm{C}(\mathrm{u})=\mathbb{C}, \text { con } \mathbb{C} \text { constante, } \\
& \mathrm{C}(\mathrm{u})=\mathrm{u}
\end{aligned}
$$

Reemplazando en la Ec. [A5.1.6] resultan:

$$
\begin{aligned}
& \omega_{1}+\omega_{2}=1 \\
& \mathrm{u}_{1} \omega_{1}+\mathrm{u}_{2} \omega_{2}=3 / 5
\end{aligned}
$$

Resolviendo se tiene: $\omega_{1}=0.416$ y $\omega_{2}=0.584$.

Para $\mathrm{N}$ genérico, Villadsen y Michelsen (1978) presentan subrutinas que permiten determinar las raíces del polinomio de Jacobi $\mathrm{P}_{\mathrm{N}}(\mathrm{u})$ y los factores de peso. 


\section{Evaluación de propiedades termodinámicas y de transporte}

Teniendo en cuenta la baja concentración de acetato de etilo y etanol, para la determinación de la viscosidad, la densidad, la conductividad térmica y capacidad calorífica se consideró a la corriente compuesta exclusivamente por aire.

\section{Viscosidad}

Para la determinación de la viscosidad $(\mu)$ se empleó la siguiente expresión (Perry, 1999):

$$
\mu_{g}=\frac{\mathbf{A ~ \mathrm { T } _ { \mathrm { r } }}}{\left[1+0.36 \mathrm{~T}_{\mathrm{r}}\left(\mathrm{T}_{\mathrm{r}}-1\right)\right]^{1 / 6}} \quad[\mathrm{~Pa} \mathrm{~s}]
$$

donde:

$$
\mathrm{A}=1.610410^{-10} \frac{\mathrm{M}^{1 / 2} \mathrm{P}_{\mathrm{c}}^{2 / 3}}{\mathrm{~T}_{\mathrm{c}}^{1 / 6}}
$$

$\mathrm{T}_{\mathrm{c}}$ : temperatura crítica $[\mathrm{K}]$

$\mathrm{T}_{\mathrm{r}}$ : temperatura reducida

$\mathrm{P}_{\mathrm{c}}$ : presión crítica $[\mathrm{Pa}]$

M: peso molecular $[\mathrm{g} / \mathrm{mol}]$

Para el aire:

$$
\mathrm{T}_{\mathrm{c}, \text { aire }}=132.45 \mathrm{~K} \quad \mathrm{P}_{\mathrm{c}, \text { aire }}=3.7910^{6} \mathrm{~Pa} \quad \mathrm{M}_{\text {aire }}=28.9 \mathrm{~g} / \mathrm{mol}
$$

\section{Densidad}

Para la determinación de la densidad $\left(\rho_{g}\right)$ se trató al aire como un gas ideal.

\section{Conductividad térmica}

Se utilizó la siguiente expresión, empleada por López (2002), para evaluar la conductividad térmica $\left(\lambda_{\mathrm{g}}\right)$ :

$$
\lambda_{\mathrm{g}}=4.18910^{3} \mu\left(\hat{\mathrm{c}}_{\mathrm{p}} 2.39410^{-4}+\frac{2.48}{\mathrm{M}}\right)\left[\mathrm{W} / \mathrm{m}^{\circ} \mathrm{C}\right]
$$


donde:

M: peso molecular $[\mathrm{g} / \mathrm{mol}]$

$\hat{\mathrm{c}}_{\mathrm{p}, \mathrm{g}}:$ capacidad calorífica másica $\left[\mathrm{J} / \mathrm{kg}{ }^{\circ} \mathrm{C}\right]$

$\mu_{\mathrm{g}}: \operatorname{viscosidad}[\mathrm{Pa} \mathrm{s}]$

\section{Capacidad calorífica}

$\hat{\mathrm{c}}_{\mathrm{p}, \mathrm{g}}=\hat{\mathrm{c}}_{\mathrm{p}, \text { aire }}=\frac{0.21 \tilde{\mathrm{c}}_{\mathrm{p}, \mathrm{O}_{2}}+0.79 \tilde{\mathrm{c}}_{\mathrm{p}, \mathrm{N}_{2}}}{\mathrm{M}_{\text {aire }}},\left[\mathrm{J} / \mathrm{kg}{ }^{\circ} \mathrm{C}\right]$

Se supuso una dependencia lineal de la capacidad calorífica con la temperatura,

$\tilde{\mathrm{c}}_{\mathrm{p}, \mathrm{i}}=a_{\mathrm{i}}+b_{\mathrm{i}} \mathrm{T},\left[\mathrm{J} / \mathrm{mol}{ }^{\circ} \mathrm{C}\right]$

Los coeficientes $a_{\mathrm{i}} \mathrm{y} b_{\mathrm{i}}$ se obtuvieron de Cengel y Boles (1996):

$$
\begin{aligned}
& a_{\mathrm{O}_{2}}=25.48 \mathrm{~J} / \mathrm{mol}^{\circ} \mathrm{C} \\
& b_{\mathrm{O}_{2}}=1.5210^{-2} \mathrm{~J} / \mathrm{mol}^{\circ} \mathrm{C}^{2} \\
& a_{\mathrm{N}_{2}}=28.90 \mathrm{~J} / \mathrm{mol}^{\circ} \mathrm{C} \\
& b_{\mathrm{N}_{2}}=-1.5710^{-3} \mathrm{~J} / \mathrm{mol}^{\circ} \mathrm{C}^{2}
\end{aligned}
$$

\section{Determinación de la difusividad efectiva}

La difusividad efectiva del componente i $\left(\mathrm{D}_{\mathrm{e}, \mathrm{i}}\right)$ se evaluó con la expresión de Bosanquet, la cual resulta aplicable porque los componentes que difunden están muy diluidos:

$$
\mathrm{D}_{\mathrm{e}, \mathrm{i}}=\frac{\varepsilon_{\mathrm{p}(\text { macro })}}{\tau} \frac{1}{\frac{1}{\mathrm{D}_{\mathrm{m}, \mathrm{i}}}+\frac{1}{\mathrm{D}_{\mathrm{K}, \mathrm{i}}}},\left[\mathrm{m}^{2} / \mathrm{s}\right]
$$

donde:

$\varepsilon_{\mathrm{p}(\text { macro) }}:$ macroporosidad del adsorbente

$\tau:$ tortuosidad de los macroporos

$\mathrm{D}_{\mathrm{m}, \mathrm{i}}$ : coeficiente de difusión molecular de la especie i, $\left[\mathrm{m}^{2} / \mathrm{s}\right]$

$\mathrm{D}_{\mathrm{K}, \mathrm{i}}$ : difusividad Knudsen de la especie i, $\left[\mathrm{m}^{2} / \mathrm{s}\right]$ 
Para la evaluación del coeficiente de difusión molecular se consideró la difusión de los VOCs, acetato de etilo o etanol, en aire, empleando la siguiente expresión, que surge a partir de la expresión de Chapman-Enskog considerando gas ideal:

$$
\mathrm{D}_{\mathrm{m}, \mathrm{i}}=\frac{0.00266 \mathrm{~T}^{3 / 2}}{\mathrm{P} \mathrm{M}_{\mathrm{i} \text { aire }}^{1 / 2} \sigma_{\mathrm{i} \text { aire }}{ }^{2} \Omega_{\mathrm{d}, \mathrm{i}}}
$$

donde:

$\mathrm{M}_{\mathrm{i} \text { aire }}=2\left[\left(1 / \mathrm{M}_{\mathrm{i}}\right)+\left(1 / \mathrm{M}_{\text {aire }}\right)\right]^{-1}, \mathrm{M}_{\mathrm{i}} \mathrm{y} \mathrm{M}_{\text {aire }}$ : pesos moleculares de la especie i y del aire respectivamente.

T: temperatura absoluta $[\mathrm{K}]$,

P: presión [bar],

$\sigma_{\mathrm{i} \text { aire }}$ : longitud característica $(A)$, dada por :

$\sigma_{\mathrm{i} \text { aire }}=\frac{\sigma_{\mathrm{i}}+\sigma_{\text {aire }}}{2}$

$\Omega_{\mathrm{d}, \mathrm{i}}:$ integral de colisión (adimensional)

$\Omega_{\mathrm{d}, \mathrm{i}}=\frac{\mathrm{A}}{\left(\mathrm{T}^{0}\right)^{\mathrm{B}}}+\frac{\mathrm{C}}{\mathrm{e}^{\mathrm{DT}}}+\frac{\mathrm{E}}{\mathrm{e}^{\mathrm{FT}}}+\frac{\mathrm{G}}{\mathrm{e}^{\mathrm{HT}^{0}}}$

donde: $\mathrm{T}^{0}=\frac{k_{B} \mathrm{~T}}{\varepsilon_{\mathrm{i} \text { aire }}}$

siendo:

$k_{B}:$ constante de Boltzmann

$\varepsilon_{\mathrm{i} \text { aire }}$ : energía característica, evaluada como $\varepsilon_{\mathrm{i} \text { aire }}=\sqrt{\varepsilon_{\mathrm{i}} \varepsilon_{\text {aire }}}$

Los parámetros $\sigma_{\mathrm{i}}, \sigma_{\text {aire }}, \varepsilon_{\mathrm{i}}, \varepsilon_{\text {aire }}$ y A, B, C, D, E, F, G, y H se obtienen de Tablas (Poling y col., 2001).

Para la evaluación del coeficiente de difusión Knudsen, para cada sustancia, se empleó la expresión:

$$
D_{\mathrm{K}, \mathrm{i}}=\frac{2}{3} \sqrt{\frac{8 \mathrm{R} \mathrm{T}}{\pi \mathrm{M}_{\mathrm{i}}}} \mathrm{r}_{\text {poro }}
$$

donde el radio de poro, $\mathrm{r}_{\text {poro }}$, se evaluó a partir del modelo de poros en paralelo, como: 


$$
\mathrm{r}_{\text {poro }}=\frac{2 \varepsilon_{\mathrm{p}(\text { macro })}}{\mathrm{S}_{\mathrm{g}(\text { macro })} \rho_{\mathrm{pap}}}
$$

Con la información sobre las propiedades físicas del adsorbente, densidad aparente $\left(\rho_{\text {pap }}\right)$, macroporosidad ( $\left.\varepsilon_{\mathrm{p}(\text { macro) }}\right)$ y superficie específica presentadas en la Tabla 3 , se calculó un radio de poro $\mathrm{r}_{\text {poro }}=1.610^{-8} \mathrm{~m}$.

Determinación de los coeficientes de transferencia de calor y materia fluidoadsorbente según el tipo de lecho

\section{Lecho Fijo Relleno:}

- Coeficiente de transferencia de materia

$$
\mathrm{k}_{\mathrm{m}, \mathrm{i}}=\frac{S h_{\mathrm{i}} \mathrm{D}_{\mathrm{m}, \mathrm{i}}}{\mathrm{d}_{\mathrm{p}}}
$$

donde $\mathrm{D}_{\mathrm{m}, \mathrm{i}}$ es la difusividad molecular, $\mathrm{d}_{\mathrm{p}}$ el diámetro de pastilla de adsorbente y $S h$ el número de Sherwood.

Para el Sh se adoptó la siguiente expresión propuesta por Gunn (1978):

$$
S h_{\mathrm{i}}=\left(7-10 \varepsilon_{\mathrm{L}}+5 \varepsilon_{\mathrm{L}}^{2}\right)\left(1+0.7 \operatorname{Re}^{0.2} \mathrm{Sc}_{i}^{1 / 3}\right)+\left(1.33-2.4 \varepsilon_{\mathrm{L}}+1.2 \varepsilon_{\mathrm{L}}^{2}\right) \operatorname{Re}^{0.7} \mathrm{Sc}_{i}^{1 / 3}
$$

Las expresiones para los números adimensionales de Reynolds (Re) y Schmidt (Sc) son:

$$
\begin{aligned}
& \mathrm{Re}=\frac{\rho_{g} \mathrm{v}_{\mathrm{s}} \mathrm{d}_{\mathrm{p}}}{\mu_{g}} \\
& \mathrm{Sc}_{\mathrm{i}}=\frac{\mu_{\mathrm{g}}}{\rho_{\mathrm{g}} \mathrm{D}_{\mathrm{m}, \mathrm{i}}}
\end{aligned}
$$

donde $\mathrm{v}_{\mathrm{s}}$ es la velocidad superficial de la corriente de fluido.

\section{- Coeficiente de transferencia de calor}

$$
\mathrm{h}_{\mathrm{t}}=\frac{N u \lambda_{\mathrm{g}}}{\mathrm{d}_{\mathrm{p}}}
$$

Para el $N u$, número de Nusselt, se adoptó la siguiente expresión, también propuesta por Gunn (1978), que es análoga a la de transferencia de de materia ([A5.2.9]):

$$
N u=\left(7-10 \varepsilon_{\mathrm{L}}+5 \varepsilon_{\mathrm{L}}^{2}\right)\left(1+0.7 \operatorname{Re}^{0.2} \operatorname{Pr}^{1 / 3}\right)+\left(1.33-2.4 \varepsilon_{\mathrm{L}}+1.2 \varepsilon_{\mathrm{L}}^{2}\right) \operatorname{Re}^{0.7} \operatorname{Pr}^{1 / 3}
$$


La expresión para el número adimensional Prandt (Pr) es:

$$
\operatorname{Pr}=\frac{\hat{\mathrm{c}}_{\mathrm{p}, \mathrm{g}} \mu_{\mathrm{g}}}{\lambda_{\mathrm{g}}}
$$

\section{Monolito}

- Coeficiente de transferencia de materia

$$
\mathrm{k}_{\mathrm{m}, \mathrm{i}}=\frac{S h_{\mathrm{i}} \mathrm{D}_{\mathrm{m}, \mathrm{i}}}{\mathrm{d}_{\mathrm{h}}}
$$

donde $\mathrm{D}_{\mathrm{m}, \mathrm{i}}$ es la difusividad molecular, $\mathrm{d}_{\mathrm{h}}$ el diámetro hidráulico de los canales (Tabla 4) y Sh el número de Sherwood.

Para el Sh se adoptó la siguiente aproximación de Barreto (2007):

$$
S h_{\mathrm{i}}=S h_{\infty}+\frac{5.4 \alpha_{\mathrm{i}} \mathrm{Pe}_{\mathrm{i}}}{\left(12 \alpha_{\mathrm{i}}+0.519 \mathrm{Pe}_{\mathrm{i}}^{1.5}\right)^{1 / 3}}
$$

donde $S h_{\infty}$ es un valor asintótico del $S h$ cuando el perfil de velocidad y concentración se encuentran desarrollados. Para canales cuadrados se tiene: $S h_{\infty}=2.977$ (Barreto, 2007).

El número de Peclet $\left(\mathrm{Pe}_{\mathrm{i}}\right)$ y $\alpha_{\mathrm{i}}$ se definen como:

$$
\mathrm{Pe}_{\mathrm{i}}=\frac{2 \mathrm{~d}_{\mathrm{h}}^{2} u}{16 \Delta Z_{\mathrm{M}} \mathrm{D}_{\mathrm{m}, \mathrm{i}}}
$$

donde $u$ es la velocidad lineal en el canal y $\Delta Z_{\mathrm{M}}$ es la longitud de unidad monolítica. (Para los cálculos realizados en este trabajo, se adoptaron, en general, valores de $\Delta Z_{\mathrm{M}}$ entre 20 y $30 \mathrm{~cm}$ dependiendo de la longitud del lecho (L). Para los valores de $\Delta Z_{\mathrm{M}}$ empleados, el $S h$ alcanzó prácticamente su valor asintótico)

$$
\alpha_{\mathrm{i}}=\left((\mathrm{fRe} / 100)^{4} \mathrm{~b}_{\infty}{ }^{12}+\left(\mathrm{Sc}_{\mathrm{i}} / \mathrm{b}_{1}{ }^{6}+1 / \mathrm{b}_{0}{ }^{6}\right)^{-2}\right)^{1 / 12}
$$

con

$\mathrm{fRe}=14.2$ 
$\mathrm{b}_{\infty}=0.4273$
$\mathrm{~b}_{1}=0.3392$
$\mathrm{~b}_{0}=0.5642$

La expresión para el número adimensional de Reynolds (Re) es:

$$
\operatorname{Re}=\frac{u \mathrm{~d}_{\mathrm{h}}}{\mu_{\mathrm{g}}}
$$

- Coeficiente de transferencia de calor

$\mathrm{h}_{\mathrm{t}}=\frac{N u \lambda_{\mathrm{g}}}{\mathrm{d}_{\mathrm{h}}}$

Para el $N u$, número de Nuselt, se adoptó la siguiente expresión por analogía a la de materia [A5.2.16]:

$$
N u=S h_{\infty}+\frac{5.4 \alpha_{\mathrm{T}} \mathrm{Pe}_{\mathrm{T}}}{\left(12 \alpha_{\mathrm{T}}+0.519 \mathrm{Pe}_{\mathrm{T}}^{1.5}\right)^{1 / 3}}
$$

Para canales cuadrados se tiene: $S h_{\infty}=2.997$

$\mathrm{Pe}_{\mathrm{T}} \mathrm{y} \alpha_{\mathrm{T}}$ están definido como:

$$
\mathrm{Pe}_{\mathrm{T}}=\frac{\mathrm{d}_{\mathrm{h}}^{2} u \rho_{\mathrm{g}} \hat{\mathrm{c}}_{\mathrm{p}, \mathrm{g}}}{16 \Delta Z_{\mathrm{M}} \lambda_{\mathrm{p}}}
$$

donde $\lambda_{\mathrm{p}}$ es la conductividad térmica del material adsorbente.

$\alpha_{\mathrm{T}}=\left((\mathrm{fRe} / 100)^{4} \mathrm{~b}_{\infty}{ }^{12}+\left(\operatorname{Pr} / \mathrm{b}_{1}{ }^{6}+1 / \mathrm{b}_{0}{ }^{6}\right)^{-2}\right)^{1 / 12}$

Para fRe, $b_{\infty}, b_{1}, b_{0}$ se toman los mismos valores que los utilizados para determinar el coeficiente transferencia de materia. 




\section{6/ ANALISIS DE LOS CICLOS DE ADSORCION/DESORCION Y CAUDAL MINIMO DE DESORCION}

Sistema de dos lechos que operan alternativamente vs. concentrador rotativo Determinación del período que define el ciclo de adsorción/desorción Determinación del caudal mínimo $\left(\boldsymbol{W}_{\text {des }}^{\text {min }}\right)$

Influencia de la relación $D / L$ en el valor de $\boldsymbol{W}_{\text {des }}^{\text {min }}$

Efecto de la relación entre áreas de adsorción y desorción

Influencia de la cantidad de adsorbente sobre el caudal mínimo de desorción Criterio de diseño: Zona de Transferencia de Masa (ZTM)

Método aproximado de cálculo

Conclusiones 



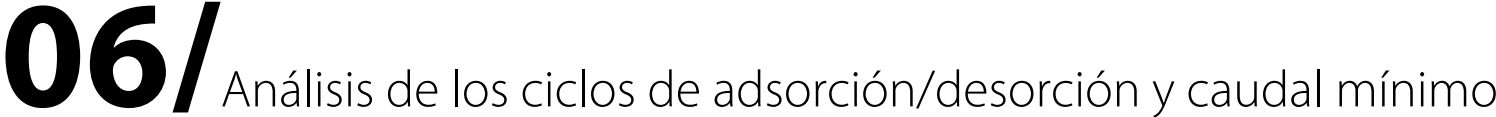 de desorción}

A partir del análisis sobre las resistencias a la transferencia de materia y energía, realizado en el Capítulo 5, Sección 5.4.2, se consideró apropiado optar por un lecho monolítico para el adsorbedor incluido en el sistema de eliminación propuesto en el Capítulo 2. Como fuera presentado en el Capítulo 3, dentro de los monolitos es factible considerar un sistema de dos lechos que operen alternativamente o un concentrador rotativo. En la Sección 6.1 se selecciona la configuración más apropiada para nuestro caso particular en estudio en base a las ventajas que presenta una de las alternativas sobre la otra.

Se analiza el ciclo de adsorción/desorción y se presenta una modalidad de cálculo para la determinación del período que define dicho ciclo. Esta modalidad consiste en un proceso iterativo que permite determinar los ciclos estacionarios, lo que sucede cuando los mismos se repiten permanentemente a lo largo del tiempo.

Se introduce el concepto de caudal mínimo de desorción, $\mathrm{W}_{\mathrm{des}}^{\min }$. Se evalúa la influencia que presentan, sobre los valores de caudal mínimo de desorción, la relación diámetro/longitud del equipo y la relación entre las secciones que se destinan para las etapas de adsorción y desorción.

Por otro lado, se estudia el efecto que causa la cantidad de adsorbente y la temperatura de regeneración sobre los valores de caudal mínimo de desorción. Los resultados obtenidos se comparan con los del caso ideal, o sea cuando no se consideran las resistencias a la transferencia de calor y materia.

A partir de seleccionar un volumen adecuado de adsorbente que determine junto con la pérdida de carga las dimensiones del sistema de adsorción/desorción ( $D, L)$, resulta definido el porcentaje que representa la Zona de Transferencia de Masa (ZTM) en el lecho una vez finalizada la etapa de adsorción.

En base a las características del caso ideal (Capítulo 4), surge un método aproximado de cálculo que, considerando la ZTM como criterio de diseño y la concentración de VOC estipulada a la salida, permite determinar el tiempo correspondiente a la etapa de adsorción y volumen de adsorbente requerido para el sistema de adsorción/desorción. 


\subsection{Sistema de dos lechos que operan alternativamente vs. concentrador rotativo}

Una vez definido el tipo de lecho (i.e., lecho relleno o monolito), se debe determinar la configuración del mismo. En el Capítulo 5 se optó por un monolito, por lo que teniendo en cuenta lo mencionado en el Capítulo 3, se tienen dos configuraciones: un sistema de dos lechos que operan alternativamente o un concentrador rotativo.

Una particularidad que presenta el concentrador rotativo es que, a la salida del mismo, la corriente posee una concentración y temperatura constantes en el tiempo. Esta característica no sucede para un sistema de dos lechos que operan alternativamente. Esto último trae inconvenientes al enfrentar el diseño del sistema de eliminación de VOCs propuesto en el Capítulo 2. La variación de la concentración y temperatura de la corriente a la salida del desorbedor durante la regeneración del lecho (ver Figs. 13 y 14 en el Capítulo 5), implica que las condiciones operativas a la entrada del reactor e intercambiadores de calor no resulten constantes. Para el caso particular del reactor, a medida que la concentración en la corriente a tratar, va disminuyendo, el salto adiabático de temperatura también disminuye. Para las concentraciones más bajas resulta necesario disponer de reactores voluminosos si se pretende satisfacer la concentración límite recomendada a la salida del mismo. Al diseñar para la situación más desfavorable, se tiene que, para las concentraciones más altas, no solo se contará con un exceso de catalizador para cumplir con los requerimientos, sino que los niveles de temperatura alcanzados en el interior del reactor se elevarán demasiado lo que puede perjudicar al material empleado como catalizador. Un efecto similar surge en el intercambiador de calor donde la fuerza impulsora resulta afectada a lo largo de la operación. Como consecuencia del estado no estacionario, surge que al diseñar para la situación adversa, se tienen intercambiadores de calor y reactor bastante más voluminosos que los obtenidos al emplear un concentrador rotativo.

Los concentradores rotativos presentan ciertas características, que pueden ser ventajosas para el sistema en estudio. Las mismas se describen a continuación:

- Alternativas de diseño, como la posición vertical (forma de disco, "pancake", ver Fig. 2 del Capítulo3), que permiten utilizar espacios pequeños debido a que el área proyectada es relativamente baja.

- Permiten una simplificación del diseño de las cañerías por las cuales circulan las corrientes de adsorción y desorción, dado que la circulación de las mismas se produce siempre en una única dirección.

- Evita el uso de válvulas empleado en el sistema de dos lechos. 
- Las zonas correspondientes a la etapa de adsorción y desorción pueden ser de distinto tamaño permitiendo minimizar la pérdida de carga.

Teniendo en cuenta todas las ventajas que presenta el concentrador rotativo y, en especial la particularidad de la constancia de la concentración y temperatura de la corriente a la salida del mismo, se optó por dicha configuración para el sistema de eliminación propuesto en el Capítulo 2. Como fuera mencionado en el Capítulo 3, tanto el Método de las Características presentado en el Capítulo 4 como el de Colocaciones Ortogonales conjuntamente con el Cuasi-analítico propuestos en el Capítulo 5, son aplicables a este tipo de lecho.

En la actualidad, los concentradores rotativos se encuentran ampliamente comercializados para la concentración de corrientes gaseosas con VOCs. Algunas de las compañías que comercializan este tipo de sistemas de concentración son: Anguil Environmental Systems, INC; Chemisch Thermische Prozesstechnik GMBH; Formia Emissions Control Oy; Munters Corporation - Zeol Division; Tecam Soluciones Medioambientales S.L.; Fusion Environmental Corporation; Catalytic Combustion.

\subsubsection{Características geométricas del concentrador rotativo}

A partir de haber seleccionado la configuración del adsorbedor (concentrador rotativo), se deben definir las características geométricas del mismo. Las características del monolito, i.e., forma y dimensiones de los canales, son las mismas que las presentadas en la Tabla 4 del Capítulo 5, por lo tanto solo resta definir las dimensiones globales del lecho, sección (S) y longitud (L).

Dado que los concentradores rotativos presentan una sección circular (Fig. 2, Capítulo3), será necesario definir su diámetro. La selección de las características geométricas, diámetro y longitud, se realizarán en este capítulo a partir de considerar ciertos criterios de diseño.

Como material adsorbente se emplea carbón activado, cuyas características fueron presentadas en la Tabla 3 del Capítulo 5.

La utilización de un lecho fijo, de tipo monolítico o particulado, conduce a que la sección destinada a la etapa de adsorción $\left(\mathrm{S}_{\mathrm{ads}}\right)$ coincida con la correspondiente a la etapa de desorción $\left(\mathrm{S}_{\mathrm{des}}\right)$. En el caso de un concentrador rotativo esto significa que se divide la sección circular del equipo en dos porciones idénticas de igual ángulo $\theta$ $\left(\theta_{\text {ads }}=\theta_{\text {des }}\right)$, Figura 1a. Sin embargo, a diferencia de un sistema de dos lechos, no 
necesariamente debe ser $\mathrm{S}_{\mathrm{ads}} / \mathrm{S}_{\mathrm{des}}=1\left(\theta_{\mathrm{ads}} / \theta_{\mathrm{des}}=1\right)$, sino que es posible proporcionar secciones distintas para cada una de las etapas, como se muestra en la Figura 1b. En la Sección 6.4 se analizará el efecto de la relación $\mathrm{S}_{\mathrm{ads}} / \mathrm{S}_{\mathrm{des}}$ sobre el proceso de adsorción/desorción.

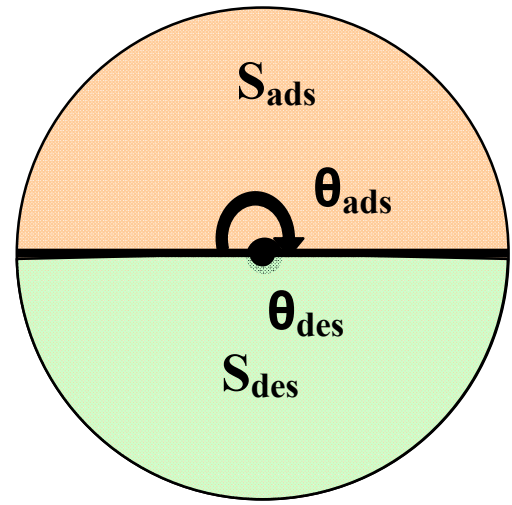

Fig. 1a - Sección axial del rotor con $\theta_{\mathrm{ads}}=\theta_{\text {des }}$

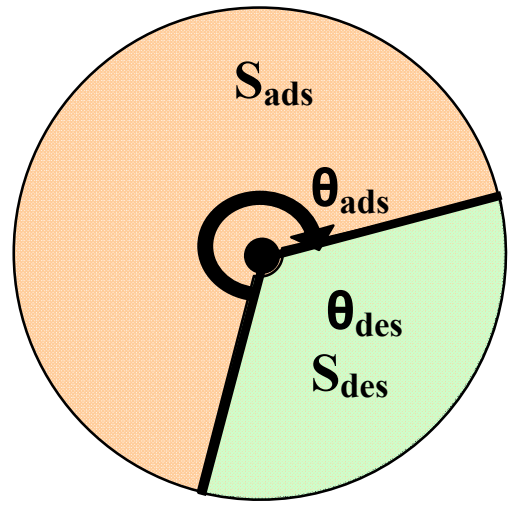

Fig. 1b - Sección axial del rotor $\operatorname{con} \theta_{\mathrm{ads}}>\theta_{\text {des }}$

\subsection{Determinación del período que define el ciclo de adsorción/desorción}

Una vez definida la geometría del lecho (masa de adsorbente), establecidas las condiciones operativas de las corrientes a adsorber y de desorción y teniendo en cuenta el valor establecido para la concentración de VOCs en la corriente a la salida del adsorbedor, se procede a calcular el período que define el ciclo de adsorción/desorción. Dicho período debe determinarse iterativamente, estableciéndose como condición, que ambas etapas, de adsorción y desorción, se extiendan hasta una relación entre los tiempos tal que $\frac{t_{\text {ads }}}{t_{\text {des }}}=\frac{S_{\text {ads }}}{S_{\text {des }}}$ y los mismos se repitan, sin sufrir variaciones, de un ciclo al siguiente. Para el caso en particular en que $S_{a d s}=S_{\text {des }}$, se tiene $t_{a d s}=t_{\text {des. }}$.

A continuación se describe la modalidad de cálculo empleada para la determinación del período del ciclo de adsorción/desorción a partir de considerar la evolución del proceso desde su estado inicial hasta alcanzar el estado de "ciclo estacionario".

En primer lugar se establece que la zona del rotor correspondiente a la etapa de adsorción se encuentra inicialmente limpia y a una temperatura equivalente a la temperatura de la corriente utilizada para desorber. Como fuera recientemente mencionado, las condiciones operativas de la corriente a adsorber, la geometría del equipo y el valor establecido para la concentración de VOCs en la corriente de salida determinan el tiempo en el cual transcurre la etapa de adsorción mediante el empleo de los balances de conservación presentados en el capítulo anterior (Ecs. [44], [45] y [46]). 
Al finalizar la primera iteración de adsorción, se continúa con la etapa de desorción, para la cual se utiliza una corriente caliente de aire puro. El tiempo correspondiente a dicha etapa se determina a partir de la siguiente relación: $t_{\text {des }}=t_{\text {ads }} \frac{S_{\text {des }}}{S_{\text {ads }}}$.

Las condiciones en las que queda el lecho, luego de una etapa de desorción, dependen del estado en el que se encuentra el mismo al finalizar la etapa previa de adsorción, de las condiciones operativas de la corriente empleada para desorber y del tiempo en que se lleva a cabo dicha etapa.

Dado que cada ciclo comprende una etapa de adsorción seguida de una etapa de desorción, luego de transcurrido el primer ciclo de adsorción/desorción (ciclo 1), se procede a una segunda etapa de adsorción dando comienzo al ciclo 2. Para el caso en que no se haya alcanzado una desorción total de los VOCs en el ciclo previo de desorción, no se podrá considerar al lecho completamente limpio al comenzar la etapa de adsorción correspondiente al ciclo 2. Esto hace que el tiempo en el cual se lleva a cabo dicha etapa disminuya con respecto al obtenido en el ciclo anterior, debido a que la concentración de VOCs máxima permitida en la corriente de salida es alcanzada más rápidamente.

Siendo que el tiempo de desorción es proporcional al tiempo de adsorción $\left(\mathrm{t}_{\mathrm{des}}=\mathrm{t}_{\mathrm{ads}} \frac{\mathrm{S}_{\mathrm{des}}}{\mathrm{S}_{\mathrm{ads}}}\right)$, éste también resulta menor al tiempo considerado en el ciclo 1. Esto implica que se desorba menos cantidad de VOCs, por lo que al inicio de la siguiente etapa de adsorción (ciclo 3) el lecho se encontrará con una concentración de VOCs mayor que al inicio de la etapa de adsorción en el ciclo 2 .

Esta modalidad de cálculo se repite sucesivamente hasta que los perfiles de concentración correspondientes a cada una de las etapas no cambien de manera significativa de un ciclo a otro. Cuando esto sucede queda definido el período del ciclo de adsorción/desorción. Existen situaciones en las que el sistema de resolución no converge debido a que el caudal de desorción establecido resulta inapropiadamente pequeño y no permite concebir un ciclo estacionario. Para que converja, la diferencia entre las etapas sucesivas de adsorción debe ir disminuyendo.

En la Figura 2 se muestra como se estabiliza el proceso de adsorción/desorción a lo largo de los ciclos. Para la obtención de los resultados se consideró un concentrador rotativo con un diámetro (D) de $4 \mathrm{~m}$ y un largo (L) de $0.5 \mathrm{~m}$, con la siguiente relación 
entre secciones: $\mathrm{S}_{\mathrm{ads}}=1.7 \mathrm{~S}_{\mathrm{des}}$ (la razón para tomar esta relación se presentará en el punto 6.5). Las características de la corriente de alimentación al adsorbedor, el cual se considera inicialmente limpio y a $150{ }^{\circ} \mathrm{C}$, son las mismas que las presentadas en el Capítulo 2, pero considerando una sobrepresión, debido a la pérdida de carga en todo el sistema, necesaria para realizar una descarga a la atmósfera. El incremento de presión se genera en el soplador presente en el sistema de eliminación (Capítulo 2, Fig. 3). Teniendo en cuenta esta situación se adoptó una presión de 1.3 atm a la entrada del adsorbedor. Dichas características se presentan en la Tabla 1. Para la desorción del lecho se utilizó una corriente de aire puro a $150{ }^{\circ} \mathrm{C}$ con un caudal de desorción de $3.82 \mathrm{~kg} / \mathrm{s}$.

De la Fig. 2 se obtiene que, para el sistema establecido, el proceso de adsorción/ desorción prácticamente se estabiliza a partir del ciclo 6 con un período de aproximadamente 846 s. Vale la pena aclarar que para un concentrador de características geométricas diferentes a las establecidas, el proceso de estabilización de los ciclos resulta cualitativamente similar.

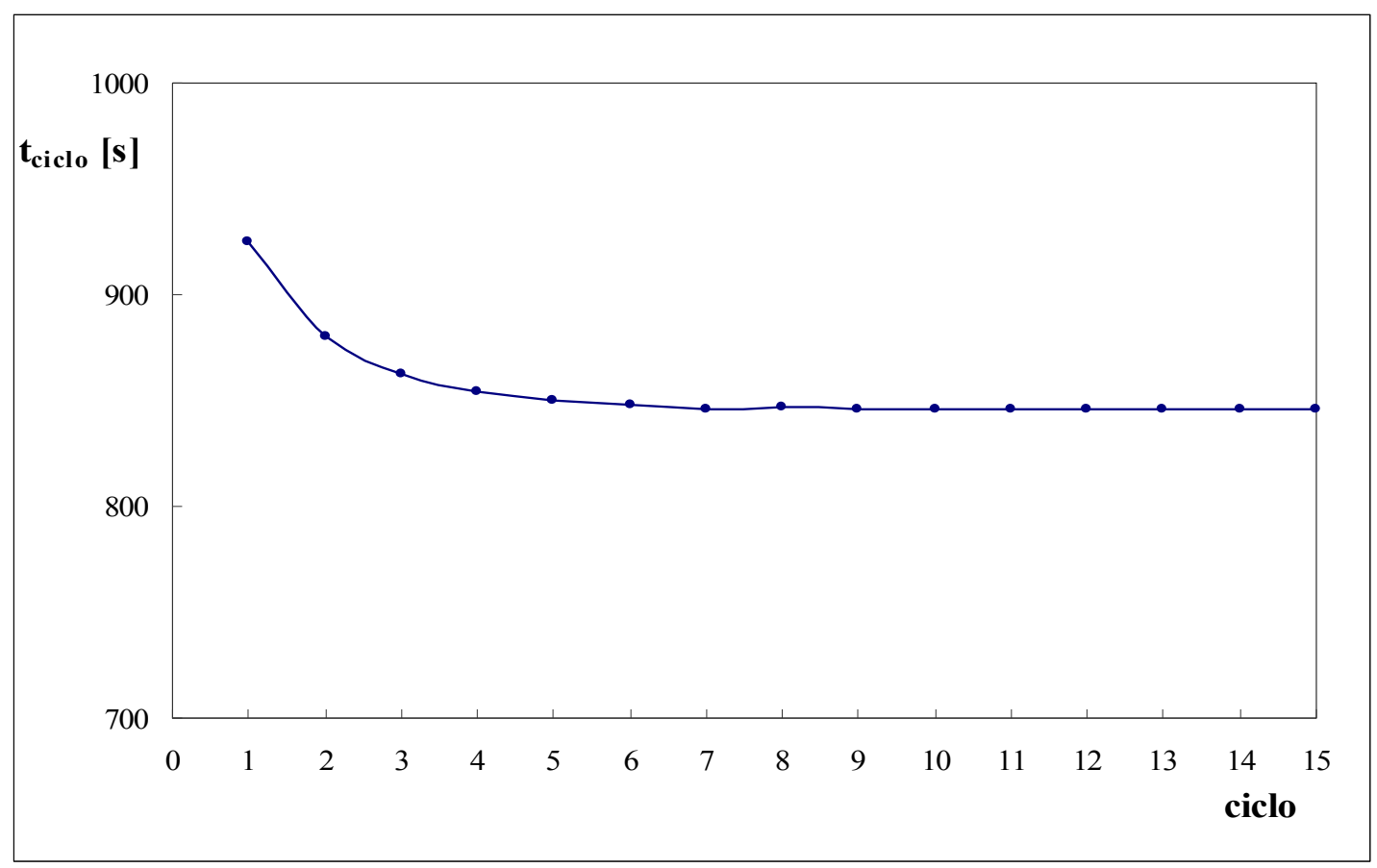

Fig. 2 - Variación del período del proceso de adsorción/desorción en función del número de ciclos. 
Tabla 1: Características de la corriente a tratar

\begin{tabular}{|c|c|c|}
\hline \multicolumn{2}{|c|}{ Caudal $\left[\mathbf{m}^{3} / \mathbf{s}\right]$} & 9 \\
\hline $\begin{array}{c}\text { Composición } \\
{\left[\mathbf{m o l} / \mathbf{m}^{3}\right]}\end{array}$ & Acetato de Etilo & $3.0610^{-2}$ \\
\cline { 2 - 3 } & Etanol & $1.2510^{-2}$ \\
\hline \multicolumn{2}{|r|}{ Tipo de emisión } & Permanente \\
\hline \multicolumn{2}{|c|}{ Presión $[\mathbf{a t m}]$} & 1.3 \\
\hline \multicolumn{2}{|r|}{ Temperatura $\left[{ }^{\circ} \mathbf{C}\right]$} & 50 \\
\hline
\end{tabular}

\subsection{Determinación del caudal mínimo $\left(W_{\text {des }}^{\text {mín }}\right)$}

En la Figura 3 se puede observar la evolución de los ciclos según el valor del caudal de desorción, $\mathrm{W}_{\text {des }}$, para el mismo rotor y condiciones operativas mencionadas en la sección anterior (Fig. 2). De la Fig. 3 se desprende que, para valores de caudal de desorción que superen o igualen un cierto valor mínimo, se puede determinar el período del ciclo de adsorción/desorción. El valor mínimo posible del caudal másico de desorción $\left(\mathrm{W}_{\text {des }}\right)$ para el cual el período puede ser estabilizado se denomina caudal mínimo de desorción, $\mathrm{W}_{\mathrm{des}}^{\mathrm{min}}$. Para el sistema establecido, $\mathrm{W}_{\mathrm{des}}^{\min }$ es aproximadamente $3.69 \mathrm{~kg} / \mathrm{s}$ (ver Fig.3). Para valores de $\mathrm{W}_{\text {des }}$ por debajo de este valor, no es posible obtener una estabilización del ciclo de adsorción/desorción para ningún valor del tiempo, como para el caso de $\mathrm{W}_{\text {des }}=3.56 \mathrm{~kg} / \mathrm{s}$ en la Fig. 3. Para valores mayores a $\mathrm{W}_{\text {des }}^{\text {min }}$ (e.g. $\mathrm{W}_{\text {des }}=3.82 \mathrm{~kg} / \mathrm{s}$ en la Fig. 3) el período se estabiliza más rápidamente. A medida que crece el caudal de desorción, el período, correspondiente al ciclo de adsorción/desorción, aumenta hasta un dado valor asintótico, que corresponde al evaluado en el ciclo 1 (ver Fig. 3). 


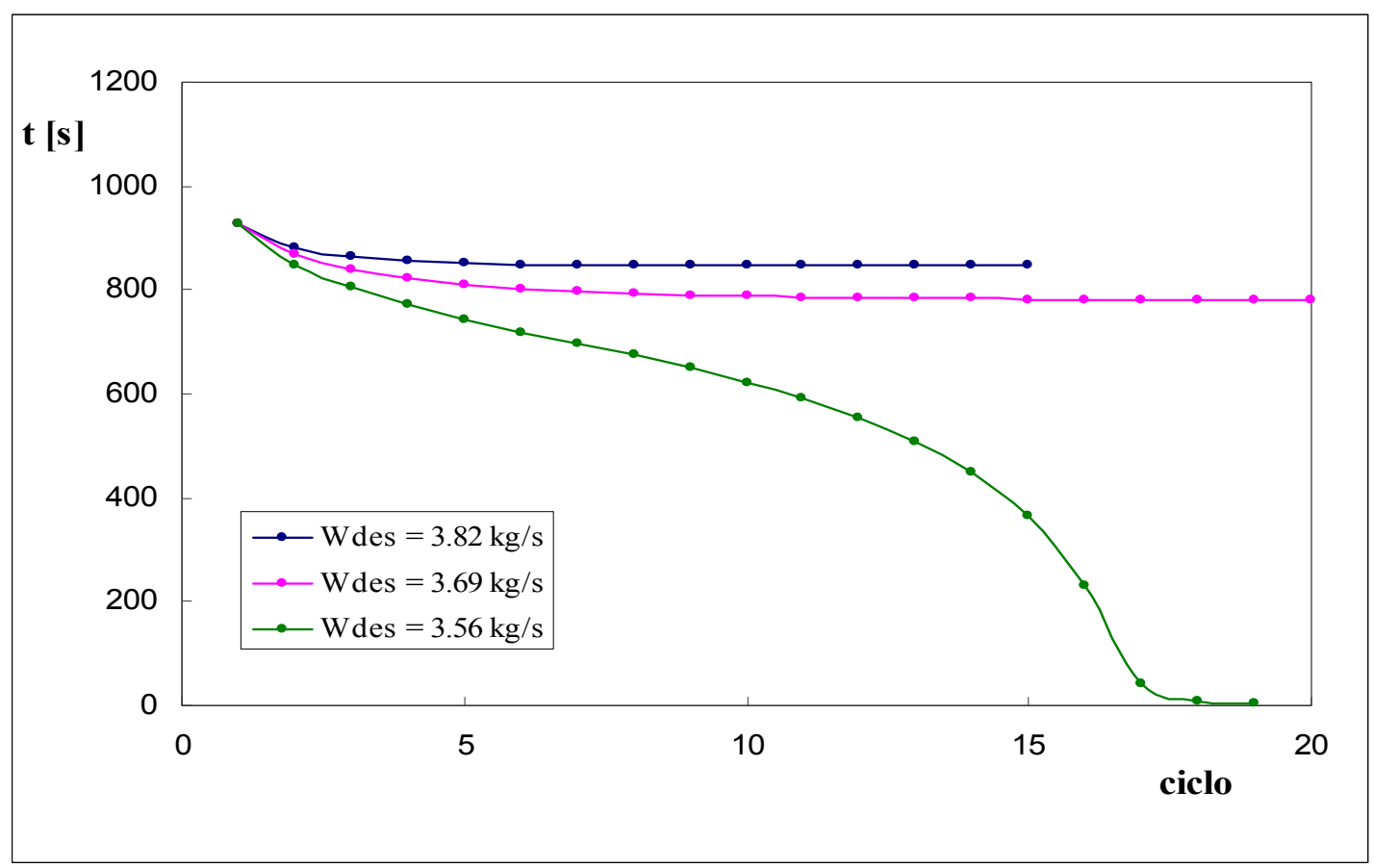

Fig. 3 - Variación del período del proceso de adsorción/desorción en función del número de ciclos según el valor de $\mathrm{W}_{\text {des }}$.

Para una alternativa de diseño diferente $u$ otras condiciones operativas el análisis es análogo, aunque, obviamente, el valor de caudal mínimo de desorción puede cambiar.

Se recuerda que cuanto menor sea el caudal de la corriente empleada para la regeneración del lecho, más concentrada resulta dicha corriente cuando abandona el sistema de adsorción/desorción. Esto implica un reactor e intercambiadores de menor volumen. En el Capítulo 4 se determinó la velocidad mínima de la corriente de desorción para el caso ideal de no considerar las resistencias a la transferencia de calor y materia.

\subsection{Influencia de la relación $D / L$ en el valor de $W_{\text {des }}^{\operatorname{mí}}$}

Se estudió el impacto que causa la relación diámetro/longitud del lecho (D/L) sobre el valor de $\mathrm{W}_{\mathrm{des}}^{\mathrm{min}}$. Se varió la relación $\mathrm{D} / \mathrm{L}$ manteniendo constante el volumen del lecho (V). A modo de ejemplo conservamos las características geométricas definidas en las secciones anteriores donde: $\mathrm{D}=4 \mathrm{~m}$ y $\mathrm{L}=0.5 \mathrm{~m}$, por lo que resulta: $\mathrm{D} / \mathrm{L}=8 \mathrm{y}$ $\mathrm{V}=6.28 \mathrm{~m}^{3}$. Comparamos el valor de $\mathrm{W}_{\text {des }}^{\mathrm{min}}($ i.e. $3.69 \mathrm{~kg} / \mathrm{s}$ en Fig. 3) con el obtenido para un lecho de igual volumen pero con las siguiente dimensiones: $\mathrm{D}=3 \mathrm{~m}$ y $\mathrm{L}=0.89 \mathrm{~m}(\mathrm{D} / \mathrm{L}=3.37)$. Se observó que la disminución de la relación $\mathrm{D} / \mathrm{L}$ de más de 
un $50 \%$ no altera el valor de $\mathrm{W}_{\mathrm{des}}^{\mathrm{min}}$. Esto se puede demostrar a partir de los balances de conservación.

Teniendo en cuenta lo visto en el Capítulo 5, el balance de materia en el lecho para un componente i, despreciando la acumulación en el gas es la siguiente:

$$
\mathrm{v}_{\mathrm{s}} \frac{\partial \mathrm{C}_{\mathrm{i}}}{\partial \mathrm{Z}}=-\left(1-\varepsilon_{\mathrm{L}}\right) \mathrm{a}_{\mathrm{v}} \mathrm{k}_{\mathrm{m}, \mathrm{i}}\left(\mathrm{C}_{\mathrm{g}, \mathrm{i}}-\mathrm{C}_{\mathrm{s}, \mathrm{i}}\right)
$$

Adimensionalizamos la variable espacial $\mathrm{Z}$ como $\mathrm{Z}^{*}=\mathrm{Z} / \mathrm{L}$ y definiendo la velocidad superficial $\mathrm{v}_{\mathrm{s}}$ en función del caudal resulta:

$$
\frac{\mathrm{W}}{\rho_{\mathrm{g}} \mathrm{V}} \frac{\partial \mathrm{C}_{\mathrm{i}}}{\partial \mathrm{Z}^{*}}=-\left(1-\varepsilon_{\mathrm{L}}\right) \mathrm{a}_{\mathrm{v}} \mathrm{k}_{\mathrm{m}, \mathrm{i}}\left(\mathrm{C}_{\mathrm{g}, \mathrm{i}}-\mathrm{C}_{\mathrm{s}, \mathrm{i}}\right)
$$

La Ec. [2] demuestra claramente que mientras el volumen del concentrador rotativo no cambie resulta indistinta la relación $\mathrm{D} / \mathrm{L}$ adoptada. Se podrían llegar a observar pequeñas diferencias de un caso a otro debido a que el cambio de sección provoca una variación en la velocidad y por ende en los coeficientes de transferencia de calor y materia. Sin embargo, al encontrarse en la región asintótica del $S h$ y $N u$ (Apéndice 5.1), los coeficientes resultan ser bastante similares. Obviamente, la magnitud que sí se verá modificada es la pérdida de carga, la cual aumenta al disminuir la relación D/L.

\subsection{Efecto de la relación entre áreas de adsorción y desorción}

Teniendo en cuenta que para el concentrador rotativo la sección destinada a la adsorción, $\mathrm{S}_{\mathrm{ads}}$, puede ser diferente a la de desorción, $\mathrm{S}_{\mathrm{des}}$, (Sección 6.1), resulta interesante analizar la influencia de la relación $\mathrm{S}_{\mathrm{ads}} / \mathrm{S}_{\mathrm{des}}\left(\theta_{\mathrm{ads}} / \theta_{\mathrm{des}}\right)$ en el proceso de adsorción/desorción y particularmente qué efecto tiene sobre el valor del caudal mínimo de desorción.

A continuación se estudia la influencia del ángulo $\left(\theta_{\mathrm{des}}\right)$ que determina la fracción de lecho destinada a la etapa de desorción sobre la cantidad desorbida, q, suponiendo el caso ideal en que no existen resistencias a la transferencia de calor y materia. En este caso, teniendo en cuenta lo visto en el Capítulo 4 (Sección 4.2), el balance de materia en un canal resulta:

$$
\mathrm{Q}_{\text {canal }} \frac{\partial \mathrm{C}_{\mathrm{i}}}{\partial \mathrm{V}_{\text {canal }}}=-\left(1-\varepsilon_{\mathrm{L}}\right) \frac{\partial \mathrm{q}_{\mathrm{i}}}{\partial \mathrm{t}}
$$


Para simplificar el análisis reparametrizamos el producto $\mathrm{Q}_{\text {canal }} \mathrm{t}$ por la variable $\varphi$, resultando:

$$
\frac{1}{\mathrm{~S}_{\text {canal }}} \frac{\partial \mathrm{C}_{\mathrm{i}}}{\partial \mathrm{Z}}=-\left(1-\varepsilon_{\mathrm{L}}\right) \frac{\partial \mathrm{q}_{\mathrm{i}}}{\partial \varphi}
$$

La variable $\varphi$ varía entre 0 y $\varphi_{\text {final }}=Q_{\text {canal }} t_{\text {des }}$

Según la Ecuación [4] la cantidad desorbida (q) resulta ser una función de la nueva variable $\varphi$, de la posición a lo largo del lecho $(Z)$ y de las condiciones en las que se encuentra el lecho una vez finalizada la etapa previa de adsorción. Restaría verificar si q tiene algún tipo de dependencia con el ángulo correspondiente a la etapa desorción $\left(\theta_{\text {des }}\right)$ a través de $\varphi$. Para eso definimos el caudal volumétrico que ingresa al canal en función del caudal total de desorción:

$$
\mathrm{Q}_{\text {canal }}=\frac{\mathrm{Q}_{\text {des }}}{\mathrm{N}_{\text {canales }}}
$$

donde $\mathrm{N}_{\text {canales }}^{\text {des }}=\frac{\theta_{\text {des }} \mathrm{N}_{\text {canales }}^{t}}{2 \pi}$, con $\mathrm{N}_{\text {canales }}^{t}$ : número total de canales

Dado que el número de canales correspondientes a la etapa de desorción ( $\left.\mathrm{N}_{\text {canales }}^{\text {des }}\right)$ es una función del ángulo $\theta_{\text {des }}$, reemplazando en [5] resulta:

$$
\mathrm{Q}_{\text {canal }}=\frac{\mathrm{Q}_{\text {des }} 2 \pi}{\theta_{\text {des }} \mathrm{N}_{\text {canales }}^{t}}
$$

Por su lado, el tiempo en que el canal recorre la porción correspondiente a la etapa de desorción, $t_{\text {des }}$, también resulta ser una función del ángulo $\theta_{\text {des }}$ a través de la siguiente expresión:

$$
\mathrm{t}_{\mathrm{des}}=\frac{1}{r e v}\left(\frac{\theta_{\mathrm{des}}}{2 \pi}\right)
$$

donde rev representa las revoluciones por unidad de tiempo.

Se tiene:

$$
\mathrm{Q}_{\text {canal }} \mathrm{t}_{\mathrm{des}}=\frac{\mathrm{Q}_{\text {des }}}{\mathrm{N}_{\text {canales }}^{t} r e v}
$$


De la Ec. [8] surge que el producto $\mathrm{Q}_{\text {canal }} \mathrm{t}_{\mathrm{des}}=\varphi_{\text {final }}$, resulta independiente del valor del ángulo recorrido y por lo tanto q también lo es.

En el caso real no se cumple exactamente la independencia de q con del ángulo que delimita la zona de desorción. Sin embargo no se observaron grandes diferencias al incluir las resistencias al sistema de cálculo. Esto se puede observar en la Figura 4 donde se presenta la variación de los períodos correspondientes al ciclo estacionario, y los valores de $\mathrm{W}_{\text {des }}^{\min }$ según la relación $\mathrm{S}_{\mathrm{ads}} / \mathrm{S}_{\mathrm{des}}$. Para la obtención de las curvas se consideraron las condiciones geométricas definidas en la Sección 6.1.1, las condiciones operativas de la Tabla 1 y las siguientes dimensiones globales: $\mathrm{D}=4 \mathrm{~m}, \mathrm{~L}=0.7 \mathrm{~m}$. Se fijó este valor de L para la determinación de áreas, dado que será el utilizado en la continuidad del trabajo como se analizará en la Sección 6.6.

A partir de los resultados obtenidos se observa que para relaciones de área superiores a 1 , el valor de caudal mínimo $\left(\mathrm{W}_{\mathrm{des}}^{\mathrm{min}}\right)$ resulta prácticamente independiente de la relación entre secciones. Para el caso en que $\mathrm{S}_{\mathrm{ads}} / \mathrm{S}_{\mathrm{des}}<1$, el caudal mínimo aumenta, por lo que estas relaciones de áreas no resultan convenientes.

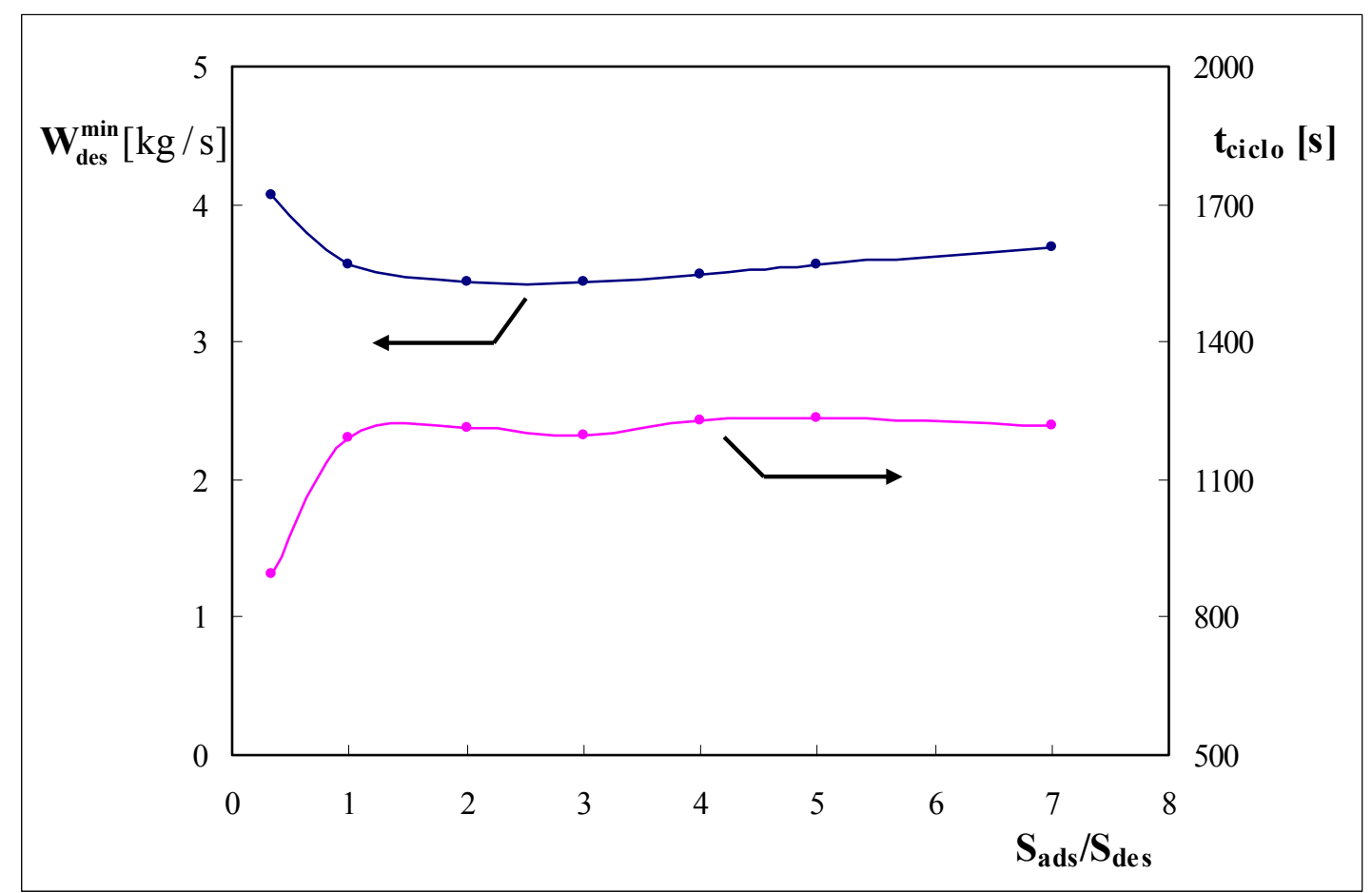

Fig. 4 - Variación de los períodos correspondientes a cada etapa y al ciclo, como también los valores de $\mathrm{W}_{\mathrm{des}}^{\min }$ según la relación $\mathrm{S}_{\mathrm{ads}} / \mathrm{S}_{\mathrm{des}}$. 
En la Tabla 2 pueden observarse los valores del tiempo $\left(\mathrm{t}_{\mathrm{des}}\right)$, caudal mínimo $\left(\mathrm{W}_{\mathrm{des}}^{\mathrm{min}}\right)$, sección $\left(\mathrm{S}_{\mathrm{des}}\right)$ y velocidad superficial $\left(\mathrm{v}_{\mathrm{s}}\right)$ correspondientes a la etapa de desorción para dos de los casos presentados en la Fig. 4.

Tabla 2: Análisis de la influencia de las secciones del rotor

\begin{tabular}{ccc}
\hline & CASO I & CASO II \\
& $\mathrm{S}_{\mathrm{ads}} / \mathrm{S}_{\mathrm{des}}=1$ & $\mathrm{~S}_{\mathrm{ads}} / \mathrm{S}_{\mathrm{des}}=3$ \\
\hline $\mathbf{t}_{\text {des }}[\mathbf{s}]$ & 596.25 & 298.65 \\
$\mathbf{W}_{\mathrm{des}}^{\mathbf{m i n}}[\mathbf{k g} / \mathbf{s}]$ & 3.56 & 3.43 \\
$\mathbf{S}_{\text {des }}\left[\mathbf{m}^{2}\right]$ & 6.28 & 3.14 \\
$\mathbf{v}_{\mathbf{s}}[\mathbf{m} / \mathbf{s}]$ & 0.53 & 1.01 \\
$\mathbf{v}_{\mathbf{s}} \mathbf{t}_{\text {des }}[\mathbf{m}]$ & 313.26 & 302.35 \\
\hline
\end{tabular}

El producto $\mathrm{v}_{\mathrm{s}} \mathrm{t}_{\text {des }}\left(=\frac{\mathrm{Q}_{\text {canal }}}{\mathrm{S}_{\text {canal }}} \mathrm{t}_{\text {des }}\right)$ resulta ser prácticamente el mismo con un error menor al 4\%. Las mínimas diferencias observadas, tanto en la Fig. 4 como en la Tabla 2, surgen, como fuera mencionado en la sección 6.4, debido a que los coeficientes de transferencia de calor y materia no son exactamente iguales en una y otra situación (varían con la velocidad). De todas maneras, al encontrarse en la región asintótica del $S h$ y $N u$ (ver Apéndice 5.1), los coeficientes resultan ser bastante similares.

Considerando estos resultados, el valor de la relación $\mathrm{S}_{\mathrm{ads}} / \mathrm{S}_{\mathrm{des}}$ no sería relevante (siempre que $S_{a d s} / S_{\text {des }}>1$ ). Sin embargo, se podría optimizar la selección a partir de minimizar la pérdida de carga del sistema. Para dicha optimización se considera gas ideal, flujo laminar y viscosidad de la corriente constante con la temperatura. La expresión correspondiente a la pérdida de carga para el sistema monolítico es la siguiente (Barreto, 2007):

$$
\Delta \mathrm{P}=\mu_{\mathrm{g}} \frac{\mathrm{v}_{\mathrm{s}}}{8} \mathrm{fRe} \mathrm{R}_{\mathrm{f}} \mathrm{L}
$$

donde el factor de pérdida de carga (para régimen laminar), $\mathrm{R}_{\mathrm{f}} \mathrm{y} f \mathrm{fe}$, dependen de la geometría del canal. Para un canal de sección cuadrada se tiene:

$$
\mathrm{R}_{\mathrm{f}}=\frac{\mathrm{a}_{\mathrm{v}}{ }^{2}}{\varepsilon_{\mathrm{L}}{ }^{3}} \text { y } \mathrm{fRe}=14.2(\operatorname{para} \mathrm{R}=0)
$$


Dado que: $\Delta \mathrm{P}_{\mathrm{T}}=\Delta \mathrm{P}_{\text {ads }}+\Delta \mathrm{P}_{\text {des }}$, resulta:

$$
\Delta \mathrm{P}_{\mathrm{T}}=\frac{\mathrm{R} \mu_{\mathrm{g}}}{8 \mathrm{P} \mathrm{M}_{\text {aire }}} \quad \text { fRe } \mathrm{R}_{\mathrm{f}} \mathrm{L}\left(\frac{\mathrm{W}_{\mathrm{ads}} \mathrm{T}_{\mathrm{ads}}}{\mathrm{S}_{\mathrm{ads}}}+\frac{\mathrm{W}_{\text {des }} \mathrm{T}_{\text {des }}}{\mathrm{S}_{\text {des }}}\right)=g^{*}\left(\frac{\mathrm{W}_{\mathrm{ads}} \mathrm{T}_{\mathrm{ads}}}{\mathrm{S}_{\mathrm{ads}}}+\frac{\mathrm{W}_{\text {des }} \mathrm{T}_{\text {des }}}{\mathrm{S}_{\mathrm{T}}-\mathrm{S}_{\text {ads }}}\right)
$$

De [10] se tiene que: $g^{*}=\frac{\mathrm{R} \mu_{\mathrm{g}}}{8 \mathrm{PM}_{\text {aire }}}$ fRe $\mathrm{R}_{\mathrm{f}} \mathrm{L} \quad \mathrm{y} \quad \mathrm{S}_{\mathrm{T}}=\mathrm{S}_{\mathrm{ads}}+\mathrm{S}_{\mathrm{des}}$.

Se debe encontrar un valor de $\mathrm{S}_{\text {ads }}^{*}$ que minimice $\Delta \mathrm{P}_{\mathrm{T}}$. Con dicho propósito derivamos la Ec. [10] en función de $\mathrm{S}_{\mathrm{ads}}$ e igualamos a cero, obteniéndose la siguiente relación:

$$
\mathrm{S}_{\mathrm{ads}}^{*}=\frac{\mathrm{S}_{\mathrm{T}} \sqrt{\mathrm{W}_{\mathrm{ads}} \mathrm{T}_{\mathrm{ads}}}}{\sqrt{\mathrm{W}_{\mathrm{ads}} \mathrm{T}_{\mathrm{ads}}}+\sqrt{\mathrm{W}_{\mathrm{des}} \mathrm{T}_{\text {des }}}}
$$

Adoptando un diámetro de $4 \mathrm{~m}\left(\mathrm{~S}_{\mathrm{T}}=12.57 \mathrm{~m}^{2}\right)$, las condiciones operativas presentadas en la Tabla 1 y considerando, a modo de ejemplo, $\mathrm{W}_{\text {des }}=3.4 \mathrm{~kg} / \mathrm{s}$ y $\mathrm{T}_{\mathrm{des}}=150{ }^{\circ} \mathrm{C}$, se tiene que $\left(\frac{\mathrm{S}_{\mathrm{ads}}}{\mathrm{S}_{\mathrm{des}}}\right)^{*}=1.7$.

\subsection{Influencia de la cantidad de adsorbente sobre el caudal mínimo de desorción:} $\mathbf{W}_{\text {des }}^{\text {mín }}$

A continuación se analiza la influencia de la cantidad de adsorbente $\left(\mathrm{V}_{\mathrm{ads}}\right)$ sobre el caudal mínimo de desorción, $\mathrm{W}_{\mathrm{des}}^{\mathrm{min}}$. Para este análisis se consideran las características geométricas mencionadas en la Sección 6.1.1, estableciéndose que la relación entre las secciones es la recientemente establecida como óptima para las condiciones estipuladas $\left(\frac{\mathrm{S}_{\mathrm{ads}}^{*}}{\mathrm{~S}_{\mathrm{des}}}=1.7\right)$. Recordamos que esta relación no afecta los resultados obtenidos (caudal mínimo es independiente de dicha relación) (Fig. 4). Para los cálculos se mantuvo la sección del equipo constante $(\mathrm{D}=4 \mathrm{~m})$ y se varió su longitud $(\mathrm{L})$.

En la Figura 5 se puede observar el impacto que causa la cantidad de adsorbente en los valores de $\mathrm{W}_{\text {des }}^{\text {mín }}$, para dos temperaturas de la corriente de desorción, y se comparan ambos casos con el valor de $\mathrm{W}_{\text {des }}^{\text {mín }}$ obtenido al no considerar las resistencias a la transferencia de calor y materia (caso ideal). 


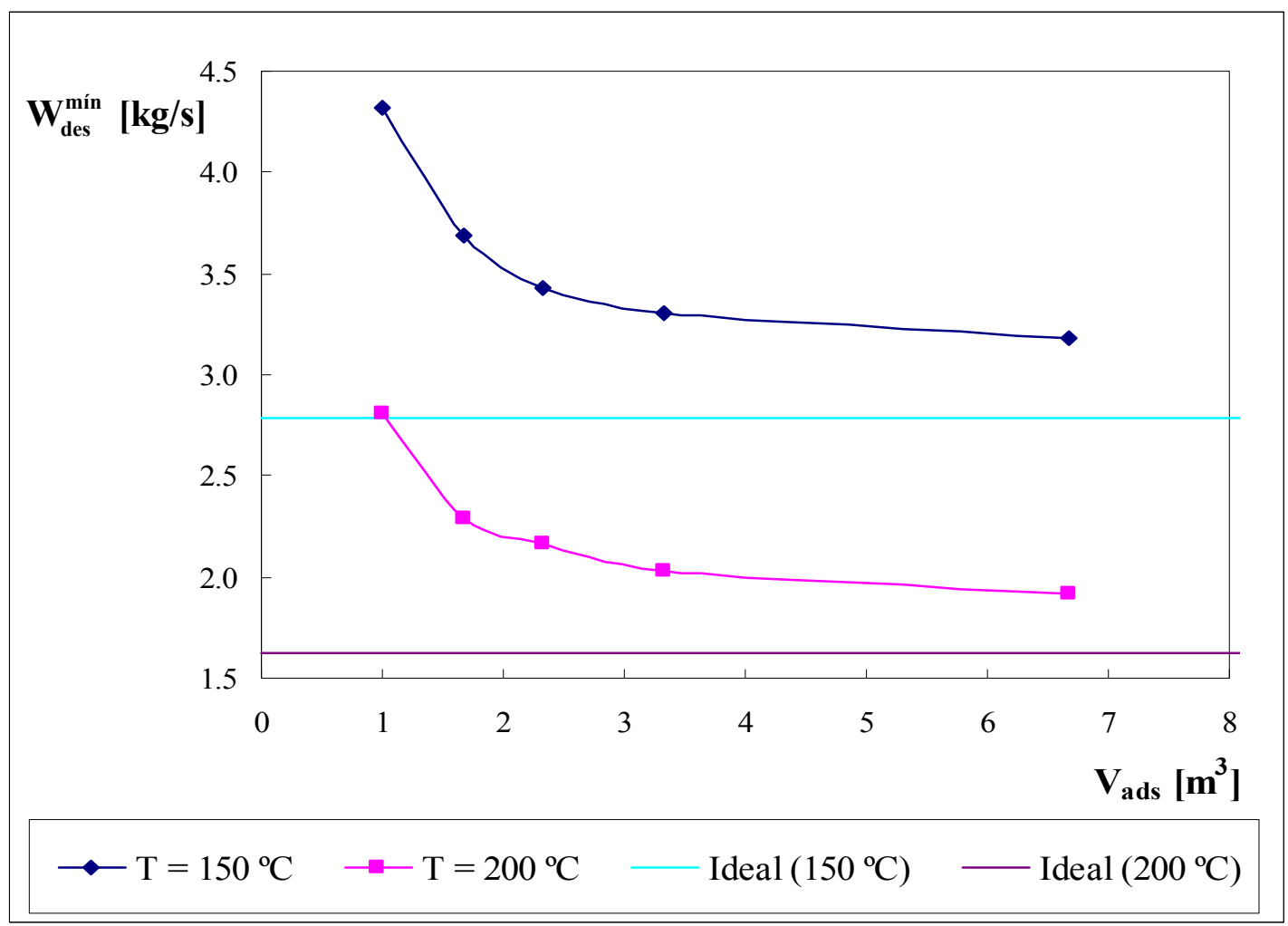

Fig. 5 - Variación del valor de $\mathrm{W}_{\mathrm{des}}^{\mathrm{min}}$ en función de la cantidad de adsorbente.

De la Fig. 5 se desprende que al aumentar el volumen de adsorbente, disminuye el valor de $\mathrm{W}_{\mathrm{des}}^{\mathrm{min}}$. A medida que la cantidad de adsorbente aumenta, la disminución del caudal mínimo de desorción resulta ser cada vez menos significativa y tiende al valor teórico que surge del caso ideal. Para los valores máximos ensayados se obtienen diferencias del orden del 15\%.

Se debe destacar que, para volúmenes de adsorbente inferiores a un dado valor crítico (en Fig. 5: $1.5 \mathrm{~m}^{3}$ aprox.), el valor correspondiente al caudal mínimo de desorción se encuentra altamente influenciado por la cantidad de adsorbente disponible. Como consecuencia, para un volumen de adsorbente determinado, el comportamiento del sistema de eliminación resulta afectado de manera significativa frente a alguna perturbación de las variables operativas de la corriente a tratar. Por lo tanto, resulta conveniente operar con cantidades de adsorbente por encima del valor crítico en cuestión. Por otro lado, para volúmenes relativamente grandes, si bien el valor de caudal mínimo prácticamente se mantiene inalterado, los costos de inversión se vuelven innecesariamente más elevados.

Teniendo en cuenta estas observaciones se puede seleccionar un volumen de adsorbente apropiado para las condiciones operativas establecidas (Tabla 1). A partir de 
pretender un volumen de adsorbente relativamente pequeño y un valor de caudal mínimo de desorción lo más cercano posible al valor teórico obtenido del caso ideal, surge, para una temperatura de desorción de $150^{\circ} \mathrm{C}$, un rango de volumen de adsorbente entre aproximadamente 1.5 y $3 \mathrm{~m}^{3}$ (ver Fig. 5). Para volúmenes de adsorbente menores a $1.5 \mathrm{~m}^{3}$, el caudal mínimo comienza a aumentar de manera significativa a medida que la cantidad de adsorbente disminuye, y para volúmenes mayores a $3 \mathrm{~m}^{3}$, el caudal mínimo de desorción prácticamente no cambia. Teniendo en cuenta el rango de volumen de adsorbente establecido como óptimo, se propone una diferencia entre el caudal mínimo de desorción a utilizar y el obtenido para el caso ideal de aproximadamente un $25 \%$. Esto implica un volumen de adsorbente de alrededor de $2.3 \mathrm{~m}^{3}\left(\mathrm{~W}_{\mathrm{des}}^{\mathrm{min}}=3.43 \mathrm{~kg} / \mathrm{s}\right)$.

A partir de haber definido un volumen de adsorbente, y especificar un valor para la pérdida de carga, se establecen las dimensiones globales del lecho, diámetro (D) y longitud (L). Conservando un diámetro de $4 \mathrm{~m}$ y considerando la porosidad del lecho establecida en la Tabla 4 del Capítulo $5\left(\varepsilon_{\mathrm{L}}=0.73\right)$, resulta una longitud de $0.7 \mathrm{~m}$.

Llevando a cabo el mismo razonamiento para el sistema de dos lechos presentado en el Capítulo 5, se requiere de un volumen de adsorbente de $3.58 \mathrm{~m}^{3}$. Manteniendo la sección establecida en el capítulo anterior, i.e. $2.25 \mathrm{~m}^{2}$, resulta una longitud de $3 \mathrm{~m}$. La diferencia que existe con la cantidad de adsorbente requerida para el concentrador rotativo surge debido a que la presión de la corriente a tratar, utilizada en los cálculos, difiere en un 30\% (1.3 atm en el caso del concentrador rotativo y $1 \mathrm{~atm}$ en el sistema de dos lechos).

En la Fig. 5 también se observa que, al modificar la temperatura de la corriente de desorción $\left(\mathrm{T}=200^{\circ} \mathrm{C}\right)$, el efecto que causa la variación de la cantidad de adsorbente sobre el caudal mínimo de desorción resulta similar al discutido para una corriente de desorción a $150{ }^{\circ} \mathrm{C}$. Consecuentemente podría adoptarse un rango de volumen de adsorbente similar para el diseño del sistema de adsorción/desorción.

Sin embargo, el valor de la temperatura a la cual se lleva a cabo la etapa de desorción influye en los valores de caudal mínimo. Al aumentar la temperatura de $150{ }^{\circ} \mathrm{C}$ a $200{ }^{\circ} \mathrm{C}$, los valores del caudal mínimo de desorción disminuyen aproximadamente un $40 \%$. En cambio, el período de ciclo para un dado volumen prácticamente no cambia, (se observa un aumento del $5 \%$ ). Esto se debe esencialmente a que el período del ciclo 
de adsorción/desorción está determinado por la etapa de adsorción, la cual es análoga en ambas situaciones.

\subsection{Criterio de diseño: Zona de Transferencia de Masa (ZTM)}

En la sección anterior se determinó un rango de volumen de adsorbente apropiado para el diseño del sistema de adsorción/desorción, a partir de analizar el efecto del mismo sobre el caudal mínimo de desorción (Fig. 5). Para las condiciones operativas establecidas en la Tabla 1 se propuso un volumen de adsorbente equivalente a $2.3 \mathrm{~m}^{3}$. En la Figura 6 se presenta el perfil de concentración del acetato de etilo al finalizar la etapa de adsorción una vez alcanzado el estado de ciclo estacionario.

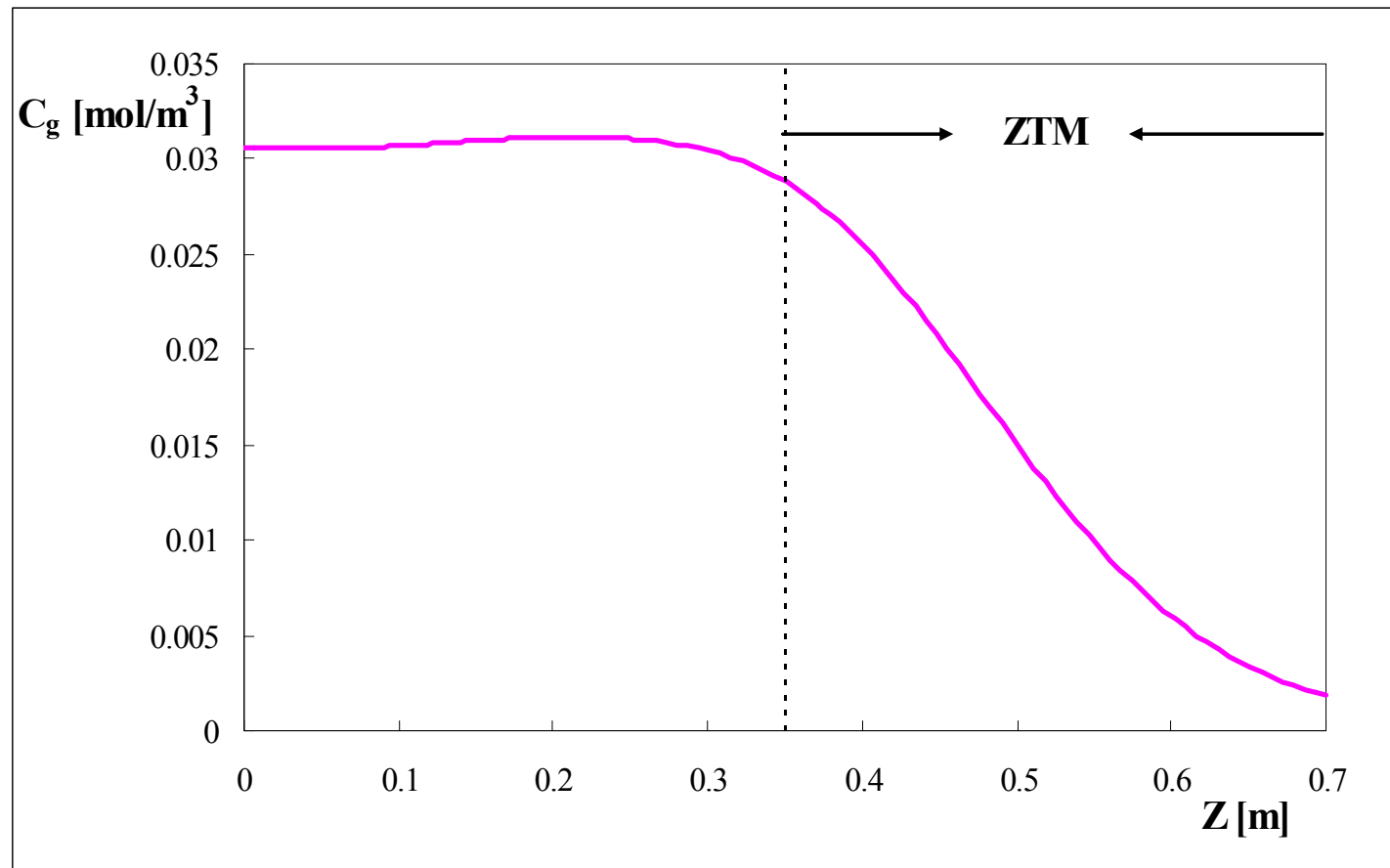

Fig. 6 - Perfil de concentración del acetato de etilo al finalizar la etapa de adsorción una vez alcanzado el estado de ciclo estacionario $(\mathrm{D}=4 \mathrm{~m}, \mathrm{~L}=0.7 \mathrm{~m})$.

Si definimos a la Zona de Transferencia de Masa (ZTM) como aquella zona donde las concentraciones límites se encuentran entre 0.95 y 0.05 del valor de la concentración de alimentación, surge que dicha zona representa aproximadamente un $50 \%$ de la longitud del rotor, mientras que el otro $50 \%$ se encuentra saturado.

Para los extremos del rango de volumen de adsorbente determinado en la sección anterior, $1.5-3 \mathrm{~m} 3$, la ZTM representa entre un 60 y un $40 \%$ de la longitud del rotor respectivamente. Esto implica que, al finalizar la etapa de adsorción, un porcentaje significativo de lecho se encuentre saturado. 
Resulta factible pensar que, en general, variando las condiciones operativas, ya sea de la corriente a tratar o de desorción, el rango establecido para el tamaño de la ZTM, se mantiene aproximadamente entre el 40 y $60 \%$. En base a estas observaciones, resulta adecuado considerar como criterio de diseño del sistema de adsorción/desorción, que al finalizar la etapa de adsorción, la ZTM represente aproximadamente un $50 \%$ de la longitud del rotor.

\subsection{Método aproximado de cálculo}

Como fuera demostrado en el Capítulo 5, el caso ideal, sin resistencias a la transferencia de calor y materia, para el cual fue empleado el Método de las Características, resultó ser una buena aproximación para simular el comportamiento del sistema de adsorción /desorción. Sin embargo, siendo que para el caso ideal, el caudal mínimo de desorción se mantiene constante frente a la variación de volumen de adsorbente (Fig. 5), no es posible discriminar un rango de volumen de adsorbente para el diseño del sistema de adsorción/desorción (Sección 6.6). Además, por definición, cuando las resistencias a la transferencia de calor y materia no son consideradas, no existe una ZTM, por lo que no es posible adoptar esta característica como criterio de diseño. No obstante, teniendo en cuenta las características del caso ideal presentadas en el Capítulo 4, es factible, mediante el empleo de una aproximación, determinar el volumen de adsorbente adecuado para el diseño del sistema de adsorción/desorción.

En el Capítulo 4 se demostró que la onda térmica se desplaza más rápidamente que el frente de concentración, por lo tanto el proceso se comporta como si fuera prácticamente isotérmico. Por otro lado, se tiene que a la temperatura de adsorción $\left(50{ }^{\circ} \mathrm{C}\right)$, el acetato de etilo posee una constante de adsorción bastante más chica que la del etanol (Sección 5.6, Tabla 13), por lo que el etanol se adsorbe más fácilmente dentro del lecho induciendo a una separación de los componentes. Esto implica que el acetato de etilo se evidencie en primer lugar a la salida, determinando el tiempo de adsorción.

Teniendo en cuenta estas observaciones, resulta apropiado considerar al acetato de etilo como un único componente, cuyo frente de concentración se traslada isotérmicamente a lo largo del lecho en forma de escalón. El perfil de concentración del acetato de etilo obtenido a partir del Método de las Características, se puede observar en la Figura 7 (caso ideal). 


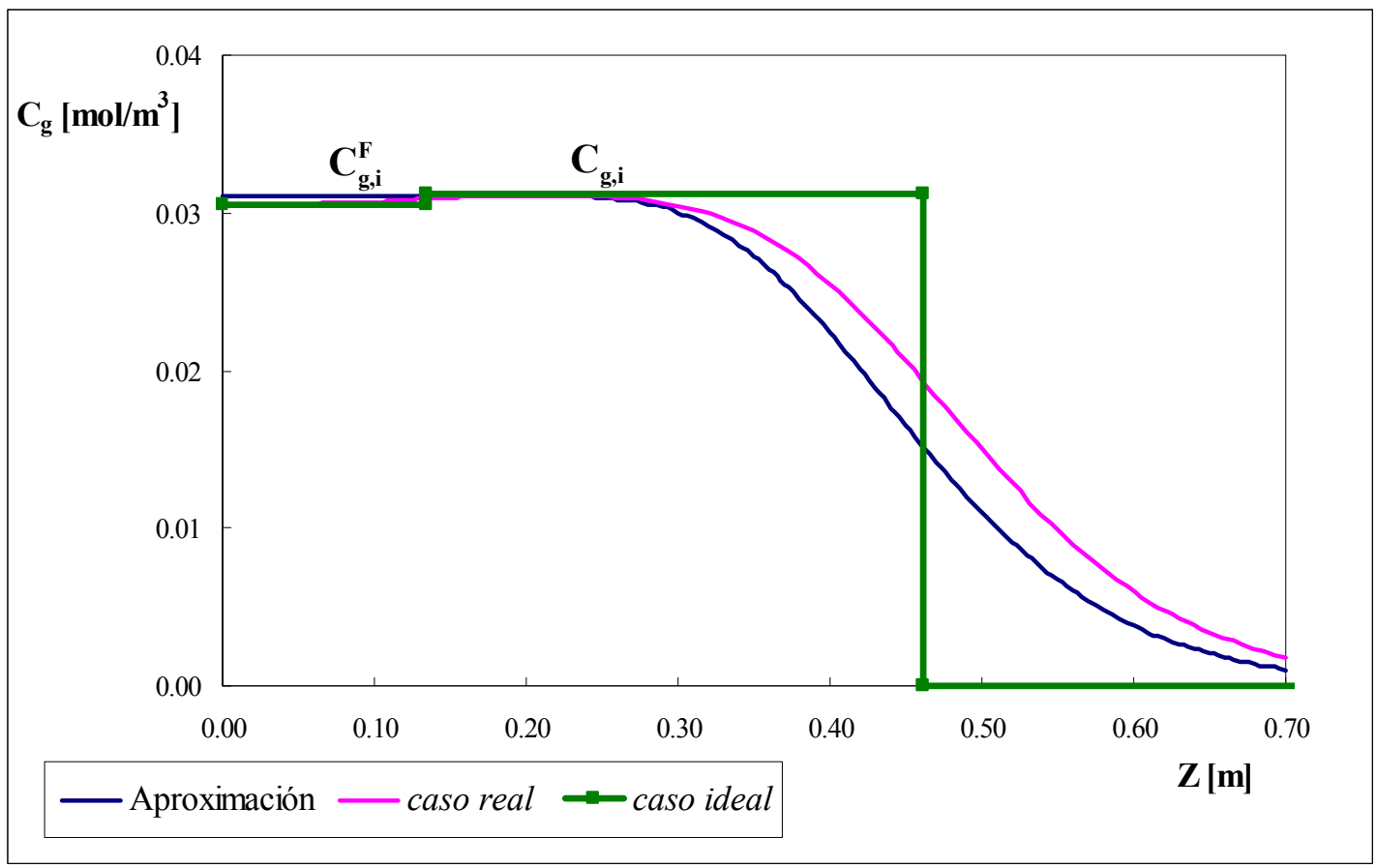

Fig. 7 - Perfil de concentración del acetato de etilo a los $753 \mathrm{~s}$ de la etapa de adsorción para el caso ideal, la aproximación de la Ec. [17] y el caso real. La curva que representa el caso real corresponde al ciclo estacionario.

Por otro lado, teniendo en cuenta que para nuestro caso particular de estudio, la corriente a tratar es muy diluida, la isoterma de adsorción resulta aproximadamente lineal.

Para un componente que se adsorbe isotérmicamente siguiendo una isoterma de adsorción lineal, con flujo pistón y teniendo en cuenta las resistencias a la transferencia de masa, la respuesta de un escalón de concentración en la alimentación puede representarse mediante la siguiente expresión de la varianza (Antos y col., 2003) ( $\left.\vartheta^{2}\right)$ :

$$
\vartheta^{2}=2\left[\kappa\left(1-\varepsilon_{\mathrm{L}}\right)\right]^{2} \frac{\mathrm{Z}}{\mathrm{v}_{\mathrm{s}}} \frac{1}{\mathrm{~K}_{\mathrm{v}}}
$$

donde $K$ es la constante de equilibrio de adsorción correspondiente a la siguiente isoterma de adsorción lineal:

$$
\mathrm{q}=\kappa \mathrm{C},
$$

$\varepsilon_{\mathrm{L}}$ es la porosidad del lecho, $\mathrm{Z}$ la posición axial, $\mathrm{v}_{\mathrm{S}}$ la velocidad superficial y $\mathrm{K}_{\mathrm{V}}$ es el coeficiente de transferencia de materia por unidad de volumen de lecho incluyendo las resistencias interna y externa. 
Teniendo en cuenta lo visto en el Capítulo 5 (Sección 5.4.2), para un lecho monolítico el coeficiente $\mathrm{K}_{\mathrm{v}}$ resulta:

$$
\mathrm{K}_{\mathrm{v}}=\mathrm{K}^{*}\left(1-\varepsilon_{\mathrm{L}}\right)=\frac{1}{\frac{1}{\mathrm{a}_{\mathrm{v}} \mathrm{k}_{\mathrm{m}}}+\frac{\ell_{e}^{2}}{3 \mathrm{D}_{\mathrm{e}}}}\left(1-\varepsilon_{\mathrm{L}}\right)
$$

A medida que el escalón de concentración aplicado en la alimentación avanza en el lecho, el mismo comienza a distorcionarse debido a la presencia de las resistencias a la transferencia de materia. Cuando dicho escalón alcanza valores suficientemente altos de $\mathrm{Z}$ es posible suponer que el escalón disperso responde a la "Función de Distribución Normal de Gauss" dada por:

$$
F(\mathrm{Z}, \mathrm{t})=\int_{-\infty}^{t} \frac{1}{\vartheta \sqrt{2 \pi}} \exp \left[-\left(\frac{\tau-\varpi}{2 \vartheta^{2}}\right)^{2}\right] d \tau
$$

donde $F$ es la concentración en la fase fluida adimensionalizada con el valor de concentración de alimentación a un dado $\mathrm{Z}$ y t, y $\varpi$ es el tiempo de residencia medio correspondiente a una posición Z:

$$
\varpi=\left[\kappa\left(1-\varepsilon_{\mathrm{L}}\right)+\varepsilon_{\mathrm{L}}\right] \frac{\mathrm{Z}}{\mathrm{v}_{\mathrm{s}}}
$$

La integración de la expresión [15] puede escribirse en la forma:

$$
F(\mathrm{Z}, \mathrm{t})=\frac{1}{2}\left[1+\operatorname{erf}\left(\frac{\mathrm{t}-\varpi}{\vartheta \sqrt{2}}\right)\right]
$$

donde erf es la función error.

Winitzky (2003, 2008) propone la siguiente aproximación de la función error, que se comporta de manera altamente precisa (error máximo $=0.00012$ ):

$$
[\operatorname{erf}(\mathrm{x})]^{2} \simeq 1-\exp \left[-\mathrm{x}^{2} \frac{4 / \pi+0.147 \mathrm{x}^{2}}{1+0.147 \mathrm{x}^{2}}\right]
$$

En la Ec. [18], se debe tomar el signo de la raíz positivo para $\mathrm{x}>0$ y negativo para $\mathrm{x}<0$.

Para aplicar la formulación descrita a nuestro caso, y con el objetivo de obtener una mayor precisión de los resultados, si bien se trata de una corriente diluida, no se consideró una isoterma estrictamente lineal, sino que en base a la isoterma de Langmuir 
generalizada (Ec. [38a], Capítulo 4) se tomó en cuenta el efecto de inhibición del acetato de etilo en el denominador, resultando: $\boldsymbol{K}_{\mathrm{i}}=\frac{Q_{\mathrm{i}} \mathrm{K}_{\mathrm{i}}}{1+\mathrm{K}_{\mathrm{i}} \mathrm{C}_{\mathrm{i}}}, \mathrm{i}=$ acetato de etilo. La concentración adoptada para $\mathrm{C}_{\mathrm{i}}$, obtenida a partir del Método de las Caracteríticas, se encuentra indicada en la Fig. $7\left(\mathrm{C}_{\mathrm{g}, \mathrm{i}}\right)$. Se podría haber tomado la concentración de alimentación, $\mathrm{C}_{\mathrm{g}, \mathrm{i}}^{\mathrm{F}}$, o un promedio entre ambas, sin embargo, en este caso, la concentración seleccionada prácticamente no difiere de la concentración de alimentación, $\mathrm{C}_{\mathrm{g}, \mathrm{i}}^{\mathrm{F}}$, y resulta ser la más representativa (Fig. 7).

El perfil de concentración correspondiente al acetato de etilo, para las mismas condiciones que las empleadas en la Fig. 6, obtenido mediante el uso de la Ec. [17], se representa en la Fig. 7, donde también se muestra el perfil de concentración correspondiente al caso real de la Fig. 6. De la comparación de ambas curvas se infiere que la Ec. [17] resulta ser una muy buena aproximación de la curva representada en la Fig. 6.

La curva correspondiente al caso real se encuentra desplazada hacia valores de $\mathrm{Z}$ algo mayores con respecto a la curva obtenida con la aproximación dada por la Ec. [17]. Esto se debe a que para el caso ideal y la aproximación (Ec. [17]), se considera al lecho inicialmente limpio, situación que en realidad no sucede al alcanzar el estado de ciclo estacionario.

En la Figura 8 se puede observar la precisión de la aproximación cuando se la compara con el perfil de concentración del caso real al finalizar la etapa de adsorción correspondiente al primer ciclo, en este caso el lecho se encuentra inicialmente limpio. Se observa que la aproximación solo difiere ligeramente del caso real.

Teniendo en cuenta la concentración de VOC permitida a la salida del lecho y el criterio de diseño expuesto en la sección anterior, por el cual se propone que la ZTM alcance alrededor de un $50 \%$ una vez finalizada la etapa de adsorción, es factible estimar el tiempo de adsorción y el volumen de adsorbente requerido mediante el uso de la aproximación brindada por la Ec. [17]. 


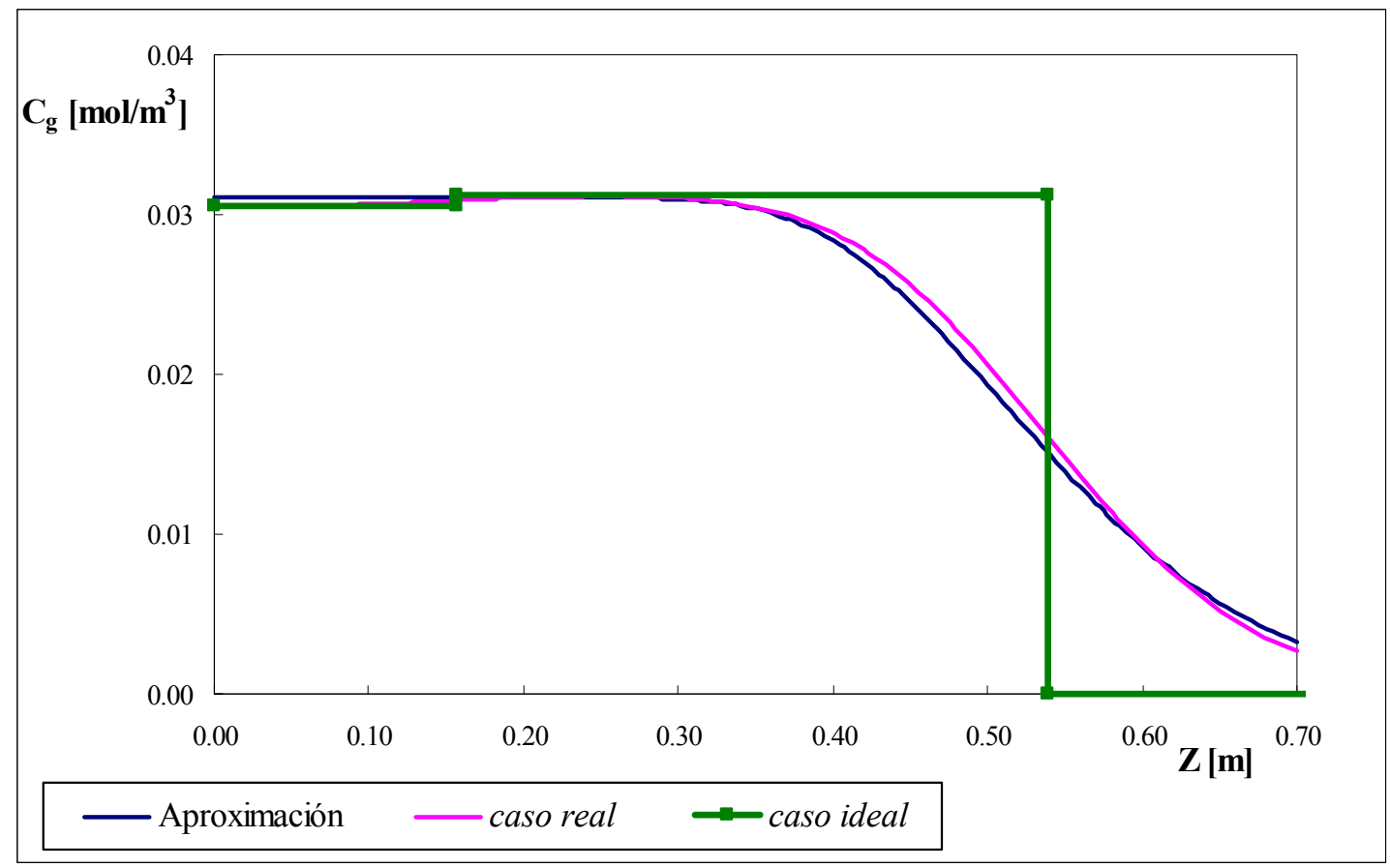

Fig. 8 - Perfil de concentración del acetato de etilo a los $880 \mathrm{~s}$ de la etapa de adsorción para el caso ideal, la aproximación de la Ec. [17] y caso real. La curva que representa el caso real corresponde al ciclo 1 .

Por ejemplo, para las condiciones de las Fig. 6, 7 y 8, tras un proceso iterativo de cálculo se determinó un tiempo de adsorción de aproximadamente $830 \mathrm{~s}$ y un volumen de adsorbente de $2.3 \mathrm{~m}^{3}$. Por lo tanto, se observa una diferencia de alrededor del $10 \%$ en el tiempo de adsorción con respecto al obtenido para el caso real (753 s), mientras que el volumen resulta prácticamente el mismo (ver Sección 6.6). La diferencia en el tiempo se debe principalmente, como ya fuera mencionado, a que para la aproximación el lecho es considerado inicialmente limpio.

Si bien fue necesario el análisis realizado en la Sección 6.6 para el estudio del comportamiento del sistema de adsorción/desorción según la cantidad de material adsorbente disponible, mediante el uso de la aproximación (Ec. [17]) y el criterio de considerar que la ZTM representa alrededor de un $50 \%$ del lecho al finalizar la etapa de adsorción, el esfuerzo de cálculo para la determinación de la cantidad de material adsorbente requerida resulta ser mucho menor.

Por otro lado, se tiene que el valor de caudal mínimo puede ser estimado a partir de considerar el valor teórico que surge del caso ideal, dado que este valor se encuentra muy próximo al valor obtenido al considerar las resistencias a la transferencia de calor y materia (Sección 6.6). Siendo que no conviene operar con un caudal estrictamente igual 
al mínimo, se opta por un caudal un 25\% mayor al valor teórico. Dicho valor coincide con el valor adoptado en la Sección 6.6 para el caso real.

\subsection{Conclusiones}

A partir de considerar las ventajas del empleo de concentradores rotativos frente a sistemas de dos lechos que operan alternativamente, resultó apropiado optar por dicha configuración para el sistema de eliminación propuesto en el Capítulo 2 (Fig. 3).

Para dimensiones $(\mathrm{D}, \mathrm{L})$ definidas del concentrador rotativo, condiciones operativas estipuladas y un valor establecido para la concentración de VOCs en el efluente, se determinó el caudal mínimo de desorción que permite alcanzar el estado de ciclo estacionario.

En primer lugar se analizó el impacto que causa la relación diámetro/longitud del rotor $(\mathrm{D} / \mathrm{L})$ sobre el valor de caudal mínimo de desorción. Se comprobó que, mientras el volumen del equipo no cambie, resulta virtualmente indistinta la relación $\mathrm{D} / \mathrm{L}$ adoptada.

Asimismo, se analizó el efecto de la relación entre áreas destinadas a la etapa de adsorción y desorción sobre el valor del caudal mínimo de desorción (Fig. 4). De los resultados obtenidos se tuvo que, para valores mayores a uno de relación de áreas, el valor del caudal mínimo resulta prácticamente independiente de la relación entre secciones. Para el caso en que $\mathrm{S}_{\mathrm{ads}} / \mathrm{S}_{\mathrm{des}}<1$, el caudal mínimo adopta valores más elevados, por lo que esta alternativa no resulta una buena opción.

Se pudo optimizar la relación $\mathrm{S}_{\mathrm{ads}} / \mathrm{S}_{\mathrm{des}}$ a partir de minimizar la pérdida de carga del sistema.

Por otro lado, se estudió la influencia de la cantidad de material adsorbente sobre el valor de caudal mínimo de desorción. De los resultados obtenidos (Fig. 5) surge la posibilidad de estimar un rango de cantidad de material adsorbente adecuado para el diseño del sistema de adsorción/desorción. Para las condiciones establecidas, y para una temperatura de regeneración de $150{ }^{\circ} \mathrm{C}$, se estimó un rango entre 1.5 y $3 \mathrm{~m}^{3}$ de adsorbente. Para volúmenes de adsorbente menores a $1.5 \mathrm{~m}^{3}$, el caudal mínimo comienza a aumentar de manera significativa a medida que la cantidad de adsorbente disminuye, y para volúmenes mayores a $3 \mathrm{~m}^{3}$, el caudal mínimo de desorción prácticamente no cambia con la cantidad de adsorbente.

Para los extremos del rango de volumen de adsorbente determinado a partir del análisis de la Fig. 5, la Zona de Transferencia de Masa (ZTM) representa entre un 60 y 
un $40 \%$ del adsorbedor al finalizar la etapa de adsorción. Estos resultados pueden ser considerados como un criterio de diseño del sistema de adsorción/desorción para un caso general.

Un análisis similar se realizó para una temperatura de regeneración de $200{ }^{\circ} \mathrm{C}$ (Fig. 5). En principio, el efecto que causa la variación de la cantidad de adsorbente sobre el caudal mínimo de desorción resulta similar al discutido para una corriente de desorción a $150{ }^{\circ} \mathrm{C}$. Consecuentemente podría adoptarse un rango de volumen de adsorbente similar para el diseño del sistema de adsorción/desorción. Por otro lado, se verificó que el valor de la temperatura a la cual se lleva a cabo la etapa de desorción influye en los valores de caudal mínimo. Al aumentar la temperatura de $150{ }^{\circ} \mathrm{C}$ a $200{ }^{\circ} \mathrm{C}$, el valor del caudal mínimo de desorción disminuye aproximadamente un 40 \%. Esto confirma que cuanto mayor es la temperatura de regeneración, más eficiente resulta el proceso de desorción. Sin embargo, a mayores temperaturas aumenta el grado de consumo del carbón, lo que implica una desventaja ya que a temperaturas por encima de los $300{ }^{\circ} \mathrm{C}$ el carbón pierde estabilidad térmica (Yates y col., 2000).

A partir de haber definido un volumen de adsorbente y especificar un valor para la pérdida de carga, se establecen las dimensiones globales del lecho, diámetro (D) y longitud (L).

En resumen, teniendo en cuenta el análisis realizado sobre el comportamiento del sistema de adsorción/desorción, se puede determinar el diseño y condiciones operativas adecuadas del sistema.

En base a las características que presenta el caso ideal (Capítulo 4), resulta apropiado considerar al acetato de etilo como un único componente presente en la corriente a tratar, cuyo frente de concentración se traslada isotérmicamente a lo largo del lecho. A partir de estas observaciones, se propone un método aproximado de cálculo que, teniendo en cuenta el valor de la concentración de VOC permitida a la salida y el porcentaje que debe alcanzar la ZTM al finalizar la etapa de adsorción, permite determinar el tiempo de adsorción y la cantidad de material adsorbente requerida para el diseño del sistema de adsorción/desorción.

Finalmente, es factible estimar el valor de caudal mínimo a partir de considerar el valor teórico que surge del caso ideal. Para el caso real se opta por un caudal un $25 \%$ mayor al valor teórico.

Por lo tanto, la resolución del caso ideal junto con el empleo de la aproximación presentada por la Ec. [17], proporcionan un procedimiento de cálculo que requiere un 
esfuerzo significativamente menor con respecto al presentado para el caso real, con resultados satisfactoriamente precisos. 


ESTUDIO DE SISTEMAS

COMBINADOS DE

COMBUSTION

CATALITICA DE VOCs

\section{7/ COMBUSTION CATALITICA: DETERMINACION DE PARAMETROS CINETICOS}

Estudio cinético de la combustión de acetato de etilo y etanol sobre un catalizador de MnCu Conclusiones 

La incineración es el método destructivo más importante en la actualidad, ya sea por vía térmica o catalítica. Teniendo en cuenta las consideraciones mencionadas en el Capítulo 2, para nuestro caso particular de estudio, la oxidación catalítica surge como una alternativa adecuada.

Con un catalizador adecuado y un correcto diseño del reactor es posible eliminar los VOCs en forma prácticamente completa con elevada selectividad a $\mathrm{CO}_{2}$, lo cual es altamente positivo desde el punto de vista ambiental.

Los catalizadores usados para la eliminación de VOCs incluyen como materiales activos a los óxidos metálicos (Everaert y Baeyens, 2004) o metales nobles, como platino $(\mathrm{Pt})$ o paladio $(\mathrm{Pd})$, los cuales generalmente poseen una mayor vida útil y son más resistentes al envenenamiento, pero en general son bastante más costosos (Moretti, 2002). Se han estudiado diferentes catalizadores para la eliminación de los compuestos específicos, acetato de etilo y etanol (Kaskantzis Neto y Moura, 1997; Papaefthimiou y col., 1999, Larsson y Andersson, 2000). Sin embargo, dichos trabajos se encuentran orientados a la comparación de diferentes catalizadores, pero no suministran la información cinética requerida para llevar a cabo el análisis y diseño de un reactor. En la búsqueda de catalizadores que permitan trabajar a menores temperaturas, en el INTEQUI (CONICET/UNSL) se han desarrollado catalizadores en base a óxidos de $\mathrm{Cu}$ y Mn, que demostraron ser altamente eficientes para la oxidación de etanol y acetato de etilo (Morales y col., 2008). En virtud del vínculo entre los grupos de trabajo del INTEQUI y del CINDECA, se dispone de información experimental de ensayos cinéticos realizados sobre uno de los catalizadores, lo que permitió emplear la misma para la obtención de parámetros cinéticos.

En este capítulo se presenta un estudio cinético de la combustión del acetato de etilo y el etanol sobre un catalizador de $\mathrm{MnCu}$. El objetivo es determinar un esquema cinético de reacción, proponer las expresiones para las velocidades de reacción de cada componente y obtener los valores de los parámetros cinéticos a partir de la regresión de los datos experimentales presentados por Pramparo (2010). 
Teniendo en cuenta este objetivo en primer lugar se describe brevemente el sistema experimental empleado para la obtención de los datos experimentales. Luego se presenta el análisis cualitativo de los mismos a partir del cual se proponen las expresiones cinéticas capaces de representar el sistema experimental. Se plantea el modelo de dicho sistema experimental y se describe brevemente el procedimiento de regresión empleado. Por último, se presentan los valores de los parámetros cinéticos obtenidos a partir de la regresión de los datos experimentales, analizando la capacidad del modelo propuesto, y parámetros obtenidos, para representar la información experimental empleada. Finalmente se realiza una breve comparación de las energías de activación obtenidas con la, limitada, información reportada en bibliografía.

\subsection{Estudio cinético de la combustión de acetato de etilo y etanol sobre un catalizador de $\mathrm{MnCu}$.}

\subsubsection{Descripción del sistema experimental}

El catalizador utilizado para el estudio cinético, identificado como $\mathrm{Mn}_{9} \mathrm{Cu}_{1}$, fue sintetizado por el método de co-precipitación descrito por Morales y col. (2008), a partir de soluciones acuosas de $\mathrm{Cu}\left(\mathrm{NO}_{3}\right)_{2} \cdot 4 \mathrm{H}_{2} \mathrm{O}$ y $\mathrm{Mn}\left(\mathrm{NO}_{3}\right)_{2} \cdot 4 \mathrm{H}_{2} \mathrm{O}$. La relación másica $\mathrm{Mn}: \mathrm{Cu}$ fue de 9:1. Detalles adicionales de la preparación y caracterización física del catalizador se encuentran en Morales y col. (2008) y Pramparo (2010). Propiedades inherentes a la caracterización física del catalizador se presentan en la Tabla A7.1.1 del Apéndice 7.1.

Los ensayos experimentales para el estudio cinético fueron llevados a cabo en un reactor tubular de cuarzo, de lecho fijo, que trabaja a presión atmosférica. Un diagrama del rector y las dimensiones del mismo se presentan en la Figura 1. La corriente de reactivos ingresa por la Zona 1, que es anular, donde se precalienta, atraviesa la Zona 2, donde se ubica el catalizador y luego retorna, a través de la Zona 3. En el interior del reactor, en la zona de puesta en contacto de la corriente reactiva con el catalizador, se ubica una vaina de vidrio, donde se aloja una termocupla. El reactor se encuentra dentro de un horno que permite alcanzar la temperatura de trabajo deseada. Se empleó el catalizador molido (tamaño de partícula: 500-841 $\mu \mathrm{m}$ ), diluido con una cantidad de vidrio molido (de igual tamaño que el catalizador) que permitiera obtener una altura de lecho de $1.3 \mathrm{~cm}$. 
La mezcla reactiva consistió de helio, oxígeno y el, o los, VOCs que se deseaban estudiar. La relación oxígeno/helio fue la misma que la de oxígeno/nitrógeno en el aire. Se trabajó con un caudal fijo de $100 \mathrm{ml} / \mathrm{min}$, medido en condiciones ambiente. Los VOCs fueron incorporados a la alimentación a través de un sistema de columnas rellenas para cada componente, las que están sumergidas en baños termostáticos. De esta forma la corriente absorbe la cantidad de VOCs definida por el equilibrio a la temperatura en la columna de saturación respectiva. Detalles adicionales del equipo experimental se encuentran en Pramparo (2010). Los reactivos y productos de la reacción fueron analizados por cromatografía en fase gaseosa.

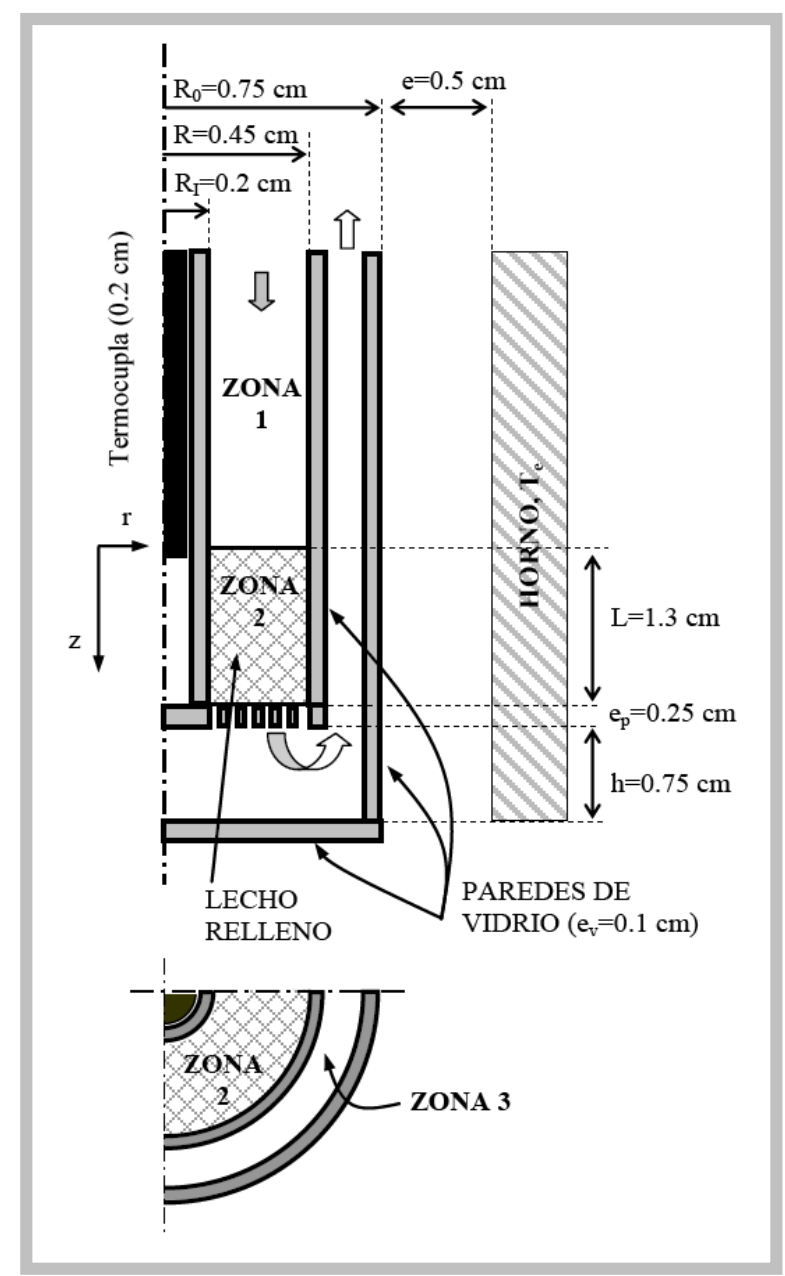

Fig. 1 - Reactor tubular de cuarzo de lecho fijo

Se realizaron tres tipos de ensayos. En primer lugar se utilizó como alimentación sólo uno de los VOCs en estudio, acetato de etilo (1) o etanol (2). Estos ensayos permitieron analizar el comportamiento de cada sustancia al ser alimentada individualmente, y realizar un análisis simplificado del sistema cinético, lo que sirvió 
de punto de partida para el análisis del sistema completo. Este último, incluyendo acetato de etilo y etanol, en diferentes proporciones, constituyó el segundo grupo de ensayos y abarca la mayor parte de estudios experimentales empleados en la regresión. En ambos grupos de ensayos se emplearon tres masas de catalizador (100, 200 y $300 \mathrm{mg})$. Dada la densidad del catalizador $\left(2750 \mathrm{~kg} / \mathrm{m}^{3}\right)$, los valores de GHSV (CNTP) fueron 55000, 82500 y $165000 \mathrm{~h}^{-1}$. Asimismo, se emplearon diferentes concentraciones de alimentación, siendo el rango de las mismas de, aproximadamente, 0 a 4000 ppm para el etanol y de 0 a 2000 ppm para el acetato de etilo.

En cada ensayo se midieron composiciones de entrada y salida. Para una dada condición experimental (composición de VOCs en la alimentación y masa de catalizador) se comenzó operando con temperaturas bajas (conversión casi nula), y se realizaron sucesivos ensayos, incrementando progresivamente la temperatura de operación, hasta alcanzar condiciones de conversión completa de los VOCs.

De los datos experimentales disponibles, se retuvieron aquellos en los cuales el balance de carbono "cerraba" dentro de un rango aceptable (menor al 10\%). El número de datos que no contempló está condición resultó ser pequeño. La Tabla 1 presenta la concentración de VOCs en la alimentación en cada una de las condiciones experimentales analizadas, así como las masas de catalizador empleadas y los rangos de temperaturas utilizados.

Adicionalmente, se realizaron ensayos complementarios, no empleados en la regresión, que tuvieron como objetivo analizar la incidencia de ciertas sustancias, particularmente los productos de reacción, sobre el comportamiento cinético del sistema. 
Tabla 1: Condiciones experimentales. Acetato de Etilo (1); Etanol (2).

\begin{tabular}{ccccc}
\hline Corrida \# & $\mathbf{y}_{1}^{0}[\mathbf{p p m}]$ & $\mathbf{y}_{2}^{0}[\mathbf{p p m}]$ & $\mathbf{m}_{\text {cat }}[\mathbf{m g}]$ & Rango de $\mathbf{~}\left[{ }^{\circ} \mathbf{C}\right]$ \\
\hline R1 & 0.0 & 3981.1 & 100 & $88.0-229.0$ \\
R2 & 0.0 & 2491.9 & 100 & $88.0-213.8$ \\
R3 & 0.0 & 3972.9 & 200 & $80.0-214.6$ \\
R4 & 0.0 & 2367.3 & 200 & $89.1-202.1$ \\
R5 & 2008.2 & 0.0 & 200 & $125.1-220.1$ \\
R6 & 1273.8 & 0.0 & 200 & $117.0-208.1$ \\
R7 & 2036.7 & 0.0 & 100 & $107.5-247.6$ \\
R8 & 1273.8 & 0.0 & 100 & $103.0-235.1$ \\
R9 & 1476.2 & 840.5 & 100 & $84.2-236.6$ \\
R10 & 1526.6 & 738.1 & 200 & $81.0-220.4$ \\
R11 & 1474.6 & 871.0 & 300 & $80.7-218.7$ \\
R12 & 1019.6 & 1912.7 & 100 & $85.0-237.1$ \\
R13 & 991.0 & 1723.1 & 200 & $80.3-232.4$ \\
R14 & 1019.6 & 1809.7 & 300 & $81.1-222.9$ \\
R15 & 508.6 & 2963.9 & 100 & $85.2-234.7$ \\
R16 & 477.0 & 2896.7 & 200 & $80.3-222.6$ \\
R17 & 508.6 & 3371.7 & 300 & $82.0-215.4$ \\
\hline
\end{tabular}

\subsubsection{Análisis cualitativo de los resultados experimentales}

El análisis cualitativo de los resultados experimentales tuvo por objetivo establecer el esquema de reacciones que se tiene al tratar los VOCs individualmente o en mezclas. Asimismo, sirvió como una primera orientación para determinar la forma que pudieran presentar las expresiones cinéticas. A continuación se presentan algunos ensayos que son representativos del conjunto de los mismos.

En la Figura 2 se presentan resultados de dos ensayos, donde el etanol es el único VOC alimentado, para una misma masa de catalizador y dos concentraciones iniciales diferentes. Tales ensayos corresponden a las corridas R1 y R2 indicadas en la Tabla 1. Las curvas de fracción molar de acetaldehído (3) sugieren que en ambos ensayos hay una formación apreciable del mismo, el cual se convierte en la sustancia más difícil de eliminar. 


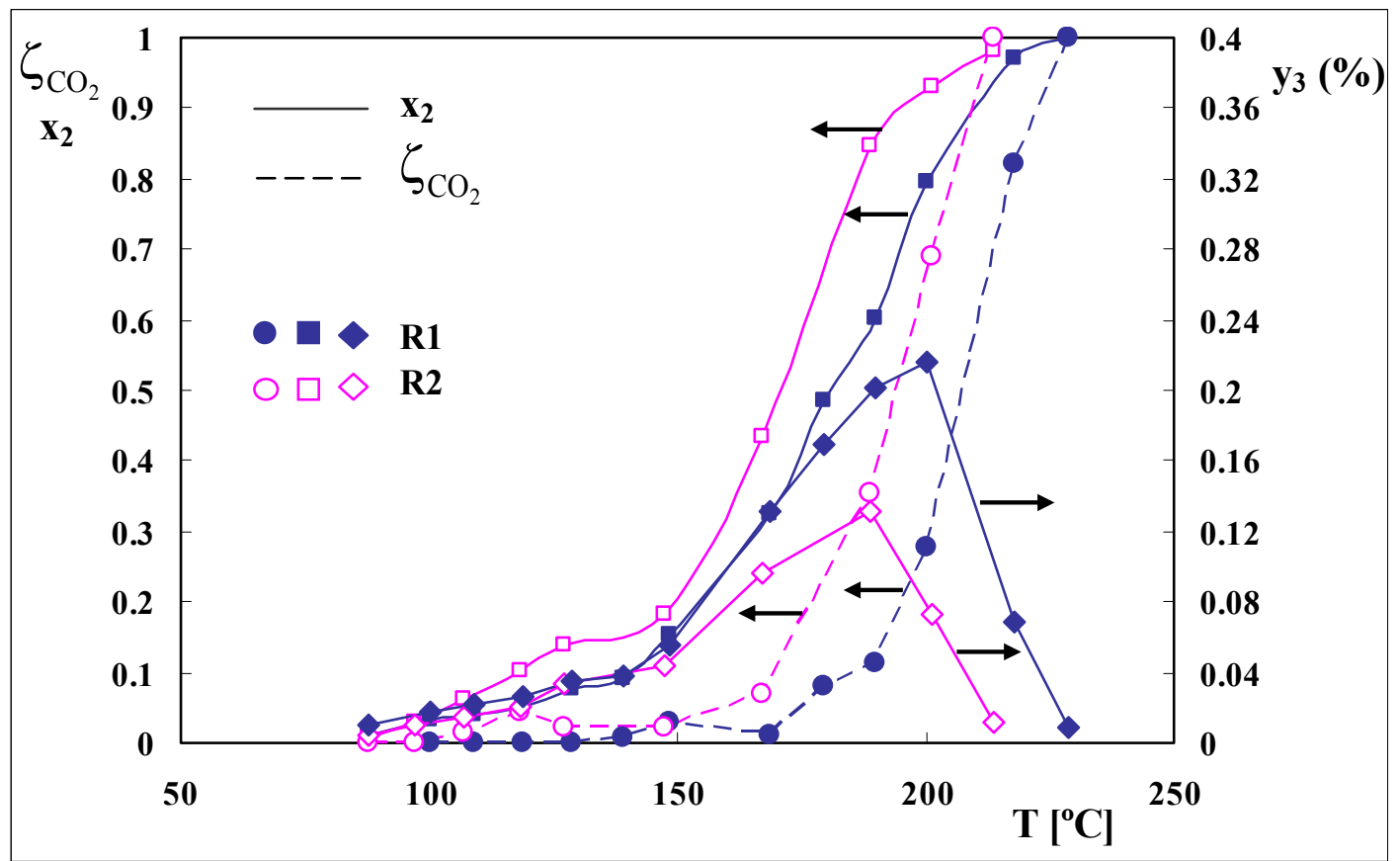

Fig. 2 - Variación de la conversión de etanol $\left(\mathrm{x}_{2}\right)$, fracción molar de acetaldehído $\left(\mathrm{y}_{3}\right)$ y producción de $\mathrm{CO}_{2}\left(\zeta_{\mathrm{CO}_{2}}\right)$ en función de la temperatura para dos concentraciones de alimentación de etanol correspondientes a las corridas R1 y R2 en la Tabla $1\left(\mathrm{~m}_{\mathrm{cat}}=100 \mathrm{mg}\right)$.

La diferencia de niveles alcanzada por la fracción molar de acetaldehído se debe a la diferencia en la concentración inicial de etanol. La aparición de acetaldehído al llevarse a cabo la oxidación de etanol ha sido informada en numerosos trabajos con diferentes tipos de catalizadores (Rajesh y Ozkan, 1993; Larsson y Andersson, 1998, 2000; Delimaris e Ioannides, 2008, 2009).

En estos ensayos no se ha detectado ácido acético ni monóxido de carbono, reportado este último en algunos trabajos (Rajesh y Ozkan, 1993). Esto sugirió un sistema de reacciones en serie, donde el etanol produce acetaldehído y éste el producto final, $\mathrm{CO}_{2}$. Otra cuestión que se trató de dilucidar a partir de estos resultados fue la posibilidad de incluir un camino de oxidación directa de etanol a $\mathrm{CO}_{2}$, como ha sido señalado por algunos autores (Rajesh y Ozkan, 1993). Con este fin se incluyeron en la Fig. 2 las curvas de la siguiente relación:

$$
\zeta_{\mathrm{CO}_{2}}=\left(\frac{\mathrm{y}_{\mathrm{CO} 2}}{\mathrm{y}_{\mathrm{CO} 2}^{\max }}\right)
$$

donde $\mathrm{y}_{\mathrm{CO} 2}^{\max }$ es la máxima fracción molar que se puede obtener para el $\mathrm{CO}_{2}$, de acuerdo a la concentración y tipo de VOC alimentado. Esta relación debería ser coincidente con la curva de conversión de etanol si este se oxidase directamente a 
$\mathrm{CO}_{2}$ a través de un único mecanismo. En este caso es obvio que esto no sucede por la existencia del mecanismo de formación de acetaldehído mencionado previamente, pero también es útil para inferir si existe un mecanismo de oxidación directa del etanol. Es claro que en ambos ensayos $\zeta_{\mathrm{CO}_{2}}$ permanece prácticamente nula, aún para conversiones superiores al $50 \%$ de etanol. Asimismo, $\zeta_{\mathrm{CO}_{2}}$ comienza a aumentar aproximadamente en coincidencia con el máximo de acetaldehído. Estos resultados sugieren que, para este catalizador, la oxidación directa de etanol a $\mathrm{CO}_{2}$ es irrelevante, lo que permite plantear el siguiente esquema para la oxidación de etanol:

$$
\begin{array}{ll}
\mathrm{C}_{2} \mathrm{H}_{6} \mathrm{O}+0.5 \mathrm{O}_{2} & \rightarrow \mathrm{C}_{2} \mathrm{H}_{4} \mathrm{O}+\mathrm{H}_{2} \mathrm{O} \\
\mathrm{C}_{2} \mathrm{H}_{4} \mathrm{O}+2.5 \mathrm{O}_{2} & \rightarrow 2 \mathrm{CO}_{2}+2 \mathrm{H}_{2} \mathrm{O}
\end{array}
$$

En la Figura 3 se presentan resultados de dos ensayos, donde el acetato de etilo es el único VOC alimentado, para una misma masa de catalizador y dos niveles de concentración inicial. Tales ensayos corresponden a las corridas R7 y R8 indicadas en la Tabla 1. La masa de catalizador es igual a la de la Fig. 2, lo que permite observar que, más allá de la influencia de la concentración de VOC empleada, el etanol reacciona a temperaturas apreciablemente menores que el acetato de etilo. Nuevamente se ha graficado la relación dada por la Ec. [1]. A diferencia de lo que se observó para la oxidación de etanol, en este caso, la conversión de acetato de etilo y $\zeta_{\mathrm{CO}_{2}}$ son, dentro del error experimental, prácticamente coincidentes, en particular para los valores más altos de conversión de acetato de etilo.

El análisis cromatográfico mostró que en ciertos ensayos se detectaban pequeñas cantidades de etanol y acetaldehído, pero en ninguna condición eran valores relevantes. Sustancias intermedias entre el acetato de etilo y el $\mathrm{CO}_{2}$ han sido reportadas para estudios con diferentes catalizadores. Normalmente las sustancias más detectadas han sido etanol y acetaldehído, pero las cantidades producidas han sido muy variables de acuerdo al tipo de catalizador. Por ej., en los resultados de Delimaris e Ioannides (2009) prácticamente no se observa etanol y muy poco acetaldehído al emplear un catalizador de $\mathrm{CuO}$, mientras que hay una apreciable formación de etanol, y algo de acetaldehído, al utilizar $\mathrm{CeO}_{2}$. 


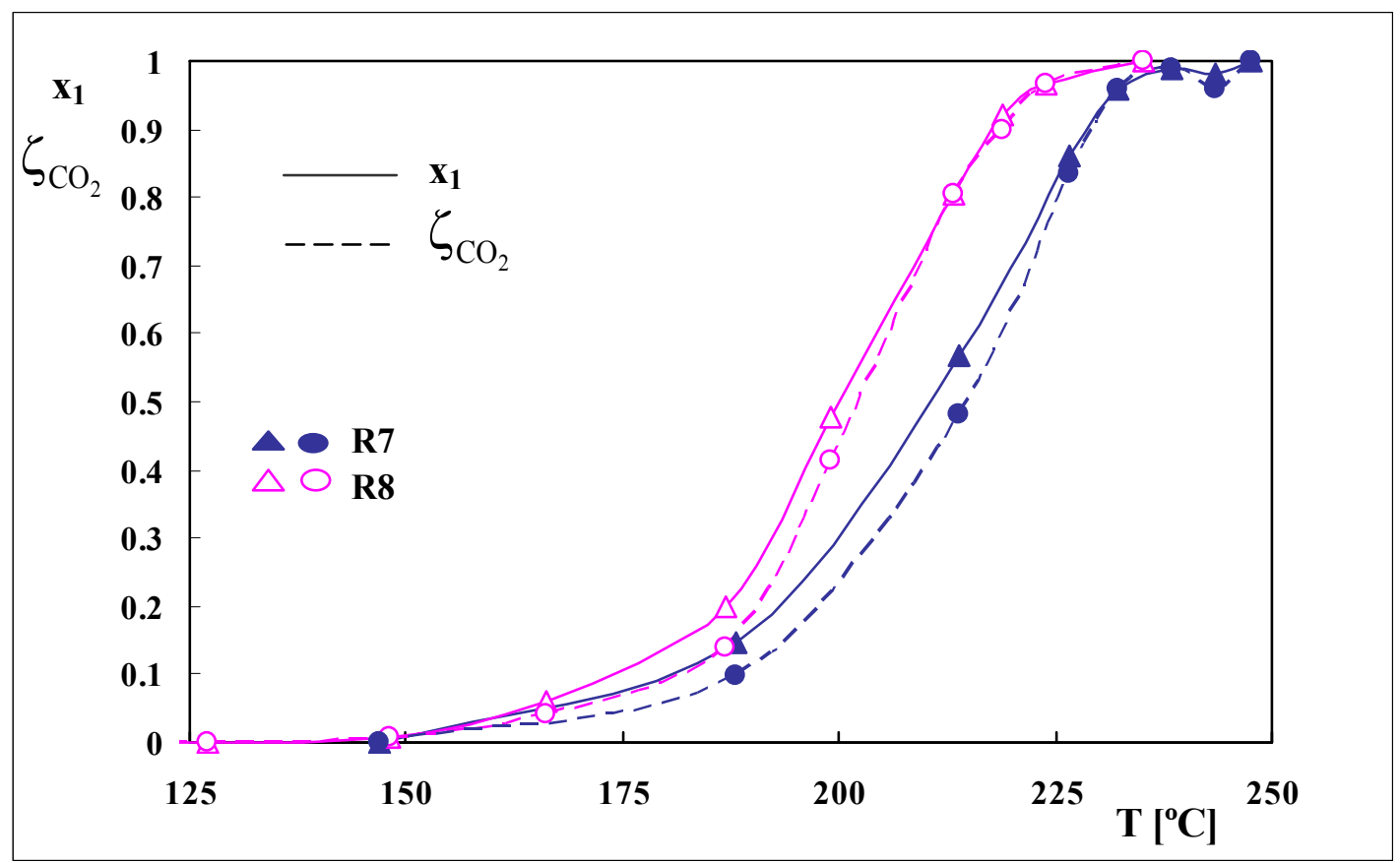

Fig. 3 - Variación de la conversión de acetato de etilo $\left(\mathrm{x}_{1}\right)$ y producción de $\mathrm{CO}_{2}\left(\zeta_{\mathrm{CO}_{2}}\right)$ en función de la temperatura para dos concentraciones de alimentación de acetato de etilo correspondientes a las corridas $\mathrm{R} 7$ y R8 en la Tabla $1\left(\mathrm{~m}_{\text {cat }}=100 \mathrm{mg}\right)$.

La oxidación del acetato de etilo presumiblemente, y como lo señalan algunos trabajos de la literatura (Gandhe y col., 2007), podría tener mecanismos más complejos que la del etanol y en consecuencia formarse intermediarios antes de alcanzar el producto final $\mathrm{CO}_{2}$. Una conclusión similar alcanzaron Lintz y Wittstock (2001) al analizar la oxidación de acetato de butilo sobre un catalizador de CuMn, pero la concentración de compuestos intermediarios es baja y siempre se plantea un mecanismo de oxidación directa del éster. Aunque para este catalizador la formación de compuestos intermediarios no se puede descartar, las sustancias que se forman, particularmente etanol y acetaldehído, son difíciles de detectar por su baja concentración. El etanol se oxida a temperaturas mucho más bajas que el acetato de etilo, en consecuencia si se forma a partir de acetato de etilo, reacciona rápidamente. Una situación similar se produce para el acetaldehído. De esta forma, es aceptable asumir que el acetato de etilo reaccione dando directamente los productos finales de la oxidación a través de la reacción:

$$
\mathrm{C}_{4} \mathrm{H}_{8} \mathrm{O}_{2}+5 \mathrm{O}_{2} \rightarrow 4 \mathrm{CO}_{2}+4 \mathrm{H}_{2} \mathrm{O}
$$

la que permite completar el esquema de reacciones que ocurren en el sistema en estudio. 
El segundo aspecto que se puede abordar a partir del análisis cuantitativo es de tipo cinético. En las Figs. 2 y 3 la conversión de cualquiera de los VOCs alimentado, se desplaza hacia mayores temperaturas a medida que aumenta la concentración inicial del mismo. En otras palabras, para la misma masa de catalizador y la misma temperatura de trabajo, la conversión es menor al ser mayor la concentración inicial. Este comportamiento puede asimilarse al de una reacción con orden de reacción menor que uno en el reactivo limitante, en una expresión cinética de tipo potencial. Esta hipótesis fue corroborada cuantitativamente por Pramparo (2010) al analizar solamente el comportamiento del acetato de etilo. Por su parte, Rajesh y Ozkan (1993), sobre un catalizador de $\mathrm{Cu}$ encontraron un orden de reacción de 0.2 para el etanol al estudiar su oxidación. La alternativa cinética que puede conducir a resultados similares a órdenes de reacción menores que uno, es asumir que las reacciones son de tipo Langmuir-Hinshelwood-Hougen-Watson (LHHW) con un primer orden irreversible e incluyendo términos de inhibición.

\subsubsection{Interacción en la combustión conjunta de etanol y acetato de etilo}

Aunque en muchos casos se estudian todos los VOCs separadamente, es muy factible encontrar en la práctica (como en nuestro caso particular de estudio) la necesidad de eliminar más de un VOC simultáneamente. En consecuencia, resulta relevante estudiar el comportamiento del sistema de reacción cuando ambos VOCs se encuentran presentes en la mezcla reactiva. Se ha observado que el comportamiento cinético de un dado VOC puede alterarse por la presencia de otros compuestos en la mezcla (Dangi y Abraham, 1997; Irusta y col., 1998; Abdullah y col., 2003; Delimaris y col., 2008; Agüero y col., 2009; Kim y col., 2010; Chi He y col., 2010; Santos y col., 2011). Por ejemplo, Lahousse y col. (1998) estudiaron el comportamiento de un sistema con benceno, acetato de etilo y hexano sobre dos catalizadores diferentes encontrando que la conversión del acetato de etilo no se altera por la presencia de los otros compuestos. Abdullah y col. (2003) estudiaron la combustión de acetato de etilo y benceno sobre Cr-ZSM-5, encontrando que la combustión del acetato de etilo se ve favorecida por la presencia de benceno. Esto demuestra que el comportamiento cinético depende fuertemente del tipo de catalizador y de la composición de la mezcla reactiva. 
El comportamiento de la mezcla de etanol y acetato de etilo fue estudiado por Agüero y col. (2009) sobre un catalizador de $\mathrm{MnOx} / \mathrm{Al}_{2} \mathrm{O}_{3}$, y por Santos y col. (2011) quienes emplearon criptomelano como catalizador. Agüero y col. (2009) encontraron que la presencia de acetato de etilo inhibe la combustión del etanol. Por otra parte, Santos y col. (2011) concluyeron que existe un efecto de inhibición mutuo entre el etanol y el acetato de etilo, resultando relativamente más perjudicado el acetato de etilo. Una complejidad adicional al análisis del sistema es la formación del acetaldehído a partir de la combustión del etanol (Fig. 2). Al respecto, Santos y col. (2011) mencionan que el acetaldehído puede también jugar un rol importante dentro de los efectos de inhibición observados, por el hecho de ser un intermediario significativo en la oxidación del etanol.

La interacción entre los VOCs, cuando se tratan simultáneamente etanol y acetato de etilo en el sistema en estudio, se puede analizar a partir de las Figuras 4 y 5 . En la Fig. 4 se presenta la variación de los valores experimentales de la fracción molar $\left(\mathrm{y}_{1}\right)$ y conversión $\left(\mathrm{x}_{1}\right)$ del acetato de etilo con la temperatura para tres condiciones de trabajo diferentes, con una única masa de catalizador (200 mg). En dos de dichas condiciones el acetato de etilo es el único VOC, con dos concentraciones de alimentación diferentes (R5 y R6 en la Tabla 1), mientras que en una tercera condición (R16 en Tabla 1) se alimentó etanol y acetato de etilo.

El comportamiento de las curvas R5 y R6 es similar al observado en la Fig. 3: a mayor concentración de acetato de etilo en la alimentación (1274 ppm para R6 y 2008 ppm para R5), las curvas correspondientes a la conversión se desplazan hacia mayores temperaturas. Para las curvas R16, la fracción molar de acetato de etilo en la alimentación tiene el valor más bajo (477 ppm), pero se tiene una fracción molar de etanol relativamente alta (2897 ppm). Si no hubiese una interacción entre VOCs, la curva correspondiente a la conversión del acetato de etilo (R16) debería verse desplazada hacia menores temperaturas. Sin embargo la Fig. 4 muestra lo contrario, indicando una fuerte influencia del etanol y/o acetaldehído en el comportamiento cinético de la reacción [4]. Teniendo en cuenta únicamente las curvas R5 y R6 queda claro que debe considerarse un efecto de inhibición del acetato de etilo (el único VOC presente en estas corridas) en la expresión cinética de la reacción [4]. Teniendo en cuenta los resultados que expresan las curvas R16, también debe ser considerado un efecto inhibitorio del etanol y/o acetaldehído en dicha expresión cinética. 


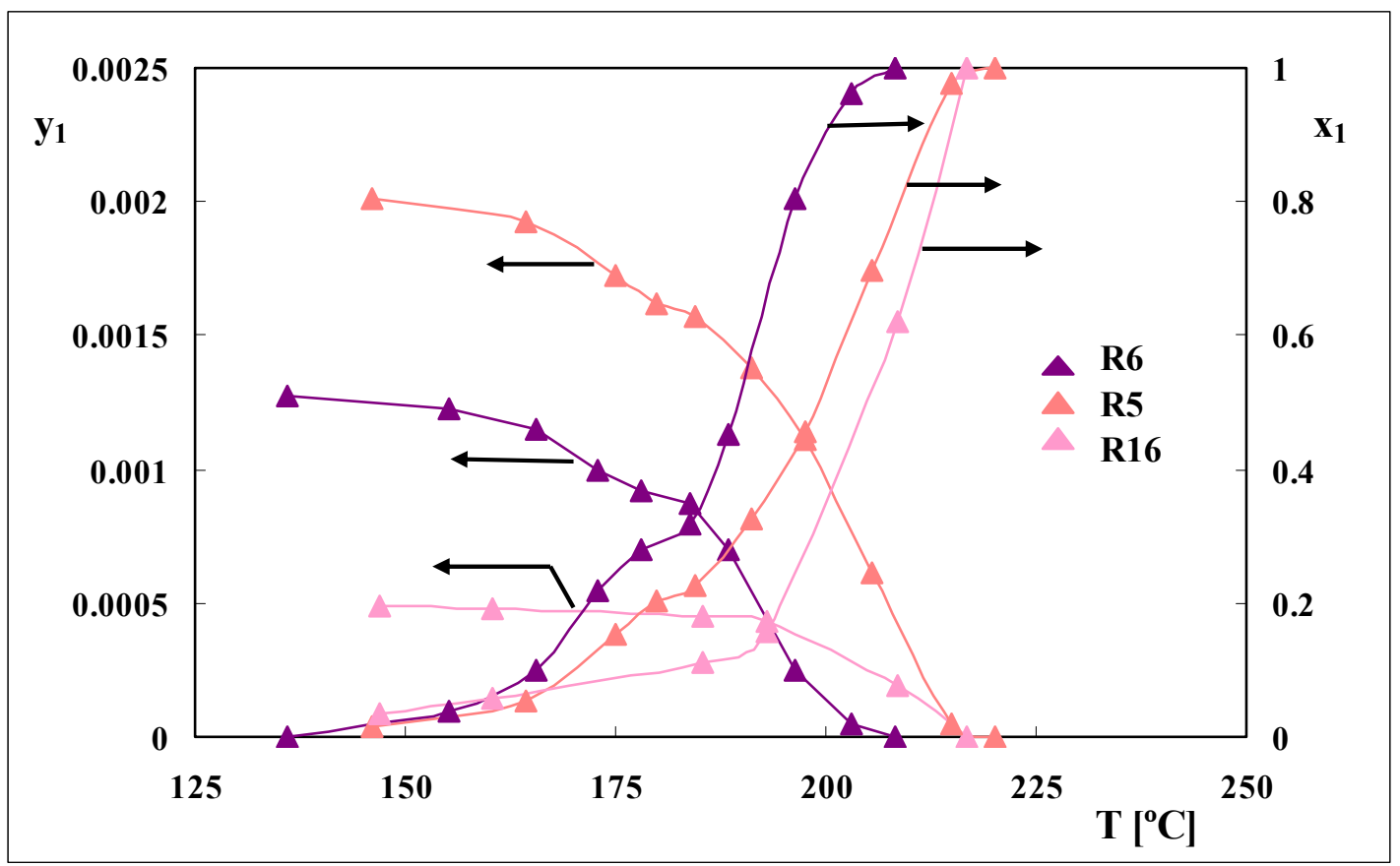

Fig. 4 - Variación de los valores experimentales de $\mathrm{y}_{1} \mathrm{y}_{1}$ con la temperatura para tres condiciones diferentes: R5, R6 y R16 (Tabla 1) $\left(\mathrm{m}_{\text {cat }}=200 \mathrm{mg}\right)$.

La Fig. 5 presenta la variación de los valores experimentales de fracción molar del acetato de etilo $\left(\mathrm{y}_{1}\right)$ y acetaldehído $\left(\mathrm{y}_{3}\right)$, y la conversión de etanol $\left(\mathrm{x}_{2}\right)$, con la temperatura, para dos condiciones diferentes (R4 y R10 en la Tabla 1). Para las curvas correspondientes a la condición R4 solo se tiene etanol como VOC presente en la alimentación al reactor con una concentración de 2367 ppm, mientras que en el caso de las curvas correspondientes a la condición R10 se empleó una mezcla de etanol $(738.1 \mathrm{ppm})$ y acetato de etilo $(1527 \mathrm{ppm})$ con una concentración total de VOCs de 2265 ppm.

En la Fig. 5 se puede apreciar que ambas curvas R4 y R10 que representan la conversión de etanol son prácticamente coincidentes. Sin embargo, considerando la diferencia de la concentración de etanol en la alimentación (2367 ppm para la curva correspondiente a R4 y 738.1 ppm para la curva R10) y los resultados presentados en la Fig. 2, la curva R10 correspondiente a la conversión de etanol debería verse desplazada hacia menores temperaturas. Esto demuestra que la influencia del acetato de etilo sobre el comportamiento cinético de la mezcla no puede ser ignorada. Una conclusión similar acerca de los efectos de inhibición puede obtenerse a partir de analizar el comportamiento del acetaldehído. Dado que la concentración de etanol en la alimentación al reactor es mayor para R4 que R10, la curva R4 correspondiente a la producción de acetaldehído alcanza un valor máximo mayor al que se observa en 
la curva R10. Sin embargo, luego de haber alcanzado el valor máximo en la curva R4 (aproximadamente a $\operatorname{los} 178^{\circ} \mathrm{C}$ ), la velocidad de consumo del acetaldehído es mayor que la observada en la curva R10, a pesar de encontrarse el máximo prácticamente a la misma temperatura. Esto último indica que la presencia del acetato de etilo influye sobre el comportamiento cinético de la reacción [3].

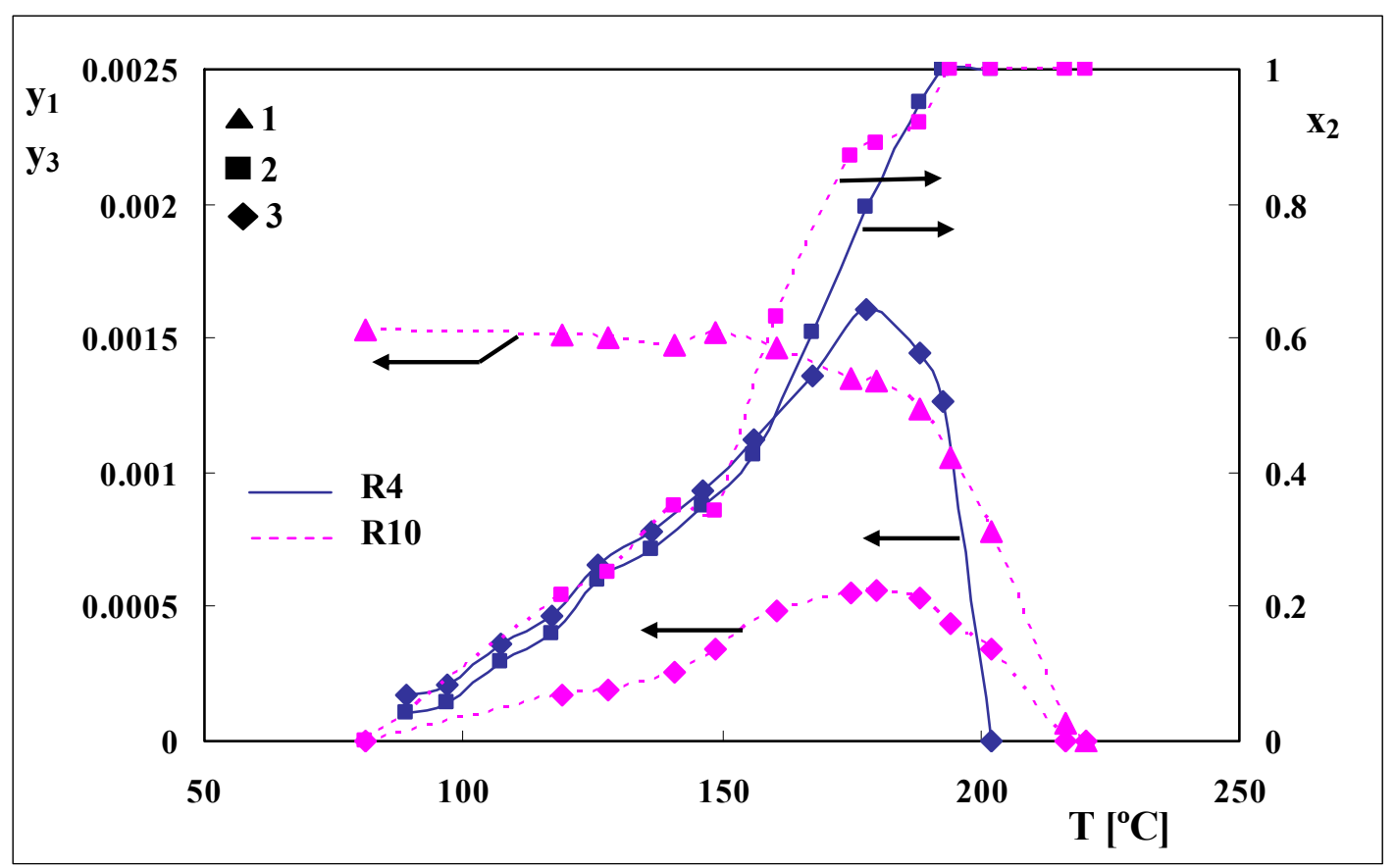

Fig. 5 - Variación de los valores experimentales del $\mathrm{y}_{1}, \mathrm{y}_{3}, \mathrm{x}_{2}$ con la temperatura para dos condiciones diferentes: R4 y R10 (Tabla 1$)\left(\mathrm{m}_{\text {cat }}=200 \mathrm{mg}\right)$.

En la Fig. 5 se puede apreciar que el acetato de etilo es más difícil de oxidar que el acetaldehído, dado que no se consume a temperaturas menores a $180^{\circ} \mathrm{C}$ aproximadamente.

Considerando los resultados experimentales es factible concluir que para este catalizador no pueden ignorarse los efectos de inhibición entre las especies presentes (etanol, acetato de etilo y acetaldehído). Este comportamiento, también reportado por Santos y col. (2011), debe ser considerado en el momento de proponer las expresiones de las velocidades de reacción.

Una información adicional, a efectos de postular las expresiones cinéticas, se obtuvo a partir de los ensayos complementarios. En la literatura se suele señalar que en algunos casos los productos finales de la oxidación, particularmente el agua, pueden afectar el comportamiento cinético del catalizador (Pan y col., 2009), mientras que en otros su influencia es despreciable (Larsson y Andersson, 2000). En 
este contexto, se realizaron ensayos adicionales, incluyendo agua y $\mathrm{CO}_{2}$ en la alimentación, en concentraciones equivalentes a las que producirá la oxidación completa del VOC alimentado. No se observaron variaciones en las respuestas alcanzadas, se incluyesen o no en la alimentación, los productos finales de reacción, $\mathrm{CO}_{2}$ y agua.

Teniendo en cuenta estos resultados, y considerando que el objetivo propuesto es obtener expresiones cinéticas capaces de representar el sistema experimental estudiado, sin abordar un estudio de tipo mecanístico para obtener las mismas, se propusieron expresiones cinéticas para las tres reacciones [2], [3] y [4], según se describe a continuación.

Por una cuestión de ser consistente con la nomenclatura que se adoptará en el próximo capítulo, se designará como reacciones (a), (b) y (c) a las anteriormente denominadas [4], [2] y [3] respectivamente.

$$
\begin{array}{lll}
\mathrm{C}_{4} \mathrm{H}_{8} \mathrm{O}_{2}+5 \mathrm{O}_{2} & \rightarrow & 4 \mathrm{CO}_{2}+4 \mathrm{H}_{2} \mathrm{O} \\
\mathrm{C}_{2} \mathrm{H}_{6} \mathrm{O}+0.5 \mathrm{O}_{2} & \rightarrow & \mathrm{C}_{2} \mathrm{H}_{4} \mathrm{O}+\mathrm{H}_{2} \mathrm{O} \\
\mathrm{C}_{2} \mathrm{H}_{4} \mathrm{O}+2.5 \mathrm{O}_{2} & \rightarrow & 2 \mathrm{CO}_{2}+2 \mathrm{H}_{2} \mathrm{O}
\end{array}
$$

Las expresiones cinéticas se escriben en forma reparametrizada con una temperatura de referencia $\left(\mathrm{T}_{\mathrm{ref}}=175^{\circ} \mathrm{C}\right)$, porque es la forma en que se emplearon en la regresión.

$$
\begin{aligned}
& \tau_{\mathrm{a}}=\frac{\mathrm{k}_{\mathrm{ref}, \mathrm{a}} \exp \left[-\left(\mathrm{E}_{\mathrm{a}} / \mathrm{R}\right)\left((1 / \mathrm{T})-\left(1 / \mathrm{T}_{\mathrm{ref}}\right)\right)\right] \mathrm{C}_{1}}{1+\mathrm{K}_{1} \mathrm{C}_{1}+\mathrm{K}_{2} \mathrm{C}_{2}+\mathrm{K}_{3} \mathrm{C}_{3}} \\
& \boldsymbol{z}_{\mathrm{b}}=\frac{\mathrm{k}_{\mathrm{ref}, \mathrm{b}} \exp \left[-\left(\mathrm{E}_{\mathrm{b}} / \mathrm{R}\right)\left((1 / \mathrm{T})-\left(1 / \mathrm{T}_{\mathrm{ref}}\right)\right)\right] \mathrm{C}_{2}}{1+\mathrm{K}_{1} \mathrm{C}_{1}+\mathrm{K}_{2} \mathrm{C}_{2}+\mathrm{K}_{3} \mathrm{C}_{3}} \\
& \boldsymbol{\tau}_{\mathrm{c}}=\frac{\mathrm{k}_{\mathrm{ref}, \mathrm{c}} \exp \left[-\left(\mathrm{E}_{\mathrm{c}} / \mathrm{R}\right)\left((1 / \mathrm{T})-\left(1 / \mathrm{T}_{\mathrm{ref}}\right)\right)\right] \mathrm{C}_{3}}{1+\mathrm{K}_{1} \mathrm{C}_{1}+\mathrm{K}_{2} \mathrm{C}_{2}+\mathrm{K}_{3} \mathrm{C}_{3}}
\end{aligned}
$$

donde $\mathrm{C}_{1}, \mathrm{C}_{2} \mathrm{y} \mathrm{C}_{3}$ son las concentraciones molares de acetato de etilo (1), etanol (2) y acetaldehído (3) dentro de la partícula de catalizador, $\mathrm{E}_{\mathrm{a}}, \mathrm{E}_{\mathrm{b}} \mathrm{y} \mathrm{E}_{\mathrm{c}}$ son las energías de activación correspondientes a las reacciones a, b y c respectivamente, $\mathrm{k}_{\mathrm{ref}, \mathrm{a}}, \mathrm{k}_{\mathrm{ref}, \mathrm{b}} \mathrm{y}$ $\mathrm{k}_{\mathrm{ref,c}}$ son las constantes específicas de velocidad de las reacciones a, b y c, y $\mathrm{K}_{1}, \mathrm{~K}_{2}$ y $\mathrm{K}_{3}$ representan las constantes de adsorción de cada uno de los componentes. 
La concentración de oxígeno no fue incluida porque su variación en los ensayos no fue lo suficientemente significativa como para apreciar su influencia. Este punto no constituye una falencia del modelo, ya que concentraciones similares se encontrarían al tratar una corriente industrial.

\subsubsection{Modelo del sistema experimental}

Las expresiones dadas por las Ecs. [5] a [7] corresponden a las respectivas velocidades de reacción intrínsecas. En consecuencia, para la obtención de los parámetros mediante la regresión de los datos experimentales se debió definir el modelo del sistema experimental en el cual se obtuvieron dichos datos. Dadas las características de los ensayos, el reactor se consideró como un reactor integral, puesto que se alcanzan incluso conversiones totales a las mayores temperaturas. En estos casos se define como "situación ideal" a aquella en la cual no existe ningún tipo de resistencia a la transferencia de calor y materia, dentro y fuera de la pastilla de catalizador, y el reactor es isotérmico y con flujo pistón. Para definir el modelo experimental se debió verificar el cumplimiento de dichas hipótesis o, de no cumplirse, incorporar en el mismo las resistencias correspondientes. En la literatura (Weisz y Prater, 1954; Mears, 1971) se han presentado criterios que permiten analizar si algunas de estas hipótesis se cumplen o no. Se verificó que pueden despreciarse las resistencias externas de calor y materia y que puede aceptarse que cada partícula es isotérmica, pero la resistencia a la transferencia de materia en el interior del catalizador no puede ignorarse (Pramparo, 2010).

El sistema experimental empleado ha sido analizado detalladamente para verificar si podría considerarse isotérmico, a través de un estudio que simula los procesos de transferencia de calor en la geometría exacta del equipo experimental (Mariani y col., 2009). La conclusión obtenida fue que la temperatura se mantuvo prácticamente uniforme, excepto para aquellos ensayos en donde la concentración de VOCs en la alimentación resultó relativamente alta y para las temperaturas más altas. En consecuencia, para estos casos particulares, la temperatura leída tuvo que ser corregida de manera tal de obtener una mejor estimación de los parámetros.

Teniendo en cuenta el análisis previo, las ecuaciones utilizadas para representar el reactor experimental fueron: 


$$
\begin{aligned}
& \frac{\mathrm{dC}_{\mathrm{g}, 1}}{\mathrm{dV}}=-\delta\left(1-\varepsilon_{\mathrm{L}}\right) \frac{\mathrm{r}_{1}^{\mathrm{ef}}}{\mathrm{Q}} \\
& \frac{\mathrm{dC}_{\mathrm{g}, 2}}{\mathrm{dV}}=-\delta\left(1-\varepsilon_{\mathrm{L}}\right) \frac{\mathrm{r}_{2}^{\mathrm{ef}}}{\mathrm{Q}} \\
& \frac{\mathrm{dC}_{\mathrm{g}, 3}}{\mathrm{dV}}=-\delta\left(1-\varepsilon_{\mathrm{L}}\right) \frac{\mathrm{r}_{3}^{\text {ef }}-\mathrm{r}_{2}^{\text {ef }}}{\mathrm{Q}}
\end{aligned}
$$

Con las condiciones iniciales:

$\operatorname{Para} \mathrm{V}=0 \quad \mathrm{C}_{\mathrm{g}, 1}=\mathrm{C}_{\mathrm{g}, 1}^{0} ; \quad \mathrm{C}_{\mathrm{g}, 2}=\mathrm{C}_{\mathrm{g}, 2}^{0} ; \quad \mathrm{C}_{\mathrm{g}, 3}=0 ; \quad[8 \mathrm{a}, 9 \mathrm{a}, 10 \mathrm{a}]$

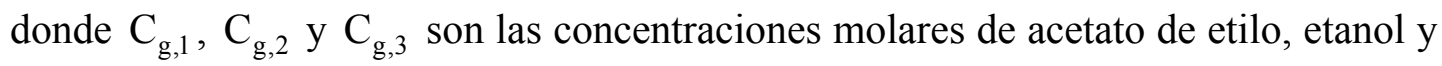
acetaldehído en la corriente de fluido, $\mathrm{V}$ el volumen de lecho, $\delta$ el factor de dilución y Q el caudal volumétrico.

Para obtener las velocidades de consumo efectivas $\left(r_{1}^{\text {ef }}, r_{2}^{\text {ef }}\right.$ y $\left.r_{3}^{\text {ef }}\right)$ se debieron resolver las ecuaciones de difusión-reacción en el interior de la partícula para las tres reacciones. Se consideró geometría esférica y difusividad efectiva constante en el interior de la partícula, por lo que las ecuaciones a resolver resultaron:

$$
\begin{aligned}
& \mathrm{D}_{\mathrm{e}, 1}\left[\frac{1}{\mathrm{z}^{2}} \frac{\mathrm{d}}{\mathrm{dz}}\left(\mathrm{z}^{2} \frac{\mathrm{dC}_{1}}{\mathrm{dz}}\right)\right]=r_{\mathrm{a}} \\
& \mathrm{D}_{\mathrm{e}, 2}\left[\frac{1}{\mathrm{z}^{2}} \frac{\mathrm{d}}{\mathrm{dz}}\left(\mathrm{z}^{2} \frac{\mathrm{dC}_{2}}{\mathrm{dz}}\right)\right]=r_{\mathrm{b}} \\
& \mathrm{D}_{\mathrm{e}, 3}\left[\frac{1}{\mathrm{z}^{2}} \frac{\mathrm{d}}{\mathrm{dz}}\left(\mathrm{z}^{2} \frac{\mathrm{dC}_{3}}{\mathrm{dz}}\right)\right]=r_{\mathrm{c}}-r_{\mathrm{b}}
\end{aligned}
$$

Con las condiciones de borde:

$$
\begin{array}{lll}
\mathrm{z}=0 & \mathrm{dC}_{\mathrm{j}} / \mathrm{dz}=0 & \mathrm{j} \equiv 1,2,3 \\
\mathrm{z}=\mathrm{r}_{\mathrm{p}} & \mathrm{C}_{\mathrm{j}}=\mathrm{C}_{\mathrm{g}, \mathrm{j}} & \mathrm{j} \equiv 1,2,3
\end{array}
$$

donde $\mathrm{D}_{\mathrm{e}, 1}, \mathrm{D}_{\mathrm{e}, 2} \mathrm{y}_{\mathrm{e}, 3}$ son las difusividades efectivas de cada VOC (Apéndice 7.1).

El sistema de ecuaciones se ve reducido a las Ecs. [8] y [11] cuando sólo se alimenta acetato de etilo y a las Ecs. [9], [10], [12] y [13] cuando sólo se alimenta etanol. 


\subsubsection{Procedimiento de regresión}

El conjunto de subrutinas empleado para realizar la regresión de los datos experimentales puede dividirse en dos subprogramas. El subprograma GREGPAK (Stewart y col., 1992), permite obtener las estimaciones óptimas de los parámetros cinéticos comparando los valores predichos por el modelo, con los experimentales, de las fracciones molares de salida del lecho para cada componente. Rutinas propias de este programa fueron utilizadas para la resolución de las ecuaciones diferenciales ordinarias (Ecs. [8] a [10]) que representan el lecho. Para esta resolución se requiere de valores de velocidades de reacción efectivas, las cuales se obtienen resolviendo las ecuaciones de reacción-difusión (Ecs. [11] a [13]) en el interior de la pastilla. Este sistema se resolvió mediante un algoritmo presentado anteriormente (Bressa y col., 2001).

No se incluyó la variación de las constantes de adsorción con la temperatura, en consecuencia se calculó un único valor para el rango de temperatura estudiado.

Se utilizaron un total de diecisiete conjuntos de ensayos experimentales (Tabla 1), incluyendo cada uno entre diez y quince niveles de temperatura de operación. Nueve de los conjuntos correspondían a mezclas de etanol y acetato de etilo en la alimentación, para tres niveles de masa de catalizador (100, 200 y $300 \mathrm{mg}$ ). Tomando como base los moles de carbono que se encuentran en cada molécula, las relaciones etanol/acetato de etilo nominales fueron 3,1 y $1 / 3$ para cada uno de los tres niveles de masa de catalizador (en la práctica hubo pequeñas desviaciones de estos valores ya que la carga de los VOCs incorpora cierto error experimental). Los otros ocho conjuntos de ensayos correspondían a alimentaciones de uno solo de los VOCs, cuatro de etanol y cuatro de acetato de etilo. En estos ensayos las masa de catalizador y niveles de concentración se ubicaron en los mismos rangos que al emplear mezclas.

Se realizó un balance de carbono en cada ensayo, especialmente en el caso de mezclas, donde podrían existir los mayores errores experimentales. La mayoría de los ensayos cerraba el balance entre $\pm 5 \%$, lo cual se consideró aceptable.

En principio se debieron obtener nueve parámetros: tres energías de activación, tres constantes de adsorción y tres constantes específicas de reacción a la temperatura de referencia. Este elevado número de parámetros requirió llevar a cabo una estrategia de cálculo para alcanzar un adecuado ajuste de los datos experimentales. 
En primer lugar se obtuvieron los parámetros a partir de ajustar los datos experimentales para un único ensayo correspondiente a una mezcla de acetato de etilo y etanol (R9 en la Tabla 1). Los parámetros obtenidos se fueron reajustando a medida que se fue incorporando cada uno de los ensayos hasta alcanzar la totalidad de los mismos (Tabla 1).

\subsubsection{Resultados}

Al avanzar en la regresión se verificó que la constante de adsorción del etanol conducía a valores muy pequeños y que no se podía ajustar adecuadamente. En consecuencia se adoptó $\mathrm{K}_{2}=0$. De esta forma, se determinaron los ocho parámetros restantes. Los valores óptimos de los parámetros y sus respectivos intervalos de confianza se presentan en la Tabla 2.

Tabla 2: Valores óptimos de los parámetros cinéticos e intervalos de confianza

\begin{tabular}{ccc}
\hline Parámetro & Unidades & Valores óptimos e intervalos de confianza \\
\hline $\mathrm{k}_{\mathrm{ref}, \mathrm{a}}$ & $\mathrm{s}^{-1}$ & $6.6210^{1}(1 \pm 0.11)$ \\
$\mathrm{k}_{\mathrm{ref}, \mathrm{b}}$ & $\mathrm{s}^{-1}$ & $1.8110^{3}(1 \pm 0.17)$ \\
$\mathrm{k}_{\mathrm{ref}, \mathrm{c}}$ & $\mathrm{s}^{-1}$ & $1.2210^{2}(1 \pm 0.14)$ \\
$\mathrm{E}_{\mathrm{a}}$ & $\mathrm{J} \mathrm{mol}^{-1}$ & $1.4810^{5}(1 \pm 0.05)$ \\
$\mathrm{E}_{\mathrm{b}}$ & $\mathrm{J} \mathrm{mol}^{-1}$ & $1.1010^{5}(1 \pm 0.04)$ \\
$\mathrm{E}_{\mathrm{c}}$ & $\mathrm{J} \mathrm{mol}^{-1}$ & $1.6910^{5}(1 \pm 0.05)$ \\
$\mathrm{K}_{1}$ & $\mathrm{~m}^{3} \mathrm{~mol}^{-1}$ & $4.1910^{2}(1 \pm 0.15)$ \\
$\mathrm{K}_{3}$ & $\mathrm{~m}^{3} \mathrm{~mol}^{-1}$ & $6.7510^{2}(1 \pm 0.19)$ \\
\hline
\end{tabular}

En las Figuras 6, 7 y 8 se presenta la comparación de valores experimentales con el modelo propuesto, evaluado con los parámetros obtenidos, para diversas condiciones de alimentación, incluyendo etanol y acetato de etilo, para diferentes relaciones de los mismos y una misma masa de catalizador (300 mg). 


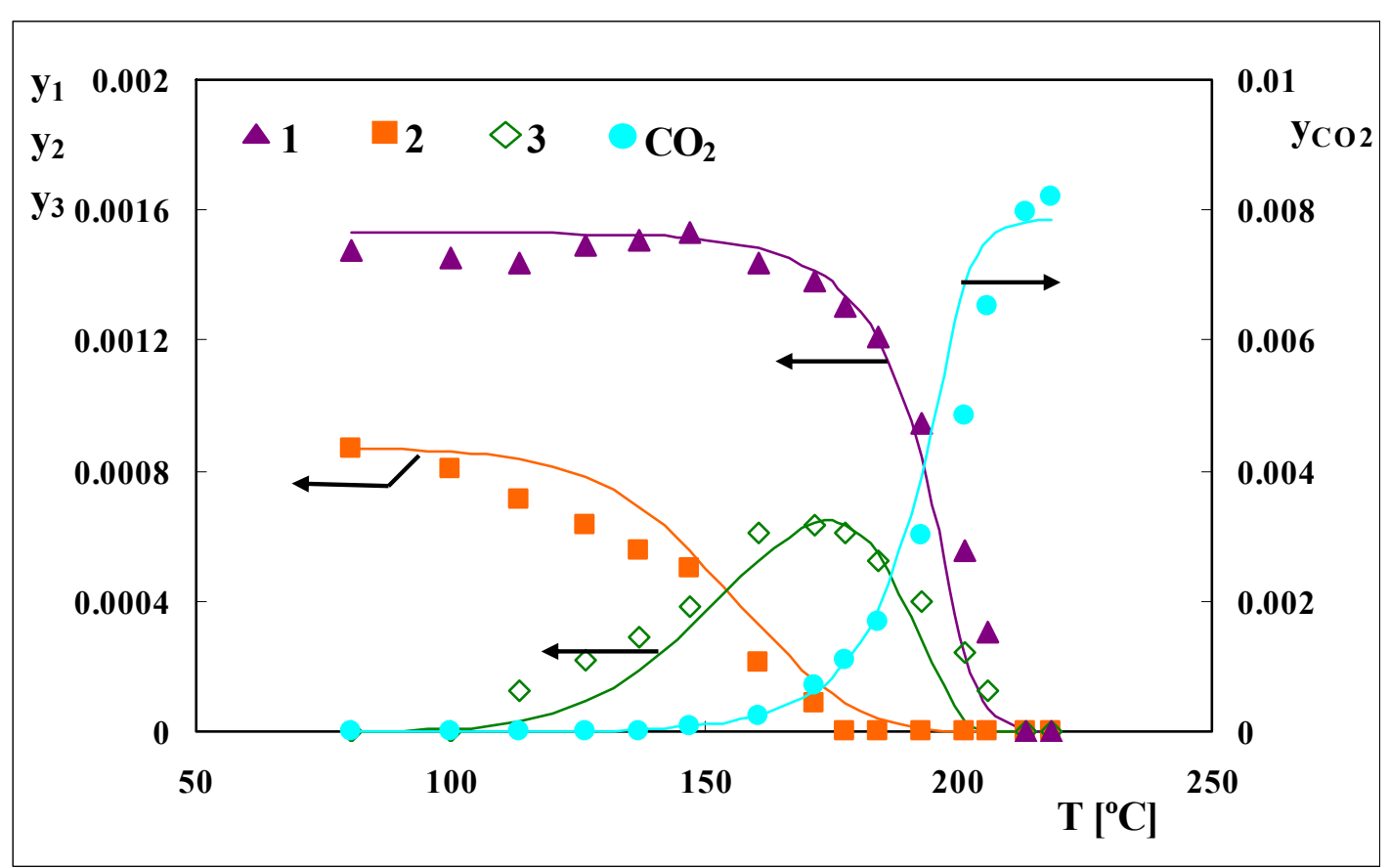

Fig. 6 - Comparación de datos experimentales (símbolos) y calculados con el modelo (líneas) para la condición experimental R11 en Tabla 1 ( $\left.\mathrm{m}_{\mathrm{cat}}=300 \mathrm{mg}\right)$.

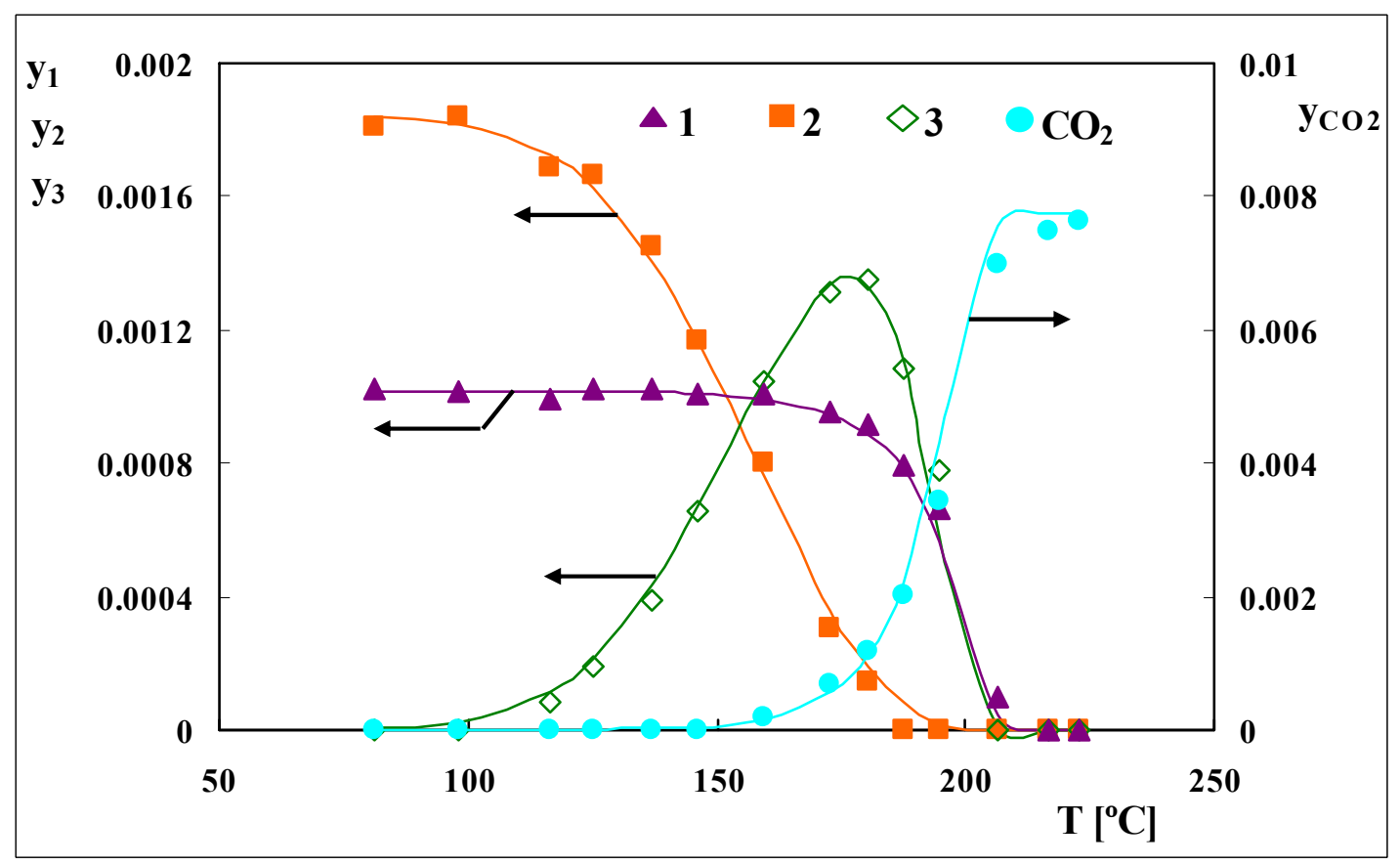

Fig. 7 - Comparación de datos experimentales (símbolos) y calculados con el modelo (líneas) para la condición experimental R14 en Tabla 1 ( $\left.\mathrm{m}_{\mathrm{cat}}=300 \mathrm{mg}\right)$. 


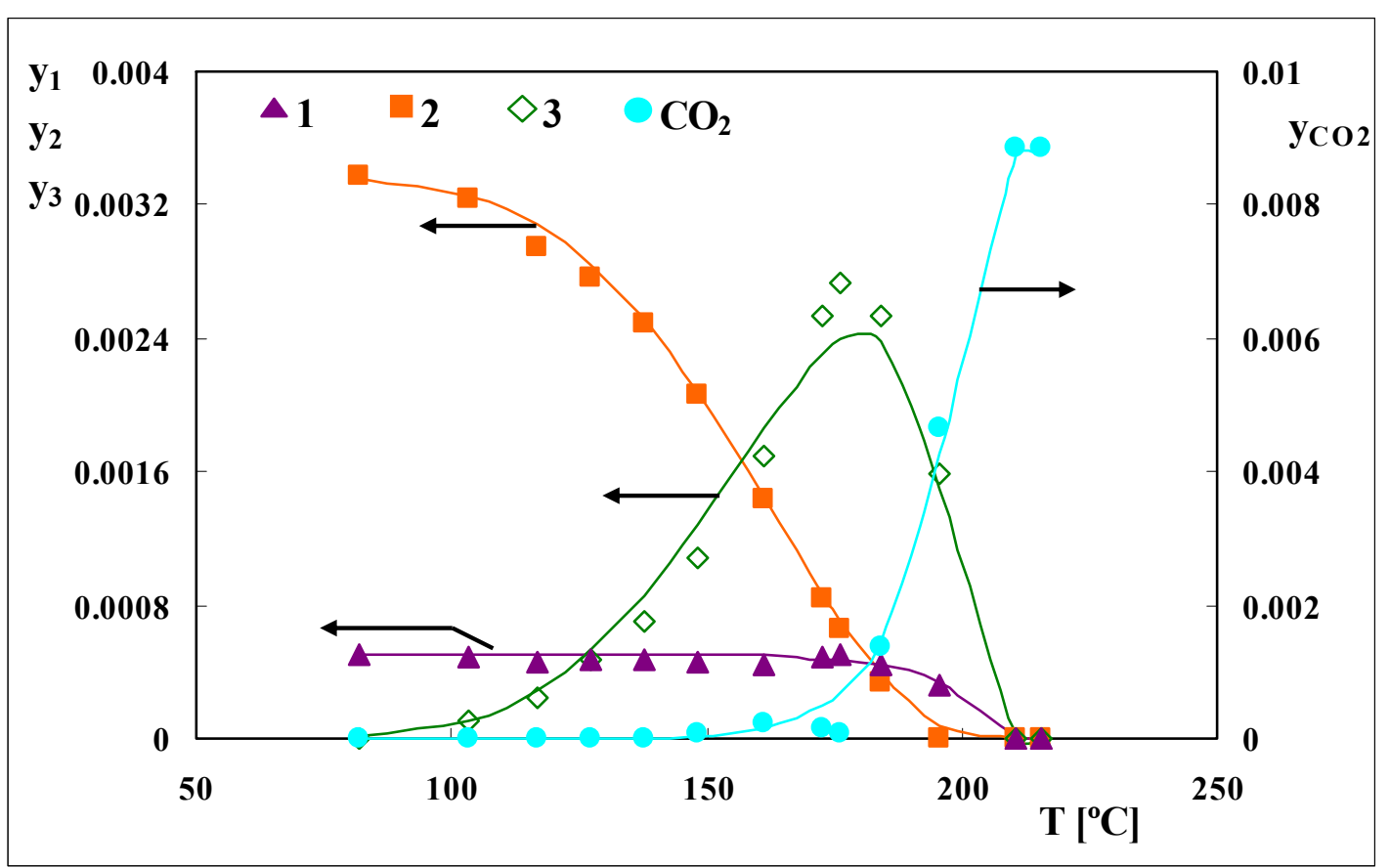

Fig. 8 - Comparación de datos experimentales (símbolos) y calculados con el modelo (líneas) para la condición experimental R17 en Tabla $1\left(\mathrm{~m}_{\text {cat }}=300 \mathrm{mg}\right)$.

Se aprecia que, a pesar de las diferencias en las condiciones de alimentación el modelo empleado, y los parámetros obtenidos, permiten representar de forma muy satisfactoria el comportamiento del sistema estudiado.

Las Figuras 9, 10 y 11 muestran los Parity Plot de las fracciones molares de salida, obtenidas en forma experimental $\left(\mathrm{y}_{\exp }\right)$ o evaluadas con el modelo $\left(\mathrm{y}_{\bmod }\right)$, para el acetato de etilo (1), el etanol (2) y el $\mathrm{CO}_{2}$ respectivamente. Los puntos se diferencian cuando corresponden a ensayos con uno solo o ambos VOCs en la alimentación. Se aprecia que la comparación del conjunto de valores experimentales y calculados con el modelo es satisfactoria, con errores del $10.43 \%$ (142 ensayos) para el etanol y de $15.20 \%$ (142 ensayos) para el acetato de etilo. 


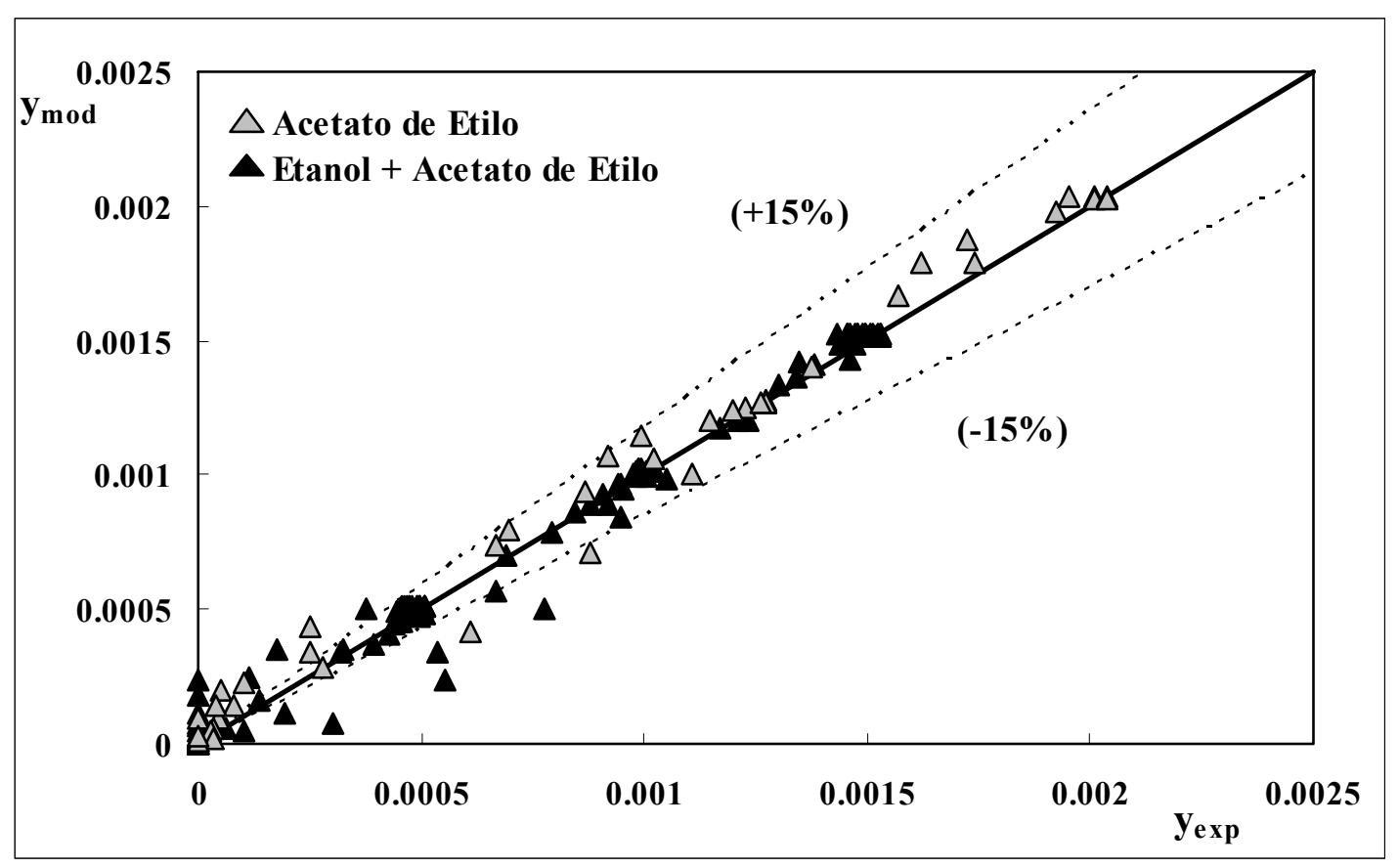

Fig. 9 - Parity Plot para el acetato de etilo. $(\bmod =$ modelo, $\exp =$ experimental $)$

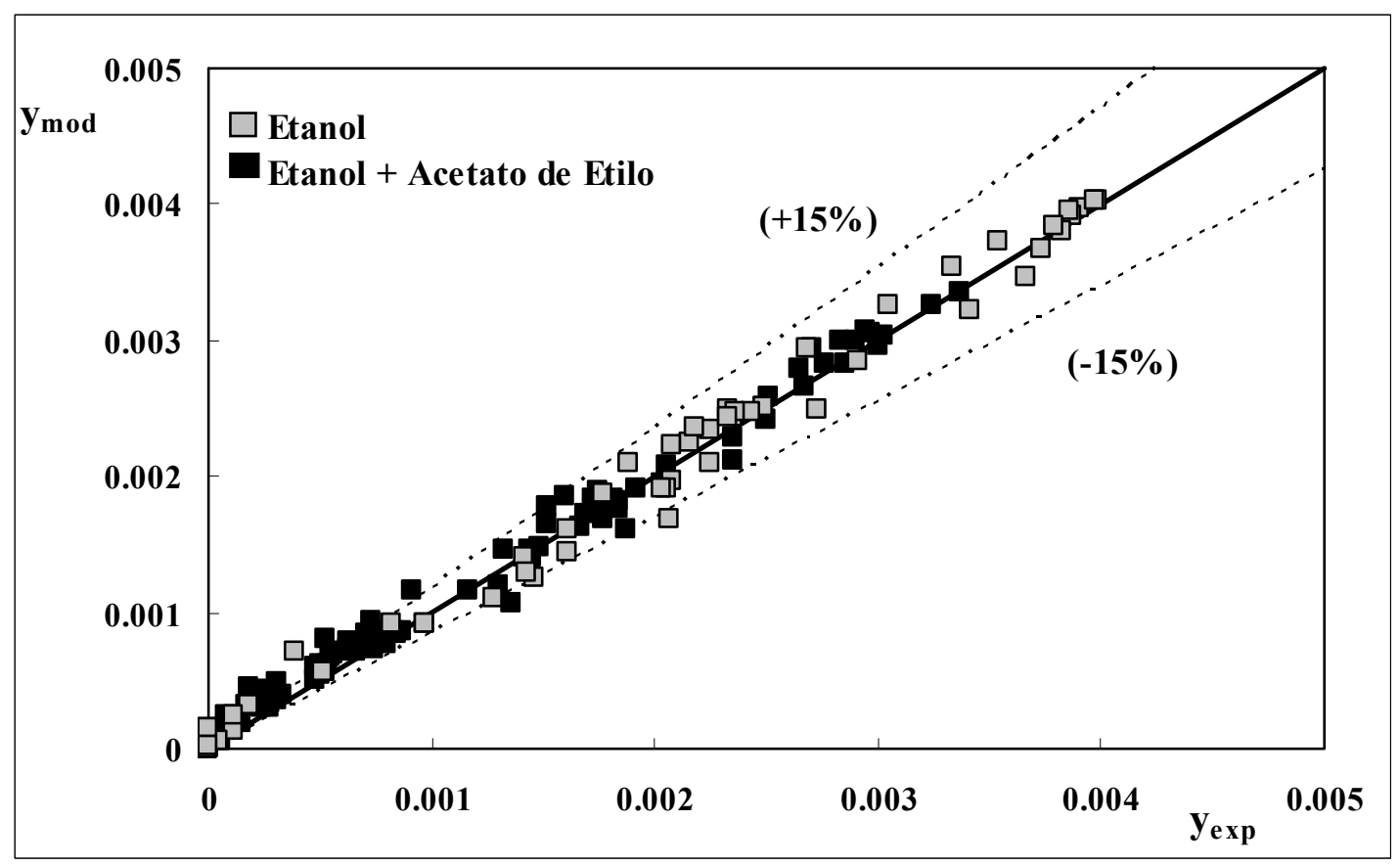

Fig. 10 - Parity Plot para el etanol. $(\bmod =$ modelo, $\exp =$ experimental $)$ 


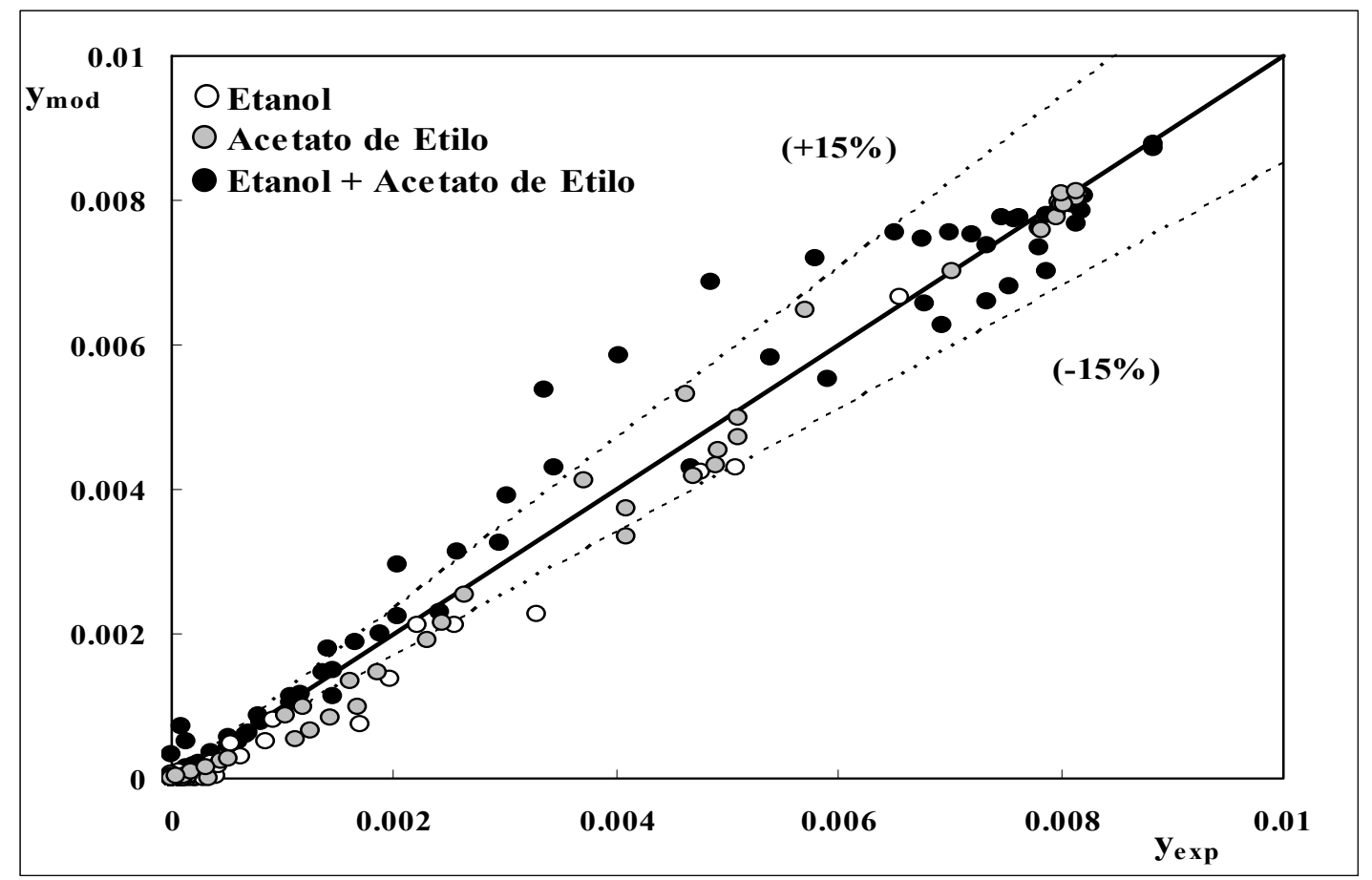

Fig. 11 - Parity Plot para el $\mathrm{CO}_{2} .(\bmod =$ modelo, exp $=$ experimental $)$

A partir de lo resultados obtenidos en la regresión, se pudo verificar una fuerte influencia de la transferencia de materia en el interior del catalizador. La Figura 12 presenta la variación del factor de efectividad $(\eta)$ de la reacción de combustión del acetato de etilo (i.e., reacción (a)) a lo largo del reactor.

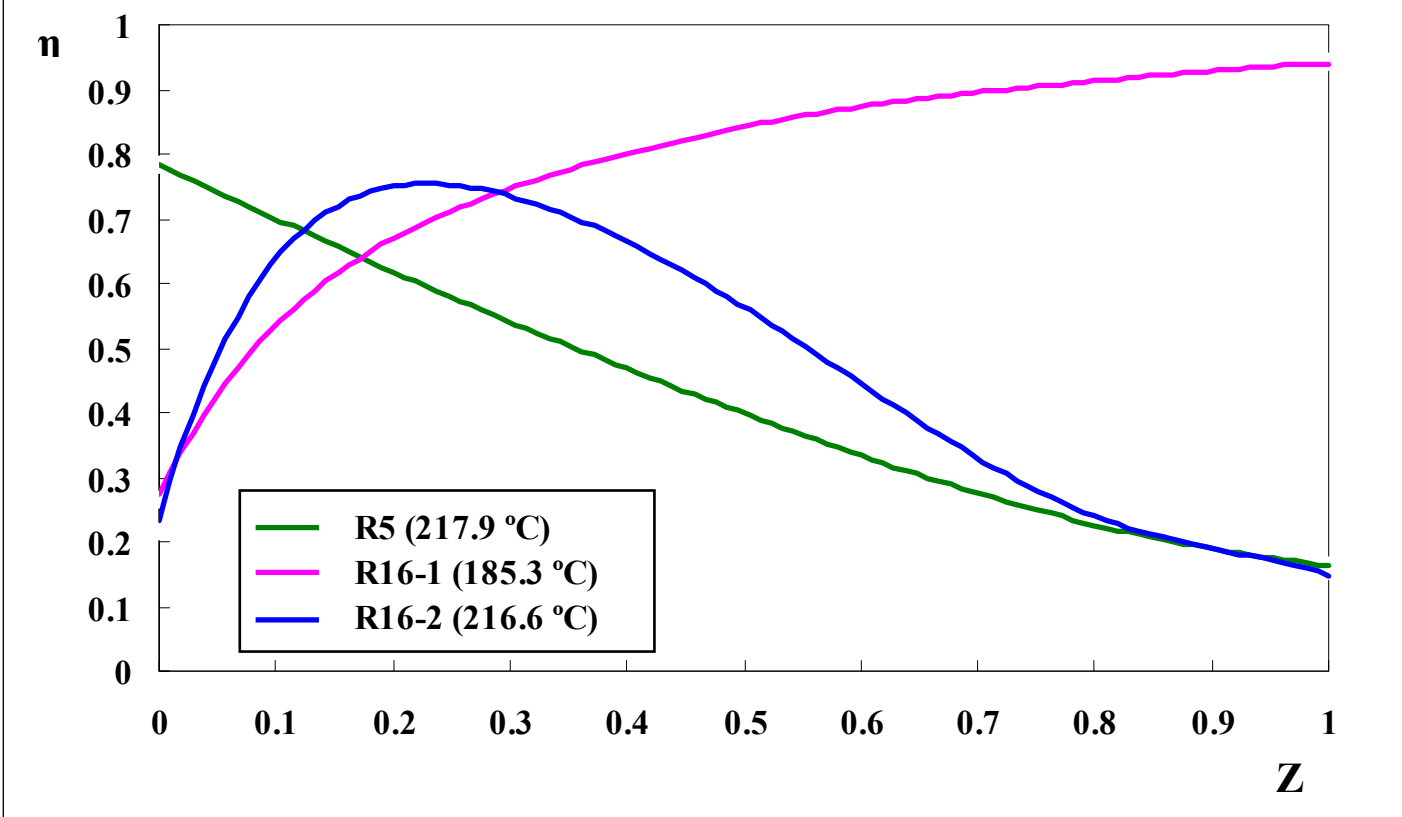

Fig. 12 - Variación del factor de efectividad ( $\eta$ ) de la reacción (a) a lo largo del reactor para las condiciones $\mathrm{R} 5\left(217.9^{\circ} \mathrm{C}\right)$ y R16 $\left(185.3{ }^{\circ} \mathrm{C}(1)\right.$ y $\left.216.6^{\circ} \mathrm{C}(2)\right)$ de la Tabla 1. 
Previamente a analizar las curvas presentadas en la Fig. 12, resulta apropiado conocer la funcionalidad del factor de efectividad con la concentración de las especies presentes en el reactor.

Como es de esperar y, en nuestro caso particular se verifica, el factor de efectividad de cada reacción disminuye con el aumento del módulo de Thiele $(\Phi)$ de cada una de las mismas. El módulo de Thiele, en general, relaciona la capacidad de reacción química con la capacidad de difusión según la siguiente expresión:

$$
\Phi_{\mathrm{j}}=\ell_{e}\left(\frac{\mathrm{r}_{\mathrm{j}}^{\mathrm{s}}}{\mathrm{D}_{\mathrm{e}, \mathrm{j}} \mathrm{C}_{\mathrm{s}, \mathrm{j}}}\right)^{1 / 2}
$$

donde $\ell_{e}$ es la longitud efectiva del catalizador $\mathrm{y}_{\mathrm{j}}^{\mathrm{s}}$ es la velocidad de consumo de la especie $\mathrm{j}$ evaluada en la superficie del catalizador.

Considerando que los datos representados en la Fig. 12 corresponden a la reacción de combustión del acetato de etilo, reemplazando $r_{j}^{s}$ por $r_{1}^{s}$ en la Ec. [14] se tiene:

$$
\Phi_{1}=\ell_{e}\left[\frac{\mathrm{k}_{\mathrm{a}}}{\mathrm{D}_{\mathrm{e}, 1}\left(1+\mathrm{K}_{1} \mathrm{C}_{\mathrm{s}, 1}+\mathrm{K}_{3} \mathrm{C}_{\mathrm{s}, 3}\right)}\right]^{1 / 2}
$$

siendo :

$$
\mathrm{k}_{\mathrm{a}}=\mathrm{k}_{\text {ref }, \mathrm{a}} \exp \left[-\left(\mathrm{E}_{\mathrm{a}} / \mathrm{R}\right)\left((1 / \mathrm{T})-\left(1 / \mathrm{T}_{\text {ref }}\right)\right)\right]
$$

Teniendo en cuenta la variación del factor de efectividad con el módulo de Thiele y considerando la Ec. [15], se tiene que un aumento en la concentración del acetato de etilo $\left(\mathrm{C}_{\mathrm{s}, 1}\right)$ o en la concentración del acetaldehído $\left(\mathrm{C}_{\mathrm{s}, 3}\right)$, dada su aparición en los términos de inhibición en las expresiones cinéticas, provoca una disminución en el módulo de Thiele $\mathrm{y}$, por lo tanto, un aumento en el factor de efectividad correspondiente a la reacción (a). A continuación se analizan las curvas presentes en la Fig. 12, teniendo en cuenta esta situación.

Para la curva R5 (Tabla 1) el reactor opera a $217.9{ }^{\circ} \mathrm{C}$ y solo se tiene acetato de etilo como VOC presente en la alimentación. A medida que la reacción avanza a lo largo del reactor, la concentración de acetato de etilo disminuye y el módulo de Thiele, $\Phi_{1}$, aumenta (ver Ec. [15]), consecuentemente el factor de efectividad disminuye como se puede apreciar en la Fig. 12. 
Para la curva R16-1 (Tabla 1) el reactor opera a $185.3{ }^{\circ} \mathrm{C}$ y se tiene una mezcla de acetato de etilo y etanol presentes en la alimentación. En consecuencia, a medida que la reacción avanza a lo largo del reactor, no solo se consume acetato de etilo (reacción (a)), sino que, según la reacción (b), se produce acetaldehído. Para la temperatura de operación del reactor $\left(185.3^{\circ} \mathrm{C}\right)$, la velocidad neta de producción del acetaldehído es positiva, por lo que su concentración aumenta monótonamente a lo largo del reactor (Figura 13). Dicho aumento resulta más significativo que la disminución de la concentración del acetato de etilo, provocando una disminución del módulo de Thiele, $\Phi_{1}$, y por ende un aumento monótono en el factor de efectividad (ver curva R16-1 en la Fig. 12).

En cambio, si para las mismas condiciones de R16 (ver Tabla1), la temperatura de operación del reactor aumenta, por ejemplo a $216.6{ }^{\circ} \mathrm{C}$, se tiene que a partir de una determinada posición en el reactor la velocidad de consumo del acetaldehído resulta más rápida que su velocidad de producción, presentando un máximo en su perfil de concentración el cual puede observarse en la Fig. 13. Dicho comportamiento de cambio de concentración genera un máximo en el factor de efectividad representado por la curva R16-2 en la Fig. 12.

Un comportamiento similar se obtiene al analizar el factor de efectividad de la reacción (b).

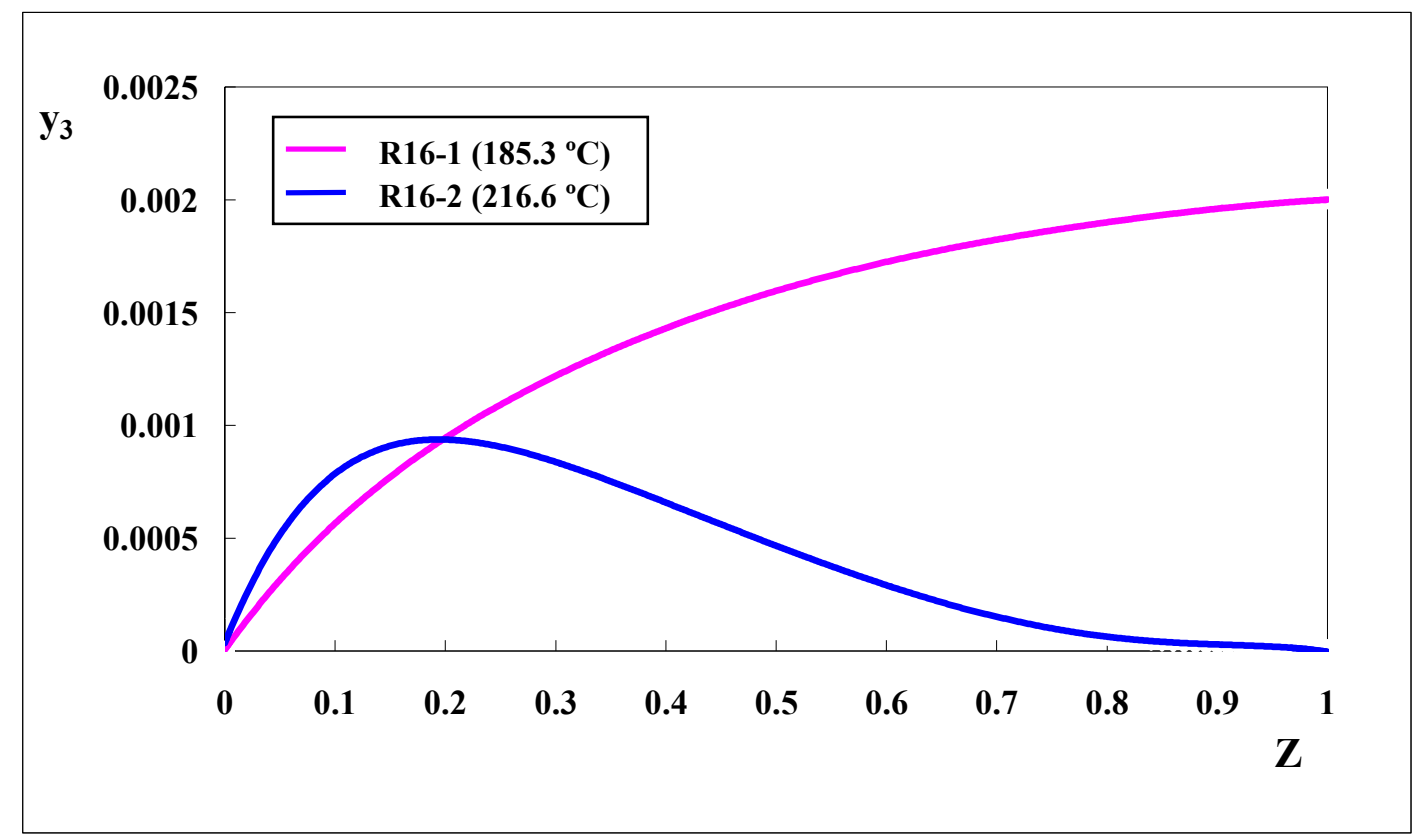

Fig. 13 - Variación de la fracción molar de acetaldehído $\left(\mathrm{y}_{3}\right)$ a lo largo del reactor para las condiciones R16-1 $\left(185.3{ }^{\circ} \mathrm{C}\right)$ y R16-2 $\left(216.6^{\circ} \mathrm{C}\right)$ de la Tabla 1. 
Por último resulta interesante analizar la producción total de $\mathrm{CO}_{2}$ cuando ambos VOCs se encuentran presentes en la alimentación al reactor. La Figura 14 presenta los puntos experimentales (símbolos) de $\zeta_{\mathrm{CO}_{2}}$ para tres relaciones de etanol/acetato de etilo diferentes y dos masas de catalizador (ensayos R11, R14 y R17 en Tabla 1, $\mathrm{m}_{\text {cat }}=300 \mathrm{mg}$, y R9, R12 y R15 en Tabla $1, \mathrm{~m}_{\text {cat }}=100 \mathrm{mg}$ ). Se aprecia que las diferencias entre los puntos experimentales para cada masa de catalizador son pequeñas $\mathrm{y}$, al estar dentro del error experimental, podrían interpretarse como una única curva. Con el modelo teórico, se aprecian pequeñas diferencias, pero por simplicidad se ha representado una única curva para cada masa de catalizador (Fig. 14), que muestra la capacidad del modelo empleado para captar el comportamiento de una mezcla con diferentes relaciones de los dos VOCs estudiados.

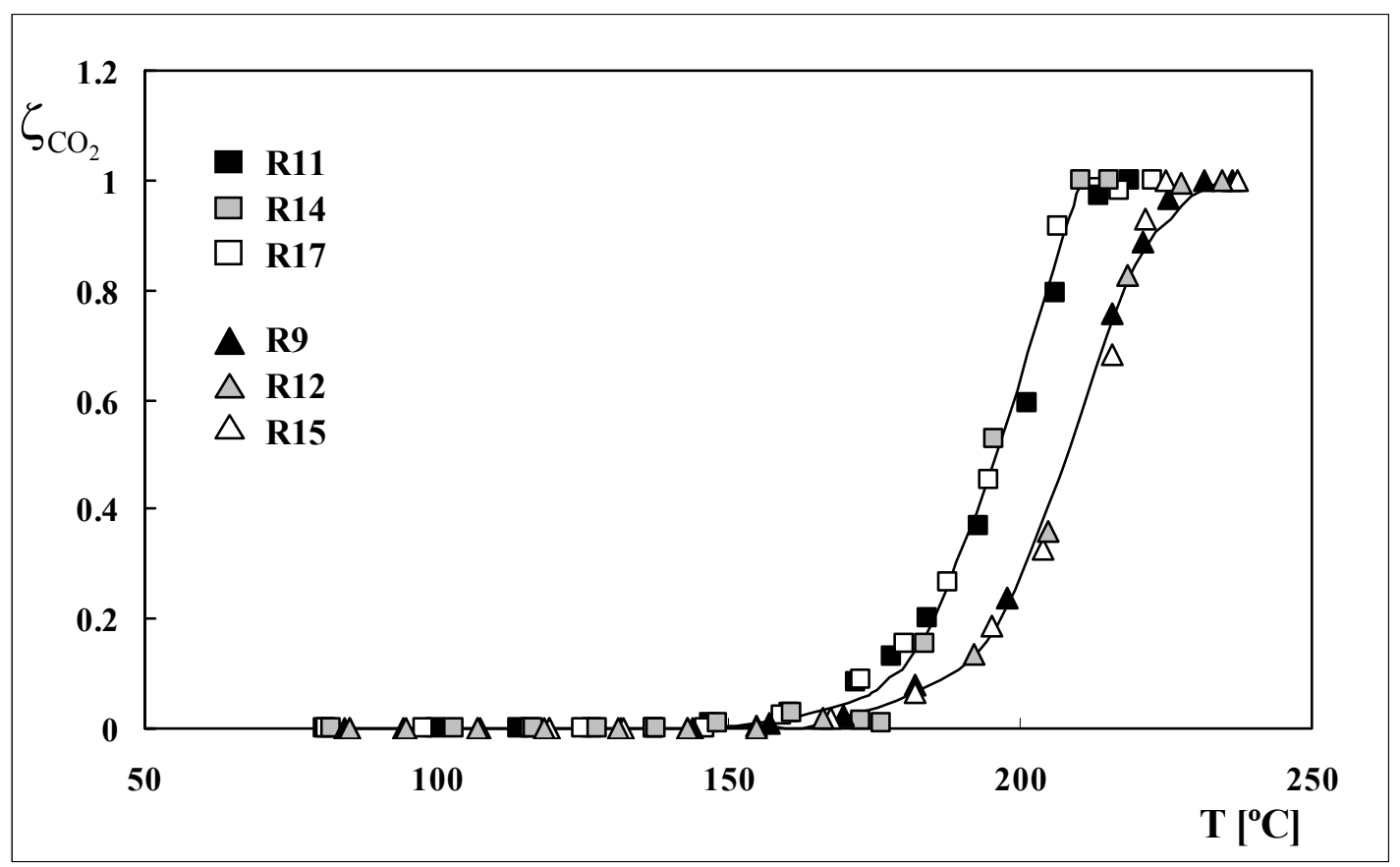

Fig. 14 - Valores de $\zeta_{\mathrm{CO}_{2}}$ obtenidos a partir del modelo (líneas) y experimentalmente (símbolos) para dos masas de catalizador: $\mathrm{m}_{\text {cat }}=300 \mathrm{mg}$ (R11, R14 Y R17 en Tabla 1) y $\mathrm{m}_{\text {cat }}=100 \mathrm{mg}$ (R9, R12 y R15 en Tabla 1).

La comparación de los parámetros obtenidos con valores de la literatura no puede realizarse en forma directa, ya que debería tratarse de un estudio sobre el mismo catalizador. Por otra parte, no se conoce un trabajo donde se reporte un conjunto completo de parámetros cinéticos, correspondientes a un esquema como el aquí planteado. Los valores que se informan con mayor frecuencia son los de energía de activación y, aunque obtenidas con otros catalizadores, pueden servir como marco de 
referencia. La revisión se ha limitado a situaciones en las cuales se emplean catalizadores de óxidos metálicos, por esa razón no se incluye en esta comparación estudios sobre metales nobles, por ej., Pd o Pt.

Para bajas conversiones de etanol, produciendo principalmente acetaldehído, Idriss y Seebauer (2000) reportan un amplio rango de energías de activación (50 a $130 \mathrm{~kJ} / \mathrm{mol}$ ). Esto se debe a que han empleando materiales catalíticos muy variados.

Por su parte, Delimaris e Ioannides (2008) indican un rango de energías de activación aparentes para el etanol entre 82 y $126 \mathrm{~kJ} / \mathrm{mol}$ sobre catalizadores en base a Mn (generalmente combinado con Ce), mientras que Delimaris e Ioannides (2009) reportan un rango de energías de activación aparentes entre aproximadamente $101 \mathrm{y}$ $136 \mathrm{~kJ} / \mathrm{mol}$ para catalizadores en base a $\mathrm{Cu}$ (generalmente combinado con $\mathrm{Ce}$ ). Es importante mencionar que en estos trabajos se detectaron acetaldehído y acetato de etilo como productos intermediarios y que los valores reportados están basados en la velocidad de producción de $\mathrm{CO}_{2}$, esto significa que no se encuentran discriminadas las reacciones consecutivas (b) y (c) propuestas en nuestro trabajo. Esto trae un cierto grado de incertidumbre al momento de comparar los resultados publicados con los obtenidos a partir de la regresión.

Finalmente, Oyama y col. (1999) informan una energía de activación de 89 kJ/mol para la oxidación de etanol sobre catalizadores en base a Mn.

El valor reportado en este trabajo para la energía de activación correspondiente a la oxidación parcial del etanol (reacción (b)) es de $110 \mathrm{~kJ} / \mathrm{mol}$. A pesar de no poder realizarse una comparación directa debido a las diferencias establecidas, el valor resultante de nuestro estudio se encuentra dentro del rango de valores reportados en la literatura.

Para el acetaldehído no se encuentra una información bibliográfica que resulte, al menos, comparable.

Para el acetato de etilo Delimaris e Ioannides (2008), quienes identifican etanol, acetaldehído y ácido acético como productos intermediarios, indican un rango de energías de activación aparentes entre 95 y $119 \mathrm{~kJ} / \mathrm{mol}$ sobre catalizadores en base a Mn (generalmente combinado con Ce), mientras que Delimaris e Ioannides (2009) reportan un rango de energías de activación aparentes entre aproximadamente $121 \mathrm{y}$ $145 \mathrm{~kJ} / \mathrm{mol}$ para catalizadores en base a $\mathrm{Cu}$ (generalmente combinado con Ce). Aquí también los valores reportados se encuentran basados en la velocidad de producción del $\mathrm{CO}_{2}$. Por su parte Papaefthimiou y col. (1997) indican un valor de $151 \pm 12 \mathrm{~kJ} / \mathrm{mol}$ 
para la oxidación de acetato de etilo sobre un catalizador en base Co. El valor obtenido en la regresión para la energía de activación correspondiente a la oxidación de acetato de etilo (reacción (a)) de $148 \mathrm{~kJ} / \mathrm{mol}$, se ubica razonablemente en los valores de bibliografía. Asimismo, y al igual que para el acetaldehído, es obvio, a partir de los resultados experimentales de las Figs. 5, 6 y 7, que la energía de activación del acetato de etilo debe ser sustancialmente mayor a la del etanol.

\subsection{Conclusiones}

Se ha estudiado la combustión catalítica de etanol y acetato de etilo sobre un catalizador denominado $\mathrm{Mn}_{9} \mathrm{Cu}_{1}$ en un reactor de laboratorio de lecho fijo. Se verificó que la oxidación de etanol produce acetaldehído, el que pasa directamente a dióxido de carbono, constituyendo un sistema de reacciones en serie, y se descartó un posible camino de combustión directa de etanol a dióxido de carbono. Por otro lado, se puede asumir razonablemente que la combustión del acetato de etilo produce directamente $\mathrm{CO}_{2}$.

Se observó que los productos finales de reacción no tienen incidencia en la velocidad de combustión de los VOCs.

Se analizó la interacción entre los diferentes VOCs al oxidarse una mezcla de los mismos (Figs. 4 y 5), verificándose que existe una influencia mutua en el comportamiento cinético, generada por los términos de inhibición en los cuales se incluyen el acetaldehído y el acetato de etilo.

Los datos experimentales se ajustaron satisfactoriamente empleando expresiones de tipo LHHW, tanto para la oxidación de etanol a acetaldehído como para las combustiones totales de este último y de acetato de etilo (Figs. 6, 7 y 8). Se ajustaron satisfactoriamente las energías de activación y constantes específicas de todas las reacciones y las constantes de equilibrio de adsorción del acetaldehído y acetato de etilo (Tabla 2). La constante de equilibrio de adsorción del etanol condujo a valores muy pequeños y se fijó un valor nulo para la misma. Las energías de activación obtenidas para la oxidación de etanol y acetato de etilo se encuentran en el rango de valores reportados en bibliografía para catalizadores similares.

A partir de los resultados obtenidos en la regresión, y mediante el análisis de la variación del factor de efectividad $(\eta)$ a lo largo del reactor, se pudo confirmar una fuerte influencia de la transferencia de materia en el interior del catalizador (Fig. 12), la 
cual había sido estimada empleando criterios de bibliografía y por lo tanto, incorporada en el modelo de reactor utilizado para la regresión de los datos experimentales. 
Presentación de las características del catalizador y evaluación de la difusividad efectiva

\section{Características del catalizador}

El catalizador utilizado para el estudio cinético, identificado como $\mathrm{Mn}_{9} \mathrm{Cu}_{1}$, fue sintetizado por el método de co-precipitación descrito por Morales y col. (2008), a partir de soluciones acuosas de $\mathrm{Cu}\left(\mathrm{NO}_{3}\right)_{2} \cdot 4 \mathrm{H}_{2} \mathrm{O}$ y $\mathrm{Mn}\left(\mathrm{NO}_{3}\right)_{2} \cdot 4 \mathrm{H}_{2} \mathrm{O}$. En la Tabla A7.1.1 se presentan las características de la pastilla catalítica. Dicha información fue empleada para la evaluación de la difusividad efectiva.

Tabla A7.1.1: Características de la pastilla catalítica

\begin{tabular}{ccccc}
\hline $\mathbf{d}_{\mathbf{p}}[\mathbf{m}]$ & $\mathbf{r}_{\text {poro }}[\mathbf{m}]$ & $\boldsymbol{\varepsilon}_{\mathbf{p}}$ & $\tau$ & $\boldsymbol{\rho}_{\mathbf{p}}\left[\mathbf{k g} / \mathbf{m}^{3}\right]$ \\
\hline $6.3010^{-4}$ & $6.2510^{-9}$ & 0.41 & 1.6 & 2750 \\
\hline
\end{tabular}

donde:

$\mathrm{d}_{\mathrm{p}}$ : diámetro de pastilla de catalizador

$\mathrm{r}_{\text {poro }}$ : radio de poro de la pastilla de catalizador

$\varepsilon_{\mathrm{s}}:$ porosidad del catalizador

$\tau:$ tortuosidad

\section{Determinación de la difusividad efectiva}

Las difusividades efectivas de las especies involucradas en la reacción, i.e. acetato de etilo, etanol y acetaldehído $\left(\mathrm{D}_{\mathrm{e}, \mathrm{i}}\right)$ en la mezcla oxígeno/helio, se evaluaron con la expresión de Bosanquet. Dicha expresión resulta apropiada debido a la dilución de las especies reactivas, lo cual justifica despreciar la fricción entre las mismas y la contribución viscosa. Por otro lado, la interacción Knudsen con las paredes en el interior de los poros, resulta adecuada para diámetros de poro del orden de $10 \mathrm{~nm}$ (Tabla A7.1.1).

$$
\mathrm{D}_{\mathrm{e}, \mathrm{i}}=\frac{\varepsilon_{\mathrm{p}}}{\tau} \frac{1}{\frac{1}{\mathrm{D}_{\mathrm{m}, \mathrm{i}}}+\frac{1}{\mathrm{D}_{\mathrm{K}, \mathrm{i}}}},\left[\mathrm{m}^{2} / \mathrm{s}\right]
$$


donde:

$\mathrm{D}_{\mathrm{m}, \mathrm{i}}$ : coeficiente de difusión molecular de la especie i en la mezcla oxígeno/helio, $\left[\mathrm{m}^{2} / \mathrm{s}\right]$

$\mathrm{D}_{\mathrm{K}, \mathrm{i}}$ : difusividad Knudsen de la especie $\mathrm{i},\left[\mathrm{m}^{2} / \mathrm{s}\right]$

Para la evaluación del coeficiente de difusión molecular se consideró la difusión de los VOCs, acetato de etilo, etanol o acetaldehído, en la mezcla oxígeno(20\%)/helio(80\%). En consecuencia, resulta:

$$
\frac{1}{D_{m, i}}=\frac{0.2}{D_{m, i-O_{2}}}+\frac{0.8}{D_{m, i-H e}}
$$

donde:

$\mathrm{D}_{\mathrm{m}, \mathrm{i}-\mathrm{O}_{2}}:$ coeficiente de difusión molecular de la especie i en oxígeno, $\left[\mathrm{m}^{2} / \mathrm{s}\right]$

$\mathrm{D}_{\mathrm{m}, \mathrm{i}-\mathrm{He}}:$ coeficiente de difusión molecular de la especie i en helio, $\left[\mathrm{m}^{2} / \mathrm{s}\right]$

La difusividad binaria, $\mathrm{D}_{\mathrm{m}, \mathrm{i}-\mathrm{j}}$, para mezclas gaseosas a presiones por debajo de (alrededor) de 10 atm no dependen de la composición, resultan inversamente proporcional a $\mathrm{P}$ y pueden estimarse con relativa facilidad a partir de expresiones derivadas de la aplicación de la teoría cinética de los gases. Una de tales expresiones es la propuesta por Fuller y col. (1966).

$$
\mathrm{D}_{\mathrm{m}, \mathrm{i}-\mathrm{j}}=3.1610^{-8} \frac{(\mathrm{T})^{1.75}}{\mathrm{P}\left(\mathrm{v}_{\mathrm{D}, \mathrm{i}}^{1 / 3}+\mathrm{v}_{\mathrm{D}, \mathrm{j}}^{1 / 3}\right)^{2}}\left(\frac{1}{\mathrm{M}_{\mathrm{i}}}+\frac{1}{\mathrm{M}_{\mathrm{j}}}\right)^{1 / 2}
$$

donde:

$\mathrm{M}_{\mathrm{i}}$ y $\mathrm{M}_{\mathrm{j}}$ : pesos moleculares de la especie i y de $\mathrm{j}\left(\mathrm{He}, \mathrm{O}_{2}\right)$ respectivamente, $[\mathrm{kg} / \mathrm{mol}]$ $\mathrm{v}_{\mathrm{D}, \mathrm{i}} \mathrm{Y} \mathrm{v}_{\mathrm{D}, \mathrm{j}}$ : volumen de difusión, que resultan aproximadamente dos tercios del volumen líquido molar en el punto normal de ebullición, [ $\left.\mathrm{m}^{3} / \mathrm{mol}\right]$.

Los valores del volumen de difusión para el acetato de etilo, etanol, acetaldehído, oxígeno y helio se dan en la siguiente tabla. También se informa el del aire, que se empleará para la simulación del reactor en el Capítulo 8. 
Tabla A7.1.1: Volumen de difusión

\begin{tabular}{c|cccccc} 
& Acetato de Etilo & Etanol & Acetaldehído & He & $\mathbf{O}_{\mathbf{2}}$ & Aire \\
\hline $\mathbf{v}_{\mathbf{D}}\left[\mathbf{m}^{\mathbf{3}} / \mathbf{m o l}\right]$ & $65.7310^{-6}$ & $39.0810^{-6}$ & $37.6610^{-6}$ & $2.910^{-6}$ & $16.610^{-6}$ & $20.110^{-6}$
\end{tabular}

Para la evaluación del coeficiente de difusión Knudsen, para cada sustancia (acetato de etilo, etanol o acetaldehído), se empleó la siguiente expresión:

$$
D_{\mathrm{K}, \mathrm{i}}=\frac{2}{3} \sqrt{\frac{8 \mathrm{R} \mathrm{T}}{\pi \mathrm{M}_{\mathrm{i}}}} \mathrm{r}_{\text {poro }}
$$




ESTUDIO DE SISTEMAS

COMBINADOS DE

$\bigcirc$ COMBUSTION

CATALITICA DE VOCs

08/ simulacion del ReActor

Modelo del reactor catalítico

Resultados y discusión

Conclusiones 



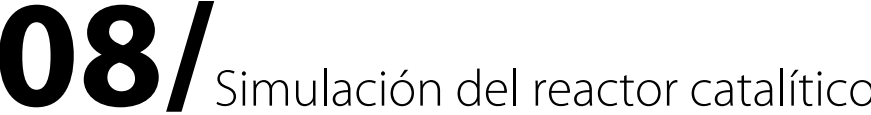

Una vez establecida la información cinética correspondiente a nuestro caso particular de estudio (Capítulo 7), es posible llevar a cabo el diseño y análisis del reactor catalítico presente en el sistema de eliminación propuesto en el Capítulo 2.

Con el propósito de obtener una pérdida de carga tolerable, el caudal de la corriente de alimentación al reactor resulta importante para la elección del tipo de lecho, relleno o estructurado. En nuestro caso, el caudal volumétrico a tratar es del orden de los $10 \mathrm{Nm}^{3} / \mathrm{s}(12.7 \mathrm{~kg} / \mathrm{s})$, un valor suficientemente elevado como para buscar alternativas que tiendan a generar bajas pérdidas de carga.

En general, los lechos estructurados, conocidos como reactores monolíticos, son recomendables para la incineración catalítica de VOCs (Kolaczkowski, 2006) debido a que permiten satisfacer los requerimientos hidráulicos. Sin embrago, este tipo de lecho requiere de costos adicionales, en cuanto a su desarrollo y su manufactura, que deben ser considerados al momento de seleccionar el tipo de lecho a emplear.

Por otro lado, teniendo en cuenta que el empleo del concentrador rotativo en el sistema combinado de eliminación (Capítulo 2), permite reducir el caudal de ingreso al reactor, es posible concebir el uso de un lecho relleno. Se debe destacar que mientras el lecho relleno con flujo axial resulta el esquema más habitual, el flujo radial resulta una opción de interés cuando se opera con caudales relativamente elevados, a fin de lograr bajas pérdidas de carga (Rase, 1990).

De manera tal de cumplir con los requerimientos hidráulicos del sistema de eliminación propuesto, y considerando los costos y sencillez operativa, se optó por un lecho relleno de flujo radial para el diseño del reactor catalítico.

Habiendo optado por un lecho relleno, se debe seleccionar un tamaño de pastilla de catalizador tal que permita mantener una pérdida de carga tolerable. De esta manera el diámetro de la pastilla debe superar aproximadamente los $3 \mathrm{~mm}$, lo que resulta en una resistencia a la transferencia de materia en el interior de la misma muy elevada, por lo que se deben resolver numéricamente los balances de conservación de las especies involucradas en la reacción en el interior de las pastillas en cada posición a lo largo del lecho. A fin de sortear ciertos inconvenientes que surgieron al emplear rutinas de uso general se propone una aproximación que resulta altamente precisa, disminuye el 
tiempo de cálculo en más de un orden de magnitud y evita dificultades de convergencia encontradas con el procedimiento numérico. El desarrollo de la aproximación propuesta, junto con el análisis de los resultados obtenidos, fueron presentados por Campesi y col. (2012b).

\subsection{Modelo del reactor catalítico}

El sistema de reacciones globales, presentadas en el capítulo previo, consiste en la combustión del acetato de etilo (a), oxidación parcial de etanol para producir acetaldehído (intermediario) (b) y la combustión del acetaldehído (c):

$$
\begin{aligned}
& \mathrm{C}_{4} \mathrm{H}_{8} \mathrm{O}_{2}+5 \mathrm{O}_{2} \rightarrow 4 \mathrm{CO}_{2}+4 \mathrm{H}_{2} \mathrm{O} \\
& \mathrm{C}_{2} \mathrm{H}_{6} \mathrm{O}+1 / 2 \mathrm{O}_{2} \rightarrow \mathrm{C}_{2} \mathrm{H}_{4} \mathrm{O}+\mathrm{H}_{2} \mathrm{O} \\
& \mathrm{C}_{2} \mathrm{H}_{4} \mathrm{O}+5 / 2 \mathrm{O}_{2} \rightarrow 2 \mathrm{CO}_{2}+2 \mathrm{H}_{2} \mathrm{O}
\end{aligned}
$$

El reactor propuesto es un lecho fijo con catalizador granular de forma esférica; el sistema de flujo adoptado es de flujo radial, lo que permite reducir sustancialmente la pérdida de carga (Rase, 1990). Con la misma finalidad, se adoptó un tamaño de catalizador con $\mathrm{d}_{\mathrm{p}}=3 \mathrm{~mm}$ y se asumió una porosidad de lecho de $\varepsilon_{\mathrm{L}}=0.4$.

El modelo del reactor se planteó bajo las siguientes hipótesis: reactor adiabático, flujo pistón, gas ideal y pastilla catalítica isotérmica. Se tuvieron en cuenta las resistencias a la transferencia de calor y materia entre el seno de la corriente y la superficie de las partículas catalíticas y la resistencia a la transferencia de materia en el interior de la pastilla.

Las ecuaciones de conservación resultan en cinco ecuaciones diferenciales ordinarias, describiendo la variación de las variables de estado a lo largo del lecho (concentración, temperatura, presión), cuatro ecuaciones algebraicas para el transporte hacia la superficie externa del catalizador y tres balances de conservación para las especies reactivas en el interior del catalizador.

Balances de materia en el lecho:

$$
\frac{\mathrm{dF}_{\mathrm{j}}}{\mathrm{dV}}=-\left(1-\varepsilon_{\mathrm{L}}\right) \mathrm{r}_{\mathrm{j}}^{\mathrm{ef}}, \quad \mathrm{j}=1,2,3
$$


donde se indica con " 1 " al acetato de etilo, con " 2 " al etanol y con " 3 " al acetaldehído. $F_{j}$ representa el caudal molar de la especie $j, \mathrm{~V}$ es el volumen de lecho $\mathrm{y}_{\mathrm{j}}^{\mathrm{ef}}$ son las velocidades efectivas de consumo de las especies $\mathrm{j}$.

El balance de energía en el lecho es:

$$
\frac{\mathrm{dT}_{\mathrm{g}}}{\mathrm{dV}}=\frac{\left(1-\varepsilon_{\mathrm{L}}\right)}{\mathrm{W} \hat{\mathrm{c}}_{\mathrm{p}, \mathrm{g}}}\left[\left(-\Delta \mathrm{H}_{1}\right) \mathrm{r}_{1}^{\mathrm{ef}}+\left[\left(-\Delta \mathrm{H}_{2}\right)-\left(-\Delta \mathrm{H}_{3}\right)\right] \mathrm{r}_{2}^{\mathrm{ef}}+\left(-\Delta \mathrm{H}_{3}\right) \mathrm{r}_{3}^{\mathrm{ef}}\right]
$$

donde $T_{g}$ es la temperatura de la fase fluida, $\mathrm{W}$ es el caudal másico de la corriente a tratar, $\hat{c}_{\mathrm{p}, \mathrm{g}}$ es la capacidad calorífica por unidad de masa de la corriente a tratar $\mathrm{y}$ $\Delta \mathrm{H}_{\mathrm{i}}$ son los calores de combustión de las especies $\mathrm{i}$.

Las resistencias externas se contabilizan mediante los balances:

$$
\begin{aligned}
& \mathrm{k}_{\mathrm{m}, \mathrm{j}}\left(\mathrm{C}_{\mathrm{g}, \mathrm{j}}-\mathrm{C}_{\mathrm{s}, \mathrm{j}}\right)-\ell_{e} \mathrm{r}_{\mathrm{j}}^{\mathrm{ef}}=0, \quad \mathrm{j}=1,2,3 \\
& \mathrm{~h}_{\mathrm{t}}\left(\mathrm{T}_{\mathrm{s}}-\mathrm{T}_{\mathrm{g}}\right)-\ell_{e}\left[\left(-\Delta \mathrm{H}_{1}\right) \mathrm{r}_{1}^{\mathrm{ef}}+\left[\left(-\Delta \mathrm{H}_{2}\right)-\left(-\Delta \mathrm{H}_{3}\right)\right] \mathrm{r}_{2}^{\mathrm{ef}}+\left(-\Delta \mathrm{H}_{3}\right) \mathrm{r}_{3}^{\mathrm{ef}}\right]=0
\end{aligned}
$$

donde $\mathrm{k}_{\mathrm{m}} \mathrm{y} \mathrm{h}_{\mathrm{t}}$, son los coeficientes de transferencia de materia y energía fluidopartícula, respectivamente y $\ell_{e}$ es la longitud efectiva del catalizador. El subíndice "s" se refiere a la superficie del catalizador. Las $r_{j}^{\text {ef }}$ dependen de $T_{s}$ y $C_{s, j}(j=1,2,3)$.

El flujo radial asumido para el tipo de reactor afecta, en los balances previos (Ecs. [3] y [4]), la evaluación de los coeficientes de transferencia $\mathrm{k}_{\mathrm{m}} \mathrm{y} \mathrm{h}_{\mathrm{t}}$; dado que los mismos dependen de la velocidad másica y esta varía punto a punto en dirección radial, se deben evaluar los mencionados coeficientes a medida que se avanza en el reactor. Para la obtención de tales coeficientes se empleó la correlación de Gunn (1978); la misma se encuentra en el Apéndice 5.2 junto a las expresiones para la evaluación de las propiedades termodinámicas (i.e., densidad, viscosidad, capacidad calorífica y conductividad).

Las concentraciones molares en la corriente de fluido, $\mathrm{C}_{\mathrm{g}, \mathrm{j}}$, se relacionan con el caudal molar $F_{j}$ mediante la siguiente expresión de gas ideal: $C_{g, j}=\left(F_{j} / F\right) P /(R T)$. El caudal molar total (F) es esencialmente constante debido a la dilución de las especies reactivas.

La presión local, P, fue calculada a partir de la expresión de Ergun (1952) de pérdida de carga: 


$$
\frac{\mathrm{dP}}{\mathrm{dV}}=-\left\{\left[150 \frac{\left(1-\varepsilon_{\mathrm{L}}\right)^{2}}{\varepsilon_{\mathrm{L}}^{3}} \frac{\mu_{\mathrm{g}}}{\rho_{\mathrm{g}}} \frac{1}{\mathrm{~d}_{\mathrm{p}}} \frac{\mathrm{W}}{2 \pi \mathrm{H}}\right] \frac{1}{\rho^{2}}+\left[1.75 \frac{\left(1-\varepsilon_{\mathrm{L}}\right)}{\varepsilon_{\mathrm{L}}^{3}} \frac{1}{\rho_{\mathrm{g}}} \frac{1}{\mathrm{~d}_{\mathrm{p}}}\left(\frac{\mathrm{W}}{2 \pi \mathrm{H}}\right)^{2}\right] \frac{1}{\rho^{3}}\right\} \frac{1}{2 \pi \mathrm{H}}
$$

donde $\mathrm{H}$ y $\rho$ corresponden a la altura del lecho y a la coordenada radial respectivamente. Esta última se encuentra vinculada con el volumen (V) mediante la siguiente expresión: $\mathrm{V}=\pi\left(\rho^{2}-\mathrm{R}_{\text {in }}{ }^{2}\right) \mathrm{H}, \quad\left(\mathrm{R}_{\mathrm{in}}\right.$ : radio interno del reactor $)$. Tal expresión resulta válida cuando el flujo circula desde la pared interna hacia la pared externa del lecho.

Se asumió como diámetro interno del reactor $\mathrm{D}_{\mathrm{in}}=2 \mathrm{R}_{\text {in }}=0.25 \mathrm{~m}$. El diámetro externo $\left(D_{\text {ex }}\right)$ y la altura $(H)$ del mismo fueron ajustados en base a la cantidad de catalizador requerida para la obtención de la conversión de VOCs deseada y asegurando que no se superen valores admisibles de la pérdida de carga. Se estableció que el flujo circule desde el diámetro interno del reactor hacia el externo con el fin de que el aumento de la sección de flujo $(2 \pi \rho \mathrm{H})$ pueda compensar el aumento de temperatura, lo que implica un aumento de la viscosidad y disminución de la densidad del gas, dos efectos que favorece un aumento de la pérdida de carga (Ec. [5]). El hecho de obtener una menor pérdida de carga en el reactor resulta interesante al momento de evaluar la pérdida de carga global del sistema combinado (Capítulo 2, Fig. 3), teniendo en cuenta que, en general, el reactor es el equipo que presenta mayor pérdida de carga en comparación con el concentrador rotativo e intercambiadores.

El tratamiento para los balances de materia en el interior del catalizador se describirá en la próxima sub-sección.

\subsubsection{Expresiones cinéticas y evaluación de las velocidades efectivas de consumo}

Como se describió en el capítulo previo, las velocidades intrínsecas para las reacciones $(a, b, c)$ fueron evaluadas y ajustadas para un catalizador en base a óxidos de $\mathrm{Mn}$ y $\mathrm{Cu}$ (Morales y col., 2008), habiéndose empleado expresiones del tipo LHHW, con un término común de inhibición (Campesi y col., 2012a):

$$
\begin{aligned}
& r_{\mathrm{a}}=\frac{\mathrm{k}_{\mathrm{a}} \mathrm{C}_{1}}{1+\mathrm{K}_{1} \mathrm{C}_{1}+\mathrm{K}_{3} \mathrm{C}_{3}} \\
& r_{\mathrm{b}}=\frac{\mathrm{k}_{\mathrm{b}} \mathrm{C}_{2}}{1+\mathrm{K}_{1} \mathrm{C}_{1}+\mathrm{K}_{3} \mathrm{C}_{3}}
\end{aligned}
$$




$$
r_{\mathrm{c}}=\frac{\mathrm{k}_{\mathrm{c}} \mathrm{C}_{3}}{1+\mathrm{K}_{1} \mathrm{C}_{1}+\mathrm{K}_{3} \mathrm{C}_{3}}
$$

siendo :

$\mathrm{k}_{\mathrm{a}}=\mathrm{k}_{\mathrm{ref}, \mathrm{a}} \exp \left[-\left(\mathrm{E}_{\mathrm{a}} / \mathrm{R}\right)\left((1 / \mathrm{T})-\left(1 / \mathrm{T}_{\mathrm{ref}}\right)\right)\right]$,

$\mathrm{k}_{\mathrm{b}}=\mathrm{k}_{\mathrm{ref}, \mathrm{b}} \exp \left[-\left(\mathrm{E}_{\mathrm{b}} / \mathrm{R}\right)\left((1 / \mathrm{T})-\left(1 / \mathrm{T}_{\mathrm{ref}}\right)\right)\right]$,

$\mathrm{k}_{\mathrm{c}}=\mathrm{k}_{\mathrm{ref}, \mathrm{c}} \exp \left[-\left(\mathrm{E}_{\mathrm{c}} / \mathrm{R}\right)\left((1 / \mathrm{T})-\left(1 / \mathrm{T}_{\mathrm{ref}}\right)\right)\right]$.

Se puede apreciar que el etanol no participa del término de inhibición, debido a que se encontró que su efecto es insignificante (Capítulo 7). Los valores resultantes de los parámetros cinéticos (Campesi y col., 2012a) se reproducen en la Tabla 1, destacándose que las tres reacciones $(\mathrm{a}, \mathrm{b}, \mathrm{c})$ presentan elevadas energías de activación.

Tabla 1: Parámetros Cinéticos $(\alpha=a, b, c)$

\begin{tabular}{cccc}
\hline Parámetros & Reacción (a) & Reacción (b) & Reacción (c) \\
\hline $\mathbf{K}_{\mathrm{ref}, \boldsymbol{\alpha}}[\mathbf{1} / \mathbf{s}] ;\left(\mathbf{T}_{\mathrm{ref}}=\mathbf{1 7 5}^{\mathbf{}} \mathbf{C}\right)$ & $6.6210^{1}$ & $1.8110^{3}$ & $1.2210^{2}$ \\
$\mathbf{E}_{\boldsymbol{\alpha}}[\mathbf{J} / \mathbf{m o l}]$ & $1.4810^{5}$ & $1.1010^{5}$ & $1.6910^{5}$ \\
\hline $\mathbf{K}_{\mathbf{1}}\left[\mathbf{m}^{\mathbf{3}} / \mathbf{m o l}\right]=4.1910^{2}$ & $\mathbf{K}_{\mathbf{3}}\left[\mathbf{m}^{\mathbf{3}} / \mathbf{m o l}\right]=6.7510^{2}$ & \\
\hline
\end{tabular}

Las propiedades más relevantes de la pastilla catalítica, concernientes a la simulación del reactor, se informan en la Tabla 2.

Tabla 2: Características de la pastilla catalítica

\begin{tabular}{cccccc}
\hline $\mathbf{d}_{\mathbf{p}}[\mathbf{m}]$ & $\mathbf{r}_{\text {poro }}[\mathbf{m}]$ & $\boldsymbol{\varepsilon}_{\mathbf{p}}$ & $\tau$ & $\ell_{e}[\mathbf{m}]$ & $\rho_{\mathbf{p}}\left[\mathbf{k g} / \mathbf{m}^{3}\right]$ \\
\hline $3.0010^{-3}$ & $6.2510^{-9}$ & 0.41 & 1.6 & $5.010^{-4}$ & 2750 \\
\hline
\end{tabular}

Se adoptó un tamaño de pastilla catalítica (Tabla 2), que permitiera mantener tolerable la caída de presión, en tales condiciones, la resistencia a la transferencia de materia en el interior de la misma resulta muy significativa. En consecuencia, a fin de cuantificar las velocidades efectivas de consumo, se deben resolver numéricamente los balances de conservación de las tres especies (acetato de etilo, etanol y acetaldehído) en el interior de las pastillas en cada posición a lo largo del lecho. Para el problema planteado en este trabajo, teniendo en cuenta la 
estequiometría de las reacciones $(a, b, c)$ y las expresiones cinéticas [6], los balances de conservación se pueden escribir, siguiendo una ley de tipo Fick y asumiendo que la difusividad efectiva es constante en el interior de la pastilla, como se muestra a continuación:

$$
\begin{aligned}
& \nabla^{2} \mathrm{C}_{1}=\frac{1}{\mathrm{D}_{\mathrm{e}, 1}} \mathrm{C}_{1} \mathrm{k}_{\mathrm{a}} /\left(1+\mathrm{K}_{1} \mathrm{C}_{1}+\mathrm{K}_{3} \mathrm{C}_{3}\right) \\
& \nabla^{2} \mathrm{C}_{2}=\frac{1}{\mathrm{D}_{\mathrm{e}, 2}} \mathrm{C}_{2} \mathrm{k}_{\mathrm{b}} /\left(1+\mathrm{K}_{1} \mathrm{C}_{1}+\mathrm{K}_{3} \mathrm{C}_{3}\right) \\
& \nabla^{2} \mathrm{C}_{3}=\frac{1}{\mathrm{D}_{\mathrm{e}, 3}}\left(-\mathrm{C}_{2} \mathrm{k}_{\mathrm{b}}+\mathrm{C}_{3} \mathrm{k}_{\mathrm{c}}\right) /\left(1+\mathrm{K}_{1} \mathrm{C}_{1}+\mathrm{K}_{3} \mathrm{C}_{3}\right)
\end{aligned}
$$

donde $\mathrm{D}_{\mathrm{e}, \mathrm{j}}$ son las difusividades efectivas, evaluadas con la expresión de Bosanquet, $\mathrm{D}_{\mathrm{e}, \mathrm{j}}=\frac{\varepsilon_{\mathrm{p}}}{\tau}\left(\frac{1}{\mathrm{D}_{\mathrm{m}, \mathrm{j}}}+\frac{1}{\mathrm{D}_{\mathrm{K}, \mathrm{j}}}\right)^{-1} ; \mathrm{j}=1,2,3$ (Apéndice 7.1 , evaluando la difusividad de cada componente en el aire). Dicha expresión resulta apropiada debido a la dilución de las especies reactivas, lo cual justifica despreciar la fricción entre las mismas y la contribución viscosa. Por otro lado, la interacción Knudsen con las paredes en el interior de los poros, resulta adecuada para diámetros de poro del orden de $10 \mathrm{~nm}$ (Tabla 2).

Como fue mencionado previamente, para el modelo del reactor se asumió pastilla catalítica isotérmica (Sección 8.1) al valor de la temperatura en la superficie, $\mathrm{T}_{\mathrm{s}}$.

Una vez que se resuelven las Ecs. [7a, b, c], para una dada composición y temperatura en la superficie del catalizador, se pueden evaluar las velocidades efectivas de consumo de cada una de las especies $\left(\mathrm{r}_{\mathrm{j}}^{\mathrm{ef}}\right)$, a partir de la integración de las velocidades locales en el volumen de pastilla.

En una primera instancia, para este último fin se resolvieron numéricamente las expresiones de balance de los tres VOCs (Ecs. [7a, b, c]) según el procedimiento iterativo desarrollado en Bressa y col. (2001). El mismo fue incorporado a la rutina de integración numérica del sistema de ecuaciones diferenciales/algebraicas ([1] [4]), para lo cual se empleó el programa DDASAC incluido en el paquete GREGPAK (Stewart y col., 1992).

Se evidenció en numerosos casos que la resolución se detenía, impidiendo completar la simulación. Este efecto fue provocado por la elevada sensibilidad de las reacciones con la temperatura (elevadas energías de activación, Tabla 1) que 
provocan el abrupto encendido del sistema. La situación física incide en el procedimiento de resolución al grado de producir la detención, aún cuando los parámetros de control de las rutinas fueran manipulados para evitarla.

La alternativa para solucionar el problema fue emplear una aproximación explícita para las velocidades efectivas de consumo. El procedimiento aproximado se basa en la aplicación de las soluciones analíticas disponibles para reacciones únicas con expresiones cinéticas lineales, como se detalla a continuación.

8.1.1.1 Procedimiento aproximado para evaluar las velocidades efectivas de consumo $\left(\mathrm{r}_{\mathrm{j}}^{\mathrm{ef}}\right)$

Considerando una única reacción en la que una especie A se consume con velocidad de $\mathrm{r}_{\mathrm{A}}\left(\mathrm{C}_{\mathrm{A}}\right)$ y que el flujo de materia se expresa con una expresión tipo Fick de difusividad constante, el balance de conservación en el interior de una pastilla catalítica resulta,

$$
\mathrm{D}_{\mathrm{e}, \mathrm{A}} \nabla^{2} \mathrm{C}_{\mathrm{A}}=\mathrm{r}_{\mathrm{A}}\left(\mathrm{C}_{\mathrm{A}}\right)
$$

Si la resistencia difusional es tan elevada como para que A solo alcance a penetrar una delgada capa en el interior del catalizador, su factor de efectividad tiende al valor límite (Froment y Bischoff, 1990):

$$
\eta_{\mathrm{A}, \lim }=1 / \Phi_{\mathrm{A}}
$$

donde el módulo de Thiele modificado $\Phi_{\mathrm{A}}$, asumiendo que la reacción de A sea irreversible, resulta:

$$
\Phi_{\mathrm{A}}^{2}=\ell_{e}^{2} \frac{\mathrm{r}_{\mathrm{A}}^{\mathrm{s}}\left(\mathrm{C}_{\mathrm{s}, \mathrm{A}}\right)^{2}}{\mathrm{D}_{\mathrm{e}, \mathrm{A}} 2 \int_{0}^{\mathrm{C}_{\mathrm{s}, \mathrm{A}}} \mathrm{r}_{\mathrm{A}}\left(\mathrm{C}_{\mathrm{A}}\right) \mathrm{dC}_{\mathrm{A}}}
$$

Si la expresión $r_{A}\left(C_{A}\right)$ aumenta con $C_{A}, \eta_{A}$ es una función decreciente con $\Phi_{A}$ que razonablemente se puede aproximar mediante la expresión:

$$
\eta_{\mathrm{A}}=\eta^{*}\left(\Phi_{\mathrm{A}}\right)
$$

donde “*” indica la función correspondiente al factor de efectividad para una cinética lineal $\left(\mathrm{r}_{\mathrm{A}}=\mathrm{kC}_{\mathrm{A}}\right)$, que se dispone analíticamente para formas 
convencionales de la pastilla de catalizador. En particular, para pastillas esféricas, $\eta^{*}\left(\Phi_{\mathrm{A}}\right)=\left[1 / \tanh \left(3 \Phi_{\mathrm{A}}\right)-1 / 3 \Phi_{\mathrm{A}}\right] / \Phi_{\mathrm{A}}$.

De esta manera, la velocidad efectiva de consumo $\mathrm{r}_{\mathrm{A}}^{\text {ef }}$ se evalúa como:

$$
\mathrm{r}_{\mathrm{A}}^{\mathrm{ef}}=\mathrm{r}_{\mathrm{A}}^{\mathrm{s}} \eta^{*}\left(\Phi_{\mathrm{A}}\right)
$$

Retomando los balances de conservación para el sistema en estudio, Ecs. [7 a, b, c], se puede apreciar que la Ec. [7a] para el acetato de etilo y la Ec. [7b] para el etanol tienen una estructura similar, pero la Ec. [7c] para el acetaldehído difiere, dado que el mismo es un producto intermediario. Combinando la [7b] y la [7c] es posible hacer una transformación para uniformar los tres balances. A tal fin multiplicamos la Ec. [7c] por un factor " $\Omega$ ” y le sumamos la Ec. [7b] resultando:

$\nabla^{2}\left(\mathrm{C}_{3} \Omega+\mathrm{C}_{2}\right)=\left[\mathrm{C}_{3} \Omega\left(\mathrm{k}_{\mathrm{c}} / \mathrm{D}_{\mathrm{e}, 3}\right)+\mathrm{C}_{2} \mathrm{k}_{\mathrm{b}}\left(1 / \mathrm{D}_{\mathrm{e}, 2}-\Omega / \mathrm{D}_{\mathrm{e}, 3}\right)\right] /\left(1+\mathrm{K}_{1} \mathrm{C}_{1}+\mathrm{K}_{3} \mathrm{C}_{3}\right)$

Exigimos que la relación entre los coeficientes de $\mathrm{C}_{2}$ y $\mathrm{C}_{3}$ en el primer miembro y en el término del corchete del segundo sea la misma, de donde surge,

$$
\Omega=\left(\mathrm{D}_{\mathrm{e}, 3} / \mathrm{D}_{\mathrm{e}, 2}\right)-\left(\mathrm{k}_{\mathrm{c}} / \mathrm{k}_{\mathrm{b}}\right)
$$

Sustituyendo $\Omega$ en [13] se tiene,

$$
\nabla^{2}(\mathrm{Y})=\frac{1}{D_{\mathrm{e}, 3}} \mathrm{Y} \mathrm{k}_{\mathrm{c}} /\left(1+\mathrm{K}_{1} \mathrm{C}_{1}+\mathrm{K}_{3} \mathrm{C}_{3}\right)
$$

donde:

$$
\mathrm{Y}=\mathrm{C}_{3} \Omega+\mathrm{C}_{2}
$$

La nueva variable $\mathrm{Y}$ representa la concentración de un reactivo ficticio que se consume con una velocidad equivalente a $\boldsymbol{r}_{\mathrm{c}}$ (Ec. [6c]). Se adopta como sistema de trabajo al conjunto de Ecs. [7a], [7b] y [15], representando cada una el balance para un reactivo que se consume con velocidad proporcional a su concentración y con un término de inhibición común.

De forma análoga que para una única reacción, las expresiones del factor de efectividad límite para cada uno de los reactivos del sistema [7a], [7b], [15] con elevadas restricciones difusionales, resultan:

$$
\eta_{1, \lim }=1 / \Phi_{1} ; \quad \eta_{2, \lim }=1 / \Phi_{2} ; \quad \eta_{\mathrm{Y}, \lim }=1 / \Phi_{\mathrm{Y}}
$$


donde:

$$
\begin{aligned}
& \Phi_{1}^{2}=\ell_{e}^{2} \frac{\mathrm{k}_{\mathrm{a}} \mathrm{C}_{\mathrm{s}, 1}^{2}}{\mathrm{D}_{\mathrm{e}, 1}\left(1+\mathrm{K}_{1} \mathrm{C}_{\mathrm{s}, 1}+\mathrm{K}_{3} \mathrm{C}_{\mathrm{s}, 3}\right)^{2} 2 \int_{0}^{\mathrm{C}_{\mathrm{s}, 1}} \frac{\mathrm{C}_{1}}{1+\mathrm{K}_{1} \mathrm{C}_{1}+\mathrm{K}_{3} \mathrm{C}_{3}} \mathrm{dC}_{1}} \\
& \Phi_{2}{ }^{2}=\ell_{e}^{2} \frac{\mathrm{k}_{\mathrm{b}} \mathrm{C}_{\mathrm{s}, 2}{ }^{2}}{\mathrm{D}_{\mathrm{e}, 2}\left(1+\mathrm{K}_{1} \mathrm{C}_{\mathrm{s}, 1}+\mathrm{K}_{3} \mathrm{C}_{\mathrm{s}, 3}\right)^{2} 2 \int_{0}^{\mathrm{C}_{\mathrm{s}, 2}} \frac{\mathrm{C}_{2}}{1+\mathrm{K}_{1} \mathrm{C}_{1}+\mathrm{K}_{3} \mathrm{C}_{3}} \mathrm{dC}} \\
& \Phi_{\mathrm{Y}}{ }^{2}=\ell_{e}^{2} \frac{\mathrm{k}_{\mathrm{c}} \mathrm{Y}_{\mathrm{s}}^{2}}{\mathrm{D}_{\mathrm{e}, 3}\left(1+\mathrm{K}_{1} \mathrm{C}_{\mathrm{s}, 1}+\mathrm{K}_{3} \mathrm{C}_{\mathrm{s}, 3}\right)^{2} 2 \int_{0}^{\mathrm{Y}_{\mathrm{s}}} \frac{\mathrm{Y}}{1+\mathrm{K}_{1} \mathrm{C}_{1}+\mathrm{K}_{3} \mathrm{C}_{3}} \mathrm{dY}}
\end{aligned}
$$

Para [18c], a partir de [16] se obtiene:

$$
\mathrm{Y}_{\mathrm{s}}=\mathrm{C}_{\mathrm{s}, 3} \Omega+\mathrm{C}_{\mathrm{s}, 2}=\mathrm{C}_{\mathrm{s}, 3}\left[\left(\mathrm{D}_{\mathrm{e}, 3} / \mathrm{D}_{\mathrm{e}, 2}\right)-\left(\mathrm{k}_{\mathrm{c}} / \mathrm{k}_{\mathrm{b}}\right)\right]+\mathrm{C}_{\mathrm{s}, 2}
$$

Notamos que $\mathrm{Y}_{\mathrm{s}}$, y por ende el perfil de $\mathrm{Y}$, puede adoptar valores negativos.

A fin de calcular las integrales que se presentan en las Ecs. [18], combinamos las Ec. [7a] y [7b] con la [15] resultando:

$$
\begin{aligned}
& \frac{\mathrm{D}_{\mathrm{e}, 1}}{\mathrm{k}_{\mathrm{a}}} \frac{\nabla^{2} \mathrm{C}_{1}}{\mathrm{C}_{1}}=\frac{\mathrm{D}_{\mathrm{e}, 3}}{\mathrm{k}_{\mathrm{c}}} \frac{\nabla^{2} \mathrm{Y}}{\mathrm{Y}} \\
& \frac{\mathrm{D}_{\mathrm{e}, 2}}{\mathrm{k}_{\mathrm{b}}} \frac{\nabla^{2} \mathrm{C}_{2}}{\mathrm{C}_{2}}=\frac{\mathrm{D}_{\mathrm{e}, 3}}{\mathrm{k}_{\mathrm{c}}} \frac{\nabla^{2} \mathrm{Y}}{\mathrm{Y}}
\end{aligned}
$$

Las Ecuaciones [20] son exactamente iguales que las que resultan si el sistema [7a], [7b], [15] fuese lineal, es decir, cuando el término de inhibición es unitario. En tal caso, se conoce analíticamente la solución de los balances en condiciones de fuertes efectos difusionales:

$$
\begin{aligned}
& \mathrm{C}_{1}=\mathrm{C}_{\mathrm{s}, 1} \exp \left[-\left(\mathrm{k}_{\mathrm{a}} / \mathrm{D}_{\mathrm{e}, 1}\right)^{0.5} \mathrm{z}\right] \\
& \mathrm{C}_{2}=\mathrm{C}_{\mathrm{s}, 2} \exp \left[-\left(\mathrm{k}_{\mathrm{b}} / \mathrm{D}_{\mathrm{e}, 2}\right)^{0.5} \mathrm{z}\right] \\
& \mathrm{Y}=\mathrm{Y}_{\mathrm{s}} \exp \left[-\left(\mathrm{k}_{\mathrm{c}} / \mathrm{D}_{\mathrm{e}, 3}\right)^{0.5} \mathrm{z}\right]
\end{aligned}
$$

donde $\mathrm{z}$ es la distancia desde la superficie de la pastilla catalítica.

Si en la Ecuación [21c] usamos las Ecs. [16] y [19] se obtiene, adicionalmente: 


$$
\mathrm{C}_{3} \Omega+\mathrm{C}_{2}=\left(\mathrm{C}_{\mathrm{s}, 3} \Omega+\mathrm{C}_{\mathrm{s}, 2}\right) \exp \left[-\left(\mathrm{k}_{\mathrm{c}} / \mathrm{D}_{\mathrm{e}, 3}\right)^{0.5} \mathrm{z}\right]
$$

Ejemplificamos el uso de las Ecs. [21] para calcular la integral en [18a]. Para cada valor de la variable de integración, $\mathrm{C}_{1}$, con el cual se deba calcular el subintegral, se calcula el valor $\mathrm{z}$ de [21a], y con este valor se calcula $\mathrm{C}_{2}$ de [21b] y $\mathrm{C}_{3}$ de [21d]. Intercambiando el rol de las variables, se puede proceder en forma análoga para evaluar las integrales de [18b] y [18c].

Si los módulos de Thiele $\Phi_{1}, \Phi_{2}$ y $\Phi_{\mathrm{Y}}$ resultan suficientemente elevados, las [17] permiten el cálculo de los factores de efectividad. Caso contrario, valores pequeños o intermedios de los mismos, resta la posibilidad de emplear el mismo tipo de aproximación que para una reacción única, como resulta expresado por la Ec. [11]:

$$
\eta_{1}=\eta^{*}\left(\Phi_{1}\right), \eta_{2}=\eta^{*}\left(\Phi_{2}\right), \eta_{\mathrm{Y}}=\eta^{*}\left(\Phi_{\mathrm{Y}}\right)
$$

Para la función $\eta^{*}(\Phi)$ se utilizó la expresión para geometría esférica:

$$
\eta^{*}=[1 / \tanh (3 \Phi)-1 /(3 \Phi)] / \Phi \text {. }
$$

Las Ecs. [22] suponen que el módulo de Thiele modificado es el parámetro dominante para determinar el valor de cada factor de efectividad.

Una vez calculados $\Phi_{1}, \Phi_{2}$ y $\Phi_{Y}$, calculamos $\eta_{1}, \eta_{2}$ y $\eta_{Y}$ de las Ecs. [17] o [22], según corresponda. Las velocidades efectivas para el acetato de etilo (1), el etanol (2) y el reactivo ficticio $(Y)$ serán:

$$
\begin{aligned}
& \mathrm{r}_{1}^{\mathrm{ef}}=\mathrm{r}_{1}^{\mathrm{s}} \eta_{1} \\
& \mathrm{r}_{2}^{\mathrm{ef}}=\mathrm{r}_{2}^{\mathrm{s}} \eta_{2} \\
& \mathrm{r}_{\mathrm{Y}}^{\text {ef }}=\mathrm{r}_{\mathrm{Y}}^{\mathrm{s}} \eta_{\mathrm{Y}}=\mathrm{Y}_{\mathrm{s}} \mathrm{k}_{\mathrm{c}} /\left(1+\mathrm{K}_{1} \mathrm{C}_{\mathrm{s}, 1}+\mathrm{K}_{3} \mathrm{C}_{\mathrm{s}, 3}\right) \eta_{\mathrm{Y}}
\end{aligned}
$$

Para evaluar $\mathrm{r}_{3}^{\text {ef }}$, es decir la velocidad neta de consumo de acetaldehído, notamos que [16] nos permite calcular su flujo en la superficie externa en función de los correspondientes flujos de etanol y del reactivo ficticio. Surge que

$$
\mathrm{r}_{3}^{\mathrm{ef}}=\left[\mathrm{r}_{\mathrm{Y}}^{\mathrm{ef}}-\left(\frac{\mathrm{D}_{\mathrm{e}, 3}}{D_{\mathrm{e}, 2}}\right) \mathrm{r}_{2}^{\mathrm{ef}}\right] \frac{1}{\Omega}
$$


El procedimiento reseñado no requiere ninguna iteración para calcular los $r_{j}^{\text {ef }}$, supuesto se especifican los valores $\mathrm{C}_{\mathrm{s}, \mathrm{j}}$.

Debe señalarse que el uso de las aproximaciones [22] requiere que la velocidad de consumo en cada uno de los balances aquí considerados, Ecs. [7a], [7b] y [15], decrezca desde la superficie externa del catalizador hacia su interior. Esta condición no resulta fácil de comprobar, pero puede exigirse, con elevada probabilidad de suficiencia, que las velocidades sean al menos decrecientes sobre la superficie externa. A tal fin debe comprobarse para las $\boldsymbol{r}_{\alpha}$ (Ecs. [6]), $\alpha=a, b, c$ :

$$
-\left.\frac{1}{\ell_{e}} \frac{\mathrm{d} \boldsymbol{r}_{\alpha}}{\mathrm{dz}}\right|_{\mathrm{z}=0}=\sum_{\mathrm{j}=1}^{3}\left(\partial \boldsymbol{r}_{\alpha} / \partial \mathrm{C}_{\mathrm{j}}\right)^{\mathrm{s}}\left(\mathrm{r}_{\mathrm{j}}^{\mathrm{ef}} / \mathrm{D}_{\mathrm{e}, \mathrm{j}}\right)>0
$$

Los valores $\mathrm{r}_{\mathrm{j}}^{\text {ef }}$ se evalúan de las Ecs. [23 a,b] y [24] y si [25] se verifica, pueden aceptarse como válidos.

Se debe destacar que para la evaluación de las velocidades efectivas de consumo planteada en esta sección, se adoptaron dos aproximaciones. La primera se desprende de las Ecs. [20], a partir de las cuales se supone al sistema de reacciones como lineal, permitiendo emplear las Ecs. [21]. La segunda está ligada a la determinación de los factores de efectividad mediante las expresiones [22] correspondientes al factor de efectividad para una única reacción de primer orden.

También debe mencionarse que existen ciertas singularidades que se deben tener en cuenta a la hora de utilizar la aproximación que se acaba de describir. La primera es cuando $\mathrm{Y}_{\mathrm{s}}$, Ec. [19], es cero. Resulta en este caso $\mathrm{Y}=0$ (es decir, se cumple que $\mathrm{C}_{3}=-\mathrm{C}_{2} / \Omega$ ), en toda la pastilla y consecuentemente $r_{\mathrm{Y}}^{\mathrm{ef}}=0$. Sin embargo, no debe llevarse a cabo el cálculo de $\Phi_{\mathrm{Y}}$, dado que la Ec. [18c] se indetermina. En este caso, $\mathrm{r}_{3}^{\text {ef }}$ es directamente evaluada a partir de la Ec. [24] tomado $\mathrm{r}_{\mathrm{Y}}^{\mathrm{ef}}=0$.

La segunda singularidad surge cuando $\Omega=0$. Según la Ec. [14], esto implica que $\mathrm{k}_{\mathrm{c}} / \mathrm{D}_{3}=\mathrm{k}_{\mathrm{b}} / \mathrm{D}_{2}$. Luego, de la Ec. [16] resulta $\mathrm{Y}=\mathrm{C}_{2}$ y la evaluación de $\mathrm{C}_{3}$ se indetermina. En la práctica existe una baja probabilidad de que $\Omega$ tome exactamente un valor nulo, sin embargo, valores muy pequeños de $\Omega$ causan inconvenientes debido a errores de redondeo. Para evitar este problema, se puede 
fijar un intervalo convenientemente pequeño de $\Omega$ alrededor de cero: $-\wp\left(D_{3} / D_{2}\right)<\Omega<\wp\left(D_{3} / D_{2}\right)$, donde $\wp$ es un número pequeño, pero suficientemente grande para evitar errores de redondeo (en la práctica se adoptó un valor de $\wp=10^{-4}$ con 16 cifras significativas). Si el valor real de $\Omega$ pertenece al intervalo indicado, se llevan a cabo dos grupos de cálculos para la obtención de las $\mathrm{r}_{\mathrm{j}}^{\mathrm{ef}}$ adoptando como valores límites de $\mathrm{k}_{\mathrm{c}}$ (ver Ec. [15]): $\mathrm{k}_{\mathrm{c}, \mathrm{L}}=\mathrm{k}_{\mathrm{b}}(1-\wp)\left(\mathrm{D}_{3} / \mathrm{D}_{2}\right)$ y $\mathrm{k}_{\mathrm{c}, \mathrm{U}}=\mathrm{k}_{\mathrm{b}}(1+\wp)\left(\mathrm{D}_{3} / \mathrm{D}_{2}\right)$, i.e. con los valores de $\Omega$ correspondientes a los valores extremos del intervalo. Luego, los resultados se interpolan linealmente para obtener los valores reales de $r_{j}^{e f}$.

Vale la pena mencionar que el procedimiento puede ser extendido a otros problemas generales reducidos a un conjunto de reacciones paralelas de la forma:

$$
\mathrm{D}_{\mathrm{e}, \mathrm{j}} \nabla^{2} \mathrm{C}_{\mathrm{j}}=\Re\left(\mathrm{C}_{\mathrm{j}}\right) / \mathrm{DEN},
$$

donde DEN puede depender de todas las concentraciones de las especies involucradas $\left\{\mathrm{C}_{\mathrm{j}}\right\}$, pero resulta un término común para todas las especies, y $\{\mathfrak{R}\}$ es un conjunto de funciones positivas que representan la fuerza impulsora para la reacción de cada una de las especies. Para el caso presentado en este capítulo resulta: $\mathfrak{R}\left(\mathrm{C}_{\mathrm{j}}\right)=\mathrm{k}_{\mathrm{j}} \mathrm{C}_{\mathrm{j}}$. La forma que adopta cualquiera de los perfiles equivalente a las Ecs. [21] dependerá de las funciones específicas $\mathfrak{R}$.

\subsection{Resultados y discusión}

\subsubsection{Comparación de la aproximación con el procedimiento numérico}

En primer lugar, se presentará la convalidación de la aproximación propuesta en la sección anterior para la evaluación de las velocidades efectivas de consumo.

En la Figura 1 se realiza la comparación entre los factores de efectividad calculados con el procedimiento numérico descrito en Bressa y col. (2001) (identificado como ETA en la Fig. 1) y los obtenidos con la aproximación anteriormente detallada. Esta comparación se realiza para las tres sustancias consideradas (acetato de etilo, etanol y compuesto ficticio $\mathrm{Y}$ ), para $\mathrm{T}_{\mathrm{g}}^{0}=180{ }^{\circ} \mathrm{C} \mathrm{y}$ $\mathrm{W}=6.36 \mathrm{~kg} / \mathrm{s}$. Se demuestra que la aproximación resulta razonablemente precisa para cualquiera de las tres especies. El rango de temperatura que abarcan los 
resultados en la Fig. 1 es $180-290^{\circ} \mathrm{C}$, virtualmente cubre todas las temperaturas de interés práctico.

Asimismo, la Fig. 1 permite apreciar los bajos valores resultantes de los factores de efectividad y su disminución a lo largo del reactor, como consecuencia del aumento de la temperatura.

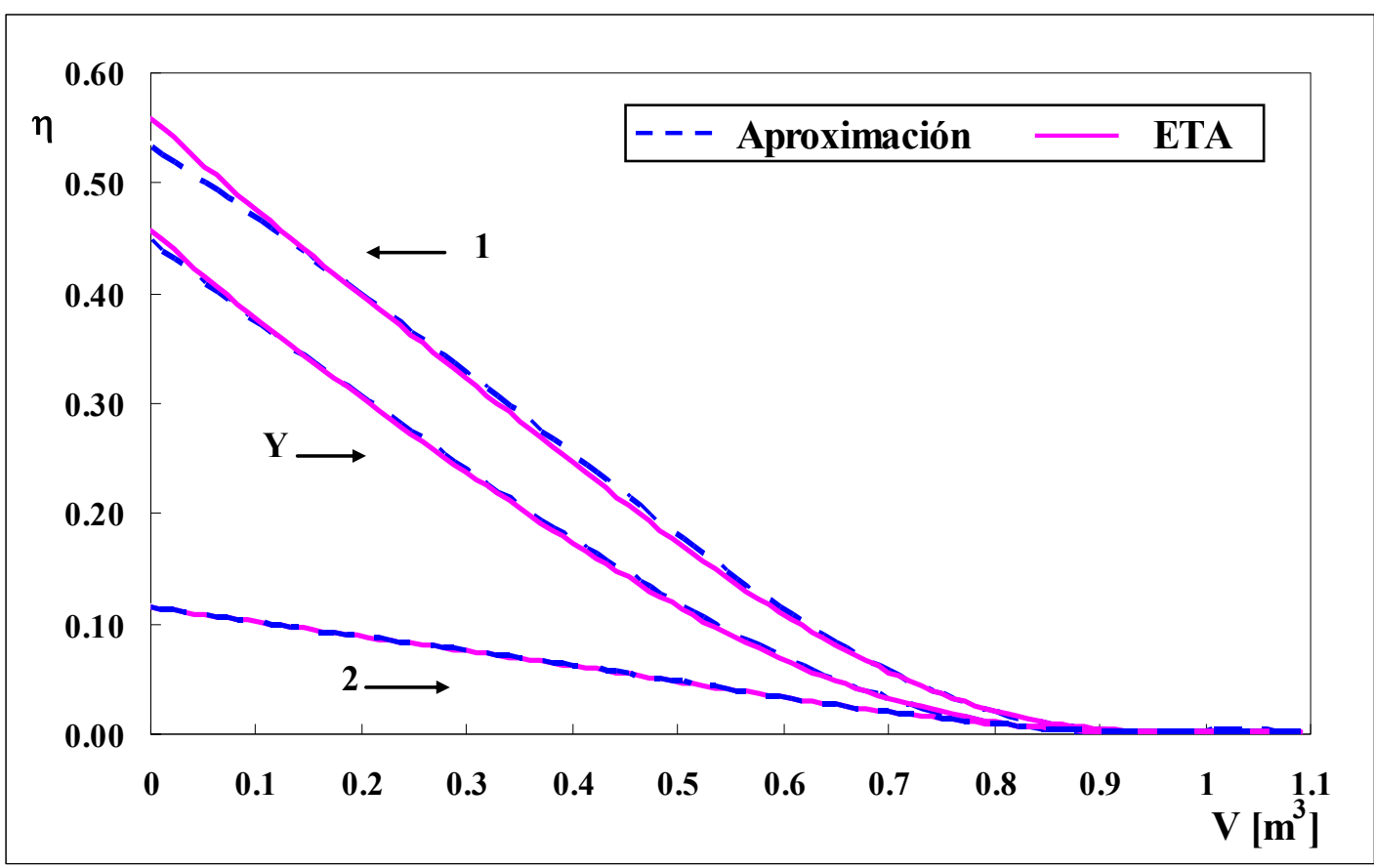

Fig. 1 - Variación de $\eta_{\mathrm{j}}$ a lo largo del volumen de reactor para $\mathrm{W}=6.36 \mathrm{~kg} / \mathrm{s} \mathrm{y} \mathrm{T}_{\mathrm{g}}^{0}=180{ }^{\circ} \mathrm{C}$ (1: Acetato de Etilo - 2: Etanol - Y: compuesto ficticio).

Resulta interesante mencionar que las diferencias más grandes, a pesar de seguir siendo tolerables, surgieron para los factores de efectividad más altos, particularmente para el acetato de etilo en la zona más próxima a la entrada del lecho. Para factores de efectividad bajos, la aproximación se comporta de manera muy precisa (i.e. para la mayoría de los datos en la Fig. 1). Los errores más elevados pueden asociarse al uso de la aproximación [22] más que a la propia evaluación del módulo de Thiele, por lo que los perfiles representados por las Ec. [21] se comportan de manera muy satisfactoria.

Es importante resaltar que la aproximación permitió llevar a cabo todos los cálculos necesarios para el presente estudio sin encontrar problemas numéricos en el curso de la resolución. Por otra parte, el uso de la misma disminuye el tiempo de simulación en más de un orden de magnitud, hecho que facilitará acciones futuras tendientes a optimizar condiciones operativas y variables de diseño del sistema. 


\subsubsection{Análisis del comportamiento del reactor}

En la Figuras $2 \mathrm{a}$ y $2 \mathrm{~b}$ se presentan perfiles típicos de temperatura en la corriente y en la superficie externa del catalizador para distintos caudales de la corriente a tratar. Teniendo en cuenta el esquema del sistema combinado de eliminación de VOCs, presentado en el Capítulo 2 (Fig. 3), se observa que la corriente tratada en el reactor catalítico proviene de la zona donde se lleva a cabo la desorción. Esto implica que cuanto menor sea el caudal de la corriente empleada para la regeneración, mayor es su concentración en VOCs, lo cual favorece al proceso de combustión. De las Figs. 2a y 2 b, se aprecia claramente que a medida que aumenta la concentración de la corriente a tratar, las resistencias externas a la transferencia de calor comienzan a tomar mayor importancia.

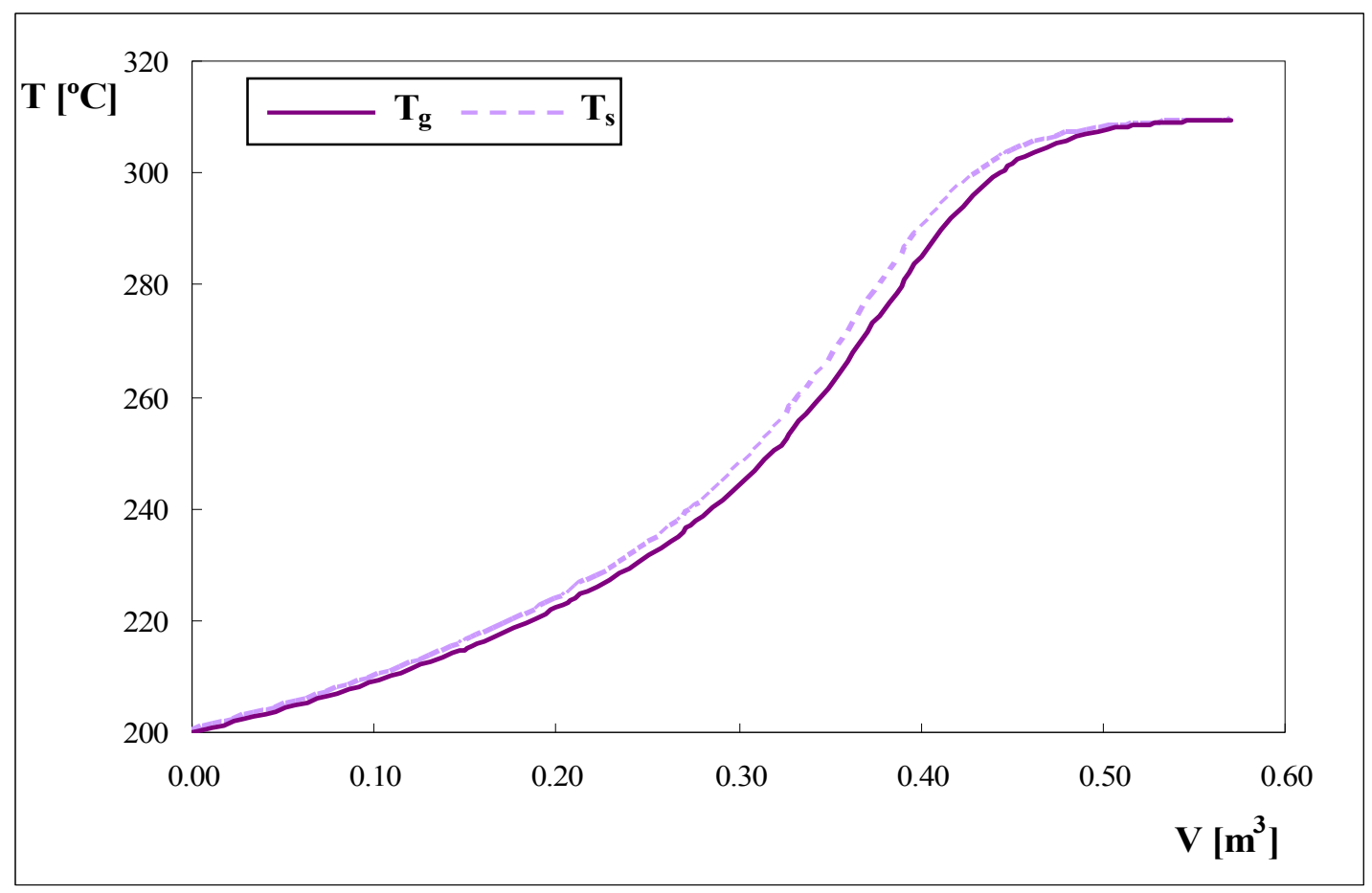

Fig. 2a - Perfiles de temperatura en el reactor para $\mathrm{W}=6.36 \mathrm{~kg} / \mathrm{s} \quad\left(\mathrm{T}_{\mathrm{g}}^{0}=200{ }^{\circ} \mathrm{C}\right.$, $\mathrm{C}_{\mathrm{g}, 1}^{0}=4.1410^{-2} \mathrm{~mol} / \mathrm{m}^{3}, \mathrm{C}_{\mathrm{g}, 2}^{0}=1.7110^{-2} \mathrm{~mol} / \mathrm{m}^{3}, \mathrm{C}_{\mathrm{g}, 3}^{0}=0 \mathrm{~mol} / \mathrm{m}^{3}$ ) 


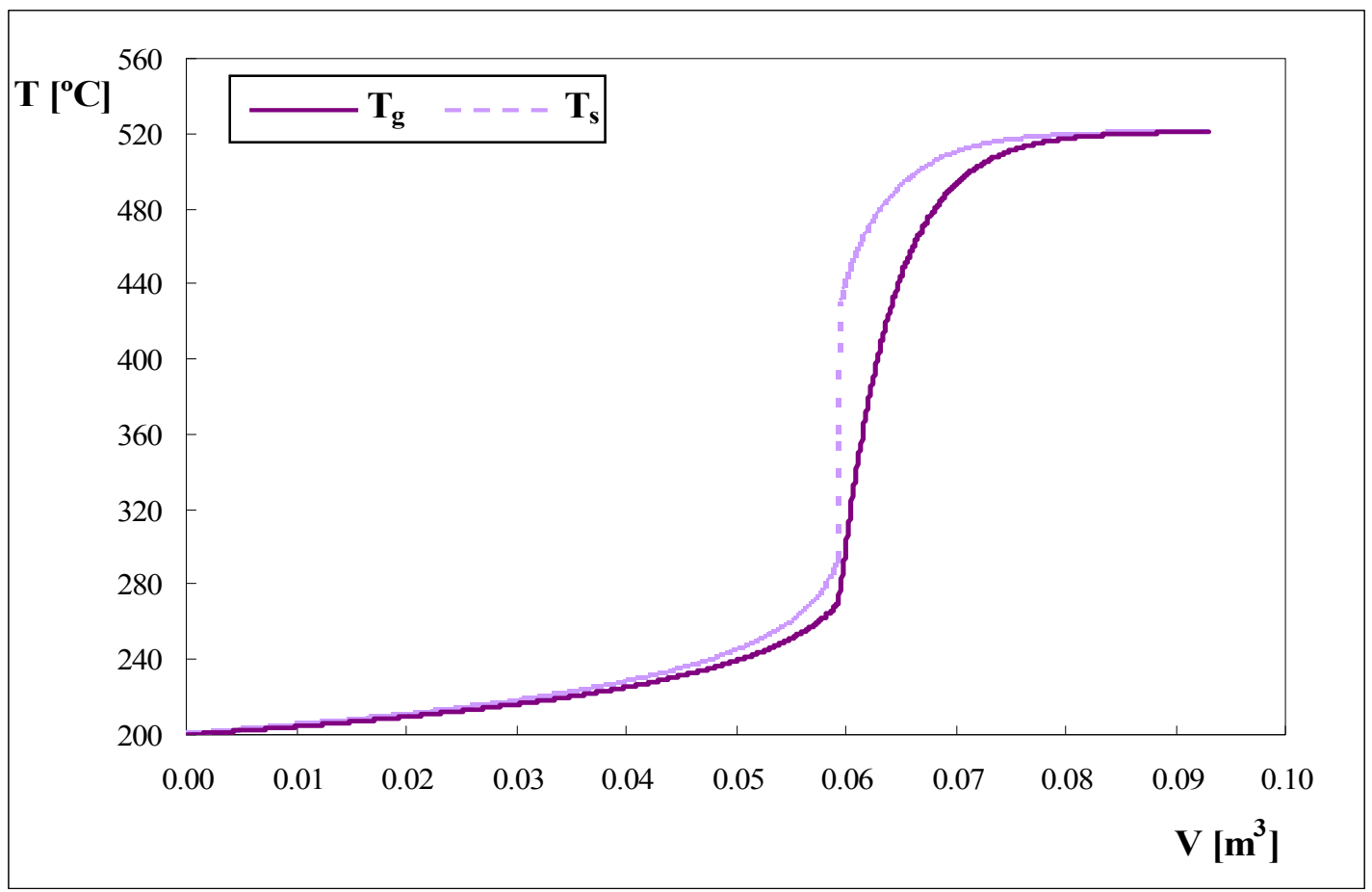

Fig. 2b - Perfiles de temperatura en el reactor para $\mathrm{W}=2.16 \mathrm{~kg} / \mathrm{s}\left(\mathrm{T}_{\mathrm{g}}^{0}=200{ }^{\circ} \mathrm{C}\right.$, $\mathrm{C}_{\mathrm{g}, 1}^{0}=1.2210^{-1} \mathrm{~mol} / \mathrm{m}^{3}, \mathrm{C}_{\mathrm{g}, 2}^{0}=5.0310^{-2} \mathrm{~mol} / \mathrm{m}^{3}, \mathrm{C}_{\mathrm{g}, 3}^{0}=0 \mathrm{~mol} / \mathrm{m}^{3}$ )

Una conclusión análoga puede obtenerse respecto a las resistencias externas a la transferencia de masa, como resulta de la inspección de las Figura 3a y 3b, en donde se presentan perfiles de concentración molar de los VOCs en la corriente de fluido y sobre la superficie externa del catalizador. 


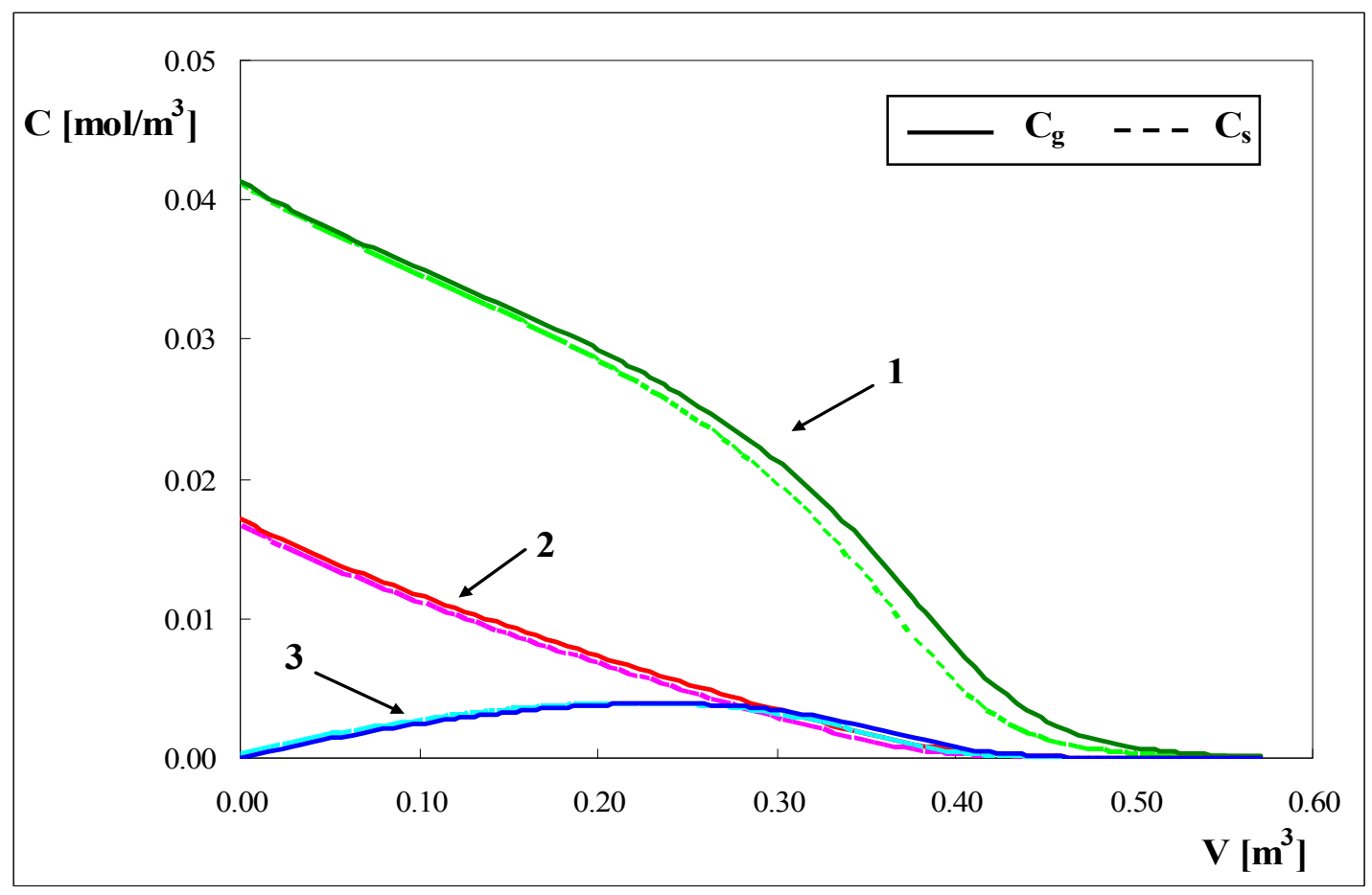

Fig. 3a - Perfiles de composición en el reactor para $\mathrm{W}=6.36 \mathrm{~kg} / \mathrm{s}$. (1: Acetato de Etilo 2: Etanol-3: Acetaldehído) $\left(\mathrm{T}_{\mathrm{g}}^{0}=200^{\circ} \mathrm{C}\right)$.

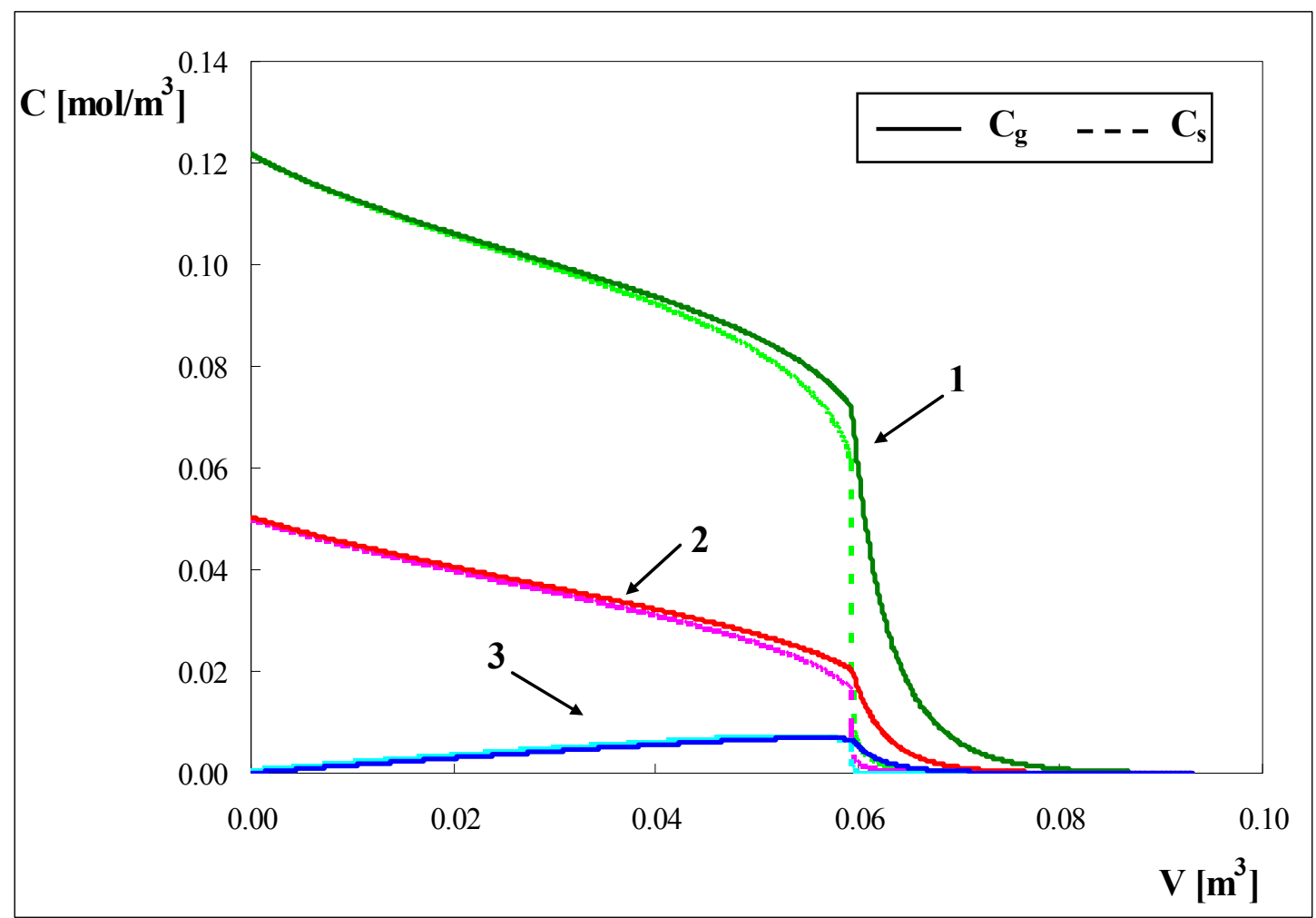

Fig. $3 \mathbf{b}$ - Perfiles de composición en el reactor para $\mathrm{W}=2.16 \mathrm{~kg} / \mathrm{s}$. (1: Acetato de Etilo 2: Etanol-3: Acetaldehído) $\left(\mathrm{T}_{\mathrm{g}}^{0}=200^{\circ} \mathrm{C}\right)$.

En la Fig. 3b se puede destacar que, a medida que el consumo del acetato de etilo se acelera bruscamente, debido al aumento de la temperatura (Fig. 2b), la 
concentración en la superficie cae de manera significativa. Este efecto, tan pronunciado para corrientes más concentradas, no permitió que la subrutina DDASAC funcionara correctamente, impidiendo completar la simulación del reactor. Para estos casos, en donde los perfiles de temperatura y concentración presentan cambios tan abruptos, se reemplazó la subrutina DDASAC por un sistema de tanques agitados en serie, lo que permitió llevar a cabo la simulación completa del reactor. Esta alternativa de cálculo, igual que para el adsorbedor (Capítulo 5), supone que el lecho puede dividirse en una serie de $\mathrm{N}_{t}$ tanques de igual tamaño, $\Delta \mathrm{V}$, con composición uniforme en cada tanque. De esta forma resultan 9 ecuaciones con 9 incógnitas a determinar en cada tanque (temperatura y concentración de cada una de las especies en la corriente de fluido, temperatura y concentración de cada una de las especies en la superficie del catalizador y la presión local). El sistema de ecuaciones queda definido por las Ecs. [26], [27] y [28] presentadas a continuación, más las Ecs. [3] y [4].

$$
\begin{aligned}
& \frac{\left(\mathrm{F}_{\mathrm{j}}^{\mathrm{i}}-\mathrm{F}_{\mathrm{j}}^{\mathrm{i}-1}\right)}{\Delta \mathrm{V}}+\left(1-\varepsilon_{\mathrm{L}}\right) \mathrm{r}_{\mathrm{j}}^{\mathrm{ef}}=0, \quad \mathrm{j}=1,2,3, \quad \mathrm{i}=1, \ldots, \mathrm{N}_{\mathrm{t}} \\
& \frac{\left(\mathrm{T}_{\mathrm{g}}^{\mathrm{i}}-\mathrm{T}_{\mathrm{g}}^{\mathrm{i}-1}\right)}{\Delta \mathrm{V}}-\frac{\left(1-\varepsilon_{\mathrm{L}}\right)}{\mathrm{W} \mathrm{\hat {c } _ { \mathrm { p } , \mathrm { g } }}}\left[\left(-\Delta \mathrm{H}_{1}\right) \mathrm{r}_{1}^{\mathrm{ef}}+\left[\left(-\Delta \mathrm{H}_{2}\right)-\left(-\Delta \mathrm{H}_{3}\right)\right] \mathrm{r}_{2}^{\mathrm{ef}}+\left(-\Delta \mathrm{H}_{3}\right) \mathrm{r}_{3}^{\mathrm{ef}}\right]=0, \quad \mathrm{i}=1, \ldots, \mathrm{N}_{\mathrm{t}}
\end{aligned}
$$

$$
\underset{i=1, \ldots, N_{t}}{\Delta V}+\left\{\left[150 \frac{\left(1-\varepsilon_{L}\right)^{2}}{\varepsilon_{L}^{3}} \frac{\mu_{g}}{\rho_{g}} \frac{1}{d_{p}} \frac{\mathrm{W}}{2 \pi \mathrm{H}}\right] \frac{1}{\rho^{2}}+\left[1.75 \frac{\left(1-\varepsilon_{\mathrm{L}}\right)}{\varepsilon_{\mathrm{L}}{ }^{3}} \frac{1}{\rho_{\mathrm{g}}} \frac{1}{\mathrm{~d}_{\mathrm{p}}}\left(\frac{\mathrm{W}}{2 \pi \mathrm{H}}\right)^{2}\right] \frac{1}{\rho^{3}}\right\} \frac{1}{2 \pi \mathrm{H}}=0
$$

Vale la pena mencionar que la aproximación presentada previamente para las velocidades efectivas de reacción no causó ningún tipo de inconveniente al operar en conjunto con el sistema de tanques en serie.

En las Figs. 3a y 3 b también se observa que la combustión del acetato de etilo resulta ser la reacción clave para determinar el tamaño del reactor catalítico. Sin embargo, la presencia de etanol causa dos efectos en la velocidad de consumo del acetato de etilo. Por un lado, el acetaldehído formado a partir del etanol inhibe, hasta cierto grado, la combustión del acetato de etilo a través del denominador en las expresiones de las velocidades de reacción. Por otro lado, y de mayor importancia, es que las reacciones de etanol y acetaldehído contribuyen al incremento de la 
temperatura, lo que ayuda a la aceleración de la velocidad de reacción del acetato de etilo.

\subsection{Conclusiones}

Se propuso un reactor de lecho fijo con catalizador granular de forma esférica; el sistema adoptado es de flujo radial, lo que permitió reducir sustancialmente la pérdida de carga. Con la misma finalidad, se adoptó un tamaño de catalizador con $\mathrm{d}_{\mathrm{p}}=3 \mathrm{~mm}$.

El modelo del reactor se planteó bajo las siguientes hipótesis: reactor adiabático, flujo pistón, gas ideal y pastilla catalítica isotérmica. Se tuvieron en cuenta las resistencias a la transferencia de calor y materia entre el seno de la corriente y la superficie de las partículas catalíticas y la resistencia a la transferencia de materia en el interior del catalizador. Esta última resultó ser muy elevada debido al tamaño de pastilla adoptado.

Se debieron resolver numéricamente los balances de conservación de las tres especies en el interior de las pastillas en cada posición a lo largo del lecho, a fin de cuantificar las velocidades efectivas de consumo. Tal procedimiento resulta acoplado con el de resolución de los balances en la corriente de gas. Para ambos problemas, interno y en la corriente de gas, se emplearon rutinas de uso general. Se evidenció en numerosos casos que el procedimiento numérico global se detenía, impidiendo completar la simulación. Este efecto fue provocado por la elevada sensibilidad de las reacciones con la temperatura (elevadas energías de activación) que provocan el encendido del sistema. La situación física incide en el procedimiento de resolución al grado de producir la detención de la simulación, aún cuando los parámetros de control de ambas rutinas fueran manipulados para evitarla.

La alternativa para solucionar el problema fue emplear una aproximación explícita para las velocidades efectivas de las tres reacciones principales que tienen lugar en la combustión, que evidenció ser altamente precisa y eficiente, dado que permitió eliminar los problemas numéricos enunciados, como así también disminuir el tiempo de cálculo en más de un orden de magnitud. Cabe destacar que la aproximación desarrollada puede aplicarse para una familia de problemas cinéticos similares.

A medida que el consumo del acetato de etilo se acelera debido al aumento de la temperatura (Fig. 3b), la concentración en la superficie cae de manera significativa. Este efecto, muy pronunciado para las corrientes más concentradas, no permitió que la 
subrutina DDASAC funcionara correctamente, impidiendo completar la simulación del reactor. Para estos casos, en donde los perfiles de temperatura y concentración presentan cambios tan abruptos, se reemplazó la subrutina DDASAC por un sistema de tanques agitados en serie, lo que permitió llevar a cabo la simulación completa del reactor. 

ESTUDIO DE SISTEMAS

COMBINADOS DE

COMBUSTION

CATALITICA DE VOCs

\section{9/ SISTEMA DE RECUPERACION TERMICA}

Selección del sistema de intercambio térmico

Características geométricas y modelo de cálculo de un intercambiador de flujo cruzado de tipo "plate fin" (con ambos fluidos unmixed)

Definición de las variables geométricas

Conclusiones 



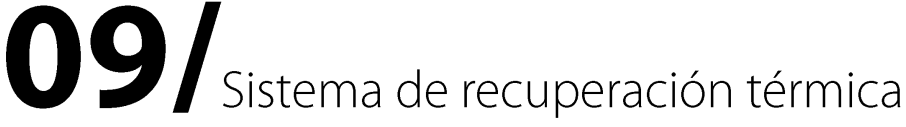

El esquema del sistema de eliminación de VOCs propuesto en el Capítulo 2 (Fig. 3), incluye dos etapas de recuperación del calor generado por la reacción. Considerando la mejor opción en cuanto al aprovechamiento energético, surge el esquema presentado en la Figura 1.

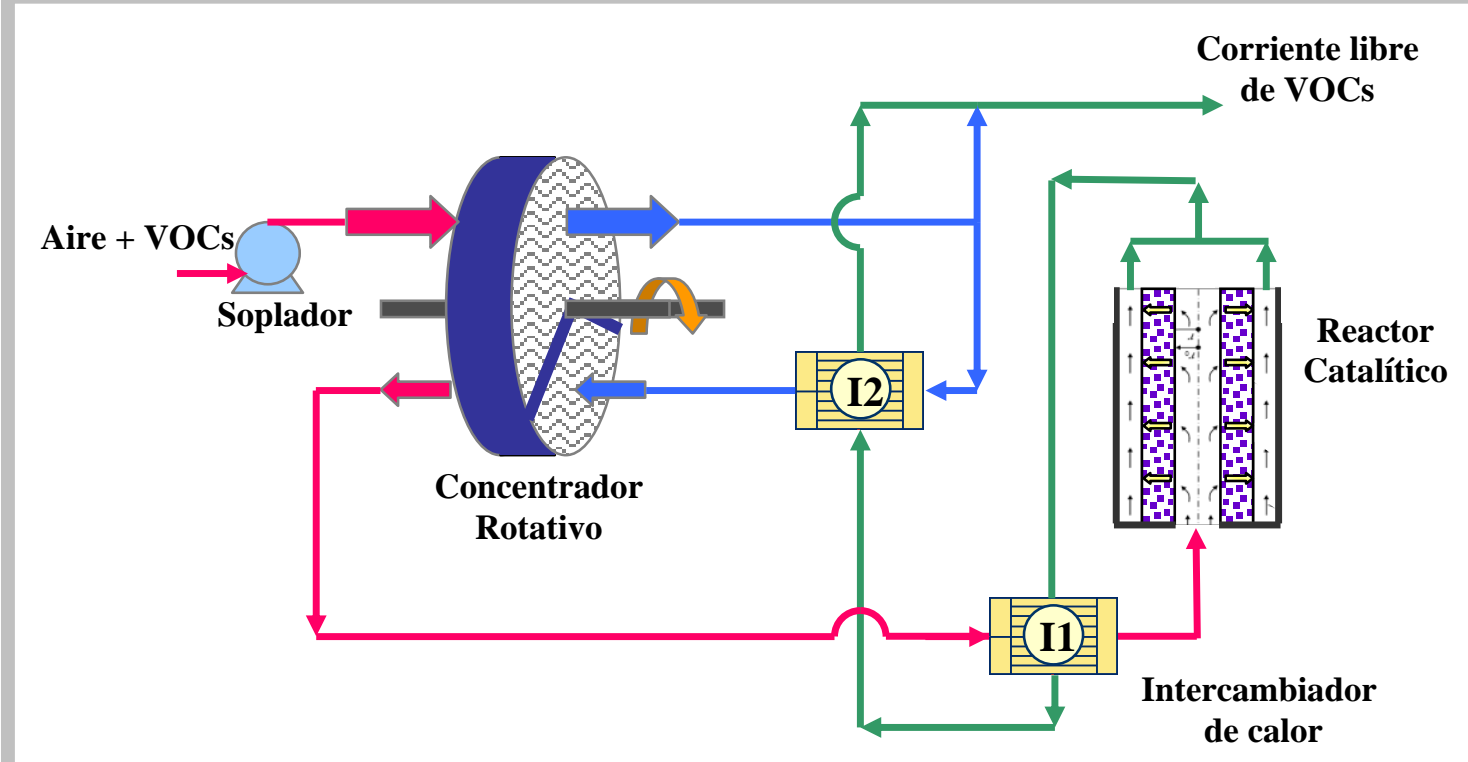

Fig. 1 - Sistema combinado: combustión catalítica de VOCs con concentración de los mismos en un sistema de adsorción/desorción.

Uno de los intercambiadores permite elevar la temperatura de la corriente previo a su ingreso al reactor (I1 en Fig. 1). El segundo intercambiador permite el precalentamiento del aire utilizado para la regeneración del adsorbedor (I2 en Fig. 1).

En este capítulo se propone el sistema de intercambio térmico a utilizar a partir de analizar las características que presentan las corrientes a tratar. En base al tipo de intercambiador gas - gas seleccionado, se describen las características geométricas del mismo y se presenta un modelo de cálculo para llevar a cabo su simulación. 


\subsection{Selección del sistema de intercambio térmico}

Los intercambiadores pueden ser de tipo recuperativo o regenerativo. Estos últimos pueden ser potencialmente más eficientes que los primeros, sin embargo, presentan una mayor complejidad operativa.

Los sistemas regenerativos pueden operar de una manera similar al concentrador rotativo (Capítulo 3) o mediante un sistema de dos lechos con inversión de flujo. Con el primero se corre el riesgo de que las corrientes de intercambio tomen contacto entre sí, pudiéndose contaminar la corriente limpia que sale del reactor. Por otro lado, mediante el empleo de dos lechos, no es posible obtener uniformidad de la concentración y, esencialmente, de la temperatura de la corriente a la salida del mismo, lo cual constituye un aspecto de importancia para la operación del reactor en estado estacionario.

Otra alternativa consiste en emplear lechos catalíticos con regeneración térmica por inversión de flujo. Estos procesos constituyen una alternativa de interés (Matros y Bunimovich, 1995), pero con un tipo de operación marcadamente diferente al planteado para este trabajo, por lo que su estudio no será considerado en la continuidad de la Tesis.

La opción más sencilla, desde el punto de vista operativo, es un intercambiador de tipo recuperativo. Esto garantizará que no se pongan en contacto en ningún momento las corrientes, así como la uniformidad de la concentración y temperatura de la corriente a la salida del mismo. Dentro de los intercambiadores de tipo recuperativo, existe una amplia variedad de alternativas entre las cuales se puede elegir el equipo a utilizar (Hewitt y col., 1994). Vale la pena mencionar que para ambos sistemas de recuperación presentes en el esquema de la Fig. 1, el análisis y el tipo de intercambiador seleccionado son similares.

Resulta pertinente destacar algunas características de las corrientes a tratar, y orientar el análisis de las mismas hacia la selección de alternativas de intercambiadores de calor:

$\Rightarrow$ Tanto la corriente fría como la caliente son, esencialmente, la misma corriente, con una diferencia de temperatura.

$\Rightarrow$ Tanto la corriente fría como la caliente son gaseosas. Esta situación normalmente conducirá a coeficientes peliculares de transferencia de calor bajos o muy bajos.

$\Rightarrow$ Los caudales másicos de las corrientes a tratar son prácticamente iguales. Esta situación, junto con el punto anterior, conduce a que sean esperables coeficientes 
peliculares no muy diferentes (en el orden de magnitud) y bajos; por lo tanto la posibilidad de emplear superficies extendidas debería contemplarse como una alternativa de interés para ambas corrientes.

$\Rightarrow$ Los caudales de las corrientes a tratar pueden ser relativamente altos, según el grado de concentración de la corriente (los caudales más altos corresponden al caso en el cual no se emplea el preconcentrador). Esta situación normalmente conducirá a pérdidas de carga importantes en el equipo.

$\Rightarrow$ La presión de trabajo es baja. Esta condición permite ampliar el espectro de alternativas, ya que no limita la elección de, prácticamente, ningún tipo de intercambiador

$\Rightarrow$ Las temperaturas de trabajo pueden ser relativamente altas. Esta variable representa un condicionamiento significativo en la elección del tipo de intercambiador y, además, en el material a emplear en el mismo.

$\Rightarrow$ Las corrientes no presentan problemas de toxicidad o problemas de corrosión significativos. Los VOCs a tratar, en los niveles considerados, no representan un problema crucial en caso de un escape.

$\Rightarrow$ El ensuciamiento no debería ser un problema significativo. Normalmente las resistencias de ensuciamiento de las corrientes de aire son bajas (normas TEMA) y, al compararse con los bajos valores relativos de los coeficientes peliculares que se obtienen al tratar una corriente gaseosa, no tendrán un peso significativo.

Analizando las características mencionadas previamente y teniendo en cuenta la fracción de energía a recuperar, surgieron diferentes diseños de intercambiadores (Campesi, 2005). Considerando la similitud del caso planteado en este trabajo con el presentado por Campesi (2005), resultó adecuado seguir el mismo tipo de análisis para la selección de un tipo de intercambiador.

El empleo del método $\varepsilon$-NTU (el cual permite obtener la efectividad del tipo de intercambiador de calor a partir del Número de Unidades de Transferencia), permitió realizar un rápido escrutinio dentro de las distintas alternativas de intercambiadores y eventualmente rechazar algunas. La condición recomendada (Hewitt y col, 1994) para un diseño confiable es que el valor operativo de $\varepsilon$, i.e. la eficiencia del intercambiador, sea menor o igual a 0.95 de la eficiencia máxima $\varepsilon_{\text {máx }}$, la cual se obtiene para NTU tendiendo a infinito. Bajo estas condiciones, Campesi (2005) retuvo diferentes diseños de intercambiadores que permitiesen minimizar la pérdida de carga. Según el análisis 
realizado, los intercambiadores de flujo cruzado, tipo "plate-fin", aparecen como la mejor alternativa por la baja pérdida de carga $\mathrm{y}$, dado que son altamente compactos, su pequeño volumen. Tomando como referencia este análisis se consideró, para nuestro caso particular de estudio, un intercambiador de flujo cruzado, tipo "plate-fin", con ambos fluidos "unmixed" para enfrentar el diseño.

\subsection{Características geométricas y modelo de cálculo de un intercambiador de flujo cruzado tipo "plate-fin" (con ambos fluidos "unmixed")}

El intercambiador de flujo cruzado, con superficies extendidas ("plate-fin heat exchanger") es una alternativa de interés para ser empleado en sistemas de recuperación energética (Hewitt y col., 1994; Ogulata y col., 1999; Picón-Nuñez y col., 1999). Este tipo de intercambiador compacto consiste en un conjunto de placas planas, entre las cuales se colocan placas corrugadas, que constituyen una superficie o superficies extendidas, como se muestra en la Figura 2.

Ambos tipos de placas están soldadas, constituyendo de esa forma, el intercambiador, un bloque único. De esta manera, se alcanza un área de transferencia por unidad de volumen de equipo elevada. Los espacios entre placas permiten el paso de fluido en forma alternada en cada uno de los sentidos de flujo, constituyendo el flujo cruzado, como se aprecia en la Figura 3.

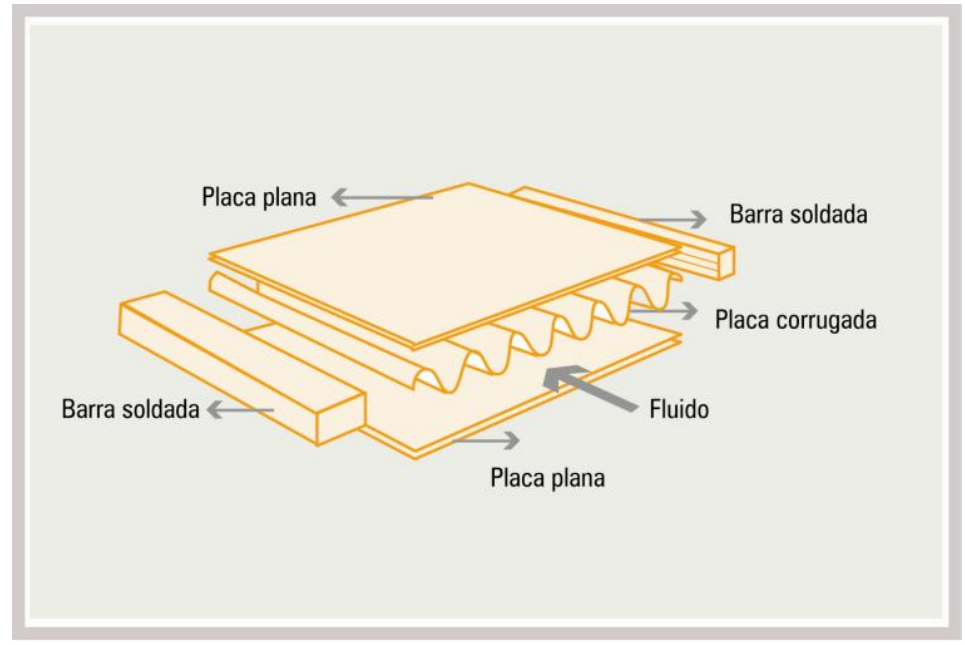

Fig. 2 - Intercambiador de flujo cruzado 1-1 (ambos fluidos unmixed)

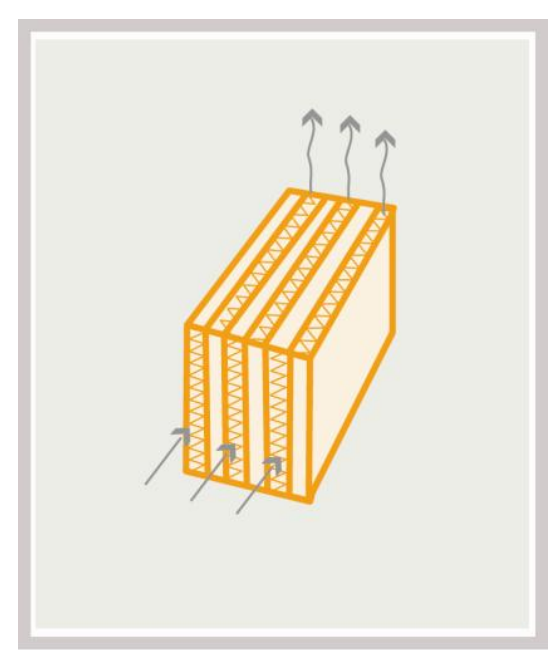

Fig. 3 - Arreglo: Flujo Cruzado

Originalmente fueron desarrollados para sistemas que requerían bajo peso y volumen, construyéndose en aluminio, lo que representa una limitación en la temperatura. Posteriormente, se construyeron en diferentes tipos de aceros, lo que les 
permite trabajar hasta temperaturas de $800{ }^{\circ} \mathrm{C}$. No son adecuados para sistemas a altas presiones o con fluidos que tiendan a generar un ensuciamiento importante (Hewitt y col., 1994), pero no es el caso del sistema en estudio.

Existen numerosas alternativas de superficies extendidas, tanto por la forma que tiene la sección transversal de los canales que se forman, como por la posibilidad de generar canales alternados en la dirección de flujo o superficies con protuberancias a efectos de incrementar la turbulencia (Shah y Mueller, 1985). Dado que la selección del tipo de superficie extendida constituye un tópico específico, que excede la extensión de este trabajo, se decidió emplear un único tipo de superficie extendida, consistente en canales rectangulares, con dimensiones definidas como se indica en la Figura 4.

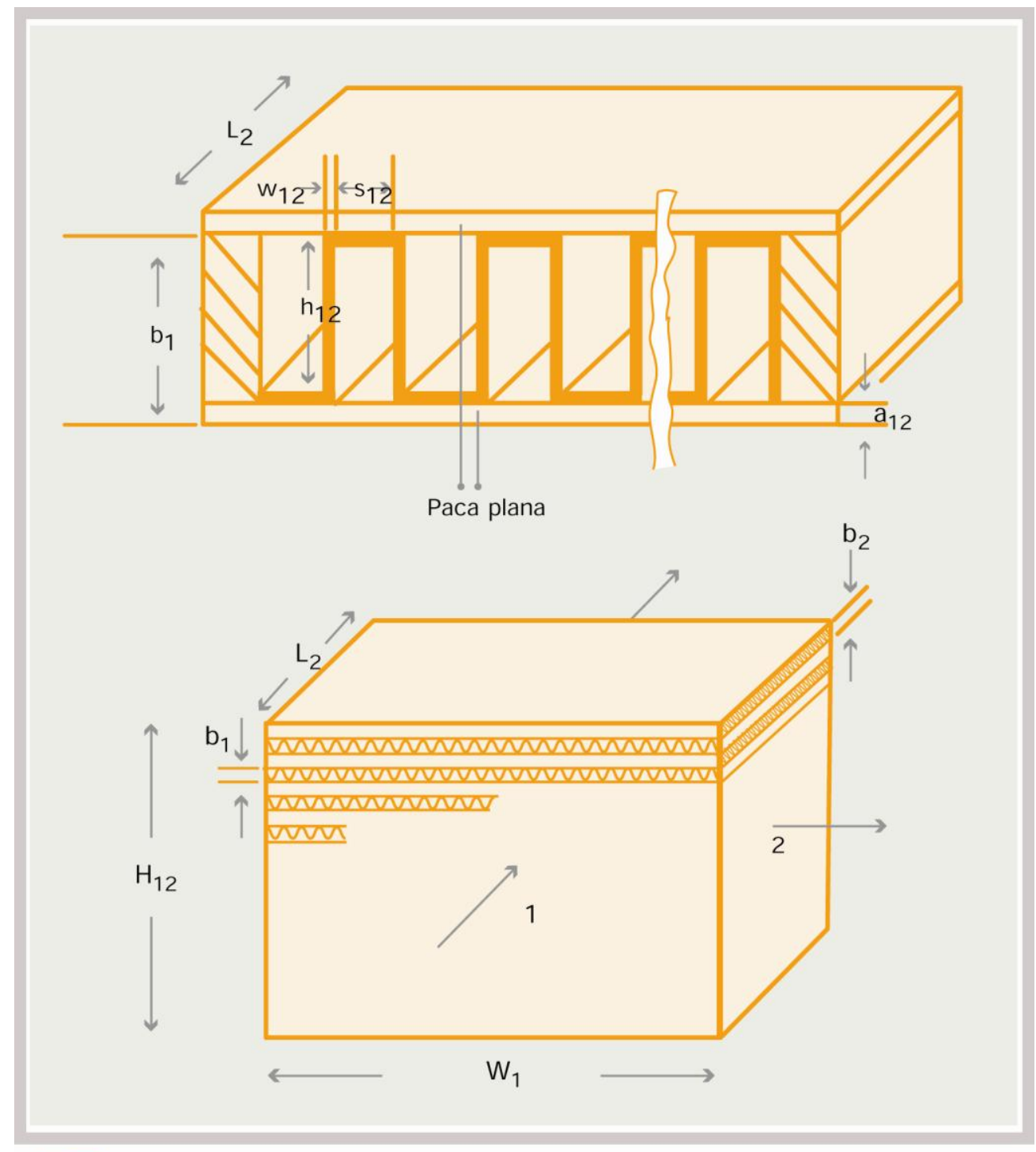

Fig. 4 - Características geométricas de un intercambiador de flujo cruzado con ambos fluidos "unmixed". 
En la Fig. 4 se presentan las características geométricas del sistema. En este caso los fluidos se identifican como "1" y " 2 ". En general se plantea esta diferencia para permitir la selección de características geométricas diferentes para las corrientes fría y caliente. Dada la similitud de las corrientes se empleará la misma geometría para todos los canales. En consecuencia, al agregar un 1 a una letra se refiere a la corriente "1", al agregar un 2, a la corriente " 2 " y al agregar un 12 a valores comunes a ambas corrientes. Para el diseño se debe especificar:

- Altura del equipo $\left(\mathrm{H}_{12}\right)$

- Ancho del equipo para la corriente 1 y profundidad para la corriente $2\left(\mathrm{~W}_{1}\right)$

- Ancho del equipo para la corriente 2 y profundidad para la corriente $1\left(\mathrm{~L}_{2}\right)$

- Espesor de las placas planas $\left(\mathrm{a}_{12}\right)$

- Espesor de las placas corrugadas $\left(\mathrm{w}_{12}\right)$

- Ancho de cada canal $\left(\mathrm{s}_{12}\right)$

- Alto de cada canal $\left(\mathrm{h}_{12}\right)$

- Conductividad del material de las placas planas $\left(\mathrm{k}_{\mathrm{p}, 12}\right)$

- Conductividad del material de las placas corrugadas $\left(\mathrm{k}_{\mathrm{f}, 12}\right)$

Esta información permite estimar una cantidad de características geométricas derivadas, que se señalan en el Apéndice 9.1. Una de estas características es el número de filas que atraviesa cada fluido, que se denominarán $\mathrm{NF}_{12}$. Por ser $\mathrm{H}_{12}$ un valor común para ambas corrientes, resulta el mismo número de filas para ambos fluidos.

El diseño comprende tanto las características de los canales como las dimensiones de todo el equipo. Para este tipo de intercambiadores no se posee un simulador comercial, razón por la cual se desarrolló un programa de cálculo en Matlab para llevar a cabo el diseño.

La resolución de los siguientes balances entálpicos (Ecs. [1] y [2]) permite obtener el calor a intercambiar y la temperatura de salida de una de las corrientes. Dado que el objetivo de estos equipos, en este caso particular, será elevar la temperatura de una corriente (la empleada para la desorción en un caso (I2) y la de entrada al reactor en el otro (I1)), la temperatura de salida que se calculará será la de la corriente caliente. El resto de las variables operativas se encuentran definidas.

$$
\begin{aligned}
& Q_{\mathrm{int}}=\mathrm{W}_{\mathrm{c}} \hat{\mathrm{c}}_{\mathrm{p}, \mathrm{c}}\left(\mathrm{T}_{\mathrm{c}}^{\mathrm{S}}-\mathrm{T}_{\mathrm{c}}^{0}\right) \\
& Q_{\mathrm{int}}=\mathrm{W}_{\mathrm{h}} \hat{\mathrm{c}}_{\mathrm{p}, \mathrm{h}}\left(\mathrm{T}_{\mathrm{h}}^{0}-\mathrm{T}_{\mathrm{h}}^{\mathrm{S}}\right)
\end{aligned}
$$


donde $Q_{\text {int }}$ es el calor intercambiado, W el caudal másico, $\hat{\mathrm{c}}_{\mathrm{p}}$ la capacidad calorífica por unidad de masa y $\mathrm{T}^{0}$ y $\mathrm{T}^{\mathrm{S}}$ las temperaturas de entrada y salida del intercambiador. Los subíndices "c" y "h" corresponden a las corrientes fría y caliente respectivamente.

El diseño se realizó empleando el método $\varepsilon$-NTU. Las variables de diseño son las dimensiones globales del equipo, alto, ancho y profundidad, y las dimensiones del canal, alto y ancho. Definido el tamaño de los canales, las dimensiones globales determinarán la cantidad de canales en los cuales se dividirá cada corriente de fluido. Dado que el número de canales por fila $\left(\mathrm{n}_{\mathrm{p} 1}\right.$ y $\left.\mathrm{n}_{\mathrm{p} 2}\right)$ y el número de filas, $\mathrm{NF}_{12}$, para cualquier fluido, deben ser enteros, se proponen valores aproximados de $\mathrm{H}_{12}, \mathrm{~W}_{1}$ y $\mathrm{L}_{2}$ y luego se ajustan de tal forma de llegar a un número entero de canales por fila y de filas para cada fluido.

Un diseño satisfactorio, desde el punto de vista térmico, será aquel que permita cumplir con la relación:

$$
\varepsilon_{\text {real }}>\varepsilon_{\text {req }}
$$

La evaluación de $\varepsilon_{\text {real }}$ (eficiencia real del intercambiador) se realizó con una expresión aproximada (Incropera y de Witt, 1990) que es ampliamente utilizada y recomendada, siendo:

$$
\varepsilon_{\text {real }}=1-\exp \left\{\frac{\mathrm{NTU}^{0.22}}{R^{\prime}}\left[\exp \left(-R^{\prime} \mathrm{NTU}^{0.78}\right)-1\right]\right\}
$$

donde para $\mathrm{W}_{\mathrm{c}} \hat{\mathrm{c}}_{\mathrm{p}, \mathrm{c}}<\mathrm{W}_{\mathrm{h}} \hat{\mathrm{c}}_{\mathrm{p}, \mathrm{h}}$ (situación dada en nuestro caso debido, esencialmente, a la variación de los $\hat{\mathrm{c}}_{\mathrm{p}}$ con la temperatura, resultando: $\hat{\mathrm{c}}_{\mathrm{p}, \mathrm{c}}<\hat{\mathrm{c}}_{\mathrm{p}, \mathrm{h}}$ ),

$$
R^{\prime}=\frac{\mathrm{W}_{\mathrm{c}} \hat{\mathrm{c}}_{\mathrm{p}, \mathrm{c}}}{\mathrm{W}_{\mathrm{h}} \hat{\mathrm{c}}_{\mathrm{p}, \mathrm{h}}} \quad, \quad \mathrm{NTU}=\frac{\mathrm{U} \mathrm{A}_{\mathrm{T}}}{\mathrm{W}_{\mathrm{c}} \hat{\mathrm{c}}_{\mathrm{p}, \mathrm{c}}}
$$

donde $\mathrm{U}$ es el coeficiente global de transferencia de calor y $\mathrm{A}_{\mathrm{T}}$, el área de transferencia.

En este sistema se pueden tomar distintas superficies como referencia del área de transferencia, A $\mathrm{A}_{\mathrm{T}}$. La alternativa normalmente adoptada (Hewitt y col., 1994) es emplear el área de las placas planas $\left(\mathrm{A}_{\mathrm{TP}}\right)$, por lo tanto

$$
\mathrm{A}_{\mathrm{T}}=\mathrm{A}_{\mathrm{TP}}=2 \quad \mathrm{~W}_{1} \mathrm{~L}_{2} \quad \mathrm{NF}_{12}
$$

La evaluación del coeficiente global de transferencia de calor, U, es: 


$$
\frac{1}{\mathrm{U}}=\frac{1}{\mathrm{U}_{\mathrm{p}}}=\frac{1}{\mathrm{~h}_{\mathrm{p}, 1}}+\frac{\mathrm{a}_{12}}{\mathrm{k}_{\mathrm{p}, 12}}+\frac{1}{\mathrm{~h}_{\mathrm{p}, 2}}
$$

Los coeficientes $h_{p, j}$ tienen en cuenta la eficiencia de la superficie extendida generada por la placa corrugada, en consecuencia se calculan como:

$$
\mathrm{h}_{\mathrm{p}, \mathrm{j}}=\left(\frac{\mathrm{A}_{\mathrm{ef}, \mathrm{j}}}{\mathrm{A}_{\mathrm{p}}}\right) \frac{1}{\mathrm{R}_{\mathrm{f}, \mathrm{j}}+\frac{1}{\mathrm{~h}_{\mathrm{j}}}} \quad \mathrm{j}=1,2
$$

siendo:

$$
\mathrm{A}_{\mathrm{ef}, \mathrm{j}}=\eta_{\mathrm{t}, \mathrm{j}} \mathrm{A}_{\mathrm{f}, \mathrm{j}}+\left(\mathrm{A}_{\mathrm{S}, \mathrm{j}}-\mathrm{A}_{\mathrm{f}, \mathrm{j}}\right) \quad \mathrm{j}=1,2
$$

Las definiciones de $A_{f, j}$ y $A_{S, j}$ se encuentran en el Apéndice 9.1. La eficiencia de la superficie extendida se evalúa como:

$$
\eta_{\mathrm{t}, \mathrm{j}}=\frac{\tanh \left(\omega_{\mathrm{j}}\right)}{\omega_{\mathrm{j}}} \quad \mathrm{j}=1,2
$$

siendo:

$$
\omega_{\mathrm{j}}=\left(\frac{\mathrm{h}_{12}}{2}\right)\left[\frac{\mathrm{h}_{\mathrm{j}}}{\mathrm{k}_{\mathrm{f}, 12} \cdot\left(\mathrm{w}_{12} / 2\right)}\right]^{1 / 2} \quad \mathrm{j}=1,2
$$

Dadas las características de la superficie extendida, que no presentan un extremo libre, sino que están comprendidas entre dos placas planas, la longitud de las mismas es la mitad de su altura.

Las correlaciones para evaluar los coeficientes peliculares $h_{j}$ se presentan en el Apéndice 9.1.

La evaluación de la pérdida de carga total se lleva a cabo a partir de la expresión:

$$
\Delta \mathrm{P}_{\mathrm{j}}=\Delta \mathrm{P}_{\mathrm{e}, \mathrm{j}}+\Delta \mathrm{P}_{\mathrm{c}, \mathrm{j}}+\Delta \mathrm{P}_{\mathrm{s}, \mathrm{j}} \quad \mathrm{j}=1,2
$$

donde:

$\Delta \mathrm{P}_{\mathrm{c}, \mathrm{j}}$ : es la pérdida de carga por fricción en los tramos rectos de cada canal.

$\Delta \mathrm{P}_{\mathrm{e}, j}$ : es la pérdida de carga en la entrada del intercambiador (contracción abrupta).

$\Delta \mathrm{P}_{\mathrm{s}, \mathrm{j}}$ : es la pérdida de carga en la salida del intercambiador (expansión abrupta). 
En estos sistemas el factor dominante en la pérdida de carga suele ser el generado por la fricción en los tramos rectos de los canales.

Asimismo, algunos autores incluyen, además, un término adicional que tiene en cuenta la variación de la velocidad del fluido por el cambio en su densidad. En esta instancia se ha desestimado este término porque es normalmente muy pequeño.

La evaluación de cada término en la ecuación [12] es:

$$
\Delta P_{\mathrm{c}, \mathrm{j}}=\left(\frac{\mathrm{v}_{\mathrm{s}, \mathrm{j}}^{2} \rho_{g, \mathrm{j}}}{2}\right)\left(4 f_{\mathrm{j}} \frac{\mathrm{L}_{\mathrm{j}}}{\mathrm{d}_{\mathrm{h}, \mathrm{j}}}\right) \quad \mathrm{j}=1,2
$$

donde $v_{s, j}$ es la velocidad superficial del fluido $j, L_{j}=L_{2}$ para el fluido " 1 " $y L_{j}=W_{1}$ para el fluido "2", $f_{\mathrm{j}}$ representa un factor de fricción y $\mathrm{d}_{\mathrm{h}, \mathrm{j}}$ es el diámetro hidráulico.

Las pérdidas de carga en la entrada se evalúan a partir de la expresión:

$$
\Delta P_{\mathrm{e}, \mathrm{j}}=\left(\frac{\mathrm{v}_{\mathrm{s}, \mathrm{j}}{ }^{2} \rho_{\mathrm{g}, \mathrm{j}}}{2}\right) K e_{\mathrm{j}} \quad \mathrm{j}=1,2
$$

Las pérdidas de carga en la salida se evalúan a partir de una expresión análoga a esta última, reemplazando $K e_{\mathrm{j}}$ por $K s_{\mathrm{j}}$.

Las correlaciones para evaluar $f, K e$ y $K s$ se presentan en el Apéndice 9.1.

\subsection{Definición de las variables geométricas}

En la Sección 9.1, se optó por un intercambiador de flujo cruzado con superficies extendidas tipo "plate-fin", tanto para precalentar la corriente de regeneración (I2), como para precalentar la corriente previo a su ingreso al reactor (I1). Según las especificaciones que requieren cada una de estas corrientes y considerando ciertos criterios de diseño, se definieron las dimensiones generales del equipo y de los canales para cada situación.

Para poder reducir la cantidad de variantes de diseño para el intercambiador propuesto, se fijaron, teniendo en cuenta la información disponible en la bibliografía, las siguientes características geométricas:

$$
\begin{aligned}
& \text { * } \quad \text { Espesor de las placas planas }\left(\mathrm{a}_{12}\right) \\
& \text { * } \quad \text { Espesor de las placas corrugadas }\left(\mathrm{w}_{12}\right)
\end{aligned}
$$

Asimismo, al seleccionar el material de las placas, se define: 
- Conductividad del material de las placas planas $\left(\mathrm{k}_{\mathrm{p}, 12}\right)$

- Conductividad del material de las placas corrugadas $\left(\mathrm{k}_{\mathrm{f}, 12}\right)$

En este caso particular se seleccionó un acero al carbono. Se debe destacar que en este tipo de intercambiadores el material seleccionado es particularmente importante para su evaluación térmica porque las placas corrugadas conforman una superficie extendida y la transferencia de calor en las mismas es determinante en el diseño.

Los valores adoptados para tales magnitudes se presentan en la Tabla 1. Básicamente, los datos geométricos corresponden a un caso analizado por Hewitt y col. (1994).

TABLA 1: Características del intercambiador

\begin{tabular}{cccc}
\hline $\begin{array}{c}\mathbf{a}_{12} \\
{[\mathrm{~mm}]}\end{array}$ & $\begin{array}{c}\mathbf{w}_{12} \\
{[\mathbf{m m}]}\end{array}$ & $\begin{array}{c}\mathbf{k}_{\mathbf{p}, \mathbf{1 2}} \\
{[\mathbf{J} / \mathbf{m ~ s ~ K}]}\end{array}$ & $\begin{array}{c}\mathbf{k}_{\mathbf{f}, 12} \\
{[\mathbf{J} / \mathbf{m ~ s ~ K}]}\end{array}$ \\
\hline 0.30 & 0.15 & 40 & 40 \\
\hline
\end{tabular}

De esta manera, para alcanzar el diseño del equipo restan definir las dimensiones globales, $\mathrm{H}_{12}, \mathrm{~W}_{1}$ y $\mathrm{L}_{2}$, y el ancho y alto de cada canal, $\mathrm{s}_{12}$ y $\mathrm{h}_{12}$ respectivamente. Para la determinación de las dimensiones globales, siendo que los valores de la pérdida de carga no resultaron significativos, se consideró, como criterio de diseño, alcanzar velocidades del orden de los valores máximos recomendados para gases (Serth, 2007). De esta manera se obtienen coeficientes de transferencia más elevados y se requiere de una menor área de transferencia. En este caso se adoptó una velocidad de aproximadamente $20 \mathrm{~m} / \mathrm{s}$ para ambas corrientes.

Por otro lado, para poder definir el ancho y alto de un canal, se partió de los valores correspondientes al caso analizado por Hewitt y col. (1994) y se lo fue modificando de manera tal de no superar el 5\% de exceso de área. Como resultado, para definir la geometría completa del intercambiador, se debe seguir un procedimiento de tipo iterativo.

En el capítulo siguiente se presentarán distintas alternativas de diseño para los intercambiadores I1 y I2 (Fig. 1) considerando la variación de los caudales de las corrientes de intercambio y se llevará a cabo un análisis de los resultados obtenidos. 


\subsection{Conclusiones}

El esquema de eliminación de VOCs propuesto del Capítulo 2 presenta dos recuperadores de calor. Uno permite elevar la temperatura de la corriente previo a su entrada al reactor y el otro precalienta la corriente empleada para la regeneración del sistema de adsorción/desorción. Para ambos sistemas de intercambio térmico se emplea un intercambiador gas - gas que utiliza el calor generado por la reacción. El hecho de utilizar la energía proveniente de la corriente que sale del reactor para calentar, en dos etapas, la corriente que lleva a cabo el proceso de desorción y luego ingresa al reactor, implica no solo un ahorro energético importante sino que permite liberar a la atmósfera una corriente a temperaturas más tolerables para el ambiente (ver Capítulo 10).

De manera tal de garantizar que no haya contacto entre las corrientes a tratar, así como la uniformidad de concentración y temperatura de la corriente de salida, y teniendo en cuenta su sencillez operativa, se optó por un intercambiador de tipo recuperativo. Para este tipo de intercambiadores, existe una amplia variedad de alternativas.

A partir de analizar las características que presentan las corrientes a tratar y mediante el análisis realizado por Campesi (2005), se retuvieron diferentes diseños de intercambiadores aptos para el caso de estudio y que permitiesen minimizar la pérdida de carga. Dentro de los mismos se destacaron los intercambiadores de flujo cruzado con superficies extendidas tipo "plate-fin". Estos últimos aparecen como la mejor alternativa por la baja pérdida de carga y su alta relación área/volumen. Por lo tanto, se optó por un intercambiador de este tipo para enfrentar el diseño.

Según las especificaciones que requieren cada una de las corrientes y considerando ciertos criterios de diseño se definieron las características geométricas y se presentaron las herramientas de cálculo para el tipo de intercambiador seleccionado.

El diseño de ambos intercambiadores y análisis de resultados se llevará a cabo en el próximo capítulo. 


\section{$9.1 /_{\text {Moende }}$}

\section{Intercambiadores de flujo cruzado con ambos fluidos unmixed}

Para evaluar los coeficientes peliculares y el factor de fricción se deben calcular relaciones geométricas auxiliares, obtenidas a partir de la información que se debe definir para el diseño, como fue señalado en el texto principal. Estas características son:

Diámetro hidráulico, $\mathrm{d}_{\mathrm{h}}$

$$
\mathrm{d}_{\mathrm{hj}}=\frac{4 \mathrm{~h}_{12} \mathrm{~s}_{12}}{2\left(\mathrm{~h}_{12}+\mathrm{s}_{12}\right)} \quad \mathrm{j}=1,2
$$

Número de canales por fila, $\mathbf{n}_{\mathrm{p}}$

$$
\mathrm{n}_{\mathrm{p} 1}=\frac{\mathrm{w}_{1}}{\left(\mathrm{w}_{12}+\mathrm{s}_{12}\right)} ; \quad \mathrm{n}_{\mathrm{p} 2}=\frac{\mathrm{L}_{2}}{\left(\mathrm{w}_{12}+\mathrm{s}_{12}\right)}
$$

Dado que $n_{\mathrm{p} 1}$ y $\mathrm{n}_{\mathrm{p} 2}$ deben ser enteros, estas relaciones se emplean para ajustar los valores de $\mathrm{W}_{1}$ y $\mathrm{L}_{2}$.

Altura de una fila, incluyendo los espesores de las placas plana y corrugada, b

$$
\mathrm{b}_{\mathrm{j}}=\mathrm{a}_{12}+\mathrm{w}_{12}+\mathrm{h}_{12} \quad \mathrm{j}=1,2
$$

Número de filas para cada corriente, $\mathrm{NF}$

$$
\mathrm{NF}_{12}=\frac{\mathrm{H}_{12}}{\mathrm{~b}_{12}+\mathrm{b}_{12}}
$$

Esta expresión se emplea para ajustar el valor final de $\mathrm{H}_{12}$ teniendo en cuenta que $\mathrm{NF}_{12}$ debe ser un número entero. En este caso se empleará la misma geometría de canales para ambas corrientes y por ello aparece la suma de $b_{12}$ en el denominador; si la geometría fuese diferente se debería emplear el valor de b para cada corriente. 
Área de flujo para cada corriente, $\mathrm{S}$

$$
\mathrm{S}_{\mathrm{j}}=\mathrm{NF}_{12} \mathrm{n}_{\mathrm{pj}} \mathrm{h}_{12} \mathrm{~s}_{12} \quad \mathrm{j}=1,2
$$

Área total de intercambio para cada corriente, $\mathbf{A}_{\mathrm{S}}$

$$
A_{\mathrm{S} 1}=2\left(\mathrm{~h}_{12}+\mathrm{s}_{12}\right) \mathrm{L}_{2} \mathrm{n}_{\mathrm{p} 1} \mathrm{NF}_{12} \quad ; \quad \mathrm{A}_{\mathrm{S} 2}=2\left(\mathrm{~h}_{12}+\mathrm{s}_{12}\right) \mathrm{W}_{1} \mathrm{n}_{\mathrm{p} 2} \mathrm{NF}_{12}
$$

Área de las superficies extendidas, $\mathbf{A}_{\mathrm{f}}$

$$
\mathrm{A}_{\mathrm{f} 1}=2 \mathrm{~h}_{12} \mathrm{~L}_{2} \mathrm{n}_{\mathrm{p} 1} \mathrm{NF}_{12} \quad ; \quad \mathrm{A}_{\mathrm{f} 2}=2 \mathrm{~h}_{12} \mathrm{~W}_{1} \mathrm{n}_{\mathrm{p} 2} \mathrm{NF}_{12}
$$

Relación entre el área de flujo y el área frontal para cada corriente, $\sigma$

$$
\sigma_{1}=\frac{\mathrm{S}_{1}}{\mathrm{H}_{12} \mathrm{~W}_{1}} \quad ; \quad \sigma_{2}=\frac{\mathrm{S}_{2}}{\mathrm{H}_{12} \mathrm{~L}_{2}}
$$

Teniendo en cuenta estas definiciones, el número de Reynolds se calcula como:

$$
\operatorname{Re}_{\mathrm{j}}=\frac{\mathrm{v}_{\mathrm{s}, \mathrm{j}} \rho_{\mathrm{g}, \mathrm{j}} \mathrm{d}_{\mathrm{h}, \mathrm{j}}}{\mu_{\mathrm{g}, \mathrm{j}}} \quad \mathrm{j}=1,2
$$

donde la velocidad lineal se calcula como:

$$
\mathrm{v}_{\mathrm{s}, \mathrm{j}}=\frac{\mathrm{W}_{\mathrm{j}}}{\rho_{\mathrm{g}, \mathrm{j}} \mathrm{S}_{\mathrm{j}}} \quad \mathrm{j}=1,2
$$

\section{Expresiones para calcular el factor de fricción, $f$}

Se presentan en primer lugar las expresiones de factor de fricción debido a que algunas de las mismas se emplearán para determinar los coeficientes peliculares.

$$
\mathrm{Re}_{\mathrm{j}}<2100 \quad f_{\mathrm{l}, \mathrm{j}}=\frac{f_{\mathrm{LF}}}{\mathrm{Re}_{\mathrm{j}}} \quad \mathrm{j}=1,2
$$

Donde el valor de $f_{\mathrm{LF}}$ depende de la geometría del canal. A efectos de poder emplear diferentes relaciones de ancho y alto del canal se ajustaron los valores discretos de $f_{\mathrm{LF}}$ proporcionados por Hewitt y col. (1994) con la expresión: 


$$
f_{\mathrm{LF}}=12.56 \mathrm{~Eb}^{4}-35.83 \mathrm{~Eb}^{3}+46.03 \mathrm{~Eb}^{2}-32.53 \mathrm{~Eb}+24
$$

donde $E b=s_{12} / h_{12}$.

Para valores mayores del número de Reynolds se adoptaron las correlaciones de Bhatti y Shah (1987) sugeridas por Shah y Sekulic (2003):

$$
\begin{array}{lll}
2100 \leq \operatorname{Re}_{\mathrm{j}} \leq 4000 & \mathrm{f}_{\mathrm{Ij}}=0.0054+2.310^{-8} \operatorname{Re}_{\mathrm{j}}^{3 / 2} & \mathrm{j}=1,2 \\
\operatorname{Re}_{\mathrm{j}}>4000 & \mathrm{f}_{\mathrm{Tj}}=0.00128+0.1143 \operatorname{Re}_{\mathrm{j}}^{-1 / 3.2154} & \mathrm{j}=1,2
\end{array}
$$

\section{Expresiones para calcular los factores en entrada y salida de cada corriente, Ke y Ks:}

Estos factores están generalmente informados en gráficos en función de $\sigma$, con dos tipos de curvas, una para régimen laminar $(\operatorname{Re}<2100)$ y otras para régimen turbulento, para diferentes valores de Re. Asimismo, existen gráficos diferentes de acuerdo a la geometría del canal.

Teniendo en cuenta que para desarrollar el programa del modelo se requiere una expresión matemática, se buscó realizar un ajuste de la información obtenida de los gráficos. En esta situación, y teniendo en cuenta que la influencia de estos términos en la pérdida de carga no es dominante, se realizaron los ajustes con las siguientes simplificaciones:

- $\quad$ se consideraron solo los valores del gráfico de canales rectangulares.

- se condensaron, en una única expresión, todos los valores de $\mathrm{Ke}$ o $K s$ correspondientes a diferentes Re cuando el régimen no es laminar.

Las expresiones resultantes para $\mathrm{Ke}$ son:

$$
\begin{aligned}
& \operatorname{Re}_{\mathrm{j}}<2100 K e_{\mathrm{j}}=-0.119 \sigma_{\mathrm{j}}^{3}-0.199 \sigma_{\mathrm{j}}^{2}-0.082 \sigma_{\mathrm{j}}+1.1994 \mathrm{j}=1,2 \quad \text { [A9.1..15] } \\
& \operatorname{Re}_{\mathrm{j}}>2100 K e_{\mathrm{j}}=-0.4946 \sigma_{\mathrm{j}}^{3}+0.2627 \sigma_{\mathrm{j}}^{2}-0.2168 \sigma_{\mathrm{j}}+0.551 \mathrm{j}=1,2 \quad \text { [A9.1.16] }
\end{aligned}
$$

mientras que las expresiones resultantes para $K s$ son:

$$
\begin{array}{llll}
\operatorname{Re}_{\mathrm{j}}<2100 K s_{\mathrm{j}}=0.116 \sigma_{\mathrm{j}}^{3}+0.764 \sigma_{\mathrm{j}}^{2}-2.681 \sigma_{\mathrm{j}}+1.00 & \mathrm{j}=1,2 & \text { [A9.1.17] } \\
\mathrm{Re}_{\mathrm{j}}>2100 K s_{\mathrm{j}}=-0.031 \sigma_{\mathrm{j}}^{3}+1.138 \sigma_{\mathrm{j}}^{2}-2.207 \sigma_{\mathrm{j}}+0.999 & \mathrm{j}=1,2 & \text { [A9.1.18] }
\end{array}
$$




\section{Expresiones para calcular los coeficientes peliculares:}

Para la zona laminar el número de Nusselt alcanza un valor constante si el flujo se encuentra completamente desarrollado, dependiendo este valor asintótico de la geometría del canal. Hewitt y col (1994) proporcionan valores de Nusselt asintótico discretos, correspondiente a ciertas relaciones alto/ancho del canal. Asimismo, proporcionan relaciones que permiten verificar si es razonable aceptar que el sistema tiene un flujo totalmente desarrollado sin introducir un error significativo. Como esta situación se verificó para la generalidad de los casos analizados, solo se retuvieron las expresiones asintóticas de $\mathrm{Nu}$. Nuevamente, se ajustaron con un polinomio los valores discretos disponibles, resultando:

$$
\operatorname{Re}_{\mathrm{j}}<2100 \quad N u_{1 \mathrm{j}}=6.015 \mathrm{~Eb}^{4}-19.019 \mathrm{~Eb}^{3}+25.196 \mathrm{~Eb}^{2}-16.819 \mathrm{~Eb}+8.235 \mathrm{j}=1,2
$$

Para régimen turbulento se empleó la correlación de Gnielinski (1976), recomendada por Shah y Sekulic (2003):

$$
\operatorname{Re}_{\mathrm{j}}>8000 \quad N u_{\mathrm{Tj}}=\frac{\left(f_{\mathrm{Tj}} / 2\right)\left(\mathrm{Re}_{\mathrm{j}}-1000\right) \operatorname{Pr}_{\mathrm{j}}}{1+12.7\left(f_{\mathrm{Tj}} / 2\right)^{0.5}\left(\operatorname{Pr}_{\mathrm{j}}^{2 / 3}-1\right)} \quad \mathrm{j}=1,2
$$

Para la zona intermedia Shah y Sekulic (2003) sugieren una expresión propuesta por Taborek (1990) que es un promedio pesado entre los valores de Nusselt de zona laminar y turbulenta:

$$
2100 \leq \mathrm{Re}_{\mathrm{j}} \leq 4000 \quad N u_{\mathrm{Ij}}=\varphi_{\mathrm{I}} N u_{1 \mathrm{j}}+\left(1-\varphi_{\mathrm{I}}\right) N u_{\mathrm{T} j} \quad \mathrm{j}=1,2
$$

donde $\varphi_{\mathrm{I}}=1.33-\left(\operatorname{Re}_{\mathrm{j}} / 6000\right)$. 



\section{0/ ANALISIS DEL IMPACTO QUE CAUSA LA INCORPORACION DE UN CONCENTRADOR SOBRE EL REACTOR CATALITICO E INTERCAMBIADOR DE CALOR}

Factor de concentración, Fc

Impacto del Fc sobre el reactor catalítico

Presentación de tres sistemas de eliminación de VOCs

Evaluación de resultados

Selección de la unidad impulsora

Conclusiones 



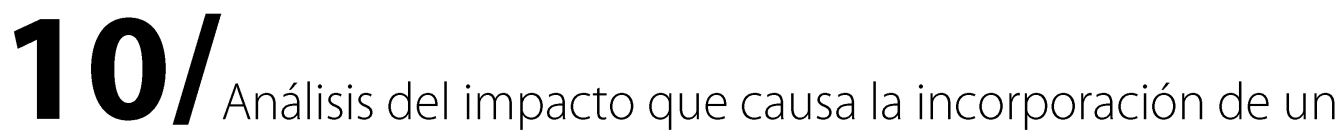
concentrador sobre el reactor catalítico e intercambiadores de calor

Un objetivo fundamental de la incorporación de una etapa previa de concentración de VOCs al sistema de eliminación catalítica (Capítulo 2, Fig. 3), es el de reducir los requerimientos de catalizador y superficie de intercambio térmico.

Se define el factor de concentración Fc como la relación entre el caudal del efluente a tratar y el alimentado al reactor. En este capítulo se intenta cuantificar el impacto del Fc sobre el volumen necesario de lecho catalítico granular para la incineración de acetato de etilo y etanol, y sobre la superficie de intercambio requerida para alcanzar la temperatura de ingreso al reactor y la temperatura de desorción. El estudio se lleva a cabo mediante la simulación del reactor, cuyas características fueron presentadas en el Capítulo 8, y la simulación del sistema de recuperación de calor propuesto en el Capítulo 9. Para completar el análisis de resultados, se presentan tres alternativas de eliminación de VOCs.

Además, para un Fc determinado, se analiza la influencia de la temperatura de alimentación al reactor sobre la cantidad de catalizador a emplear.

Finalmente de acuerdo al caudal del efluente a tratar y los requerimientos de pérdida de carga de todo el sistema, se propone un soplador adecuado a partir de catálogos comerciales.

\subsection{Factor de concentración, Fc}

La incorporación de un sistema de adsorción/desorción al sistema catalítico de eliminación de VOCs permite concentrar la corriente a tratar previo a su entrada al reactor. El grado de concentración puede cuantificarse a través del factor de concentración, Fc. Este factor se define como la relación entre el caudal másico del efluente primario $\left(\mathrm{W}_{\mathrm{ads}}\right.$ ) y el caudal másico de ingreso al reactor. El caudal másico de ingreso al reactor, o lo que es lo mismo, el caudal de salida del proceso de desorción, equivale a la suma del caudal empleado para la desorción $\left(\mathrm{W}_{\mathrm{des}}\right)$ y el caudal másico de los VOCs desorbidos $\left(\mathrm{W}_{\mathrm{VOCs}}\right)$. Por lo tanto resulta: 


$$
\mathrm{Fc}=\frac{\mathrm{W}_{\mathrm{ads}}}{\mathrm{W}_{\mathrm{des}}+\mathrm{W}_{\mathrm{vOCs}}}
$$

Cuando el caudal de desorción equivale al valor mínimo, i.e., $\mathrm{W}_{\mathrm{des}}^{\mathrm{min}}$ (Capítulo 6), el factor de concentración, Fc, alcanza su valor máximo. En este caso se lo denomina $\mathrm{Fc}^{\text {máx }}$. Por otro lado, el mínimo valor que Fc puede adoptar es 1 , y equivale a la situación de no concentrar la corriente a tratar previo a su ingreso al reactor.

En base a los resultados obtenidos en el Capítulo 6 (Fig. 5), el factor de concentración Fc puede alcanzar valores de hasta aproximadamente 6 para una temperatura de desorción de $200{ }^{\circ} \mathrm{C}$. Se recuerda que este valor conservativo de temperatura surge de la necesidad de evitar pérdidas significativas del carbón activado por combustión (Yates et al., 2000). El factor de concentración aumenta con el aumento de la temperatura de desorción. En la Tabla 1 se presentan los valores de $\mathrm{Fc}^{\text {máx }}$ para cada uno de los puntos de las curvas de la Fig. 5 del Capítulo 6, destacándose la situación recomendada según el análisis realizado en el capítulo en cuestión.

Tabla 1: Variación del valor de $\mathrm{Fc}^{\text {máx }}$ en función del volumen de adsorbente para dos temperaturas de desorción

\begin{tabular}{c|rcccc}
\hline $\mathbf{1 5 0}$ & $\mathbf{1 . 0 0}$ & $\mathbf{1 . 6 7}$ & $\mathbf{2 . 3 4}$ & $\mathbf{3 . 3 4}$ & $\mathbf{6 . 6 7}$ \\
\hline $\mathbf{2 0 0}$ & 2.92 & 3.42 & 3.67 & 3.81 & 3.96 \\
\hline $\mathbf{T}_{\text {des }}{ }^{\circ}{ }^{\mathrm{Cds}}\left[\mathrm{m}^{3}\right]$ & 4.53 & 5.56 & 5.88 & 6.25 & 6.64 \\
\hline
\end{tabular}

\subsection{Impacto del Fc sobre el reactor catalítico}

Según los resultados reportados en la Tabla 1 y considerando la posibilidad de no incorporar un concentrador al sistema de eliminación, se toma un rango de 1 - 6 de Fc para evaluar el impacto del mismo sobre el tamaño del reactor catalítico. El estudio se lleva a cabo mediante la simulación del reactor radial cuyas características fueron presentadas en el Capítulo 8, considerando un catalizador de laboratorio en base de óxidos de $\mathrm{Mn}$ y $\mathrm{Cu}$ descrito en Morales y col. (2008) y con expresiones cinéticas presentadas en el Capítulo 7.

Para la determinación del volumen de reactor se fijó el diámetro interno en $0.25 \mathrm{~m}$. El diámetro externo y el alto del mismo se definieron de manera tal de mantener una 
pérdida de carga menor a la admisible ( $0.3 \mathrm{~atm}$ para todo el sistema) y no superar la máxima cantidad de VOCs permitida a la salida. Como se mencionó en capítulos previos se asume una tolerancia de $20 \mathrm{mg} / \mathrm{kg}(5 \mathrm{ppm})$ de VOCs en el efluente descargado a la atmósfera (European Commission). Estas condiciones de base del reactor se emplearán en todos los análisis a realizar en este capítulo.

Previo al análisis del impacto que causa el Fc sobre el volumen del reactor, se debe definir la temperatura de entrada al reactor, $\mathrm{T}^{0}$, que permita iniciar las reacciones de combustión de los VOCs presentes en la corriente a tratar. Se encontró que, de acuerdo a la cinética desarrollada en el Capítulo 7, temperaturas de entrada al reactor superiores a $\operatorname{los} 180^{\circ} \mathrm{C}$, han resultado adecuadas para el inicio de las reacciones de combustión. Teniendo en cuenta lo dicho, se adopta un rango de $180-220^{\circ} \mathrm{C}$ para estudiar la influencia de $\mathrm{T}^{0}$ sobre el volumen de reactor.

Como es de esperar, para un sistema con elevadas energías de activación (Capítulo 7, Tabla 2), el valor de la temperatura $\mathrm{T}^{0}$ presenta un impacto importante sobre el volumen requerido de catalizador. Este efecto se observa claramente en la Figura 1, para la cual se consideró $\mathrm{Fc}=1$. Para temperaturas inferiores a $180^{\circ} \mathrm{C}$ el volumen de lecho aumenta rápidamente.

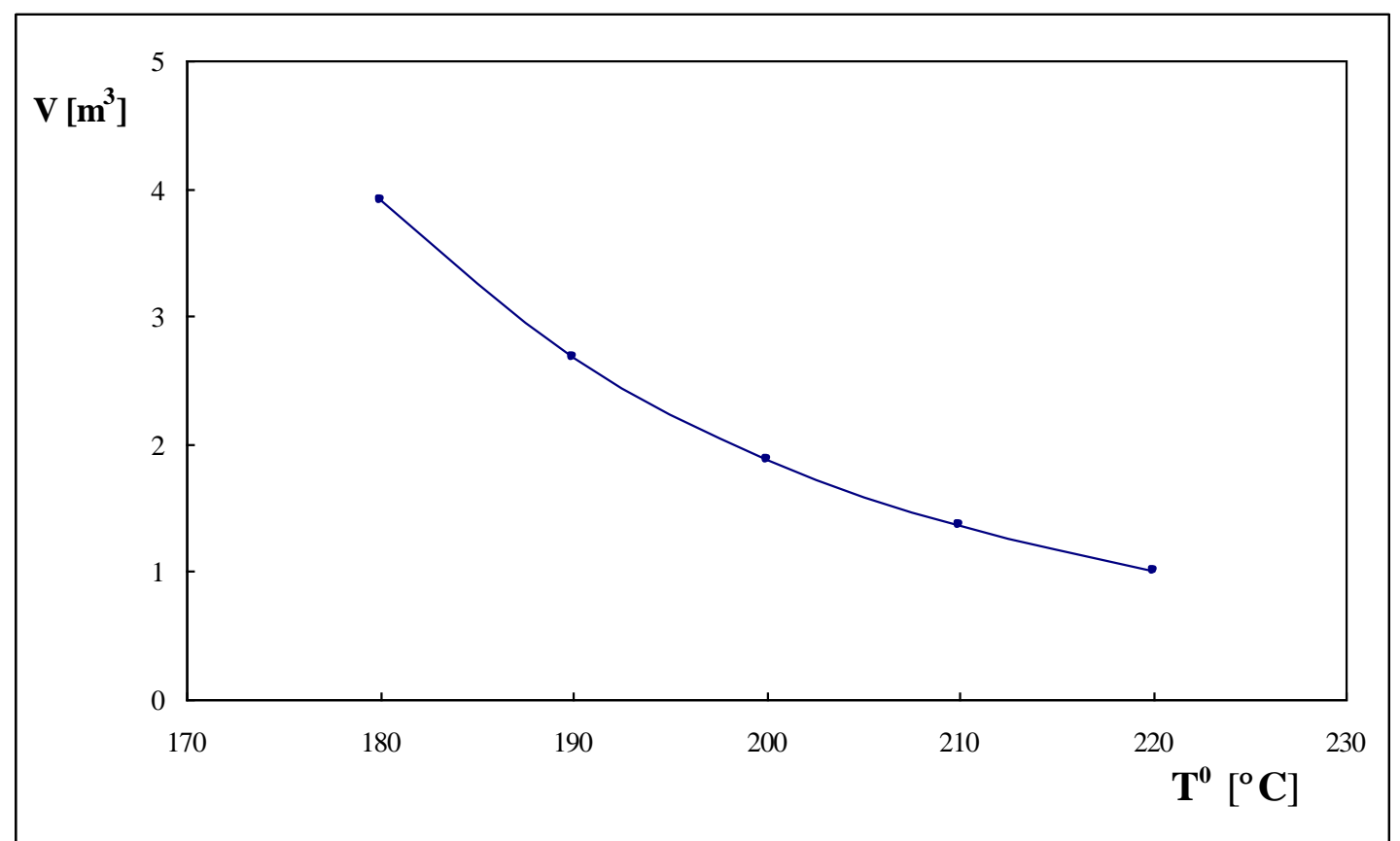

Fig. 1 - Impacto de la temperatura de entrada al reactor $\left(\mathrm{T}^{0}\right)$ sobre el volumen de lecho catalítico granular para un $\mathrm{Fc}=1$. 
La Figura 2 permite cuantificar el efecto del factor de concentración, Fc, sobre el volumen requerido del reactor catalítico (V) para las temperaturas extremas del intervalo adoptado para $\mathrm{T}^{0}$ (i.e., 180 y $220^{\circ} \mathrm{C}$ ). En la Fig. 2 el volumen indicado como $\mathrm{V}_{\text {ref }}$ representa el volumen de lecho requerido cuando $\mathrm{Fc}=1$, lo que equivale a considerar que la corriente a tratar no circula por el adsorbedor.

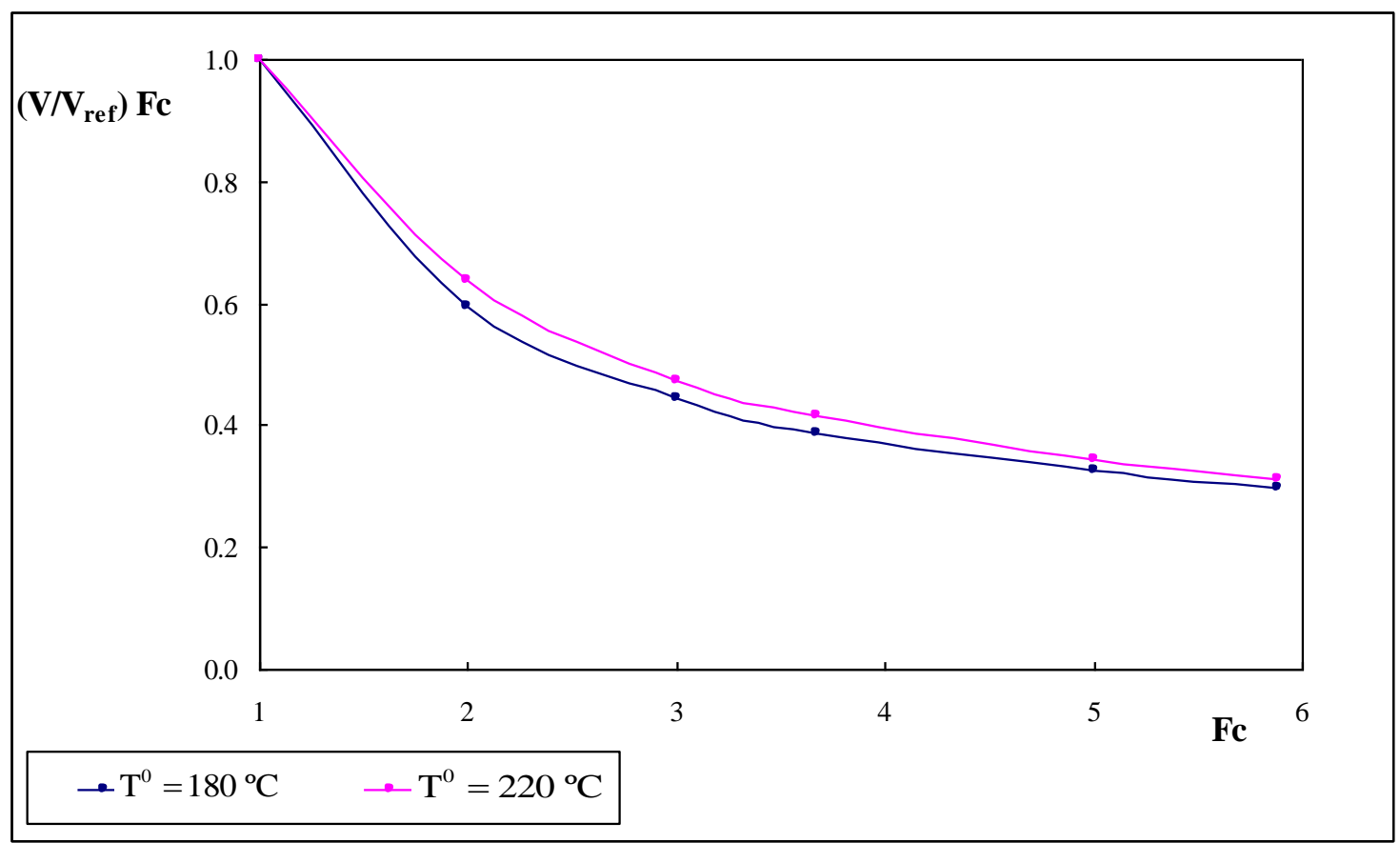

Fig. 2. Impacto de Fc sobre el volumen del reactor catalítico, a distintas temperaturas de alimentación, $\mathrm{T}^{0}$.

La relación $\left(\mathrm{V} / \mathrm{V}_{\text {ref }}\right) \mathrm{Fc}$ graficada en la Fig. 2 podría ser igual a la unidad si la reducción del volumen de lecho se debiera únicamente a la disminución del caudal de alimentación. Sin embargo, el hecho de que tal relación disminuya con Fc se debe al efecto adicional que provoca el mayor salto adiabático cuando Fc aumenta, lo que permite acelerar el incremento de la temperatura en el lecho a medida que la conversión de VOCs progresa y por lo tanto disminuir aún más el volumen de lecho necesario. Como ejemplo, el volumen de lecho necesario para $\mathrm{Fc}=2$ representa alrededor del $30 \%$ de $\mathrm{V}_{\text {ref }}$, mientras que para $\mathrm{Fc}=5.88$ resulta solo el $5 \%$. Para $\mathrm{T}^{0}=200{ }^{\circ} \mathrm{C}$ y $\mathrm{Fc}=5.88$, se requiere de un volumen de lecho de aproximadamente $0.09 \mathrm{~m}^{3}$, lo que representa un valor muy bajo para el tratamiento de un efluente gaseoso del orden de $10 \mathrm{Nm}^{3} / \mathrm{s}$.

Una característica notable que surge de los datos representados en la Fig. 2, es el hecho de que la relación $\left(\mathrm{V} / \mathrm{V}_{\text {ref }}\right) \mathrm{Fc}$ resulte virtualmente independiente de la temperatura de entrada al reactor, al menos para el rango de trabajo $\left(180-220^{\circ} \mathrm{C}\right)$. Es importante 
mencionar que para valores intermedios de $\mathrm{T}^{0}$, prácticamente no se observaron diferencias respecto a los extremos representados en la Fig. 2. Esto implica que el efecto del aumento de la temperatura adiabática (i.e., para un Fc determinado) es, en términos relativos, independiente del valor de $\mathrm{T}^{0}$. Dicha relación, $\mathrm{V} / \mathrm{V}_{\text {ref, }}$, puede expresarse de manera aproximada como: $\mathrm{V}=\mathrm{V}_{\text {ref }} \mathrm{Fc}^{(-1.7)}$, cuando ambos volúmenes, $\mathrm{V}$ y $\mathrm{V}_{\text {ref }}$ son evaluados a la misma temperatura $\mathrm{T}^{0}$. Esta relación nos permite ahorrar una gran cantidad de cálculos numéricos en la búsqueda de las variables operativas de diseño óptimas, debido a que el efecto de $\mathrm{T}^{0}$ tendría que ser evaluado numéricamente solo a $\mathrm{Fc}=1$ para la obtención de $\mathrm{V}_{\text {ref. }}$.

Analizando los resultados obtenidos a partir de la simulación del reactor, se tiene que el rango de temperatura de alimentación al reactor para el cual se realizaron los ensayos (i.e., $180-220^{\circ} \mathrm{C}$ ) resultó ser adecuado y, además, es compatible con las temperaturas de desorción analizadas en el Capítulo $6, \mathrm{~T}_{\mathrm{des}}\left(150-200{ }^{\circ} \mathrm{C}\right)$.

\subsection{Presentación de tres sistemas de eliminación de VOCs}

A continuación se presentan tres alternativas de sistemas de eliminación de VOCs a modo de poder evaluar (en la Sección 10.4) los diferentes requerimientos de catalizador y superficies de intercambio. En una de estas alternativas la corriente a tratar no será concentrada previo a su entrada al reactor, por lo que $\mathrm{Fc}=1$. Las otras dos situaciones corresponden a dos de los casos presentados en la Tabla 1 y fueron seleccionados a partir del análisis realizado en el Capítulo 6 (Sección 6.6.). Estos casos corresponden a $\mathrm{Fc}=3.67$ para una temperatura de desorción de $150{ }^{\circ} \mathrm{C}$ y $\mathrm{Fc}=5.88$ para una temperatura de desorción de $200^{\circ} \mathrm{C}$. Para todas las situaciones planteadas se adoptó una temperatura de $200{ }^{\circ} \mathrm{C}$ a la entrada del reactor catalítico (valor intermedio del rango analizado).

Las características de la corriente a tratar (efluente primario) son las mismas que han sido consideradas en capítulos previos. Las recordamos en la Tabla 2. 
Tabla 2: Características de la corriente a tratar

\begin{tabular}{|c|c|c|}
\hline \multicolumn{2}{|c|}{ Caudal $\left[\mathrm{m}^{3} / \mathbf{s}\right]$} & 11.7 \\
\hline $\begin{array}{c}\text { Composición } \\
{\left[\mathbf{m o l} / \mathbf{m}^{3}\right]}\end{array}$ & Acetato de Etilo & $2.3510^{-2}$ \\
\cline { 2 - 3 } & Etanol & $9.6310^{-3}$ \\
\hline \multicolumn{2}{|c|}{ Tipo de emisión } & Permanente \\
\hline \multicolumn{2}{|c|}{ Presión [atm] } & 1 \\
\hline \multicolumn{2}{|r|}{ Temperatura $\left[{ }^{\circ} \mathbf{C}\right]$} & 50 \\
\hline
\end{tabular}

En cada una de las alternativas mencionadas, la corriente a tratar ingresa a un soplador, cuyas características se describirán posteriormente (Sección 10.5), y sale a una presión equivalente a 1.3 atm.

\subsection{1 $F_{C}=1$}

En principio se darán a conocer las características del sistema de eliminación que no incluye la etapa previa de concentración. En este caso el sistema consta de un reactor catalítico y un intercambiador de calor (I1 en la Fig. 1 del Capítulo 9). Este último permite elevar la temperatura de la corriente a tratar $\left(50^{\circ} \mathrm{C}\right)$ hasta la temperatura de entrada al reactor $\left(200^{\circ} \mathrm{C}\right)$ empleando el calor generado por la propia reacción.

Retomando la situación particular de $\mathrm{Fc}=1(\mathrm{~W}=12.72 \mathrm{~kg} / \mathrm{s})$, las características geométricas resultantes para el reactor se presentan en la siguiente tabla:

Tabla 3: Características del reactor catalítico $(\mathrm{Fc}=1)$

\begin{tabular}{c|c}
\hline Diámetro interno $\left(\mathbf{D}_{\text {in }}\right)$ & $0.25 \mathrm{~m}$ \\
Diámetro externo $\left(\mathbf{D}_{\mathbf{e x}}\right)$ & $0.90 \mathrm{~m}$ \\
Altura $(\mathbf{H})$ & $3.18 \mathrm{~m}$ \\
\hline Masa de catalizador $\left(\mathbf{m}_{\text {cat }}\right)$ & $3080.4 \mathrm{~kg}$ \\
\hline Pérdida de Carga $(\mathbf{\Delta P})$ & $0.17 \mathrm{~atm}$ \\
\hline
\end{tabular}

A la salida del reactor la corriente alcanza una temperatura de $255^{\circ} \mathrm{C}$.

Como intercambiador de calor se seleccionó un intercambiador de flujo cruzado tipo “plate-fin” (Capítulo 9). Ciertas características del mismo fueron definidas en el Capítulo 9 (Tabla 1). Como fuera mencionado en el capítulo previo, las dimensiones 
globales del mismo y de los canales se definieron a partir de considerar los valores recomendados de velocidad del aire $(20 \mathrm{~m} / \mathrm{s})$ y no superar el exceso de área en más de un $5 \%$. Tales dimensiones junto a la masa de acero al carbono empleada y la pérdida de carga, se presentan en la Tabla 4 (densidad del acero al carbono: $\left.7850 \mathrm{~kg} / \mathrm{m}^{3}\right)$.

Tabla 4: Características del intercambiador I1 $(\mathrm{Fc}=1)$

\begin{tabular}{|c|c|}
\hline \multicolumn{2}{|l|}{ Dimensiones Globales } \\
\hline Altura del intercambiador $\left(\mathbf{H}_{12}\right)$ & $0.60 \mathrm{~m}$ \\
\hline Ancho del intercambiador $\left(W_{1}\right)$ & $2.15 \mathrm{~m}$ \\
\hline Profundidad de intercambiador $\left(L_{2}\right)$ & $2.45 \mathrm{~m}$ \\
\hline \multicolumn{2}{|c|}{ Dimensiones de los Canales } \\
\hline Alto del canal $\left(h_{12}\right)$ & $5.55 \mathrm{~mm}$ \\
\hline Ancho del canal $\left(s_{12}\right)$ & $1.85 \mathrm{~mm}$ \\
\hline Masa de acero al carbono $\left(\mathbf{m}_{\mathrm{ac}}\right)$ & $4239 \mathrm{~kg}$ \\
\hline Pérdida de Carga $(\Delta P)$ & $0.12 \mathrm{~atm}$ \\
\hline
\end{tabular}

Puesto que se requiere que la corriente a tratar $\left(50^{\circ} \mathrm{C}\right)$ alcance la temperatura de entrada al reactor $\left(200^{\circ} \mathrm{C}\right)$ y conociendo la temperatura de la corriente a la salida del mismo $\left(255^{\circ} \mathrm{C}\right)$, a partir de los balances entálpicos, es posible determinar la temperatura de la corriente caliente a la salida del intercambiador I1. En este caso se tiene una temperatura de salida de la corriente caliente de $105.55^{\circ} \mathrm{C}$.

Para todas las configuraciones presentadas, vinculamos " 1 " con la corriente fría y "2" con la corriente caliente.

\subsubsection{Fc $=3.67$}

Para la situación en la cual se considera $\mathrm{Fc}=3.67$ se debe incluir un sistema de adsorción/desorción al sistema de eliminación de VOCs. Las características geométricas del concentrador se encuentran definidas en el Capítulo 6 para uno de los puntos evaluados en la Fig. 5. Las mismas se recuerdan en la Tabla 5. 
Tabla 5: Características del concentrador rotatorio

\begin{tabular}{c|c}
\hline Diámetro del rotor $(\mathbf{D})$ & $4 \mathrm{~m}$ \\
Longitud del rotor $(\mathbf{L})$ & $0.70 \mathrm{~m}$ \\
Relación entre áreas $\left(\mathbf{S}_{\text {ads }} / \mathbf{S}_{\text {des }}\right)$ & 1.7 \\
\hline Masa de carbón activado $\left(\mathbf{m}_{\text {ads }}\right)$ & $1751.6 \mathrm{~kg}$ \\
\hline Pérdida de Carga $(\boldsymbol{\Delta P})$ & $0.035 \mathrm{~atm}$ \\
\hline
\end{tabular}

Las características del material adsorbente y de los canales de la matriz monolítica, fueron presentadas en las Tablas 3 y 4 del Capítulo 5 respectivamente. La relación entre las áreas correspondientes a los procesos de adsorción y desorción fue seleccionada con el propósito de minimizar la pérdida de carga (Capítulo 6).

La corriente a tratar abandona la zona de adsorción a una temperatura de $58.26{ }^{\circ} \mathrm{C}$, alcanzando una pérdida de carga de 0.02 atm.

En esta alternativa la temperatura de regeneración del lecho es de $150{ }^{\circ} \mathrm{C}$ y el caudal empleado $\left(\mathrm{W}_{\mathrm{des}}^{\mathrm{mín}}\right)$ es de $3.47 \mathrm{~kg} / \mathrm{s}\left(\mathrm{Fc}=\mathrm{Fc}^{\text {máx }}=3.67\right)$, de esta manera resulta que la corriente de aire + VOCs abandona la zona de desorción a una temperatura de $119.56^{\circ} \mathrm{C}$ con una pérdida de carga de $0.015 \mathrm{~atm}$.

Las características geométricas del reactor se presentan en la Tabla 6.

Tabla 6: Características del reactor catalítico $(\mathrm{Fc}=3.67)$

\begin{tabular}{c|c}
\hline Diámetro externo $\left(\mathbf{D}_{\text {in }}\right)$ & $0.25 \mathrm{~m}$ \\
Diámetro externo $\left(\mathbf{D}_{\text {ex }}\right)$ & $0.64 \mathrm{~m}$ \\
Altura $(\mathbf{H})$ & $0.72 \mathrm{~m}$ \\
\hline Masa de catalizador $\left(\mathbf{m}_{\text {cat }}\right)$ & $328.7 \mathrm{~kg}$ \\
\hline Pérdida de Carga $(\Delta \mathbf{P})$ & $0.21 \mathrm{~atm}$ \\
\hline
\end{tabular}

A la salida del reactor la corriente alcanza una temperatura de $400.7^{\circ} \mathrm{C}$.

Para esta alternativa se deben emplear dos intercambiadores, uno que permita elevar la temperatura de la corriente previo a su ingreso al reactor (I1 en Capítulo 9), y otro para precalentar el aire utilizado para la regeneración del adsorbedor (I2 en Capítulo 9). En la siguiente tabla se describen las características geométricas de I1 junto a la masa de acero al carbono empleada y la pérdida de carga total, es decir, $\Delta \mathrm{P}_{1}+\Delta \mathrm{P}_{2}$. 
Tabla 7: Características del intercambiador I1 $(\mathrm{Fc}=3.67)$

\begin{tabular}{|c|c|}
\hline \multicolumn{2}{|l|}{ Dimensiones Globales } \\
\hline Altura del intercambiador $\left(\mathrm{H}_{12}\right)$ & $0.86 \mathrm{~m}$ \\
\hline Ancho del intercambiador $\left(W_{1}\right)$ & $0.42 \mathrm{~m}$ \\
\hline Profundidad de intercambiador $\left(L_{2}\right)$ & $0.61 \mathrm{~m}$ \\
\hline \multicolumn{2}{|c|}{ Dimensiones de los Canales } \\
\hline Alto del canal $\left(h_{12}\right)$ & $11.1 \mathrm{~mm}$ \\
\hline Ancho del canal $\left(s_{12}\right)$ & $3.7 \mathrm{~mm}$ \\
\hline Masa de acero al carbono $\left(\mathbf{m}_{\mathrm{ac}}\right)$ & $130.3 \mathrm{~kg}$ \\
\hline Pérdida de Carga $(\Delta P)$ & $0.01 \mathrm{~atm}$ \\
\hline
\end{tabular}

Dado que la temperatura de la corriente de salida del proceso de regeneración es de $119.56{ }^{\circ} \mathrm{C}$ y se debe alcanzar una temperatura de $200{ }^{\circ} \mathrm{C}$ previo a su ingreso al reactor, conociendo la temperatura de la corriente a la salida del reactor $\left(400.7^{\circ} \mathrm{C}\right)$, a partir de los balances entálpicos se determina la temperatura de la corriente caliente a la salida del intercambiador. En este caso es de $321.33^{\circ} \mathrm{C}$.

Las características geométricas correspondientes al intercambiador I 2 se presentan en la Tabla 8.

Tabla 8: Características del intercambiador I2 $(\mathrm{Fc}=3.67)$

\begin{tabular}{|c|c|}
\hline \multicolumn{2}{|l|}{ Dimensiones Globales } \\
\hline Altura del intercambiador $\left(\mathbf{H}_{12}\right)$ & $0.76 \mathrm{~m}$ \\
\hline Ancho del intercambiador $\left(\mathbf{W}_{1}\right)$ & $0.41 \mathrm{~m}$ \\
\hline Profundidad de intercambiador $\left(L_{2}\right)$ & $0.60 \mathrm{~m}$ \\
\hline \multicolumn{2}{|c|}{ Dimensiones de los Canales } \\
\hline Alto del canal $\left(h_{12}\right)$ & $8.33 \mathrm{~mm}$ \\
\hline Ancho del canal $\left(s_{12}\right)$ & $2.78 \mathrm{~mm}$ \\
\hline Masa de acero al carbono $\left(\mathbf{m}_{\mathrm{ac}}\right)$ & $147 \mathrm{~kg}$ \\
\hline Pérdida de Carga $(\Delta P)$ & $0.02 \mathrm{~atm}$ \\
\hline
\end{tabular}

Restaría definir la temperatura de la corriente caliente a la salida de I2; mediante el empleo de los balances entálpicos resulta $231.56{ }^{\circ} \mathrm{C}$. Según el esquema propuesto en la Fig. 3 del Capítulo 2, una vez que esta corriente abandona el intercambiador I2, 
se mezcla con la fracción de la corriente limpia que no fue empleada para la regeneración del concentrador. De esta manera la corriente es liberada a la atmósfera a una temperatura de $105.6^{\circ} \mathrm{C}$ (igual que para $\mathrm{Fc}=1$ ). Esta corriente de aire caliente podría utilizarse en sistemas de calefacción de las instalaciones industriales.

\subsubsection{Fc $=5.88$}

Igual que para la alternativa anterior $(\mathrm{Fc}=3.67)$, para el caso donde $\mathrm{Fc}=5.88$ las características geométricas del sistema de asorción/desorción ya están definidas (Capítulo 6) y son las mismas que las previamente descriptas (Tabla 5). Por lo tanto, la temperatura de la corriente a la salida de la zona de adsorción y la pérdida de carga resultan iguales.

Sin embargo, en esta alternativa la temperatura de regeneración del lecho es de $200{ }^{\circ} \mathrm{C}$ y el caudal empleado es de $2.16 \mathrm{~kg} / \mathrm{s}(\mathrm{Fc}=5.88)$, por lo tanto la temperatura de la corriente al abandonar la zona de desorción y la pérdida de carga alcanzada, son diferentes. En este caso la temperatura de la corriente a la salida de la zona de desorción es de $136.1^{\circ} \mathrm{C}$ y la pérdida de carga es de $0.01 \mathrm{~atm}$. En consecuencia, la pérdida de carga total para el concentrador es $0.03 \mathrm{~atm}$.

Las características geométricas del reactor se encuentran en la siguiente tabla:

Tabla 9: Características del reactor catalítico $(\mathrm{Fc}=5.88)$

\begin{tabular}{c|c}
\hline Diámetro externo $\left(\mathbf{D}_{\text {in }}\right)$ & $0.25 \mathrm{~m}$ \\
Diámetro externo $\left(\mathbf{D}_{\mathbf{e x}}\right)$ & $0.60 \mathrm{~m}$ \\
Altura $(\mathbf{H})$ & $0.40 \mathrm{~m}$ \\
\hline Masa de catalizador $\left(\mathbf{m}_{\text {cat }}\right)$ & $153.4 \mathrm{~kg}$ \\
\hline Pérdida de Carga $(\boldsymbol{\Delta} \mathbf{P})$ & $0.27 \mathrm{~atm}$ \\
\hline
\end{tabular}

A la salida del reactor la corriente alcanza una temperatura de $520.78^{\circ} \mathrm{C}$.

Para esta alternativa también deben emplearse dos intercambiadores, I1 y I2. Las características geométricas de I1 se presentan en la Tabla 10. 
Tabla 10: Características del intercambiador I1 $(\mathrm{Fc}=5.88)$

\begin{tabular}{|c|c|}
\hline \multicolumn{2}{|l|}{ Dimensiones Globales } \\
\hline Altura del intercambiador $\left(\mathrm{H}_{12}\right)$ & $0.88 \mathrm{~m}$ \\
\hline Ancho del intercambiador $\left(W_{1}\right)$ & $0.25 \mathrm{~m}$ \\
\hline Profundidad de intercambiador $\left(L_{2}\right)$ & $0.44 \mathrm{~m}$ \\
\hline \multicolumn{2}{|c|}{ Dimensiones de los Canales } \\
\hline Alto del canal $\left(h_{12}\right)$ & $14.7 \mathrm{~mm}$ \\
\hline Ancho del canal $\left(s_{12}\right)$ & $4.9 \mathrm{~mm}$ \\
\hline Masa de acero al carbono $\left(\mathbf{m}_{\mathrm{ac}}\right)$ & $44.6 \mathrm{~kg}$ \\
\hline Pérdida de Carga $(\Delta P)$ & $0.01 \mathrm{~atm}$ \\
\hline
\end{tabular}

Dado que la temperatura de la corriente de salida del proceso de regeneración es de $136.1{ }^{\circ} \mathrm{C}$ y se debe alcanzar una temperatura de $200{ }^{\circ} \mathrm{C}$ previo a su ingreso al reactor, conociendo la temperatura de la corriente a la salida del reactor $\left(520.78^{\circ} \mathrm{C}\right)$, a partir de los balances entálpicos se determina la temperatura de la corriente caliente a la salida del intercambiador. En este caso es de $458.23^{\circ} \mathrm{C}$.

Las características del intercambiador I2 se presentan en la siguiente tabla:

Tabla 11: Características del intercambiador $\mathrm{I} 2(\mathrm{Fc}=5.88)$

\begin{tabular}{c|c}
\hline \multicolumn{2}{c}{ Dimensiones Globales } \\
\hline Altura del intercambiador $\left(\mathbf{H}_{\mathbf{1 2}}\right)$ & $0.65 \mathrm{~m}$ \\
Ancho del intercambiador $\left(\mathbf{W}_{\mathbf{1}}\right)$ & $0.33 \mathrm{~m}$ \\
Profundidad de intercambiador $\left(\mathbf{L}_{\mathbf{2}}\right)$ & $0.54 \mathrm{~m}$ \\
Volumen de acero al carbono & $0.012 \mathrm{~m}^{3}$ \\
\hline \multicolumn{2}{c}{ Dimensiones de los Canales } \\
\hline Alto del canal $\left(\mathbf{h}_{\mathbf{1 2}}\right)$ & $8.33 \mathrm{~mm}$ \\
Ancho del canal $\left(\mathbf{s}_{\mathbf{1 2}}\right)$ & $2.78 \mathrm{~mm}$ \\
\hline Masa de acero al carbono $\left(\mathbf{m}_{\mathbf{a c}}\right)$ & $92 \mathrm{~kg}$ \\
\hline Pérdida de Carga $(\mathbf{\Delta P})$ & $0.02 \mathrm{~atm}$ \\
\hline
\end{tabular}

Restaría definir la temperatura de la corriente caliente a la salida de I2; mediante el empleo de los balances entálpicos resulta $321.63{ }^{\circ} \mathrm{C}$. Al mezclarse con la fracción de aire limpio no empleada para la regeneración del concentrador, la corriente, al 
igual que para las alternativas propuestas previamente, es liberada a la atmósfera a una temperatura de $105^{\circ} \mathrm{C}$ aproximadamente.

\subsection{Evaluación de resultados}

Resulta apropiado abordar una discusión de tipo económica que nos permita realizar una comparación entre las alternativas planteadas en la sección anterior. Para dicho análisis se debe tener en cuenta el tipo de material a emplear en cada uno de los equipos que integran el sistema combinado propuesto (concentrador, reactor e intercambiadores) y la forma en que se los debe proveer.

Como material adsorbente se empleó carbón activado, el mismo es muy utilizado industrialmente y, comparado con otros materiales adsorbentes, es significativamente más económico. Su costo dependerá de cómo se lo provea. El hecho de que se lo requiera en una estructura monolítica implica un costo de manufactura más alto que si se lo obtiene en forma particulada. Sin embargo, debe tenerse en cuenta que para el caso de emplear el carbón activado en forma particulada se requiere mayor cantidad de adsorbente, si se pretende alcanzar las mismas especificaciones de pérdida de carga y concentración a la salida. Dicha comparación se realizó en el Capítulo 5, donde se demostró que resulta una diferencia de aproximadamente el $50 \%$.

Por otro lado, el catalizador empleado para nuestro sistema de estudio, en base a óxidos de cobre y manganeso, fue sintetizado en el laboratorio, por lo que no se comercializa ni se emplea a nivel industrial. Su falta de comercialización implica que no se pueda obtener información precisa en cuanto al costo del mismo y nos induce a pensar que podría ser relativamente más costoso que el carbón activado.

Por último, el acero al carbono, empleado para los intercambiares de calor, es un material con un amplio grado de comercialización y su costo, al igual que el carbón activado, está fuertemente ligado a su manufactura.

Si bien no fue posible abordar un análisis económico detallado del sistema debido a la falta de información de los costos comerciales de todos los materiales a emplear y los costos de manufactura de los equipos correspondientes, es factible estimar la conveniencia que genera la incorporación de un concentrador, a partir de evaluar la disminución de los requerimientos de catalizador y superficie de intercambio.

En la Tabla 12 se presenta la información que resulta conveniente destacar a partir de las alternativas de sistemas de eliminación de VOCs planteadas en la sección anterior. 
Tabla 12: Impacto del Fc sobre los equipos a emplear en el sistema de eliminación

\begin{tabular}{l|ccc}
\hline Fc & $\mathbf{1}$ & $\mathbf{3 . 6 7}$ & $\mathbf{5 . 8 8}$ \\
Masa de Carbón Activado $[\mathrm{kg}]$ & 0 & 1751.6 & 1751.6 \\
Masa de Catalizador $\left(\mathbf{M n}_{9} \mathbf{C u}_{\mathbf{1}}\right)[\mathrm{kg}]$ & 3080.4 & 328.7 & 153.4 \\
Masa de Acero al Carbono $[\mathrm{kg}]$ & 4239 & 277.3 & 136.6 \\
\hline
\end{tabular}

Analizando las masas de cada uno de los materiales en forma individual (Tabla 12), se puede concluir que la incorporación de un concentrador permite obtener un reactor e intercambiadores de calor mucho más pequeños. Cuanto mayor es el grado de concentración de la corriente $(\mathrm{Fc})$, mayor es el efecto que causa sobre el tamaño de los equipos; para el reactor catalítico, este efecto también se observa en la Fig. 2. Para $\mathrm{Fc}=3.67$, se requiere de un $10.7 \%$ del catalizador y $6.5 \%$ del acero al carbono empleados para el caso de no incluir un sistema de adsorción/desorción $(\mathrm{Fc}=1)$. Para el mayor Fc evaluado (5.88) se requiere solo un 5 y $3 \%$ del catalizador y acero al carbono, respectivamente.

Si bien es cierto que para el caso de incluir un sistema de adsorción/desorción, se deben incorporar alrededor de $1750 \mathrm{~kg}$ de carbón activado, la cantidad de catalizador y acero al carbono a emplear son bastante menores que para cuando $\mathrm{Fc}=1$. Para $\mathrm{Fc}=3.67$, se requiere de aproximadamente $2750 \mathrm{~kg}$ menos de catalizador $\mathrm{y}$ $4000 \mathrm{~kg}$ menos de acero al carbono.

La significativa reducción de material catalítico y superficie de intercambio a emplear, y el hecho de que el costo comercial del catalizador podría ser más elevado que el del carbón activado, indicarían que la inclusión del sistema de adsorción/ desorción implica un ahorro económico que puede ser importante. Como se dijo anteriormente, a medida que el Fc aumenta, mayor es el efecto que causa sobre el tamaño del reactor e intercambiadores y, por lo tanto, mayor es el beneficio económico obtenido.

Para obtener valores de Fc cercanos a 6 se debe incrementar la temperatura de desorción, $\mathrm{T}_{\text {des }}$, a $200^{\circ} \mathrm{C}$. Para el caso de los intercambiadores, el mayor precalentamiento requerido para alcanzar esta temperatura será compensado largamente por un caudal menor y una mayor fuerza impulsora.

Vale la pena mencionar que para temperaturas de desorción más elevadas, que permitan alcanzar temperaturas a la salida del desorbedor superiores a $180{ }^{\circ} \mathrm{C}$, resultaría 
factible prescindir del intercambiador I1. Sin embargo, deben tenerse en cuenta ciertas consideraciones al momento de seleccionar la temperatura de desorción. Las mismas se encuentran relacionadas con el efecto de la temperatura sobre los materiales de adsorbente y catalizador. Por un lado, la velocidad con la cual se oxida el carbón activado con la temperatura (ver Capítulo 5), deberá ser incluido en el análisis, debido a que la frecuencia con la que se debe reemplazar el material adsorbente será un factor relevante a considerar desde el punto de vista económico. Por otro lado, las consecuencias del aumento de temperatura en el reactor catalítico también deberán ser tenidas en cuenta. Para $\mathrm{Fc}=5.88$, el aumento de temperatura en el reactor es de aproximadamente $320^{\circ} \mathrm{C}$, y la temperatura a la salida del mismo alcanza alrededor de los $520^{\circ} \mathrm{C}$. En consecuencia, la posibilidad de una disminución de la actividad catalítica para esos niveles térmicos también debería ser analizada.

Como fuera mencionado en la sección anterior, se fijó, para las tres alternativas presentadas, un valor de $200{ }^{\circ} \mathrm{C}$ para la temperatura de entrada al reactor, sin embrago, para definir este valor de temperatura se debe ponderar económicamente la disminución de la cantidad de catalizador con el aumento de área de intercambio de I1 para el precalentamiento de la corriente $\mathrm{y}$, adicionalmente, como ya se dijo, se debe realizar un análisis más detallado del impacto de las temperatura de salida del lecho (el nivel térmico más alto en el sistema) sobre los materiales a emplear.

\subsection{Selección de la unidad impulsora}

Para las alternativas de sistemas de eliminación de VOCs propuestas se obtuvo una pérdida de carga (incluyendo reactor, concentrador rotativo e intercambiadores de calor) de $0.3 \mathrm{~atm}$ para la corriente de aire conteniendo los VOCs. Debe aclararse que en el cálculo de la pérdida de carga no se contabilizó la asociada a cañerías y conexiones.

La selección de la unidad impulsora presenta alguna dificultad en virtud del valor relativamente grande del caudal a manejar y la baja elevación de presión requerido. Un equipo que puede funcionar adecuadamente es el soplante (también conocido como turbosoplante). Los soplantes son máquinas rotativas destinadas a comprimir generalmente aire, que pueden manejar relativamente altos caudales y en las que la relación de compresión, $\mathrm{R}$ (cociente entre la presión a la salida y a la entrada) se encuentra en el rango 1,1 - 4; no tienen ningún tipo de refrigeración incorporada y, en 
general, son de una única etapa. Se pueden clasificar en función de la relación de compresión de la siguiente forma:

Soplantes de baja presión: $\mathrm{R}=1,1$ a 1,5

Soplantes de media presión: $\mathrm{R}=1,5$ a 2,5

Soplantes de alta presión: $\mathrm{R}=2,5$ a 4

Para relaciones de compresión mayores se denominan turbocompresores o compresores rotativos. En nuestro caso sería conveniente emplear un soplante de baja presión, siendo que $\mathrm{R} \simeq 1.3$.

Para las condiciones específicas del problema la firma Howden Industrial (http://www.howdenindustrial.com) dispone de unidades como las mostradas en la Figura 3 con capacidad de hasta $6000 \mathrm{~m}^{3} / \mathrm{min}$ y elevaciones de presión de hasta $3 \mathrm{~atm}$ $\left(3.10^{5} \mathrm{~Pa}\right)$ en simple etapa. La Figura 4 proporcionada por la empresa permite seleccionar el tamaño del equipo y la potencia necesaria en función del caudal a la entrada y la elevación de presión requerida; en la misma se indican los resultados para el caso específico (teniendo en cuenta que el caudal volumétrico se debe evaluar a la temperatura indicada en el diagrama):

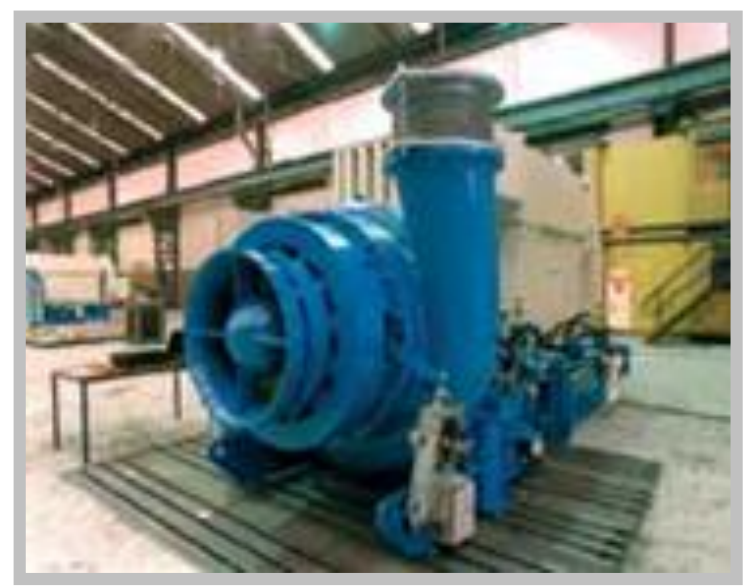

Caudal de $625.9 \mathrm{~m}^{3} \mathrm{~min}^{-1}(1 \mathrm{~atm}-$ $\left.15^{\circ} \mathrm{C}\right)$

Elevación de presión 0.3 atm $(0.3$ bar) : tamaño 75 , potencia $400 \mathrm{KW}$

Fig. 3. Turbo-soplante 


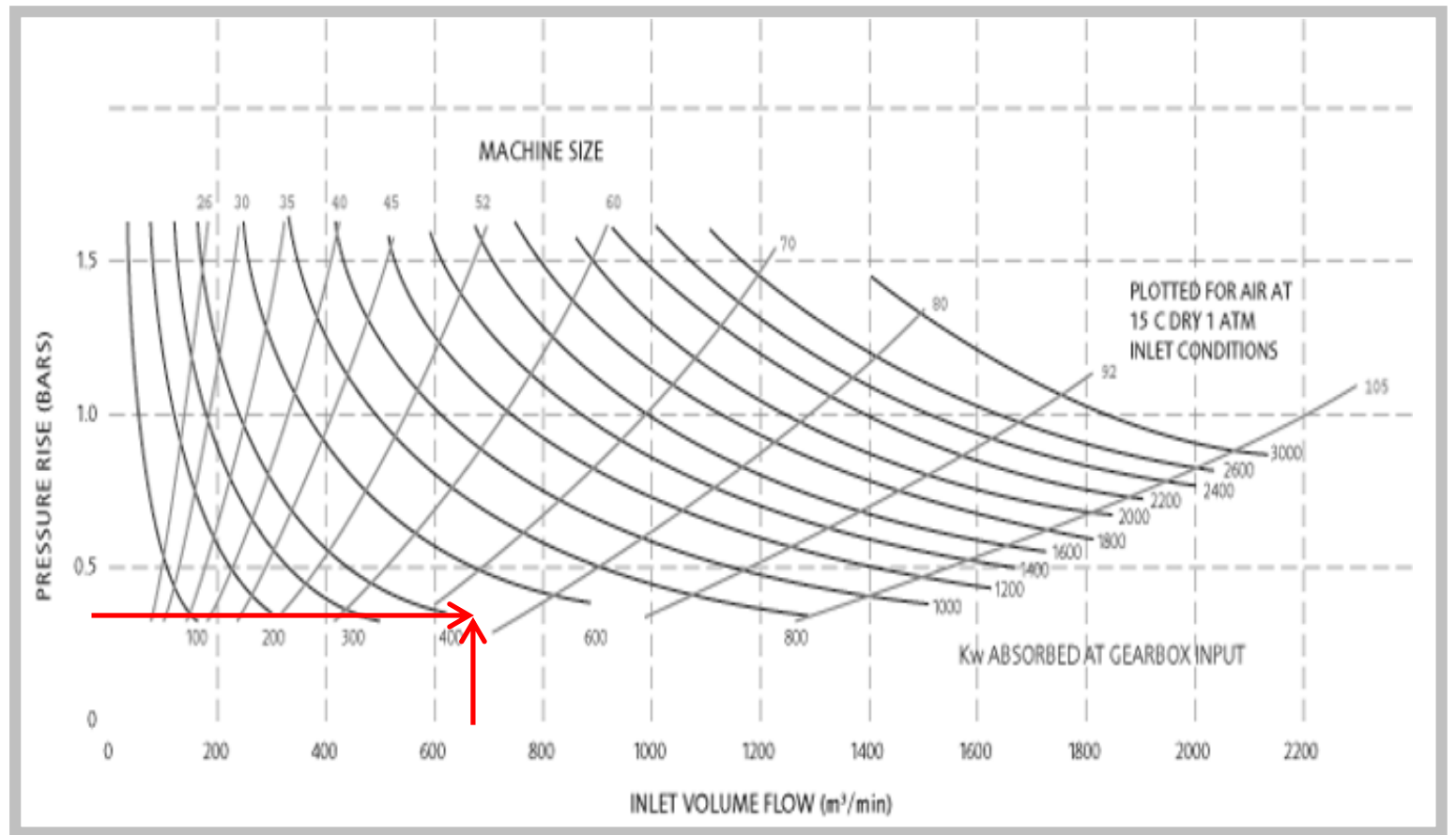

Fig. 4. Selección del tamaño de equipo y potencia en función del caudal a la entrada de la elevación de presión requerida ( $\rightarrow$ caso específico).

Otra alternativa pueden ser los denominados turbo-compresores $\mathrm{HV}$, ofrecidos por la empresa Aktiengesellschaft Kühnle, Kopp \& Kausch (http://www.agkkk.de/english/index.htm), que se muestran en la Figura 5. Los modelos de equipo adecuados conforme a la Figura 6 provista por la empresa son:

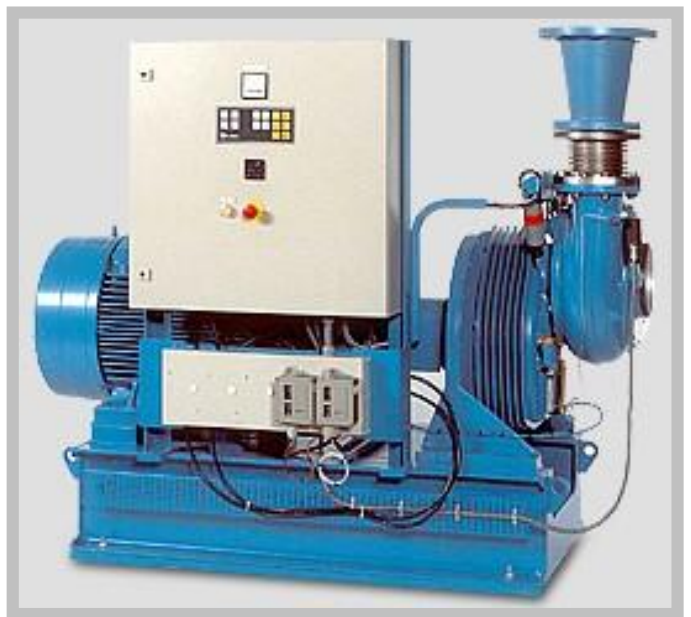

Fig. 5. Turbocompresor HV.
Caudal $702 \mathrm{~m}^{3} / \mathrm{min}\left(42120 \mathrm{~m}^{3} / \mathrm{h}\right)$

Elevación de presión $3.0310^{4} \mathrm{~Pa}(0,3$ bar): Modelo KA 66, potencia $450 \mathrm{KW}$ 


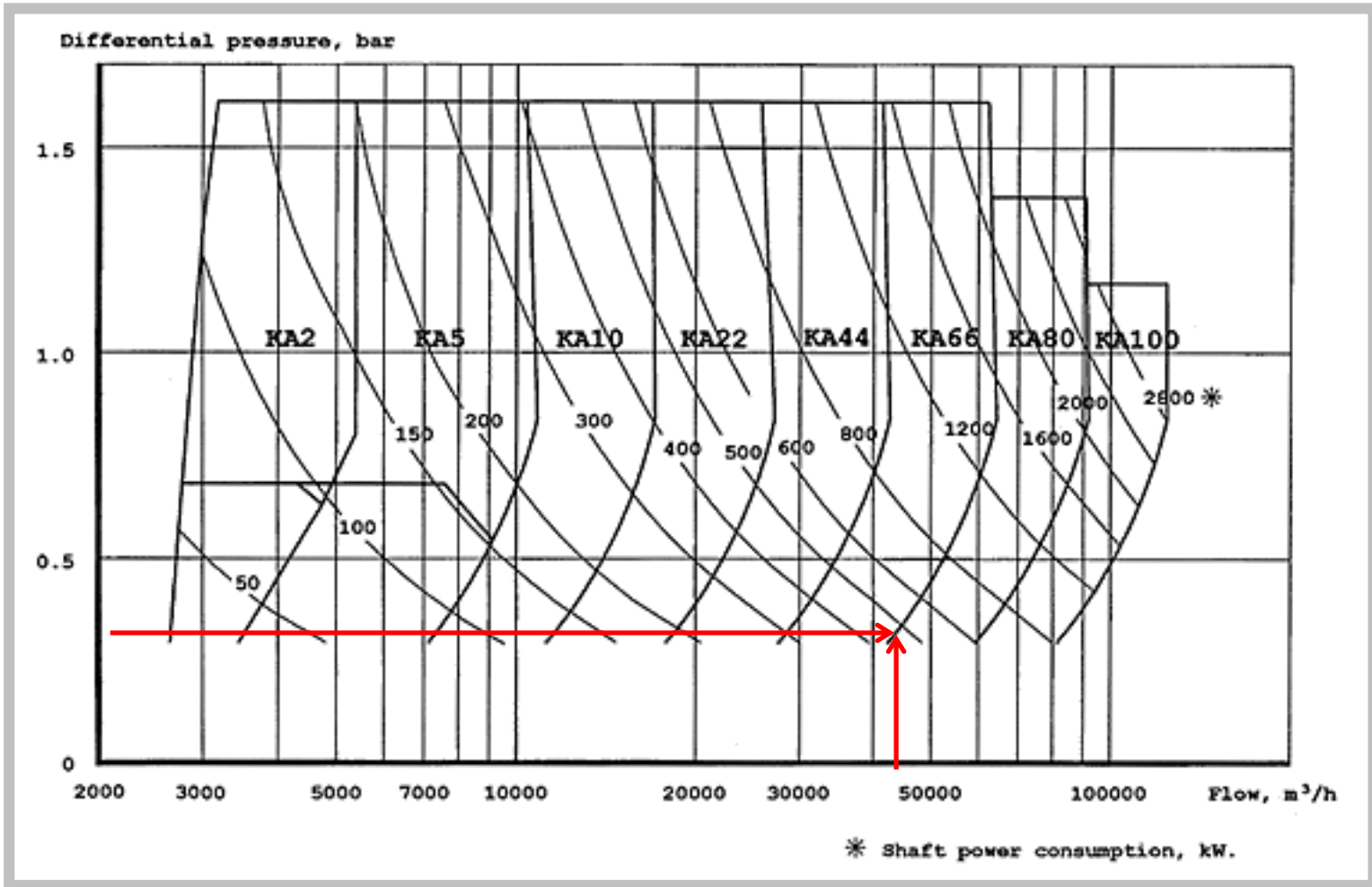

Fig. 6. Selección del tamaño de equipo y potencia en función del caudal a la entrada de la elevación de presión requerida ( $\rightarrow$ caso específico).

Es importante mencionar que, a los fines de la selección del impulsor se deben considerar posibles variaciones que puedan surgir en la corriente a tratar.

\subsection{Conclusiones}

Se evaluó el impacto que causa la incorporación de una etapa previa de concentración al sistema de eliminación catalítica de VOCs, sobre los requerimientos de catalizador y superficie de intercambio. Para dicha evaluación se definió el concepto de factor de concentración, Fc, como la relación entre el caudal del efluente a tratar y el alimentado al reactor. El análisis de los resultados indica que, en el rango de valores factibles de alcanzar para el factor Fc (menor que alrededor de seis), la disminución del volumen de lecho catalítico es altamente significativa. Por ejemplo, con $\mathrm{Fc}=3.67 \mathrm{la}$ cantidad de catalizador a utilizar es de solo un $10.5 \%$ de la requerida cuando no se concentran los VOCs (Tabla 12). En cuanto a los requerimientos de superficie de intercambio, el impacto es aun mayor, siendo que para $\mathrm{Fc}=3.67$, la cantidad de acero al carbono a emplear es del $6.5 \%$ de la requerida para $\mathrm{Fc}=1$ (Tabla 12).

Si bien es cierto que para el caso de incluir un sistema de adsorción/desorción, se deben incorporar alrededor de $1750 \mathrm{~kg}$ de carbón activado, la significativa reducción de material catalítico y superficie de intercambio a emplear, y el hecho de que el costo 
comercial del catalizador podría ser más elevado que el del carbón activado, indicarían que la inclusión del sistema de adsorción/desorción implica un ahorro económico que puede ser importante. A medida que el grado de concentración de la corriente $(\mathrm{Fc})$ aumenta, mayor es el efecto que causa sobre el tamaño de los equipos.

Cuanto mayor es la temperatura de la corriente empleada para la regeneración del adsorbedor, mayor es el Fc que se puede alcanzar. Sin embargo, al seleccionar la temperatura de desorción debe ser tenido en cuenta el efecto que este valor puede causar sobre el material adsorbente y el catalizador.

De acuerdo a la cinética desarrollada en el Capítulo 7, temperaturas de entrada al reactor $\left(\mathrm{T}^{0}\right)$ superiores a los $180{ }^{\circ} \mathrm{C}$, han resultado adecuadas para el inicio de las reacciones de combustión, por lo tanto, se adoptó un rango de $180-220^{\circ} \mathrm{C}$ para estudiar la influencia de $\mathrm{T}^{0}$ sobre el volumen de reactor. Como era de esperar para un sistema con elevadas energías de activación (Capítulo 7, Tabla 2), la temperatura, $\mathrm{T}^{0}$, presenta un importante efecto sobre el volumen requerido de catalizador (Fig. 1).

Finalmente se llevó a cabo la elección de un soplador adecuado, a partir de catálogos comerciales, para satisfacer los requerimientos de pérdida de carga de todo el sistema. 


N ESTUDIO DE SISTEMAS

COMBINADOS DE

COMBUSTION

CATALITICA DE VOCs

CONCLUSIONES Y RECOMENDACIONES 

El objetivo propuesto para este trabajo fue el estudio de sistemas de eliminación de Compuestos Orgánicos Volátiles (VOCs) por medio de la oxidación catalítica de los mismos. Se estudió en forma específica la destrucción catalítica de acetato de etilo y etanol producidos en corrientes efluentes de plantas de impresión de envases para alimentos. A partir de este estudio particular, se pretendió alcanzar conclusiones y desarrollos metodológicos que puedan llegar a extenderse a la eliminación de VOCs diferentes a los específicamente aquí considerados.

Las corrientes a tratar suelen tener altos caudales y temperaturas cercanas a la ambiente. Si bien el desarrollo de nuevos materiales catalíticos tiene un progreso permanente, permitiendo operar a menores temperaturas, estas son lo suficientemente elevadas como para que el consumo energético represente un desafío de importancia en el análisis de este tipo de procesos. Teniendo en cuenta que las reacciones de oxidación son altamente exotérmicas, la posibilidad del aprovechamiento de la energía liberada por las reacciones, a través de un intercambio térmico, constituye un camino atractivo de ahorro energético. Sin embargo, las corrientes con VOCs pueden ser relativamente diluidas, por lo que el salto térmico en el reactor es bajo, y por lo tanto también lo es la fuerza impulsora para el intercambio de calor. La posibilidad de realizar una preconcentración de la corriente a tratar, utilizando un sistema de adsorción/desorción representa una variante, que conduce a la utilización de sistemas combinados, que presentan una mayor complejidad, pero un camino alternativo para la disminución de costos, especialmente de tipo operativo. En este contexto de análisis de sistemas combinados, los estudios se orientaron hacia la propuesta de distintas opciones empleando un reactor catalítico, recuperación térmica en un intercambiador de calor y la inclusión o no de un sistema de adsorción/desorción.

\section{Sistema de adsorción/desorción}

La incorporación de un sistema de adsorción/desorción proporciona una corriente más concentrada y de menor caudal en la alimentación al reactor, lo que permite una mayor liberación de energía mediante su oxidación y consecuentemente mayor elevación de la temperatura en el reactor, disminuyendo en gran medida los 
requerimientos de catalizador y superficie de intercambio.

Entre las diferentes técnicas para llevar a cabo los procesos de adsorción y desorción, las más convencionales son la Temperature Swing Adsorption (TSA) y la Pressure Swing Adsorption (PSA). Teniendo en cuenta el sistema combinado propuesto, es factible el uso del calor liberado durante la reacción de oxidación para la obtención de la energía necesaria que permita el calentamiento de la corriente empleada para la desorción. Esto permitiría orientarse a un proceso autosustentable energéticamente para cuando se lleva a cabo el proceso de adsorción/desorción mediante el empleo de la técnica TSA. En cambio, cuando la técnica a emplear es la PSA necesariamente se debe recurrir a requerimientos adicionales de compresión, y por ende de energía, para la presurización de la corriente a tratar. Dado que se pretende alcanzar el menor requerimiento de energía posible, se decidió optar por la TSA.

Para la adsorción de VOCs, se han realizado estudios empleando adsorbentes de diferentes tipos, como carbones activados, zeolitas o adsorbentes poliméricos. Teniendo en cuenta el tipo (etanol y acetato de etilo) y concentración de VOCs y el costo del adsorbente, se optó por el uso de carbón activado. El mismo puede emplearse en forma particulada o estructurada (monolito). A partir de información bibliográfica se obtuvieron las características y los parámetros de adsorción de los VOCs en estudio, acetato de etilo y etanol, sobre carbón activado.

Para el caso del monolito, el adsorbente se supuso depositado sobre las paredes de la matriz monolítica, o bien, constituyendo el material de las mismas. El análisis realizado permitió concluir que resulta conveniente la segunda alternativa.

Para la simulación del sistema de adsorción/desorción se tuvieron en cuenta dos enfoques. La diferencia de los mismos estriba en incluir, o no, las resistencias a la transferencia de calor y materia dentro y fuera del material adsorbente.

En el caso de la inclusión de las resistencias a la transferencia de calor y materia dentro y fuera del sólido (caso real), se deben resolver los correspondientes balances de calor y materia. Para la resolución de los mismos en el medio poroso (adsorbente), se propuso el Método de Colocaciones Ortogonales. Para la resolución de los balances de materia y energía en la corriente de fluido se retuvo el Método Cuasi-Análitico propuesto por Matros y col. (1999).

Para nuestro caso particular de estudio se analizó el efecto de las resistencias a la transferencia de calor y materia según el tipo de lecho considerado, i.e., lecho relleno o monolito. Para un valor de pérdida de carga y tiempo de adsorción similares, se observó 
que para el lecho relleno se requiere aproximadamente el doble de masa de material adsorbente. Esto se debe principalmente a la disposición del material adsorbente en cada uno de los lechos, lo que genera que la longitud característica para la transferencia de materia en el medio poroso sea mucho mayor en el lecho relleno que en el monolito y, por lo tanto, resulta en una mayor resistencia a la transferencia de materia. Como consecuencia de este efecto, en el lecho relleno se genera una mayor dispersión del frente de concentración. Este tipo de comportamiento da lugar a que, para el lecho relleno analizado, ningún sector del mismo se encuentra completamente saturado al finalizar la etapa de adsorción, mientras que una gran porción del monolito si lo está. De esta forma, el lecho relleno resultó claramente menos eficiente que el monolito.

Considerando los efectos que proporciona la disposición del material adsorbente, el monolito resultó ser el tipo de lecho más apropiado para el sistema de eliminación propuesto.

Se estudiaron dos alternativas de diseño para los sistemas de adsorción/desorción, consistentes en un sistema de dos lechos que operan alternativamente o un concentrador rotativo. Este último, garantiza una uniformidad de la concentración y temperatura de la corriente a la salida del mismo, lo cual constituye un aspecto de importancia para la operación en estado estacionario del reactor y los sistemas de intercambio. Teniendo en cuenta esta particularidad, se optó por un concentrador rotativo para el sistema de eliminación propuesto.

Una variable operativa de importancia es el caudal empleado en el proceso de desorción, dado que cuanto menor es el caudal empleado se alcanza una mayor concentración de VOCs. Se verificó que el caudal másico de desorción presenta un valor mínimo, $\mathrm{W}_{\mathrm{des}}^{\min }$, para el cual es posible sostener en el tiempo los ciclos de adsorción/desorción. Por debajo de este valor el sistema se torna inestable. Se analizó el impacto que causan ciertas variables geométricas sobre el valor del caudal mínimo de desorción, comprobándose que, para un valor de volumen constante, resulta virtualmente indistinta la relación diámetro/longitud (D/L) adoptada. Lo mismo se observó para valores mayores a uno de relación de áreas destinadas a los procesos de adsorción y desorción, $S_{\text {ads }} / S_{\text {des }}$. Tal valor pudo optimizarse a partir de minimizar la pérdida de carga del sistema. Para el caso en que $\mathrm{S}_{\mathrm{ads}} / \mathrm{S}_{\mathrm{des}}<1$, el caudal mínimo adopta valores más elevados, por lo que esta alternativa no resulta una buena opción. 
Se observó que, al aumentar la temperatura de regeneración del lecho, de150 ${ }^{\circ} \mathrm{C}$ a $200{ }^{\circ} \mathrm{C}$, el valor del caudal mínimo de desorción disminuye aproximadamente un 40 \%. Esto verifica que a mayores temperaturas de regeneración, más eficiente resulta el proceso de desorción. Sin embargo a mayores temperaturas aumenta el grado de consumo del carbón (a temperaturas por encima de los $300{ }^{\circ} \mathrm{C}$ el carbón pierde estabilidad térmica) lo que provoca que se deba acortar el tiempo de recambio del material adsorbente.

Otro aspecto analizado fue el impacto de la cantidad de material adsorbente sobre el valor del caudal mínimo. Para las condiciones establecidas para el análisis se observó una significativa sensibilidad del caudal mínimo con la cantidad de adsorbente a emplear. Se obtuvieron resultados para dos temperaturas de desorción, 150 y $200{ }^{\circ} \mathrm{C}$. Para el diseño del sistema de adsorción/desorción, se consideró adecuado adoptar un rango entre 1.5 y $3 \mathrm{~m}^{3}$ de adsorbente. Dicho rango resultó ser independiente de la temperatura de regeneración del lecho y para los extremos del mismo, la Zona de Transferencia de Masa (ZTM) representó entre un 60 y un $40 \%$ del adsorbedor al finalizar la etapa de adsorción. Estos resultados pueden ser considerados como un criterio de diseño del sistema de adsorción/desorción para un caso general.

A partir de haber definido un volumen de adsorbente y especificar un valor para la pérdida de carga, se establecieron las dimensiones globales del lecho, diámetro (D) y longitud (L).

Para resolver el sistema sin resistencias (caso ideal) se aplicó el Método de las Características. Este método permite reemplazar el sistema de ecuaciones diferenciales de derivadas parciales de primer orden con dos variables independientes (tiempo y espacio) por un sistema de ecuaciones diferenciales ordinarias con una única variable independiente.

Se compararon los perfiles de concentración obtenidos a partir de considerar o no las resistencias a la transferencia de calor y materia. A pesar de que las resistencias provocan una dispersión sobre el frente de avance de la concentración, se observó una gran similitud entre los perfiles de concentración, tanto para la etapa de adsorción como para la de desorción. Esto indicaría que es factible adoptar el caso ideal como una buena aproximación para simular el comportamiento del sistema.

En base a las características que presentó el caso ideal, resulta apropiado considerar al acetato de etilo como un único componente presente en la corriente a tratar, cuyo frente de concentración se traslada isotérmicamente a lo largo del lecho. A partir de 
estas observaciones, se propuso un método aproximado que, teniendo en cuenta el valor de la concentración de VOC permitida a la salida y el porcentaje que debe alcanzar la ZTM al finalizar la etapa de adsorción, permite determinar el tiempo de adsorción y la cantidad de material adsorbente requerida para el diseño del sistema de adsorción/desorción.

Finalmente se tiene que el valor de caudal mínimo puede ser estimado a partir de considerar el valor teórico que surge del caso ideal, se opta por un caudal un 25\% mayor al valor teórico. La resolución del caso ideal junto con el empleo de la aproximación propuesta, proporcionan un esfuerzo de cálculo significativamente menor con respecto al presentado para el caso real, con resultados relativamente precisos.

\section{Reactor Catalítico}

Los catalizadores usados para la eliminación de VOCs incluyen como materiales activos a los óxidos metálicos o metales nobles, como platino (Pt) o paladio (Pd). Se han estudiado en bibliografía diferentes catalizadores para la eliminación de los compuestos específicos, acetato de etilo y etanol, sin embargo, dichos trabajos no suministran la información cinética requerida para llevar a cabo el análisis y diseño de un reactor. En la búsqueda de catalizadores que permitan trabajar a menores temperaturas, en el INTEQUI (CONICET/UNSL) se han desarrollado catalizadores en base a óxidos de $\mathrm{Cu}$ y $\mathrm{Mn}$, que demostraron ser altamente eficientes para la oxidación de etanol y acetato de etilo. En virtud del vínculo entre los grupos de trabajo del INTEQUI y CINDECA, se dispuso de la información experimental, lo que permitió emplear la misma para determinar un esquema cinético de reacción, proponer las expresiones para las velocidades de reacción de cada componente y obtener los valores de los parámetros cinéticos.

Se verificó que la oxidación de etanol produce acetaldehído, el que pasa directamente a dióxido de carbono, constituyendo un sistema de reacciones en serie, y se descartó un posible camino de combustión directa de etanol a dióxido de carbono. Por otro lado la combustión del acetato de etilo produce directamente $\mathrm{CO}_{2}$.

Se observó que los productos finales de reacción no tienen incidencia en la velocidad de combustión de los VOCs.

Se analizó la interacción entre los diferentes VOCs al oxidarse una mezcla de los mismos, verificándose que existe una influencia mutua en el comportamiento cinético 
generada por los términos de inhibición en los cuales se incluyen el acetaldehído y el acetato de etilo.

Los datos experimentales se ajustaron satisfactoriamente empleando expresiones de tipo LHHW, tanto para la oxidación de etanol a acetaldehído como para las combustiones totales de este último y de acetato de etilo. Se ajustaron satisfactoriamente las energías de activación y constantes específicas de todas las reacciones y las constantes de equilibrio de adsorción del acetaldehído y acetato de etilo. La constante de equilibrio de adsorción del etanol condujo a valores muy pequeños y se fijo un valor nulo para la misma.

Una vez establecida la información cinética correspondiente a nuestro caso particular de estudio, fue posible llevar a cabo el diseño y análisis del reactor catalítico presente en el sistema de eliminación. Se propuso un reactor de lecho fijo con catalizador granular de forma esférica y se adoptó un sistema de flujo radial, lo que permitió reducir sustancialmente la pérdida de carga. Con la misma finalidad, se adoptó un tamaño de catalizador con $\mathrm{d}_{\mathrm{p}}=3 \mathrm{~mm}$.

Se tuvieron en cuenta las resistencias a la transferencia de calor y materia entre el seno de la corriente y la superficie de las partículas catalíticas y la resistencia a la transferencia de materia en el interior del catalizador. Esta última resultó ser muy elevada debido al tamaño de pastilla adoptado.

Se debieron resolver numéricamente los balances de conservación de las tres especies en el interior de las pastillas en cada posición a lo largo del lecho, a fin de cuantificar las velocidades efectivas de consumo. Tal procedimiento, que se encuentra acoplado con el de resolución de los balances en la corriente de gas, presentó, en numerosos casos, problemas de estabilidad, presumiblemente debido a la alta sensibilidad de las reacciones con la temperatura, por lo que no fue posible completar la simulación del reactor. La alternativa para solucionar el problema fue emplear una aproximación explícita para las velocidades efectivas de las tres reacciones principales que tienen lugar en la incineración. La misma evidenció ser altamente precisa y eficiente, dado que permitió eliminar los problemas numéricos enunciados, como así también disminuir el tiempo de cálculo en más de un orden de magnitud. Cabe destacar que la aproximación desarrollada puede aplicarse para una familia de problemas cinéticos similares. 


\section{Sistema de recuperación de calor}

El esquema del sistema de eliminación de VOCs propuesto, incluye dos recuperadores de calor que emplean el calor generado por la reacción. A partir de analizar las características que presentan las corrientes a tratar, se seleccionó un intercambiador de flujo cruzado con superficies extendidas tipo “plate-fin” para enfrentar el diseño, utilizando acero al carbono como material para los mismos. El modelo y los parámetros empleados para su simulación han sido extensamente desarrollados en la literatura específica, de donde se extrajo la información necesaria.

\section{Análisis y discusión del sistema combinado}

Se propusieron tres alternativas de diseño y operación del sistema combinado, con el fin de evaluar el impacto que causa la incorporación de una etapa previa de concentración al sistema de eliminación catalítica de VOCs, sobre los requerimientos de catalizador y superficie de intercambio. Para dicha evaluación se definió el concepto de factor de concentración, Fc, como la relación entre el caudal del efluente original a tratar y el alimentado al reactor. El análisis de los resultados indicó que, en el rango de valores factibles de alcanzar para el factor Fc (hasta seis), la disminución del volumen de lecho catalítico y de la superficie de intercambio es altamente significativa.

Analizando las masas de cada uno de los materiales en forma individual (i.e, catalizador, carbón activado y acero al carbono de los intercambiadores), se tuvo que, para un $\mathrm{Fc}=3.67$, se requiere solo del $10.7 \%$ de la masa de catalizador y $6.5 \%$ de la masa de acero al carbono (material de los intercambiadores) necesaria cuando no se incluye un sistema de adsorción/desorción $(\mathrm{Fc}=1)$. Si bien es cierto que para el caso de incluir un sistema de adsorción /desorción, se deben incorporar alrededor de 1750 kg de carbón activado, la cantidad de catalizador a emplear es bastante menor que para cuando $\mathrm{FC}=1$

Si bien no fue posible abordar un análisis económico del sistema debido a la falta de información de los costos comerciales de todos los materiales a emplear y los costos de manufactura de los equipos correspondientes, es posible discutir el beneficio económico obtenido a partir la fuerte disminución de los requerimientos de materiales.

Considerando que el catalizador empleado no es comercializado industrialmente, es factible pensar que su costo será bastante mayor que el costo comercial del carbón activado. Por lo tanto, la significativa reducción de material catalítico a emplear 
indicaría que la inclusión del sistema de adsorción/desorción implica un ahorro económico que puede ser importante. A medida que el grado de concentración de la corriente $(\mathrm{Fc})$ aumenta, mayor es el efecto que causa sobre el tamaño del reactor e intercambiadores y, por ende, mayor es el beneficio económico obtenido.

Para alcanzar mayores valores de Fc, es necesario aumentar la temperatura de la corriente empleada para la regeneración del adsorbedor. Sin embargo, al seleccionar la temperatura de desorción debe ser tenido en cuenta el efecto que este valor puede causar sobre el material adsorbente y el catalizador.

\section{Recomendaciones para trabajos futuros}

Se han identificado algunos aspectos que resultan importantes para el estudio de estos sistemas y que, aunque se encuentran fuera del alcance de este trabajo, constituyen recomendaciones para trabajos futuros.

- Las zeolitas pueden ser manufacturadas de manera tal de proveer una adsorción más eficiente que al emplear carbón activado. Esta característica puede resultar interesante si se pretende reducir el tamaño del adsorbedor. Aunque el costo original puede ser mayor que el del carbón activado, un mayor tiempo de recambio y la posibilidad de operar a una mayor temperatura en el proceso de desorción posicionan a las zeolitas como un material potencialmente competitivo con el carbón activado.

- En el caso de no emplearse un sistema de adsorción/desorción (Fc = 1), la utilización de otra configuración del reactor, como los monolitos, puede constituir una alternativa de interés para reducir la pérdida de carga y la cantidad de catalizador a emplear.

- Los intercambiadores de tipo regenerativos pueden ser potencialmente más eficientes que los recuperativos. Por lo tanto, el empleo de los mismos puede resultar una alternativa apropiada pese a su mayor complejidad de operación. Nuevamente, esta alternativa podría ser más atractiva cuando no se preconcentra la corriente y el requerimiento de área de intercambio es mucho mayor.

- Dado que cuando $\mathrm{Fc}_{\mathrm{C}}=1$, los requerimientos de catalizador y superficie de intercambiado resultaron ser bastante significativos, otra alternativa a considerar, es el empleo de lechos catalíticos con regeneración térmica por inversión de flujo.

- Al momento de seleccionar una de las alternativas de sistemas de eliminación de VOCs consideradas en este trabajo, es necesario disponer de los costos operativos y de 
inversión para poder discriminar de una manera cuantitativa. De esta manera, se podría obtener una optimización de las variables operativas y de los distintos esquemas de eliminación. 

ESTUDIO DE SISTEMAS

COMBINADOS DE

$\checkmark$ COMBUSTION

V catalitica de VOCs

nOMENCLATURA 

$\mathrm{a}_{\mathrm{v}}$ : área específica (área externa por unidad de volumen de sólido), $\mathrm{m}^{2} / \mathrm{m}^{3}$

$\mathrm{a}_{12}$ : espesor de placas planas del intercambiador, $\mathrm{m}$

$\mathrm{A}_{\mathrm{ef}}$ : $\quad$ área efectiva del intercambiador, $\mathrm{m}^{2}$

$\mathrm{A}_{\mathrm{f}}$ : $\quad$ área de las superficies extendidas del intercambiador, $\mathrm{m}^{2}$

$\mathrm{A}_{\mathrm{p}}$ : $\quad$ área total de placas del intercambiador, $\mathrm{m}^{2}$

$\mathrm{A}_{\mathrm{S} 1}, \mathrm{~A}_{\mathrm{S} 2}$ : área total de intercambio de cada corriente, $\mathrm{m}^{2}$

$\mathrm{A}_{\mathrm{T}}$ : $\quad$ área de transferencia de calor del intercambiador, $\mathrm{m}^{2}$

$\mathrm{A}_{\mathrm{TP}}$ : $\quad$ área de transferencia de placas planas, $\mathrm{m}^{2}$

$\mathrm{b}_{1}, \mathrm{~b}_{2}$ : altura de una fila del intercambiador incluyendo los espesores de placa plana y corrugada, $m$

$\mathrm{B}_{\mathrm{i}}$ : $\quad$ número de Biot para la transferencia de materia

$\mathrm{c}_{\mathrm{p}}$ : $\quad$ capacidad calorífica volumétrica, $\mathrm{J} / \mathrm{m}^{3}{ }^{\circ} \mathrm{C}$

$\hat{\mathrm{c}}_{\mathrm{p}}$ : $\quad$ capacidad calorífica másica, $\mathrm{J} / \mathrm{kg}{ }^{\circ} \mathrm{C}$

$\tilde{\mathrm{c}}_{\mathrm{p}}: \quad$ capacidad calorífica molar, $\mathrm{J} / \mathrm{mol}{ }^{\circ} \mathrm{C}$

C: $\quad$ concentración molar, $\mathrm{mol} / \mathrm{m}^{3}$

$\overline{\mathrm{C}}: \quad$ concentración media en el sólido, $\mathrm{mol} / \mathrm{m}^{3}$

$\mathrm{d}_{\mathrm{h}}$ : diámetro hidráulico, $\mathrm{m}$

$\mathrm{d}_{\mathrm{p}}$ : diámetro de pastilla, $\mathrm{m}$

D: diámetro de rotor, $m$

$D_{\mathrm{e}}$ : difusividad efectiva, $\mathrm{m}^{2} / \mathrm{s}$

$\mathrm{D}_{\mathrm{ex}}$ : diámetro externo del reactor radial, $\mathrm{m}$

$\mathrm{D}_{\text {in }}$ : diámetro interno del reactor radial, $\mathrm{m}$

$\mathrm{D}_{\mathrm{K}}$ : difusividad Knudsen, $\mathrm{m}^{2} / \mathrm{s}$

$\mathrm{D}_{\mathrm{m}}$ : $\quad$ coeficiente de difusión molecular, $\mathrm{m}^{2} / \mathrm{s}$

E: energía de activación, $\mathrm{J} / \mathrm{mol}$

$f$ : $\quad$ factor de fricción en el intercambiador

F: $\quad$ caudal molar, $\mathrm{mol} / \mathrm{m}^{3}$

Fc: $\quad$ Factor de concentración 
$h_{p, 1}, h_{p, 2}$ : coeficientes de transferencia de calor efectivos de cada corriente, $\mathrm{J} / \mathrm{m}^{2} \mathrm{~s}{ }^{\circ} \mathrm{C}$

$\mathrm{h}_{\mathrm{t}}$ : $\quad$ coeficiente de transferencia de calor fluido-sólido, $\mathrm{J} / \mathrm{m}^{2} \mathrm{~s}{ }^{\circ} \mathrm{C}$

$\mathrm{h}_{12}$ : altura de cada canal del intercambiador, $\mathrm{m}$

$\mathrm{H}: \quad$ altura del reactor, $\mathrm{m}$

$\mathrm{H}_{12}$ : altura del intercambiador, $\mathrm{m}$

$\mathrm{J}_{\mathrm{i}}$ : $\quad$ relación entre calor de adsorción y capacidad calorífica $\left(-\Delta \mathrm{H}_{\mathrm{i}} / \mathrm{c}_{\mathrm{p}, \mathrm{g}}\right),{ }^{\circ} \mathrm{C}$

$\mathrm{k}$ : $\quad$ constante específica de velocidad de reacción, $\mathrm{mol} / \mathrm{s} \mathrm{m}^{3}$

$\mathrm{k}_{\mathrm{B}}$ : $\quad$ constante de Boltzman $\left(1,38.10^{-6} \mathrm{erg} / \mathrm{K}\right)$

$\mathrm{k}_{\mathrm{f}, 12}$ : conductividad del material de las placas corrugadas del intercambiador, $\mathrm{J} / \mathrm{m} \mathrm{s}^{\circ} \mathrm{C}$

$\mathrm{k}_{\mathrm{m}}$ : coeficiente de transferencia de materia fluido-sólido, $\mathrm{m} / \mathrm{s}$

$\mathrm{k}_{\mathrm{p}, 12}$ : conductividad del material de las placas planas del intercambiador, $\mathrm{J} / \mathrm{m} \mathrm{s}^{\circ} \mathrm{C}$

$\mathrm{k}_{\text {ref: }} \quad$ constante específica de velocidad de reacción evaluada a la temperatura $\mathrm{T}_{\text {ref, }}$, $\mathrm{mol} / \mathrm{s} \mathrm{m}^{3}$

$\mathrm{K}: \quad$ constante de equilibrio de adsorción, $\mathrm{m}^{3} / \mathrm{mol}$

$\mathrm{K}^{*}: \quad$ coeficiente global de transferencia de materia, $1 / \mathrm{s}$

$\mathrm{K}_{\mathrm{T}}^{*}$ : coeficiente global de transferencia de calor, $1 / \mathrm{s}$

$\mathrm{K}_{\mathrm{v}}$ : $\quad$ coeficiente global de transferencia de materia por unidad de volumen de lecho $\left(\mathrm{K}^{*}\left(1-\varepsilon_{\mathrm{L}}\right)\right), 1 / \mathrm{s}$

L: $\quad$ longitud del adsorbedor, $\mathrm{m}$

$L_{c}: \quad$ longitud de la arista del canal cuadrado del monolito, $\mathrm{m}$

$\mathrm{L}(\mathrm{u})$ : polinomios interpolatorio de Lagrange para el conjunto de nodos $\{\mathrm{u}\}$

$\mathrm{L}_{2}$ : $\quad$ ancho del intercambiador para la corriente " 2 " y profundidad para la corriente "1", m

$\ell_{e}: \quad$ longitud de difusión efectiva en el sólido (longitud característica), $\mathrm{m}$

$\mathrm{M}: \quad$ peso molecular, $\mathrm{kg} / \mathrm{mol}$

$\mathrm{m}: \quad$ masa, $\mathrm{kg}$

$\mathrm{n}_{\mathrm{c}}$ : número de canales por unidad de superficie transversal total del monolito, canales $/ \mathrm{m}^{2}$

$\mathrm{n}_{\mathrm{p} 1}, \mathrm{n}_{\mathrm{p} 2}$ : número de canales por fila del intercambiador

$\mathrm{N}_{\text {canales }}^{\text {des }}$ : número de canales del monolito correspondientes a la etapa de desorción

$\mathrm{N}_{\text {canales }}^{t}:$ número total de canales del monolito 
$\mathrm{NF}_{12}$ : número de filas para cada corriente en el intercambiador

$\mathrm{N}_{\mathrm{t}}$ : número de tanques agitados en serie

Nu: $\quad$ número de Nusselt

P: $\quad$ presión, atm, $\mathrm{kg} / \mathrm{m} \mathrm{s}^{2}$

$\mathrm{P}_{\mathrm{c}}$ : $\quad$ presión crítica, $\mathrm{kg} / \mathrm{m} \mathrm{s}^{2}$

Pe: número de Peclet

$\mathrm{P}_{\mathrm{N}}$ : polinomio de Jacobi

q: $\quad$ moles adsorbidos por unidad de volumen de adsorbente, $\mathrm{mol} / \mathrm{m}^{3}$

Q: $\quad$ caudal volumétrico, $\mathrm{m}^{3} / \mathrm{s}$

$Q: \quad$ cantidad de saturación del componente a adsorber por unidad de volumen de adsorbente, $\mathrm{mol} / \mathrm{m}^{3}$

$Q_{\text {int }}$ : calor intercambiado, $\mathrm{J} / \mathrm{s}$

$\mathrm{r}: \quad$ radio del reactor, $\mathrm{m}$

$\mathrm{r}^{\mathrm{ef}}: \quad$ velocidad de consumo efectiva, $\mathrm{mol} / \mathrm{s} \mathrm{m}^{3}$

$\mathrm{r}_{\mathrm{p}}$ : $\quad$ adio de la pastilla, $\mathrm{m}$

$\mathrm{r}_{\text {poro }}$ : radio de poro, $\mathrm{m}$

$\mathrm{r}^{\mathrm{s}}$ : $\quad$ velocidad de consumo evaluada en la superficie del sólido, $\mathrm{mol} / \mathrm{s} \mathrm{m}^{3}$

$r$ : de reacción intrínseca, $\mathrm{mol} / \mathrm{s} \mathrm{m}^{3}$

$\mathrm{R}: \quad$ constante universal de los gases, $\mathrm{J} /{ }^{\circ} \mathrm{C}$ mol

$\mathrm{R}_{\mathrm{c}}$ : $\quad$ radio de curvatura del canal del monolito, $\mathrm{m}$

Re: número de Reynolds

$\mathrm{R}_{\mathrm{in}}$ : $\quad$ radio interno del reactor radial, $\mathrm{m}$

Rf: factor de pérdida de carga del monolito (régimen laminar)

$R_{f}$ : $\quad$ resistencia de ensuciamiento del intercambiador, $\mathrm{m}^{2} \mathrm{~s}{ }^{\circ} \mathrm{C} / \mathrm{J}$

$\mathrm{s}_{12}$ : ancho de cada canal del intercambiador, $\mathrm{m}$

S: $\quad$ sección transversal, $\mathrm{m}^{2}$

Sc: $\quad$ número de Schmidt

$\mathrm{S}_{\mathrm{g}}: \quad$ superficie específica, $\mathrm{m}^{2} / \mathrm{kg}$

Sh: $\quad$ número de Sheerwood

$\mathrm{t}: \quad$ tiempo, $\mathrm{s}$

$t: \quad$ espesor del tabique del monolito, $\mathrm{m}$

T: $\quad$ temperatura absoluta, ${ }^{\circ} \mathrm{C}$ o K

$\mathrm{T}_{\mathrm{c}}$ : $\quad$ temperatura crítica, $\mathrm{K}$

$\mathrm{T}_{\mathrm{r}}: \quad$ temperatura reducida 


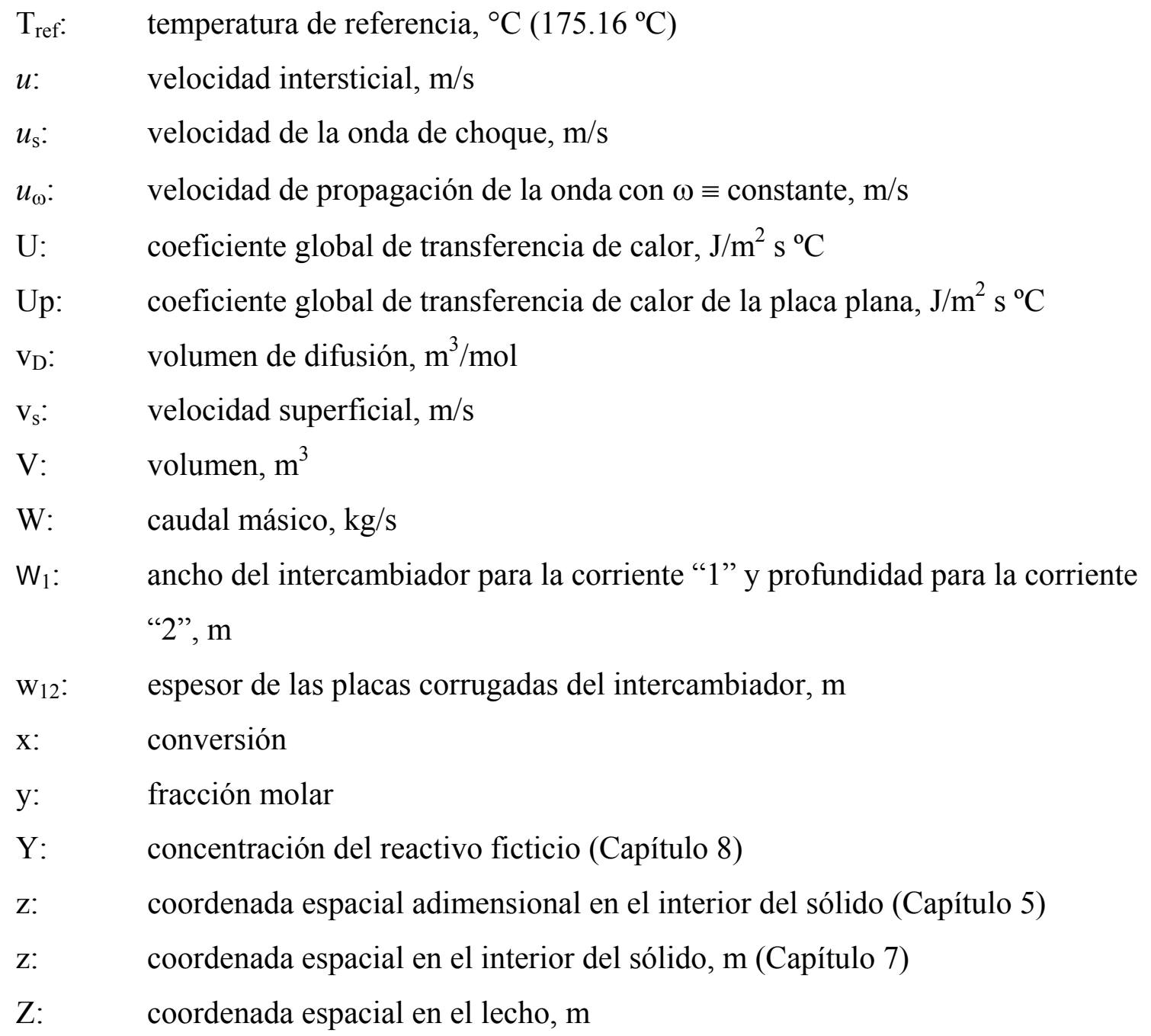

\section{Letras Griegas}

$\gamma: \quad$ relación entre capacidades caloríficas $\left(\mathrm{c}_{\mathrm{p}, \mathrm{p}} / \mathrm{c}_{\mathrm{p}, \mathrm{g}}\right)$

$\gamma^{*}: \quad$ coeficiente que define la geometría del material adsorbente

$\delta$ : $\quad$ factor de dilución del reactor

$\Delta \mathrm{H}: \quad$ calor adsorción o calor de reacción (combustión), $\mathrm{J} / \mathrm{mol}$

$\Delta \mathrm{P}: \quad$ pérdida de carga, $\mathrm{Pa} \mathrm{o}$ atm

$\Delta \mathrm{P}_{\mathrm{c}}$ : pérdida de carga por fricción en los tramos rectos de cada canal del intercambiador, $\mathrm{Pa}$ o atm

$\Delta$ Pe: $\quad$ pérdida de carga en la entrada del intercambiador (contracción abrupta), $\mathrm{Pa}$ o atm

$\Delta$ Ps: pérdida de carga en la salida del intercambiador (expansión abrupta), $\mathrm{Pa}$ o atm

$\Delta \mathrm{Z}_{\mathrm{M}}: \quad$ longitud de unidad monolítica, $\mathrm{m}$ 
$\varepsilon_{\mathrm{L}}: \quad$ porosidad del lecho

$\varepsilon_{\mathrm{p}}: \quad$ porosidad del sólido

$\varepsilon_{\text {real }}$ : eficiencia real del intercambiador

$\varepsilon_{\text {req. }}: \quad$ eficiencia requerida del intercambiador

$\zeta_{\mathrm{CO}_{2}}: \quad$ producción de $\mathrm{CO}_{2},\left(\mathrm{y}_{\mathrm{CO} 2} / \mathrm{y}_{\mathrm{CO} 2}^{\max }\right)$

$\eta: \quad$ factor de efectividad

$\eta_{t}$ : $\quad$ eficiencia de la superficie extendida del intercambiador

$\theta_{\text {ads }}$ ángulo destinado al proceso de adsorción en el rotor

$\theta_{\text {des: }} \quad$ ángulo destinado al proceso de desorción en el rotor

$\lambda$ : $\quad$ conductividad térmica, $\mathrm{J} / \mathrm{m} \mathrm{s}^{\circ} \mathrm{C}$

$\mu: \quad$ viscosidad, $\mathrm{kg} / \mathrm{m} \mathrm{s}$

$\rho: \quad$ coordenada radial, $\mathrm{m}$

$\rho: \quad$ densidad, $\mathrm{kg} / \mathrm{m}^{3}$

$\rho_{\text {pap }}: \quad$ densidad aparente del sólido, $\mathrm{kg} / \mathrm{m}^{3}$

$\sigma: \quad$ autovalor(de la matriz $\mathcal{F}$, Capítulo 4)

$\tau: \quad$ factor de tortuosidad

v: relación volumétrica entre las fases sólida y fluido en función de la porosidad, $\left(1-\varepsilon_{\mathrm{L}}\right) / \varepsilon_{\mathrm{L}}$

$\Phi: \quad$ módulo de Thiele

$\omega: \quad$ factores de peso (Capítulo 5)

$\Gamma: \quad$ curva en el espacio de las variables $C_{i}(i=1, \ldots, m+1)$

\section{Subíndices:}

ads: $\quad$ adsorbedor / adsorbente / adsorción

c: $\quad$ corriente fría

cat: catalizador

des: desorbedor / desorción

exp: experimental

g: $\quad$ fase gaseosa

h: corriente caliente

macro: macroporos

mod: modelo 


$\begin{array}{ll}\text { p: } & \text { fase sólida } \\ \text { s: } & \text { superficie del sólido } \\ \text { T: } & \text { total } \\ \text { 1: } & \text { acetato de etilo } \\ \text { 2: } & \text { etanol } \\ \text { 3: } & \text { acetaldehído }\end{array}$

\section{Superíndices:}

S: $\quad$ salida

0: $\quad$ entrada / inicial (Capítulo 4)

F: $\quad$ alimentación (Capítulo 4)

mín: mínimo

máx: máximo 


ESTUDIO DE SISTEMAS

COMBINADOS DE

COMBUSTION

$\checkmark$ CATALITICA DE VOCs

BiblografiA 



\section{Bibliografía}

Abdullah A.Z., Bakar M.Z.A., Bhatia S., A kinetic study of catalytic combustion of ethyl acetate and benzene in air stream over Cr-ZSM-5 catalyst, Ind. Eng. Chem. Res. 42, 6059-6067 (2003).

Agüero F.N., Barbero B.P., Gambaro L., Cadús L.E., Catalytic combustion of volatile organic compounds in binary mixtures over $\mathrm{MnOx} / \mathrm{Al}_{2} \mathrm{O}_{3}$ catalyst, Applied Catalysis B: Environmental 91, 108-112 (2009).

Ambrozek B., Theoretical studies of volatile organic compounds (VOCs) recovery from waste air streams in cyclic thermal swing adsorption (TSA) system with fixed bed of polymeric adsorbent, Paper 1379, Proc. of CHISA $2004-16^{\text {th }}$ Int. Congress of Chemical and Process Eng., Praga, Zchec Republic (2004).

Antos D., Kaczmarski K., Wojciech P., Seidel-Morgenstern A., Concentration dependence of lumped mass transfer coefficients. Linear versus non-linear chromatography and isocratic versus gradient operation, J. of Chromatography A 1006, 61-76 (2003).

Barreto G.F., Reactores/Catalizadores Estructurados, (2007).

Bathen D., Physical waves in adsorption technology-an overview, Separation and Purification Technology 33, 163-177 (2003).

Bhatia S., Abdullah A.Z., Wong C.T., Adsorption of butyl acetate in air over silverloaded Y and ZSM-5 zeolites: experimental and modeling studies, J. of Hazardous Materials 163, 73-81 (2009).

Bhatti M.S., Shah R.K., Laminar convective heat transfer in ducts, in Handbook of Single-Phase Convective Heat Transfer, Kakac S., Shah R.K., Aung W., Eds., Wiley, New York, Chapter 3 (1987).

Boger T., Heibel A.K., Sorensen C.M., Monolithic Catalysts for the Chemical Industry, Ind. Eng. Chem. Res. 43, 4602-4611 (2004).

Bressa S.P., Mariani N.J., Ardiaca N.O., Mazza G.D., Martínez O.M., Barreto G.F., An algorithm for evaluating reaction rates of catalytic reaction networks with strong diffusion limitations, Comp. Chem. Eng. 25, 1185-1198 (2001).

Brosillon S., Manero M-H., Foussard J-N., Mass transfer in VOC adsorption on zeolita: experimental and theoretical breakthrough curves, Environ. Sci. Technol. 35, 3571-3575 (2001).

Buzek L., Wamuzinski K., Tanczyk M., Januz-Cygan A., Cost analysis for the removal of volatile organic compounds from air using hybrid systems: membrane separation/condensation versus membrane separation/combustion, Chem. Eng,. Process. 38, 273-279 (1999). 
Campesi M.A., Estudio de reactores catalíticos para la eliminación de VOCs en una corriente gaseosa, Tesis del Trabajo Final de la Carrera Ing. Química (F.I.U.N.L.P), La Plata, 2005.

Campesi M.A., Mariani N.J., Martínez O.M., Barreto G.F., Eliminación de VOCs mediante un proceso de oxidación catalítica con recuperación térmica, Ing. Mecánica, Tecnología y Desarrollo 2 (4), 111-119 (2007).

Campesi M.A., Mariani N.J., Pramparo M.C., Barbero B., Cadús L., Martínez O.M., Barreto G.F., Combustion of volatile organic compounds on a $\mathrm{MnCu}$ catalyst, Catalysis Today 176 (1), 225-228 (2011).

Campesi M.A., Mariani N.J., Bressa S.P., Pramparo M.C., Barbero B., Cadús L., Martinez O.M., Barreto G.F., Kinetic study of the combustion of ethanol and ethyl acetate mixtures over a $\mathrm{MnCu}$ catalyst, Fuel Processing Technology 103, 84-90 (2012a).

Campesi A., Luzi C.D., Martínez O.M., Barreto G., Effect of concentration by thermal swing adsorption on the catalytic incineration of VOCs, International Journal of Chemical Reactor Engineering 10 (1), Art.A54 (2012b). DOI: $\underline{10.1515 / 1542-6580.3026}$

Carsten K.W.M., Prins R., Sorption of volatile organic compounds on hydrophobic zeolites, Microporous and Mesoporous Materials 35-36, 349-365 (2000).

Çengel Y.A., Boles M.A., Termodinámica, McGraw-Hill, 2da Ed., (1996).

Chang F-T., Lin Y-C., Bai H., Pei BS., Adsorption and desorption characteristics of semiconductor volatile organic compounds on the thermal swing honeycomb zeolite concentrator, J. of Air \& Waste Management Association 53, 1384-1390 (2003).

Chi He, Peng Li, Jie Cheng, Zheng-Ping Hao, Zhi-Ping Xu, A comprehensive study of deep catalytic oxidation of benzene, toluene, ethyl acetate, and their mixtures over Pd/ZSM-5 catalyst: Mutual effects and kinetics, Water Air Soil Pollut 209, 365-376 (2010).

Cho S.J., Ryoo M.W., Song K.S., Lee J.H., Kang S.K., A catalytic combustion technology of concentrated VOCs in textile coating process, Korean J. Chem. Eng. 16(4), 478-481 (1999).

Choung J-H., Lee Y-W., Choi D-K., Adsorption equilibria of toluene on polymeric adsorbents, J. Chem. Eng. Data 46, 954-958 (2001).

Crittenden B., Patton A., Juin C., Perera S., Tennison S., Botas Echevarría J.A., Carbon monoliths: a comparison with granular materials, Adsorption 11, 537-541 (2005).

Dangi S., Abraham M.A., Kinetics and modeling of mixture effects during complete catalytic oxidation of benzene and methyl tert-butyl ether, Ind. Eng. Chem. Res. 36, 1979-1988 (1997). 
de la Fuente García D., Gutiérrez Martín F., Optimización de sistemas de destrucción de VOCs, Ingeniería Química, 151-158 (1998).

Delimaris D., Ioannides T., VOC oxidation over $\mathrm{MnOx}-\mathrm{CeO}_{2}$ catalysts prepared by a combustion method, Appl. Catal. B:Environ. 84, 303-312 (2008).

Delimaris D., Ioannides T., VOC oxidation over $\mathrm{CuO}-\mathrm{CeO}_{2}$ catalysts prepared by a combustion method, Appl. Catal. B:Environ. 89, 295-302 (2009).

EPA - Choosing an adsorption system for VOC: Carbon, zeolite, or polymers, Technical Bulletin EPA, 456/F-99-004 (1999).

http://www.epa.gov/ttn/catc/dir1/fadsorb.pdf

Ergun S., Flow through Packed Columns, Chemical Engineering Progress 48 (2), 89 (1952).

Everaert K., Baeyens J., Creemers C., A comparative study of abatement techniques for volatile organic compounds (VOCs), Proc. of CHISA 2002 - 15th Int. Congress of Chemical and Process Eng., Praga, Zchec Republic (2002).

Everaert K., Baeyens J., Catalytic combustion of volatile organic compounds, J. of Hazardous Materials B109, 113-139, (2004).

Friday D.K., Le Van Mahle, J.J. Buettner L.C., PSA for air purification: experiments and modeling, Studies in Surface Science and Catalysis 80, 169-176 (1993).

Froment G.F., Bischoff K.B., Chemical Reactor Analysis and Design, $2^{\text {nd }}$ Edition, John Wiley \& Sons (1990).

Fuller E. N., Schettler P.D, Giddings J.C., A new method for prediction of binary gas-phase diffusion coefficients, Industrial and Engineering Chemistry 58 (5), 18-27 (1966).

Gadkaree K. P., Carbon honeycomb structures for adsorption applications, Carbon 36 (7-8), 981-989 (1998).

Gales L., Mendes A., Costa C., Hysteresis in the cyclic adsorption of acetone, ethanol and ethyl acetate on activated carbon, Carbon 38, 1083-1088, (2000).

Gales L., Mendes A., Costa C., Recovery of acetone, ethyl acetate and ethanol by thermal pressure swing adsorption, Chemical Engineering Science 58, 5279-5289, (2003).

Gandhe A.R., Rebello J.S., Figueiredo J.L., Fernandes J.B., Manganeso oxide OMS2 as an effective catalyst for total oxidation of ethyl acetate, Applied Catálisis B: Environmental 72, 129-135 (2007).

Ghoshal A.K., Manjare S.D., Selection of appropriate adsorption technique for recovery of VOCs: an analysis, Journal of Loss Prevention in the Process Industries 15, 413-421 (2002). 
Gnielinski G., New equation for heat and mass transfer in turbulent pipe and channel flow, Int. Chem. Eng. 16, 359-368 (1976).

Greene H.L., Combined sorbent/catalyst system, U.S.Patent 5.414.201 (1995).

Gunn D. J., Transfer of heat or mass to particles in fixed and fluidized beds, International J. of Heat and Mass Transfer 21 (4), 467-476 (1978).

Hewitt G.F., Shires G.L., Bott T.R., Process heat transfer, CRC Press (1994).

Hodgson A.T., Destaillats H., Sullivan D.P., Fisk. W.J., Performance of ultraviolet photocatalytic oxidation for indoor air cleaning applications, Indoor Air 17, 305316 (2007).

Huang Z-H., Kang. F., Liang K-M., Hao J., Breakthrough of methyethylketone and benzene vapors in activated carbon fiber beds, J. of Hazardous Materials 98, 107115 (2003).

Idriss H., Seebauer E.G., Reactions of ethanol over metal oxides, J. of Molecular Catalysis A: Chemical 152, 201-212 (2000).

Incropera F.P., de Witt D.P., Fundamentals of Heat and Mass Transfer, $3^{\text {ra }}$ Ed., Wiley (1990)

Irusta S., Pina M.P., Menéndez M., Santamaría J., Catalytic combustion of volatile organic compounds over La-based perovskites, J. of Catal. 179, 400-412 (1998).

Jacoby W.A., Blake D.M., Fennell J.A., Boulter J.E., Vargo L.M., George M.C., Dolberg S.K., Heterogenous photocatalysis for control of volatile organic compounds in indoor air, J. Air \& Waste Manag. 46, 891-898 (1996).

Kaskantzis Neto G, Moura J.C., Parametric evaluation of VOC conversion via catalytic incineration, Braz. J. Chem. Eng. 14 (2), (1997).

$<$ http://www.scielo.br/scielo.php?script=sci_arttext\&pid=S0104-

66321997000200006\&lng=en\&nrm=iso>

Khan F.I., Goshal A.Kr., Removal of Volatile Organic Compounds from polluted air, Journal of Loss Prevention in the Process Industries 13, 527-545 (2000).

Kim S.C., Shim W.G., Catalytic combustion of VOCs over a series of manganese oxide catalysts, Applied Catalysis B: Environmental 98, 180-185 (2010).

Ko D., Kim M., Moon I., Choi D-k, Analysis of purge gas temperature in cyclic TSA process. Chemical Engineering Science 57, 179-195 (2002).

Kolaczkowski S., Treatment of Volatile Organic Carbon Emissions from Stationary Sources: Catalytic Oxidation of the Gaseous Phase, Chap. 5 in Structured Catalysts and Reactors, $2^{\text {nd }}$ Edition, A. Cybulski and J. A. Moulijn Eds., 2006, Taylor and Francis. 
Lahousse C., Bernier A. Grange P., Delmon B., Papaefthimiou P., Ioannides T., Verykios X., Evaluation of $\gamma-\mathrm{MnO}_{2}$ as a VOC Removal Catalyst: Comparison with a Noble Metal Catalyst, J. of Catalysis 178, 214-225 (1998).

Larsson P.O., Andersson A., Complete oxidation of CO, ethanol, and ethyl acetate over copper oxide supported on titania and ceria modified titania, Journal of Catalysis 179 (1), 72-89 (1998).

Larsson P-O., Andersson A., Oxides of copper, ceria promoted copper, manganese and copper manganese on $\mathrm{Al}_{2} \mathrm{O}_{3}$ for the combustion of $\mathrm{CO}$, ethyl acetate and ethanol, Appl. Catal. B: Environ. 24, 175-192 (2000).

Leson G., Winer A.M., Biofiltartion: an innovative air pollution control Technology for VOC emissions, J. Air Waste Manage. Assoc., 41 (8), 1045-1054 (1991).

Lewandowski, D.A., Design of Thermal Oxidation Systems for Volatile Organic Compounds, CRC Press (2000).

Lintz H-G., Wittstock K., The oxidation of solvents in air on oxidic catalyst formation of intermediates and reaction network, Applied Catalysis A: General 216, 217-225 (2001).

López E., Estudio de Reactores Monolíticos Autotérmicos para la Generación Primaria de Calor, Tesis Doctoral en Ingeniería Química, Universidad Nacional del Sur (2002).

Manjare S.D., Ghoshal A.K., Studies on Dynamic Adsorption Behaviour of Ethyl Acetate from Air on $5 A$ and $13 X$ Molecular Sieves, The Canadian Journal of Chemical Engineering 83 (2), 232-241 (2005).

Mariani N.J., Martínez O.M., Pramparo M.C., Barbero B., Cadús L., Barreto G.F., Una alternativa simple para analizar el comportamiento térmico de reactores catalíticos de lecho fijo escala laboratorio, $5^{\circ}$ Congresso de Catálise do Mercosul, Río de Janeiro, Brasil, paper 01143 (2009).

Matros Yu. Sh., Bunimovich G. A., Control of volatile organic compounds by the catalytic reverse process, Ind. Eng. Chem. Res. 34, 1630-1640 (1995).

Matros Yu. Sh., Bunimovich G. A., Strots V. O., Mirosh E. A., Reversed flow converter for emission control after automotive engines. Chemical Engineering Science 54, 2889-2898 (1999).

Mears D.E., Tests for Transport Limitation in Experimental Catalytic Reactors, Ind. Eng. Chem. Proc. Des. Dev. 10, 541, (1971).

Morales M.R., Barbero, B.P, Cadús, L.E., Evaluation and characterization of $\mathrm{Mn-}$ $\mathrm{Cu}$ mixed oxide catalysts for ethanol total oxidation: Influence of copper content, Fuel 87, 1177-1186 (2008).

Moreno-Castilla C., Pérez-Cadenas A.F., Carbon-based honeycomb monoliths for environmental gas-phase applications, Materials 3, 1203-1227 (2010). 
Moretti E. C., Reduce VOC and HAP Emissions, Chem. Eng. Prog. 98, 30-40 (2002).

Ogulata R.T., Doba F.,Yilmaz T., Second-law and experimental analysis of a crossflow heat exchanger, Heat Transfer Eng. 20, 20-27 (1999).

Oyama S.T., Li W., Zhang W., A comparative study of ethanol oxidation with ozone on supported molybdenum and manganese oxide catalysts, Studies in Surface Science and Catalysis 121, 105-110 (1999).

Pan H., Xu M., Li Z., Huang S., He C., Catalytic combustion of styrene over copper based catalyst: Inhibitory effect of water vapor, Chemosphere, 76, 721-726 (2009).

Papaefthimiou P., Ioannides T., Verykios X.E., Combustion of non-halogenated volatile organic compounds over group VIII metal catalysts, Appl. Cat. B: Env. 13, 175-184 (1997).

Papaefthimiou P., Ioannides T., Verykios X.E., VOC removal: investigation of ethylacetate oxidation over supported Pt catalysts, Catalysis Today 54, 81-92 (1999).

Passalia C., Alfano O.M., Brandi R.J., A methodology for modeling photocatalytic reactors for indoor pollution control using previously estimated kinetic parameters, J. Hazardous Material 211-212, 357-365 (2012).

Patton A., Crittenden B.D., Perera S.P., Use of the linear driving force approximation to guide the design of monolithic adsorbents, Chem. Eng. Res. Des. 82, 999-1009 (2004).

Perry R.H. y Green D. W., Perry's Chemical Engineer's Handbook, McGraw-Hill, 7ma Ed. (1999).

Picon-Nuñez M., Polley G.T., Torres-Reyes E., Gallegos-Muñoz A., Surface selection and design of plate-fin heat exchangers, Applied Thermal Eng. 19, 917931 (1999).

Poling B. E., Prausnitz J. M., O'Connell J.P., The Properties of Gases and Liquids, $5^{\text {th }}$ Edition, McGraw-Hill, New York (2001).

Pramparo M.C., Estudio de sistemas de destrucción catalítica de compuestos orgánicos volátiles, Doctoral Thesis, U.N.L.P., Argentina (2010).

Rajesh H., Ozkan U.S., Complete oxidation of ethanol, acetaldehyde, and ethanol/methanol mixtures over copper oxide and copper-chromium oxide catalysts, Ind. Eng. Chem. Res. 32, 1622-1630 (1993).

Rase H.F., Fixed-bed reactor design and diagnostics: gas-phase reactions, Butterworth Publishers (USA) (1990).

Ray M.B., Photodegradation of the Volatile Organic Compounds in the Gas Phase: A Review, Developments in Chemical Engineering and Mineral Processing 8, 405439 (2000). 
Rene E.R., Veiga M.C., Kennes C., Combined biological and physicochemical waste-gas cleaning techniques, Journal of Environmental Science and Health, Part A 47, 920-939 (2012).

Rezaei F., Webley P., Structured adsorbents in gas separation processes, Separation and Purification Technology 70, 243-256 (2010).

Rhee H., Amundson N. R., An Analisis of an Adiabatic Adsorption Column: Part 1. Theorical Development, Chemical Engineering Journal 1 (3), 241-254 (1970a).

Rhee H., Heerdt, E. D., Amundson N. R., An Analisis of an Adiabatic Adsorption Column: Part II. Adiabatic adsorption of a single solute, Chemical Engineering Journal 1(4), 279-290 (1970b).

Rhee H., Heerdt, E. D., Amundson N. R., An Analisis of an Adiabatic Adsorption Column: Part III: Adiabatic Adsorption of two Solutes, Chemical Engineering Journal 3, 22-34 (1972).

Ritter J.A., Yang R.T., Pressure swing adsorption: experimental and theoretical study on air purification and vapor recovery, Ind. Eng. Chem. Res. 30 (5), 10231032 (1991).

Ritter J.A., Liu Y., Tapered pressure swing adsorption columns for simultaneous air purification and solvent vapor recovery, Ind. Eng. Chem. Res. 37 (7), 2783-2791 (1998).

Ruthven D. M., Principles of Adsorption and Adsorption Processes, John Wiley \& Sons, (1984).

Santos V.P., Pereira M.F.R., Órfão J.J.M., Figueiredo J.L., Mixture effects during the oxidation of toluene, ethyl acetate and ethanol over a cryptomelane catalyst, J. of Hazardous Material 185, 1236-1240 (2011).

Serth, R.W., Process heat transfer. Principles and applications, Elsevier (2007).

Shah R.K., Mueller A.C., Heat Exchangers, Capítulo 4 de Handbook of Heat Transfer Applications, 2da Ed., Rohsenow W.M., Harnett J.P., Ganic E.N., Eds. Mc Graw Hill (1985).

Shah R.K., Thonon B., Benforado D.M., Opportunities for heat exchanger applications in environmental systems, Applied Thermal Engineering 20, 631-650 (2000).

Shah R.K., Sekulic D.P., Fundamentals of Heat Exchanger Design, Ed. John Wiley \& Sons (2003).

Shepherd P.E., Activated carbon adsorption for treatment of VOC emissions, Paper presented at the $13^{\text {th }}$ Annual EnviroExpo, Boston Massachusetts (2001). 
Shiraishi F., Yamaguchi S., Ohbuchi Y., A rapid treatment of formaldehyde in a highly tight room using a photocatalytic reactor combined with a continuous adsorption and desorption apparatus, Chemical Engineering Science 58, 929-934 (2003).

Shiraishi F., Nomura T., Yamaguchi S., Ohbuchi Y., Rapid removal of trace HCHO from indoor air by an air purifier consisting of a continuous concentrator and photocatalytic reactor and its computer simulation, Chemical Engineering J. 127, 157-165 (2007).

Shiraishi F., Ishimatsu T., Toluene removal from indoor air using a miniaturized photocatalytic air purifier including a preceding adsorption/desorption unit, Chemical Engineering Science 64, 2466-2472 (2009).

Sramek M., Ditl P., Neumanová E., Gas purification by combined method adsorption - catalytic oxidation, Proceeding of the 16th International Congress of Chemical and Process Engineering (CHISA), Paper N\# 0580, editado en CD, (2004).

Stewart W.E., Caracotsios M., Sørensen J.P., Parameter estimation from multiresponse data, A.I.Ch.E. J. 38, 641-650 (1992).

Taborek J., Design method for tube-side laminar and transition flow regime with effects of natural convection, Paper presented in the Open Forum at the $9^{\text {th }}$ Int. Heat Transfer Conf., Jerusalem, Israel (1990).

Van de Beld B., Borman R.A., Derkx O.R., Van Woezik B.A.A., Westerterp K.R., Removal of volatile organic compounds from polluted air in a reverse flow reactor: an experimental study, Ind. Eng. Chem. Res. 33, 2946-2956 (1994).

Villadsen J., Michelsen M. L., Solution of Differential Equation Models by Polynomial Approximation, Prentice - Hall (1978).

Weisz P.B., Prater C.D., Interpretation of Measurement in Experimental Catalysis, Adv. Catal. 6, 143 (1954).

Winitzki S., Uniform approximations for transcendental functions, Proc. ICCSA, LNCS 2667/2003, 962 (2003).

Winitzky S., A handy approximation for the error function and its inverse, on line (2008). http://en.wikipedia.org/wiki/Error_function

Yang R. T., Gas Separation by Adsorption Processes, Imperial College Press (1997).

Yang R. T., Adsorbents: Fundamentals and Applications, John Wiley \& Sons (2003).

Yates M., Blanco J., Avila P., Martin M. P., Honeycomb monoliths of activated carbons for effluent gas purification, Microporous and Mesoporous Materials 37, 201-208 (2000). 
